MWT $\backsim \cup 1992 \quad$ ILLINOIS HISTORY

LBRaAl

SUR VEY

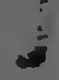

A Nomenclator of Leptosphaeria V. Cesati \& G. de Notaris

(Mycota-Ascomycotina-Loculoascomycetes)

J.L. Crane and C.A. Shearer

Illinois Natural History Survey Bulletin

Volume 34, Article 3

March 1991 

ILLINOIS

NATURAL

HISTORY

S UR VEY

\section{A Nomenclator of Leptosphaeria V. Cesati \& G. de Notaris (Mycota-Ascomycotina-Loculoascomycetes)}

\section{J.L. Crane}

Center for Biodiversity

Illinois Natural History Survey

\section{C.A. Shearer}

Department of Plant Biology

University of Illinois at Urbana-Champaign

Illinois Natural Hislory Survey Bulletin

Volume 34, Article 3

March 1991 
Illinois Natural History Survey, Lorin I. Nevling. Chief

A Division of the lllinois Department of Energy and Natural Resources

A catalog of the publications of the Illinojs Natural History Survey is available without charge from the address below. A price list and an order blank are included with the catalog.

Illinois Natural History Survey

Distribution Center

Natural Resources Building

607 East Peabody Drive

Champaign, Illinois 61820

Citation:

Crane, J.L., and Shearer, C.A. 1991. A Nomenclator of Leprosphaeria V. Cesati \& G. de Notaris (Mycota-Ascomycotina-Loculoascomycetes). Illinois Natural History Survey Bulletin 34(3):195-355.

Editor: John P. Ballenot

Designer: Gail Glende Rost

US ISSN 0073-4918

Printed by Authority of the State of Illinois (X1128I-MD-3-9l) 


\section{Contents}

Acknowledgments iv

Introduction and Historical Background 195

List of Leptosphaeria Species, Varieties, and Forms 198

Host Index 291

Host Family Index 308

Substrate Index 318

Geographic Index 328

Appendix 1. Taxonomic Division of Leptosphaeria 339

Appendix 2. Genera Historically Allied to Leptosphaeria

Appendix 3. Synonyms of Leptosphaeria 347

Appendix 4. Anamorphs of Leptosphaeria 348

Literature Cited 351 


\section{Acknowledgments}

We express sincere appreciation to our colleagues at the Illinois Natural History Survey and the University of Illinois at UrbanaChampaign who assisted us in the completion of this nomenclator. Carla G. Heister and Monica A. Lusk located many references in the University of Illinois library system from cryptic citations. Martha Landis is noted for her ability to locate references that cannot be found by others. Helen F. Sullivan and Susan Burke assisted us with many of the slavic references. Dr. Ken Robertson assisted with the verification of host nomenclature.

Mycological literature is so diverse that no one library can encompass it all. Therefore, we are especially indebted to Dr. S. Udagawa at the National Institute of Hygienic Sciences in Tokyo, Japan, who so kindly supplied copies of the Japanese papers. Dr. Robbin C. Moran at the Missouri Botanical Garden in St. Louis provided articles from journals not available in the University of Illinois library system. Drs. E. Müller of Switzerland, F.A. Stafleu of the Netherlands, J. Rammeloo of Belgium, P. Fanton of the University of Padua, Italy, Clark T. Rogerson and G.J. Samuels of the New York Botanical Garden, Bronx, and Richard P. Korf of Cornell University, Ithaca, New York, supplied copies of species descriptions from rare mycological books. journals, and exsiccati labels. We are most grateful to Drs. Lekh Batra and David Farr at the National Fungus Collections in Beltsville. Maryland, and to Drs. Donald Pfister and Jean Boise at the Farlow' Herbarium and Library of Harvard University in Cambridge, Massachusetts, for their kind hospitality during our visits, and for making available to us their rich mycological libraries and collections.

Several individuals helped us in a variety of ways, and without their willingness to contribute time and expertise this project would not have been completed. Betty A. Nelson typed the original manuscript and its several revisions and checked for inconsistencies in author and joumal citations. She and Patty L. Duzan spent many hours on computer-related problems in sorting this nomenclator. We also thank Sabine M. Huhndorf, who supplied several names of Leptosphaeria species.

We gratefully acknowledge Dr. Richard T. Hanlin of the University of Georgia. Athens. for critically reviewing the manuscript and John P. Ballenot for editing the manuscript. Support for this study was provided by the National Science Foundation. Systematic Biology Program, grant NSF-BSR-87-00065. 


\section{Introduction and Historical Background}

This nomenclator of Leprosphaeria is an index to names published in the genus through 1989 and includes the source of publication, nomenclatural history, substrate and/or host, and geographical location for each species name. Orthographic errors of specific epithets were corrected, with the original spelling given in brackets. Full names and initials of authorities are given. Standardized, complete names of periodicals are cited following the system of Brown and Stratton (1963). Porter and Koster (1970), Koster and Gascoigne (1971), and Smits (1968). The titles of books and pamphlets are cited according to Stafleu and Cowan (1976-1988). Obligate synonomy is indicated by the symbol $\equiv$. Articles of the International Code of Botanical Nomenclature cited in this work follow Greuter et al. (1988). Several authors have described new species in Leptosphaeria as trinomials in which the subgenus or group to which the new species belongs is indicated as a middle name in parentheses; an example is Leptosphaeria (Clypeosphaeria) hendersonice. Such trinomials are alphabetized by specific epithet; however, the group name precedes the epithet.

The scientific names of host plants are given as reported in the protologue of each Leptosphaeria species, with the currently accepted name in parentheses. Several host epithets reported in the original descriptions were never published or could not be verified. These are changed to "sp." Plant host families and genera were verified in Willis (1973), Farr et al. (1979), and Cronquist (1981). Specific epithets of vascular plants were confirmed in Halliday and Beadle (1983), Kartesz and Kartesz (1980), the Gray Herbarium Card Index (1894-present), and Index Kewensis (1895-present). Names of pteridophytes were substantiated in Christensen (1905-1906), and names of mosses, in Wijk et al. (1959-1960). Lichenized fungi names follow Zahlbruckner (1921-1940) and Lamb (1963), and fungal host names were verified in Saccardo (1882-1931) and the Index of Fungi (1920-1987). For convenience, Leptosphaeria species reported from Algae, Fungi, Lichenes, and Musci are listed under these headings and their respective hosts in the host index. When available, specific substrate information, such as leaf, stem, water, etc., is given.

The geographical location of species is usually described by country and is based on information in the protologue and new combinations. Geographical names were verified in Seltzer (1952).

Cesati and de Notaris (1863) established the genus Leptosphaeria and included 26 species; among these was Leptosphaeria hira (G.L. Rabenhorst) V. Cesati \& G. de Notaris, the type species of an earlier genus. Nodulosphaeria G.L. Rabenhorst, 1858. Leptosphaeria was conserved against Nodulosphaeria with Leptosphaeria doliolum (C.H. Persoon:E.M. Fries) V. Cesati \& G. de Notaris as the type species (Greuter et al. 1988). The original description of Leptosphaeria was superficial by modern taxonomic standards, and the genus was delimited largely by ascospore characteristics. The ascospore characteristics selected (oblong or fusoid, two- to manycelled, hyaline, becoming yellowish or dark brown) and the poor characterization of other structural features resulted in the inclusion of a wide range of Ascomycetes in this genus.

Leptosphaeria now comprises approximately 1.689 taxa. These taxa represent, according to present-day concepts of ascomycete classification, a melange of Euascomycetes and Loculoascomycetes.

Few mycologists have attempted to deal with the systematics of Leprosphaeria. Saccardo $(1878,1883,1891,1895,1899,1913$, 1928) recognized 800 species. which he grouped primarily according to host (parasites of dicotyledons, monocotyledons, and cryptogams) and habit (species on stems and/or branches and leaves). Species were further subdivided. based on the external features of 
the pseudothecium (glabrous, hairy, setose) and ascospore septation. Saccardo's concept of Leprosphaeria was broad, and his emended description specified the presence of pseudoparaphyses (as paraphyses).

Höhnel (1907a) was the first to use information on centrum structure for the classification of Leptosphaeria-like fungi. He established the family Pseudosphaeriaceae for species in which the asci grow up into a cellular tissue that occupies the inner space of the fruiting body. As the asci develop, the cellular tissue becomes compressed and at fruitingbody maturity simulates paraphyses similar to those found in the Sordariales (Pyrenomycetes). In the Sordariales, however, the paraphyses originate prior to the asci, and the asci grow up among them. Höhnel included four genera in the Pseudosphaeriaceae [Psewdosphaeria F. v. Höhnel, Pyrenophora F. v. Höhnel, Scleroplea (P.A. Saccardo) C.A. Oudemans, and Wettsteinina F. v. Höhnel]. Later, Höhnel (1918a, 1918b) divided Leptosphaeria into three genera based on centrum structure (Leptosphaeria, Scleropleella F. v. Höhnel, and Nodulosphacria G.L. Rabenhorst) (Appendix 1). Petrak (1923) subsequently provided evidence that Leptosphaeria is related to Wettsteinina and belongs in the Pseudosphaeriaceae.

Wehmeyer (1942) reported the occurrence of 13 species of Leptosphaeria from North America and described two new species. He noted, "Many of the species show minor differences of spore structure, olten correlated with the host. In the descriptive literature. these details are not always given. Large numbers of species have been described, largely on host distinction. on the one hand, whereas many host varieties have been obscured by inclusion in one of the ubiquitous species on the other hand. As a result it is difficult to be sure of species determinations without a comparative study of the entire genus." This statement remains valid today. Wehmeyer (1946) also redescribed seven species of Leprosphoria and discussed evolutionary trends within the genus. He noted that 382 species have the 3 -septate type of ascospore and believed that the 3-septate, dark brown spore type is the primitive state. According to Welmeyer, the spores of more recently evolved species are lighter in color, longer, narrower, and more septate. These trends lead directly, without a break. to Ophiobolus H. Riess.

Müller (1950), circumscribing Leptosphaeria broadly, considered 114 species occurring in Switzerland. Within his broad generic concept, Müller divided Leptosphaeria into four sections (Appendix I). These secrions were circumscribed based on pseudothecial and centrum structure and ascospore morphology:

Three of his sections correspond, in part, to the three groups of Höhnel (Appendix 1).

Munk (1957) accepted Müller"s sections with certain reservations and divided Leptosphaeria into four somewhat different sections (Appendix 1). Sections I (Eu-Leptosphaeria). III (Scleropleella), and IV (Nodulosphaeria)which correspond to Müller"s Sections II. I. and $I V$, respectively - were more restricted than those of Müller. The remaining species of Leptosphaeria were treated in Section II (ParaLeptosphaeria).

Holm (1957), in a treatment of 62 Swedish species of Leptosphaeria, rejected Müller's broad concept of Leprosphaeria and limited the genus to those species most closely. related to Leptosphactia doliolum. the type of the genus (Appendix 1). Excluded species were distributed primarily in Nodulosphaeria H. Riess, Phaeosphacria I. Miyake. and Entodesmium H. Riess. Holm placed much emphasis on substrata and relation of the pseudothecium to the substratum. Holm's disbursement of Leptosphaeria species was rejected by both Dennis (1978) and Sivanesan (1984) but was accepted by Hedjaroude (1969). v. Arx and Müller (1975). Eriksson (1967). and Shoemaker (1984).

In the past 50 years, there have been a large number of intergeneric transfers of Leptosphaeria species. Many of these transfers have come about by the partitioning of species groups into new (Paraphacosphacria $\mathrm{O}$. Eriksson) or existing (Entodesmium H. Riess and Phacosphueria I. Miyake) genera. Other species have been transferred to genera very similar to and integrating with Leprosphaeria (Lidophia J.C. Walker \& B.C. Sutton. Massaria G. de Notaris. Massarima P.A. Saccardo.

Melanomma T.R.J. Nitschke ex L. Fuchel. Ophiobolus H. Riess, and Wetssteinina F. r. Höhnel). Descriptions of related generat and a synopsis of their relationships to Lepesphlaeria 
are summarized in Appendix 2. Genera synonymous with Leprosphaeria are listed in Appendix 3.

In recent years, Leptosphaeria has been included in two orders in the Bitunicatae or Loculoascomycetes. Luttrell (1973) placed Leptosphaeria in the Pleosporales under the Pleosporaceae. Von Arx and Müller (1975) retained Leptosphaeria in the Pleosporaceae under the Dothideales. Hawksworth et al. ( 1983 ) placed Leptosphaeria in the Dothideales in either the Phaeosphaeriaceae or the Pleosporaceae. Eriksson and Hawksworth (1986) classified the genus in Dothideales under Phaeosphaeriaceae. Most recently, Barr (1987a) has placed Leptosphaeria in the
Leptosphatriaceae of the Pleosporales in the Loculoascomycetes.

One of the most intriguing aspects of Leprosphacria is the diversity of anamorphic states attributed to this genus. Anamorphs of Leptosphaeria have been demonstrated for 70 species, and most of these associated ananorphs are Coelomycetes (Appendix 4). The largest numbers of Leptosphaeria associations are with Phoma P.A. Saccardo and Siagonospora (P.A. Saccardo) P.A. Saccardo.

It is intended that this nomenclator wil] be the base for a reappraisal of Leptosphaeria using modern concepts and techniques applicable to Loculoascomycetes. 


\section{List of Leptosphaeria Species, Varieties, and Forms}

ahbreviata (M.C. Cooke) P.A. Saccardo, Sylloge Fungorum Omnium Hucusque Cognitorum Digessit P.A. Saccardo 2:26. 1883.

Esphaeria abhreviata M.C. Cooke. Handbook of British Fungi, p. 893. 1871. On dead stems of Ruhus sp., Rosaceae. Great Britain.

ahuensis K.S. Panwar \& S.J. Kaur, Kavaka, Madras 3:67-68. 1975. On dead wood of unknown host, unknown family. India.

ahutilonis M. Chochrjakov in V. Tranzschel, L. Gutner, and M. Chochrjakov. Trudȳ Instituta Novogo Lubyanogo Sỹr ya. Moskva 4:133. 1933. On leaves of Abutilon avicemae Gaentner, Malvaceae. U.S.S.R.

ahutilonis L.E. Wehmeyer \& S. Ahmad, Biologia. Biological Society of Pakistan, Lahore 10:12. 1964. Nom, illegit. Ant. 64.1. On unknown substrate of Abutilon indicum (L.) Sweet, Malvaceae. Pakistan.

acamthi N.T. Patouillard, Revue Mycologique, Toulouse 8:181. 1886. On dead stems of Adhatoda sp. Acanthaceae. China.

aceris N.N. Woronichin, Vestnik Tiflisskogo Botanicheskogo Sada. Tiflis (Moniteur du Jardin Botanique de Tiflis) 35:5. 1914. On living leaves of Acel laetum C.A. Mey.. Aceraceae. U.S.S.R. (Caucasus).

acheniarum K. Starbäck [see Leprosphaeria agnita var. acheniarmm K. Starbäck].

achilleae (B. Auerswald) V. Cesati \& G. de Notaris. Commentario della Società Crittogamologica Italiana, Milan 1:236. 1863. ESphaeria achilleae B. Auerswald in G.L. Rabenhorst. Klotzschii Herbarium Vivum Mycologicum Sistens Fungorum Per Totan Germaniam Cresentium Collectionem Perfectam. Dresden. Edition 1, Century 15, No. 1448. Anno 1850. On stems of Achillea millefolinm auct., Compositae. Germany.

acicola (L. Fuckel) P.A. Saccardo, Sylloge Fungorum Omnium Hucusque Cognitorum Digessit P.A. Saccardo 2:55. 1883. EPleospora? acicola L. Fuckel, Symbolae Mycologicae, Erster Nachtrag, p. 301. 1871 On fallen needles of Pimis syliesmis L.. Pinaceae. Germany. acomiri P.A. Saccardo, Nuovo Giomale Botanico Italiano e Bolletino della Società Botanica
Italiana, Firenze 7:315. 1875. On dry stems of Aconitum napellus L., Ranunculaceae. Italy.

(Mefasphaeria) acarella M.C. Cooke, Grevillea, London 13:99. 1885. 三Metasphaeria acorella (M.C. Cooke) A.N. Berlese \& P. Voglino. Sylloge Fungorum Omnium Hucusque Cognitorum Digessit P.A. Saccardo Additamenta Ad Volumina I-IV.p. 158. I886. On leaves of Acorus calamus L., Araceae. Great Britain.

acori P.A. Karsten. Hedwigia, Dresden 22:179. 1883. [Ad interim.] Nom. inval. Art. 34.1. On decaying leaves of Acorus calamus L.. Araceae. Finland.

aculeorum G. Passerini. Atti della R. Accademia dei Lincei Memoriae. Rome. Series 4, 6:458-459. (1889) 1890. On stems of Rosa canina L.. Rosaceae. Italy.

acuta H. Rehm, Ascomyceten. Fascicle 16. No. 783. Anno 1884. Nom. illegit. An. 64.1. 三Leprosphaeria acusiuscula A.N. Berlese.

acula (G.F. Hoffmann:E. M. Fries) P.A. Karsten. Mycologia Fennica Pars 2. Pyrenomycetes. p. 98. 1873. ESphaeria acnia G.F. Hoffmann:E. M. Fries. Vegetabilia Cryptogama. Fascicle 1. p. 22. 1787: E.M. Fries. Systema Mycologicum Sistens Fungorum 2:507. 1823. EPleospora acura (G.F. Hoffmann:E.M. Fries) L. Fuckel. Symbolae Mycologicae. p. 135.1870. EAmpullina acura (G.F. Hoffmann:E.M. Fries) L. Quélet. Mémoires de la Société d'Émulation de Montbéliard. Series 2. No. 5. pp. 523-524. 1875; Les Champignons du Jura et des Vosges 3:95. 1875. On fallen stems of Urica dioica L.. Urticaceae. Austria, Belgium, Finland, France, Gemmany, Great Britain. ltaly, U.S.A.

acuta (G.F. Hoffmann:E.M. Fries) P.A. Karsten forma insignis F. Fautrey in C. Rouneguère. Fungi Selecti Gallici Exsiccati, Century 72. No. 7137. Anno 1897: Revue Mycologique. Toulouse 19:149. 1897. On dry. deconicated stems of Urica dicica L., Urticaceae. France.

acula (G.F. Hofmann:E.M. Fries) P.A. Karsten forma micue F. Fautrey in C. Rouncguère. Revue Mycologique. Toulouse 16:7. 1894: 
Fungi Selecti Gallici Exsiccati. Century 65. No. 6434. Anno 1894. On diseased stalks of Urrica dioica L.. Unicaceae. France. acurispora S. Tóth, Omagiu Lui Traian Săvulescu cu Prilejul Implinirii A 70 De Ani. Academia Republicii Populare Romane, Bucharest. p. 778. 1959. On dry stems of Erysimum diffusum Ehrh., Erysimum erysimoides (L.) Fritsch ex Janchen. Sisvmbrimm strictissimm L. Crucilerae. Romania.

acutiuscula A.N. Berlese, Icones Fungorum

Omnium Hucusque Cognitorum ad usum Sylloges Saccardianae Adcommodatae 1:86. 189.4. ELeprosphaeria acura H. Rehm. On stems of Urrica sp., Urticaceae. Gernany. adesmicola (C.L. Spegazzini) L. Holm, Svensk Botanisk Tidskrilt, Stockholm 62:224-225. 1968. EGibberidea adesmicola C.L.

Spegazzini. Anales del Museo Nacional de Historia Natural de Buenos Aires 19(Series 3, 12):385. 1909. On dead branches of Adesmics sp. Leguminosae. Argentina.

adiermla (W. Nylander) P.A. Saccardo \& D.

Saccardo in P.A. Saccardo, Sylloge Fungorum Omnium Hucusque Cognitorum Digessit P.A. Saccardo 17:731. 1905. On thallus of Lecided excentrica Roehling, Lichenes (Lecideaceac). Ireland. Italy.

degira P.A. Saccardo \& C.L. Spegazzini in P.A. Saccardo. Michelia Commentarium Mycologicum Fungos in Primis Itilicos Illustrans 1:37. 1877. On wilting leaves ol'Populus alba L.. Salicaceae. Italy.

ucharopolis A.1. Lobik. Materialȳ po Floristicheskim i Faunisticheskim Obsledovaniyam Terskogo Okruga. pp. 22-23. 1928 [as achropi]. On leaves of Achropus limoralis (Gouan.) Parl.. Gramineae. U.S.S.R.

aerea C.L. Spegazzini. Anales de la Sociedad Cientifica Argentina. Buenos Aires 12:178-179. 1881. Fungi Argentini Pugillus 4. No. 166. 1881. ESphuerulina (Lepeosp)/uerella) acrea (C.L. Spegazıini) M.C. Cooke, Grevillea, London 18:80. 1890. =P/cospora pellira (E.M. Fries) G.L. Rahenhorst var. pellita, Fide L.E. Wehmeyer, A World Monograph of Pleospora. p. 47. 1961. On branches and dead leaves of Ervhrina cristagalli L.. Tillandsia bicolor Brongn., Leguminosie. Bromeliaceae. Argentina.

aemensis G. Scalia, Prima Contribuzione allat Conoscenza delle Flora Micologica della Provincia di Catania. p. 16. 1899. On dry branches of Similax aspera L.. Smilacaceac. Italy.

affinis P.A. Karsten, Fungi Fenniae Exsiccati. Century 9. No. 896. Anno 1869. 三Mef(1.5pluaria affinis (P.A. Karsten) P’.A. Saccardo. Sylloge Fungorum Omnium Hucusque Cognitorum Diggesit P.A. Saccardo 2:159.
1883. On deud stems of Rhinamhus $\mathrm{sp}$. Scrophulariaceae. Finland.

africana A.M. Saccas, Étude de la Flore Crylogil mique des Cafëiers en Afrique Centrale.

Bulletin de Institut Français du Café du Cacao et d'Autres Plantes Stimulantes (Bulletin IFCC No. 16), pp. 219-221. 1981. Nom. inval. Art. 37.1. On dead branches of Coffea robusta L. Linden (=Coffea canephora Pierre ex Froehn.). Rubiaceae. South Africa.

agraces H. Sydow, P. Sydow, \& E.J. Butler, Annales Mycologici, Berlin 9:409. 1911. On wilted leaves of Agave rigida Mill. var, sisalama (Perrine) Engelm., Agavaceae. India.

aglaja P.A. Saccardo. Nuovo Giomale Botanico Italiano e Bolletino della Societa Botanica Italiana, Firenze 7:310. 1875. On leaves of Oralis sricta L.. Oxalidaceae. Italy.

agminalis P.A. Saccardo \& P. Morthier in P.A. Saccardo. Michelia Commentarium Mycologicum Fungos in Primis Italicos Illustrans 1:498-499. 1879. DDothideopsella ugminalis (P.A. Saccardo \& P. Morthier) F. v. Höhnel, Sitzungsberichte der Akademie der Wissenschalten in Wien, Mathematischnatturwissenschaltiche Klasse. Abt. I, 124(1 \& 2):70. 1915. On stems of Clematis vitalbes L.. Ranunculaceac. France, Italy.

agminulis P.A. Saccardo \& P. Morthier forma minos. F. Fautrey in C. Roumeguère, Revue Mycologique. Toulouse 13:168. 1891; Fungi Selecti Gillici Exsiccati, Century 59. No. 5849. Anno 1891. On unknown substrate of Clematis vitulba L., Ranunculaceac. France.

agnita (J. Desmazières) V. Cesati \& G. de Notaris, Commentario della Società Crittogamologica Italiana, Milan 1:236. 1863. 三Splueria (Carlicola) agnita J. Desmazières. Annales des Sciences Naturelles, Paris, Botanique, Series 3. 16:313.1851. On stems of Valeriana officinalis L., Valerianaceae. Italy.

asnita (J. Desmazières) V. Cesati \& G. de Notaris subsp. lebens: P.A. Saccardo \& G. Scalia in P.A. Saccardo, C.H. Peck, and W. Trelease, Hartinan Alanka Expedition 5:330. 1904. On dead herbaceous stems of unhnown host. U.S.A.

agnikn (J. Desmazières) V. Cesati \& G. de Notaris var. achemarmm K. Starbäck. Arkiv för Bolanik, Uppsala, Stockholm 5(7):23. 1905. (Raised to species rank: Leptosplicaria achenicarum K. Starbick in reprint on same page.| On dry achenes of Mikania sp.. Compositas. Bolivial.

agnitu P.A. Saccardo var. ambigua A.N. Berlese, Atti dell Accalemia Scientifica VenetoTrentino-Istriana, Padova, Series 1 , 9:247-248. 1886: Ricerche intorno alla Leprospharia aguita (Desm.) Ces. et de Not.. ed allat L. "gilviensis (B. et Br.) Ces. el de 
Not., pp. 4-5. 1886. On dry stems of Eupatorium cannabinum L., Compositae. Germany.

agnita (J. Desmazières) V. Cesati \& G. de Notaris var. bupleuri P.A. Saccardo, Bulletin. Société R. de Botanique de Belgique, Bruxelles 28:91 1889. On dead stems of Bupleurum falcantum L., Umbelliferac. U.S.S.R.

agnita P.A. Saccardo var. chrysanthemi A.N. Berlese. Atti dell' Accademia Scientifica Veneto-Trentino-Istriana, Padova, Series 1, 9:246-247. 1886; Ricerche intorno alla Leptosphacria agnita (Desm.) Ces. et de Not.. ed alla Leptosphaeria ogiliensis (B. et $\mathrm{Br}$.) Ces. et de Not., pp. 3-4. 1886. On dry stems of Cluysumthemum corymbosum L., Compositae. Germany.

agnita (J. Desmazières) V. Cesati \& G. de Notaris var. crigerontis A.N. Berlese, Atti dell Accademia Scientifica Veneto-TrentinoIstriana, Padova, Series 1, 9:247. 1886; Ricerche intorno alla Leptosphaeria agnita (Desm.) Ces. et de Not., ed alla Leptosphaeria ogilviensis (B. et Br.) Ces. et de Not., p. 4. 1886. On dead stems of Erigeron canadensis L.. Compositae. U.S.A.

agnita (J. Desmazières) V. Cesati \& G. de Notaris var. major P.A. Saccardo \& A.N. Berlese, Atti del Istituto Veneto di Scienze, Lettere ed Arti. Venezia, Series 6, 3:730. 1885. On stems of Chacrophyllum aureum L., Umbelliferac. Switzerland.

agnita P.A. Saccardo var. major A.N. Berlese, Atti dell' Accademia Scientifica Veneto-TrentinoIstriana, Padova. Series 1, 9:247. 1886: Ricerche intorno alla Leptosphacria agnita (Desm.) Ces. et de Not. ed alla Leprosphacria ogilviensis (B. et Br.) Ces. et de Not.. p. 4. 1886. On dry stems of Chaerophy/lmm anreum L., Umbelliferae. Germany.

agnita (J. Desmazières) V. Cesati \& G. de Notaris var. "rifolii R. González Fragoso. Memórias de la R. Sociedad Española de Historia Natural. Madrid 11:90. 1919. On dry stems of Trifolium angustifolium L., Leguminosite. Spain.

ahmadii F. Petrak, Sydowia. Annales Mycologici, Horn, N.Ö. 8:167. 1954. On dry branches of Capparis apliylla Roth. Capparaceate. Pakistan.

ailamthi P.A. Karsten \& P.A. Hariot, Joumal de Botanique, Paris 3:206. 1889. On branches of Ailamhus glandulosa Dest., Simaroubaceae. France.

akagicusis E.A. Vainio, Botanical Magazine, Tokyo 35:79. 1921. On tree bark of unknown hosi. unknown lamily. Japan.

alboplanctala (G.D. Westendorp) J.B. Ellis d B.M. Everhart, The North American Pytenomycetes. A Contribution to Mycologic Botany, p. 375. 1892. ESphaeria albopunciata G.D. Westendorp.

alhopunctaia (G.D. Westendorp) P.A. Saccardo, Sylloge Fungorum Omnium Hucusque Cognitorum Digessit P.A. Saccardo 2:72.

1883. ESphueria alhopunctata G.D.

Westendorp. Bulletins de L'Académie Royale des Sciences. des Letres et des Beaux-Arts de Belgique, Brussels, Series 2, 7:87-88. 1859. =Heptameria (Lepiosphaeria) alhopunciata (G.D. Westendorp) M.C. Cooke, Grevillea. London 17:32. 1889. EPhaeosphaeria alhopunctata (G.D. Westendorp) R.A. Shoemaker, Canadian Journal of Botany. Ottawa 67:1566. 1989. On seeds of Phragnites commmnis Trin., Gramineae. Belgium.

alhulae E. Müller, Sydowia. Annales Mycologici. Horn, N.Ö. 4(1-6):281. 1950. On dead stems of Lonicera alpigena L., Caprifoliaceae. Switzerland.

(Leptosphaerella) alcides P.A. Saccardo, Nuovo Giornale Botanico ltaliano e Bolletino della Società Botanica Italiana. Firenze 8:176. 1876. On underside of leaves of Populus alba L., Salicaceae. Italy.

ulcides P.A. Saccardo forma quercina R. Cifenri. Annales Mycologici. Berlin 20:51. 1922. On dry leaves of Quercus rohur L.. Fagaceae. Italy.

alexandrinis A. Negru, Mycopathologia et Mýcologia Applicata. Den Haag 33:365-366. 1967. On leaves of Cucumis sariuss L.. Cucurbitaceae. Romania.

algarbicnsis M.T. Lucas. Memórias da Sociedade Broteriana. Coimbra 21:34. 1970. On dead leaves of Chamuerops humilus L.. Palmae. Portugal.

algida E. Rostrup. Meddelelser om Gronland. Kjobenhavn 3:558. 1888. On leaves of Catabrosa algida Fr.. Gramineae. Greenland.

alhagii I.P. Frolov, Novosti Sistematiki Nizshikh Rastenij. Novitates Systematicae Plantarum Non Vascularium 7:185-186. 1970. On dry branches of Alhagi sp.. Leguminosac. U.S.S.R.

alhaginis I.P. Frolov in E.N. Koschkelova, 1.P. Frolov, and Z. Dzhuraeva. Mikoflora Badkhyza, Karabilya i Yuzhnoi Chasti Murgahskogo Oazisa (Mikromitsety) [The Mycotlora of Badkhyz. Karabil and the Southern Part of the Murgab Oasis (Micromy(etes)|. P. \$6. 1970. Nom. nud. Art. 32.1. ELeptosphacria alhagii 1.P. Frolos.

aliena H. Sydow. Annales Mlycologici. Berlin 35:28-30. 1937. On kaves and culms of Cladimu articulatum R. Br.. Cyperaceae. Austratia (Neu South Wales).

alliariac (L. Fuckel) J. Schröter in F.J. Colnn. Kryptogamen-Flora Von Schlesien. Im Namen Der Selhlesischen Gesellschaft fiir 
vaterländische Cultur herausgegeben von Prof. Dr. Ferdinand Cohn, Secretair der Botanischen Section, Breslatu 3(2):364. 1894. $\equiv$ Splaeria aliriae L. Fuckel. Symbolac Mycologicae, p. 135. 1870, sub. Pleospora maculans. On stems of Alliaria officimalis Andrz ex Bieh., Cruciferae. Poland.

alliariae (B. Auerswald) H. Rehm. Ascomycelen, Fascicle 14. No. 686. Anno 1882; Hedwigia, Dresden 22:56. 1883. ESphaeria alliariae B. Auerswald in G.L. Rabenhorst, Fungi Europaei Exsiccati, Klotzschii Herbarii vivi Mycologici Continuatio, Edition 3, Century 3. No. 261. Anno 1860. On dry stems of Sisymbrium alliaria (L.) Scop., Cruciferae. Germany.

alliariae (B. Auerswald) G. Linhart, Fungi Hungarici Exsiccati [Magyarorszang Gombai], Century 2. No. 164. Anno 1883. Nom. illegit. Art. 64.1. ESphaeria alliariae B. Auerswald in G.L. Rabenhorst, Fungi Europaei Exsiccati. Klotzschii Herbarii vivi Mycologici Continuatio, Edition 3, Century 3. No. 261. Anno 1860. On dry stems of Brassica campestris L.. Cruciferac. Germany, Hungary.

allorgei A.L. Guyot, Revue de Mycologie, Paris 14:69. 1949. On dry stems of /satis tincturia L.. Cruciferae. France.

almeidae M. de Sousa da Camara. Revista Agronómica. Lisbon, No. 1:11-12. 1928. [From reprint.] On leaves of Cimamommm zeylanicum Blume, Lauraceac. Portugal. almeidana M. de Sousa da Camara. Boletim de Agricultura. Lisboa 2(1):14. 1936. [Pagination on reprint, p. 21.] On branchlets of Pinecenectria sp.. Agavaceate. Portugal. aloes M.R. Sousa de Dias \& M. de Sousa da Camara, Agronomia Lusitana, Sacavém 16:8. 1954. On leaves of Aloe arhorescens Miller. Liliaceae. Portugal.

alopecuri N.N. Lavrov. Trudȳ Tomskogo Gosudarstvennogo Universiteta. Tomsk. Series Biologicheskii 1 10:76. 1951. On dead leaves of Alopecmus borealis Trin., Gramineate. U.S.S.R.

alpiniae A. Maublanc, Bulletin de la Société Mycologique de France, Paris 21:89. 1905. On leaves of Alpinia speciosa K. Sch., Zingiberaceac. Brazil.

allaica A. Nannizzi, Atli dell' Accademia dej Fisiocritici di Siena, Series 10, 3:961. 1928. On dried sheaths of leaves on Polygonmm historla L.. Polygonaceae. Yugoslavia.

alvariensis K. Starbaick. Bihang till K. Svenska Vetenskapsakademiens Handlingar, Stockholm. Series 3. 15(2):28. 1889. [Raised to specific rank in legend of Figure 4.] $\equiv$ Leplosphaeria vagabunda P.A. Saccardo subsp. alvariensis K. Starbäck. ambiens H. Rehm, Philippine Journal of Science. Manila. Section C, 8:257. 1913. On dead branches of unknown host. unknown family. Philippines.

ammophilae' (W.G. Lasch) V. Cesati \& G. de Notaris. Commentario della Societa Crittogamologica Italiana. Milan 1:236. 1863. Ephuaria ammophilac W.G. Lasch in G.L. Rabenhorst, Klolzschii Herbarium Vivum Mycologicum Sistens Fungorum Per Totan Germaniam Cresentium Collectionem Perfectam, Dresden, Edition 1, Century 14. No. 1340. Anno 1850; Flora, Jena und Regensburg 8:282. 1850; Botanische Zeitung, Berlin \& Leipzig 8:439. 1850.

EPhacosphaeria ammophilae (W.G. Lasch) J. Kohlmeyer \& E. Kohlmeyer, Icones Fungorum Maris Liel. 3, Table 55. 1965. 三Amarenomyes ammophilae (W.G. Lasch)

O. Eriksson, Nordic Journal of Botany, Copenhagen [Opera Botanica] 60:124. 1981. On leaves of Ammopthila arenaria (L.) Link, Elytrigia juncea (L.) Nevski. Gramineae. Denmark, Finland, Germany, Netherlands, U.S.S.R.

ammophilae H. Rehm ex H. Rehm, Ascomyceten, Fascicle 14. No.691. Anno 1882. Nom. nud. 32.1 [as (Lasch) Cesati \& de Notaris]: Hedwigia. Dresden 22:57. 1883. Non Leprosphacria ammophilae (W.G. Lasch) V. Cesati \& G. de Notaris. On dry leaves of Ammophika arenaria (L.) Link. Gramineae. Germany.

ammophilae H. Rehm, lalsely so cited in A.N. Berlese and P. Voglino, Sylloge Fungorum Omnium Hucusque Cognitorum Digessit P.A. Saccardo Additamenta Ad Voluminal I-IV, p. 139. 1886; P.A. Saccardo, Sylloge Fungorum Omnium Hucusque Cognitorum Digessit P.A. Saccardo 9:790. 1891. ELeptosphaeria ammophilae (W.G. Lasch) V. Cesati \& G. de Notaris.

ammothamni E.N. Koschkelova in E.N. Koschkelovit, I.P. Frolov, and Z. Dzhuraeva. Mikoflora Badkhyza, Karabilya i Yuzhnoŏ Chasti Murgabskogo Oalzisa (Mikromitsety) [The Mycoflora of Badkhyz, Karabil and the Southern Part of the Murgab Oasis (Micronyceles)1, p. 87. 1970. Nom. nud. Ant. 32.1. On dry branches of Ammothammus lehmamii Bunge, I,eguminosac. U.S.S.R. (Badkhyza, Karabilial).

ammothammi E.N. Koschkelova, Novosti Sistematiki Nizshikh Rastenij. Novitates Systematicac Plantarum Non Vascularium 7:176-177. 1970. On dry branches of Ammothammis lelmannii Bunge. leguminosae. U.S.S.R. amorphac E. Mitroshina, Notulae Systematicate Sectione Cryptoganica Instituti Botanici Nomine V.L. Komarov Academiate Sciential- 
rum U.R.S.S.. Petropolis 6:82. 1949. On dead branches of Amorpla fruticosa L.. Leguminosae. U.S.S.R.

ampelina M. Curzi \& M. Barbaini, Atti dell" Istituto Botanico della Università e Laboratorio Crittogamica di Pavia, Milano, Series 3. 3:160. 1927. On dry vines of Viris vinifera L.. Vitaceae. lialy.

amplibola P.A. Saccardo. Nuovo Giomale Botanico Italiano e Bolletino della Società Botanica Italiana, Firenze 7:322. 1875. ELeprosphaeria culmifiaga P.A. Saccardo non (E.M. Fries) V. Cesati \& G. de Notaris. On stems of Sorghum lulgare Pers. Gramineae. Italy.

amphiloga F. Petrak in H. Sydow and F. Petrak.

Annales Mycologici, Berlin 29:202. 1931. On dead culms of Bambusa sp., Gramineae. Philippines.

anacycli R. González Fragoso, Asociación Española Para el Progresso de las Ciencias, Congreso de Coimbra 1925:11-12. 1925. Nom. inval. Art. 34.1. On dry stems of Anacylus radiams Loisel., Compositae. Portugal.

anarithma (M.J. Berkeley \& C.E. Broome?) P.A. Saccardo, Michelia Commentarium Mycologicum Fungos in Primis Italicos Illustrans 1:395. 1878: Fungi Italici autographice delineati (additis nonnullis extra-italicis asterisco notatis), Patavii, Table 400. 1878. $\equiv$ Splaceria (Folicolac) anarithma M.J. Berkeley \& C.E. Broome, Annals and Magazine of Natural History. London. Series 3. 3:374. 1859. ESphaerella anarihma (M.J. Berkeley \& C.E. Broome) M.C. Cooke, Handbook of British Fungi. p. 920. 1871. ESphacrella anarithma P.A. Saccardo, Nuovo Giomale Botanico Italiano e Bolletino della Società Botanica Italiana. Firenze 8:175. 1876 [as (B. \& Br.) Cooke]. =Metasphacria amarithma (M.J. Berkeley \& C.E. Broome) P.A. Saccardo, Sylloge Fungorum Omnium Hucusque Cognitorum Digessit P.A. Saccardo 2:175. 1883. This new combination or new species is based on material collected on dried leaves of Aira cespitosa L. from Montello (Treviso), September 1873, and may be identical to the species described by MI.J. Berkeley and C.E. Broome, also on Aira cespirosa from Batheaston. March 1853. and Bowood. October 1853. On dried leaves of Aira cespirosa L., Gramineate. Great Britain. Italy.

anarillmoides P.A. Saccardo \& C.L. Spegazzini in P.A. Saccardo. Michelia Commentarium Mycologicum Fungos in Primis Italicos Illustrans 1:395. 1878. EMetusphaeriu amarirhmoides (P.A. Salcardo \& C.L.

Spegazzini) P.A. Saccardo, Sylloge Fungorum Omnium Hucusque Cognitorum Digessit P.A. Saccardo 2:175. 1883. On decaying leaves of Portsp.? Gramincace. Italy. anarrhini R. González Fragoso. Boletim da Sociedade Broteriana. Coimbra. Series 2. 2:22-23. 1923. On dry stems of Anarrhinum bellidifolium (L.) Willd., Scrophulariaceae. Portugal.

anceps P.A. Saccardo. Michelia Commentarium Mycologicum Fungos in Primis ltalicos Illustrans 1:120.1878. On not yet dead branches of Ribes nigrum L.. Saxifragaceae. Germany.

andrijevicensis F. Bubák. Bulletin de l'Herbier Boissier. Genève and Bâle. Series 2. 6:400-401. 1906. On old stems of Ranunculus villarsii DC.. Ranunculaceae. Yugoslavia. andromedae (B. Auerswald) P.A. Saccardo. Sylloge

Fungorum Omnium Hucusque Cognitorum

Digessit P.A. Saccardo 2:49-50. 1883.

ESphaerella andromedae B. Auerswald in W.

Gonnermann and G.L. Rabenhorst. Mycologia

Europaea, Abbildungen Sämmtlicher Pilze

Europa 's. Hefte 5 und 6. Synopsis Pyrenomycetum Europaeorum, p. 12. 1869 [as Spuerella]. On dry leaves of Andromeda terragona L.. Ericaceae. Canada, Norway

anemones L. Hollós. Annales Historico-Naturales

Musei Nationalis Hungarici, Budapest $4: 334$.

1906. [Anamorph: Rhahdospora anemones L. Hollós.] On dry petioles of Anemone syliestris L.. Ranunculaceae. Hungary.

(misomeres L.E. Wehmeyer. Canadian Jourmal of

Research. Ottawa 20(Section C):582. 1942.

On unknown substrate of Agropiron repens

(L.) Beauv., Gramineae. Canada (Nova Scotia).

anomala J.B. Ellis \& B.M. Everhart. Journal of Mycology. Columbus. Ohio 3:117. 1\$\$7. $\equiv$ Didymosphaeria anomala (J.B. Ellis \& B.M. Everhart) P.A. Saccardo, Sylloge Fungorum Omnium Hucusque Cognitorum Digessit P.A. Saccardo 9:730. 1891. EMicrothelia anomala (J.B. Ellis \& B.M1. Everhart) O. Kuntze. Revisio Generum Plantarum 3(.3):498. 189\$. Elletsteinina anomala (J.B. Ellis of B.M.

Everhart) M.E. Barr. Contributions from the University of Michigan Herbarium. Ann Arbor 9:548. 1972. On dead herbaceous stems of unknown host. unknows tamily. U.S.A. (Utah).

amardica C.L. Spegazzini. Boletin de la Academia Nacional de Ciencian en Cordoba 11:218. 1888: Fungi Fuegani. No. 228. Ann 18\$8. On leaves of unknown host. unknown family. Argentina (Tierra del Fuego).

ambilmimica (M.C. Cooke) P.A. Saccardo. Sylloge Fungorum Omnium llucusque Cognitorum Digessit F.A. Saccardo 2:21. 18\$3.

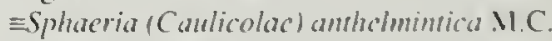
Coohe. Grevillea, London 7:54. 1878. On stems of Choneprolium anhelminticum Bert. ex Steud., Chenopondiaceae. U.S.A. 
antherici L. Hollós, Matematikai és Természetudományi Közlemenyek Vonatkozólag a Hazai Viszonyokra, Budapest 35:31. 1926. On dry stems of Anthericum romosum L.. Liliaceac. Hungary.

anthophila P.A. Saccardo \& C.L. Spegazzini, Michelia Commentarium Mycologicum Fungos in Primis Italicos Illustrans 1:396. 1878. On lower portion of fallen, rotting capitulum of Carlina acaulis L., Compositae. Italy.

amhostomella C.L. Spegazzini. Anales del Museo Nacional de Historia Natural de Buenos Aires 6:281. 1898; Fungi Argentini Novi vel Critici. No. 624. Anno 1898. On fallen decayed culms of Panicum grumosum Nees, Gramineae. Argentina.

anthostomoides (H. Rehm) H. Rehm, Bericht des Naturwissenschafulichen Vereins für Schwaben und Neuberg, Augsburg 26:84. 1881. 三Massaria anthostomoides H. Rehm, Ascomyceten, Fascicle 7, No. 339. Anno 1876. 三Monragnula anthostomoides (H.

Rehm) A. Leuchtmann, Sydowia. Annales Mycologici, Horn, N.Ö. 37:175. 1984. On decayed stems of Aconitum napellus $\mathrm{L}$.. Ranunculaceae. Italy.

aparines (L. Fuckel) P.A. Saccardo, Sylloge Fungorum Omnium Hucusque Cognitorum Digessil P.A. Saccardo 2:21. 1883. 三Pleospora uparines L. Fuckel, Symbolae Mycologicae, p. 136. 1870 [as aparinae]. On dry stems of Galium aparine L., Rubiaceae. Austria.

apios M. Miura ex I. Hino \& K. Katumoto, Transactions of the Mycological Society of Japan [Nihon Kin Gakkai Kaiho] 7:47. 1966 [as apiosis]. On living leaves of Apios fortmei Maxim., Leguminosae. Japan.

apios-fortunei [M. Miura] ex I. Hino \& K. Katumoto. Transactions of the Mycological Society of Japan [Nihon Kin Gakkai Kaiho] 7:46-47. 1966 [as apiosis-fortunei (M. Miura) 1. Hino \& K. Katumoto comb. nov.]. 三Guignardia apios-fortumii M. Miura, Report of the Akita Prefectural Agricultural Experiment Station 8:9. 1957. On living leaves of Apios fortunei Maxim., Leguminosae. Japan.

apocalypta (H. Rehm) ex H.G. Winter, Die Pilze Deutschlands, Oesterreichs und der Schweiz, Abt. Il. (Ascomyceten) in G.L. Rahenhorst, Kryptogamen-Flora von Deutschland, Oesterreich und der Schweiz 1(2):442. 1885 [as Leplosphaeria? apocalypta]. 三Xenosphaeria apocalypla H. Rehm. Ascomyceten. Fascicle 7, No. 346. Anno 1876. Nom. nud. Ant. 32.1. On thallus of Stereocaulon alpinum Laur. Lichenes (Stereocaulaceac). Jialy. apogon P.A. Saccardo \& C.L. Spegazzini in P'A. Saccardo. Michelia Conmentarium Mycol- ogicum Fungos in Primis Italicos Illustrans 1:398. 1878. ELeptosphaeria culmornm P.A. Saccardo \& C.L. Spegazzini forma apogon (P.A. Saccardo \& C.L. Spegazzini) H. Rehm. \#Heptameria apogon (P.A. Saccardo \& C.L. Spegazzini) M.C. Cooke, Grevillea, London 18:32. 1889 [as apogon Sacc.]. ĐLeprosphacria eustoma (E.M. Fries:E.M. Fries) P.A. Saccardo forma aprogon (P.A. Saccardo \& C.L. Spegazzini) A.N. Berlese, Icones Fungorum Omnium Hucusque Cognitorum ad usum Sylloges Saccardianae Adcommodatae 1:56. 1892. On culms of Scirpus holo. schoenus L.. Cyperaceae. Italy.

appendiculata R. Pirotta, Atti dell Istituto Botanico della Università e Laboratorio Crittogamica di Pavia, Milano 2 \& 3:161-162. 1879.

ELeptosphaeria vitis S. Schulzer v.

Müggenburg. Nom, illegit. Art. 63.1. [A superfluous name change.]

aquatica S.T. Tilak \& R.L. Kulkarni, Beihefte zur Nova Hedwigia Weinheim 47:456-457. 1974. On living leaves of Typha angustata Bory \& Chaub., Typhaceae. India.

aquilana D. Saccardo ex D. Saccardo in P.A.

Saccardo, Mycotheca Italica, Sistens Fungos Venetos Exsiccatos, Century 15, No. 1485. Anno 1881. Nom. nud. Art. 32.1; Sylloge Fungorum Omnium Hucusque Cognitorum Digessit P.A. Saccardo 17:724-725. 1905. $\equiv$ Nodulosphaeria aquilana (D. Saccardo) L. Holm, Symbolae Botanicae Upsalienses 14(3):83. 1957. On dry stems of unknown host, Scrophulariaceae. Italy.

aquilegiae (A.N. Berlese \& G. Bresadola) F Hazslinszky, Matematikai és Természettudományi Közlemenyek Vonatkozólag a Hazai Viszonyokra, Budapest 25(2):145. 1892. 三Merasphaeria sepincola (E.M. Fries:E.M. Fries) P.A. Saccardo var. aquilegiae A.N. Berlese \& G. Bresadola, Micromycetes Tridentini Contribuzione Allo Studio dei Funghi Microscopici del Trentino Per Cura Del Dr. A.N. Berlese e dell' Ab. G. Bresadola, p. 39. 1889. EMetasp/areria aquilegiae A.N. Berlese \& G. Bresadola, Revue Mycologique, Toulouse 12:185. 1890. On siems of Aquilegia rw/garis L.. Ranunculaceac. Germany, Hungary.

aquilina (B. Auerswald) G. Passerini, Atti della Società Crittogamologica ltaliana. Milano. Series 2. 2:47. 1879. ESphacrella aquilina B. Auerswald in W. Gonnermann and G.L. Rahenhorst, Mycologia Europaea. Abbildungen Sämmtlicher Pilze Europa's, Hefte 5 und 6. Synopsis l'yrenomycetum Europacorum, p. 20. 1869. On dry frond of Perris aquilina L.. P'teridaceae. Italy. arabidis A. Allescher, Bericht der Bayerischen Botanischen Gesellschaft sur Erforschung der 
Heimischen Flora, München 5:14. 1897. On dead stems of Arahis alpina L., Cruciferae. Germany.

arburi A. Clotilde dos Santos \& M. de Sousa da Camara, Agronomia Lusitana, Sacavém 17:139-140. 1955. On branches of Arbutus unedo L., Ericaceae. Portugal.

arctalaskana Y. Kobayasi. Annual Report of the Institute for Fermentation Osaka 3:32. 1967. On bract of inflorescence on Lycopodium selago L. var. appressum Desv., Lycopodiaceae. U.S.A.

arecae G. Mariani, Atti della Società Italiana di Scienze Naturali e del Museo Civile di Storia Naturale, Milano 50:165-166. 1911. On wilted and dead leaves of Areca sapida Soland. ex Hook. f., Palmae. Portugal. arenaria A.L. Guyot, Revue de Mycologie, Paris 14:69, 71. 1949. Nom. illegit. Art. 64.1. $\equiv$ Phaeosphaeria arenaria (A.L. Guyot) R.A. Shoemaker, Canadian Journal of Botany, Ottawa 67:1538. 1989. On dry leaves; on sheaths and culms of Festuca arenaria Osbeck, Phleum arenarium L., Gramineae. France.

arenaria (E.C. Bommer, M.H. Rousseau \& P.A. Saccardo) J. Lind, Danish Fungi as Represented in the Herbarium of E. Rostrup, Nordisk Forlag, Copenhagen, p. 220. 1913 [as arenariae]. ĐMetasphaeria arenaria E.C. Bommer, M.H. Rousseau \& P.A. Saccardo, Annales Mycologici, Berlin 3:509. 1906. On dead leaves of Elymus arenarius L., Hordemm arenarium Ascherson, Gramineae. Belgium, Denmark.

argentina C.L. Spegazzini. Anales de la Sociedad Cientifica Argentina, Buenos Aires 9:183. 1880. =Heptameria argentina (C.L. Spegazzini) M.C. Cooke, Grevillea, London 18:29. 1889. On fallen stems of Oenothera longiflora L., Onagraceae. Argentina.

argentinensis (C.L. Spegazzini) F. Petrak in F. Petrak and H. Sydow, Annales Mycologici, Berlin 33:171. 1935. EVenturiella argentinensis C.L. Spegazzini, Anales del Museo Nacional de Historia Natural de Buenos Aires 19(Series 3, 12), p. 379. 1909; Mycetes Argentinenses, Series IV, No. 619. 1909. „Neoventuria argentinensis (C.L. Spegazzini) H. Sydow \& P. Sydow, Annales Mycologici, Berlin 17:44. 1910. On wood of Pircumia dioica Moq. (=Phyrolacca), Phytolaccaceate. Argentina.

arnoldii H. Rehm, Österreichische Botanische Zeitschrift, Wien 54:84. 1904 [as arnoldi]. On thallus of Pelrigera malacea (Ach.) Funck., Lichenes (Peltigeraceae). Italy arhonaheri F. Hazslinszky, Matematikai és Természettudományi Közlemenyck Vonatkozólag a Hazai Viszonyokra, Budapest
25(2):132. 1892. On culms of Arrhenatherum avenaceum Beauv., Gramineae. Hungary.

arrhenatheri $\mathrm{F}$. Hazslinszky var. italica C. Massa in

T. Ferraris and C. Massa. Annales Mycologici, Berlin 10:287. 1912. On leaves of Arhenatherum elatius (L.) Beauv. ex J. Presl. \& C. Presl., Gramineae. Italy.

artemisiae (L. Fuckel) B. Auerswald in G.L. Rabenhorst, Fungi Europaei Exsiccati, Klotzschii Herbarii vivi Mycologici Continuatio Edita Nova, Series 2, Century 18, No. 1725. Anno 1874; Hedwigia, Dresden 17:46-47. 1878 [as in G. Niessl v.

Mayendorf]. 三Sphaeria artemisiae L. Fuckel, Fungi Rhenani Exsiccati A Leopoldo Fuckel Collecti, Fascicle 9. No. 896. Anno 1864. झPleospora helminthospora L. Fucke]. Symbolae Mycologica, p. 138. 1870; Symbolae Mycologicae Zweiter Nachtrag, p. 24. 1873. [Erroneously cited in both these works as (Ces.) Fuckel. Not based on Sphaeria helminthospora V. Cesati.] On dry stems of Artemisia campestris L. . Compositae. Austria, Germany.

arthrophyma (C.E. Fairman) L. Holm. Svensk Botanisk Tidskrift. Stockholm 62:228-229. 1962. EGibberidia arthrophyma C.E. Fairman. Mycologia, Lancaster. Pennsylvania 10:246-247. 1938. On old stems of Chrysothamnus graveolens (Nutt.) Greene. Compositae. U.S.A.

arunci S.M. Zeller, Mycologia, Lancaster, Pennsy]vania 19:134-135. 1927. On dead stems of Aruncus silvestris Kostel. [=Aruncus dioicus (Walter) Fernald], Rosaceae. U.S.A. (Oregon).

anundinacea (M.J. Sowerby:E.M. Fries) P.A. Saccardo, Atti dell' Accademia Scientifica Veneto-Trentino-Istriana, Padova 2(2):155. 1873. ESphaeria arundinacea M.J.

Sowerby:E.M1. Fries, Coloured Figures of English Fungi or Mushrooms. Table 336. 1803: E.M. Fries. Systema Mycologicum Sistens Fungorum 2:429. 1823. 三Plenspora arundinacea (M.J. Sowerby:E.M. Fries) L. Fuckel. Symbolae Mycologicae. p. 137. 1870. EMelogramma (?) arundinacea (M.J.

Sowerby:E.M. Fries) G. Niessl v. Mayendorf in G.L. Rabenhorst. Fungi Europaei Exsiccati. Klotzschii Herharii vivi Mycologici Continuatio. Edition 3. Century 19. No. 1840. Anno 1874; Hedwigia, Dresden 13:185. 1874. $\equiv$ Splatia striaeformis Auctores var. arumdinis J.B. Albertini \& L.D. v. Schweiniz. Conspecus Fungorum in Lusatiae Superioris Agro Niskiensi Crescentium e Methodo Persoonia, p. 15. 1805. TThis variety is considered a synony'm hy E.M. Fries.] EPhacosphacria arundinacea (M1.J. Sowerby) A. Hedjaroude. Sydowia. Annales Myeol- 
ogici, Horn. N.Ö. 22:78. (1968) 1969.

=Massarina armindinacere (MI.J.

Sowerby:E.M. Fries) A. Leuchtmann,

Sydowia. Annales Mycologici, Horn, N.Ö.

37:179. 1984. On lallen culms of Phragmites communis Trin., Gramineae. France, Great Britain, Italy.

armulinacea (M.J. Sowerby:E.M. Fries) H. Rehm, Bericht des Naturwissenschaftlichen Vereins für Schwaben und Neuberg. Augsburg 26:50. 1881. Nom, illegit. Art. 64.1. [Combination previously made as Leprosphaeria arundinacea (M.J. Sowerby:E.M1. Fries) P.A. Saccardo.] On fallen culns of Phragmites communis Trin., Granineae. Great Britain. Italy.

arundinacea (M.J. Sowerby:E.M. Fries) P.A.

Saccardo var. godini (J. Desmazières) P.A.

Saccardo, Sylloge Fungorum Omnium

Hucusque Cognitorum Digessit P.A. Saccardo 2:63. 1883. ESphacria godini J. Desmazières, Plantes Cryptogames Du Nord De La France. Edition 1. Fascicle 9. No. 439. Anno 1829; Annales des Sciences Naturelles, Paris, Botanique, Series 3, 5:49. 1846. On culms: on stubble of Phragmites commmis Trin.. Phragmises sp., Gramineae. France. arundinacea (M.J. Sowerby:E.M. Fries) P.A.

Saccardo var. godini (J. Desmazières) P.A. Saccardo, Michelia Commentarium Mycologicum Fungos in Primis Italicos Illustrans 2:65. 1880. ESphaeria godini J. Desmazières. Plantes Cryptogames Du Nord De La France. Edition 1, Fascicle 9. No. 439. Anno 1829: Annales des Sciences Naturelles. Paris, Botanique. Series 3. 5:49. 1846. 三Leptosphareria godini (J. Desmazières) B. Auerswald. On stubble of Phragnires commumis Trin., Gramineae. France.

areensis C.L. Spegazzini in P.A. Saccardo, Michelia Commentarium Mycologicum Fungos in Primis Italicos Illustrans 1:459. 1879. On dead, rotting stems of Equisentrm ariense L., Equisetaceae. Italy.

aselepiadis R.A. Shoemaker. Canadian Journal of Botany. Ottawa 62:2693-2694. 1984. On stems of Asclepias sp. Asclepiadaceate. Canada. U.S.A.

asparagi C.H. Peck. Bulletin of the New York State Museum. Albany 219-22():68. (1919) 1920. On dead stems of A.sparagus officinalis L., Liliace 1 e. U.S.A. (New York).

aspenagi G. Passerini. Rendiconti della Sedute della R. Accademia dei Lincei. Classe di Scien/e Fisiche, Matematiche e Natural (=Aui dell Accademia Nazionale dei Lincei, Rendiconti, Roma), Series 4, 3:90. 1887. ELeptosphacria passerimiana P.A. Saccardo. Sylloge

Fungorum Omnium Ilucusque Cognitorum Digessit P.A. Saccardo 9:785. 1891. On dry stems of Asparagus officinalis L. Liliaceac. Italy. asparagi C.H. Peck, Report. New York State Museum of Natural History, Albany 40:70. (1886) 1887. On dead stems of Asparagus officimalis L., Liliaceac. U.S.A.

asparagina P.A. Karsten, Hedwigia, Dresden 23:1. 1884: Acla Societatis pro Fauna et Flora Fennica, Helsingforsiae 2(6):55. 1885. On stens of Asparagus officinalis L., Liliaceae. Finland.

asperellac K. Hara, Byôchu-gai Zasschi (Journal of Plant Protection), Tokyo 5:883-884. 1918. On leaves of Asperella japonica Hack. Gramineae. Japan.

aspidisnac F. Tassi, Bolletino del R. Orto Botanico, Siena 3:14-15. 1900. On leaves of Aspidisira elarior Blume, Liliaceae. China.

asplenii (G.L. Rabenhorst) P.A. Saccardo, Sylloge Fungorum Omnium Hucusque Cognitorum Digessit P.A. Saccardo 2:82, 1883. ㅍ Sphaerella asplenii G.L. Rabenhorst in G. Niessl v Mayendorf, Verhandlungen des Naturforschenden Vereins in Brünn 3:178. 1865: Vorarbeiten zu einer Kryptogamenflora von Mähren und Oesterr II. Pilze und Myxomyceten, p. 121. 1865. On dry tronds of Asplenium septentrionale (L.) Hotfm., Aspleniaceue. Gennany.

associara H. Rehm. Annales Mycologici, Berlin 10:356-357. 1912: Ascomyceten, Fascicle 50. No. 1994. Anno 1912. EPhacosphaeria associara (H. Rehm) O. Eriksson, Arkiv för Botanik, Uppsala, Stockholm. Series 2, 6:412. 1967. Parasitic on Epichloe typhina (Pers.:Fr.) Tul.. Fungi (Clavicipitaceae). Canada, Sweden, U.S.A.

astericola J.B. Ellis \& B.M. Everhart. Fungi Columbiani, Century 16. No. 1537. Anno 1901. Nom. nud. Art. 32.1. On dead stems of Aster multiflorus Ait. (=Aster ericoides L.), Compositie. U.S.A.

astericula J.B. Ellis \& B.M. Everhart ex J.B. Ellis \& B.M. Everhart, Journal of Mycology,

Columbus, Ohio 8:17. 1902. On deind stems ol Aster multiflorus Ait. (=Aster ericoides L.). Composituc. U.S.A.

asteris J.H. Miller \& G. Burton, Mycologia,

Lancister, Pennsylvania 34:3-4. 1942. On dead stems of Aster sagirifolius Wedem. ex Willd., Compositue. U.S.A.

arraphavidis M.P. Vasyagina in M.P. Vasyagina,

Z.M. Byzova, and M.A. Tartenova, Flora

Sporovykh Rastenii Kazaklistana 12

Sumchatye Griby 2. Lokuloaskomitsely (Loculoascomycetes) Alna Alta: "Nauka" All Kazakhskoi SSR, p. 125. 1987. On unknown suhsirate of Arraphaxis muschkequii Krasnov. Polygonaceac. U.S.S.R.

arriplicis A.L. Lobih. Materialȳ po Floristicheskim i Faunisticheskim Obsledovaniyam Tershogo Okruga [Data from Investigations on the Flora and Fauna of the Ter Region]. p. 23. 1928. 
On unknown substrate of Atriplex verrucifera Bieb., Chenopodiaceae. U.S.S.R.

atropurpurea F. Petrak, Annales Mycologici, Berlin 25:286-287. 1927. On stems of Urtica dioica L., Unicaceae. Germany.

aucta G. Niessl v. Mayendorf in G.L. Rabenhorst, Fungi Europaei Exsiccati, Klotzschii Herbarii vivi Mycologici Continuatio, Series 2, Century 23, No. 2240. Anno 1876; Hedwigia, Dresden 16:1 18. 1877. 三Nodulosphaeria aucta (G. Niessl v. Mayendorf) L. Holm. Svenska Botanisk Tidskrift, Stockholm 55:73. 1961. On stems of Clematis recta L., Ranunculaceae. Czechoslovakia.

alcerswaldii P.A. Saccardo \& J.B. Traverso in P.A. Saccardo, Sylloge Fungorum Omnium Hucusque Cognitorum Digessit P.A. Saccardo 19:1096. 1910. Nom. illegit. Art. 63.1. Name erroneously changed to avoid a tautonym. $\equiv$ Sphaerella leptosphaerioides B. Auerswald in W. Gonnermann and G.L. Rabenhorst, Mycologia Europaea, Abbildungen Sämmtlicher Pilze Europa's, Hefte 5 und 6, Synopsis Pyrenomycetum Europaeorum, p. 13. 1869. On either side of dry leaves and sepals of Arenaria ciliata L., Caryophyllaceae. Europe. aulica (M.C. Cooke \& J.B. Ellis) J.B. Ellis in N.L. Britton, Catalogue of Plants Found in New Jersey. Geological Survey of New Jersey, Final Report of the State Geologist, Trenton 2(1):525. 1889. ESphaeria (Obiecta) aulica M.C. Cooke \& J.B. Ellis, Grevillea, London 6:95. 1878. On dead stems of Lonicera sp., Solidago sp., Caprifoliaceae, Compositae. U.S.A. (New Jersey).

australiensis (A.B. Cribb \& J.W. Cribb) G. Hughes, Syesis, British Columbia Provincial Museum, Victoria, British Columbia 2:132. 1969. $\equiv$ Metasphacria australiensis A.B. Cribb \& J.W. Cribb, Papers from the Department of Botany, University of Queensland, Brisbane 3:79. 1955. On roots of Avicennia marina (Forsk.) Vierh, var. resiniferae (Forst.) Bakh., Avicenniaceae. Australia, Canada.

australis (L. Crié) P.A. Saccardo, Sylloge Fungorum Omnium Hucusque Cognitorum Digessit P.A. Saccardo 2:54. 1883. EDepazea australis L. Crié, Annales des Sciences Naturelles, Paris, Botanique, Series 6, 7:46. 1878. On living leaves of Eustephia sp., Amaryllidaceae. Australia, New Caledonia.

austro-americana C.L. Spegazzini, Annales de la Sociedad Cientifica Argentina, Buenos Aires 10:20. 1880: Fungi Argentini Pugillus 2, No. 82. Anno 1880. On living or wilting leaves of Plantago macrostachys Decne., Pluntaginaceac. Argentina.

arellanae J.H. Fabre, Annales des Sciences Naturelles, Paris, Botanique, Scries 6, 9:89. 1878. On dead branches of Corylus arellana L., Betulaceae. France. avenae B. Auerswald in W. Gonnermann and G.L. Rabenhorst, Mycologia Europaea, Abbildugen Sämmtlicher Pilze Europa's, Hefte 5 und 6. Synopsis Pyrenomycetum Europaeorum, Table 12. Figure 165. 1869. Nom. nud. Ar. 32.1. EMetasphaeria avenae (B. Auerswald) ex P.A. Saccardo, Sylloge Fungorum Omnium Hucusque Cognitorum Digessit P.A. Saccardo 2:176. 1883 [as (Auersw.) Sacc.]. On leaves of Avena sp., Gramineae. Germany.

avenaria G.F. Weber, Phytopathology, Lancaster, Pennsylvania 12:454-455. 1922. 三Phaeosphaeria avenaria (G.F. Weber) O. Eriksson, Arkiv för Botanik, Uppsala, Stockholm, Series 2, 6:408. 1967. On leaves of Agropyron repens (L.) Beauv., Bromus inermis Leysser, Poa pratensis L.. Gramineae. U.S.A.

avenaria G.F. Weber form sp. triticea T. Johnson. Canadian Joumal of Botany. Ottawa 25: 262-263. 1947. EPhaeosphaeria avenaria (G.F. Weber) O. Eriksson form sp. triticea (T. Johnson) R.A. Shoemaker, Canadian Joumal of Botany, Ottawa 67:1522. 1989. On leaves and sheaths of Triticum durum Desf., Triticum v'ulgare Vill., Gramineae. Canada.

avicenniae J. Kohlmeyer \& E. Kohlmeyer, Nova Hedwigia. Zeitschrift für Kryptogramenkunde, Weinheim 9:98-99. 1965. On bark of living pneumatophores of Avicennia nitida Jacq., Avicenniaceae. U.S.A.

azaleae L. Hollós, Annales Historico-Naturales Musei Nationalis Hungarici. Budapest 6:529. 1908. On dead branches of Azalea sp. (=Rhododendron sp.). Ericaceae. Hungary.

bacillifera (P.A. Karsten) A. Munk, Dansk Botanisk Arkiv. Kjobenhavn 17(1):378. 1957. Nom. inval. Ant. 33.2. ESphacrella bacillifera P.A. Karsten, Hedwigia. Dresden 22:179. 1883. On dead leaves of Scheuchzeria palustris L.. Scheuchzeriaceae. Denmark.

baconnccaria (W. Lindsay) P.A. Saccardo \& A. Trotter in P.A. Saccardo, Sylloge Fungorum Omnium Hucusque Cognitorum Digessit P.A. Saccardo 22:235. 1913. EMicrothelia baeomycearia W.L. Lindsay. Transactions of the Royal Society of Edinburgh 25:541-542. 1869; Proceedings of the Royal Society of Edinburgh 6:535. 1869. Nomen. On thallus of Baeomyces rufus (Huds.) Reben!.. Lichenes (Baeonycetaceae). Great Britain.

haggei (B. Auerswald ex G. Niessl v. Mayendorf) P.A. Saccardo. Sylloge Fungorum Omnium Hucusque Cognitorum Digessit P.A. Saccardo 2:35. I883. 三Cryptospora baggei (B.

Auerswald) ex G. Niess] v. Mayendorf. Verhandlungen des Naturforschenden Vereins in Brünn 10:200. 1872. ESphacria luggei B.

Auerswald. Tauschverein (unpublished manuscript?). Nom, nud. Art. 32.1c? On branches of Sulix sp.. Salicaceale. Austria. Czechoslovakia, Gemmany. 
balcarica Z.D. Savintzeva, Novosti Sistematiki Nizshikh Rastenij, Novitates Systematicate Plantarum Non Vascularium 8:109-110. 1971. On dead stems of Trifotium richocephalum Bieb., Leguminosae. U.S.S.R.

baldingerae F. Fautrey \& J.B. Lambotte in F. Fautrey, Revue Mycologique, Toulouse 19:53. 1897. EPhaeosphaeria haldingerae (F. Fautrey \& J.B. Lambotte) A. Hedjaroude. Sydowia. Annales Mycologici, Horn, N.Ö. 22:87. (1968) 1969. On dry culms of Baldingera armedinacea (L.) Dumort. Verbenaceae. France.

haldratiana P. Baccarini, Annali di Botanica, Roma 4:274. 1906. On leaves of Sansevieria elrenhergii Schweinf. ex Baker, Agavaceae. Ethiopia.

ballotae J. Politis, Akademia Athēnōn, Pragmateiai, Athens 1935:16. 1935. On dried stems of Ballota acetabulosa (L.) Bentham, Labiatae. Greece.

hambusae (1. Miyake \& K. Hara) P.A. Saccardo, Sylloge Fungorum Omnium Hucusque Cognitorum Digessit P.A. Saccardo 24:995-996. 1928. झPhacosphaeria hambusac K. Miyake \& K. Hara, Botanical Magazine. Tokyo 24:340. 1910. 三Leptosphaerella hambusae (1. Miyake \& K. Hara) I Miyake \& K. Hara, Botanical Magazine. Tokyo 24:(249). 1913. ETrematosphacrella bambusae (K. Miyake \& K. Hara) I. Hino \& K. Katumoto, Icones Fungorum Bambusicolorum Japonicorum, p. 160. 1961. Non Tremarosphaerella bambusae (K. Miyake \& K. Hara) F.L. 'Tai, Sylloge Fungorum Sinicorum, Science Press, Academia Sinica, Peking, p. 330. 1979. Nom. illegit. Art. 64.1. On leaves of Phyllostachys hambuoides Siehold \& Zucc.. Pleiohlassus hindsii (Munro) Nakai, Pleioblasıs simoni (Carriere) Nakai. Psendosasa japonica (Siebold \& Zucc. ex Steudel) Makino, Sasa paniculata (F. Schmidt) Makino $[=S$. senanensis (Fr. \& Sav.) Rehd.], Sasaella ramosa (Makino) Makino, Semiarmindinaria tatebeana Muroi. Gramineae. Japan.

bambusae L. Roljand. Bulletin de la Société Mycologique de France. Paris 12:4, 1896 [as hanbusae]. On cortex (culm) of Bambusa sp.. Gramineae. France.

bambusicola S.C. Teng \& S. Ou. Sinensia. Special Bulletin of the Metropolitan Museum of Natural History, Nanking 7:500. 1936. On decaying culms of Bambusu sp., Gramineae. China (Chekiang).

hardanae (K.F.W. Wallroth) P.A. Saccardo, Sylloge Fungorum Omnium Hucusque Cognitorum Digessit P.A. Saccardo 2:34. 1883. ESphaeria hardanae K.F.W. Wallroth, Flora Cryptoganica Germaniae 2:805. 1833. On dried stems of Bardana sp.. Compositae. Germany.

barriae R.A. Shoemaker, Canadian Journal of Botany, Ottawa 62:2694. 1984. On stems of Lupimis sp., Leguminosae. Canada.

basalduai C.L. Spegazzini, Anales del Museo Nacional de Historia Natural de Buenos Aires 8(Series 3, 1):71. 1902; Mycetes Argentinenses, Series 11, No. 133. Anno 1902. On decaying, falten branches of Lippia seriphioides A. Gray, Verhenaceac. Argentina.

hataricola M. Chochrjakov \& M.S. Dunin in M.S. Dunin and E.D. Yakimovick, Bolezni Batata i Mery Bor'by s Nimi, pp. 89-90. 1934. On leaves of ipomoca batatas (L.) Lam., Convolvulaceae. U.S.S.R.?

hatumensis W. Siemaszko. Acta Societatis Botanicorum Poloniae. Warszawa 1:21. 1923. On living leaves of Phoenix canariensis Hort. ex Chabaud, Palmae. U.S.S.R.

baumii J.A. v. Arx \& E. Mülter, Studies in Mycology, Baam, Netherlands 9:76. 1975. $\equiv$ Baumiella caespitosa P.C. Hennings in $\mathrm{H}$. Baum, Kunene-Sambesi Expedition, Berlin, p. 165. 1903. Non Leptosplaeria caespitosa G. Niessl v. Mayendorf. On leaves of Monores dusyamthus Gilg., Dipterocarpaceae. Angola. beaumonii (M.J. Berkeley \& M.A. Curtis) P.A. Saccardo, Sylloge Fungorum Omnium Hucusque Cognitorum Digessit P.A. Saccardo 2:87. 1883. ESphaeria beaumontii M.J. Berkeley \& M.A. Curtis in M.J. Berkeley, Grevillea, London 4:145. 1876. On grass culms of unknown host of Gramineae, Gramineae. U.S.A.

bella G. Passerini, Rendiconti della Sedute della R. Accademia dei Lincei, Classe di Scienze Fisiche, Matematiche e Natural (=Atti dell Accademia Nazionale dei Lincei, Rendiconti, Roma), Series 4, 3:90. 1887. On branchlets of Chondrilla juncea L.. Compositae. Italy.

bellunckii (G.D. Westendorp) B. Auerswald. General-Doubl. Verzeichn-tauschverein, p. 4. 1870. Non vidi. ESphaeria bellynckii G.D. Westendorp, Bulletins de L'Académie Royale des Sciences. des Lettres et des Beaux-Arts de Belgique, Brussels, Series 2, 7:86. 1859. $\equiv$ Metasphaeria bellynckii (G.D. Westendorp) P.A. Saceardo, Sylloge Fungorum Omnium Hucusque Cognitorum Digessit P.A. Saccardo 2:178. 1883. $\equiv$ Scleropleella bellynckii (G.D. Westendorp) A. Munk. Dansk Botanisk Arkiv, Kjobenhavn 15(2):107. 1953. On dead stems of Comallaria polygonatum L., Liliaceas. Betgium, Germany, Italy, Sweden. berberidicola C.L. Spegazzini, Boletin de la Academia Nacional de Ciencias en Córdoba 27:369-370. 1923. On leaves of Berberis ilicifolia Forst., Berberidaceae. Argentina. berheridis C. Richon, Catalogue Ralisonné des Champignons Qui Croissent Dans le Départe- 
ment de la Marne, p. 300. 1889. On young. living branches of Berheris vulgaris $L$., Berberidaceae. France.

berlesei P. Larsen \& A. Munk, Dansk Botanisk Arkiv, Kjøbenhavn 14(7):23. 1952. $\equiv$ Phaeosphaeria berlesei (P. Larsen \& A. Munk) A. Hedjaroude, Sydowia. Annales Mycologici, Horn, N.Ö. 22(1-4):87. (1968) 1969. On dead stems of Equisetum hyemale L., Equisetaceae. Canada, Denmark, Germany, Hungary, U.S.A.

hetulina F. Hazslinszky, Matematikai és Természettudományi Közlemenyek Vonatkozólag a Hazai Viszonyokra, Budapest 25(2):152. 1892. $\equiv$ Metasphacria betulina (F.A. Hazslinszky) P.A. Saccardo \& P. Sydow in P.A. Saccardo, Sylloge Fungorum Omnium Hucusque Cognitorum Diggesit P.A. Saccardo 14:583-584. 1899. On branches of Betulu sp.. Betulaceae. Hungary.

betulina I.E. Brezhnev. Notulae Systematicae e Sectione Cryptogamica Instituti Botanici Nomine V.L. Komarov Academiae Scientiarum U.R.S.S., Petropolis 7:184. 1951. Nom. illegit. Art. 64.1. On leaf spots of Betula verrucosa Ehrh., caused by Phyllosticta betulina Sacc., Betulaceae. U.S.S.R. (Kursk).

bicolor D. Hawksworth, W. Kaiser \& B.N. Ndimande. Mycologia, Lancaster, Pennsylvania 71:483. 1979. On leaves of Saccharum officinarum L., Gramineae. Kenya.

bicuspidata (M.C. Cooke \& W.H. Harkness) A.N. Berlese \& P. Voglino, Sylloge Fungorum Omnium Hucusque Cognitorum Digessit P.A. Saccardo Additamenta Ad Volumina I-IV, p. 133. 1886. 三Sphaeria (Leptosphaeria) bicuspidala M.C. Cooke \& W.H. Harkness. Grevillea, London 13:19-20. 1884. On twigs of Baccharis sp.. Compositae. U.S.A.

hiebersteinii S.A. Gucevicz, Novosti Sistematiki Nizshikh Rastenij. Novitates Systematicae Plantarum Non Vascularium 6:128-129. 1969. On dry branches of Cerastium hichersteinii DC., Caryophyllaceae. U.S.S.R.

biseptata B. Auerswald [eited without place of publication in P.A. Saccardo, Sylloge Fungorum Omnium Hucusque Cognitorum Digessit P.A. Saccardo 2:58. 1883, sub. Leptosphueria michotii (G.D. Westendorp) P.A. Saccardol. On unknown substrate of unknown host, unknown family. Unknown country.

bispora (P. Larsen) A. Munk, Dansk Botanisk Arkiv, Kjobenhavn 17(1):376. 1957. EMcrasphaeria hispora P. Larsen, Dansk Botanisk Arkiv, Kjøbenhavn 14(7-8):33-34. 1952. On dead leaves of Typha latifolia L.. Typhaceac. Denmark.

bitorulosa [erroneously so cited by P.A. Siccardo, Sylloge Fungorum Omnium Hucusque Cognitorum Digessit P.A. Saccardo 15:190.
1901: and as Metasphaeria birorulosa for Leptosphacria tritorulosa (M.J. Berkeley \&

C.E. Broome) V. Cesati \& G. de Notaris].

bhomeri E. Müller. Sydowia. Annales Mycologici. Horn. N.Ö. 4(1-6):275. 1950. On dead stems of Medicago falcata L., Leguminosae. Switzerland.

hocconiae (M.C. Cooke \& J.B. Ellis) P.A. Saccardo. Sylloge Fungorum Omnium Hucusque Cognitorum Digessit P.A. Saccardo 2:16. 1883. ESphaeria (Caulicolae) bocconiae M.C. Cooke \& J.B. Ellis, Grevillea, London 7:10. 1876 [as bokoniae]. 三Heptameria bocconiac (M.C. Cooke \& J.B. Ellis) M.C. Cooke, Grevillea, London 18:29. 1889. On stems of Bocconia sp.. Papaveraceae. U.S.A. (New Jersey).

bonureae F. Petrak, Sydowia. Annales Mycologici. Horn. N.Ö. 4(1-6):494-496. 1950. On leaves of Bomarea caldasii (HBK) Asch. \& Graebn.. Alstroemeriaceae. Ecuador.

bondari A.A. Bitancourt \& A.E. Jenkins. Phytopathology, Lancaster, Pennsylvania 25:886. 1935. On leaves and twigs of Cirrus aurantium L.. Cirrus grandis Osb., Cirus limonia Osb.. Citrus medica L.. Citrus sinensis Osb.. Cirrus sp.. Rutaceae. Brazil.

horealis J.B. Ellis \& B.M. Everhart. The North American Pyrenomycetes. A Contribution to Mycologic Botany, p. 353. 1892. On decorticated wood of Salix sp.. Salicaceae. U.S.A.

borealis J.B. Ellis \& B.M. Everhart var. populi J. Deamess \& H.D. House, Bulletin of the New York State Museum. Albany 266:74. 1925. On decorticated branch of Populus halsamifcra L., Salicaceae. U.S.A. (New York).

hormmucllcri O. Jaap. Annales Mycologici. Berlin 14:15. 1916. On dry leaves of Rubia peregrina L., Rubiaceae. Italy.

horziana P.A. Saccardo \& F. Cavara. Nuovo Giomale Botanico Italiano e Bolletino della Società Botanica Italiana. Firenze. New Series 7:283. 1900. On dead branches of Sparrina juncer auct. Gramineae. Italy.

boncera (M.C. Cooke \& J.B. Ellis) J.B. Ellis in N.L. Britton. Catalogue of Plants Found in New Jersey. Geological Survey of New. Jersey, Final Report of the State Geologist. Trenton 2(1):525. 1889. ESphaeria (Caulicolac) honcera M.C. Cooke \& J.B. Ellis. Gresillea. London 8:15. 1879 [as bucera]. 三Merasphaeria boucera (M.C. Cooke \& J.B. Ellis) P.A. Saccardo. Sylloge Fungorum Omnium Hucusque Cognitonum Digessit P.A. Saccardo 2:161. 1\$83 [as /nucera]. On dead herbaceous stems of unknown host, unknow $n$ family. U.S.A. (New Jersey).

brachyasca E. Rostrup, Meddelelser om Gronland. Kjobenhavn 3(3):618. 1893 [published 1892?]. On stems and leaves of Saxifraga ofpositifolia L.. Savilragaceac. Greenland. 
brachypodii G. Passerini in G.L. Rabenhors, Fungi Europaei Exsiccati, Klotzschii Herharii vivi Mycologici Continuatio Edita Nova. Series 2, Century 24, No. 2334. Anno 1876: Hedwigia. Dresden 17:45. 1879. EMetasphueria brachypodii (G. Passerini) P.A. Saccardo. Sylloge Fungorum Omnium Hucusque Cognitorum Digessit P.A. Saccardo 2:176 1883. On dry leaves of Brachypodium sylraricum (Hudson) Beauv., Gramineate Italy.

brachysperma A.N. Berlese, Bulletin Trimestriel de la Société Mycologique de France, Paris 5:41-42. 1889. On dead herbaceous stems of unknown host, unknown family. Italy.

bractearum (P.A. Saccardo) P.A. Saccardo, Sylloge Fungorum Omnium Hucusque Cognitorum Digessit P.A. Saccardo 2:58. 1883. ELeprosphaeria carpophila P.A. Saccardo var. bractearm P.A. Saccardo, Michelia Commentarium Mycologicum Fungos in Primis Italicos Illustrans 1:397. 1878. On rotting bracts of heads on Dipsacus syliestris Hudson, Dipsacaceae. Italy.

brasiliensis C.L. Spegazzini. Boletin de la Academia Nacional de Ciencias en Córdoba J1:521. 1889; Fungi Puiggariani 1:143, No. 280. 1889. On living leaves of Xamhoxy/um sp. (=Zumhory/um sp.), Anacardiaceae. Brazil.

brauni E. Müller, Sydowia. Annales Mycologici. Hom, N.Ö. 4(1-6):246. 1950. On stems of Hieracium murorum L., Compositae. Switzerland.

bresadolacana O. Jaap, Annales Mycologici, Berlin 14:14-15. 1916. On dry stems of preceding year on Euphorbia witfemii Hoppe ex Koch, Euphorbiaceae. Yugoslavia.

brightonensis R.A. Shoemaker, Canadian Joumal of Botany, Ottawa 62:2695. 1984. On stems of Agastache urricifolia (Benth.) Kıze.. Labiatae. U.S.A.

briosiana G. Pollacci, Atti dell’ Istituto Botanico della Università e Laboratorio Crittogamica di Pavia. Milano. Series 2, 5:36. 1896. On living leaves of Jubaed spectabilis Humb., Bonpl. \& Kunth, Palmae. Italy.

brizae G. Passerini, Rendiconti della Seclute della R. Accademia dei Lincei, Classe di Scienze Fisiche, Matematiche e Natural (=Atti dell' Accademia Narionale dei Lincei, Rendiconti, Roma), Series 4. 3:91. 1887. EPhaeosphaeria brizue (G. Passerini) R.A. Shocmaker, Canadian Joumal of Botany, Ottawa 67:1523. 1989. On dry culms and peduncles of Briza media L., Gramineae. Italy.

brunellac J.B. Ellis \& B.M. Everhart. Proceedings of the Academy of Natural Sciences of l'hiladelphia 1890:237. 1890. On dead stems of Prunella vm/garis L.. Labiatac. Canada. bryophila P.A. Saccardo, Nuovo Giomale Butanico Italiano e Bolletino della Socictà Botanica Jaliana. Firence 7:319. 1875. On stalks of
Tortula cormosa Ehrh. ex Iledw. [=Tortella mruosa (Hedw.) Limpr. . Musci (Pottiaceac). Italy.

bryzae G. Passerini. vide Lepeosphaeria brizae G. Passerini.

Inubakii J. Klika. Annales Mycologici, Berlin 24:134 1926. On dead stems of Le'a mays L.. Gramineae. Bulgaria.

budllejae 1. Hino \& K. Kattumoto, Bulletin of the Faculty of Agriculture, Yamaguchi University. Shimonoseki 8:64t-645. 1957. On leaves of Buddleja davidii Franch., Loganiaceac. Japan.

buldllejac S.A. Gucevicz. Trudȳ Gosudarstvennogo Nikitskogo Botanicheskogo Sada, Yalta 29:190. 1959. Nom. illegit. Art. 64.1. On dried stems of Buddleja davidii Franch., Loganiaceae. U.S.S.R. (Yalta).

bulgarica F. Petrak. Annales Mycologici, Berlin 29:368. 1931. On dry stems of Rura grateolens L., Rutaceac. Bulgaria.

bupleuri P. Sydow. Hedwigia, Dresden 38:(142). 1899. On dry stems of Bupleurum perraeum L.. Unbelliferae. France.

buxina M. de Sousa da Camara, Revista Agronómica. Lisbon 20(1):21-22. 1932. On bark of trunks of Buxus sempervirens L., Buxaceae. Portugal.

byssincola R.A. Shoemaker, Canadian Journal of Bolany, Ottawa 62:2695. (1984) 1985. On unknown substrate of Lupinus sp. Leguminosae. U.S.A. (Colorado).

cahalleroi R. González Fragoso, Memórias de la R. Sociedad Española de Historia Natural. Madrid 11:90. 1919. On dry stems of Damara strumonium L., Solanaceae. Spain.

cacuminispora C.E. Fairman, Proceedings of the Rochester Academy of Science, Rochester. New York 6:98-99. 1921. On nuts of Curva sp., Juglandaceae. U.S.A.

cadubriac C.I. Spegazzini, Atti della Società Crittogamologica ltaliana, Milano, Series 2. 3(1):55. 1881. Elleptameria cadubiae (C.L Spegazzini) M.C. Cooke, Grevillea, London 18:31. 1889. \#Nodulosphaseria esdubrice (C.L. Spegazzini) L. Holm., Svensk Botanisk Tidskrilt, Stockholm 55:73-74. 1961. On rotten, fallen stems of Epilobium dodomaei Vill., Onagraceas. Italy.

caespirosa G. Niessl v. Mayendorf in J. Kunze, Fungi Sclccti Exsiccati, Century 1. No. 77. Anno 1876. Nom. nud. Art. 32.1. On dry stems and humid tissues ol Artemisia campestris L.. Compositac. Germany.

caespirosa G. Niessl v. Mayendorf ex P.A. Saccardo, Sylloge Fungorm Ommium Hucusque Cognitorum Digessit P.A. Saccardo 2:35. 1883. EPhaconlerris caespiossa (G. Niessl v. Mayendorf ex P.A. Saccardo) F. v. Höhnel. Sitzungsherichte der Akalemie der Wissenschaften in Wien, Mathematisch-naturwissenschaltliche Klasse. Aht. 1, 120:462. 19)11. 
On dry stems and humid tissues of Artemisia campestris L., Compositae. Germany.

caespirosa G. Niessl v. Mayendorf forma salviae J. Feltgen, Vorstudien zu einer Pilz-Flora des

Grossherzogthums, Luxemburg 1(2):161. 1901. [Description effectively published: Vorstudien zu einer Pilz-Florades Grossherzogthums, Luxemburg 1:262. 1899.] On dry stems of Salvia pratensis L., Labiatae. Luxembourg.

caffra F. v. Thümen, Flora, Jena und Regensburg 59:363-364. 1876: Mycotheca Universalis, Century 4, No. 351. Anno 1876. On living fronds of Marattia sp., Marattiaceae (Fem). South Africa.

californica (M.C. Cooke \& W.H. Harkness) A.N.

Berlese \& P. Voglino, Sylloge Fungorum Omnium Hucusque Cognitorum Digessit P.A. Saccardo Additamenta Ad Volumina I-IV, p. 137. 1886. ĐSphaeria (Leprosphacria) californica M.C. Cooke \& W.H. Harkness, Grevillea, London 13:20. 1886. On twigs and leaves of Araucaria imbricata Pav.,

Euomymus sp., Rhododendron sp., Sarothammus sp.. Araucariaceae, Celastraceae, Ericaceae, Leguminosae. U.S.A.

calligoni 1.P. Frolov in E.N. Koschkelova, I.P. Frolov, and Z. Dzhuraeva, Mikoflora Badkhyza, Karabilya i Yuzhnoi Chasti Murgabskogo Oazisa (Mikromitsety) [The Mycoflora of Badkhyz, Karabil and the Southern Part of the Murgab Oasis (Micromycetes)]. p. 87. 1970. Nom. nud. Art. 32.1. On bare, unsheathed (uncorticated?) wood of Calligonum sp., Polygonaceae. U.S.S.R. (Karabilia).

calligoni I.P. Frolov, Novosti Sistematiki Nizshikh Rastenij, Novitates Systentaticae Plantarum Non Vascularium 7:192-193. 1970. On decorticated twig of Calligonum sp., Polygonaceae. U.S.S.R.

calopogonii R. González Fragoso \& R. Ciferri, Boletin de la R. Sociedad Española de Historia Natural, Madrid 27:272. 1927; Publicaciones Estación Agronómica de Moca, Santa Domingo. Series B, Botanica, No. 11:21-22. 1928. Nom. inval. Art. 34.1. On living or wilted leaves of Calopogonimm mucunoides Desv., Leguminosite. Dominican Republic.

caliescens (E.M. Fries) P.A. Saccardo, Nuovo Giornale Botanico ltaliano e Bolletino della Società Botanica ltaliana, Firenze 7:315. 1875. ESphaeria calvescens E.M. Fries. Scleromyceti Sueciac. Collegit. Digessit et Evulgaviti No. 401. [Apparently not published: specimens of number 401 may he scattered in herbaria.] EPleospora calvescens (E.M. Fries) L.R. Tulasnc \& C. Tulasnc. Selecta Fungorum Carpologica 2:266. 186?. EPrenophora colvescens (E.M. Fries) P.A. Saccardo, Sylloge Fungorum Omnium
Hucusque Cognitorum Diggesit P.A. Saccardo 2:279. 1883. ECucurbitaria calvescens (E.M. Fries) V. Cesati \& G. de Notaris. Commentario della Società Crittogamologica Italiana. Milan 1:215. 1863. EChaetoplea calvescens (E.M. Fries) F.E. Clements in F.E. Clements and C.L. Shear. The Genera of Fungi, p. 275. 1931 [as (Fr.) Sacc.]. On dry stems of Alriplex hortensis L., Chenopodium alhum L., Chenopodiaceae. France.

calvescens (E.M. Fries) P.G. Crivelli, Ueber die

Heterogene Ascomycetengattung Pleospora

Rabh.; Vorschlag für eine Aufteilung.

Abhandlung zur Erlangung des Titels eines

Doktors der Naturwissenschaften der

Eidgenössischen Technischen Hochschule.

Zürich No. 7318:177. 1983. Nom. illegit. Art. 64.1.

camelliae M.C. Cooke \& G.E. Massee, Grevillea. London 16:5. 1877. On living leaves of Camellia sp.. Theaceae. Australia.

camelliae-japonicae W. Siemaszko, Acta Societatis

Botanicorum Poloniae, Warszawa 1:21. 1923

On leaves of Camellia japonica L.. Theaceae. U.S.S.R.

camilla P.A. Saccardo. Nuovo Giomale Botanico Italiano e Bolletino della Società Botanica Italiana, Firenze 7:312. 1875. On wilted leaves of Scrophularia aguatica L.. Scrophulariaceae. Italy:

camphorata G. Passerini. Atti dell Reale Accademia Nazionale dei Lincei. Rendiconti, Rome. Series 4. 7(2):44. 1891. On dry stems of Artemisia camphorara Vill.. Compositae. Italy.

camphorosmae A.I. Lobik, Materialȳ po Floristicheskim i Faunisticheskin Obsledovaniyam Terskogo Okruga [Data from Investigations on the Flora and Fauna of the Ter Regionl. p. 23. 1928. On leaves of Camphorosma perennis Pall., Chenopodiaceae. U.S.S.R. campisilii C.L. Spegazzini. Revue Mycologique. Toulouse 2:32, 1880; Michelia Commentarium Mycologicum Fungos in Primis ltalicos Illustrans 2:162. 1880. On dead leaves of Lycopodium amnotimum L.. Lycopodiaceae. ltaly.

canudensis G. de Notaris [as Leprosphaeria canadensis G. de Notaris in P.A. Saccardo. Sylloge Fungorum Omnium Hucusque Cognitorum Digessit P.A. Saccardo 2:163. 18531. Nom. nud. Art. 32.1. 三Melasphaerio canadensis P.A. Saccardo. Sylloge Fungorum Onmium Hucusque Cognitorum Digessit P.A. Saccarde) 2:163. IS8.3 [as (De Not.) Sacc.]. On dry stens of Erigeron camadensis L.. Compositae. Canada. Jtaly.

cancphorac R.L. Steyactt, Bulletin. Société R. de Botanique de Belgique. Bruvelles So(Series a. 301:22. 1948. On leaves of Coffea camephora Pierte ex Frochn., Rubiaccac. Zaire. 
caminae (WV. Phillips \& C.B. Plowright) P.A. Saccardo, Sylloge Fungorum Onmium Hucusque Cognitorum Digessit P.A. Sitcardo 2:81. 1883. ESphaeria caninae IV. Phillips \& C.B. Plowright, Grevillea, London 6:27. 1877. On thallus of Peligera camina (L.) Willd.. Lichenes (Peltigeraceate). Great Britain. cannahina T. Ferraris \& C. Massa. Annales Mycologici. Berlin 10:286. 1912. On wilted leaves of Cammabis saria L.. Cannabaceac. Italy.

cammac D. McAlpine. Proceedings of the Linnean Society of New South Wales, Sydney 27:375. 1902. On upper surface of wilted leaves of Camma indica L., Cannaceae. Australia. ('uppuridicola B.B. Mundkur \& S. Ahnıad, Mycological Papers. Commonwealth Mycological Institute, Kew 18:4. 1946. On dead branches ol Capparis aphylla Roth. Capparaceae. India.

capparidis G. Passerinj. Revue Mycologique. Toulouse 2:34-35. 1880. On rotten branches of Capparis spinosa L.. Capparaceale. Italy.

caprifolii P. Brunaud, Bulletin de la Société

Botanique de France, Paris 34:429. 1887. On dead vines of Lonicera caprifolium L., Caprifoliaceae. France.

cupsularum F. Cavara. Fungi Longobardiae Exsiccati Sive Mycetum Specinnina in Longobardia Colfecta, exsiccati et Speciebus Novis Vel Criticis, Jconibus Illustrata, Pugillus 4. No. 174. Anno 1894. On dry capsules of Oenuhere hiemis L.. Onagraceae. Italy.

capsularum F. Cavara, Hedwigia, Dresden 34:(37).

1895: Botanisches Zentralblatt. Jena \& Dresden 61:316. 1895. On dried capsules of Ocnothers biemis L., Onagraceae. Italy

'arduima G. Patsserini, Auti del Reale Istituto Veneto di Lincei Rendiconti. Rome. Series 4. $4(2): 58-59.1888$. On bracts of Cardurs nurans L.. Compositac. Italy.

calduormm (K.F.W. Wallroth) V. Cesati \& G. de Notaris, Conmentario della Società Crittogamologica lialiana, Milan 1:235. 1863.

$\equiv$ Splucria carduorum K.F.W. Wallroth, Flora Crypogamica Germaniate 2:805. 1833. On dry stems of Cardums sp., Compositae. Belgium. France. Germany. Great Britain.

caricicola F. Finutrey in C. Roumeguère, Revue Mycologique. Toulouse 15:20. 1893: Fungi Selecti Gallici Exsiccati. Century 6.3, No. 6243. Anno 1893. EPhacosphaeria caricicola (F. Fautrey) A. Leuchtmann, Syclowia. Annales Mycologici. Horn. N.Ö. 37:109. 1984. On leaves of Carex pendula Hudson. Carex riparia Curtis, Cyperaceae. France. Swikerland.

caricina J. Schröter in F.J. Cohn. KryptogamenFlora Von Schlesien. Im Namen Der Schlesischen Gesellschaft fur vaterländische Cultur herausgegehen von Prol. Dr. Ferdinand Cohn. Secretair Der Botanischen Section, Breslats 3(2):361. 1894. On leaves of Carcipunicea

L. Carer siliarica atuct. Cyperaceate. Poland. caricinella P.A. Karsten, Ölversigt al K. Vetenshapsakademiens Forhandlingar, Stockhoim 29(2):100-101. 1872. EPhaeosphueria caricinella (P.A. Karsten) O. Eriksson, Arkiv för Botanik. Uppsala, Stockholn, Serics 2. 6:414. 1967. On dried leaves of Carex pulla Good. Cyperaceae. Canada. Norway.

cariciphila C.A. Oudemans, Nederlandsch kruidkundig Archief. Verslagen en Mededeelingen der Nederlandsche Botanische Vereeniging. Leiden, Series 3. 2:870. 1903. On sheaths of Carcx aremaria L.. Cyperaceac. Netherlands. ('aricis J. Schröter, Jahresbericht der Schlesischen Gesellschafı für vatterländische Kultur. Breshatu 58:175. (1880) 1881.

EPhaeosphaeria caricis (J. Schröter) A. Leuchtmann, Sydowia. Annales Mycologici. Horn, N.Ö. 37:147. 1984. On leaves of Carex hirla L. Carex pendula Hudson. Carex iaginafa Tausch, Dactylis glomerafa L.. Lu=ula sylvarica (Hudson) Gaudin, Typha larifolia L., Cyperaceae. Granineae. Juncaceae, Typhaceae. Canada, Czechostovakia, Sweden, Switzerland, U.S.A.

caricis-fimac F. Petrak, Sydowia. Annales Mycologici. Horn, N. Ö. 1(4-6):315. 1947. On living or dead leaves of Carex firma Host. Cyperaceae. Austria.

caricis-ıulpinuc A.1. Lobik, Bolezni Rastenii S. Peterburg (Morbi Plantarum Scripta Sectionis Phytopathologiae Horti Botanici Principalis) 17:161. 1928. On leaves of Carex rulpina L.. Cyperaceae. U.S.S.R.

(arlinoides M. Losia. Anales del Jardin Botánico de Madrid 8:305. (1947) 1948. On dead sems of Curdums carlimoides Gouan, Compositae. Spain.

cameomaculans F. Pearak. Annales Mycologici, Berlin 25:287-288. 1927. On dry stems of V'aleriana officimalis L. Valerianaceae. Czechoslovakia.

(anpogena P.A. Saccardo. Nuovo Giormate Botanico Italiano e Boltenino della Società Botanica Jaliana, Firenze 7:313. 1875. On dried capsules of leromica mricifolia Jace.. Scrophulariaccae. Italy.

(arpepplila P'A. Saccardo. Nuovo Giornate Botanico Italiano e Bolletino delfa Società Botanica Jalliana, Firenze 7:31.3-314. 1875. On rotsing capsules of \%ecomer ralicans Juss., Bignoniaceac. Haly.

(arprophila P'.A. Saccardo var. bruetearmm P.A. Saccardo, Michelia Commentariun Mycologicum Fungos in Primis Italicos Illustrans

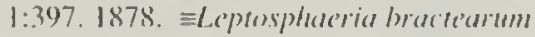
(P.A. Saccardo) P.A. Salccardo. Sylloge Fungorum Omnium Ilucusque Cognitorum Digessit P.A. Salccardo 2:58. 1883. On rotting 
bracts of heads of Dipsacus syliestris Hudson. Dipsacaceae. Italy.

cassiaecala J.B. Ellis \& B.M. Everhart, Journal of Mycology, Columbus, Ohio 2:41. 1886. On dead stems of Cassia sp., Leguminosae. U.S.A.

custa lu. Voronov, Leningrad. Vsesoiuznyi Institut Zashchity Rastenii. Otdel. Fitopatologii Materialy po Mikologii i Fitopatologii 4(1):60. 1922. On twigs of Pitex agmuscustus L., Verbenaceae. U.S.S.R.

casiugnei (M.C. Durieu de Maisonneuve \& J.P. Montagne) P.A. Saccardo. Nuovo Giomale Botanico ltaliano e Bolletino della Società Botanica Italiana, Firenze 7:317. 1875. Ephaeria castagnei M.C. Durieu de Maisonneuve \& J.P. Montagne in M.C. Durieu de Maisonneuve, Exploration Scientifique de L'Algérie Publiće Par Ordre du Gouvernement. Series Naturelles. Botanique I, Premiere Partie. p. 528. 1869. ESphaeria jasmini J.L. Castagne, Catalogue Des Plantes qui Croissent Naturellement aux Environs de Marseille, p. 167. 1845. Non Splacria jasmini L.D. v. Schweinitz. EPleospora jasmini (J.L. Castagne) L. Fuckel, Symbolae Mycologicae, p. 138. 1870. EParaphaeosphaeria castagnei (M.C. Durieu de Maisonneuve \& J.P. Montagne) $O$. Eriksson, Arkiv för Botanik, Uppsala, Stockholm, Series 2, 6:406, 1967. On hranches of Euonymus europacus L., Celastracene. France.

casrilleiae F.E. Clements, Bulletin of the Torrey Botanical Club (and Torreya), New York 30:85. 1903. On dead stems of Castilleja pallida (L.) Sprengel, Scrophulariaceae. U.S.A.

castillejae R.A. Shoenaker, Canadian Journal of Botany, Ottawa 62:2695-2696. 1984. On stems of Castilleja miniara Dougl. ex Hook.. Scrophulariaceae. U.S.A.

castrensis R.A. Shoemaker, Canadian Journal of Botany, Ottawa 62:2696. 1984. On stems of Lupimus obusilobus Heller, Leguminosae. U.S.A.

culalumica R. González Fragoso, introducción al Estudio de la Flórula de Micronicetos de Cataluña, Musei Barcinonensis Scientiarum Naturalium Opera. Series Botanica 2, pp. 89-90. 1917. Nom. inval. Art. 34.1; Flórula de Micromicetos de Cataluña (Publicacions de la Junta de Ciències Naturals de Barcelonat). pp. 89-90. 1917. Nom. inval. Art. 34.1. On thin hrancles of Smiler aspera L., Smilacaceae. Spain.

caltanci F. v. Thümen, Dic P'ilye der Reispllanze (Oryza soriva Lin.). Einc Monograplic, p. 5. I889 [as calaneil: Verlag der K.K. VersuchsStation lür Wein-tund Ohsthat zu KIos- terneuburg bei Wein. No. 12. p. 5. 1889.

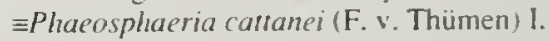

Miyake. Journal of the College of Agriculture. Imperial University of Tokyo $2(4): 247.1910$. ETremasosphaerella catranci (F. v. Thümen) G. Padwick, Manual of Rice Diseases. Commonwealth Mycological Institute, Kew: Surrey. p. 153. 1950. On wilted culms. sheaths, and leaves of Orya sativa L.. Gramineae. Italy.

cartunei (P.A. Saccardo) A. Jaczewski. Opredielitel* Gribov 2. Fungi Imperfecti (Petrograd). p. 608. 1917. EMerasphaeria carlanei P.A. Saccardo. Sylloge Fungorum Omnium Hucusque Cognitorum Digessit P.A. Saccardo 2:176. 1883. 三Pleospara endiusae (L. Fuckel) A. Cattaneo var, majar A. Cattaneo, Atti dell 'Istituto Botanico della Università e Laboratorio Crittogamica di Pavia. Mlilano. Series 1, 2:125. 1879. On wilted leaves of Oryza sativa L.. Granineae. Italy.

caucana F. Petrak. Sydowia. Annales Mycologici. Horn. N.Ö. 5(3-6):244. 1951. In the siromata of Plyllachara sp.. Fungi (Phyllachoraceae). Colombia.

caulincola (L.D. v. Schweinitz) M.A. Sherwood. Mycotaxon. An Intemational Joumal Designed to Expedite Publication of Research on Taxonomy \& Nomenclature of Fungi \& Lichens, Ithaca, New York 5:165. 1977. $\equiv$ Stictis caulincola L.D. v. Schweinitz. Synopsis Fungorum in America Boreali Media Digentium. Secundum Observationes Ludovici Davidis de Schweinitz. p. 179. 1832. EPhragmonue viacaulicola (L.D. v. Schweinitz) P.A. Saccardo, Sylloge Fungorum Onunium Hucusque Cognitorum Digessit P.A. Saccardo 8:676. 1889. On stems of unknown host, unknown fanily. U.S.A. (Pennsylvanial.

canlium P.A. Saccardo. Nonı, nud. [Falsely cited in literature as published in Reliquiae Libertianae II. No. 119. Anno 1881.] Vide Lephosphaeria vagabunda P.A. Saccardo var. cunlium C. Rounxeguère \& P.A. Saccardo.

comillesii M.J. de Urries y Azara. Anales del Jardin Botánico de Madrid 6:337-\$97. 1946. Nont. inval. Art. 36.1. On branch of Lavandula sp., Labiatae. Spain.

calvere M. Curzi. Atti dell Intituto Botanico della Universitia e Laboratorio Critogamica di Pavia. Milano, Series 3. 3:62. 1927. In living leaves of The sinensis L.. Theacese. Ital! commoli (N.C. Cooke of 11. W. Harkness) A.N. Berlese of P. Voglino, Sylloge Fungorum Ommium Hucusque Cognitorum Digensit P.A. Siccardo Additamenta Ad Volumina 1-IV. p. 136. ISs6. 三Sphueria (Didimosphacria) ceamolhi M.C. Coohe \& H.W. Harkness. Grevillea. London 13:19. 1895. On twiss of Commorhms yp.. Rhamnaceac. U.S.A. 
cehallosi L.M. Unamuno Yrigoyen. Boletin de la R. Sociedad Española de Historia Natural. Madrid 33:230-231. 1933. On dried leaves of Cyperus schenoides Griseb. Cyperaceac. Spain.

(ecreplace C.E. Chardon. Farlowia. A Journal ol Cryptogamic Botany. Cambridge, Massachusetts 2:466. 1946. On leaves of Cecropia pelsara L.. Unticaceae. Dominican Republic.

cencameae E. Müller. Sydowia. Annales Mycologici, Horn, N.Ö. 4(1-6):299. 1950. 三Nodulosphacria centaureae (E. Müller) L. Holm, Symbolae Botanicae Upsalienses. Uppsala 14(3):85. 1957. On dead stems of Cardarrs: defleratus L.. Ceminuea scabiosa L., Compositae. Switzerland.

cemmaficama A.M. Siccas, Étude de la Flore Cryptogamique des Caféiers en Alrique Centrale. Bulletin de Institut Français du Calé du Cacao et d'Autres Plantes Stimulantes (Bulletin IFCC No. 16). pp. 223-225. 1981. Nom. inval. Art. 37.1. On dead stems of Coffea excelsa Cheval. Rubiaceae. Central Alrican Republic.

cephalariai-mralensis [as cephalariae-uralensis]

N.A. Naumov \& T.L. Dobrozrakova,

Materialy po Mikologii i Fitopatologii Rossi. Petrograd 8(2):134. 1929. On dry stems ol Ceplalaria irulensis (Murray) Roemer \& Schultes, Dipsacaceac. U.S.S.R.

cerastii J. Felıgen, Vorstudien zu einer Pilz-Flora des Grossherzogthums, Luxemburg ](3):221. 1903. On dry sems of Elparorium cannahimam L., Compositae. Luxembourg.

crarispera (M.J. Berkeley \& M.A. Curtis) P.A. Succardo. Sylloge Fungorum Omnium Hucusque Cognitorum Digessit P.A. Saccardo 2:87. 1883. ESphaeria cerarispora M.J. Berkeley \& M.A. Curtis in M.J. Berkeley. Grevillea, London 4:150-151. 1876. On herbaceous stem of Zea sp.. Gramineae. U.S.A.

cercocupi H. Sydow \& P. Sydow, Annales Mycologici. Berlin 5:339. 1907. On dead leaves of Cercocarpus ledifolius Nutı. ex Torr. \& Gray. Rosaceae. U.S.A.

ceri-permitui W. Sienaszko. Acta Societatis

Botanicorum Poloniae. Warszawa 1:22. 1923.

On cladodes of Cereus perwiamus (I..) Miller. Cactaceac. Poland, U.S.S.R.

cerlenii C.L. Spegarcini, Michelia Commentarium Mycologicum Fungos in Prinis Italicos lllustrans 1:398. 1878: Rivista di Viticoltura. Enologia ed Agraria, Conegliano 3:54-56.

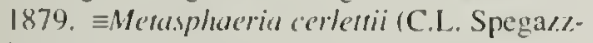
ini) P.A. Saccardo, Sylloge Fungorum Omnium Ilucusque Cognitorum Digessit P.A. Siccardo 2:167.1883. 三Chactopyrena cerleni (C.L. Spegazini) A. Trotter in P.A. Saccardo, Sylloge Fungorum Omnium
Hucusgue Cognitorum Digessit P.A. Saccardo 24:961. 1928. On dead vines ol l'inis vinifera L.. Vitaceac. Italy.

cenispere (K. Killchbremer \& M.C. Cooke) P.A.

Saccirdo, Sylloge Fungorum Omnium

Ihcuscue Cognitorum Digessit P.A. Saccardo 2:44. 1883, ESphacria comisyora K.

Kallibrenner \& M.C. Cooke. Grevillea.

London 9:29. 1880. On dead stems of

Arremisia sp.. Compositae. South Africa.

c'sarama (J.I'. Montagne ex V. Cesati \& G. de

Notaris) L. Holm, Symbolae Botanicate

Upsalienses, Uppsala 14(3):39. 1957.

$\equiv$ Whaphidospora cesatiana J.P. Montigne ex

V. Cesati \& G. de Notaris, Commentario della Società Crittogamologica Italiana, Milan 1:234. 186.3. EOphioholus c'esurianus (J.P.

Montagne ex V. Cesati \& G. de Notaris) P.A. Siccardo, Sylloge Fungorum Omnium Hucusque Cognitorum Digessil P.A. Saccardo 2:44. 1883. On stems of Echium v'tgare L.. occasionally on Brassica oleracea L..

Hypericam perforalmm L., Lycopus e'uropaews L., Boraginaceae, Cruciferac, Guttiferae.

Labiatae. Austria, Great Britain, ltaly.

chacossoma P.A. Saccardo, Michelia Commentarium Mycologicum Fungos in Primis Italicos lllustrans 2:533-534. 1882. 三Metasphaeria clicelosioma (P.A. Saccardo) P.A. Saccardo, Sylloge Fungorum Omnium Hucusque Cognitorum Digessit P.A. Saccardo 2:165. 1883. On vines (stems) of Viris vinifer L.. Vitaceate. lialy.

chamaeropis M. Chochrjakov. Notulae Systemalicae e Sectione Cryptogamica Instituti Botanici Nomine V.L. Komarov Academiae Scientiarum U.R.S.S., Petropolis 7:143-144. 1951. On living leaves of Chamuerops humilis L., Palmac. U.S.S.R.

\%hlidonii F. Fautrey in F. Fautrey and J.B. Latmbotte. Revue Mycologique. Toulouse 17:168. 1895. On dried stems of Chelidomimm majus L.. Papaveraceac. France.

(henopodii-albi W. Siemaszho. Acta Societatis

Botanicorum Poloniac. Warszawa 1:21. 1923.

On leaves of Chenopodium allom L..

Chenopodiaceac. U.S.S.R.

chilensis C.L. Spegazzini. Fungi Chilenses. p. 78. 1910: Revista de La Faculad de Agronomia Y Veterinaria, Universidad Nacional de Lal Plata. Series 2, 6:78. 1910. On dead branches of Lomatia obliqua R. Br.. Proteaceate. Chile. chorhjuhorii (L.N, Vasil'eva) L.N, Vasil'eva. Pirenomitsety i Lohuloaskomisety Severa Dal "nego Vostokal (I_eningrad:"Nauka”): 125.

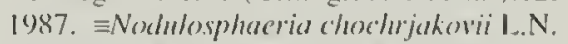
Vasil eva, Mikologiia i Fitopatologia Akademiia Nauh SSSR, Leningrald 13:278. 1979. On dead leaves ol /ledysumm ohrcumm L., Leguminosice. U.S.S.R. 
chondri L.K. Rosenvinge. Botanisk Tidsskrift. Kjobenhavn 27:XXX111-XXXV. 1906. $\equiv$ Leptosphaeria marina E. Rostrup. Non Leptosphueria marina J.B. Ellis \& B.M. Everhan.

chrysumhemi F.E. Clements \& E.S. Clements. Cryptogamae Formationum Coloradensium. Century 1, No. 24. Anno 1906. Nom. inval. Art. 36.1. On aged stems of Curdmus scopulormm Greene, Compositae. U.S.A.

chusqueue H. Sydow. Annales Mycologici. Berlin 37:376-377. 1939. On dead, deciduous leaves of Clusquea semwlua Pilger. Gramineae. Philippines.

cibostii (G. de Notaris) V. Cesati \& G. de Notaris. Commentario della Società Crittogamologica Italiana, Milan 1:235. 1863. ESphaeria cibostii G. de Notaris. Memorie della Accademia delle Scienze di Torino, Series 2. 13:103. 1853. On dead stems of unknown host. Umbelliferae. Italy.

cinclidoti A. Racovitza. Mémoires du Muséum Nationale d'Histoire Naturelle, Paris, Series B. Botanique 10:150. 1959. On dead phylloids of Cicclidoms fontinaloides (Hedw.) P. Beauv. [Cinclidotus is an orthographic variant.]. Musci (Pottiaceate). France.

(Metasphaeria) cincrea (L. Fuckel) H.G. Winter, Hedwigia, Dresden 26:58. 1887. 三Curbifaria cinerea L. Fuckel, Fungi Rhenani Exsiccati A Leopoldo Fuckel Collecti Supplement, Fascicle 6, No. 20+4. Anno 1867. $\equiv$ Sphaeria cinerea (L. Fuckel) L.

Fuckel, Symbolae Mycologicae, p. 117. 1870. $\equiv$ Metasphaeria cinerea (L. Fuckel) P.A. Saccardo. Sylloge Fungorum Omnium Hucusque Cognitorum Digessit P.A. Saccardo 2:166. 1883. On branches of Salit auria L.. Salicaceae. Austria.

cimnamomi M. Shirai \& K. Hara. Botanical Magazine. Tokyo 25:72. 1911. On diseased branches of Cimnamomum samphora T. Nees ex Eberm., Lauraceae. Japan.

circinans (L. Fuchel) P.A. Saccardo, Sylloge Fungorum Onnium Hucusque Cognitorum Digessit P.A. Saccardo 2:88. 1883. =Bvissothecium circinans $\mathrm{L}$. Fuchel, Botanische Zeitung, Berlin \& Leipzig 19:251. 1861. ETrematosphaeria circinans (L. Fuchel) H.G. Winter, Dr. L. Rabenhorst 's KryptogamanFlora von Deutschland, Oesterteich und der Schweiz, Sccond edition. 1(2) Ascomyceten: Gymnoasceen und Pyrenomyceten. p. 277. 1885. $\equiv$ Passeriniella circinans (L. Fuckel) A.N. Berlese in P.A. Saccardo, Sylloge Fungorum Omniun Hucusque Cognitorum Digessit P.A. Saccardo 1 1:326. 1895. On roots and stems of Medicager sativa L.. leguminosie. Germany.

cinteola G. Passerini. Rendiconti della Sedute della R. Accademia dei Lincei. Classe da Scienze
Fisiche, Matematiche e Natural (= Atti dell Accademia Nazionale dei Lincei. Rendiconti. Roma), Series 4. 3:90. 1887. On dny denuded stems of Iivis vinifera L.. Vitaceae. Italy:

cirsii-an'ensis M. Losa. Anales del Jardin Botänico de Madrid 8:306. (1947) 1948. On dry culms of Cirsium arvense (L.) Scop.. Compositac. Spain.

cissi L. Celotti. Miceti del Parco e Dintomi della Scuola Nazionale di Agricoltura di Montpellier Raccolti e Studiati. Congliano. p. 32. 1887. On branches of Cistus alhidus L.. Cistaceae. France.

cisticola M. de Sousa da Camara, Agronomia Lusitana, Sacavém 9:93-94. 1947. On branches of Cistus monspeliensis L.. Cistaceae. Portugal.

cistina M.J. de Urries y Azara. Boletin de la R. Sociedad Española de Historia Natural. Madrid 33:95. 1933. On dead branchlets of Cistus ladanifer L.. Cistaceae. Spain.

cirricola P.A. Saccardo. Nichelia Commentarium Nycologicum Fungos in Primis Italicos Illustrans 2:416. 1882. On living leaves of Cirrus sp.. Rutaceae. Italy.

cladii D. Cruchet. Bulletin de la Société Vaudoive des Sciences Naturelles, Lausanne 55:161. 1923. On roots of Cladium mariscus (L.) Pohl. Cyperaceae. Switzerland.

cladophila J. Schröler, Jahresbericht der Schlesischen Gesellschaft für vaterländische Kultur. Bresliau 58:174. (1880) 1881. On living stems of Rihes alpimum L.. Saxifragaceae. Sweden.

clum (B. Auerswald) P.A. Saccardo. Sylloge Fungorum Omnium Hucusque Cognitorum Digessit P.A. Saccardo 2:73. 1883. Esplaeria clara B. Auerswald in M.C. Cooke. Grevillea, London 5:121. 1877. On leaves of Carex sp.. Spurganim sp.. Cyperaceac. Sparganiaceae. Germany. Great Britain.

Clarkii D. Hawksworth. Transactions of the British Mycological Society: London 74:376. 1980. On thallus of Pelnigera cf. rufescens (Weis.) Humb.. Lichenes (Peltigeraceae). Great Britain.

clavala A.L. Guyot. Revue de Mycologgie. Paris

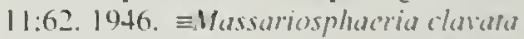
(A.L. Guyol) R.A. Shoemaker, Canadian Journal of Botany. Ottawa 67:1582. 1989. On dry stems and leaven of Kocleria allesecns DC.. Koclerios gracilis Pers.. Gramineae. France.

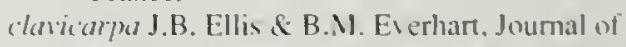
Mcology. Columbus. Ohio 1:4 i-44. 1855: Hedwigia. Dresden 25:109. 1886.

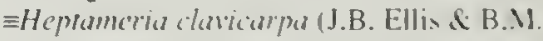
Everhart) M.C. Coohe. Grevillea. London 18:32. 1859. On dead culmo of Phragmites (onmmunis Trin.. Granincade. U.S.A. 
clavigera (M.C. Cooke \& J.B. Ellis) P.A. Saccardo, Sylloge Fungorum Omnium Hucusque Cognitorum Digessit P.A. Saccardo 2:44. 1883. ISphacria (Caulicolae) clavigera M.C. Cooke \& J.B. Ellis, Grevillea, London 6:16. 1877. On stems of Phytalacca americana L., Phytolaccaceae. U.S.A.

clavispora J.H. Miller \& M.G. Burton, Mycologia, Lancaster, Pennsylvania 34:1-2. 1942. On dead stems of Eupatorium capillifolium (Lam.) Small, Compositae. U.S.A.

clelandii C.G. Hansford, Proceedings of the Linnean Society of New South Wales, Sydney 82:216. 1957. On dead branches of Acacia kempeana F. Muell., Leguminosae. Australia.

clerodendri S.A. Gucevicz, Novosti Sistematiki Nizshikh Rastenij, Novitates Systematicae Plantarum Non Vascularium 7:159. 1970. On bark and denuded wood of Clerodendrum foctidi Bunge, Verbenaceae. U.S.S.R. clivensis (M.J. Berkeley \& C.E. Broome) G.L. Rabenhorst, Fungi Europaei Exsiccati, Klotzschii Herbarii vivi Mycologici Continuatio. III Ausgabe (Editio nova, Series Secunda), Century 10, No. 947. Anno 1866. ESphaeria clivensis M.J. Berkeley \& C.E. Broome. Annals and Magazine of Natural History, London, Series 2, 9:379. 1852. EPleospora bardanae G. Niessl v. Mayendorf, Verhandlungen des Naturforschenden Vereins in Brünn 14:178. 1876. On stems of Senecio atratus Greene, Compositae. U.S.A.

clivensis (M.J. Berkeley \& C.E. Broome) P.A. Saccardo, Sylloge Fungorum Omnium Hucusque Cognitorum Digessit P.A. Saccardo 2:16. 1883. Nom. illegit. Art. 64.1. ESphaeria (Caulicolae) clivensis M.J. Berkeley \& C.E. Broome. Annals and Magazine of Natural History, London. Series 2, 9:379. 1852. EDiapleella clivensis (M.J. Berkeley \& C.E. Broome) A. Munk, Dansk Botanisk Arkiv, Kjøbenhavn 15(2):75. 1953. „Kalmusia clisensis (M.J. Berkeley \& C.E. Broome) M.E. Barr, Mycotaxon. An International Journal Designed to Expedite Publication of Research on Taxonomy \& Nomenclature of Fungi \& Lichens, Ithaca, New York 29:504. 1987. On dead stems of Pastinaca sativa L., Senccio atratus Greene, Umbelliferae, Compositae. Germany, Great Britain, U.S.A.

clivensis (M.J. Berkeley \& C.E. Broome) P.A.

Saccardo var. constricta F.E. Clements \& E.S. Clements, Cryptogamac Formationum Coloradensium, Century 5, No. 433. Anno 1908. Nom. nud. Art. 32.I. On aged stems of Senecio atratus Greene, Compositae. U.S.A. coccodes P.A. Karsten, Fungi Fenniae Exsiccati, Century 10, No. 963. Anno 1870. इMetasphaeria coccodes (P.A. Karsten) P.A.
Saccardo. Sylloge Fungorum Omnium Hucusque Cognitorum Digessit P.A. Saccardo 2:174. 1883. On culms of Calamagrostis sp., Gramineae. Finland.

coccothrinacis R. Ciferri \& R. González Fragoso.

Boletin de la R. Sociedad Española de

Historia Natural, Madrid 26:251-252. 1926.

On living leaves of Coccothrinax argentata

(Jacq.) Bailey, Palmae. Dominican Republic.

cocoes J. Verissimo d Almeida \& M. de Sousa da

Camara, Revista Agronómica, Lisbon

2(12):384. 1904; Boletim da Sociedade

Broteriana, Coimbra 24:163-164. 1909. On

leaves of Cocos romanzaffiana Cham.

[=Arccastrum romanzoffianum (Cham.)

Beccari], Palmae. Portugal.

coffaeicida C.L. Spegazzini, Boletin de la Academia Nacional de Ciencias en Córdoba 23:560-561. 1918. On living leaves of Coffea sp.,

Rubiaceae. Costa Rica.

coffeigena (M.J. Berkeley \& M.A. Curtis) P.A.

Saccardo, Sylloge Fungorum Omnium

Hucusque Cognitorum Digessit P.A. Saccardo 2:51 . 1883. ESphaeria (Foliicolae) cof-

feigena M.J. Berkeley \& M.A. Curtis, Joumal of the Linnean Society (Botany) London 10:389. 1868: Fungi Cubenses No. 862. Anno 1868. On leaves of Coffea arabica L..

Rubiaceae. Cuba.

coffeigena (M.J. Berkeley \& M.A. Curtis) P.A.

Saccardo var. longirostrata C. Moreau \& M.

Moreau, Revue de Mycologie, Paris (Supplement Colonial No. 1) 16:21-25. 1951. On trunk and branches of Coffea canephora Pierre ex Froehn., Rubiaceae. Ivory Coast.

coic is K. Sawada, Report. Government Research Institute, Department of Agriculture, Formosa 87:30. 1944. [Descriptive catalogue of the Formosan Fungi...Taihoku, Formosa 10:30. 1944.] Nom. inval. Art. 36.1. On leaves of Coix lacryma-johi L. var. susutama Honda, Gramineae. Taiwan.

colcosanthi C.E. Fairman, Mycologia, Lancaster.

Pennsylvania 10:246. 1918. On old stems of Colcosunthus reniformis (Gray) Rydb. $(=$ Brickellia), Compositae. U.S.A. (New Mexico).

collinsoniae J. Dearness \& H.D. House, Bulletin of the New York State Museum, Albany 233-234:36-37. (1920) 1921. On dead. usually blackened stems of Collinsonia canadensis L., Labiatae. U.S.A.

collumiae (M.J. Berkeley \& M.A. Curtis) P.A

Saccardo, Sylloge Fungorum Omnium

Hucusque Cognitorum Digessit P.A. Saccardo 2:50. 1883. =Sphacria collumiae M.J.

Berkeley \& M.A. Curtis, Proccedings of the American Academy of Arts and Sciences, Boston 4:130. 1860 [as cullumiae]. On leaves of Collomia squarrosa Nuti., Polemoniaceac. South Africa. 
colocasiae L.M. Unamuno Yrigoyen, Memórias de la R. Sociedad Española de Historia Natural, Madrid 15:346. 1929. On living leaves of Colocasia antiquorum Schott, Araceae. Spain.

comatella (M.C. Cooke \& J.B. Ellis) P.A. Saccardo, Sylloge Fungorum Omnium Hucusque Cognitorum Digessit P.A. Saccardo 2:32. 1883. ESphaeria (Caulicolae) comatella M.C. Cooke \& J.B. Ellis, Grevillea, London 5:52. 1876. On stems of Asparagus sp., Baptisia sp., Daucus sp., Solanum sp., Liliaceae, Leguminosae, Umbelliferae, Solanaceae. U.S.A.

complanata (H.J. Tode:E.M. Fries) V. Cesati \& G. de Notaris, Commentario della Società Crittogamologica Italiana, Milan I:236. 1863. EShaeria complanata H.J. Tode:E.M. Fries, Fungi Mecklenburgenses Selecti 2:21 . 1791; E.M. Fries, Systema Mycologicum Sistens Fungorum 2:508. 1823. 三Metasphaeria complanata (H.J. Tode:E.M. Fries) P.A. Saccardo, Sylloge Fungorum Omnium Hucusque Cognitorum Digessit P.A. Saccardo 2:161. 1883. On herbaceous stems of unknown host, unknown family. Great Britain, U.S.A.

compositarum E. Müller, Sydowia. Annales Mycologici, Horn, N.Ö. 4(1-6):213. 1950. =Massariosphaeria compositarum (E. Müller) A. Leuchtmann, Sydowia. Annales Mycologici, Horn, N.Ö. 37:173. 1984. On dead stems of unknown host, Compositae. Switzerland.

compressa (H. Rehm) L. Holm, Symbolae Botanicae Upsalienses 14(3):29. 1957. 三Ophiobolus compressus H. Rehm, Bericht des Naturwissenschaftlichen Vereins für Schwaben und Neuberg, Augsburg 26:49. 1881.

ERhaphidospora compressa $\mathrm{H}$. Rehm. Ascomyceten, Fascicle 4, No. 189. Anno 1873. Nom. nud. Art. 32.1. On dry stems of Achillea millcfolium auct., Artemisia austriaca Jacq., Arremisia campestris L., Cirsium altissimum (L.) Spreng., Compositae. Hungary, Sweden, U.S.A.

concentrica J.B. Ellis \& B.M. Everhart. The North American Pyrenomycetes. A Contribution to Mycologic Botany, p. 354. 1892. On leaves of Malus sp., Rosaceae. U.S.A.

concimna J.B. Ellis \& B.M. Everhart, Proceedings of the Academy of Natural Sciences of Philadelphia 1895:423. 1895. On dead stems of Castillcja miniata Doug. ex Hook., Scrophulariaceae. U.S.A.

conferta G. Niessl v. Mayendorl in P.A. Saccardo. Sylloge Fungorum Onnium Hucusque Cognitorum Digessit P.A. Saccardo 2:20. 1883. On stems of Farsetia incana (L.) R. Br., Cruciferae. Czechoslovakia. congesta M.T. Lucas. Transactions of the British Mycological Society. London 46:362. 1963. On stems of Erigeron canadensis L., Compositae. Portugal.

coniformis (E.M. Fries:E.M. Fries) J. Schröter in F.J. Cohn, Kryptogamen-Flora Von Schlesien. Im Namen Der Schlesischen Gesellschaft für vaterländische Cultur herausgegeben von Prof. Dr. Ferdinand Cohn, Secretair der Botanischen Section, Breslau 3(2):369. 1894 [as conformis]. On dead stems of Urica dioica L., Urticaceae. Poland.

coniformis (E.M. Fries:E.M. Fries) H.G. Winter, Dr. L. Rabenhorst's Kryprogaman-Flora von Deutschland, Oesterreich und der Schweiz, Second edition. 1(2):469. 1885 [as (L. Fuckel) Winter]. ESphaeria coniformis E.M. Fries:E.M. Fries, Systema Mycologicum Fungorum 2:508. 1823. EPleospora coniformis (E.M. Fries:E.M. Fries) L. Fuckel. Symbolae Mycologicae, p. 136. 1870. =Metasphaeria coniformis (E.M1. Fries:E.M1. Fries) P.A. Saccardo. Sylloge Fungorum Omnium Hucusque Cognitorum Digessit P.A. Saccardo 2:159. 1883. On dry stems of Eluphrasia Iutea L., Scrophulariaceae. Austria.

(Merasphaeria) coniformis (E.M. Fries:E.M. Fries) H.G. Winter, Hedwigia, Dresden 26:58. 1887 [as (L. Fuckel) Winter]. Nom. illegit. Ar. 64.1. On stems of Euphrasia lutea L.. Scrophulariaceae. Austria.

conii C.L. Spegazzini, Anales del Museo Nacional de Historia Natural de Buenos Aires 19(Series 3, 12):382-383. 1909: Mycetes Argentinenses, Series 4, p. 382. No. 628. Anno 1909. Nom. illegit. Art. 64.1. Non Leptosphaeria conii E. Rostrup. ELeprosphaeria coniigena P.A. Saccardo \& A. Trotter. On decaying stems of Conium maculatum L., Umbelliferae. Argentina.

conii E. Rostrup. Botanisk Tidsskrift, Kjobenhavn 26:311. 1905. On stems of Conium maculatum L., Unbelliferae. Denmark.

coniigena P.A. Saccardo \& A. Trotter. Sylloge Fungorum Omnium Hucusque Cognitorum Digessit P.A. Saccardo 22:220. 1913. $\equiv$ Leprosphacria comii C.L. Spegazzini. nec Leptosphacria conii E. Rostrup. On stems of Conium maculatum L.. Unbelliferae. Argentina.

conimbricensis A.N. Berlese \& F. Saccardo, Revue Mycologique. Toulouse 11:121. 1859. On dead culms of unknown host of ?grass. ?Gramineae. Portugal.

coniothyrium (L. Fuchel) P.A. Saccardo. Nuovo Giomale Botanico Italiano e Bolletino della Società Botanica ltaliana. Firenzc 7:317. 1875. ESpharvia conioshwium L. Fuckel. Symbolae Mycologicae. p. 115. 1870. 
$\equiv$ Melanomma coniothyrium (L. Fuckel) L. Holm, Symbolae Botanicae Upsalienses, Uppsala 14(3):56. 1957. On stems of Almus glutinosa (L.) Gaertner, Broussometiasp.. Chimonumluss sp., Kerria sp., Menispermum canadense L., Rosa sp., Rubus fruticosis L.. Salix vitellina L., Samhucus nigra L.. Betulaceac, Moraceae, Calycanthaceae, Rosaceae, Menispermaceae, Salicaceac, Caprifoliaceae. Austria, France, Germany, ltaly, Portugal.

(o)miothrium (L. Fuckel) P.A. Saccardo forma berberidis (C.E. Richon) R. Ciferri, Annales Mycologici, Berlin 20:51. 1922. ELeptosphacria herberidis C.E. Richon, Catalogue Raisonné des Champignons Qui Croissent Darrs le Département de la Mame, p. 300. 1889. On leaves and branchlets of Berheris rulgaris L., Berberidaceae. France. Italy. coniothyrium P.A. Saccardo var. folicola N.N. Woronichin. Vestnik Tiflisskogo Botanicheskogo Sada, Tiflis (Moniteur du Jardin Botanique de Tiflis) 28:17. 1913. On living leaves of Lumis sp., Lauraceae. U.S.S.R. (Caucasus).

conoidea (V. Cesati \& G. de Notaris) P.A. Saccardo, Nuovo Giornale Botanico ltaliano e Bolletino della Societa Botanica ltaliana. Firenze 7:314. 1875. ESphaeria doliolum (C.H. Persoon: E.M. Fries) V. Cesati \& G. de Notaris var. conoidea G. de Notaris, Memorie della Accademia delle Scienze di Torino, Series 2. 16:466. 1856. On stems of Angelica syliestris L., Umbellilerae. Italy.

conoidea (V. Cesatti \& G. de Notaris) P.A. Saccardo forma angelicae $\mathrm{F}$. Fautrey in $\mathrm{C}$.

Roumeguère, Fungi Selecti Gallici Exsiccati, Century 56, No. 5536. Anno 1891; Revue Mycologique. Toulouse 13:8. 1891. On dry stems of Angelica sylvestris L., Umbelliferae. France.

conoidea (V. Cesati \& G. de Notaris) P.A. Saccardo forma asteris F. Fautrey, Revue Mycologique, Toulouse 16:112. 1894: Fungi Selecti Gallici Exsiccati, Century 66, No. 6552. Anno 1894. On dry stems of Aster sulignus Willd.. Compositae. France.

conoidea (V. Cesati \& G. de Notaris) P.A. Saccardo forma macrospora F. Fautrey, Revue Mycologique, Toulouse I 1:152. 1889. On rotten stems of Urtica divica L.. Unticaceac. France.

consessa (M.C. Cooke \& J.B. Ellis) P.A. Saccardo, Sylloge Fungorum Omnium llucusque Cognitorum Digessit P.A. Saccardo 2:17. 1883. ESphaeria (Canlicolae) consessa M.C. Cooke \& J.B. Ellis, Grevillea, London 6:95. 1878. On stems of lletianthus sp.. Compositae. U.S.A. consimilis J.B. Ellis \& B.M. Everhart, Joumal of Mycology, Columbus, Ohio 2:41. 1886. On dead limbs of Salix sp., Salicaceae. U.S.A.

consobrina P.A. Karsten, Översigt af K. Vetenskapsakademiens Forhandlingar, Stockholm 29(2):102-103. 1872. EP/haeosphaeria consolrina (P.A. Karsten) O. Eriksson, Arkiv för Botanik. Uppsala, Stockholm. Series 2. 6:415. 1967. On dried leaves ol Carex pulla Good., Cyperaceae. Norway.

consocians: (W. Nylander) P.A. Saccardo \& D.

Saccardo in P.A. Saccardo, Sylloge Fungorum Onnium Hucusque Cognitorum Digessit P.A. Saccardo 17:730. 1905. $\equiv$ Mycopormm consocians W. Nylander, Flora, Jena und Regensburg 55:364. 1872. On thalli and apothecia of Lecidea vermalis (L.) Ach., Lichenes (Lecideaceae). U.S.S.R.

ronsociata H. Rehm. Hedwigia, Dresden 35:149. 1896: Ascomyceten, Fascicle 24, No. 40. Anno 1896. 三Monographella consociata (H. Rehm) O. Eriksson \& J. Yue, Mycotaxon. An International Journal Designed to Expedite Publication of Research on Taxonomy \& Nomenclature of Fungi \& Lichens, lthaca, New York 38:205. 1990. On leaves of Chusquea sp., Gramineae. Ecuador.

constricta (G. Bresadola) F. Hazslinszky, Matematikai és Természeltudományi Közlemenyek Vonatkozólag a Hazai Viszonyokra, Budapest 25(2):148. 1892. EMefasphacria constricta G. Bresadola, Revue Mycologique, Toulouse 12:185. 1890. On branches of Euonymus curopacus L.. Celastraceae. Hungary.

comecta J. Kohlmeyer, Nova Hedwigia. Zeitschrili für Kryptogramenkunde, Weinheim 6:314. 1963. On rotten wood in marine waters on unknown host, unknown family. Germany.

controversu (J. Desmazières) V. Cesati \& G. de Notaris, Commentario della Società Crittogamologica Ltaliana, Milan 1:235. 1863. ESphaeria comroversa J. Desmazières, Anuales des Sciences Naturelles, Paris, Botanique. Series 2. 17:102. 1842. $\equiv D i$ aporthe controtersa (J. Desmazières) T.R.J. Nitschke in L. Fuckel. Symbolae Mycologicae, Erster Nachtrag, p. 319. 1871. =Diaporthe eres T.R.J. Nitschke fide L.E. Wehmeyer, The Genus Diaporthe Nitschke and Its Segregates, p. 63. 1933. On plant stems, on dry branches of Acer sp.. Fravimus sp., Rubus sp.. Aceraceac. Oleaceae, Rosaceac. France.

comallarias' J.V. d'Almeida \& M. de Sousa da Camara, Boletim da Sociedade Broteniana, Coimbra 24:164. 1908/1909. Nom. illegit. Art. 64.1. ELeptosphaeria dracaenae M. de Sousa da Camara. On dead leaves of Dracaena draco (L.) L.. Agavaceae. Portugal. 
convallariae (L. Fuckel) P.A. Saccardo, Michelia Commentarium Mycologicum Fungos in Primis ltalicos Illustrans 1:38. 1877. EPleospora convallariae L. Fuckel, Symbolae Mycologicae, pp. 138-139. 1870. On dry stems of Convallaria multiflora L., Liliaceae. France.

comvallariae J.V. d'Almeida \& M. de Sousa da Camara forma dracaenae J.V. d'Almeida \& M. de Sousa da Camara, Boletim da Sociedade Broteriana, Coimbra 24:164. 1908/ 1909. On dead leaves of Dracaena draco (L.) L., Agavaceae. Portugal.

comvallariae J.V. d'Almeida \& M. de Sousa da Camara forma rusci J.V. d'Almeida \& M. de Sousa da Camara, Boletim da Sociedade Broteriana, Coimbra 24:164. 1908/1909. On dead leaves of Ruscus aculeatus L., Ruscaceae. Portugal.

cookei R. Pirotta, Atti dell' Istituto Botanico della Università e Laboratorio Crittogamica di Pavia. Milano 2 \& 3:162-163. (1877) 1879 [as cookii]. On cortex of living runners on Vitis vinifera L., Vitaceae. Great Britain.

coorgica K.H. Anahosur, Sydowia. Annales Mycologici, Horn, N.Ö. 24(1-6):286-287. (1970) 1971. On living twigs of Pteridium aquilimum (L.) Kuhn, Dennstaedıiaceae. India.

corac N. Patouillard, Journal de Botanique, Paris 2:150. 1888. On thallus of Cora pavonia (Swartz) Fries (=Dictyonema), Lichenes (Thelephoraceae). Central America.

corallorhizae C.H. Peck, Report. New York State Museum of Natural History, Albany 38:105. 1885. On dead stems of Corallorrhiza multiflora Nutt., Orchidaceae. U.S.A.

cordylines (C.L. Spegazzini) P.A. Saccardo \& A. Trotter in P.A. Saccardo, Sylloge Fungorum Omnium Hucusque Cognitorum Digessit P.A. Saccardo 22:231. 1913. [Incorrectly cited as a comb. nov. based on Leptosphaerella cordylines C.L. Spegazzini. Nom. inval. Art. 43.1.] On dry leaves of Cordyline dracaenoides Kunth, Agavaceae. Argentina.

cornuta E. Müller, Sydowia. Annales Mycologici, Horn, N.Ö. 7(1-4):273-274. 1953. On dead stems of Lascrpitium siler L., Unbelliferae. Switzerland.

coromillac G. Moesz, Botanikai Közlemények. Budapest 28:164. 1931. On dead stems of Coronilia sp. [as C. comata L.], Leguminosae. Hungary.

comugans H. Rehm, Österreichische Botanische Zeitschrift. Wien 54:84. 1904. On living leaves of Cytisus alpinus Miller, leguminosae. Yugoslavia.

corticola (L. Fuckel) P.A. Saccardo. Michelia Commentarium Mycologicum Fungos in Primis Italicos Illustrans 1:342. 1878; Fungi
Italici autographice delineati (additis nonnullis extra-italicis asterisco notatis). Patavii, Table 288. 1878. ESphacria corticala L. Fuckel. Symbolae Mycologicae, p. 114. 1870. =Metasphaeria carticola (L. Fuckel) P.A. Saccardo. Sylloge Fungorum Omnium Hucusque Cognitorum Digessit P.A. Saccardo 2:166. 1883. EPlensphaerulina corticala (L. Fuckel) H. Rehm. Annales Mycologici. Berlin 10:538. 1912. EClathridium corticola ( $\mathrm{L}$. Fuckel) R.A. Shoemaker \& E. Müller, Canadian Journal of Botany. Otrawa 42:404. 1964. 三Griphosphaeria corticola (L. Fuckel) F. v. Höhnel, Annales Mycologici, Berlin 16:87. 1918. EDiscostroma corricola (L. Fuckel) 1. Brockmann, Sydowia. Annales Mycologici, Horn, N.Ö. 28:313. (]975) 1976. On cortex of branchlets, on dry stems of Cornus sanguinea L., Lonicera caprifolium

L., Prunus baccata Borkh.. Prumus domestica L., Prumus spinosa L., Rosa sp., Caprifoliaceae, Rosaceae. Austria, Canada, Czechoslovakia, Germany, Hungary, ltaly, Switzerland.

conina (E. Rostrup) J. Lind. Danish Fungi as Represented in the Herbarium of E. Rostrup. Nordisk Forlag, Copenhagen, p. 217. 1913. =Metasphaeria comina E. Rostrup. Dansk Botanisk Forening. Copenhagen, Meddelsher 2(4):90-91. 1888. On rotten feathers of Conus comix [Animalia]. Corvidae. Denmark.

corymispora A.N. Berlese \& G. Bresadola. Annuario della società Degli Alpinisti Tridentini 14:328-329. 1889; Micromycetes Tridentini Contribuzione Allo Studio dei Funghi Microscopici del Trentino, pp. 32-33. 1899. On dead stems of Cirsium lanceolatum (L.) Scop., Compositae. Italy.

cosmicola A. Chiplonkar, Sydowia. Annales Mycologici, Horn, N.O. 22(1-1):27+-275. (1968) 1969 [as cosmosicola]. On dried stems of Cosmos sulphurcus Cav.. Compositae. India.

coumarnumae R. González Fragoso \& R. Ciferri. Boletin de la R. Sociedad Española de Historia Natural, Madrid 27:326-327. 1927. Nom. nud. Art. 34.1. On living leaves of Coumarouna punctata S.F. Blake. Leguminosae. Dominican Republic.

commaromac R. González Fragoso \& R. Ciferri. Publicaciones Estación Agronómica de Moca. Santa Domingo. Series B. Botanica. No. 11:22. 1928. Nom. inval. Art. 34.1. On living leaves of Coumarouna puncrara S.F. Blake. Leguminosae. Dominican Republic.

crastophila (P.A. Saccardo) P.A. Saccardo. Nuovo Giomale Botanico Italiano e Bolletino della Socicta Botanica Italiana, Firenze 7:321. 1875. Esphacrella crastophila P.A. Sac- 
cardo, Atti dell Accademia Scientifica Veneto-Trentino-1striana. Padova 2(2): 142. 1873. On leaves of Fesuca sp., Gramineae. ltaly.

crastophila P.A. Saccardo forma rofieldiae P.A. Saccardo in G. Bresadola and P.A. Saccardo. Malpighia. Rassegna Mensuale di Botanica. Messina, Genova 11:289. 1897. On stems and leaves of Toficldia calyculata (L.) Wahlenb., Liliaceae. Italy.

crepini (G.D. Westendorp) G. de Notaris, Commentario della Società Crittogamologica ltaliana. Milan 2:486. 1867. ESphaeria crepini G.D. Westendorp. Bulletins de L'Académie Royale des Sciences, des Lettres et des Beaux-Arts de Beigique, Brussels, Series 2, 7:88. 1859. On bracteoles of spikes on Lycopodium annotinum L., Lycopodiaceae. Belgium, Italy. crozalsiana R.C. Maire, Annales Mycologici, Berlin 11:352. 1913. On dead branches of Tamarix sp., Tamaricaceae. Algeria.

crozalsii L. Vouaux, Bulletin de la Société Mycologique de France, Paris 29:120. 1913 [as crozalsi]. On thallus of Aspicilia calcarea (L.) Korb [=Lecanora calcarea (L.) Sommerf.]. Placodium reicholytum (Ach.) DC., Lichenes (Teloschistaceae). France. crucheni E. Muiller, Sydowia. Annales Mycologici, Horn, N.Ö. 4(1-6):255-256. 1950. 三Melasphaeria cladii D. Cruchet, Bullerin de la Société Vaudoise des Sciences Naturelles. Lausanne 55:166-167. 1923. Non Leptosphacria cladii D. Cruchet, 1923. On branches of Cladium mariscus (L.) Pohl. Araceae. Switzerland.

crucnta P.A. Saccardo. Michelia Commentarium Mycologicum Fungos in Primis lialicos Illustrans 2:318-319. 1881. On dead stems of Thalictum flavum L.. Ranunculaceae. France.

crustacea J. Schröter in F.J. Cohn, KryptogamenFlora Von Schlesien. Im Namen Der Schlesischen Gesellschaft für vaterländische Culıur herausgegeben von Prof. Dr. Ferdinand Cohn. Secretair der Botanischen Section. Breslau 3(2):365. 1894. On dead stems of Artemisia sp., Compositae. Poland.

cryprica H. Sydow. Annales Mycologici, Berlin 28:85-86. 1930. Parasitic on stromata of Phyllachora sp., Fungi (Phyllachoraceae). Venezuela.

cururbitae L. Montemartini. Rivista di Patologia Vegetale, Padova 8:185. 1916. On leaves of

Cucurbita pepo L., Cucurbitaceae. Italy. cucurbitarioides J.H. Fabre, Annales des Sciences Naturelles, Paris, Botanique, Scries 6. 15:50. 1883. On more or less dry stems of Dorycnium suffruticosum Vili., Leguminosae. France. culmicola (E.M. Fries:E.M. Fries) G.L. Rabenhorst, Botanische Zeitung, Berlin \& Leipzig 24:412. 1866. ĐSphaeria culmicola E.M. Fries:E.M. Fries, Systema Mycologicum Sistens Fungorum 2:430. 1823. On grass culms of unknown host of Gramineae, Gramineae. Italy.

culmicola (E.M. Fries:E.M. Fries) P.A. Karsten, Mycologia Fennica Pars 2. Pyrenomycetes. p. 108. 1873. Nom, illegit. Art. 64.1. ESphaeria culmicola E.M. Fries:E.M. Fries, Systema Mycologicum Fungorum 2:430. 1823. On dried culms of unknown host of Gramineae, Gramineae. Italy.

culmicola (E.M. Fries) P.A. Karsten forma major P.A. Saccardo, Nuovo Giornale Botanico ltaliano e Bolletino della Società Botanica Italiana, Firenze 7:323. 1875. On stems of grass, Gramineae. ltaly.

culmicola (E.M. Fries:E.M. Fries) P.A. Karsten forma melicae $\mathrm{F}$. Fautrey in $\mathrm{C}$. Roumeguère. Revue Mycologique, Toulouse 17:176. 1895; Fungi Selecti Gallici Exsiccati, Century 69. No. 6847. Anno 1895. On sheaths of dry straw of Melica altissima L., Gramineae. France?

rulmicola (E.M. Fries:E.M. Fries) P.A. Karsten var aquarica P.A. Saccardo, Michelia Commentarium Mycologicum Fungos in Primis lalicos Illustrans 2:319. 1881. On reeds of Scirpus lacustris L., Cyperaceae. France.

culmicola (E.M. Fries:E.M. Fries) P.A. Karsten var. hispalensis R. González Fragoso, Trabajos del Museo Nacional de Ciencias Naturales, Madrid, Series Botanica 10:87. 1916. On leaves and sheaths of Phragmites communis Trin., Gramineae. Spain.

culmicola (E.M. Fries:E.M. Fries) P.A. Karsten var. minor P.A. Saccardo, Nuovo Giomale Botanico Italiano e Bolletino della Società Botanica ltaliana. Firenze 7:322-323. 1875. On stems of grass, Gramineae. Haly.

culmicola (E.M. Fries:E.M. Fries) P.A. Karsten var. nigrans (M.R. Roberge) P.A. Karsten, Mycologia Fennica Pars 2, Pyrenomycetes, pp. 108-109. 1873. ESphaeria (Caulicola) nigrans M.R. Roberge in J. Desmazières. Annales des Sciences Naturelles, Paris. Botanique, Series 3, 6:79. 1846. 三Lcptosphaeria nigrans (M.R. Roberge) V. Cesati \& G. de Notaris. On sheath of Dactylis glomerata L.. Gramineae. Finland, France.

culmicola (E.M. Fries:E.M. Fries) P.A. Karsten var. rhizomatum P.A. Saccardo. Michelia Commentarium Mycologicum Fungos in Primis Italicos lllustrans 2:319. 1881. On rhizome of Phragmites sp., Gramineac. France.

culmifida P.A. Karsten, Fungi Fenniae Exsiccati, Century 10, No.961. Anno 1870; Mycologia Fennica 2:103. 1873. EMetasphacria 
culmifida (P.A. Karsten) P.A. Saccardo, Sylloge Fungorum Omnium Hucusque Cognitorum Digessit P.A. Saccardo 2:174. 1883. ETrichometasphaeria culmifida (P.A. Karsten) L. Holm, Symbolae Botanicie Upsalienses, Uppsala 14(3): I40. 1957. On culms of Phleum pratense L.. Gramineae. Finland.

culmifraga (E.M. Fries:E.M. Fries) V. Cesati \& G. de Notaris, Commentario della Società Crittogamologica Italiana, Milan I:235. 1863. ESphaeria culmifraga E.M. Fries:E.M. Fries, Systema Mycologicum Sistens Fungorum 2:510. 1823. EPlcospora culmifraga (E.M. Fries:E.M. Fries) L. Fuckel, Symbolae Mycologicae, p. 137. 1870. On culm of grass of unknown host of Gramineae, Gramineae. Italy, Sweden.

culmifraga P.A. Saccardo, Mycotheca Veneta, sistens Fungos Venetos Exsiccatos, Century I, No. 77. Anno 1875. Nom. nud. Art. 32.1 [as (Fr.) Ces. et DN.]. ELeprosphaeria amphibola P.A. Saccardo. On culms of Sorghum vulgare Pers., Gramineale. Italy.

culmifruga (E.M. Fries:E.M. Fries) V. Cesati \& G. de Notaris forma majuscula H. Rehm. Ascomyceten, Fascicle 4, No. 195. Anno 1873. Non vidi. Hedwigia. Dresden 24:67. 1885. On fallen culms of unknown host. Gramineae. Unknown country.

culmifraga (E.M. Fries:E.M. Fries) V. Cesati \& G. de Notaris forma mimuscula H. Rehm, Hedwigia, Dresden 24:66-67. 1885. $\equiv$ Phacosphacria minuscula $(\mathrm{H}$. Rehm) R.A. Shoemaker, Canadian Joumal of Botany, Ottawa 67:1545. 1989. On unknown substrate of unknown host, unknown family. Canada, Sweden.

chlmifraga (E.M. Fries:E.M. Fries) V. Cesati \& G. de Notaris forma poae F. Fautrey in $\mathrm{C}$. Roumeguère, Fungi Selecti Gallici Exsiccati, Century 58, No. 5755. Anno 1891; Revue Mycologique, Toulouse 13:129. 1891. On rachis of panicles of old straw of Poa suderica Haenke, Gramineae. France.

culmifraga (E.M. Fries:E.M. Fries) var. alpestris H. Rehm, Österreichische Botanische Zeitschrift. Wien 56:296. 1906. Nom. nud. Art. 32.I. On grass blade of unknown host. Gramincac. Austria.

culmifraga (E.M. Fries:E.M. Fries) V. Cesati \& G. de Notaris var. bromicola G. Bresadola. Revue Mycologique. Toulouse 12:184. 1890. On stems of Bromus asper Murray. Gramineae. Hungary.

culmifraga (E.M. Fries:E.M. Fries) P.A. Karsten var. herporrichoides (G. de Notaris) P.A. Karsten, Mycologia Fennica 2:108. 1873; Bidrag till Kännedom om Finlands Natur och Folk. Utgifnat al" Finskia Vetenships-Societeten.
Helsingfors 23:108. 1873. 三Leptosphaeria herpotrichoides $\mathrm{G}$. de Notaris.

culmifraga (E.M. Fries:E.M. Fries) V. Cesati \& G. de Notaris var. linearis P.A. Saccardo.

Michelia Commentarium Mycologicum Fungos in Primis ltalicos lllustrans 2:319. 1881. On stems of Brachypodium sp.. Gramineae. France.

culmifraga (E.M. Fries:E.M. Fries) V. Cesari \& G. de Notaris var. propinqua P.A. Saccardo. Michelia Commentarium Mycologicum Fungos in Primis Italicos Illustrans 2:319. 1881. On leaves of Poa aquatica L.. Gramineae. France.

culmorum B. Auerswald in H. Rehm. Bericht des Naturwissenschafulichen Vereins für Schwaben und Neuberg, Augsburg 26:60. 1881. $\equiv$ Scleropleclla culmorum (B. Auerswald) F. * Höhnel. Berichte der Deutschen Botanischen Gesellschaft, Berlin 36:135-140. 1918. [Falsely so cited.] झNodulosphaeria culmorum (B. Auerswald) G. Moesz, Magyar tudományos akadémia Balkán-kutatásainak Tudományos Eredményei. Budapest 3:1 10. 1926. 三Phaeosphaeria microscopica (P.A. Karsten) O. Eriksson var. culmorum (B. Auerswald) O. Eriksson. Arkiv för Botanik. Uppsala, Stockholm. Series 2. 6:427. 1967. EPhaeosphaeria culmorum (B. Auerswald) A. Leuchtmann, Sydowia. Annales Mycologici. Horn. N.Ö. 37:113. 1984. On sheaths and leaves of unknown host of grass. Gramineae. Canada, Czechoslovakia, Denmark. Finland. Germany, Italy. Sweden. Switzerland. U.S.A.. U.S.S.R.

culmorum B. Auerswald forma apogon (P.A. Saccardo \& C.L. Spegazzini) H. Rehm. Ascomyceten, Fascicle 15. No. 734. Anno 1883; Hedwigia. Dresden 23:71-72. 1884. On unknown substrate on unknown host. unknown fanily. Unknown country:

culmorum B. Auerswald fomm epigeii A.I. Lobik. Materialȳ po Floristicheskim i Faunisticheskim Obsledovaniyam Tershogo Okruga [Data [rom Investigations on the Flora and Fauna of the Ter Region]. p. 23. 1928. On leaves of Calamogrosis cpigejos (L.) Roth. Gramineae. U.S.S.R.

culmerum B. Auerswald forma hingarica H. Rehm. Ascomyceten. Fascicle 19. No. 9.11. Amno 1888: Hedwigia. Dresden 27:173. 1888. On dry culms ol Luzula albida (Hoffm.) DC.. Juncaceae. Hungary.

culmorum B. Auerswald forma phragmitis A.I. Lobik. Materialy po Floristicheshim i Faunisticheshim Obsledovaniyam Tershogo Ohruga [Data from Investigations on the Flora and Fauna of the Ter Region], p. 24. 1928. On leaves of Phagnites communis Trin.. Gramincate. U.S.S.R. 
culmorum B. Auerswald var. flavobrumea J.

Feltgen, Vorstudien zu einer Pilz-Flora des

Grossherzothums, Luxemhurg 1(3):205. 1903.

On dry culms of Glyceria spectubilis Mert. \&

Koch (or Phalaris sp.?), Gramineae. Luxembourg.

culmorum B. Auerswald var. paleicola P.C. Hennings, Verhandlungen des Botanischen Vereins Der Provinz Brandenburg, Berlin 44:177-178. 1903. On culms of Carex leporina L., Cyperaceae. Germany.

cumana P.A. Saccardo \& C.L. Spegazzini in P.A. Saccardo, Michelia Commentarium Mycologicum Fungos in Primis Italicos Illustrans 1:394-395. 1878. EMetasphaeria c'umana (P.A. Saccardo \& C.L. Spegazzini) P.A. Saccardo, Sylloge Fungorum Omnium Hucusque Cognitorum Digessit P.A. Saccardo 2:177. 1883. On dead leaves of Carex sp.. Cyperaceae. Italy.

cumulata W. Kirschstein. Verhandlungen des Botanischen Vereins Der Provinz Brandenburg. Berlin 48:56. (1906) 1907. On fallen culms of Phragmites sp.. Gramineae. Germany.

curlu P.A. Saccardo \& Abbé Flageolet in P.A. Saccardo, Bulletin de la Société Mycologique de France, Paris 12:66. 1896. On dead stems of Rlus ryphina L.. Anacardiaceae. France.

cycadis M. Svrŏek. Ceská Mykologie, Praha 34:177. 1980. On upper surface of living leaves of Cyeas revoluta Thunb., Cycadaceae. Iran.

cylindrospora B. Auerswald \& G. Niessl v. Mayerdorf in P.A. Saccardo, Sylloge Fungorum Omnium Hucusque Cognitorum Digessit P.A. Saccardo 2:37. 1883. On dead stems of Epilobium angustifolium L., Onagraceae. Czechoslovakia.

cylindrospora A.M. Saccas, Étude de la Flore Cryptogamique des Caféiers en Afrique Centrale. Bulletin de Institut Français du Café du Cacao et d'Autres Plantes Stimulantes (Bulletin IFCC No. 16), pp. 237-239. 1981. Nom. inval. Art. 37.1. On dead branches of Coffea robusta L. Linden (=Coffea camephora Pierre ex Froelon.), Rubiaceae. Central African Republic.

cylindrostoma K. Starhäck, Arkiv för Botanik, Uppsala, Stockholm 5(7):23. 1905. On unidentified ?stems of unknown host. unknown family. Argentina.

cynaracearm B. Auerswald \& G. Nicssl v. Mayendorf in G. Niessl v. Mayendorf, Verhandlungen des Naturforschenden Vereins in Brünn 10:174. (1871) 1872. 三Metasphateria cymerearum (B. Auerswald \& G. Niess) v. Mayendorf) P.A. Saccardo, Sylloge Fungorum Omnium Hucusque Cognitorum Digessit P.A. Saccardo 2:170. 1883. On dry leaves of Carlina acoulis I.., Compositale. Crechoslovakia. ¿ynodomis-rlactyli E. Marchal \& R.L. Steyaert. Bulletin. Société Royale de Botanique de Belgique, Bruxelles 61(Serics 2, 12):161. 1929. On living leaves of Cymodon daciy/on (L.) Pers., Gramineae. Zilire.

cynoglossi L. Hollós, Annales Historico-Naturales Musei Nationalis Hungarici, Budapest 5:455. 1907. On dried stems of Cynoglossum officinale L., Boraginaceae. Hungary.

cynops J.H.C. Fahre, Annales des Sciences Naturelles, Paris, Botanique, Series 6 , 15:50-51. 1883. On dead stems of Plamlago cynops L., Plantaginaceae. France.

(ynosuri L.M. Unamuno Yrigoyen, Anales del Jardin Botánico de Madrid 4:152. 1944. On culms of Cynosurus cristums L., Gramineat. Spain.

cyperi L. Hollós, Matematikai és Természentudományi Közlemenyek Vonatkozólag a Hazai Viszonyokra, Budapest 35:31. 1926. On dry leaves of Cyperus flavescens. L., Cyperaceac. Hungary.

'ypericola L. Hollós, Matematikai és Természettudományi Közlemenyek Vonatkozólag a Hazai Viszonyokra, Budapest 35:32. 1926. On dry leaves of Cyperus flavesiens L., Cyperaceae. Hungary.

(y)erina G. Passerini, Erbario Crittogamico Italiano. Series 2, Fascicle 22, No. 1074. Anno 1881. EPhaeosphaeria cyperina (G. Passerini) R.A. Shoemaker, Canadian Journal of Botany. Ottawa 67:1540.1989. On stubble of Cyperus moni L., Cyperaceae. Italy.

dactylina G. Passerini, Rendiconti della Sedute della R. Accademia dei Lincei, Classe di Scienze Fisiche, Matematiche e Natural (=Atti dell' Accademia Nazionale dei Lincei, Rendiconti, Roma), Series 4, 3:91. 1887. On dry culms of Dactylis glomerata L., Gramineae. Italy.

danica A.N. Berlese, Icones Fungorum Omnium Hucusque Cognitorum ad usum Sylloges Saccardianae Adcommodatae 1:87. 1892. झLeptosphaeria marina E. Rostrup. Non Leptosphacria marina J.B. Ellis \& B.M. Everhart. EDidymosphaeria danica (A.N. Berlese) 1.M. Wilson \& J.M. Knoyle. Transactions of the British Mycological Society, London 44:57. 1961. ELautitia danica (A.N. Berlese) S. Schatz, Canadian Journal of Botany, Ottawa 62:31. 1984. Parasitic on Chondrus crispus J. Stackhouse, Algae (Gigartinaceae). Denmark.

daphnes M.R. de Sousia Dias \& M. de Sousa da Camara, Agrononia Lusitana, Sacavém 15:23-24. 1953. On branchlets of Daphne gnidium L., Thymelacaceac. Portugal.

dophmiphylli C.T. Dzhalagoniya, Trudȳ Sukhumkogo Botanicheskogo Sada, Sukhum 15:118. 1964 [as dophmiphylliac']. On living leaves of Daphniphyllhm macropodium Mig., Daphniphyllaceac. U.S.S.R.

darkeri R.A. Shoemaker, Canadian Journal of Botany, Otlawa 62:2699-2700. 1984. On 
stems of Agastache wricifolia (Benth.) Kıze., Labiatae. U.S.A.

dasylirii (G.L. Rabenhorst) P.A. Saccardo, Sylloge Fungorum Omnium Hucusque Cognitorum Digessit P.A. Saccardo 2:67-68. 1883.

ESphaeria dasylirii G.L. Rabenhorst, Fungi Europaei Exsiccati, Klotzschii Herbarii vivi Mycologici Continuatio Edita Nova, Series 2 , Century 27, No. 2655. Anno 1881; Hedwigia. Dresden 21:9. 1882. On leaves of Dasylirion junceum Zucc., Agavaceae. Italy.

davidii S.A. Gucevicz, Trudȳ Gosudarstvennogo

Nikitskogo Botanicheskogo Sada. Yalta 29:191. 1959. On dried stems of Buddlcja davidii Franch., Loganiaceae. U.S.S.R.

daviesiae F. Petrak, Sydowia. Annales Mycologici,

Horn, N.Ö. 8(1-6):197. 1954. On dry

branches of Davicsia latifolia $\mathrm{R}$. Br.,

Leguminosae. Australia.

davisiana F. Petrak, Sydowia. Annales Mycologici, Horn, N.Ö. 7(1-4):20. 1953. On dry stems of Scutellaria hrevibracteata Stapf, Labiatae. Turkey.

dearnessii R.A. Shoemaker, Canadian Journal of Botany, Ottawa 62:2700. 1984. On stems of Asclepias syriaca L., Asclepiadaceae. Canada. debeanxii C. Roumeguère \& P.A. Saccardo in C. Roumeguère, Revue Mycologique. Toulouse 2:188. 1880. 三Metasphaeria debeaurii (P.A. Saccardo) P.A. Saccardo. Sylloge Fungorum Omnium Hucusque Cognitorum Digessit P.A. Saccardo 2:182. 1883. On costa of leaves of Chamaerops humilis L., Palmae. Algeria. decaisneama (L. Crié) P.A. Saccardo, Sylloge Fungorum Omnium Hucusque Cognitorum Digessit P.A. Saccardo 2:50. 1883. 三Depazea caisneana L. Crié, Annales des Sciences Naturelles, Paris, Botanique, Series 6, 7:48. 1878. On dry leaves of Populus sp., Salicaceae. France.

deficiens F. Tassi, Bolletino del R. Orto Botanico, Siena 3:121. 1900. On stems of Tellima grandiflora (Pursh) Douglas ex Lindley. Saxifragaceae. China.

defodiens (J.B. Ellis) J.B. Ellis in N.L. Britton. Catalogue of Plants Found in New Jersey. Geological Survey of New Jersey, Final Report of the State Geologist. Trenton 2(1):525. 1889. ESphacria (Leptosphaeria) defodiens J.B. Ellis. Bulletin of the Torrey Botanical Club (and Torreya), New York 8:90. 1881. On dead plant of Juncus effusus L.. Juncaceac. U.S.A. (New York).

delawayi N.T. Patouillard, Revue Mycologique.

Toulouse 8:82. 1886. On dead stems of Primula sikkimensis Hook., Primulaceae. Clinil.

dematiicola W. Kirschstein. Annales Mycologici,

Berlin 34:188. 1936. On old fruiting stem ol Typha angustifolia L.. Typhaceae. Germany. domatian F.A. Hazslinszky, Mattematikat és Természettudományi Közlemenyek Von- atkozólag a Hazai Viszonyokra. Budapest 25(2):43. 1892: Magyarország s

Társországainak Sphaeriai, Budapest, p. 107. 1892. On dead stems of Hypericum perforatum L., Guttiferae. Hungary.

demissa G. Niessl v. Mayendorf, Instituto. Revista Scientifica e Litteraria. Coimbra 31:89-90.

1883. On dry stems of Vinca media Hoffmanns. \& Link, Apocynaceae. Portugal. dennisiana (A. Leuchtmann) R.A. Shoemaker, Canadian Journal of Botany. Ottawa 67:1578. 1989. EPhaeosphaeria dennisiana A. Leuchtmann, Sydowia. Annales Mycologici. Horn, N.Ö. 37:155-156. 1984. On dry leaves of Mimuartia sedoides (L.) Hiern.. Caryophyllaceae. Great Britain.

densa J. Bresadola. Hedwigia. Dresden 35:199. 1896. On leaves of Acorus calamus L., Araceae. Germany.

depressa (L. Fuckel) H.G. Winter, Dr. L.

Rabenhorst's Kryptogaman-Flora von Deutschland. Oesterreich und der Schweiz. Second edition, 1(2):473. 1885. ESphaeria depressa L. Fuckel. Symbolae Mycologicae. p. 115. 1870. झMerasphaeria depressa (L. Fuckel) P.A. Saccardo, Sylloge Fungorum Omnium Hucusque Cognitorum Digessit P.A. Saccardo 2:166. 1883. On dry stems of Carpinus sp.. Betulaceae. Austria.

depressa L.E. Wehmeyer \& S. Ahmad. Biologia.

Biological Society of Pakistan, Lahore 10:12. 1964. Nom. illegit. Art. 64.1. [A later homonym of Leprosphaeria depressa $T$. Petch. 1926.] On stems of Euphorbia sp. (as E. oxyoidea). Euphorbiaceae. Pahistan.

depressa T. Petch, Ceylon Journal of Science. Colombo. Section A (Botany) 10:135. 1926. On stems of Camellia theifera Griff.,

Theaceae. Sri Lanka.

derasa (M.J. Berkeley \& C.E. Broome) B. Auerswald, Botanischer Tauschverein in Wien. P. 4. 1886. Non vidi. ESphaeria derasa MI.J. Berkeley \& C.E. Broome. Annals and Magazine of Natural History, London. Series 2. 9:328. 1852. झNodulosphaeria derasa (M1.J. Berkeley \& C.E. Broome) L. Holm. Symbolae Botanicae Upsalienses 14(3):89. 1957. On unknown substrate of Senecio jacobaea L. Compositae. Great Britain.

derasa (M.J. Berkeley \& C.E. Broome) F. v. Thümen. Mycotheca Universalis. Century 3. No. 269. Anno 1875. Nom. illegit. An. 64.1. Spharria derasa M.J. Berkele! \& C.E. Broome. Amals and Magazine of Natural History, London. Series 2. 9:328. 1852. $\equiv$ Nohulosphario derasa (M.J. Berheley d C.E. Broome) L. Holm, Symbolae Botanicae Upsalienses, Uppsala 1 $4(3): \$ 9.1957$. On unknown substrate of Senceio jacobaea L.. Compositae. Great Britain.

derasa (M1.J. Berkeley \& C.E. Broome) F. 1.

Thümen lorma alpe'snis H. Rehm. Heduigia. 
Dresden 24:235. 1885. On stems of unknown host of composite family, Compositae. Italy. derasa (M.J. Berkeley \& C.E. Broome) F.v. Thümen forma macrospora $\mathrm{F}$. Fautrey in $\mathrm{C}$. Roumeguère, Revue Mycologique. Toulouse 16:8. 1894; Fungi Selecti Gallici Exsiccati, Century 65. No. 6436. Anno 1894. On dry branches of Sumbucus ebulus L.. Caprifoliaceae. France?

(Pocosphaeria) derasa (M.J. Berkeley \& C.E. Broome) B. Auerswald forma robusta P. Strasser, Verhandlungen der ZoologischBotanischen Gesellschaft in Wien 57:315. 1907. ELeptosphaeria robusta (P. Strasser) E. Müller. $\equiv$ Nodulosphacria robusta (P. Strasser) L. Holm, Symbolae Botanicae Upsalienses, Uppsala 14(3):90. 1957. $\equiv$ Ophiobolus robustus (P. Strasser) R.A. Shoemaker, Canadian Journal of Botany. Ottawa 54:2389. 1976. On dry stems of Senecio nemorensis 1., Composilae. Austria. derasa (M.J. Berkeley \& C.E. Broome) B. Auerswald var. frunconicu F. Petrak, Kryptogamische Forschungen. München 2:162-163. 1931. ELeptosphaeria franconica (F. Peirak) E. Müller, Sydowia. Annales Mycologici, Horn, N.O. 4( $1-6): 298-299.1950$. On dry stems of Inula salicina L., Compositae. Romania.

desciscens C.A. Oudemans, Beihefte zum Botanischen Zentralblatt, Cassel I I:527. 1902. On stems of unknown host, unknown family. Netherlands.

desmonci H. Sydow \& P. Sydow, Hedwigia, Dresden 49:79. 1909. On branches of Desmoncuss sp., Palmae. Brazil.

(Leptosphaerella) diana P.A. Saccardo \& C.L. Spegazzini in P.A. Saccardo. Michelia Conmentarium Mycologicum Fungos in Primis Italicos Illustrans 1:398-399. 1878. ESphaerulina (Leptosphaerella) diana (P.A. Saccardo \& C.L. Spegazzini) M.C. Cooke. Grevillea, London 18:80. 1890. $\equiv$ Mycotodea diana (P.A. Saccardo \& C.L. Spegazzini) W. Kirschstein in O.C. Schmidt, Kryploganenflora der Mark Brandenburg und Angrenzender Gebiete herausgegeben von dem Botanishen Verein der Provinz Brandenburg, Leipzig 7:437. 1938. On rotten leaves of Acer negundo L.. Aceraceac. Italy.

dianthi (E. Rostrup) J. Lind. Danish Fungi as Represented in the Herbarium of E. Rostrup. Nordisk Forlag. Copenhagen, p. 222. 1913. $\equiv$ Metasphaeria dianthi E. Rostrup, Botanisk Tidsskrift. Kjobenhavn 26:311. 1905. On stems of Diauthus superbus L., Caryoplyyllaceae. Denmark.

diamthi A. Christow, Bulgaria. Ministerstvo na zemledielicto i durzhuvnitie imoti. Zemlestopanska hibliotcka (Ministerium für Landwirtschaft und Staatsdomänen Land- wirtschaftsbiblothek) 43:4-6. 1931. Nom. illegit. Art. 64.1. On unknown substrate of Dianthus sp., Caryophyllaceae. Bulgaria.

diamli L. Hollós, Matematikai és Természettudományi Közlemenyek Vonatkozólag a Hazaj Viszonyokra (Maguar tudomanyos akadémia Budapesı. Matenatikai és természettudomanyi bizottság), Budapest 35:32. 1926. Nom. illegit. Art. 64.1. On dry stems of Dianthus caryophyllus L.. Caryophyllaceae. Hungary.

diamthi N. Golovina, Notulae Systematicae e Sectione Cryptogamica Instituti Botanici Nomine V.L. Komarov Academiae Scientiarum U.R.S.S., Petropolis 12:156. 1959. Nom. illegit. Art. 64.1. On dead stems of Dianthus sp.. Caryophyllaceae. U.S.S.R. (Uzbek).

diaporthoides H.G. Winter, Boletim da Sociedade Broteriana, Coimbra 3:56. (1884) 1885. On dried stems of unknown host of Umbelliferae. Umbelliferae. Brazil, Portugal.

dichosciadii F. Petrak, Sydowia. Annales Mycologici, Horn. N.Ö. 9(1-6):561. 1955. On decaying leaves of Dichosciadium ranunculacemm (F. v. Mïller) Domin, Hydrocotylaceae. Australia.

dichroa G. Passerini, Rendiconti della Sedute della R. Accademia dei Lincei, Classe di Scienze Fisiche, Matematiche e Natural (=Atti dell' Accademia Nazionale dei Lincei, Rendiconti, Roma), Series 4. 3:90. 1887. EPasseriniella dichor (G. Passerini) A.N. Berlese, Icones Fungorum Omnium Hucusque Cognitorum ad usum Sylloges Saccardianae Adcommodatac 1:51. 1890. On dry branches of Deutzia scabra Thunb., Saxifragaceae. Italy.

didymellae-vincetovici E. Müller, Sydowia. Annales Mycologici. Horn. N.Ö. 5(3-6):51-52. 1951. On fruiting bodies on Didymella vincetoxici (de Not.) Sacc., Fungi (Phaeosphaeriaceae). Switzerland

digitalis (P.L. Crouan \& H.M. Crouan) P.A. Saccardo. Sylloge Fungorum Omnium Hucusque Cognitorum Digessit P.A. Saccardo 2:85. 1883. ESphaeria digitalis P.L. Crouan \& H.M. Crouan, Florule Du Finistc̀re Contenant Les Descriptions Dc 360 Espèces Nouvelles De Sporogames, De Nombreuses Ohservations et une synonymie des plantes Cellulaires et Vasculaires Qui Croissent Spontanément Dans ce Département, p. 28. 1867. On dead stcms of Digitalis sp.. Scrophulariaccae. France.

dioica (E.M. Fries:E.M. Fries) P.A. Saccardo, Sylloge Fungorum Omnium Hucusque Cognitorum Digessit P.A. Saccardo 2:18. 1883 [as (Moug.) Sacc.]. ESphaeria diovica E.M. Fries:E.M. Fries, Elenchus Fungorum Sistens Commentarium in Systema Mycologicum 2:82. 1828. On branches of Acer p.serudoplestamus L., Spartium sp., Aceraccate. Leguminosae. France, Great Britain. 
discors P.A. Saccardo \& J.B. Ellis in P.A. Saccardo, Michelia Commentarium Mycologicum Fungos in Primis Italicos Illustrans 2:567. 1882. $\equiv$ Metasphaeria discors (P.A. Saccardo \& J.B. Ellis) P.A. Saccardo, Sylloge Fungorum Omnium Hucusque Cognitorum Digessit P.A. Saccardo 2:173. 1883. EPasseriniella discors (P.A. Saccardo \& J.B. Ellis) A.E. Apinis \& C.G.C. Chesters, Transactions of the British Mycological Society, London 47:432. 1964. On culms of Spartina sp., Gramineae. U.S.A. (New Jersey).

disseminata G. de Notaris, Commentario della Società Crittogamologica Italiana, Milan 2:486. 1867. On unknown substrate of unknown host, Gramineae. Italy.

dissiliens (M.C. Cooke \& J.B. Ellis) J.B. Ellis in N.L. Britton, Catalogue of Plants Found in New Jersey. Geological Survey of New Jersey, Final Report of the State Geologist, Trenton 2(1):525. 1889. 三Sphaeria (Caulicolae) dissiliens M.C. Cooke \& J.B. Ellis, Grevillea, London 5:51. 1876. On dead stems of Desmodium sp., Leguminosae. U.S.A. (New Jersey).

distributa (M.C. Cooke \& J.B. Ellis) P.A. Saccardo, Sylloge Fungorum Omnium Hucusque Cognitorum Digessit P.A. Saccardo 2:18. 1883. ESphaeria (Obtectae) distributa M.C. Cooke \& J.B. Ellis, Grevillea, London 7:41. 1878. On small twigs of Desmodium sp., Leguminosae. U.S.A. (New Jersey).

dobrogica T. Săvulescu \& C. Sandu-Ville. Hedwigia, Dresden 75:170. 1935. On dead branches of Smilar excelsa L., Liliaceae. Ronania.

dodonaeue A. Canonaco, Bollettino di Studi e Informazioni. R. Giardino Botanico (Coloniale) Palermo 14:extr. p. 12. 1936: 14:20. 1937. [Note extract was published in 1936. which is date of publication for the species.] On branches of Dodonaea viscosa (L.) Jacq.. Sapindaceae. Turkey.

dolioloides (B. Auerswald) P.A. Karsten, Mycologia Fennica Pars 2, Pyrenomycetes, pp. 106-107. 1873. 三Nodulosphaeria dolioloides B. Auerswald in G.L. Rabenhorst, Fungi Europaei Exsiccati, Klotzschii Herbarii vivi Mycologici Continuatio Edita Nova, Series Secunda, Century 6. No. 547. 1863. $\equiv P / e-$ ospora dolioloides (B. Auerswald) L. Fuckel. Symbelae Mycologicae, p. 138. 1870. On stems of Achillea millefolium auct., Anthemis tinceria L., Chrysanthemum vulgaro(L.) Bernh., Senecio irwlgaris L.. Tanas'tum ru/gare L., Compositac. Finland. France. Germany, Portugal, Spain.

dolioloides (B. Auerswald) B. Auerswald, Botanischer Tausclıvereinin Wien. P. 4. 1860. Non vidi. $\equiv$ Nolulosphasion dolioloides $B$.
Auerswald in G.L. Rabenhorst, Fungi

Europaei Exsiccati, Klotzschii Herbarii vivi

Mycologici Continuatio, Edition 3, Century 6.

No. 547. Anno 1863. EPleospora dolioloides

(B. Auerswald) L. Fuckel, Symbolae

Mycologicae, p. 138. 1870.

dolioloides (B. Auerswald) P.A. Karsten. Fungi

Fenniae Exsiccati, Century 10, No. 978. Anno

1870. ENodulosphaeria dolioloides B.

Auerswald in G.L. Rabenhorst. Fungi

Europaei Exsiccati, Klotzschii Herbarii vivi

Mycologici Continuatio. Edition 3. Century 6.

No. 547. Anno 1863. EPleospora dolioloides

(B. Auerswald) L. Fuckel. Symbolae

Mycologicae, p. 138. 1870. On dry stems of

Achillea millefolium auct., Anthemis tinctoria

L.. Chrysanthemum vulgare (L.) Bermh..

Senecio v'ulgaris L., Tanacerum vulgare L..

Compositae. Finland, Germany.

dolioloides (B. Auerswald) P.A. Karsten var. cirsii

P.A. Karsten, Hedwigia, Dresden 23:4. 1884.

On dead stems of Cirsium sp., Compositae.

Finland.

dolioloides (B. Auerswald) P.A. Karsten var. inops

P.A. Karsten, Hedwigia, Dresden 23:4. 1884.

On dead stems of Anthriscus sv/vestris (L.)

Hoffm.. Celastraceae. Finland.

dolioloides (B. Auerswald) P.A. Karsten var. loihvri J. Feltgen, Vorstudien zu einer Pilz-Flora des Grossherzogthums. Luxemburg 1(2):16t-165. 1901. On dry stems of Lathyrus niger (L.)

Bernh.. Leguminosac. Luxembourg.

dolioloides (B. Auerswald) P.A. Karsten var. rhinanthi J. Feltgen. Vorstudien zu einer PilzFlora des Grossherzogthums. Luxemburg 1(2):165. 1901. On dry stems of Rhinamihus sp., Scrophulariaceae. Luxembourg.

doliolum (C.H. Persoon:E.M. Fries) V. Cesati \& G. de Notaris, Commentario della Società Crittogamologica ltaliana. Milan 1:234-235. 1863. ESphaeria doliolum C.H. Persoon. lcones et Descriptiones Fungorum Minus Cognitorum, p. 39. 1800; E.M. Fries, Systema Mycologicum Sistens Fungorum 2:509. 1823. =Bilimbiospora dolichum B. Auerswald in G.L. Rabenhorst, Fungi Europaei Exsiccati. Klotzschii Herbarii vivi Mycologici Continuatio, Ausgabe 11I. Century 3, No. 261. Anno 1860. Nom, rejicem. [Bilimbiospora doliolum is the same as Lephosphaeria doliolum but is based on different lypes.] EPleospora doliolum (C.H. Persoon:E.M. Fries) L. Tulasne \& C. Tulasne. Selecta Fungorum Carpologica 2:276. 1863. ECryprosphacria doliolum (C.H. Persoon) R.K. Greville. Scotish Cryptogamic Flora 4. Table 239. 1826. On stems of Achillea sp.. Ambrosia sp.. Anophalis sp.. Arcriums sp.. Aster sp.. Cirsium sp.. Erigeron sp.. Eupatorimm sp.. Holianthus sp. Latuca sp.. 
Polymmis sp., Senecio sp., Solidago sp., Sonchus sp. (Compositae); Aralia sp. (Araliaceae); Apocyuum sp., Asclepias sp. (Asclepiadaceac); Potentilla sp., Rubus sp. (Rosaceale); Dasyistoma sp. Gerardia sp.. Veronica sp. (Scrophulariaceare); Smilax sp. (Smilacaceae); Heracleum sp., Pustinaca sp. (Umbelliferac): Urica sp. (Unticaceae), Araliaceae. Asclepiadaceac, Compositac, Rosaceae, Scrophulariaceae, Smilacaceac. Umbellilerae, Urticaceae. Belgium, Canadal, Finland, France, Germany. Great Britain. Italy, Sweden, U.S.A.

doliolum (C.H. Persoon:E.M. Fries) V. Cesati \& G. de Notaris forma carlinae-vulgaris C. SanduVille. Academia Republicii Populare Romîne. Filiala lasi. Studii si Cercetări Stiintifice. Biologie si Stiinte Agricole 9:258. 1964; Studii Şi Cercetări de Biologie. Bucureşti. Series Botanica 16:296. 1964. On dead stems of Carlina wulgaris L., Compositae. Ronania.

doliolum (C.H. Persoon:E.M. Fries) V. Cesati \& G. de Notaris Corma carlinae-v'wlgaris C. SanduVilte, Ciuperci Pyrenomycetes-Sphaeriales Din România, p. 275. 1971. Nom. illegit. Art. 64.1. On dead stems of Carlina virgaris L., Compositae. Romania.

doliolum (C.H. Persoon:E.M. Fries) V. Cesati \& G. de Notaris forma syndoliola H. Rehm, Ascomycetes Lojkani Lecti in Hungaria. Transylvania et Galicia, Berlin. pp. 55-56. 1882. On dry stems of Pencedamum longifolium Waldst. \& Kit., Umbelliferae. Hungary.

doliolım (C.H. Persoon:E.M. Fries) V. Cesati \& G. de Notaris var. amgustispora N.T. Patouillard, Revue Mycologique. Toulouse 8:180. 1886. On dead stems of Pedicularis debilis Franch. ex Maxim., Scrophulariaceae. China.

doliolım (C.H. Persoon:E.M. Fries) V. Cesati \& G. de Notaris var. cacaliae N. Naumov,

Ural'skoe Obshchestvo Estestvoznaniia, v Ekeaterinburgic Zadipiski 35(11-12):21 1916. On dry stens of Cacalia hastata L.. Compositae. U.S.S.R.

doliolum (C.H. Persoon:E.M. Fries) V. Cesati \& G. de Notaris var. contoidea V. Cesati \& G. de Notaris, Commentario della Società Crittogamologica Italiana, Milan 1:235. 1863.

ELeprosphacria comoidea (V. Cesati \& G. de Notaris) P.A. Saccardo.

doliolum (C.H. Persoon:E.M. Fries) V. Cesati \& G. de Notaris var. dissimilis H. Rehm, Ascomyceten, Fascicle 18, No. 888. Anno 1886: Hedwigia, Dresslen 26:94. 1887. [Reprint is page 14 at FII.] On decayed stems of lleracleam palmatum Baumg. Unbelliferac. Romania.

doliolum (C.II. Persoon:E.M. Fries) V. Cesati \& G. de Notaris var. leommi C. Sandu-Ville,
Academia Republicii Populare Romîne. Filiala lasi. Studii si Cercetări Stiintificc. Biologie si Stiinte Agricole 10 (Fascicle 2):213-214. 1959. On dead stems of Leonurus cardiaca L., Labiatae. Romania. doliolum (C.H. Persoon:E.M. Fries) V. Cesati \& G. de Notaris var. pachyspora P.A. Saccardo. Michelia Commentarium Mycologicum Fungos in Primis Italicos Illustrans 2:318. 1881. On stens of Adonis pyrenaica DC. Ranunculaceae. France.

doliolum (C.H. Persoon:E.M. Fries) V. Cesati \& G. de Notaris var. pinquicula P.A. Saccardo, Revue Mycologique, Toulouse 3:44. 1881: Reliquiae Mycologicae Libertianae. Series Altera Reviserunt C. Roumeguère \& P.A. Saccardo. Toulouse, No. 121. 1881: Michelia Commentarium Mycologicum Fungos in Primis Italicos Illustrans 2:598. 1882. On stems of Foeniculum sp.. Unbelliferae. France.

doliolum (C.H. Persoon:E.M. Fries) V. Cesati \& G. de Notaris var. subdisticlla K. Starbäck \& A.Y. Grevillus in K. Starbäck. Bihang till K. Svenska Vetenskapsakademiens Handlingar. Stockholm 16(3). No. 3. pp. 6-7. 1890;

Bidrag till Kännedomen om Sveriges Ascomycetflora, pp. 6-7. 1890. On dry stems ol Aremisia inlgaris L., Compositae. Sweden.

"lonacina P.A. Saccardo, Atti dell' Accademia Scientifica Veneto-Trentino-Istriana, Padova 2(2):155. 1873. ĐPhaeosphaeria donacina (P.A. Saccardo) R.A. Shoemaker, Canadian Journal of Botany, Ottawa 67:1524. 1989. [Anamorph: Ascochyta donacina P.A. Saccardo.) On dead, decaying culms of Arundo domar L.. Gramineae. Netherlands. drabar (W. Nylander) P.A. Karsten, Mycologia Fennica Pars 2, Pyrenonycetes, p. 102. 1873. ESphacria drabae W. Nylander in W. Nylander and T. Saelan, Herbarium Musei Fennici Förteckning Öfver Finska Musei Växt samling, Utgifven Af Sällskapet Pro Fauna et Flora Fennica Och Uppgjord af W. Nylander Och Th. Saelan. Med en Karta, p. 112. 1859. On stens of Draba alpina L.. Cruciferae. U.S.S.R.

dracaenae M. de Sousa da Camara, Revista Agronómica, Lisbon 1:23. 1903. On dead leaves of Dracacha draco (L.) L., Agavaceac. Portugal.

dracuenue M. de Sousa da Camara forma rusci J.V. d’Almeida \& M. de Sousa da Camara. [An error for Leprosphacria comallarieac J.V. d'Almeida \& M. de Sousa da Camara forma rusci J.V. d'Almeida \& M. de Sousa da Camara.]

dracomis M.J. de Urríes y Azara. Anales del Instututo Botánico A.J. Cavanillo. Madrid 
14:158-159. (1955) 1956. On leaves of Dracaena draco (L.) L. Agavaceac. Spain. drechsleri (R.A. Shoemaker) M.E. Barr in M.E. Barr, C.T. Rogerson, S.J. Smith, and J.H. Haines, Bulletin of the New York State Museum. Albany 459:23. 1986. 三Ophiobolus drechsleri R.A. Shoemaker, Canadian Journal of Botany, Ottawa 54:2376-2377. 1976. On unknown substrate of Ambrosia trifida L., Helianturs anmuns L., Helianthus grosserrauts Martens, Heliopsis sp., Verbesina virginica L., Compositae. Canada, U.S.A.

dryadea P.A. Saccardo, Nuovo Giornale Botanico Italiano e Bolletino della Società Botanica Italiana, Firenze 7:311. 1875. On dried leaves of Quercus pedunculata Ehrh.. Fagaceae. Italy.

(Leptosphacrella) dryudea P.A. Saccardo subsp. lissomiensis P.A. Saccardo. Annales Mycologici. Berlin 12:304-305. 1914. On dying stems and leaves of Kigelia pimnata DC., Bignoniaceae. Philippines.

dryadis E. Rostrup, Botanisk Tidsskrilt, Kjobenhavn 25:305. 1903. On decorticated wood stems and fruits of Dryas octopefala L.. Rosaceae. Iceland.

dryadis E. Rostrup. Norske Ascomyceter i Christiania Universitetets Botaniske Museum. Christiania [Oslo] (1. Kommission Hos Jacob Dybwad), p. 24. 1904. [Issued in Skrifter Udg. af Videnskabsselskabet i Christiania. Mathematisk-naturvidenskabelig klasse. Christiania (Oslo) No. 274.] Non vidi. On upper surface of dried leaves of Dryas octopetala L., Rosaceae. Norway.

dryophila (M.C. Cooke \& H.W. Harkness) P.A. Saccardo, Sylloge Fungorum Omnium Hucusque Cognitorum Digessit P.A. Saccardo 2:51. 1883. ESphaerella dryophila M.C. Cooke \& H.W. Harkness, Grevillea. London 9:86. 1881. On leaves of Quercus sp.. Fagaceae. U.S.A.

dubia P.A. Saccardo \& J. Paoletti, Bulletin. Société R. de Botanique de Belgique, Bruxelles 28:90-91. 1889. On stems of '́aleriana dubia Turcz., Valerianaceae. U.S.S.R.

dubiosa (V. Mouton) C.A. Oudemans, Enumeratio Systematica Fungorum 1:981. 1919. ELeptosphaeria nardi (E.M. Fries) V. Cesati \& G. de Notaris var. dubiosa V. Mouton.

dumetorum G. Niessl v. Mayendorf, Verhandlungen des Naturforschenden Vereins in Brünn 10:176-177. (1871) 1872. On dried stems of Humulus lupulus L., Urticaceae. Czechoslovakia.

dumetormm G. Niessl v. Mayendorf forma ebuli H. Rehm ex 11. Rehm. Asconyceten. Fascicle 14. No. 687. Anno 1882. Nomi. nud. Art. 32.1: Hedwigia, Dresden 22:56. 1883. On dry stems of Sambucus ehulus L., Caprifoliaceae. Crechoslovakia.

dumetorum G. Niessl v. Mayendorf forma melifoti

H. Rehm, Hedwigia. Dresden 22:56. 1883.

On preceding year's stems of Melilorus alba

Medicus. Leguminosae. Germany:

dumelorum G. NiessI v. Mayendorf var. coniformis

J. Feligen. Vorstudien zu einer Pilz-Flora des

Grossherzogthums. Luxemburg 1(2):159-160.

1901. On dry stems of Senecio fuchsii C.C.

Gmelin. Compositae. Luxembourg.

dumetorum G. Niessl v. Mayendorf var. coronillae

G. Moesz, Arbeiten des Ungarischen

Biologischen Forschungs-Institutes. Tihany

3:96. 1930. On dead stems of Coronilla

coronata L.. Leguminosae. Hungary.

dumetorum G. Niessl v. Mayendorf var. doli-

chospora J. Feltgen, Vorstudien zu einer PilzFlora des Grossherzogthums. Luxemburg 1(3):210-211. 1903. On corticated branches of Sambucus racemosa L.. Caprifoliaceae.

Luxembourg.

dumetorum G. Niessl v. Mayendorf var. guliiborealis W.H. Trail, Transactions and Proceedings of the Botanical Society of Edinburgh 17:491. 1889. On dead stems of Gulium borcule L.. Rubiaceae. Norway.

dumetormm G. Niessl v. Mayendorf var. marruhii P.A. Saccardo. Sylloge Fungorum Omnium Hucusque Cognitorum Digessit P.A. Saccardo 2:15. 1883. On stems of Marrwhium vulgare L., Labiatae. France.

dumerorum G. Niessl v. Mayendorf var. swmphwri J. Feligen. Vorstudien zu einer Pilz-Flora des Grossherzogthums. Luxemburg 1(3):211. 1903. On dry stems of Symphymm caucusicum Bieb.. Boraginaceae. Luxembourg.

duplex (M.J. Sowerby:E.M. Fries) P.A. Saccardo. Sylloge Fungorum Omnium Hucusyue Cognitorum Digessit P.A. Saccardo 2:87. 1883. ESphacria duplex M.J. Sowerby:E.M1. Fries. Coloured Figures of English Fungi or Mushrooms 3. Table 375. 1803: Sistema Mycologicum Sistens Fungorum 2:520. 1823. On leaves of Sagitharia sp.. Spartina sp.. Typha sp.. Alismataceae, Gramineae.

Typhaceae. Germany. Great Britain. Sueden. chumea G. Niessl v. Mayendort. Hedwigia. Dresden 20:98. 1881. EMetasphaeria churmea (G.

Niessl V. Mayendorf ) P.A. Saccardo. Stlloge Fungorum Omnium Hucusque Cognitorum Digessit P.A. Saccardo 2:162. 1853. On dry stems of Chomdrilla juncea L. Compositae. Unknown country

echiella J. Foltgen. Vorstudien zu einer Pilz-Flora des Grossherzogthums. Luxemburg 1(3):219. 1903. On sems of Echium vulgare L.. Boraginaceas. Luxemburg.

echii J. Feltgen, Vorstudien zu einer Pilz-Flora des Grossherzogthums. Luxenıurg $1(4): 28$. 
1905. On barked stems of Echium vulgare L. Boraginaceae. Luxembourg.

echinella (M.C. Cooke) F. v. Thümen, Mycotheca Universalis, Century 3. No. 266. Anno 1875. Esphaeria echinella M.C. Cooke. Handbook of British Fungi. p. 906. 1871. On rotten stems of Atriplex sp.. Chenopodiaceae. Great Britain.

echinops (F.A. Hazslinszky) P.A. Saccardo, Sylloge Fungorum Omnium Hucusque Cognitorum Digessit P.A. Saccardo 2:32. 1883. 三Pleospsora echinops F.A. Hazslinszky, Matematikai és Természettudományi Közlemenyek Vonatkozólag a Hazaj Viszonyokra. Budapest 10:47. 1872. On stems of Verhascum phlomoides L., Scrophulariaceac. Hungary. eichhorniae R. González Fragoso \& R. Cifferi. Boletin de la R. Sociedad Española de Historia Natural, Madrid 26:473-474. 1926. Nom. inval. Art. 34.1. On dry leaves of Eichhornia crassipes (C.F.P. Mart.) SolmsLaub., Pontederiaceae. Dominican Republic. elaeidicola K.A. Pirozynski. Mycological Papers. Commonwealth Mycological Institute, Kew 129:19-20. 1972. On dead fronds of Elacis guineensis Jacq.. Palmae. Tanzania.

elaeidis C. Booth \& J.S. Robertson, Transactions of the British Mycological Society, London 44:26. 1961. On leaves of Elaeis guineensis Jacq., Palmae. Nigeria.

elacospora (P.A. Saccardo) P.A. Saccardo, Sylloge Fungorum Omnium Hucusque Cognitorum Digessit P.A. Saccardo 2:65. 1883. 三Leptosphaeria graminum P.A. Saccardo var elaeospora P.A. Saccardo. On dead leaves of Phragmites commumis Trin., Gramineae. France.

elaondi P. Rieuf, Al-Awamia; Revue de la Recherche Agronomique Marocaine 16:51. 1965. On branches of Pelargonium capitatum (L.) L'Her., Geraniaceae. Morocco.

ellisiana A.N. Berlese, Icones Fungorum Omnium Hucusque Cognitorum ad usum Sylloges Saccardianae Adcommodatae 1:68-69. 1892. ELeprosphaeria subconica J.B. Ellis \& B.M. Everhart, non Leprosphaeria subconica (G.W. Clinton \& C.H. Peck) P.A. Saccardo las Sphacria (Leptosphaeria) suhconica G.W. Clinton \& C.H. Peck in J.B. Ellis. North American Fungi, Series I, Century 7, No. 697. Anno 1881]. On dead stems of Oenothera biennis L.. Onagraceae. U.S.A.

elongata L.E. Wehmeyer, Mycologia, Lancaster, Pennsylvania 44:633. 1952. EPhaeosphaeria elougata (L.E. Wehmeyer) R.A. Shoemaker. Canadian Journal of Botany, Ottawa 67:1540. 1989. On dry stems of Elymus glancus Buckley, Gramineae. Canada, Finland. U.S.A.

elymi P. Larsen in L. Kolderup Rosenvinge and E. Wamning. The Botany of Iceland 2(Part 3):
474-475. (1931) 1932. Nom. illegit. Art. 64.1. ELeptosphaeria larseniana A. Munk. On dead plant of Elymus arenarius L., Gramineae. lceland.

clymi G.F. Atkinson, Bulletin. Comell University Agricultural Experiment Station, lthaca, New York 3(1):7. 1897. On dead portions of leaves of Elymus sp., Gramineac. U.S.A.

emiliana J.H. Fabre, Annales des Sciences

Naturelles, Paris, Botanique, Series 6, 9:90.

1878. On dry branches of Jasmimum fruricans

L., Pistacia terehinthus L., Oleaceae,

Anacardiaceae. France.

'mperri (A.P. de Candolle:E.M. Fries) H.G. Winter,

Dr. L. Rabenhorst's Kryptogaman-Flora von Deutschland, Oesterreich und der Schweiz, Second edition, 1(2):487. 1885 [as (Fuckel)]. \#yphoderma sphaerioides (J.B. Albertini \& L.D. v. Schweinitz) A.P. de Candolle var. empetri A.P. de Candolle, Flore Française 6:165. 1815. EXyloma empetri C.H. Persoon ined. =Hysterium sphaerioides J.B. Albertini \& L.D. v. Schweinitz, Conspectus Fungorum in Lusatiae Superioris Agro Niskiensi Crescentium e Methodo Persoonia, p. 57, No. 167. 1805. $\equiv$ Sphaeria emperri (A.P. de Candolle) E.M. Fries:E.M. Fries, Systema Mycologicum Sistens Fungorum 2:522. 1823. ESphaeria empetri (E.M. Fries?) L. Fuckel. Symbolae Mycologicae Dritter Nachtrag, p. 18. 1875. Nom. illegit. Ant 64.1. 三Metasphaeria emperi (A.P. de Candolle:E.M. Fries) P.A. Saccardo, Sylloge Fungorum Omnium Hucusque Cognitorum Digessit P.A. Saccardo 2:171 . 1883. On dry leaves of Emperrum nigrum L., Empetraceae. France. endiusae (L. Fuckel) P.A. Saccardo, Sylloge Fungormm Omnium Hucusque Cognitorum Digessit P.A. Saccardo 2:57. 1883. 三Pleospora endiusae L. Fuckel, Symbolae Mycologicae. pp. 136-137. 1870. In association with dried stems and pods of Endiusa hirsuta Alef. (=Vicia), Leguminosae. Germany.

endophuena E. Bonmer, M. Rousseau \& P.A.

Saccardo in P.A. Saccardo. Sylloge Fungorum Omnium Hucusque Cognitorum Digessit P.A. Saccardo 9:781. 1891. On dead branches of Ailanthus glandulosa Desf., Simaroubaceate. Bclgium.

ephedrae A. Maublanc, Bulletin de la Société Mycologique de France. Paris 21:88. 1905. On dead branches of Ephedra distachya $1 .$. Ephedraceac. France.

epiculamia (H. Riess) V. Cesati \& G. de Notaris, Commentario della Società Crittoganologica Italiana, Milan 1:236. 1863. ESphaeria epicalamia H. Riess in G.L. Rahenhorst. Klotzschii Herharium Vivum Mycologicum. I. Ausgabe, Century 19. No. 1828. Anno 1854. EPlenspora epicalamia L. Fuckel, Symbolae 
Mycologicae, p. 139. 1870. झPhaeosphaeria epicalmia (H. Riess) L. Holm, Symbolae Botanicae Upsalienses, Uppsala 14(3):114. 1957. [1llustrations as Sphaeria epicolamia H. Riess, Hedwigia, Dresden l(6), Table IV, Figure 6.] On dry culms of Luzula lutea (All.) DC., Luzula nemorosa (Pollich)

E.H.F. Meyer, Luzula sy/vatica (Hudson) Gaudin. Juncaceae. Germany, Switzerland. epicalamia V. Cesati \& G. de Notaris var. pleosporoides J. Feltgen, Vorstudien zu einer PilzFlora des Grossherzogthums, Luxemburg 1 (2):155. 1901. On dry culms of Luzula albida (Hoffm.) DC., Juncaceae. Luxembourg.

epicarecta (M.C. Cooke) P.A. Saccardo, Sylloge Fungorum Omnium Hucusque Cognitorum Digessit P.A. Saccardo 2:65. 1883. ESphaeria epicarecta M.C. Cooke, Grevillea, London 5:120. 1877. On leaves of Carex sp., Cyperaceae. Great Britain.

epilobii E. Müller, Sydowia. Annales Mycologici. Horn, N.Ö. 4(1-6):303-304. 1950. 三Leptosphaeria multiseptata H.G. Winter var. alpina H. Rehm, Hedwigia, Dresden 24:235, 1885. Non Leptosphacria alpina A. Maublanc. $\equiv$ Nodulosphaeria epilobii (E. Müller) L. Holm, Symbolae Botanicae Upsalienses, Uppsala 14(3):91. 1957. On dry stems of Epilobium flcischeri Hochst., Epilobium montanum L., Epilobium trigonum Schrank, Onagraceae. Italy. Switzerland.

equiseti P.A. Karsten, Öfversigt af K. Vetenskapsakademiens Forhandlinger. Stockholm 29(2):101-102. 1872. झPhaeosphaeria equiseti (P.A. Karsten) L. Holm \& K. Holm, Nordic Journal of Botany, Copenhagen [Opera Botanica] 1:113.1981. On aged stipes of Equisetum variegatum Schleicher ex Weber \& Mohr, Equisetaceae. Canada, U.S.S.R.

equiseficola L. Hollós, Matematikai és Természetıdományi Közlemenyek Vonatkozólag a Hazai Viszonyokra, Budapest 35:33. 1926. On dry stems of Equisetum variegatum Schleicher ex Weber \& Mohr, Equisetaceae. Hungary.

eranthemi N.T. Patouillard, Revue Mycologique. Toulouse 8:181. 1886. On peduncles of Eranthemum sp., Acanthaceae. China. eremophila (C.L. Spegazzini) P.A. Saccardo \& A. Trotter in P.A. Saccardo, Sylloge Fungorum Omnium Hucusque Cognitorum Digessit P.A. Saccardo 22:232. 1913. [Incorrectly cited as a comb. nov. based on Leprosphacrella cremophila C.L. Spegazzini.] Non. inval. Art. 43.I.

ericae (E.M. Fries:E.M. Fries) A. Malbranche in P.A. Saccardo, Syllogue Fungorum Omnium Hucusque Cognitorum Digessit P.A. Saccardo 9:780-781. 1891. ESphacria ericae E.M.
Fries:E.M. Fries, Elenchus Fungorum Sistens Commentarium in Systema Mycologicum 2:101. 1828. On trunks of Calluna vulgaris (L.) Hull, Ericaceae. France.

erigerontis F.E. Clements \& E.S. Clements.

Cryptogamae Formationum Coloradensium, Century 1, No. 28. Anno 1906. Nom. inval. Art. 32.1. On aged stems of Erigeron viscidus Rydb., Compositae. U.S.A.

erigerontis (A.N. Berlese) A.N. Berlese, Icones

Fungorum Omnium Hucusque Cognitorum ad usum Sylloges Saccardianae Adcommodatae 1:80-81. 1892. ELeptosphaeria agnita P.A. Saccardo var. erigerontis A.N. Berlese. On aged stems of Erigeron viscidus Rydb. Compositae. U.S.A. (Colorado).

erioboryae H. Sydow, P. Sydow, \& E. Butler, Annales Mycologici, Berlin 9:409. 1911. On leaves of Eriobotrya japonica (Thunb.) Lindley, Rosaceae. India.

eriophora (M.C. Cooke) P.A. Saccardo. Sylloge Fungorum Omnium Hucusque Cognitorum Digessit P.A. Saccardo 2:87-88. 1883. ESphaeria (Caulicolae) eriophora M.C. Cooke, Grevillea, London 5:153. 1877. $\equiv$ Pocosphaeria eriophora (M.C. Cooke) A.N. Berlese. Icones Fungorum Omnium Hucusque Cognitorum ad usum Sylloges Saccardianae Adcommodatae 1:89. 1892. On stems of Amaranthus sp.. Chenopodium sp.. Lappa sp. (=Arctium sp.). Amaranthaceae. Chenopodiaceae. Compositae. U.S.A.

eryngii H. Fabre, Annales des Sciences Naturelles. Paris, Botanique, Series 6, 15:49. 1883. On somewhat dry stems and petioles of Eryngium campestre L., Umbelliferae. France.

erythrinae H. Sydow, Annales Mycologici. Berlin 37:376. 1939. On dead deciduous leaves of Erythrina sp.. Leguminosae. Philippines.

espeletiae E. Muiller in E. Muiller and R.W.G.

Dennis, Kew Bulletin. Royal Botanic Gardens, Kew 19:373, 384. 1965. On dead inflorescence stalks of Espeletia (?Espcletia schuln:ii Wedd.) (=Balsamorhi=a). Compositae. Venezuela.

ettalensis A. Allescher, Bericht der Bayerischen Botanischen Gesellschaft zur Erforschung der Heimischen Flora. München 5:13-14. 1897. On dead stems of Laserpitium latifolium L.. Umbelliferae. Germany:

cumorpha (MI.J. Berkeley \& M.A. Curtis) F.S. Earle. Bulletin of the Torrey Botanical Club (and Torreya), New York 25:361-362. 1898. ESphaeria cumorpha M.J. Berkeley \& M.A. Curtis, Grevillea. London 4:145. 1876. EDidymella eumorpha (M.J. Berkeley of M.A. Curtis) P.A. Saccardo. Sylloge Fungorum Omnium Hucusque Cognitorum Digessit P.A. Saccardo 1:560. 1881. $\equiv D i-$ dymosphaeria eumorphus (M.J. Berkele! of 
M.A. Curtis) G.F. Atkinson, Bulletin. Corncll University Agricultural Experiment Station, Ithaca, New York 3(1):6. 1889. On culms of Arundinaria sp., Gramineae. U.S.A. (Alabama, South Carolina).

euphorbiac G. Niessl v. Mayendorf in G.L. Rabenhorst, Fungi Europaei Exsiccati, Klotzschii Herbarii vivi Mycologici Continuatio Edita Nova, Series 2, Century 19, No. 1841. Anno 1874; Just's Botanische Jarhesberichte, Berlin 2:319. 1876. On dried stems of Euphorbia cyparissias L., Euphorbiaceae. Czechoslovakia.

eluphorbiac G. Niessl v. Mayendorf forma esulae J. Feltgen, Vorstudien zu einer Pilz-Flora des Grossherzogthums. Luxemburg 1(3):212. 1903. On dry barked stems of Euphorbia esula L., Euphorbiaceae. Luxembourg.

eluphorbiaecola P. Brunaud, Bulletin de la Société Botanique de France 34:244. 1887. On dead stems of Euphorbia pilosa L.. Euphorbiaceae. France.

eluphorbiicolla S. Ahmad, Monographs. Biological Society of Pakistan. Lahore 8:87. 1978 (1979?) [as euphorbiaecola]. ELeplosphaeria depressa L.E. Wehmeyer \& S. Ahmad. 1964. Nec Leprosphaeria depressa T. Petch. 1926. On branches of Euphorbia osyridea Boiss., Euphorbiaceae. Pakistan.

customa (E.M. Fries:E.M. Fries) P.A. Saccardo, Aui dell' Accademia Scientifica Veneto-TrentinoIstriana, Padova 2(2):258. 1873 [as Fr.?]. Esphaeria eustoma E.M. Fries:E.M. Fries, Elenchus Fungorum Sistens Commentarium in Systema Mycologicum 2:109. 1828. On pedicels of Sorghum vulgare Pers., Gramineae. lialy.

customa (L. Fuckel) P.A. Saccardo, Fungi Italici autographice delineati (additis nonnullis extraitalicis asterisco notatis), Patavii, Table 497. 1879. Nom. illegit. Art. 64.1. 三Pleospora eustoma L. Fuckel, Symbolae Mycologicae, p. 139. 1870 [as Fr. El. II. p. 109, unter Sphaeria(?); see discussion by L. Holm. Symbolae Botanicae Upsalienses, Uppsala 14(3): 1 10-111. 1957]. EPhaeosphaeria eustomu (L. Fuckel) L. Holm. Symbolae Botanicac Upsalienses, Uppsala 14(3):109. 1957. On very rotten straw of unknown host of Gramincac. Gramineae. Austria, Canada, Switzerland, U.S.A.

customa (E.M. Fries:E.M. Fries) P.A. Saccardo forma eustomoides (P.A. Saccardo) A.N. Berlese, Icones Fungorum Omnium I Jucusque Cognitorum ad usum Sylloges Saccardianic Adcommodatae 1:56. 1890. [Vide Leptosphaeria eustomoides P.A. Saccardo.] eustoma (E.M. Fries:E.M. Fries) P.A. Saccardo forma leguminosa C.E. Fairman. Annales Mycologici, Berlin 4:327-328. 1906. On inner surface of pods of Robinia pseudacacius L., Leguminosac. U.S.A. eustomella P.A. Saccardo, Michelia Commentarium Mycologicum Fungos in Primis Italicos Illustrans 2:251. 1881. On culms of Festuca sp., Gramineae. ltaly.

eusromoides P.A. Saccardo, Nuovo Giornale Botanico Italiano e Bolletino della Società Botanica Italiana, Firenze 7:319-320. 1875. ELeptosphaeria eustoma (E.M. Fries:E.M. Fries) P.A. Saccardo forma eustomoides (P.A. Saccardo) A.N. Berlese, Icones Fungorum Omnium Hucusque Cognitorum ad usum Sylloges Saceardiannac Adcommodatae 1:56. 1890. EPhaeosphueria eustomoides (P.A. Saccardo) R.A. Shoemaker, Canadian Journal of Botany, Ottawa 67:1526. 1989. On culms of Andropogon ischaemum L., Gramineae. Italy.

euslomoides P.A. Saccardo forma lolii F. Fautrey in C. Roumeguère, Fungi Selecti Gallici Exsiccati, Century 59, No. 5847. Anno 1891; Revue Mycologique, Toulouse 13:167-168. 1891. On rachis of Lolium perenne $\mathbf{L}$.. Gramineae. France.

eustomoides P.A. Saccardo var. punctala A.C. Batisti, J.L. Bezerra \& E.A.F. da Matta, Memórias da Sociedade Broteriana, Coimbra 14:36-37. 1961. On leaves of Jambosa malaccensis DC. (=Syzygium), Myrtaceae. Brazil.

eutypoides C.H. Peck, Report. New York State Museun of Natural History, Albany 38:105. 1885. On dead stems of Chenopodium alhum L.. Chenopodiaceae. U.S.A.

cricelsu A.M. Saccas, Étude de la Flore Cryptogamique des Caféiers en Afrique Centrale. Bulletin de Institut Français du Café du Cacao et d'Autres Plantes Stimulantes (Bulletin IFCC No. 16), pp. 240-242. 1981. Nom. inval. Ari. 37.1. On dead branches of Coffer e'scelsa Cheval., Rubiaceac. Central African Republic

erocarpogena C.E. Fairman. Proceedings of the Rochester Academy of Science, Rochester. New York 6:98. 1921. On a shuck of hickory nut tree of Hicoria Raf. (=Carya Nutt.). Juglandaceae. U.S.A.

fagaricola (C.L. Spegazzini) P.A. Saccardo, Sylloge Fungorum Omnium Hucusque Cognitorum Digessit P.A. Salccardo 24:1000. 1928.

[ Incorrectly cited as a comb. nov, based on Leprosphaerella fagaricola C.L. Spegazzini.] Nom. inval. Art. 43.1.

faginea G. Passerini, Atti dell’ Reale Accademia Nazionale dei Lincei. Rendiconti, Rome. Series 4, 7(2):44. 1891. On dead stems of Fagus sp., Fagaceate. Italy.

fallaciosu A.N. Berlesc, Bulletin Trimestriel de la Société Mycologique de France, Paris 5:43. 1889. On dead stems of Samureja homensis I... Labiatae. Italy. 
fallax A.N. Berlese, Fungi Moricolae Iconographia e Descrizione dei Funghi Parassiti del Gelso. Fascicle IV, No. 3. Anno 1887. On dead stems of Morus alba L., Moraceae. Italy.

faulii G.D. Darker, Canadian Joumal of Botany, Ortawa 42:1006-1008. 1964. On dead needles of Abies halsamea Miller, Pinaceae. Canada, U.S.A.

feijoae G.V. Artemieff, Sovetskie Subtropiki. Sukhum 7:62. 1935. On living leaves of Feijoa sp. [as $F$. feijoicola] ( $=A c c a)$, Myrtaceae. U.S.S.R.

feltgeni P.A. Saccardo \& P. Sydow in P.A. Saccardo, Sylloge Fungorum Omnium Hucusque Cognitorum Digessit P.A. Saccardo 16:513. 1902. Nom. illegit. Art. 64.1. 三Leptosphaeria hemerocallidis J. Feltgen.

ferruginea M. Chochrjakov, Notulae Systematicae e Sectione Cryptogamica Instituti Botanici Nomine V.L. Komarov Academiae Scientiarum U.R.S.S., Petropolis 7:144. 1951. On living leaves and stems of Ipomoea batatas (L.) Lam., Convolvulaceae. U.S.S.R.

ferulicola E.N. Koschkelova in E.N. Koschkelova, I.P. Frolov, and Z. Dzhuraeva, Mikoflora Badkhyza, Karabilya i Yuzhnoĭ Chasti Murgabskogo, Oazisa (Mikromitsety) [The Mycoflora of Badkhyz, Karabil and the Southem Part of the Murgab Oasis (Micromycetes)]. p. 87. 1970. Nom. nud. Art. 32.1. On dry stems and stalks of Ferula badra-kema K. Pol., Umbelliferae. U.S.S.R. (Karabilia).

fibrincola $\mathrm{F}$. v. Höhnel \& H. Rehm in $\mathrm{H}$. Rehm, Annales Mycologici, Berlin 3:228. 1905; Ascomyceten, Fascicle 34, No. 1591. Anno 1905. On rotten paper. Germany.

fici-elasticae F. Petrak, Annales Mycologici, Berlin 14:164. 1916. On dead leaves of Ficus clastica Roxb., Moraceae. Czechoslovakia. fiedlaeri (G. Niessl v. Mayendorf) P.A. Saccardo, Michelia Commentarium Mycologicum Fungos in Primis Italicos Illustrans 1:39. 1877. $\equiv$ Cryptospora fiedlaeri $\mathrm{G}$. Niessl $\mathrm{v}$ Mayendorf, Hedwigia, Dresden 13:42-43. 1874. $\equiv$ Metasphacria fiedlaerii P.A. Saccardo, Sylloge Fungorum Omnium Hucusque Cognitorum Digessit P.A. Saccardo 2:165. 1883. On branches of Cormus sanguinea L., Comaceate. Italy.

filamentosa J.B. Ellis \& B.M. Everhart, Joumal of Mycology. Columbus, Ohio 4:76. 1888. On dead pieces of living leaves of Yucca filamentosa L.. Agavaceac. U.S.A.

filiformis L.E. Wehmeyer, Mycologia, Lancaster, Penusylvania 44:6+1. 1952. ĐO Phioholus filiformis (L.E. Wehmeyer) R.A. Shoemaker, Canadian Joumal of Botany. Ottawa 54:2378-2379. 1976. On stem ol unknown host of composite. Compositae. U.S.A.

fimbriata J.B. Ellis \& B.M. Everhart. The North Atnerican l'yrenomycetex. A Contribution to
Mycologic Botany, p. 357. 1892. On herbaceous stems of unknown host, unknown family. U.S.A.

fimiseda H.G. Winter, Hedwigia, Dresden 10:I63. 1871. On smoked hare (Lepus europaeus, Leporidae) in Harth Woods. Germany.

fiumana $\mathrm{F}$. Hazslinszky, Matematikai és Természettudományi Közlemenyek Vonatkozólag a Hazai Viszonyokra. Budapesı 25(2):143. 1892. On stems of unknown host of Labiatae, Labiatae. Hungary.

flotoviae C.L. Spegazzini. Boletin de la Academia Nacional de Ciencias en Córdoba 25:70-71. 1921. On dead and dry clump of Flatoria excelsa DC. (=Dasyphyllum), Compositae. Chile.

fluviatilis (W. Phillips \& C.B. Plowright) P.A. Saccardo, Sylloge Fungorum Omnium Hucusque Cognitorum Digessit P.A. Saccardo 2:84. 1883. ESphaeria fluviarilis W. Phillips \& C.B. Plowright, Grevillea, London 10:73. 1881. On unknown substrate of Lemanea fluviatilis C. Aq., Algae (Lemaneaceae). Great Britain.

foeniculacea $\mathrm{H}$. Fabre. Annales des Sciences Naturelles, Paris. Botanique. Series 6, 15:51. 1883. On dead stems of Foeniculum vulgare Miller, Umbelliferae. France.

foeniculacea H. Fabre subsp. lupina P.A. Saccardo \& G. Scalia in P.A. Saccardo. C.H. Peck, and W. Trelease, Harriman Alaska Expedition 5:29-30. 1904. On decaying stems of Lupinus sp., Leguminosae. U.S.A. (Alaska).

foeniculi R. González Fragoso. Boletim da Sociedade Broteriana. Coimbra. Series 2. 2:23. 1923. On dry stems of Foeniculum vulgare Miller. Umbelliferae. Portugal.

foliicola N.A. Naumov, Ural'skoe Obshchestvo Estestvoznaniia, v Ekeaterinburgie Zadipiski 35(11-12):21. 1916. On living leaves of Libanotis montana Crantz, Umbelliferae. U.S.S.R.

folliculata J.B. Ellis \& B.M. Everhart, Proceedings of the Academy of Natural Sciences of Philadelphia 1890:237. 1890. On leaves of Carex folliculata $\mathrm{L}$.. Cyperaceae. Canada (Ontario).

folliculata J.B. Ellis \& B.M. Everhart var. onvspora J.J. Davis. Transactions of the Wisconsin Acadeny of Science. Arts and Letters. Madison 18:87. 1915. EDidymella onspora (J.J. Davis) R.A. Shoemaker. Canadian Joumal of Botany Ottawa 67:1576. 1989. On unknown substrate of Carex gracillima Schwein.. Cyperaceae. U.S.A.

francoac (C.L. Spegazzini) P.A. Saccardo \& A. Trotter. Sylloge Fungorum Onnium Hucusque Cognitorum Digessit P.A. Saccardo 22:221. 1913. ELeprosphacerella francoae C.L. Spegazzini. Fungi Chilenses, p. 77. 1910; 
Revista de La Facultad de Agronomia Y Veterinaria, Universidad Nacional de La Plata, Series 2, 6:77. 1910. On year-old dry scapes of Francoa sonchifoliae Cav., Saxifragaceae. Chile.

franconica (F. Petrak) E. Müller, Sydowia. Annales Mycologici, Horn, N.Ö. 4( I-6):298-299. 1950. 三Leptosphaeria derasa (M.J. Berkeley \& C.E. Broome) B. Auerswald var. franconica F. Petrak, Kryplogamische Forschungen, München 2:162-163. 1931. 三Nodulosphaeria franconica (F. Petrak) L. Holm, Symbolae Botanicae Upsalienses, Uppsala 14(3):84. 1957. On unknown substrate of Iminla salicina L., Compositae. Switzerland.

fraserae J.B. Ellis \& B.M. Everhart, Bulletin of the Torrey Botanical Club (and Torreya), New York 27:52-53. 1900. On dead stems of Frasera speciosa Dougl. ex Griseb., Gentianaceae. U.S.A.

fraxini J.B. Ellis \& B.M. Everhart, Journal of Mycology, Columbus, Ohio 3:44. 1887. On living leaves of Fraximus americana L., Oleaceie. U.S.A.

frigida L.N. Vasil'eva, Pirenomitsety i Lokuloaskomitsety Severa Dal'nego Vosıoka (Leningrad:"Nauka"), p. 117. 1987 [as frigidus]. On dead leaves of Luzula sp., Juncaceae. U.S.S.R.

frondis W. Kirschstein. Annales Mycologici, Berlin 33:212.1935. On young living stems of Lonicera tatarica L., Caprifoliaceae. Germany.

fuckelii G. Niessl v. Mayendorf in W. Voss, Österreichische Botanische Zeitschrift, Wien 32:357-358. 1882. EPhacosphaeria fuckelii (G. Niessl) L. Holm. Symbolae Botanicae Upsalienses, Uppsala 14(3):123. 1957. On dead stems of Calamagrostis montana Host, Gramineae. Canada, Germany, Romania, Sweden, Switzerland, U.S.A., Yugoslavia.

fuckelii G. NiessI v. Mayendorf forma filamentifera P.A. Karsten, Hedwigia, Dresden 27:261. 1888. On sheath of Phalaris arundinacea L., Gramineae. Finland.

fuegiana C.L. Spegazzini. Boletin de la Academia Nacional de Ciencias en Córdoba 11:219. 1887; Fungi Fuegiani, No. 231. Anno 1887. On leaves and stems of Hicrochlö antarcica R. Br., Gramineae. Argentina.

fulgida (M.C. Cooke \& C.A. Peck) M.E. Barr in M.E. Burr, C.T. Rogerson, S.J. Smith, and J.H. Haines, Bulletin of the New York Stite Museum, Albany 459:23. 1986. ESphaeria (Curlicolue) fulgida M.C. Cooke \& C.A. Peck in M.C. Cooke \& J.B. Ellis, Grevillea, London 6:15. 1877 las G.W. Clinton \& C.A. Peck in C.A. Peck, Report. New York State Museum of Natural History. Albany 29:62.
(1875) 1878]. 三Ophiobolus fulgidus (M.C. Cooke \& C.H. Peck) P.A. Saccardo. Sylloge Fungorum Omnium Hucusque Cognitorum Digessit P.A. Saccardo 2:346. 1883.

$\equiv$ Rhaphidospora fulgida (M.C. Cooke \& C.H. Peck) M.C. Cooke, Grevillea, London 18:16. 1889. On stenis of Ambrosia trifida L., Compositae. U.S.A. (New York).

fungicola H.G. Winter, Hedwigia, Dresden 25:101.

1886. On aged hymenium of Sterem subpileatum Berkeley \& Broome, Fungi (Stereaceae). São Tomé.

fuscella (M.J. Berkeley \& C.E. Broome) V. Cesalti \& G. de Notaris, Commentario della Società Crittogamologica Italiana, Milan 1:236. 1863. ESphaeria (Obrectae) fuscella M.J. Berkeley

\& C.E. Broome. Annals and Magazine of Natural History, London, Series 2, 9:325. 1882. On dead twigs of Rosa sp., Rosaceae. Great Britain.

fuscella (M.J. Berkeley \& C.E. Broome) P.A.

Saccardo forma microspora C. Roumeguère. Revue Mycologique, Toulouse 12:163. 1890; Fungi Selecti Gallici Exsiccati, Century 55. No. 5438. Anno 1890. On dry branches of Sarolhammus scoparius (L.) Wimmer ex Koch, Leguminosae. France.

fuscella V. Cesati \& G. de Notaris var. hippophaes $\mathbf{J}$. Feltgen, Vorstudien zu einer Pilz-Flora des Grossherzogthums, Luxemburg 1(3):212. 1903. On barky branches of Hippophaë rhammoides L., Elaeagnaceae. Luxembourg.

fuscella (M.J. Berkeley \& C.E. Broome) V. Cesati \& G. de Notaris var. sydowiana P.A. Saccardo in H. Sydow, Mycotheca Germanica, Fascicle 10-11, No. 485. Anno 1906; Annales Mycologici, Berlin 4:484. 1907. On branches of Hippophaë rhamnoides L., Elaeagnaceae. Germany.

fuscidula G. Passerini, Rendiconti della Sedute della R. Accademia dei Lincei, Classe di Scienze Fisiche, Matematiche e Natural (=Atti dell' Accademia Nazionale dei Lincei, Rendiconti. Roma), Series 4, 3:91. 1887. On dry leaves of Melica altissima L.. Gramineae. Italy.

fuscidula G. Passerini forma magnolii R. González

Fragoso, Trabajos del Museo Nacional de Ciencias Naturales, Madrid. Series Botanica 10:88. 1916. On wilted or dry leaves of Melica magnolii Gren. \& Godron. Gramineac. Spain.

fusispora G. Niessl v. Mayendorf, Verhandlungen des Naturforschenden Vereins in Brünn 10:177-178. (1871) 1872. On fallen branchlets of Genista tinctoria L.. Leguminosae. Austria (near Gratz).

fusispora $\mathrm{G}$. Niessl v. Mayendorf forma erysimi $\mathrm{C}$. Sandu-Ville, Ciuperci PyrenomycetesSphaeriales Din România, pp. 277-278. 1971. On branches of Erysimum hicracifolium L.. Cruciferae. Romania. 
galeobdolonis J. Feltgen, Vorstudien zu einer PilzFlora des Grossherzogthums, Luxemburg 1(3):221. 1903. On dry stems of Galeobdolon lulem Hudson, Labiatae. Luxembourg. galeopsidicola F. Petrak. Annales Mycologici. Berlin 25:289. 1927. On stems of Galeopsis speciosa Miller, Labiatae. Czechoslovakia.

galii (G.H. Otth) P.A. Saccardo, Hedwigia Beiblatt, Dresden 35:XXIX. 1896; Sylloge Fungorum Omnium Hucusque Cognitorum Digessit P.A. Saccardo 11:XXIX. 1896. EPleospora galii G.H. Otth, Mitteilungen der Naturforschenden Gesellschaft in Bern 1867(660):56. 1868. On stems of Galium sp., Rubiaceae. Switzerland. galii-silvarici W. Kirschstein. Verhandlungen des Botanischen Vereins der Provinz Brandenburg, Berlin 48:56. (1906) 1907. On dry stems of Galium sylvaticum L.. Rubiaceae. Germany.

galiicola P.A. Saccardo, Atti dell' Accademia Scientifica Veneto-Trentino-Istriana, Padova 2(2): 152-153. 1873. On fallen rotten stems of Galiun mollıgo L., Rubiaceae. Italy?. Netherlands.

galicola P.A. Saccardo var. braclyyspora P.A. Saccardo, Nuovo Giornale Botanico Italiano e Bolletino della Società Botanica ltaliana, Firenze 7:315. 1875. On stems of Centranthus sp., Hesperis sp., Lappa sp., Valerianaceae, Cruciferae. Compositae. Italy.

galiormm (M.R. Roberge) V. Cesati \& G. de Notaris. Commentario della Società Crittogamologica Italiana. Milan 1:235. 1863. ESphacria (Caulicola) galiorum M.R. Roberge in J. Desmazières, Annales des Sciences Naturelles, Paris, Botanique, Series 3. 6:77. 1846. =Melasphacria galiorim (M.R. Roberge) P.A. Saccardo. Sylloge Fungorum Omnium Hucusque Cognitorum Digessit P.A. Saccardo 2:160. 1883. On old stems of Galium sp.. Rubiaceae. France.

(Metasphaeria) galiormm (M.R. Roberge) H.G. Winter. Hedwigia, Dresden 26:57-58. 1887. Nom. illegit. Art. 64.1. On old stems of Galium sp., Rubiaceae. France.

galiorum P.A. Saccardo forma cirsiorum P.A. Saccardo, Sylloge Fungorum Omnium Hucusque Cognitorum Digessit P.A. Saccardo 2:22. 1883. On stems of Cirsium peslustre (L.) Scop., Compositae. France.

galiormm P.A. Saccardo forma genfianue P.A Saccardo. Sylloge Fungorum Omnium Hucusque Cognitorum Digessit P.A. Saccardo 2:22. 1883. On dead stems of Gentiana hitea I... Gentianaceae. France.

galiorum P.A. Saccardo subsp. anrirrhini P.A. Saccardo, Nuovo Giomale Botanico Italiano e Bolletino della Società Botanica Italianat. Firenze. New Series 22:38-39. 1915. On stents of Antirhimum siculum Miller. Scrophulariaceac. Yugoslavia. galiorum P.A. Saccardo var. gentiance P.A.

Saccardo, Atti del Istituto Veneto di Scienze, Lettere ed Arti. Venezia. Series 6. 2:156-457. (1883) 1884. On stems of Gentiana lurea L.. Gentianaceae. Switzerland.

galiorum P.A. Saccardo var. lapsanae P.A. Saccardo \& P.A. Briard, Revue Mycologique. Toulouse 7:209. 1885. On dead stems of Lampsana communis L. (=Lapsana communis L.). Compositae. France.

galiorım P.A. Saccardo var. gnaphaliana C.E. Fairman in C.F. Millspaugh and L. W. Nuttall. Fieldiana:Botany, Chicago 5:352. 1923. On stems of Ginaphalium sp.. Compositae. U.S.A. (California).

galligena K. Keissler. Beihefte zum Botanischen Zentralblatt. Cassel. Abt. 11. 37:274-275. 1920. On thallus of Parmelia [as $P$. arrara Zahlbr.]. Lichenes (Parmeliaceae). U.S.A. (Hawaii).

gaubae F. Petrak, Sydowia. Annales Mycologici. Horn. N.Ö. 9(1-6):562. 1955. EPhaeosphacria gawbae (F. Petrak) R.A. Shoemaker. Canadian Journal of Botany. Otiawa 67:1514. 1989. On dry leaves of Danthonia frigida Vichery, Gramineae. Australia.

gaultheriae J. Dearness, Mycologia, Lancaster. Pennsylvania 9:349.1917. On dead stems of Gaulheria shallon Pursh. Ericaceae. Canada (British Columbia).

geasteris L. Hollós. Annales Historico-Naturales Musei Nationales Hungarici, Budapest 5:279. 1907. In internal layers of exoperidium of Geaster pseudolimbarus Hollós. Fungi (Geastraceae). Hungary.

genistae C.A. Oudemans. Verslagen van de Gew one Vergadering der Wis-en Natuurkundige

Afdeeling. K. Academic van Wetenschappen Te Amsterdam 9(1):141. 1900. On fruits of Genista anglica L.. Leguninosae. Netherlands.

genisıae C.A. Oudemans var. microspora A.L. Guyot. Revue de Mycologie, Pariv 11:68. 1946. On dry stems of Genista tinctoria L.. Leguminosae. France.

geographicola (F. Amold) P.A. Saccardo \& D.

Saccardo in P.A. Saccardo, Sylloge Fungorum Omnium Hucusque Cognitorum Digessit P.A. Saccardo 17:731. 1905. EPliaeospora geographicola F. Amold in F. W. Zopf. Hedwigia. Dresden 35:356-357. 1896. EPhaeosprora geographicola F. Amold. Verhandlungen der Zoologisch-Botanischen Gesellschaft in Wien 46:139-140. 1896. [Effecrively puhlished in Verhandlungen der Zoologisch-Botanischen Gesellschaft in Wien 24:254. 1874.] On thalli of Rhi-exarpun geographictam (L.) DC.. Lichenes (Rhizocarpaceae). Germany. 
geographicola (F. Amold) L. Vouaux, Bulletin de la Société Mycologique de France, Paris 29:118. 1913. Nom. illegit. Art. 64.1. On thallus of Rhizocarpon geographicum (L.) DC.. Lichenes (Rhizocarpaceae). Italy.

georgius-fischeri R. Sprague, Mycologia, Lancaster. Pennsylvania 50:815. 1958. On living leaves of Muchlenhergia filiformis Rydb., Gramineae. U.S.A.

gihelliana R. Pirotta, Atti dell' Istituto Botanico della Università e Laboratorio Crittogamica di Pavia, Milano $2 \&$ 3:164. (1877) 1879. On branches of Viris vinffera L., Vitaceae. Italy.

gigaspora A.M. Saccas. Étude de la Flore Cryptogamique des Caféiers en Afrique Centrale. Bulletin de Institut Français du Calé du Cacao et d'Autres Plantes Stimulantes (Bulletin IFCC No. 16). pp. 248-250. 1981. Nom. nud. Art. 37.1. On dead stems and branches of Coffea rohusta L. Linden (=Coffea canephora Pierre ex Froehn.). Rubiaceae. Central African Republic.

gigaspsora G. Niessl v. Mayendorf in P.A. Saccardo. Sylloge Fungorum Omnium Hucusque Cognitorum Digessit P.A. Saccardo 2:65. 1883; G. Niessl v. Mayendorf in G.L. Rabenhorst, Fungi Europaei Exsiccati. Klotzschii Herbarii vivi Mycologici Continuatio Edita Nova, Series 2, Century 30. No. 2998. Anno 1883. On leaves of Carex paludosa Good., Cyperaceate. Czechoslovakia.

gillotiana P.A. Saccardo \& C. Roumeguère, Revue Mycologique, Toulouse 5:236. 1885. On corticated branches of Salix sp., Salicaceae. France.

ginkgo S.A. Gucevicz, Novosti Sistematiki Nizshikh Rastenij, Novitates Systematicae Plantarum Non Vascularium 7:160. 1970. On dry branches of Ginkgo biloba L., Ginkgoaceae. U.S.S.R.

glandulosae A.I. Lobik, Bolezni Rastenii S.

Peterburg (Morbi Plantarum Scripta Sectionis Phytopathologiae Horti Botanici Principalis) 17:161. 1928. On leaves of Ailamimus glandulosa Desf., Simaroubaceae. U.S.S.R.

glaucopunctalu (R.K. Greville) B. Auerswald, Hedwigia, Dresden 7:185. 1868. 三Cryplosphaeria glancopmetata R.K. Greville, Flora Edinensis or a Description of Plants Growing Near Edinburgh, pp. 362-363. 1824.

ESphaeria glaucopunchata (R.K. Greville) F. Currey, Transactions of the Linnean Society of London 22:333. 1859. On dead leaves of Ruscus aculeams L., Ruscaceae. France. Great Britain. Italy.

glocospora (M.J. Berkeley \& F. Currey) P.A. Siccardo, Sylloge Fungorum Omnium Hucusque Cognitorum Digessit P.A. Saccardo 2:25. 1883. ESphaeria (Canliculue) glocospora M.J. Berkeley \& F. Currey in M.J.
Berkeley and C.E. Broome. Annals and Magazine of Natural History, London, Series

3, 7:454.1861. ETrichometasphaeria glocospora (M1.J. Berkeley \& F. Currey) L. Holm, Symbolae Botanicae Upsalienses, Uppsala 14(3):144. 1957. On stems of Artemisia ahsinthium L.. Compositae. Great Britain.

glyceriae L.M. Unamuno Yrigoyen, Revista de la R. Academia de Ciencias exactas, lisicas y naturales de Madrid 30:483-486. 1933. On leaves of Glyceria fluitans (L.) R. Br., Gramincae. Spain.

glvereriac-plicalae T. Săvulescu \& C. Sandu-Ville,

Hedwigia, Dresden 73:74-75. 1933.

$\equiv$ Phacosphaeria glyceriae-plicaloe (T.

Săvulescu \& C. Sandu-Ville) R.A. Shoemaker, Canadian Journal of Botany, Otawa 67:1527. 1989. On wilted leaves of Glyceria plicala (Fries) Fries, Gramineae. Romania. gnaphalii (G.D. Westendorp \& L. Fuckel) P.A.

Saccardo, Sylloge Fungorum Omnium

Hucusque Cognitorum Digessit P.A. Saccardo 2:34. 1883. EPleospora gnaphalii G.D. Westendorp? in L. Fuckel. Fungi Rhenani Exsiccati A Leopoldo Fuckel Collecti Supplement, Fascicle 7. Total Series Fascicle 22. No. 2153. Anno 1868; Symbolac Mycologicae, p. 136. 1870. On dry stems of Gnaphalium sp., Compositae. Austria. Belgium.

gossypii N.N. Woronichin. Trudy Akademiia Nauk SSSR Botanicheskii Muzei 21:125. 1927. On spots, on leaves of Gossypium sp.. Malvaceae. U.S.S.R.

graminis (L. Fuckel) P.A. Saccardo, Sylloge Fungorum Omnium Hucusque Cognitorum Digessit P.A. Saccardo 2:76-77. 1883. EPleospora graminis L. Fuckel, Symbolae Mycologicate, p. 139. 1870. EP/ucosphaeria graminis (L. Fuckel) L. Holm, Symbolae Botanicae Upsalienses, Uppsala 14(3):118. 1957. On dry culms of Plragmites communis Trin., Gramincae. Germany, Great Britain. graminum P.A. Saccardo, Michelia Commentarium Mycologicum Fungos in Primis Italicos Illustrans 1:119. 1878; Fungi Italici autographice delineati (additis nonnullis extra-italicis asterisco notatis), Patavii, Table 483. 1879. $\equiv$ Melasphaeria graminum P.A. Saccardo, Sylloge Fungorum Omnium IIucusque Cognitorum Digessit P.A. Saccardo 2:174. 1883. On dead leaves ol Calamagrossis sp.. Gramineac. Germany.

graminum P.A. Saccardo var. elueospora P.A.

Saccardo. Michelia Commentarium Mycologicum Fungos in Prinis Italicos Illustrans 2:320. 1881. ELeposphaerias elacospora (P.A. Saccardo) P.A. Saccardo. Sylloge 
Fungorum Omnium Hucusque Cognitorum

Digessit P.A. Saccardo 2:65. 1883.

grammodes (G. de Notaris) V. Cesati \& G. de Notaris, Commentario della Società Crittogamologica lraliana, Milan 1:235. 1863.

$\equiv$ Sphaeria grammodes G. de Notaris,

Memorie della Accademia delle Scienze di Torino, Series 2, 2:74-75. 1841. On dry stems of Artemisia vulgaris L., Compositae. Italy.

grandispora P.A. Saccardo, Michelia Commentarium Mycologicum Fungos in Primis Italicos Illustrans 1:341. 1878. EMctasphaeria grandispora (P.A. Saccardo) P.A. Saccardo, Sylloge Fungorum Omnium Hucusque Cognitorum Digessit P.A. Saccardo 2:181. 1883. $\equiv$ Massariosphaeria grandispora (P.A. Saccardo) A. Leuchtmann, Sydowia. Annales Mycologici, Hom, N.Ö. 37:172. 1984. =Lophiotrema grandispora (P.A. Saccardo) R.A. Shoemaker, Canadian Journal of Botany. Ottawa 67:1580. 1989. On decaying leaves of Typha Iatifolia L., Typhaceae. Italy.

gratissima P. Rieuf \& G. Teasca, Al-Awamia: Revue de la Recherche Agronomique Marocaine 34:54. 1970 [as gratissimae]. On wood and leaves of Persea americana P. Mill.. Lauraceae. Morocco.

gratissima P. Rieuf \& G. Teasca var. longispora P. Rieuf \& G.Teasca. Al-Awamia; Revue de la Recherche Agronomique Marocaine 34:56. 1970 [as gratissimae]. On branches, on irregular, marginal spots of leaves of Persea americana P. Mill., Lauraceae. Morocco. grignonnensis A.L. Guyot, Revue de Mycologie, Paris 11:66-68. 1946. On dry runners of Clematis vitalha L., Ranunculaceae. France.

grisea G. Passerini, Atti della R. Accademia dei Lincei Memorie. Rome, Series 4, 6:459. (1889) 1890. On wilted culms of Sorghum vulgare Pers., Gramineae. ltaly.

grossulariae Z. Girzitska. Universytet Botanichnyi Sad Visnik Izvestiia Kiev. I(Livr. V-VI): 167. 1927. On living branches of Ribes grossularia L., Saxifragaceac. Spain.

guazimae R. González Fragesa \& R. Ciferri. Boletin de la R. Sociedad Española de Historia Natural. Madrid 26:474. 1926. Nom. nud. Art. 34.1. On living leaves of Guazuma sp.. Sterculiaceae. Dominican Republic.

guazumae R. González Fragoso \& R. Ciferri, Publicaciones Estación Agronómica de Moca. Santa Domingo. Series B, Botanica, No. 8:22-23. 1927. Nom. inval. Art. 34.1. On living leaves of Guazuma sp.. Sterculiacente. Dominican Republic.

gynerii C.L. Spegazzini, Anales del Museo Nacional de Historia Natural de Buenos Aires 6:281-282. 1898; Fungi Argentini Novi vel Critici, No. 627. Anno 1899. On rotting dead leaves of Gynerium argeneum Nees. Gramineae. Argentina.

gypsophilae L. Hollós, Annales Historico-Naturales Musei Nationalis Hungarici. Budapest 5:45.

1907. On dry stems of Gypsophila paniculara L., Caryophyllaceae. Hungary.

hacmatites (M.R. Roberge) G. Niessl v. Mayendorf in G.L. Rabenhorst. Fungi Europaei Exsiccati. Klotzschii Herbarii vivi Mycologici Continuatio Edita Nova, Series 2. Century 28. No. 2761. Anno 1882. ESphaeria (Caulicola) haematites M.R. Roberge in J. Desmazières. Annales des Sciences Naturelles. Paris. Botanique, Series 3, 16:311. 1851. 三Didymella haematites (M.R. Roberge) P.A. Saccardo, Sylloge Fungorum Omnium Hucusque Cognitorum Digessit P.A. Saccardo 1:553. 1882. ESphaerella haemarires (M.R. Roberge) M.C. Cooke, Journal of Botany: British and Foreign, London 21:109. 1883. On dry stems of Clematis viralha L. Ranunculaceac. France.

haematites (M.R. Roberge) H.G. Winter, Hedwigia. Dresden 22:10. 1883. Nom. illegit. Ar. 64.1. ESphaeria (Caulicola) haematires M.R. Roberge in J. Desmazières. Annales des Sciences Naturelles. Paris, Botanique. Series 3, 16:311. 1851. EDidymella haematites (M.R. Roberge) P.A. Saccardo. Sylloge Fungorum Omnium Hucusque Cognitorum Digessit P.A. Saccardo 1:553.1882 [as M.R. Roberge et J. Desmazières]. ESphaerella haematites (M.R. Roberge) M.C. Cooke. Journal of Botany, British and Foreign. London 21:109. 1883. On runners of Clematis vitalba L.. Ranunculacene. Belgium. France, Germany.

haematites (M.R. Roberge) P.A. Saccardo. Sylloge Fungorum Omnium Hucusque Cognitorum Digessit P.A. Saccardo 2:36. 1883. Nom. illegit. Art. 64.1. ESphaeria (Caulicola) haematites M.R. Roberge in J. Desmazières. Annales des Sciences Naturelles. Paris. Botanique. Series 3. 16:311. 1851. On dry stems of Clematis vitalba L.. Ranunculaceae. France.

halima T. Johnson. Mycologia. Lancaster. Pennsylvania 48:502. 1956. $\equiv$ Phaeosphacria halima (T. Johnson) R.A. Shoemaker, Canadian Journal of Botany, Ottawa 67:1514. 1989. On test panel of Liriodendron tulipifere $\mathrm{L}$. submerged in salt water. Magnoliace:e. U.S.A.

haloryli B. Kravtzes. Trudȳ Instituta Botaniki Akademiya Nauk Zazaklıskoj SSR Alma-Ata 2:1.46-147. 1955. On decorticated, rotten wood of Halowlon aphy/hm (Minkw.) Hjin. Chenopodiaceice. U.S.S.R.

hamamelidis C.E. Fairman. Proceedings of the Rochester Academy of Science. Rochester. 
New York 6:127. 1929. On dead plant of Hamametis virginiana L.. Hamamelidaceae. U.S.A.

hardenbergiae C.G. Hansford. Proceedings of the Linnean Society of New South Wales. Sydney 79:120-121. 1954. On leaves of Hardenbergia violucea (Schneev.) Steam. Apocynaceae. Australia.

harknessianna J.B. Ellis \& B.M. Everhart, Journal of Mycology, Columbus, Ohio 1:91. 1885. On dead stems of Frasera? sp., Gentianaceale. U.S.A.

hausmamiana B. Auerswald. Hedwigia, Dresden 7:185. 1868; Österreichische Botanische Zeitschrift. Wien 18:241. 1868. On upper surface of dead leaves of Silene acantis (L.) Jacq.. Caryophyllaceae. Italy.

hausmanniana B. Auerswald var, cherleriae P.A. Saccardo, Sylloge Fungorum Omnium Hucusque Cognitorum Digessit P.A. Saccardo 2:47. 1883. [Based on Terry specimen $\# 6179$, published as Leptosphaeria hausmannianes $\mathbf{B}$. Auerswald in P.A. Saccardo. Michelia Commentarium Mycologicum Fungos in Primis Jalicos Illustrans 2:599. 1882.] On underside of dead leaves of Silene acanlis (L.) Jacq.. Caryophyllaceae. France.

harslinskyana A.N. Berlese, Icones Fungorum Omnium Hucusque Cognitorum ad usum Sylloges Saccardianae Adcommodatae 1:78. 1892 [as Leptosphaeria hanzslinskyana]. On culn of an unknown host of gratss, Gramineae. Germany.

hazslinszkii P.A. Saccardo, Sylloge Fungorum Omnium Hucusque Cognitorum Digessit P.A. Saccardo 2:28. 1883. [Based on Ditopella sp.. published by F.A. Hazslinszky, Verhandlungen der Zoologisch-Botanischen Gesellschaft in Wien 20:217. 1870.] On dead branches of Rosa sp., Rosaceale. Romania.

hederae (M.J. Sowerby?) P.A. Saccardo, Nuovo Giornale Botanico Italiano c Bolletino della Società Botanica Italiana, Firenze 7:313. 1875. झSphaeria hederae P.A. Saccardo, Atti dell' Accademia Scientifica Veneto-TrentinoIstriana, Padova 2(1):144-145. 1873 |as Sphaeria hedarae M.J. Sowerhy?, Sphaeria hederae M.C. Cooke?]. ESphueria hederae M.J. Sowerby, Coloured Figures of English Fungi or Mushrooms 3. Table 37l. Figure 5. 1803. झMetasphaeria hederae (M.J.

Sowerby?) P.A. Saccardo, Sylloge Fungorum Omnium Hucusque Cognitorum Digessit P.A. Saccardo 2:169. 1883. On lower surface of rotting leaves of /ledera helis L., Araliaccac. Great Britain?. Italy.

hedericola (J. Desmazières) P.A. Saccardo. Sylloge Fungorum Omnium Hucusque Cognitorum Digessit P.A. Saccardo 2:51. 1883. 三Sphuerice (Depazea) hedericola J. Desmazières.
Annales den Sciences Naturclles, Paris. Botanique. Series 3. 14:115. 1850 [as hederoecola]. On wilted and dead leaves of Hedera helit L.. Araliaceae. France.

heliamthemi B. Auerswald in W. Gonnemann and G.L. Rabenhorst, Mycologia Europacal. Abbildungen Sämmtlicher Pilze Europa's. Hefte 5 und 6, Synopsis Pyrenomycetum Europieorum, Table 12. Figure 166. 1869. Nom. nud. Art. 32.1. EMelasphaeria helianthemi (B. Auerswald) ex P.A. Saccardo, Sylloge Fungorum Omnium Hucusque Cognitorum Digessit P.A. Saccardo 2:160. 1883. On stems of Heliunthemum sp.. Compositae. Germany.

heliamhi J.B. Ellis \& B.M. Everhart in J.F. Brenckle, Mycologia, Lancaster. Pennsylvania 9:284. 1917. Nom. nud. Art. 32.1. On unknown substrate of Helianthus giguntews L., Compositae. U.S.A. (North Dikota).

heliumbi S.T. Tilak in T.S. Viswanathan and S.T.

Tilak. Mycopathologia et Mycologia Applicata. Den Haag 13:241. 1960. On dry culms of theliamthus annuus L.. Compositae. India.

helichrysi M.C. Cooke [falsely so cited in P.A. Saccardo, Sylloge Fungorum Omnium Hucusque Cognilorum Digessit P.A. Saccardo 9:783. 1891]. झLeptosphaerella helichrysi M.C. Cooke. Grevillea, London 19:7. 1890.

helicicola (J. Desmazières) G. Niessl v. Mayendorf. Verhandlungen des Naturforschenden Vereins in Brünn 10:175. (1871) 1872. ESphacria (Folicola) helicicola J. Desmazières. Annales des Sciences Naturelles. Paris, Botanique. Series 3, 11:356-357. 1849. EMerasphacria helicicola (J. Desmazières) P.A. Saccardo. Sylloge Fungorum Omnium Hucusque Cognitorum Digessit P.A. Saccardo 2:169. 1883. On dried leaves of Hedera helix L., Araliaceate. France.

heliopsidis (L.D. v. Schweinitz:E.M. Fries) L. Holm, Svensk Botanisk Tidskrift. Stockholm 62:230. 1968. ESphacria heliopsidis L.D. v. Schweinitz. Synopsis Fungorum Carolinac Superioris. Pp. 34-35. 1822. EDothidea heliopsidis (L.D. v. Schueinitz:E.M. Fries) L.D. v. Schweinit/, Synopsis Fungorum in America Boreali Media Degentium. Secundum Observationes Ludovici Davidis de Schweinitz, p. 231. 1832; E.M. Frics. Systema Mycologicum Sistens Fungorum 2:556. 1823. EMontagnella heliepsidis (L.D. v. Schweinit $\ell$ E.M. Fries) P.A. Saccardo, Sylloge Fungorum Ommium Ilucusque Cognitorum Digessit P.A. Saccardo 2:646. 188.3. $\equiv$ Phacoderris heliopsidis (L.D. v. Scliweinitz:E.M. Fries) F. v. llöhnel.

Situngsberichte der Akademie der Wissenschaften in Wien. Mathenatischnaturwissenschafiliche Klasse. Abc. I. 
118:876. 1909. 三Rosenscheldia heliopsidis (L.D. v. Schweinitz:E.M. Fries) F. v. Theissen \& H. Sydow, Annales Mycologici, Berlin 13:649. 1915. EGibberidea heliopsidis (L.D. v. Schweinitz:E.M. Fries) C.L. Shear, Mycologia, Lancaster, Pennsylvania 29:361. 1937. ESyncarpella heliopsidis (L.D. v. Schweinitz:E.M. Fries) M.E. Barr in M.E. Barr and J.R. Boise, Memoirs of the New York Botanical Garden, Bronx 49:300. 1989. On stems of Aster sp., Helianthus sp., Heliopsis sp., Compositae. U.S.A. helminthospora (V. Cesati) V. Cesati \& G. de Notaris. Commentario della Società Crittogamologica Italiana, Milan 1:235. 1863. Esphaeria helminthospora V. Cesati in G.L. Rabenhorst, Klotzschii Herbarium Vivum Mycologicum Sistens Fungorum Per Totam Germaniam Cresentium Collectionem Perfectam, Dresden. Edition 1, Century 18, No. 1735. Anno 1853: Botanische Zeitung. Berlin \& Leipzig 11:236. 1853. On unknown substrate of Imula crithmoides L.. Compositae. Germany.

helminthospora (V. Cesati) V. Cesati \& G. de Notaris forma crithmi-marinimi P.A. Saccardo, Sylloge Fungorum Omnium Hucusque Cognitorum Digessit P.A. Saccardo 2:33. 1883. On dead stems of Crithmum marifimum L., Unbelliferae. France.

heloniacfolia (M.C. Cooke \& J.B. Ellis) P.A. Saccardo, Sylloge Fungorum Omnium Hucusque Cognitorum Digessit P.A. Saccardo 2:68. 1883. =Sphaeria (Foliicola) heloniaefolia M.C. Cooke \& J.B.Ellis, Grevillea, London 8:16. 1879. On leaves of Helonias bullata L., Liliaceac. U.S.A.

(Leprosphacrella) helverica P.A. Saccardo \& C.L. Spegazzini in P.A. Saccardo, Michelia Commentariun Mycologicum Fungos in Primis ltalicos lllustrans 1:399. 1878. On upper surface of wilted leaves of Selaginclla helvetica (L.) Spring. Selaginellaceac. ltaly.

helierica P.A. Saccardo \& C.L. Spegazzini forma major H. Rehm, Ascomyceten, Fascicle 18, No. 884. Anno 1886: Hedwigia. Dresden 26:93. 1887. On unknown substrate of Sclaginella helictica (L.) Spring. Selaginellaceae. Italy.

hemerocallidis J. Feltgen, Vorstudien zu einer PilzFlora des Grossherzogthums, Luxemburg 1(2):155. 1901. [Validly published by relerence to an effectively published description in Vorstudien zu einer Pil/-Flora des Grossherzogthums, Luxemburg 1:252. 1899, as Leprospherevia sp. $1=$ Leprosphaeria felrgemi P.A. Saccardo \& 1'. Sydow, Sylloge Fungorum Omnium Hucusque Cognitorum Digessit P.A. Satceardo 16:513. 1902. Nom. illegit. Art. 64.1. On dry stems of /1 'merocallis sp.. Liliaceac. Luxembours. hemicrypta C.A. Oudemans, Nederlandsch kruidkundig Archief. Verslagen en Mededeelingen der Nederlandsche Botanische Vereeniging. Leiden, Series 2, 4:521-522. 1886. On leaves of Carex sp., Cyperaceae. Netherlands.

(Clypeosphaeria) hendersoniae (J.B. Ellis) M.C. Cooke. Grevillea. London 17:91. 1889. ESphaeria (Obtectae) hendersonia J.B. Ellis in M.C. Cooke and J.B. Ellis, Grevillea, London 6:14-15. 1877. 三Clypeosphaeria hendersoniae (J.B. Ellis) P.A. Saccardo, Sylloge Fungorum Omnium Hucusque Cognitorum Digessit P.A. Saccardo 2:91. 1883. On canes of Rubus sp., Rosaceae. U.S.A.

hendersoniae (L. Fuckel) L. Holm, Symbolae Botanicae Upsalienses, Uppsala 14(3):26. 1957. Nom. illegit. Art. 64.1. Non Leprosphaeria (Clypeosphaeria) hendersoniae M.C. Cooke, Grevillea, London 17:91. 1889. =Cucurbitaria hendersoniae L. Fuckel. Symbolae Mycologicae, p. 172. 1870. =Melanomma hendersoniae P.A. Saccardo. Sylloge Fungorum Onnium Hucusque Cognitorum Digessit P.A. Saccardo 2:109. 1883. On corticated or decorticated branches of Salix sp., Salicaceae. Italy. Sweden.

hermodactyli T. Ferraris in T. Ferraris and C. Massa. Annales Mycologici. Berlin 10:287. 1912. On leaves of Hermodacry/us tuberosus (L.) Miller, Iridaceae. Italy.

herpotrichoides G. de Notaris, Sferiacei Italici, pp. 80-81. 1863. EPhacosphaeria herpotrichoides (G. de Notaris) L. Holn, Symbolae Botanicae Upsalienses. Uppsala 14(3):115. 1957. On culms of Andropogon sp. Koeleria cristata (L.) Pers., Gramineae. Finland. ltaly. hesperia M.E. Barr, Canadian Journal of Botany: Ottawa 45:1044. 1967. ELimospora hrumellac J.B. Ellis \& B.M. Everhart. Proceedings of the Academy of Natural Sciences of Philadelphia 1894:337. 1894. [Anamorph: Phoma brumellac (J.B. Ellis \& B.M. Everhart) C.E.O. Kuntze, Revisio Generum Plantarum 3:502. 1898.] ECeuhocarpon brumellae (J.B. Ellis \& B.M. Everhart) A.N. Berlese. lcones Fungorum Onnium Hucusque Cognitorum ad usum Sylloges Saccardianae Adconmodatae 2:149. 1899. On living and dying leates of Prunella wu/garis L.. Labiatac. Canada. U.S.A.

hesperidicula R. Picbatuer, Bosanko-Khertsovachki Musey Glasnik, Sarajevo 48:104. 1936. On dead stems of Hosperis dimarica G. Beck. Cruciferac. lugoslavia.

heterospora (G. de Notaris) P.A. Saccardo, Sylloge Fungorum Omnium Hucusque Cognitorum Digessit P.A. Saccardo 2:67. I8S3 las Leprospharevia hererospora (G. de Notaris) G. 
Niessl v. Mayendorf]. ESphacria heterospora G. de Notaris, Sferiacei ltalici, Century 1, Fuscicle 2, p. 65. 1863. EBissuhecium heterosporum (G. de Notaris) F. v. Thümen, Mycotheca Universalis, Century 14. No. 1361. Anno 1879. On living. subterranean rhizome of Iris germanica L.. Iridaceate. Czechoslovakia, ltaly.

hererospora (G. de Notaris) G. Niess!v. Mayendorf. Verhandlungen des Naturforschenden Vereins in Brünn 10:173. 1872. ESphaeria heter)spora G. de Notaris, Sferiacei Italici, p. 65. 1863. EByssothecium heterospormm (G. de Notaris) F. v. Thümen, Mycotheca Universalis, Century 14, No. 1361. Anno 1879. ETrematosphaeria heterospora $(\mathrm{G}$. de Notaris) H.G. Winter, Dr. L. Rabenhorst's Kryptoganian-Flora von Deutschland, Oesterreich und der Schweiz, Second edition, 1(2) Ascomyceten: Gymoasceen und Pyrenomyceten, p. 277. 1885. EPhucosphacria heterospora (G. de Notaris) J.R. Boise. Mycologia, Lancaster, Pennsylvania 77:236. 1985. On living, subterranean rhizome of Iris germanica L., Iridaceae. Czechoslovakia, Jtaly.

he'ufleri (G. Niessl v. Mayendorf) P.A. Saccardo, Sylloge Fungorum Omnium Hucusque Cognitorum Digessit P.A. Saccardo 2:83. 1883. ESphacrella heufleri G. Niessl v. Mayendorf, Verhandlungen des Naturforschenden Vereins in Brünn 10:167. (1871) 1872. $\equiv$ Mycotodea heufleri $(\mathrm{G}$. Niessl v. Mayendorf) W. Kirschstein. Annales Mycologici, Berlin 34:201. 1936. On dry sporangia of Polvrichum formosum Hedw., Musci (Polytrichaceae). Italy.

hereue A.M. Saccas, Agronomie Tropicale, Nogentsur-Marne 8:244. 1953. On living leaves of Hevea hrasiliensis Muell. Arg., Euphorbiaceae. French Equatorial Africa.

hiemalis P.A. Saccardo \& C.L. Spegazzini in P.A. Saccardo. Michelia Commentarium Mycologicum Fungos in Primis Italicos Illustrans 1:395-396. 1878. EPhaeosphaeria hiemalis (P.A. Saccardo \& C.L. Spegazzini) R.A. Shoemaker, Canadian Journal of Botany, Ottawa 67:1527. 1989. On decaying stems of Equisetum hyemale L. [as hiemalis]. Equiseticeae. Canada. Italy. U.S.A.

hierochloae C.A. Oudemans, K. Akademie van Wetenschappen Amsterdam. Afdeeling Natuurkund Verslagen en Mededeelingen 3 Reeks, Deel 2:155. I885 [as hieroch/or's]. EPhacosphaevia hierohloae (C.A. Oudemans) O. Eriksson, Arkiv för Botanik, Uppsala, Stockholm, New Series 6:424. 1967. On unknown substrate of Hierochlor" alpino (Willd.) Roemer \& Schultes, Graminese. U.S.S.R.
(Mclanomma) hippophaes (J.H. Fahre) M.C. Cooke, Grevillea, London 17:91. 1889. =Melanomma hippophae's J.H. Fabre, Annales des Sciences Naturelles, Paris, Botanique, Series 6.9:92-93. 1878. On dry branches of llippophae rhammoides L., Elaeagnaceac. France.

hippophaes (A. Sollmann) A. Nannizzi, Atti dell Accademia dei Fisiocritici di Siena, Series 10, 5:385. 1931 [as hipphophaes]. ESphacria hippophaes A. Sollmann, Botanische Zeitung, Berlin \& Leipzig 20:379-380. 1862. $\equiv C l a-$ desphaeria hippophaes (A. Sollmann) T.R.J. Nitschke in G.H. Otth. Mitteilungen der Naturforschenden Gesellschaft in Bern 1870:105. 1871. Massaria hippophaes (A. Sollmann) A.L. Jaczewski. Bulletin de I'Herbier Boissier, Genève and Bâle 2:684. 1894. On dead branches of Hippophae thommoides L., Elaeagnaceae. Germany. ltaly, Switzerland.

hippophues (A. Sollmann) P.A. Saccardo, Sylloge Fungorum Omnium Hucusque Cognitorum Digessit P.A. Saccardo 22:222.1913 [as hippopphaes]. Nom. illegit. Art. 63.1. Sphaeria hippophaes A. Sollmann, Botanische Zeitung. Berlin \& Leipzig 20:379-380.

1862. On dead corticated primocanes of Hippophae rhammoides L., Elaeagnaceae. Germany.

hirra (E.M. Fries:E.M. Fries) V. Cesati \& G. de Notaris, Commentario della Società Crittogamologica Italiana, Milan 1:236. 1863. ESphaeria hirta E.M. Fries:E.M. Fries. Kungliga Svenska Vetenskapsakademiens Handlingar, Uppsala and Stockholm 1818:105. 1818: Systema Mycologicum Sistens Fungorum 2:483. 1823 [as Sphueria (Cyt.) hirut. $=$ Nodulosphaeria hirta (E.M. Fries:E.M. Fries) G.L. Rabenhorsı, Klotzschii Herbarium Vivum Mycologicum Sistens Fungorun Per Totam Germaniam Cresentium Collecrionem Perfectam, Dresden. Edition 11. Century 8. No. 725. Anno 1858. Nom. rejic. On dead stems of Sambucus racemosa L.. Caprifoliaceae. Germany, Sweden.

hira (L. Fuckel) W. Kirschstein, Annales Mycologici, Berlin 34:188-189. 1936. Nom. illegit. Art. 63.1. 三Massaria hira L. Fuckel, Symbolac Mycologicac, p. 155. 1870. On dead thin branches of Sambucus racemosa L.e Caprifoliaceae. Germany.

hispanica J. Checa \& G. Moreno, Canadian Journal of Botany, Ottawa 65:2096-2097. 1987. On twigs of Artemisia vulgaris L., Compositae. Spain.

hollosiano L.E. Wehmeyer, Mycologia, Lancaster. Pennsylvania 55:319. 1963. ELeptosphueria maritima L. Hollós. Non Leptosphaeria maritima J.B. Ellis \& B.M1. Everhart. Non 
Lepuosphaeria maritima P.A. Saccardo. On stems of Bichersteinia emodii Jaub. \& Spach.. Potentilla argyrophylla Wallich ex Lehm., Potentilla fragarioides L.. Triglachin maritima L.. Trisetum spicatum (L.) K. Richter, Geraniaceae, Gramineae, Juncaceae. Rosaceae. India.

hollosii G. Moesz, Magyar Botanikai Lapok. Budapest 28:54. 1929. ĐLeptosphaeria tamaricis L. Hollós, Botanikai Közlemények. Budapest 25:130. 1928. Non Leptosphaeria camaricis (R.K. Greville) P.A. Saccardo, Sylloge Fungorum Omnium Hucusque Cognitorum Digessit P.A. Saccardo 2:26. 1883. On dried branches of Tamarix gallica L.. Tamaricaceae. Hungary.

holmii M.T. Lucas, Memórias da Sociedade Broteriana. Coimbra 21:36. 1970. On dead culns of Scirpus holoschoenus L., Cyperaceae. Portugal.

holmii (L.N. Vasil'eva) L.N. Vasil"eva, Pirenomitsety i Lokuloaskomitsety Severa Dal nego Vostoka (Leningrad:"Nauka“), p. 122. 1987. Nom. illegit. Art. 64.1. 三Nadulosphueria holmii L.N. Vasil"eva, Mikologiia i Fitopatologiia Akademiia Nauk SSSR. Leningrad 13:277-278. 1979. On stems of Lagoris minor (Willd.) Standley, Scrophulariaceae. U.S.S.R.

honiaraensis T. Matsushima, Microfungi of the Solomon Islands and Papua-New Guinea, pp. 73-74. 1975. On decayed leaf of a broadleal tree of unknown tree host, unknown family. Solomon 1slands.

hordei A.A. Domaschova in A.A. Domaschova and N.A. Gamalizkaja. Notulae Systematicae e Sectione Cryptogamica Instituti Botanici Nomine V.L. Komarov Academiae Scientiarum U.R.S.S.. Petropolis 15:75-76. 1962. On unknown substrate of Hordcum nukestanicum Nevski, Gramineae. U.S.S.R.

hottai K. Hara, Byôchu-gai Zasschi (Joumal ol Plant Protection), Tokyo 6(4):37. 1918. Nom. nud. Art. 32.1. On trunks of Thea simensis L.. Theaceae. Japan.

horrai K. Hara ex K. Hara, Chagyokai (Tea Joumal) 14(9):14-15. 1919; Mycologia, Lancaster. Pennsylvania 13:24. 1921. Parasitic on trunks of Thea sinensis L.. Theaceae. Japan.

houscana P.A. Saccardo, Annales Mycologici. Berlin 13:116. 1915. On dead stems of Thalicmum dioicum L.. Ranunculaceate. U.S.A.

hrubyana F. Petrak. Annales Mycologici. Berlin 25:290-291. 1927. On dry stems of Linum tentifolitm L., Linaceae. Czechoslovakia.

hisrae N.T. Patouillard. Bulletin de la Société Mycologique de France. Paris 16:185-186. 1900. On a stem of /lura crepilans L. (=Manitia), Euphorbiaceae. Guadeloupe (West Indies). huthiana R. Staritz. Verhandlungen des Botanischen Vereins Der Provinz Brandenburg. Berlin 55:79-80. 1913. In leaf tissue of Sparganium ramosum Hudson. Sparganiaceae. Germany.

hyalina K.S. Panuar \& S.J. Kaur, Kavaka. Madras 3:68. 1975. On dead twigs of unknown host. unknown family. India.

hyalospora P.A. Saccardo, Nuovo Giomale Botanico Italiano e Bolletino della Società Botanica ltaliana, Firenze 7:323. 1875. $\equiv$ Mesasphaeria hyalospora (P.A. Saccardo) P.A. Saccardo, Sylloge Fungorum Omnium Hucusque Cognitorum Digessit P.A. Saccardo 2:179. 1883. On decayed stems of Zea mavs L.. Gramineae. Italy.

hydrangeae S.A. Gucevicz. Novosti Sistematiki Nizshikh Rustenij. Novitates Systematicae Plantarum Non Vascularium 7:161. 1970. On dry branches of $H$ ydrangea arhorescens L.. Saxifragaceae. U.S.S.R.

hydrophila P.A. Saccardo. Nuovo Giomale Botanico Italiano e Bolletino della Società Botanica 1taliana. Firenze 7:320-321. 1875. On canes of Juncus effusus L.. Juncaceae. Italy.

hyparheniae C.G. Hansford. Proceedings of the Linnean Society of London 153:24. 1941. On dead stems of Hyparhenia sp.. Gramineae. Uganda.

hyperborea (L. Fuckel) A.N. Berlese \& P. Voglino. Sylloge Fungorum Omnium Hucusque Cognitorum Digessit P.A. Saccardo Additamenta Ad Volumina 1-IV. p. 140. 1886. EPleosora Inperborea L. Fuckel. Pilze der Zweiten Deutschen Nordpolfahr 2:92. 1874. $\equiv M$ ycorodea hyperborea (L. Fuckel) W. Kirschstein. Annales Mycologici. Berlin 34:201. 1936. ELeptosphaeruhina hyperborea (L. Fuckel) M.E. Barr. Contributions from the University of Michigan Herbarium. Ann Arhor 9:5+2. 1972. On upper surtace of dried leaves of Andromeda tetragona L.. Ericaceac. Greenland, Norway.

hyperici H.G. Winter, Hedwigia, Dresden 11:147. 1872. On dry stems of Hypericum perforaum L.. Guttiferae. Germany.

hypericola S.A. Gucevicz. Notulae Systenaticae e Sectione Cryptogamica Instituti Botanici

Nomine V.L. Komarov Academiade Scientiarum U.R.S.S., Petropolis 13:183-184. 1960. On dried peremnial parts of Hypericum alpesiris Stev. ex Ledeb.. Guttiferae.

U.S.S.R. (Tauria).

hysterioides J.B. Ellis \& B.M. Everhart, Bulletin of the Washburn Lahoratory of Natural Histor [Washbun College]. Topeka I:4. 1884. On dead leaves of Jorophy/hm renas (Pursh) Nutt., Liliaceac. U.S.A.

icosigma R.C. Maire. Bulletin de la Societe d'Histoire Naturelle de l'Afrique du Nord. Alger $\$: 170.1917$. On dry stems of Rubia peregrima L., Rubiaceas. Algeria. 
immunda P.A. Karsten, Mycologia Fennica Pars 2 , Pyrenomycetes, p. 105. 1873; Bidrag till Kännedom om Findlands Natur och Folk. Utgifna af Finska Vetenskaps-Societeten, Helsingfors 23:105. 1873. EMetasphaeria immunda (P.A. Karsten) P.A. Saccardo, Sylloge Fungorum Omnium Hucusque Cognitorum Digessit P.A. Saccardo 2:170. 1883. On leaves of Populus tremula L., Salicaceae. Finland.

impressa (C.G.T. Preuss) P.A. Saccardo, Sylloge Fungorum Omnium Hucusque Cognitorum Digessit P.A. Saccardo 2:57. 1883.

ESphaeria impressa C.G.T. Preuss, Linnaea. Institut botanique de l'Université de Genève 26:713-714. 1853. On pods of Cheiranthus annuus L., Polypodiaceac. Germany.

inarensis (E.A. Vainio) K. Keissler, Annalen des Naturhistorischen Museums in Wien 42:99. 1928. ETrypethelium inarense E.A. Vainio, Societas pro Fauna et Flora Fennica, Helsingfors Meddelanden 10:197-198. 1883 三Xenosphaeria inarensis (E.A. Vainio) E.A. Vainio, Acta Societatis pro Fauna et Flora Fennica, Helsingforsiae 49(2):142-143. 1921. 三Sphaeria inarensis (E.A. Vainio) W. Nylander, Flora, Jena und Regensburg 68:300. 1885. Nom. inval. Art. 33.1. Parasitic on unknown lichen, Lichenes. Scandinavia. incarcerata (M.J. Berkeley \& M.A. Curtis) P.A. Saccardo, Sylloge Fungorum Omnium Hucusque Cognitorum Digessit P.A. Saccardo 2:86. 1883. ESphaeria incarcerata M.J. Berkeley \& M.A. Curtis in M.J. Berkeley, Grevillea, London 4:152. 1876. 三Heptameria (Leptosphaeria) incarcerata (M.J. Berkeley \& M.A. Curtis) M.C. Cooke, Grevillea, London 17:33. 1889. =Passeriniella incarcerata (M.J. Berkeley \& M.A. Curtis) A.N. Berlese, Icones Fungorum Omnium Hucusque Cognitorum ad usum Sylloges Saccardianae Adcommodatae 1:51. 1892. On stems of Spartina sp., Gramineae. U.S.A. inconspicua H. Rehm, Österreichische Botanische Zeitschrift, Wien 56:296. 1906. Nom. nud. Art. 32.1. On ashes of Berberis sp., Berberidaceae. Austria.

incruenta W. Kirschstein, Annales Mycologici, Berlin 37:108-109. 1939. On dry stems of Clematis vitalha L., Ranunculaceae. Germany.

inculta P.A. Saccardo \& A.F. Malbranche in P.A. Saccardo, Michelia Commentarium Mycologicum Fungos in Primis Italicos Illustrans 2:598. 1882. On stems of Chenopodium album L., Chenopodiaceae. France.

indeprensa (M.C. Duricu de Maisonneuve \& J.P. Montagne) P.A. Saccardo, Sylloge Fungorum Omnium Hucusque Cognitorum Digessit P.A. Saccardo 2:85. 1883. ESphaeria indeprensa
M.C. Durieu de Maisonneuve \& J.P.

Montagne in M.C. Durieu de Maisonneuve,

Exploration Scientifique de L'Algérie Publiée Par Ordre du Gouvernement. Series

Naturelles. Botanique 1, p. 538. (1848) 1849.

On dead and withered leaves of Silene

rosulata Soyer-Willem. \& Godr., Caryophyllaceae. Algeria.

indica H. Sydow, P. Sydow, \& E. Butler, Annales Mycologici, Berlin 9:409. 1911. On leaves and stems of Asparagus sp., Liliaceae. India. inecola K. Hara, Diseases of the Rice Plant, p. 157. 1918. On unknown substrate of Oryza sativa L., Gramineae. Japan.

infermalis G. Niess] v. Mayendorf, Instituto. Revista Scientifica e Litteraria, Coimbra 31:89. 1883. „Montagmula infernalis (G. Niessl v.

Mayendorf), A.N. Berlese, Icones Fungorum Omnium Hucusque Cognitorum ad usum Sylloges Saccardianae Adcommodatae 2:69. 1896. On dead leaves of Furcraea longaeva Karw. \& Zucc., Agavaceae. Portugal.

inquinans C.H. Peck, Bulletin of the New York State Museum, Albany 131:22-23. 1909. On bark of Acer saccharum auct., Aceraceae. U.S.A.

insignis P.A. Karsten, Öfversigt af K. Vetenskapsakademiens Forhandlingar, Stockholm 29(2):100. 1872. EPhaeosphaeria insignis (P.A. Karsten) L. Holm, Symbolae Botanicae Upsalienses, Uppsala 14(3):120. 1957. On leaves of Aira alpina L., Alopecurus ovatus Knapp, Dupontia fisheri R. Br., Dupontia psilosantha (Rupr.) Griseb., Gramineae. Canada, Norway.

insignis P.A. Karsten forma airae-cespitosae C. Roumeguère, Fungi Selecti Gallici Exsiccati, Century 58, No. 5754. Anno 1891; Revue Mycologique, Toulouse 13:129. 1891. On leaves of Aira cespitosa L.. Gramineae. France.

inspersa (L.D. v. Schweinitz) P.A. Saccardo, Sylloge Fungorum Omnium Hucusque Cognitorum Digessit P.A. Saccardo 2:27-28. 1883. 三Sphaeria inspersa L.D. v. Schweinitz in F. Currey, Transactions of the Linnean Society of London 22:328. 1869. Non Sphaeria inspersa M.J. Berkeley. On bark of unknown host [L.D. v. Schweinitz specimen in W.J. Hooker's herbarium], unknown host, unknown family. U.S.A.

insulana P.A. Saccardo, Nuovo Giornale Botanico Italiano e Bolletino della Società Botanica Italiana, Firenze, New Scries 22:39. 1915. On dead stems of Antirhinum siculum Miller. Scrophulariaceae. Yugoslavia.

intermedia (G. Nicssl v. Mayendorf), Hedwigia, Dresden 26:46-48. 1887. On unknown substrate of Calamagrostis sp., Gramineae. Austria.

imerspersa (M.C. Cooke) P.A. Saccardo, Sylloge Fungorum Omnium Hucusque Cognitorum 
Digessit P.A. Saccardo 2:77. 1883.

$\equiv$ Sphueria interspersa M.C. Cooke, Grevillea, London 6:146. 1878. On sheaths of Zea mays L., Gramineae. U.S.A. (Florida).

imolucralis G. Passerini, Hedwigia, Dresden 12:140. 1873. [Falsely so cited by P.A Saccardo, Sylloge Fungorum Omnium Hucusque Cognitorum Digessit P.A. Saccardo 2:172. 1883.] ESphaeria imolucralis G.

Passerini, Hedwigia, Dresden 12:140. 1873. $\equiv$ Metasphaeria involucralis (G. Passerini) P.A. Saccardo, Sylloge Fungorum Omnium Hucusque Cognitorum Digessit P.A. Saccardo 2:172. 1883. On involucre of Castanea sp., Fagaceae. Italy.

iridicola J.B. Lambotte \& F. Fautrey, Revue Mycologique, Toulouse 17:168. 1895. On frozen leaves of Iris foetidissima L., Iridaceae. France.

ividigena F. Fautrey in F. Fautrey and J.B. Lambotte, Revue Mycologique, Toulouse 17:168-169. 1895. On dry leaves of Iris pseudacorus L.. Iridaceae. France.

iridigena F. Fautrey var. typhae J. Feligen, Vorstudien zu einer Pilz-Flora des Grossherzogthums, Luxemburg I(3):206-207. 1903. On dry leaves of Typha angustifolia L.. Typhaceac. Luxembourg.

iridis L. Hollós, Annales Historico-Naturales Musei Nationalis Hungarici, Budapesı 5:45-46. 1907. On dry leaves of /ris arenuria Waldst. \& Kit. Iridaceae. Hungary.

irrepta G. Niessl v. Mayendorf in G. Linhart. Fungi Hungarici Exsiccati, Century 4, No. 368. Anno 1885. On unknown substrate of Cycas revoluta Thunb., Cycadaceae. Hungary.

isariphora (J. Desmazières) B. Auerswald in W. Gonnermann and G.L. Rabenhorst, Mycologia Europaea, Abbildungen Sämmtlicher Pilze Europa's, Hefte 5 und 6. Synopsis Pyrenonycetum Europaeorum, Table 12, Figure 170. 1869. ESphacria isariphora J. Desmazières. Annales des Sciences Naturelles, Paris, Botanique, Series 2, 19:358. 1843: Plantes Cryptogames Du Nord De La France, Edition 1, Fascicle 26, No. 1291. Anno 1843. Esphaerella isariphora (J. Desmazières) G. de Notaris, Comnentario della Società Crittogamologica Italiana, Milan 1:237. 1863. On dry leaves of Stellaria graminea L., Caryophyllaceae. France, Germany.

ischa'mi G. Passerini, Erbario Critogamico Italiano. Series 2, Fascicle 22, No. 1073. Anno ISSI. On dry culms of Andropogon ischaemum L.. Gramineat. Italy.

isocellulu K.S. Panwar \& S.J. Kaur, Kavaka, Madras 5:41. 1977. On dead wood of Lamtana camara L., Verbenaceac. India (Mit. Abu. Rajasthan).

iw momoi l. Miyake. Journal of the College of Agriculture, Imperial University of Tohyo
2:249. 1910. On leaves of Oryza sariva L., Gramineae. Korea.

jaceae L. Holm, Svensk Botanisk Tidskrift. Stockholm 42:35-36. 1952. ENodulosphaeria jaceae (L. Holm) L. Holm, Symbolae Botanicae Upsalienses, Uppsala 14(3):86. 1957. On dead stems of Centatrea jacea L.. Compositae. Sweden.

jacksonensis R.A. Shoemaker. Canadian Journal of Botany, Ottawa 62:2704-2705. 1984.

=Leplosphaeria tetonensis L.E. Wehmeyer.

On old stems of Pedicularis bracteosa Benth. ex Hook., Scrophulariaceae. U.S.A.

jacksonii R.A. Shoemaker, Canadian Journal of Botany, Ottawa 62:2705. 1984. On stems of Eupatorium maculatum L. and E. purpureum L., Compositae. Canada.

jalmii C.E. Chardon, Boletin de la Sociedad Venezolana de Ciencias Naturales, Caracas 5(40):263-264. 1939. [Exiract pp. 353-354.] EPhaeophragmocauma jahnii (C.E. Chardon) E. Müller in E. Müller \& R.W.G. Dennis. Kew Bulletin. Royal Botanic Gardens, Kew 19(3):376. 1965. On living leaves of Espeleria neriffolia Sch. Bip. ex Wedd., Compositae. Venezuela.

janus (M.J. Berkeley \& M.A. Curtis) P.A. Saccardo. Sylloge Fungorum Omnium Hucusque Cognitorum Digessit P.A. Saccardo 2:85. 1883. ESphaeria janus M.J. Berkeley \& M.A. Curtis, Grevillea. London 4:154. 1876. =Metasphaeria janus (M.J. Berkeley \& M.A. Curtis) A.N. Berlese, Icones Fungorum Omnium Hucusque Cognitorum ad usum Sylloges Saccardianae Adcommodatae 1:146. 1894 [as Metasphaeria junci (Oud.) Sacc.]. 三Dothidella jamus (M.J. Berkeley \& M.A. Curtis) F. v. Höhnel. Sitzungsberichte der Akademie der Wissenschaften in Wien. Mathematisch-naturwissenschaftliche Klasse. Abt. 1, 124:68, 1915. झMvcosphaerella janus (MI.J. Berkeley \& M.A. Curtis) F. Petrak. Sydowia. Annales Mycologici, Ноп, N.Ö. 11:340. (1957) 1958. On leaves of Quercus virens Ait. (=Q. virginiuna Mill.). Fagaceae. U.S.A.

juponica (I. Miyake) K. Hara. Report of the Sericultural Association of Japan. Tokyo 27(314):226. 1918. EMassaria japonica I. Mlyake. Sangyō Shikenjō Hōh ok u (Bulletin of the Sericultural Experiment Station) 1(5):319. 1915. On Moris sp.. Moraceac. Japan.

jasmini, Sylloge Fungorum Omnium Hucusque Cognitorum Digessit P.A. Saccardo 15:191. 1901. AAtributed to L. Fuchel by P.A. Saccardo in Sylloge Fungorum Omnium Hucusque Cognitorum Digessit P.A. Saccardo 3:309. 1884, cited L. Fuckel. Symbolae Mycologicae Zweiter Nachtrag. p. 24, 1873. The lungus listed there is Pleospora jasmini (J.L. Castagne) L. Fuchel.] 
johansonii E. Müller, Sydowia. Annales Mycologici, Horn, N.Ö. 4(1-6):238. 1950 [as johansoni]. झMetusphaeria arabidis C.J. Johanson, Hedwigia, Dresden 25:122. 1886. Non Leptosphaseria srabidis A. Allescher. On dry stems of Arabis alpina L., Cruciferae. Switzerland.

jubreae C.L. Spegazzini, Boletin de la Academia Nacional de Ciencias en Córdoba 25:71-72.

1921. On dead or decaying leaves of Jubuea spectabilis Humb.. Bonpl., \& Kunth, Palmae. Chile.

juncaginearum (J. Schröter) A. Munk, Dansk Botanisk Arkiv, Kjobenhavn 17(1):377. 1957. [Basionym not completely cited.] $\equiv M_{y C O}$. sphuerella juncaginearum J. Schröter in F.J. Cohn, Kryptogamen-Flora Von Schlesien. Im Namen Der Schlesischen Gesellschaft für vaterländische Cultur herausgegeben von Prof. Dr. Ferdinand Cohn, Secretair der Botanischen Section, Breslau 3(2):342. 1894. EPhcieosphaerella jumcaginearum (W.G. Lasch \& J. Schröter) P.A. Saccardo, Sylloge Fungorum Omnium Hucusque Cognitorum Digessit P.A. Saccardo 11:312. 1895. On dead stems of Triglochin marima $\mathrm{L}$.. Triglochin palustris L., Juncaginaceae. Germany.

junci (C.A. Oudemans) J. Lind, Danish Fungi as Represented in the Herbarium of E. Rostrup. Nordisk Forlag, Copenhagen, p. 217. 1913. ESphaeria junci C.A. Oudemans, Archives Néerlandaises des Sciences Exactes el Naturelles, Haarlem 8:405. 1873 [as Sphaeria junci Fr.(?)]. EMetasphaeria junci P.A. Saccardo, Sylloge Fungorum Omnium Hucusque Cognitorum Digessit P.A. Saccardo 2:177. 1883. On leaves and culms of Juncus glaucus Sibih., Juncaceae. Netherlands.

junci J. Feltgen, Vorstudien zu einer Pilz-Flora des Grossherzogthums, Luxemburg 1(2):156. 1901. On culms of Juncus effusus L.. Juncaceae. Luxembourg.

juncicola H. Rehm in H. Rehm. Ascomyceten, Fascicle 11, No. 533. Anno 1879. Nom. nud. Art. 32.1. On dry culms of Juncus trifidus L. Juncaceae. Italy.

juncicola H. Rehm ex H.G. Winter in H.G. Winter, Hedwigia, Dresden 19:167. 1880. ELoptospluseria juncicola H. Rehm, Ascomyceten, Fascicle II, No. 533. 1879. Nom. nud. Art. 32.1. EPhaeosphacria juncicola (1H. Rehm ex H.G. Winter) L. Holm, Symbolae Botanicae Upsalienses, Uppsala 14(3):129. 1957. On dried culms of Imucus hostii Tausch, Juncus Mifidus L., Juncaceac. Austria, Czcchoslovakia. ltaly. Switzerland.

juncicola H. Rehm ex H. Relam, Bericht des Naturwissenschaftlichen Vercins für Schwaben und Neubcrg, Augsburg 26:128. 1881. On dricd culms of Iturcus hostii Tiusch, Juncaceac. Italy. juncina (B. Auerswald) P.A. Saccardo, Sylloge Fungorum Omnium Hucusque Cognitorum Digessit P.A. Saccardo 2:66. 1883. ESphaeria jumcina B. Auerswald in G.L. Rabenhorst, Fungi Europaei Exsiccati, Klotzschii Herbarii vivi Mycologici Continuatio Edita Nova, Series Secunda, Century 8. No. 748. Anno 1865. ESphaerella juncina (B. Auerswald) B. Auerswald in W. Gonnermann and G.L. Rabenhorst, Mycologia Europaea, Abbildungen Sämmtlicher Pilze Europa's, Hefte 5 und 6, Synopsis Pyrenomycetum Europaeorum, p. 18.1869. 三Mycotodea juncina W. Kirschstein, Annales Mycologici, Berlin 37:134. 1939. EPhaeosphaeria juncina (B. Auerswald) L. Holm, Symbolac Botanicae Upsalienses, Uppsala 14(3):127. 1957. On dry culms of Juncus effusus L., Juncaceae. Czechoslovakia, Germany, Great Britain, Portugal.

juncina (B. Auerswald) P.A. Saccardo forma macrospora $\mathrm{G}$. Niessl v. Mayendorl in V. Mouton, Bulletin. Société R. de Botanique de Belgique, Bruxelles 39:43-44. 1900. On veins of leaves of Luzula alhida (Hoffm.) DC., Juncaceae. Belgium.

junciseda P.A. Karsten, Öfversigt af K. Vetenskapsakademiens Forhandlingar, Stockholm 29(2):101. 1872. On dead plant of Juncus. bighmis L., Juncaceae. Norway (Spitsbergen).

junci-acuti R. González Fragoso, Asociación Española Para el Progresso de las Ciencias. Congreso de Coimbra 1925:12. 1925. Non. inval. Art. 34.1. On dry culms of Juncus acutus L., Juncaceae. Portugal.

junci-glauci L.M. Unamuno Yrigoyen, Boletin de la R. Sociedad Española de Historia Natural, Madrid 29:394. 1929. On dry stems of funcus glaucus Sibth.. Juncaceae. Spain.

juncorum (P.L. Crouan \& H.M. Crouan) P.A. Saccardo, Sylloge Fungorum Omnium Hucusque Cognitorum Digessit P.A. Saccardo 2:86. 1883. EPleospora juncorum P.L. Crouan \& H.M. Crouan. Florule Du Finistèrc Contenant Les Descriptions De 360 Especes Nouvelles De Sporogames, De Nombreuses Ohservations el une synonymie des plantes Cellulaires et Vasculaires Qui Croissent Spontanément Dans ce Département, p. 22. 1867. On dead stems of Iuncus acuriflorus Ehrh. ex lloffm., Juncaceae. France.

jumiperi F. Fautrey, Revue Mycologique. Toulouse 17:70. 1895. On branchlets at ends of dry branches of Jumiperus communis' L., Cupressaccac. France.

juniperina F.A. Hazslinszky, Matematikai és Terınészettudományi Közlemenyek Vonatkozólag a Hazai Viszonyokra, Buclapes! 25(2): 150. 1892. On leaves of Juniperus nama Willd., Cupressaceac. Ilungary. 
kali J.H. Fabre, Annales des Sciences Naturelles, Paris, Botanique, Series 6, 15:50. 1883. =Metasphaeria kali (J.H. Fabre) P.A. Saccardo, Sylloge Fungorum Omnium Hucusque Cognitorum Digessit P.A. Saccardo 2(Addenda Ad Volumen Secundum):LXI. 1883. On dry stems of Salsola kali L.. Chenopodiaceae. France.

kalmiae C.H. Peck, Report. New York State Museum of Natural History, Albany 39:53. (1885) 1886. EGihheridea kalmiae (C.H. Peck) M.E. Barr, Canadian Joumal of Botany, Ottawa 39:311. 1961. On dead stems of Kalmia angustifolia L., Ericaceae. U.S.A.

kalmusii G. Niessl v. Mayendorf in P.A. Saccardo, Sylloge Fungorum Omnium Hucusque Cognitorum Digessit P.A. Saccardo 2:44. 1883. On stems of Artemisia vulgaris L., Compositae. Czechoslovakia.

kerguelensis P.C. Hennings in E. v. Drygalski, Deutsche Suedpolar Expedition 1901-1903. 8:11. 1906. On leaves of Festuca kerguelensis Hook. f.. Gramineae. Kerguelen Island (near Antarctica).

kochiana E. Müller, Sydowia. Annales Mycologici, Hom, N.ö. 5(3-6):50. 1951. On dead reeds of Carex haldensis L., Cyperaceae. Italy.

koerberi (B. Stein) H.G. Winter, Dr. L. Rabenhorst's Kryptogaman-Flora von Deutschland. Oesterreich und der Schweiz 1(2):443. 1885. $\equiv$ Leptorhaphis koerheri B. Stein in F. Cohn, Kryptogamen-Flora Von Schlesien. Im Namen Der Schlesischen Gesellschaft für vaterländische Cultur herausgegeben von Prof. Dr. Ferdinand Cohn, Secretair der Botanischen Section, Breslau 2(2):350. 1879. $\equiv$ Ophiobolus koerberi (B. Stein) A.N. Berlese \& P. Voglino, Sylloge Fungorum Omnium Hucusque Cognitorum Digessit P.A. Saccardo Additamenta Ad Volumina l-IV. p. 190. 1886. On thallus of Koerberiella wimmeriana (Koerber) B. Stein, Lichenes (Porpidiaceae). Czechoslovakia, Germany.

korroe J.C. Walker \& A.M. Smith. Transactions of the British Mycological Society, London 58:461. 1972. IOphiosphaerella korrac (J.C. Walker \& A.M. Smith) R.A. Shoemaker, Canadian Joumal of Botany, Ottawa 67:1591. 1989. On stolons and sheaths of Alena sativa L., Aronopsus compressus (Swartz) Beauv., Cynodon dactylon (L.) Pers., Eremochloa ophiuroides (Munro) Hach., Oryza sutica L.. Trinicum aestivum L., Gramineae. Australia (New South Wales), U.S.A.

kotschyana F. Petrak, Annalen des Naturhistorischen Museunı in IVien 50:433. 1939. On leaves of Acantholimon accrosum Boiss.. Acantholimon nclandmthum Boiss., Plumbaginaceas. Iran.

kuangfuensis J. Yen \& S.K. Sun. Mycotaxon. An Intemational Joumal Designed to Expedite
Publication of Research on Taxonomy \& Nomenclature of Fungi \& Lichens, lthaca, New York 7:396-397. 1978. On living branches of Saccharum officinarum L., Gramineae. Taiwan.

kunzeana A.N. Berlese, Icones Fungorum Omnium Hucusque Cognitorum ad usum Sylloges Saccardianae Adcommodatae 1:66. 1890. $\equiv$ Leptosphaeria typharum G. Kunze. Nom. J. Desmazières. On stems of Typha lasifolia L.. Typhaceae. Germany.

lacustris (L. Fuckel) H.G. Winter, Dr. L.

Rabenhorst's Kryptogaman-Flora von Deutschland, Oesterreich und der Schweiz, Second edition, 1(2), p. 451. 1885; Hedwigia. Dresden 26:57. 1887. ESphaeria lacustris L. Fuckel, Fungi Rhenani Exsiccati A Leopoldo Fuckel Collecti, Fascicle 25(10). No. 2436. Anno 1872: Symbolae Mycologicae Zweiter Nachtrag, p. 22. 1873. EMerasphaeria lacustris (L. Fuckel) P.A. Saccardo. Syllogue Fungorum Omnium Hucusque Cognitorum Digessit P.A. Saccardo 2:173. 1883. \#Massarina lacustris (L. Fuckel) A. Leuchtmann. Sydowia. Annales Mycologici. Hom, N.Ö. 37:181. 1984. =Wettsteinina lacustris (L. Fuckel) R.A. Shoemaker, Canadian Joumal of Botany, Ottawa 67:1595. 1989. On leaves and stems of Schoerroplectus lacustris (L.) Palla. Typha angustifolia L., Typha larifolia L., Cyperaceae, Typhaceae. Germany. Switzerland.

ladina E. Müller, Sydowia. Annales Mycologici, Hom, N.Ö. 4(1-6):293. 1950. $\equiv$ Nodulosphacria ladina (E. Müller) L. Holm. Symbolae Botanicae Upsalienses, Uppsala 14(3):83. 1957. On dead stems of Laserpirium halleri Crantz, Umbelliferae. Switzerland.

lagenoides C.L. Spegazzini. Anales del Museo Nacional de Historia Natural de Buenos Aires 23:57. 1912: Mycetes Argentinenses. Series 6. No. 1377. 1912. On dry decaying sheaths of Elymus? barbatus F. Kurtz.. Gramineae. Argentina.

lamprocarpi (G. Passerini) P.A. Saccardo, Sylloge Fungorum Omnium Hucusque Cognitorum Digessit P.A. Saccardo 2:66. $18 \$ 3$. ESphacrella lamprocarpi G. Passerini. Erbario Crittogamico ltaliano, Series 2. Fascicle $\$$. No. 392. Anno 1871. On florets and bracts of Juncus lampocarpus Ehrh. ex Hottm.. Junciceae. ltaly:

lankeana P.C. Hennings, Verhandlungen des Botanisclsen Vereins Der Provinz Brandenburg. Berlin 47:220-221. 1906. On last year"s stents of Hypericum perforum $\mathrm{L}$. Guttiferae. Sri Lanhat.

larseniana A. Munh, Dansk Bocanish Arhis. Kjobenhavn 14(7):27. 1952. ELeprosphaserio 
elymi P. Larsen. Non G.F. Atkinson, 1897. EPhaeosphaeria larseniana (A. Munk) R.A. Shoemaker, Canadian Journal of Botany, Ottawa 67:1568. 1989. On stems and leaves of Baldingera arundinacea (L.) Dumort., Elymus arenarius L., Iris pseudacorus L.. Phragmites communis Trin., Gramineae. Iridaceae. Iceland, U.S.A.

larialis P.A. Saccardo, Boletim da Sociedade Broteriana, Coimbra 21:213. 1905. On dead culms of Equisetum [as E. pallidum], Equisetaceae. Cape Verde Islands.

lasioderma J.B. Ellis \& B.M. Everhart, Proceedings of the Academy of Natural Sciences of Philadelphia 1893:135. 1893. 三Dothiodorthia lasioderma (J.B. Ellis \& B.M. Everhart) M.E. Barr, Mycotaxon. An International Joumal Designed to Expedite Publication of Research on Taxonomy \& Nomenclature of Fungi \& Lichens. New York 34:523. 1989. On living leaves of Artemisia ridentata Nutt., Compositae. U.S.A.

lasiosphaerioides K. Starbäck \& A.Y. Grevillus in K. Starbäck, Bihang till K. Svenska Vetenskapsakademiens Handlingar, Stockholm 16(3):7. 1890; Bidrag till Kännedomen om Sveriges Ascomycetflora, p. 7. 1890. On dry stems of Aconitum lycoctonum L., Ranunculaceae. Sweden.

lassenensis R.A. Shoemaker, Canadian Journal of Botany, Ottawa 67:1578. 1989. On stems of Allium validum S. Wats., Liliaceae. U.S.A. (California).

latebrosa J.B. Ellis in N.L. Britton, Catalogue of Plants Found in New Jersey. Geological Survey of New Jersey, Final Report of the State Geologist, Trenton 2(1):524. 1889. Nom. illegit. Art. 64.1. 三Leptosphaeria latebrosa (J.B. Ellis) P.A. Saccardo.

latebrosa (J.B. Ellis) P.A. Saccardo, Sylloge

Fungorum Omnium Hucusque Cognitorum Digessit P.A. Saccardo 2:86. 1883.

$\equiv$ Sphaeria latebrosa J.B. Ellis, Bulletin of the Torrey Botanical Club (and Torreya), New York 8:90. 1881. On basal sheaths of Andropogon sp., Gramineae. U.S.A.

lathonia P.A. Saccardo, Nuovo Giomale Botanico Italiano e Bolletino della Società Botanica Italiana, Firenze 7:310. 1875. On leaves of Vlelleborms viridis L., Ranunculaceae. Italy. lathonia P.A. Saccardo var. hellebori-foetidi C.B. Massalongo, Atti del Istituto Veneto di Scienze, Lettere ed Arti, Venezia 74:254. 1914. On leaves of Helle horus foetidus L., Ranunculaceae. Italy.

lathyri F. Fautrey, Fungi Selecti Gallici Exsiccati, Century 56, No. 5537. Anno 1891; Revue Mycologique, Toulouse 13:8. 1891. On stems of Lathyrus sylvestris L., Leguminosae. France. lathyrina P.A. Saccardo, Atti dell' Accademia Scientifica Veneto-Trentino-Istriana, Padova 2(2): 153-154. 1873. On dead stems of Lathyrus latifolius L., Leguminosae. Italy.

lathyri P.A. Saccardo, Atti dell' Accademia Scientifica Veneto-Trentino-Istriana, Padova 2(2):154. 1873. झMetasphaeria lathyri (P.A. Saccardo) P.A. Saccardo, Sylloge Fungorum Omnium Hucusque Cognitorum Digessit P.A. Saccardo 2:159. 1883. On fallen dead stems of Lathyrus sylvestris L., Leguminosae. Italy.

lauri A. Maublanc, Bulletin de la Société Mycologique de France, Paris 21:89. 1905. On leaves of Laurus nobilis L., Lauraceae. France.

lavandulae M. de Sousa da Camara, Agronomia Lusitana, Sacavém 13:126. 1951. On branchlets of Lavandula stoechas $\mathbf{L}$., Labiatae. Portugal.

lecanora H. Fabre, Annales des Sciences Naturelles, Paris, Botanique, Series 6, 15:49. 1883. On dry stems of Salsola kali L., Chenopodiaceae. France.

leersiae G. Passerini, Atti della Società Crittogamologica Italiana, Milano, Series 2, 2:43-44. 1879. ELeptosphaeria leersiana P.A. Saccardo nec (ut videtur) Sphaerella (Leptosphaerella) leersiae G. Passerini. On dried leaves of Leersia oryzoides (L.) Swartz. Gramineae, Italy.

leersiana P.A. Saccardo, Sylloge Fungorum Omnium Hucusque Cognitorum Digessit P.A. Saccardo 2:60. 1883. ELeptosphaeria leersiae G. Passerini. Non Sphaerella (Leptosphaerella) leersiae G. Passerini. On dried leaves of Leersia oryzoides (L.) Swartz, Gramineae. Italy.

leiostega (J.B. Ellis) J.B. Ellis, Catalogue of Plants Found in New Jersey. Geological Survey of New Jersey, Final Report of the State Geologist, Trenton 2(1):525. 1889. Esphaeria (Leprosphaeria) leiostega J.B. Ellis, Bulletin of the Torrey Botanical Club (and Torreya), New York 8:91. 1881. On various dead twigs and limbs of Carya sp., Rosa sp., Vaccinium sp., Juglandaceae, Rosaceae, Ericaceae. U.S.A. (New Jersey). lejostega (J.B. Ellis) F. Hazslinszky, Matematikai és Természettudományi Közlemenyek Vonatkozólag a Hazai Viszonyokra, Budapest 25(2): 148. 1892. ESphaeria (Leptosphaeria) leiostega J.B. Ellis, Bulletin of the Torrey Botanical Club (and Torreya), New York 8:91. 1881. EMetasphaeria lejostega (J.B. Ellis) P.A. Saccardo, Sylloge Fungorum Omnium Hucusque Cognitorum Digessit P.A. Saccardo 2:164. 1883. On branches of Carya sp., Rosa sp., V'accinium sp.. Juglandaceac, Rosaceac, Ericaceac. U.S.A. (New Jersey).

lelehae 1. Hino \& K. Katumoto, Bulletin of the Faculty of Agriculture, Yamaguchi Univer- 
sity, Shimonoseki 9:904. 1958. On dead culms of Leleba sp. [as L. simplex], Gramineae. Japan.

lemaneae (F. Cohn) P.A. Saccardo, Sylloge Fungorum Omnium Hucusque Cognitorum Digessit P.A. Saccardo 2:84. 1883. 三Sphaeria lemaneac F.J. Cohn in A. de Bary and M.S. Woronin, Beiträg zur Morphologie und Physiologie der Pilze 3:1-7. 1870. On thalli of Lemanea fluviatis C. Aq.. Algae (Rhodophyceae-Lemaneaceae). Germany. lemoinii C. Richon, Catalogue Raisonné des Champignons Qui Croissent Dans le Département de la Marne, p. 300. 1889. On an unknown monocotyledon host, unknown family. France.

leptogiophila (Minks) H.G. Winter, Dr. L. Rabenhorst's Krypytogaman-Flora von Deutschland, Oesterreich und der Schweiz, Second edition, I(2):443. 1885 [as Leptosphaeria?]. =Leptor/haphis leptogiophila Minks in H.G. Winter, Flora, Jena und Regensburg 60:211. 1877. 三? Metasphaeria leprogiophila (Minks) A.N. Berlese \& P. Voglino, Sylloge Fungorum Omnium Hucusque Cognitorum Digessit P.A. Saccardo Additamenta Ad Volumina ]-]V, p. 158. 1886. On thalli of Leptorhaphis leptogiophila Minks ex Wint., Lichenes (Arthropyreniaceae). Germany.

leptospora (G. de Notaris) P.A. Saccardo, Sylloge Fungorum Omnium Hucusque Cognitorum Digessit P.A. Saccardo 2:16. 1883. 三Cucurbitaria leptospora G. de Notaris. Sferiacei Italici. Century 1, Fascicle 2, pp. 62-63. 1863. On stems of Dianthus atrorubens All., Caryophyllaceae. Italy.

lespedezac Ziling. Trudȳ Botanicheskogo Instituta Akademiy Nauk SSSR, Leningrad, Moskva. Series 2, 3:680. 1936. On dead stems of Lespedeza bicolor Turcz., Leguminosae. U.S.S.R.

lethalis J.B. Ellis \& B.M. Everhart, Proceedings of the Academy of Natural Sciences of Philadelphia 1895:423. 1895. On dead stems of Penstemon confertus Dougl. ex Lindl.. Scrophulariaceae. U.S.A.

Iencomelaria (W. Mudd) L. Vouaux, Bulletin de la Société Mycologique de France, Paris 29:121. 1913. ESphaeria leucomelaria W. Mudd. A Manual of British Lichens, Containing Descriptions of All Species and Varieties, and Five Plates With Figures of the Spores of Onc Hundred and Thirty Species Illustrative of the Genera, p. 105. 1861. Nom. inval. Art. 34.1b. ETichothecium leucomelarium (W. Mudd) A.N. Berlese \& P. Voglino, Sylloge Fungorum Omnium Hucusque Cognitorum Digessit P.A. Saccardo Additamenta Ad Volumina 1-JV, p. 121. 1866. Parasitic on thallus of Borrera leucomela (L.) Mudd [=Anaptychia leucomela (L.) Mass.], Lichenes (Physciaceae). Great Britain.

leucoplaca (K.F.W. Wallroth) E.A. Vainio. Acta Societatis pro Fauna et Flora Fennica. Helsingforsiae 49(2): 143-144. 1921. EVerrucaria leucoplaca K.F.W. Wallroth. Flora Cryptogamica Germaniae 1:299. 1831. EPyrenula leucoplaca (K.F.W. Wallroth) G.W. Koerber, Systema Lichenum Germaniae, p. 361. 1855. EV'errucaria alha H.A. Schrader var. leucoplaca (K.F.W. Wallroth) L.E. Schaerer in Enumeratio Critica Lichenum Europaeorum. Quos Ex Nova Methodo Digerit, p. 219. 1850. EEopyremela leucoplaca (K.F.W. Wallroth) R.C. Harris. The Michigan Botanist. Ann Arbor 12:19. 1973. On bark of Acer sp., Almus sp.. Carya sp.. Corylus sp., Fraximus sp., Juglans sp.. Populus sp.. Quercus sp., Salix sp.. Sorbus sp.. Tilia sp., UImus sp., Aceraceae. Juglandaceae, Betulaceae, Oliaceae, Salicaceae. Fagaceae, Rosaceae. Tiliaceae. Ulmaceae. Finland.

lihanoris (L. Fuckel) P.A. Saccardo. Sylloge Fungorum Onnium Hucusque Cognitorum Digessit P.A. Saccardo 2:16-17. 1883. $\equiv$ Pleospora libanotis L. Fuckel. Symbolae Mycologicae Zweiter Nachtrag. p. 24. 1873. On dry stems of Lihanotis momana Crantz. Umbelliferae. Belgium. France. Switzerland. licatensis P.A. Saccardo, Sylloge Fungorum

Omnium Hucusque Cognitorum Digessit P.A. Saccardo 2:70. 1883. ELeptosphaeria ryphicola G. Passerini \& V. Beltrani. 1883. Nec Leptosphaeria typhicola P.A. Karsten. EPhaeosphaeria licatensis (P.A. Saccardo) R.A. Shoemaher. Canadian Joumal of Botany. Ottawa 67:1532. 1989. On dry leaves of Typha latifolia L., Typhaceae. Canada. ltaly: licatensis P.A. Saccardo forma rupefortensis G.

Passerini in G. Passerini and P. Brunaud.

Revue Mycologique. Toulouse 8:206. 18 S6.

On dead leaves of $T$ ypha larifolia L..

Typhaceae. France.

lichenicola F.W. Zopi. Hedwigia. Dresden 35:355. 1896; Nova Acta Academiae Caesareae Leopoldino Carolinae Germanicae Naturae Curiosorum, Halle 70:160-163. 1897:

Untersuchungen über die durch Parasitische

Pilze Hervorgerufenen Krankheiten der Flechten. pp. 64-67. 1897. On thallus and apothecia of Solorina crocea (L.) Ach.. Lichenes (Peltigeraceae). Italy:

lilirola K. Hara, Byôchu-gai Zasschi (Journal of Plant Protection). Tokyo 5:885-\$86.1918 On stems of Lilimm sp.. Liliaceae. Japan.

lilii J.B. Ellis \& J. Dearness in J.B. Ellis and B.M. Everhart. Proceedings of the Academy of Natural Sciences of Philadelphia 1893:136. 
1893. On leaves of Lilium superlnm L.. Liliaceae. Canada.

limiluta (C.H. Persoon) M.C. Cooke, Grevillea, London 17:91. 1889. ESphaeria limitata C.H. Persoon in K.F.W. Wallroth, Flora Cryptogamica Germaniae 2:783. 1833 [as Splacria limifafa C.H. Persoon ined. ex spec. Kunze! sub. Sphaeria mamillana, in E.M. Fries, Elenchus Fungorum Sistens Commentarium in Systena Mycologicum 2:99. 1828]. EClypeosphaeria limitata (C.H. Persoon) L. Fuckel, Symbolae Mycologicae, p. 117. 1870. On dry branches of Cormus allu L.. Cormus sanguinea L., Rhamuus sp., Cornaceae. Rhamnaceae. Germany.

limosa F. Fautrey in C. Roumeguère, Revue Mycologique. Toulouse 13:128. 1891; Fungi Selecti Gallici Exsiccati. Century 58, No. 5751. Anno 1891. On dry stems of Equisetum limosum L., Equisetaceae, France.

lindquistii M.J. Frezzi, Revista de Investigaciones Agropecuarias, Buenos Aires, Series 5. Pathologia Vegetal 5(7):73-79. 1968. [Latin description appended as an addendum to the offprints. May not constitute valid publication!] On fallen stems of Heliamthus ammus L., Compositae. Argentina.

linearis (P.A. Saccardo) E. Müller, Sydowia. Annales Mycologici, Horn, N.Ö. 4(1-6):258. 1950. ELeptosphaeria culmifraga (E.M. Fries:E.M. Fries) G. de Notaris var. linearis P.A. Saccardo, Sylloge Fungorum Omnium Hucusque Cognitorum Digessit P.A. Saccardo 2:75. 1883. On culms of Agropyron repens (L.) Beauv., Milium effusim L., Por nemoralis L., Gramineae. France, Switzerland.

lineolaris G. NiessI v. Mayendorf in G. Linhart, Botanisches Zentralblatt, Jena \& Dresden 26:119-120. 1886; Fungi Hungarici Exsiccati, Century 5, No. 466. Anno 1886.

EPhacosphaeria lineolaris (G. Niessl v. Mayendorf) R.A. Shoemaker, Canadian Journal of Botany, Ottawa 67:1545. 1989. On hard culms of Aira cespitosa L., Gramineate. Hungary.

lingue (C.L. Spegazzini) P.A. Saccardo \& A. Trotter, Sylloge Fungorum Omnium Hucusque Cognitorum Digessit P.A. Saccardo 22:219. 1913. Leptosphaerella ?lingue C.L. Spegazzini. Fungi Chilenses, p. 77. 1910: Revista de La Facultad de Agronomia Y Veterinaria, Universidad Nacional de La Plata, Series 2. 6:77. 1910. On decaying and wilted leaves of Persea lingue Nees, Lauraceae. Chile.

lithophilae S.A. Gucevicz. Notulate Systematicae e Sectione Cryptogamica Instituti Botanici Nomine V.L. Komarov Academiac Scientiarum U.R.S.S., Petropolis 13:196. 1960. On dry stems of Sobolewstia linhophila Bicb.. Cruciferae. U.S.S.R. linoralis P.A. Saccardo, Michelia Commentarium Mycologicum Fungos in Primis Italicos Illustrans 1:38. 1877. EPhaeosphereria liturulis (P.A. Saccardo) L. Holm, Symholac Botanicae Upsalicnses, Uppsala 14(3): 121. 1957. On culms of Scirpus sp., Cyperaceae. Italy.

linoralis P.A. Saccardo forma calamagrostidisarenariae P.A. Saccardo, Sylloge Fungorum Omnium Hucusque Cognitorum Digessit P.A. Saccardo 2:78. 1883. On culms and rhizones of Ammophila arenaria (L.) Link. Scirpus sp., Cyperaceae, Gramineae. Germany, Italy.

livida P. Voglino, Arti della R. Accademia D'Agricoltura di Torino 53:352-353. 1911. On somewhat living leaves of unknown host, unknown family. Italy.

lobavensis A.M. Saccas. Étude de la Flore Cryptogamique des Caféiers en Afrique Centrale. Bulletin de Institut Français du Café du Cacao et d'Autres Plantes Stimulantes (Bulletin IFCC No. 16), pp. 251-253. 1981. Nom. inval. Art. 37.1. On dead stems of Coffea excelsa Cheval., Rubiaceae. Central African Republic.

lobeliae K.H. Anahosur, Sydowia. Annales Mycologici, Horn, N.Ö. 24(1-6):286. (1970) 1971. On living leaves of Lobelia excelsa Lesch. Campanulaceae. India.

lolii H. Sydow \& P. Sydow, Hedwigia, Dresden 39:(1). 1900. On culms of Lolium perenne L., Gramineae. Germany.

longchampsi P.A. Saccardo, Revue Mycologique, Toulouse 7:145. 1885 [as (West.) Sacc.: based on Sphaeria longchampsi G.D. Westendorp in E. Marchal, Cryptogames de la Belgique]. Nom. inval. Art. 29.1. [E. Marchal used the above title in 1882 for the general distribution of exchange specimens (D.H. Pfister, Mycotaxon. An Intemational Journal Designed to Expedite Publication of Research on Taxonony \& Nomenclature of Fungi \& Lichens, lthaca, New York 23:123. 1885).] On dry stems of Libanotis sp., Umbelliferae. Belgium.

Iongipedicellata J.H. Miller \& G. Burton, Mycologia, Lancaster, Pennsylvania 34:2-3. 1942. On dead stems of herbaceous plants of Daticus carota L., Smallanthus medalia (L.) Mackenzie, Solidago caesia L.. Compositae. Umbelliferae. U.S.A. (Georgia).

longispora A.M. Saccas, Étude de la Flore Cryptogatmique des Calćicrs en Afrique Centrale. Bulletin de Institut Français du Café du Cacåo et d'Autres Pantes Stimulantes (Bulletin IFCC No. 16), pp. 24t-256. 1981. Nom. inval. Art. 37.1. On stems and branclies of Coffea encelsa Cheval. Rubiaceac. Central African Republic.

longispora J. Feltgen, Vorstudien zu ciner Pilz-Flora des Grossherzogthums, Luxemburg I(2): 1.59. 
1901. On dry stems of /ris pseudacorus L., Iridaceae. Luxembourg.

Ionicerae S.A. Gucevicz, Novosti Sistematiki Nizshikh Rastenij, Novitates Systematicae Plantarum Non Vascularium 7:161-162. 1970. Nom. illegit. Art. 64.1. On dry branches of Lonicera canadensis Bartr., Caprifoliaccae. U.S.S.R.

lonicerae (F. Fautrey) L. Holm, Symbolae Botanicae Upsalienses, Uppsala 14(3):46. 1957. $\equiv$ Metasphaeria lonicerae F. Fautrey in $\mathrm{C}$. Roumeguère, Fungi Selecti Gallici Exsiccati. Century 54, No. 5359. 1890. Parasitic on branches of Caprifolium sp., Lonicera rylosteum L., Caprifoliaceae. France, Germany.

lonicerina (P.A. Karsten) L. Holm, Symbolae Botanicae Upsalienses, Uppsala 14(3):22. 1957. ESphaeria lonicerina P.A. Karsten, Fungi Fenniae Exsiccati, Century 10, No. 971. Anno 1870 . $\equiv$ Melanomma lonicerinum (P.A. Karsten) P.A. Saccardo; Michelia Commentarium Mycologicum Fungos in Primis Italicos lllustrans 1:344. 1878. On stems of Almus sp.. Salix sp.. Betulaceae. Salicaceae. Germany.

lophamhi (M.J. Berkeley \& M.A. Curtis) P.A. Saccardo, Sylloge Fungorum Omnium Hucusque Cognitorum Digessit P.A. Saccardo 2:84-85. 1883. ESphaeria lophanthi M.J. Berkeley \& M.A. Curtis in M.J. Berkeley, Grevillea, London 4:152.1876. On stems of Lophesmthus sp., Labiatae. U.S.A.

lucilla P.A. Saccardo, Nuovo Giomale Botanico ltaliano e Bolletino della Società Botanica 1taliana, Firenze 7:310-311. 1875. Indiscriminately on wilted leaves of Pyrtis communis L., Rosaceae. Italy.

lucina P.A. Saccardo, Nuovo Giomale Botanico Jtaliano e Bolletino della Società Botanica 1taliana, Firenze 7:311. 1875. On dead leaves of Cytisus lahurum L., Leguminosac. Italy.

luchosa G. Niessl v. Mayendorf in P.A. Saccardo. Nuovo Giomale Botanico ltaliano e Bolletino della Società Botanica Italiana. Firenze 7:321 1875. =Phueosphacria luctuosa (G. Niess) v. Mayendorf) Y. Otani \& T. Mikawa, Memoirs of the National Science Museum, Tokyo. Japan [Kokuritsu Kagaku Hakubutsukan Senho Memoirs] 4:78. 1971. On rotten culms of Zecs malys L., Gramineae. Canada, Italy. U.S.A.

lumariae (M.J. Berkeley \& C.E. Broome) P.A. Saccardo, Sylloge Fungorum Omnium Hucusque Cognitorum Digessit P.A. Saccardo 2:57. 1883. ESphacria (Canlicolac) Lmariac M.J. Berkeley \& C.E. Brome, Amnals and Magazine of Natural History. London. Series 3. 3:374. 1859. On dried pods of Linnaria rediviva L.. Cruciferae. Great Britain.

Iupini (F.S. Earle) J.R. Boisc, On Trematosphac'rial (Loculoascomycetes, Fungi) and Disposition of Heterogenous Elements. Ph.D. dissertation. University of Massachusetts. Amherst, p. 47. 1984. Nom. provis. An. 34.1b. $\equiv$ Trematosphacria lupini F.S. Earle in E.L. Greene. Plantae Bakerianae 2(1):17. 1901. On stems of Lupinus sp., Leguminosae. U.S.A. (Colorado).

lupinicola F.S. Earle in E.L. Greene. Plantae Bakerianae 2:20. 1901. On dead stems of Lupinus sp., Leguminosae. U.S.A.

Iusitunica F. v. Thümen. Instituto. Revista Scientifica e Litteraria. Coimbra 28:29-30. (1880) 1881: Contributiones ad Floram Mycologicam Lusitanicam, Series 3. pp. 29-30. 1881. On dry stems of Spartium juncetum L., Leguminosae. Portugal.

luxemburgensis P.A. Saccardo \& D. Saccardo. Sylloge Fungorum Omnium Hucusque Cognitorum Digessit P.A. Saccardo 17:723. 1905. [Superfluous name for Leptosphaeria dumctorum J. Feltgen. Non G. Niessl v. Mayendorf, fide P.A. Saccardo. Sylloge Fungorum Omnium Hucusque Cognitorum Digessit P.A. Saccardo 17:723. 1905.

Leptosphaeria dumetorum was attributed to G. Niessl v. Mayendorf: the variety symphti was published by J. Feltgen as Leptosphaeria dumetorum G. Niessl v. Mayendorf var. symphyti J. Feltgen.]

luxemhurgenisis P.A. Saccardo \& D. Saccardo var. dolichospora (J. Feltgen) P.A. Saccardo. Sylloge Fungorum Omnium Hucusque Cognitorum Digessit P.A. Saccardo 17:723. 1905. $\equiv$ Leptosphaeria dumetorum G. Niessl v. Mayendorf var. dolichospora J. Feltgen. Vorstudien zu einer Pilz-Flora des Grossherzogthums. Luxemburg. Nacht. 3. p. 210.

1903. On branches of Sambucus racemosa L. [as $S$. nigra], Caprifoliaceae. Luxembourg.

luxemburgensis P.A. Saccardo \& D. Saccardo var. symphyzi J.Feltgen [falsely' so cited in P.A. Saccardo. Sylloge Fungorum Onnium Hucusque Cognitorum Digessit P.A. Saccardo 17:723. 1905.] =Leprosphacria dumetorum G. Niessl v. Mayendorf var. symphyti J. Feltgen.

luzulae H.G. Winter, Hedwigia, Dresden 11:146. 1872. On dry leaves of Lnzula maxima (Reichard) DC.. Juncaceae. Italy.

licii G. Passerini in P. Brunaud. Actes de la Socićté Linnéenne de Bordeaux 41:175. 18\$7. On dead roots of Prumus spinosa L.. Solanaceare. Guadeloupe (West Indies).

Irciophila C.E. Faiman. Proceedings of the

Rochester Academy of Science. Rochester. New York 6:126-127. 1922. On dead stemis of Lucium vulgare Dunal., Solanaceae.

U.S.A. (New York).

Iycopodicola C.H. Peck, Repor. New I'ork State Museum of Natural listory. Albany $3 \$: 105$. 18.5.5. EPhacosphaceria lycopodicoles (C.H. Pech) R.A. Shomaker. Canadian Joumal of Botany, Ottawa 67:1563. 1989. On dead 
peduncles of Lycopodium clavanum L..

Lycopodiaceae. Canada, U.S.A.

Iyropodicola C.H. Peck var. major H. Rehm in P.A.

Saccardo, Sylloge Fungorum Omnium

Hucusque Cognitorum Digessit P.A. Saccardo

9:796. 1891 [as Leplosphaeria helverica P.A.

Saccardo \& C.L. Spegazzini in H. Rehm.

Ascomyceten, Fascicle 18. No. 884. Anno

1886]. On unknown substrate of Selaginella

helverica (L.) Spring. Selaginellaceae.

Argentina.

lycopodina (J.P. Montagne) P.A. Saccardo, Sylloge

Fungorum Omnium Hucusque Cognitorum

Digessit P.A. Saccardo 2:81. 1883.

ESphaeria (Conferta) lycopodina J.P.

Montagne, Annales des Sciences Naturelles,

Paris, Botanique, Series 3, 12:313. 1849;

Sylloge Generum Specierumque Cryptoga-

marum Quas in Variis Operibus Descriptas

Iconobusque Illustratas, Nunc Ad Diagnosim

Reductas, Nonnullasque Novas Interjectas,

Ordine Systematico Disposiut. pp. 240-241.

1856. 三Phaeosphaeria lycopodina (J.P.

Montagne) A. Hedjaroude, Sydowia. Annales

Mycologici, Horn, N.Ö. 22:78. 1968. On

dorsal bracts of Lycopodium annotinum L.,

Lycopodiaceac. Canada, Czechoslovakia.

Finland, Poland, Sweden, Switzerland, U.S.A.

yndomillae C.E. Fairman, Annales Mycologici.

Berlin 4:326-327. 1906. On pods of Robinia psendacacia L., Leguminosae. U.S.A.

lythri C.H. Peck, Bulletin of the Torrey Botanical Club (and Torreya), New York 33:220-221.

1906. On dead stems of Lythrum alatum

Pursh, Lythraceae. U.S.A.

maclurae J.B. Ellis \& B.M. Everhart, Proceedings of

the Academy of Natural Sciences of Philadel-

phia 1890:236-237. 1890. ĐMetasphaeria

maclurae (J.B. Ellis \& B.M. Everhart) P.A.

Saccardo. Sylloge Fungorum Omnium

Hucusque Cognitorum Digessit P.A. Saccardo

9:838. 1891. On leaves of Maclura auran-

tiaca Nutt., Moraceae. U.S.A.

macrochloae L. Lacoste. Revue de Mycologie 22,

Paris (Supplement Colonial No. 1):10-12.

1957. On leaves of Stipa tenacissima L..

Gramineae. Algeria, Tunisia.

macrorostra A.M. Saccas, Étude de la Flore

Cryptogamique des Caféiers en Afrique

Centrale. Bulletin de Institut Français du Café

du Cacao et d"Autres Plantes Stimulantes

(Bulletin IFCC No. 16), pp. 254-256. 1981

Nom. inval. Art. 37.1. On dead branches of

Coffea robusta L. Linden (=Coffea camephora

Pierre ex Frochn.), Rubiaceae. Central

African Republic.

macrospora (L. Fuckel) F. v. Thümen, Mycotheca

Universilis, Century 14, No. 1359. Anno

1879; Hedwigia, Dresden 21:83. 1882.

$\equiv$ Pleospora macrospora L. Fuckel, Symbolac
Mycologicale, P. 138. 1870. 三Merasphaeria macrospora (L. Fuckel) P.A. Saccardo, Sylloge Fungorum Omnium Hucusque Cognitorum Digessit P.A. Saccardo 2:158. 1883. 三Nodulosphaeria macrospora $\mathrm{P}$. Morthier, sub. Leprosphaevia macrospora F. v. Thümen. Nom. nud. On dry stems of Senecio nemorensis L. Compositac. Austria. macrosporidium E.B. Gareth Jones. Transactions of the British Mycological Society. London 45:103. 1962. EPhaeosphaeria macrosporidium (E.B. Gareth Jones) R.A. Shoemaker. Canadian Journal of Botany, Ottawa 67:1532. 1989. On decayed stems of Spartina sp.. Graminease. Great Britain.

macrotheca (E. Rostrup) L. Holm, Svensk Botanisk

Tidskrift, Stockholm 46:38. 1952. 三Metas-

phacria macrotheca E. Rostrup in J. Lange, Conspectus Florae Groenlandicae 3(1):561. 1889: Meddelelser om Gronland. Kjobenhavn 3:561. 1889. 三Massaria macrotheca (E. Rostrup) J. Lind, Skrifter om Svalbard og Ishavet, Oslo 13:31. 1928. 三Wettsteinina macrotheca (E. Rostrup) E. Müller, Sydowia. Annales Mycologici, Horn, N.Ö. 12:203. (1958) 1959. On dry leaves of Carex hyperborea Drejer, Carex rigida Good.. Cyperaceae. Greenland, Sweden.

maculans (J. Desmazières) V. Cesati \& G. de

Notaris, Commentario della Società Crittogamologica Italiana, Milan 1:235. 1863.

ESphaeria maculans J. Desmazières, Annales des Sciences Naturelles, Paris, Botanique,

Series 3. 6:77-78. 1846. $\equiv$ Pleospora maculans (J. Desmazières) L.R. Tulasne \& C. Tulasne, Selecta Fungorum Carpologica 2:274. 1863. On dried stems of Brassica campestris L., Cruciferae. Belgium, France. Germany, Great Britain, ltaly, Portugal.

maculans (M.J. Sowerby) P.A. Karsten. Mycologia

Fennica 2:99. 1873. ESphaeria maculans

M.J. Sowerby, Coloured Figures of English

Fungi or Mushrooms 3. Table 394, Figure 9.

1803. Nec Sphaeria maculans J.

Desmazières. ESphaerella maculans (M.J.

Sowerby) B. Auerswald in W. Gonnermann and G.L. Rabenhorst. Mycologia Europaea, Abbildungen Sämmtlicher Pilze Europa's. Hefte 5 und 6. Synopsis Pyrenomycetum Europacorum, p. 18.1869. EPleospora sowerby L. Fuckel, Symbolae Mycologicale,

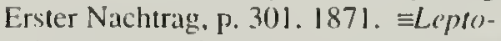
sphacria sowerby (L. Fuckel) P.A. Saccardo, Sylloge Fungorum Omnium Hucusque Cognitorum Digessit P.A. Saccardo 2:78. 1883. On leaves of Scirpus lacustris L.. Sirpus sp., Cyperaceae. Finland, Great Britain, Switzerland.

maculans (J. Desmazières) V. Cesati \& G. de Notaris forma demedata F. Fatutrey in C. 
Roumeguère, Fungi Selecti Gallici Exsiccati, Century 71, No. 7037. Anno 1896; Revue Mycologique, Toulouse 19:150. 1897. On dry roots of Brassica oleifera Moench, Cruciferae. France?

maculans (M.J. Sowerby) P.A. Karsten var. typhicola P.A. Karsten, Mycologia Fennica Pars 2, Pyrenomycetes, p. 100. 1873. [Erroneously cited as Leptosphacria typhicola P.A. Karsten in P.A. Saccardo, Sylloge Fungorum Omnium Hucusque Cognitorum Digessit P.A. Saccardo 2:79. [883.] EPhacosphaeria typhicola (P.A.Karsten) A. Hedjaroude, Sydowia. Annales Mycologici, Horn, N.Ö. 22:86. (1968) 1969. 三Massariosphaeria typhicola (P.A. Karsten) A. Leuchtmann, Sydowia. Annales Mycologici. Horn, N.Ö. 37:168. 1984. On rotting reeds of Typha latifolia L., Typhaceae. Finland.

maderensis F. Petrak, Beiblatt zu den Botanischen Jahrbüchem 62(No. 142), Heft 3:110.1928.

On dry stems of Vinca major L. Apocynaceae. Madeira Archipelago (Portugal).

magnoliae N.N. Woronichin, Vestnik Tiflisskogo Botanicheskogo Sada, Tiflis (Moniteur du Jardin Botanique de Tiflis) 35:4. 1914. On living leaves of Magnolia grandiflora L., Magnoliaceae. U.S.S.R. (Caucasus).

magnusiana A.N. Berlese \& P.A. Saccardo in A.N.

Berlese and P. Voglino, Sylloge Fungorum

Onnium Hucusque Cognitorum Digessit P.A. Saccardo Additamenta Ad Volumina 1-IV, p. 414. 1886. On dry leaves of Chamaerops Inumilis L.. Palmae. Italy.

maheui R.G. Werner, Bulletin de la Société des Sciences Naturelles du Maroc, Rabat 19(1):50-51. 1939. On apothecia of Rinodina collectica (Flk.) Am., Lichenes (Physciaceae). Morocco.

major (H. Rehm) M.L. Farr \& R.M. Horner. Nova Hedwigia. Zeitschrift für Kryptogramenkunde, Weinheim 15:248-249. 1968. ELeptosphaeria helvetica P.A. Saccardo \& C.L. Spegazzini forma major H. Rehm, Hedwigia, Dresden 26:93. 1887. On leaves of Selaginella helvetica (L.) Spring. Selaginellaceae. Italy, U.S.A. (Arizona).

malojensis W. Kirschstein, Annales Mycologici, Berlin 37:107-108. 1939. On a dry branch of Salix sp., Salicaceae. Germany.

malyi R. Picbauer, Glasnik Zemaljskog Museja u Bosni i Hercegovini Sarajevo 45:66. 1933. On stems of Pedicularis brachyodonte Schlosser \& Vuk. var. fallacis (Beck) Hayck. Scrophulariaceae. Yugoslavia.

mamillana (E.M. Fries:E.M. Fries) M.C. Cooke, Grevillea, London 17:91. 1889. 三Sphacria mamilluna E.M. Fries:E.M. Fries. Kungliga Svenska Vetenskipsakademiens Hambllingar. Uppsala and Stockholm 1818:1013. 1818:
Systema Mycologicum Sistens Fungonum 2:487. 1823. EClypeosphaeria mamillana (E.M. Fries:E.M. Fries) J.B. Lambotte. Flore Mycologique de la Belgique, Comprenant la description des espèces trouvées jusqu'a ce Jour. 2:247. 1880. On branches of Cormus sp., Cornaceae. Belgium.

mamillula M. Anzi. Atti della Società Italiana di

Scienze Naturali e del Museo Civile di Storia Naturale, Milano 11:180. 1868. EEpicymmatia mammillula (M. Anzi) P.A. Saccardo. Sylloge Fungorum Omnium Hucusque Cognitonum Digessit P.A. Saccardo 1:571. 1882. On upper surface of thallus of Peligera canina (L.) Willd., Lichenes (Peltigeraceac). Italy.

mandshurica M. Miura, Flora of Manchuria and East Mongolia, Part 111, Cryprogams, Fungi (Contributions of the Southem Manchuria Railway Company 27): 175. 1928. On leaves of Pyrus malus L. [as Malus domestica Borkh.], Rosaceae. China.

marantae H. Sydow \& P. Sydow, Annales Mycologici, Berlin 18:100. 1920. On dying leaves of Maranta arundinacea L., Marantaceae. Philippines.

marcyensis (C.H. Peck) P.A. Saccardo. Sylloge Fungorum Omnium Hucusque Cognitorum Digessit P.A. Saccardo 2:80. 1883. ESphaeria marejensis C.H. Peck. Report. New York State Museum of Natural History. Albany 31:51. 1879 [as marciensis]. =Phaeosphaeria marcyensis (C.H. Peck) L. Holm \& K. Holm. Karstenia. Suomen SieniSeura. Helsinki 21:68. 1981. On leaves of Lycopodium annotinum L., Lycopodium selago L.. Lycopodiaceae. Canada. U.S.A.

marginalis G. Passerini, Atti della Società Crittogamologica Italiana. Milano. Series 2. 2:33.

1879. ELeptosphaeria passerinii P.A. Saccardo. Non P.A. Saccardo. 1877. On wilted leaves of Achillea ptarmica L.. Compositae. Italy.

(Leptosphaeriella) marginalis P.A. Saccardo. Michelia Conmentarium Mycologicum Fungos in Primis Italicos Illustrans 1:244. 1878. On leaves of Alhizia julibrissin Durz.. Leguminosae. Italy.

marginata (K.F.W. Wallroth) G. Niessl v. Mayendorf. Verhandlungen des Naturforschenden Vereins in Brïn 10:171-172. (1871) 1872. E? Sphaeria marginata K.F. W'. Wallroth. Flora Cryplogamica Genmaniac 2:770. 1833 On fallen leaves of Pirola recunda L..

Pyrolaceas. Austria. Crechoslovakia. ltaly. marina E. Rustrup. Botanish Tidsshrift, Kjobenlaan 17:234. 1890. Nom. illegit. Art. 64.1.

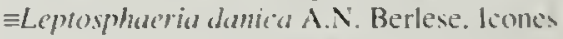
Fungorum Omnium Hucusyus Cognitorum ad 
usum Sylloges Saccardianae Adcommodatae 1:87. 1892. ELeptosphaeria chondri L.K.

Rosenvinge, Botanisk Tidsskrift, Kjobenhavn 27:XXXIII-XXXV. 1906. 三Didymosphaeria marina (E. Rustrup) J. Lind, Danish Fungi as Represented in the Herbarium of E. Rostrup, Nordisk Forlag, Copenhagen, p. 214. 1913. $\equiv$ Didymosphaeria danica (A.N. Berlese) I.M. Wilson \& J.M. Knoyle, Transactions of the British Mycological Socicty. London 44:57. 1961. 三Lautitia danica (A.N. Berlese) S. Schatz, Canadian Journal of Botany, Ottawa 63:31. 1984. Parasitic on Chondrus crispus J. Stackhouse, Algae (Gigartinaceae). Denmark. marina J.B. Ellis \& B.M. Everhart, Journal of Mycology, Columbus. Ohio 1:43. 1885. EHeptameria marina (J.B. Ellis \& B.M. Everhart) M.C. Cooke, Grevillea. London 18:32. 1889. 三Metasphaeria marina (J.B. Ellis \& B.M. Everhart) A.N. Berlese, lcones Fungorum Omnium Hucusque Cognitorum ad usum Sylloges Saccardianae Adcommodatae 1:140. 1894. EWettstcinina marina (J.B. Ellis \& B.M. Everhart) R.A Shoemaker, Canadian Joumal of Botany, Ottawa 67:1596. 1989. On dead culms of Spartina sp., Gramineae. U.S.A.

maritima L. Hollós, Annales Historico-Naturales Musei Nationalis Hungarici, Budapest 5:46. 1907. Nom. illegit. Art. 64.1. On dry siems and leaves of Triglochin maritima L., Juncaginaceae. Hungary.

maritima (M.C. Cooke \& C.B. Plowright) P.A. Saccardo, Sylloge Fungorum Omnium Hucusque Cognitorum Digessit P.A. Saccardo 2:73. 1883. ESphaeria maritima M.C. Cooke \& C.B. Plowrighi in M.C. Cooke, Grevillea, London 5:120. 1877. On culms of Juncus maritimus Lam., Juncaceae. Great Britain.

marram (M.C. Cooke) P.A. Saccardo, Sylloge Fungorum Omnium Hucusque Cognitorum Digessit P.A. Saccardo 2:60. 1883. =Sphaeria marram M.C. Cooke, Grevillea, London 5:120. 1877. 三Hepiameria marram (M.C. Cooke) M.C. Cooke, Grevillea, London 18:31. 1889. ĐPhaeosphaeria marram (M.C. Cooke) O. Eriksson, Arkiv för Botanik, Uppsala, Stockholm, Series 2, 6:425. 1967. On stems of Ammophila sp., Gramineae. Canada, Great Britain.

martagoni M. Losa, Anales del Jardin Botánico de Madrid 8:309. (1947) 1948. On dead stems of Lilium martagon L., Liliaceae. Spain.

(Melanonima) martinianum (W.L. Lindsay) M.C. Cooke. Grevillea, London 17:91. 1889. $\equiv$ Sphaseria maritinana W.L. Lindsay, Transactions of the Royal Society of Edinburgh 24:427-428. 1867. 三Melanomma marinianum (W.L. Lindsay) P.A. Saccardo, Sylloge Fungorum Omnium Hucusque
Cognitorum Digessit P.A. Saccardo 2(Addenda Ad Volumen Secundum):L1X. 1883. On cortex of not yet dead trunks of Sophora tetraptera J. Mill., Leguninosae. New Zealand.

massariella P.A. Saccardo \& C.L. Spegazzini in P.A. Saccardo, Michelia Commentarium Mycologicum Fungos in Primis Italicos lllustrans 1:393-394. 1878. On dead branches of Ulmus campestris auct., Ulmaceac. Italy.

massariella P.A. Saccardo \& C.L. Spegazzini var. brasiliensis H. Rehm, Annales Mycologici. Berlin 5:528. 1907. On branches of unknown host, unknown family. Brazil.

massariella P.A. Saccardo \& C.L. Spegazzini var. disticha D. Saccardo. Malpighia. Rassegna Mensuale di Botanica. Messina, Genova 12:208. 1898. On dead stems of Morus alba L., Moraceae. ltaly.

massarioides P.A. Saccardo \& C.L. Spegazzini, Michelia Commentarium Mycologicum Fungos in Primis ltalicos lllustrans 1:394. 1878. 三Heptameria (Leptosphaeria) massarioides (P.A. Saccardo \& C.L. Spegazzini) M.C. Cooke, Grevillea, London 18:31. 1889. 三Massariosphaeria massarioides (P.A. Saccardo \& C.L. Spegazzini) S.M. Huhndorf, J.L. Crane, \& C.A. Shearer, Mycotaxon. An International Journal Designed to Expedite Publication of Research on Taxonomy \& Nomenclature of Fungi \& Lichens, Ithaca, New York 37:204-205. 1990. On decaying stems of Heracleum sphondylium $\mathrm{L}$., Umbelliferae. Italy.

matisiae P.C. Hennings, Hedwigia, Dresden 48:107-108. 1908 [as matiaiae]. On fronds of Matisia paraensis Hub., Matoniaceac (Filicopsida). Brazil.

matritensis $\mathrm{R}$. González Fragosa, Boletin de la $\mathrm{R}$. Sociedad Española de Historia Natural, Madrid 18:368-369. 1919. On culms, leaves, and sheaths of Stipa pennata L., Gramineae. Spain.

maydis G.L. Stout, Mycologia, Lancaster, Pennsylvania $22: 275.1930$. On leaves of Zea mays L., Gramineae. U.S.A. (Illinois).

media S.A. Gucevicz, Notulae Systematicac e Sectione Cryptoganica Instituti Botanici Nomine V.L. Komarov Academiae Scientiarum U.R.S.S., Petropolis 10:183. 1955. On dead stens and sheaths of Briza media L., Gramineac. U.S.S.R.

medicaginicola M.A. Karimov, Notulae Systematicae e Sectione Cryptogamica Instituti Botanici Nomine V.L. Komarov Academiae Scientiarum U.R.S.S., Petropolis 11:122. 1956. On decaying stems of Medicago sativa L.. Leguminosac. U.S.S.R.

medicagimis P.A. Saccardo, Atti dell' Accademia Scientifica Veneto-Trentino-lstriana, Padova 
2(2):153. 1873. ELeprosphaeria medicaginum P.A. Saccardo, Michelia Commentarium Mycologicum Fungos in Primis lialicos Illustrans 2:534. 1882. Non P.A. Saccardo. 1883. On stems of Medicago sp.; not stated but presumed, Leguminosae. Austria, Germany, Italy.

medicaginis (L. Fuckel) P.A. Saccardo, Sylloge Fungorum Omnium Hucusque Cognitorum Digessit P.A. Saccardo 2:19. 1883. Nom. illegit. Art. 64.1. EPleospora medicaginis L. Fuckel, Symbolae Mycologicae, p. 134. 1870. On dried stems of Medicago sativa L., Leguminosae. Germany.

medicaginum P.A. Saccardo, Michelia Commentarium Mycologicum Fungos in Primis ltalicos lllustrans 2:534. 1881. 三Leptosphaeria medicaginis P.A. Saccardo, Atti del]' Accademia Scientifica Veneto-TrentinoIstriana, Padova 2(2):153, 1873. On stems of Medicago sp.. Leguminosae. Austria. Germany, Italy.

megalospora B. Auerswald \& G. Niess! v. Mayendorf, Verhandlungen des Naturforschenden Vereins in Brünn 10:180. (1871) 1872. $\equiv$ Nodulosphaeria megalospora (B. Auerswald \& G. Niessl v. Mayendorf) L. Holm, Svensk Botanisk Tidskrift, Stockholm 55:74. 1961. $\equiv$ Ophiobolus megalosponus (B. Auerswald \& G. Niessl v. Mayendorf) R.A. Shoemaker, Canadian Journal of Botany, Ottawa 54:2384. 1976. On dead stems of Sambucus chulus L., Caprifoliaceae. Czechoslovakia.

mclanommoides F. Fautrey in C. Roumeguère. Revue Mycologique. Toulouse 13:128-129. 1891: Fungi Selecti Gallici Exsiccati, Century 58. No. 5752. Anno 1891. On old rotting stems of Petroselinum hortense auct.,

Unbelliferae. France.

melanommoides C.L. Spegazzini. Anales del Museo Nacional de Historia Natural de Buenos Aires 6:282. 1899; Fungi Argentini Novi vel Critici, No. 628. Anno 1899. Nom. illegit. Ant. 64.1. $\equiv$ Leptosphacria subsuperficiulis P.A.

Saccardo \& P. Sydow. On fallen decaying culms of Panicum grumosum Nees. Gramineae. Chile.

melamommoides A.N. Berlese, lcones Fungorum Omnium Hucusque Cognitorum ad usum Sylloges Saccardianae Adcommoditae 1:54. 1890. ELeptosphacria revocans P.A.

Saccardo. Nec F. Fautrcy, 1891. On hranches ol Buxus sempervirens L., Buxaceae. Belgium.

melicae F. Bubák. Amuales des K.K. Naturhistorischen Hofmuseums Wien 28:198. 1914. Non. illegit. Art. 64.1. 三Massariospluaeria melicac (F. Buhák) R.A. Shocmiker. Canadian Joumal of Botany, Ottawa 67:1582. 1989. On dead stems of Nelice inaequig/mmis Boiss., Gramincae. Turkey. melicae G. Passerini, Atti della Società Crittogamologica ltaliana, Milano. Series 2. 2:44 45. 1879. On wilted leaves of Mclica uniflora Retz.. Gramineae. Italy.

melilori L. Hollós, Matematikaj és Természetrudományi Közlemenyek Vonatkozólag a Hazai Viszonyokra. Budapest 35:33. 1926. On dry stems of Melilotus alha Medicus. Leguminosae. Hungary.

mellispora M.L. Farr \& H.T. Horner. Nova Hedwigia. Zeitschrift für Kryptogramenkunde. Weinheim 15:251-252.1968. On leaves of Seluginella underwoodii Hieron.. Selaginellaceae. U.S.A. (Arizona).

mendozana F. Petrak. Sydowia. Annales Mycologici. Horn, N.Ö. 1(4-6):294-295. 1947. On dried leaves of Trisetum longighume Hackel, Gramineae. Argentina.

menthae F. Fautrey \& J.B. Lambotte. Revue Mycologique, Toulouse 17:169. 1895. On dry stems of Mentha sy/vestris L. [ Mentha longifolia (L.) Huds.], Labiatae. France.

meridionalis D. Saccardo. Mycotheca Jtalica. Century 4. No. 302. Anno 1899. Nom. nud. Art. 32.1. Ex D. Saccardo in P.A. Saccardo. Sylloge Fungorum Omnium Hucusque Cognitorum Digessit P.A. Saccardo 14:563-564. 1899. On branches of Tamarix gallica L.. Tamaricaceae. Italy

mertensiae (J.B. Ellis) P.A. Saccardo, Sylloge Fungorum Omnium Hucusque Cognitorum Digessit P.A. Saccardo 2:34. 1883. EShacria (Lepiosphaeria) mertensiae J.B. Ellis, Bulletin of the Torrey Botanical Club (and Torreya). New York 8:90-91. 1881. On dead leaves of Mertensia sp.. Boraginaceae. U.S.A.

mesoedema (M.J. Berkeley \& M.A. Curtis) J.B. Ellis \& B.M. Everhan. The North American

Pyrenomycetes. A Contribution to Mycologic Botany, p. 365. 1892. ESphueria mesoedema M.J. Berkeley \& M.A. Curtis in M.J.

Berkeley, Grevillea. London 4:151. 1876. EHeprameria mesocdema (Ml.J. Berkeley s. M.A. Curtis) P.A. Saccardo. Sylloge Fungorum Omnium Hucusque Cognitorum Digessit P.A. Saccardo 2:89. 1883. On stems of Cirsium muticum Mlich. Eupatorium coronopifolium Willd., Composinac. U.S.A. (South Carolina).

metusequoiaz S.A. Gucevicz. Trudy Gosudarswennogo Nihitshogo Botanicheshogo Sada. Ialta 32:120. 1960. On dry axillary shoots and short shoots of Melasiquoiz glyptostroboides Hu \& Cleng. Taxodiacente. U.S.S.R. (Crimea).

michorii (G.D. Westendorp) P.A. Saccardo. Sylloge Fungorum Ommium Hucusque Cognitorum Digessit P.A. Saccirdo 2:58́-59. 1 883. Ephacria michorii G.D. Westendorp. 
Bulletins de L'Académie Royale des

Sciences, des Lettres et des Beatux-Arts de Belgique, Brussels, Series 2, 7:87. 1859 ESphaerella michotii (G.D. Westendorp) B. Auerswald in W. Gonnermann and G.L. Rabenhorst, Mycologia Europaea, Abbildungen Sämmtlicher Pilze Europa's, Helte 5 und 6. Synopsis Pyrenomycelum Europaeorum. p. 18. 1869. इParaphacosphueria michorii (G.D. Westendorp) O. Eriksson. Arkiv lör Botanik, Uppsala, Stockholm. Series 2, 6:406. 1967. EScleropleella michori (G.D. Westendorp) F. v. Höhnel. Arnales Mycologici, Berlin 18:76. 1920. On dead culms of Andropogon sp.. Juncus sp., Scirpus sp., Gramineae, Juncaceae, Cyperaceae. Belgium, France. Germany, Great Britain. laly, Portugal.

micropogon P.A. Saccardo. Nuovo Giornale Botanico Italiano e Bolletino della Società Botanica Italiana, Firenze 7:320. 1875. On canes of Scirpus holoschoenus L., Cyperaceae. ltaly.

microscopica P.A. Karsten, Öfversigt af K. Vetenskapsakademiens Forhandlingar, Stockholm 29(2):102. 1872. EScleropleella microscopica (P.A. Karsten) A. Munk. Dansk Botanisk Arkiv, Kjobenhavn 15(2): 107. 1953 EPhacosphacriamicroscopica(P.A.Karsten) O. Eriksson, Arkiv för Botanik, Uppsala, Stockholm, Series 2, 6:426. 1967. EPhacosphacria microscopica(P.A. Karsten) O. Eriksson var, microscopica, Arkiv för Botanik, Uppsala, Stockholm, Series 2, 6:427. 1967. On rotting leaves of Alopecurus ovams Knapp, Poa alpina L., Poa colpodea T. Fries, Trisenum subspicatum (L.) Beauv., Gramineae. Canada. Denmark. Finland, Germany, lialy. Sweden.

microscopica P.A. Karsten forma hrachypodii E.

Niel in C. Roumeguère, Fungi Selecti Gallici Exsiccati, Century 65, No. 6437. Anno 1894: Revue Mycologique. Toulouse 16:8. 1894. On culms of Brachypodium sylvaricum (Hudson) Beauv., Gramineae. France. microscopica P.A. Karsten forma glyceriae F. Fatutrey in C. Roumeguère, Fungi Selecti Gallici Exsiccati, Century 57. No. 5651. Anno 1891: Revue Mycologique, Toulouse 13:78. 1891. On seeds and leaves of Glyceria aquarica (L.) Wahlenb., Gramineae. France. microscopica P.A. Karsten subsp. calami P.A.

Karsten. Hedwigia, Dresden 22:178. 1883:

Acta Societatis pro Fauna et Flora Fennica, Helsingforsiac 2(6):52-53. (1884) 1885. On rotten leaves of Acorns calamus L., Araceac. Finland.

microscopica P.A. Karsten var. alpina T. Ferraris.

Malpighia. Rassegna Mensuale di Botanical. Messina, Genova 16:453. 1902. On leaves of Iuzula lutea (All.) DC., Juncaceae. Italy. microscopice P.A. Karsten var. caricis-sulpinae R Staritz in P.A. Saccardo, Sylloge Fungorum Omnium Hucusque Cognitorum Digessit P.A. Saccardo 17:729. 1905. On unknown substrate of Curex izlpina L.. Cyperaceac. Germany.

micross'opica P.A. Karsten var. flaro-brumnea J. Feltgen [falsely so cited in P.A. Saccardo. Sylloge Fungorum Omnium Hucusque Cognitorum Digessit P.A. Saccardo 17:729. 1905.] =Leptosphaeria cuhnorum B. Auerswald var. flavo-brinnea J. Feltgen. microsprora J.B. Ellis \& B.M. Everhart, Bulletin of the Torrey Botanical Club (and Torreya), New York 24:461. 1897. On dead stems of Lespedeza capitata Michx., Leguminosae. Canada.

microthyrioides J. Feltgen, Vorstudien zu einer PilzFlora des Grossherzogthums, Luxemburg 1(2):154. 1901. On dry stems of litis pseudacorus L., Iridaceae. Luxembourg. mikaniae A.C. Batista \& G.E.P. Peres in A.C.

Batista, R.G.S. Falcao, and G.E.P. Peres, Atas do Instituto de Micologia, Recife 4:45-46. 1967. On leaves of Mikania sp.. Compositac. Brazil.

millefolii (L. Fuckel) G. Niessl v. Mayendorf in G.L. Rabenhorst, Fungi Europaei Exsiccati, Klotzschii Herbarii vivi Mycologici Continuatio Edita Nova, Series 2, Century 23, No. 2239. Anno 1876. 三Pleospora millefolii L. Fuckel, Symbolae Mycologicae Dritter Nachtrag, p. 20. 1875. On dry stems of Achillea millefolium auct., Compositae. Crechoslovakia.

minima (J.E. Duby) ex P.A. Saccardo, Michelia Commentarium Mycologicum Fungos in Primis ltalicos lllustrans 2:320. 1881 . ESphaeria minima J.E. Duby in C. Rouneguère, Fungi Selecti Gallici Exsiccati. Century 7, No. 694. Anno 1880. Nom. nud. \$Mycotodea minima (J.E. Duby ex P.A. Saccardo) W. Kirschstein in O.C. Schmidı. Kryptogamenflora der Mark Brandenburg und Angrenzender Gebiete herausgegeben von dem Botanishen Verein der Provinz Brandenburg, Leipzig 7(3):432. 1938. On leaves of Potenilla argentea L., Rosaceae. France.

minoensis K. Hara, Botanical Magazine. Tokyo 27(317):250. 1913. On unknown substrate of Phyllostaclyys hambusoides Sieb. \& Zucc. var. bambusoides, Gramineae. Japan.

mrabilis G. Niessl v. Mayendorf, Hedwigia, Dresden 20:97. 1881. EPleospora mirahilis (G. Niessl v. Mayendorf) F. Perrak, Annales Mycologici, Berlin 25:207. 1927. 三Wettsteinina mirabilis (G. Niessl v. Mayendorf) F. v. 1 löhnel, Sitzungsberichte der Akidemie der Wissenschaften in Wien, Mathematischnaturwissenschafiliche Klasse, Aht. 1 , 
1 16:635. 1907. On dry stenus of Chondilla juncea L.. Algae (Chondriellaceae). Unknown country.

mirandue A. Caballero, Boletin de la R. Sociedad Española de Historia Natural. Madrid 27:61-62. 1927. On living thallus of Mesugloia leveillei (J. Ag.) Mengh., Algae (Chordariaceae. Phaeophyceae). Italy. mivakeana P.A. Saccirdo, Sylloge Fungorum Omnium Hucusque Cognitorum Digessit P.A. Saccardo 24:999. 1928. EPhaensphaeria eriobomye l. Miyake, Botanical Magazine, Tokyo 27:41-42.1913. ETremulosphaerella eriobotryae (1. Miyake) F.L. Tai. Sylloge Fungorum Sinicorum, Science Press. Academia Sinica, Peking, p. 330. 1979. On leaves of Eriobotra japonica (Thunb.) Lindley, Rosaceae. China.

modesta (J. Desmazières) B. Auerswald in G.L. Rabenhorsi, Fungi Europaei Exsiccati. Klotzschii Herbarii vivi Mycologici Continuatio Edita Nova, Edition 3 (Editio Nova, Series Secunda), Century 10, No. 948. Anno 1866. ESphacria (Caulicola) modesta J. Desmazières, Annales des Sciences Naturelles, Paris, Botanique, Series 3, No. 8. p. 173. 1847. $\equiv$ Nodulosphaeria modesta (J. Desmazières) A. Munk, Dannsk Botanisk Arkiv, Kjobenhavn 15(2):136. 1953. $\equiv$ Nodulosphureria modesta (J. Desmazières) A. Munk ex L. Holm, Symbolae Botanicae Upsalienses, Uppsala 14(3):80. 1957. On dry stems of Scabiosa columbaria L., Dipsacaceae. Finland, France.

modesta (J. Desmazières) P.A. Karsten. Fungi Fenniae Exsiccati, Century 9, No. 889. 1869; Mycologia Fennica 2 (Pyrenomycetes): 106. 1873. ESphaeria modesta J. Desmazières. Annales des Seiences Naturelles, Paris, Botanique. Series 3. No. 8. p. 173. 1847. Non. illegit. Art. 64.1.

modesta (J. Desmazières) P.A. Karsten forma duaci F. Fautrey in C. Rouneguère, Fungi Selecti Gallici Exsiccati, Century 66, No. 6553. Anno 1894; Revue Mycologique, Toulouse 16:112. 1894. On llowering apex of Dancus sp.. Unbellilerae. France.

modesta (J. Desmazières) P.A. Karsten forma digitalis-luteae F. Fautrey in C. Rouneguère. Fungi Selecti Gallici Exsiccati, Century 56. No. 5538. Anno 1891: Revue Mycologique, Toulouse 13:8. 1891. On dry stems of Digilalis lafea L., Scrophulariaceae. France. modesta (J. Desmazières) P.A. Karsten forma jacobacue F. Fautrey in C. Roumeguère. Revue Mycologique. Toulouse 14:172. 1892: Fungi Selecti Gallici Exsiccati. Century 62. No. 6141. Amno 1892. On stems of Senecio jacolued 1... Compositae. France.

modesfa (J. Desmazières) P.A. Karsten forma lappac E. Nicl in C. Roumeguère, Fungi Selecti
Gallici Exsiccati. Century 65, No. 6438. Anno 1894: Revue Mycologique. Toulouse 16:8. 1894. On dead stems of Lappasp. (=Arcium sp.). Compositae. France?

modesta (J. Desmazières) P.A. Karsten forma succisac F. Fautrey in C. Roumeguère. Fungi Selecti Gallici Exsiccati, Century 56. No. 5539. Anno 1891: Revue Mycologique. Toulouse 13:8. 1891. On dri stems of Succisa sp., Dipsacaceae. France.

mudesta (J. Desmazières) P.A. Karsten forma swhestris F. Fautrey in C. Roumeguère. Fungi Selecti Gallici Exsiccati, Centun 61, No. 6034. Anno 1892: Revue Mycologique. Toulouse 14:107. 1892. On stems of Dipsacus sylestris Hudson. Dipsacaceae. France.

modesu (J. Desmazières) P.A. Karsten var. cibostii P.A. Saccardo. Sylloge Fungorum Omnium Hucusque Cognitorum Digessit P.A. Saccardo 2:40. 1883. On stems of Cinanchum sp.. and on an unknown species of Umbelliferae. Asclepiadaceae, Umbelliferae. ltaly:

modesta (J. Desmazières) B. Auerswald var. rubellula J. Desmazières in P.A. Saccardo. Sylloge Fungorum Omnium Hucusque Cognitorum Digessit P.A. Saccardo 2:40. 1883. ELeptosphaeria rubellula (J. Desmazières) F. v. Höhnel. On stems of Barkhousia taraxacifolia (Thuill.) DC.. Compositae. France.

modestula F.E. Clements \& E.S. Clements. Cryptogamae Formationum Coloradensium. Centur 3. No. 237. Anno 1907. Nom. nud. Ar. 36.1. On dead stems of Geranium richardsonii Fisch. \& Trautv. Geraniaceae. U.S.A.

molleriana J. Verissimo d'Almeida \& M. de Souza da Camara. Boletim da Sociedade Broteriana. Coinbra 24:165. 1908/1909. On leaves of Cocos romanzoffioma Cham. [=Arecustrum romanzoffiamum (Cham.) Beccari]. Palnue. Portugal.

molleriana G. Niessl v. Mayendorf, Instituto. Revista Seientilica e Litteraria, Coimbra 31:90. 1883. EMciasphaeria molleriana (G. Niessl v. Mayendorl) A.N. Berlese \& P. Voglino. Sylloge Fungorum Omnium Hucusque Cognitorum Digessit P.A. Saccardo Additamenta Ad Volumina I-IV, p. 157. 18S6. [Perhaps Sphacrella mollcriana F. V. Thümen?. lide G. Niess] v. Mayendorf.] On dead leaves of Eucalyptus globulus Labill. Myrtaceac. Portugal.

molluginis G. Passerini. Alli dell’ Accadenia Nazionale dei Lincei. Memoire. Rome. Series 4. 6:459. (1889) 1890. On dry stems of Galium mollugo L.. Rubiaceac. Italy. molydima J.P. Montagne \& J.B. Mougeot ex P.A. Saccardo. Michelia Commentarium Mycologieum Fungos in Primis Italicos Illustrans 
2:319. 1881. ESphaeria molyhdina J.P. Montagne in C. Roumeguère, Fungi Selecti Gallici Exsiccati, Century 6. No. 580. Anno 1879. Nom. nud. Art. 32.1. ESphaeria molybdina J.P. Montagne in J.B. Mougeot. J.A. Mougeot, C.G. Nestler, and W.P. Schimper, Stippes Cryptogamae VogesoRhenanae Quas in Rheni Superioris Inferiorisque, Nec Non Vogesorum Praefecturis. Collegerunt, J.B. Mougeot et C.G. Nestler. Fascicle 15, No. 1451. Anno 1860. [P.A. Saccardo's validated description cites only Fungi Gallici Exsiccati No. 580.] On willed stems of Vinceloxicum officinale Moench. Asclepiadaceae. France.

monilispora (L. Fuckel) P.A. Saccardo, Sylloge Fungorum Omnium Hucusque Cognitorum Digessit P.A. Saccardo 2:79. 1883. ESphaeria monilispora L. Fuckel, Fungi Rhenani Exsiccati A Leopoldo Fuckel Collecti, Supplement Fascicle 3, No. 1777. Anno 1866. EPleospora monilispora L. Fuckel, Symbolae Mycologicae, p. 138. 1870. On dry stalks and sheaths of Juncus lampocarpus Ehrh. ex Holfm., Juncaceae. Austria.

monilispora P.A. Saccardo forma riglochinis J. Feltgen.Vorstudien zu einer Pilz-Flora des Grossherzogthums, Luxemburg 1(3):208. 1903. On stalks of Triglochin palustris L., Juncaginaceae. Luxembourg.

monotis H. Rehm, Ascomyceten, Fascicle 18, No. 887. Anno 1886: Hedwigia, Dresden 26:94. 1887. [Reprint is page 14 at FH.] On dead tufts of Saxifiaga sp., Saxifragaceae. Italy. montana G.B. Traverso, Manipolo di Funghi Della Valle Pellina, Aosta (Tipografia cattolica), p. 20. 1912; Bulletin de la Société de la Flore Valdôtaine Aosta No. 8:34. 1912. Nom. nud. Art. 32.1c. On dry stems of Saliva glutinosa L., Labiatae. Italy.

monticola J.B. Ellis \& B.M. Everhart, Bulletin of the Torrey Botanical Cluh (and Torreya), New York 24:461-462. 1897. On dead leaves and petioles of Trifolium kingii S. Wats., Leguminosae. U.S.A.

montis-bardi F. Fautrey \& J.B. Lambotte, Revue Mycologiquc, Toulouse 18:69. 1896. On stems of Seseli memanum L.. Umhelliferae. France.

morierae F. Petrak, Sydowia. Annales Mycologici, Hom, N.Ö. 16(1-6):338. (1962) 1963. On dry stems of Moriera sp. Cruciferae. Afghanistan.

mertheriana P.A. Saccardo, Atti del Istituto Veneto di Scienze, Lettere ed Arti. Venezia, Series 6. 2:457. (1883) 1884. On rolten leaves of Succisa sp., Dipsacatceate. Switerland. mosana V. Mouton, Bulletin. Société R. de Botanique de Belgique, Bruxelles 39:45. 1900. „Massariosphaeria mosana (V. Mouton) A.
Leuchtmann, Sydowia. Annales Mycologici, 1lom, N.Ö. 37:170. 1984. On culms of Plragmites sp., Gramineae. Belgium, Great Britain.

moufan W. Siemaszko, Acta Socictatis Botanicorum Poloniac, Warszawa 1:22. 1923. On leaves of Paeonia moutan Sims. (=Pueonia suffruticosa Haw.), Paconiaceae. U.S.S.R.

molroniana P.A. Saccardo \& P. Sydow in P.A. Saccardo, Sylloge Fungorum Omnium Hucusque Cognitorum Digessit P.A. Sacearclo 16:517. 1902. ELeptosphaeria punctiformis V. Mouton. On grass culms ol unknown host, Gramineae. Belgium.

micosa V. Mouton, Bulletin. Société R. de Botanique de Belgique, Bruxelles 39:43. 1900. On leaves of Typha sp.. Typhaceae. Belgium. muchlenbeckiae B.K. Vaidehi, Mycopathologia et Mycologia Applicata Den Haag 38:135-136. 1969. On living phylloclades of Muchlenbeckia platyclados Meissn., Polygonaceae. India.

nutellenbergiae H. Rehm, Annales Mycologici. Berlin 13:5. 1915. On branches of Muehlenbergia racemosa (Michx.) Britton. Stern, \& Pogg., Gramineae. U.S.A.

muelleri (J.E. Duby) B. Auerswald in W. Gonnermann and G.L. Rabenhorst, Mycologial Europaea. Abbildungen Sämmtlicher Pilze Europa's, Hefte 5 und 6, Synopsis Pyrenomycetum Europaeorum, Table 12, Figure 167. 1869. ESphaeria muelleri J.E. Duby in G.L. Rahenhorst, Klotzschii Herbarium Vivum Mycologicum Sistens Fungorum Per Totam Germaniam Cresentium Collectionem Perfectam, Dresden, Edition 2, Century 7, No. 642. Anno 1858. On scaly bark of Acer sp.. Aceraceae. France.

mulhleubergiac (J.B. Ellis) G. Niessl v. Mayendorf, Hedwigia, Dresden 22:33. 1883. EDothidea muhlenhergiae J.B. Ellis. Bulletin ol the Torrey Botanical Club (and Torreya), New York 8:74-75. 1881. EPhyllachora muhlenhergiae (J.B. Ellis) P.A. Saccardo, Sylloge Fungorum Omnium Hucusque Cognitorum Digessit P.A. Saccardo 2:604. 1883. On culms of Mullenbergia sp., Gramineac. North America.

muirensis R. Sprague, Mycologia, Lancaster. Pennsylvania 47:249. 1955. On living leaves of Pod ammua L., Gramineae. U.S.A. (Alaska).

mulriseptala H.G. Winter. Hedwigia. Dresden 11:148. 1872. EEnodesmium multiseptum (H.G. Winter) L. Holm, Symbolae Botanicate Upsalienses, Uppsala 14(3):133. 1957. On dry stems of Latmyors sylvestris L., Leguminosac. Germany.

muliseptata H.G. Winter forma alpina H. Rehm, Hedwigia. Dresden 24:235. 1885. Nom. illegit. Art. 64.1. ELeptosphaeria epilobii E. 
Müller. On dry stems of Epilobium fleischeri Hochst., Onagraceae. Italy.

mume K. Hara, Byôchu-gai Zasschi (Journal of Plant Protection), Tokyo 5:884. 1918 [as mume in original publication]. On dead branch of Prumus mume Sieb. \& Zucc., Rosaceae. Japan.

muralis P.A. Saccardo, Michelia Commentarium Mycologicum Fungos in Primis Jtalicos Illustrans 2:64. 1880. On stems of Parietaria officinalis L., Urticaceae. France.

muricata J.B. Ellis \& B.M. Everhart, Proceedings of the Academy of Natural Sciences of Philadelphia 1893:447-448. 1894. On leaves of Andropogon muricatus Retz. [=Vetiveria zizanioides (L.) Nash], Gramineae. U.S.A.

musae T. Lin \& J. Yen, Bulletin de la Société Mycologique de France, Paris 87:427-429. 1971. On living leaves of Musa cavendishii Lamb. ex Paxton, Musaceae. Taiwan. musarum P.A. Saccardo \& A.N. Berlese, Revue Mycologique, Toulouse 11:204. 1889. On dead leaves of Musa sp., Musaceae. São Tomé.

muscari L. Hollós, Annales Historico-Naturales Musei Nationalis Hungarici, Budapest 7:51. 1909. On dry stems of Muscari comosum (L.) Miller, Liliaceae. Hungary.

musicola (C.L. Spegazzini) P.A. Saccardo \& A. Trotter in P.A. Saccardo, Sylloge Fungorum Omnium Hucusque Cognitorum Digessit P.A. Saccardo 22:229-230. 1913. [Incorrectly cited as a comb. nov. based on Leprosphaerella musicola C.L. Spegazzini.] Nom. inval. Art. 43.1.

musigena T. Lin \& J. Yen, Bulletin de la Société Mycologique de France, Paris 87:429-431. 1971. On living leaves of Musa cavendishii Lamb. ex Paxton, Musaceae. Taiwan.

myricae J. Dearness \& H.D. House, Bulletin of the New York State Museum, Albany 197:30-31. 1917. On dead twigs and branches of Myrica gale L., Myricaceae. U.S.A. (New York).

myrti S.A. Gucevicz, Trudȳ Gosudarstvennogo Nikitskogo Botanicheskogo Sada, Yalta 32:155-156. 1960. On dry branches of Myrfus conmunis L., Myrtaceae. U.S.S.R. myrticola S.A. Gucevicz, Trudȳ Gosudarstvennogo Nikitskogo Botanicheskogo Sada, Yalta 32:156-157. 1960. On dry branches of Myrtus communis L., Myrtaceae. U.S.S.R.

nanae R.A. Shoemaker. Canadian Journal of Botany, Ottawa 62:2708. 1984. On stens of Achillea nana L.. Compositae. Switzerland.

nandinae K. Togashi \& E. Tsukamoto, Annals of the Pliytopathological Society of Japan. Tokyo 17:121. 1953. On unknown substrate of Nandina domestica Thunb., Nandinaceae. Japan.

napelli E. Müller, Sydowia. Amnales Mycologici. Horn, N.Ö. 4(1-6):245. 1950. On dead stems of Aconitum napellus L., Ranunculaceae. Switzerland.

napi (L. Fuckel) P.A. Saccardo, Sylloge Fungorum Omnium Hucusque Cognitorum Digessit P.A. Saccardo 2:45. 1883. $\equiv$ Pleospora napai L. Fuckel, Symbolae Mycologicae, p. 136. 1870. On stems and pieces of Brassica napus L.. Cruciferae. Austria.

nardi (E.M. Fries) V. Cesati \& G. de Notaris, Commentario della Società Crittogamologica Italiana, Milan 1:236. 1863. ESphaeria duplex M.J. Sowerby:E.M. Fries var. nardi E.M. Fries:E.M. Fries, Systema Mycologicum Sistens Fungorum 2:520. 1823. ESphaeria nardi (E.M. Fries) E.M. Fries, Summa Vegetabilium Scandinaviae, Seu Enumeratio, Systematica et Critica, Plantarum tum Cotyledonearum, Quam Nemearum Inter Mare Occidentale et Album, Inter Eidoram et Nordkop, Hactenus Lectorum, una Cum Singulae Distributione Geographica, p. 394. 1849. 三Sphaeria nardi (E.M. Fries) G.L. Rabenhorst, Hedwigia, Dresden 1:116. 1857. $\equiv$ Pleospora nardi (E.M. Fries) L. Fuckel. Symbolae Mycologicae, p. 137. 1870. $\equiv$ Phaeosphaeria nardi (E.M. Fries) L. Holm. Symbolae Botanicae Upsalienses, Uppsala 14(3):124. 1957. On dry culms and leaves of Nardiss stricta L.. Gramineae. Belgium, France, Germany, Great Britain, Sweden, Switzerland.

nardi (E.M. Fries) V. Cesati \& G. de Notaris var. duhiosa V. Mouton, Bulletin. Société R. de Botanique de Belgique, Bruxelles 26:177. 1887. ELeprosphaeria dubiosa (V. Mouton) C.A. Oudemans. Enumeratio Systematica Fungorum 1:981. 1919. On leaves of Scirpus sylvaticus L., Gramineae. Belgium.

narmari J. Walker \& A.MI. Smith. Transactions of the British Mycological Society, London 58:459-460. 1972. EPhaeosphaeria narmari (J. Walker \& A.M. Smith) R.A. Shoemaker. Canadian Journal of Botany, Ottawa 67:1551. 1989. On stolons and sheaths of $C y$ nodon dactylon (L.) Pers., Cynodon transvaalensis Burtt-Davy, Hordeum ivulgare L.. Oryza sariva L., Pennisctum clandestinum Hochst. ex Chiov., Stenotaphrum secundatum (Walter) O. Kuntze, Triticum aestivum L.. Gramineae. Australia (New South Wales).

nashi (K. Hara) P.A. Saccardo in A. Trotter. Sylloge Fungorum Omnium Hucusque Cognitorum Digessit P.A. Saccardo 24:999. 1928. ELeprosphaerella nashi K. Hara, Kajyubyogai-ron Nihon-kankitsu-Kai. Schiznoka [Fruit Tree Diseases]. pp. 142-145. 1916. $\equiv$ Phacospora nashi $(\mathbf{K}$. Hara) $K$. Hara. Jitsuyo-Sakumotsu-Byorigaku [Manual of Plant Pathology]. p. 270. 1925. On leaves of Pyw. sinensis Hort., Rosaceae. Japan. 
nectrioides C.L. Spegazzini in P.A. Saccardo, Michelia Commentarium Mycologicum Fungos in Primis Italicos Illustrans 1:458. 1879. On decayed runners of Clematis vitalba L., Ranunculaceae. ltaly.

neglecta G. Niessl v. Mayendorf, Verhandlungen des Naturforschenden Vereins in Brünn 10:175-176. (1871) 1872. 三Metasphaeria neglecia (G. Niessl v. Mayendorf) P.A. Saccardo, Sylloge Fungorum Omnium Hucusque Cognitorum Digessit P.A. Saccardo 2:173. 1883. On dried grass leaves of unknown host, Gramineae. Italy (near Bozen. Tirolia). neomaritima R.V. Gessner \& J. Kohlmeyer, Canadian Journal of Botany, Ottawa 54:2032. 1976. 三Sphaeria maritima M.C. Cooke \& C.B. Plowright in M.C. Cooke, Grevillea, London 5:120. 1877. Non Sphaeria moritima P.L. Crouan \& H. Crouan, Florule Du Finistère Contenant Les Descriptions De 360 Espèces Nouvelles De Sporogames, De Nombreuses Observations et une synonymie des plantes Cellulaires et Vasculaires Qui Croissent Spontanément Dans ce Département, p. 27. 1867. ELeptosphaeria maritima (M.C. Cooke \& C.B. Plowright) P.A. Saccardo, Sylloge Fungorum Omnium Hucusque Cognitorum Digessit P.A. Saccardo 2:73. 1883. Non Leptosphaeria maritima L. Hollós. 1906. 三Phaeosphaeria neomaritima (R.V. Gessner \& J. Kohlmeyer) R.A.

Shoemaker, Canadian Journal of Botany, Ottawa 67:1572. 1989. On deteriorating leaf sheaths and decaying culms of Juncus maritimus Lam., Juncus roemerianus Scheele, Spartina alterniflora Loisel., Spartina lounsendii H. Groves \& J. Groves, Spartina sp.. Juncaceae, Gramineae. Canada, Great Britain, U.S.A.

neotizans (W.A. Leighton) F.W. Zopf, Hedwigia, Dresden 35:361. 1896. EVerrucaria neottizons W.A. Leighton, The Lichen-Flora of Great Britain, Ireland, and the Channel lslands, p. 497. Third edition. 1879. Parasitic on Baeomyces rufus (Huds.) Rebent., Lichenes (Baeomycetaceae). Great Britain. nervisequa H.G. Winter, Boletim da Sociedade Broteriana, Coimbra 2:42. (1883) 1884. $\equiv$ Metasphaeria nervisequa (H.G. Winter) A.N. Berlese \& P. Voglino, Sylloge Fungorum Omnium Hucusque Cognitorum Digessit P.A. Saccardo Additamenta Ad Volumina I-IV, p. 158. 1886. On leaves of Smilax pseudo-china L., Liliaceae. Portugal. nesodes (M.J. Berkeley \& C.E. Broome) P.A. Saccardo, Sylloge Fungorum Omnium Hucusque Cognitorum Digessit P.A. Saccardo 2:85. 1883. ESphaeria (Depazea) nesodes M.J. Berkeley \& C.E. Broome, Joumal of the Linnean Society (Botany), London 14:129. (1873) 1875. On leaves of /ydrocotyle asiatica L., IJydrocotylaceae. Sri Lanka. nicolai F. Bubák, Sitzungsberichte der K. Böhmischen Gesellschaft der Wissenschaften. Prag Mathematisch-Naturwissenschaftliche Klasse 1903(12): 10. 1904. On preceding year's stems of Salvia officinalis L., Labiatae. Yugoslavia.

niessleana G.L. Rabenhorst, Fungi Europaei Exiccati, Klotzschii Herbarii vivi Mycologici Continuatio. III Ausgabe (Editio nova, Series Secunda), Century 13, No. 1252. Anno 1869. Nom. nud. Art. 32.1. On dried stems and leaves of Lathyrus latifolius L., Leguminosae. Czechoslovakia.

niessleana G.L. Rabenhorst ex G. Niessl v. Mayendorf, Verhandlungen des Naturforschenden Vereins in Brünn 10:179. (1871) 1872. ĐEntodesmium niessleanum (G.L. Rabenhorst ex G. Niessl v. Mayendorf) L. Holm, Symbolae Botanicae Upsalienses, Uppsala 14(3): 133. 1957. On dried stems and leaves of Lathyrus latifolius L., Leguminosae. Czechoslovakia.

niessleana G.L. Rabenhorst ex G. Niessl v. Mayendorf forma viciae W.B. Grove, Journal of Botany, British and Foreign, London 68:74. 1930. On dead stems of Vicia sativa L., Leguminosae. Great Britain.

niessleana G.L. Rabenhorst var. starizii $\mathrm{H}$. Rehm, Hedwigia, Dresden 39: 193. 1900: Ascomyceten, Fascicle 27. No. 1335. Anno 1900. On dry stems of Seseli sp., Umbelliferae. Germany.

nigrans (M.R. Roberge) V. Cesati \& G. de Notaris, Commentario della Società Crittogamologica Italiana, Milan 1:235. 1863. 三Sphaeria culmicola E.M. Fries:E.M. Fries var. nigrans (M.R. Roberge) E.M. Fries, Summa Vegetabilium Scandinaviae, Seu Enumeratio, Systematica et Critica, Plantarum tum Cotyledonearum, Quam Nemearum Inter Mare Occidentale et Album, Inter Eidoram et Nordkop, Hactenus Lectorum, una Cum Singulae Distributione Geographica, p. 393. 1849. ESphaeria (Caulicola) nigrans M.R. Roberge in J. Desmazières, Annales des Sciences Naturelles, Paris, Botanique, Series 3, 6:79. 1846. IPhaeosphaeria nigrans (M.R. Roberge) L. Holm, Symbolae Botanicae Upsalienses, Uppsala 14(3): 1 12. 1957. On sheath of Dactylis glomerata L., Gramineae. Austria, Canada, Finland, France, Germany, Great Britain, India, Italy, Sweden, Switzerland, U.S.A.

migrans (M.R. Roberge) V. Cesati \& G. de Notaris forma arundinis C. Roumeguère, Fungi Selecti Gallici Exsiccati, Century 43, No. 4265. Anno 1887. On culms of Arundo phragmites L. (=Phragmites communis Trin.), Gramineae. France.

nigrella B. Auerswald in W. Gonnermann and G.L. Rabenhorsı. Mycologia Europaea, Abbildungen Sämmtlicher Pilze Europa's, Hefte 
5 und 6, Synopsis Pyrenomycetum Europaeorum, Table 12, Figure 163. 1869. $\equiv D i$ aporthe nigrella (B. Auerswald) G. Niessl v. Mayendorf, Verhandlungen des Naturforschenden Vereins in Brünn 10:201-202. (1871) 1872. Nec Sphaeria nigrella E.M. Fries. $\equiv$ Diaporthopsis nigrella (B. Auerswald) J.H. Fabre, Annales des Sciences Naturelles, Paris, Botanique. Series 6, 15:35-36. 1883. On stems and branches of Eryngium campestre L., Umbelliferae. Germany.

nigrella (G.L. Rabenhorst) P.A. Saccardo, Sylloge Fungorum Omnium Hucusque Cognitorum Digessit P.A. Saccardo 2:21. 1883. 三Cucurbilaria nigrella G.L. Rabenhorst, Hedwigia, Dresden 12:140. 1873. On dead stems of Brassica rapa L., Cruciferae. Germany. nigricans P.A. Karsten, Mycologia Fennica Pars 2, Pyrenomycetes, p. 107. 1873. On dry stems of Chenopodium alhum L., Chenopodiaceae. Finland.

nigricans P.A. Karsten var. grindeliae C.E. Fairman, Mycologia, Lancaster, Pennsylvania 10:245. 1918. On unknown substrate of Grindelia? sp., Compositae. U.S.A. (New Mexico). nigrificans F. Bubák \& A. Wróblewski in F. Bubák, Hedwigia. Dresden 57:329. 1916.

Presumably Eudarluca caricis (E.M. Fries:E.M. Fries) O. Eriksson, Botaniska Notiser. Lund 119:49-50. 1966. Erroneously cited as on the leaves and culms of Carex sp. on rust pustules. France.

migromaculata (H. Rehm) E. Müller. Veroeffentlichungen des Geobotanischen Institutes, Eidgenossiche Technische Hochschule Riibel in Zurich 87:24. 1986. =Ophiobolis (Plejobolus) nigromaculata H. Rehm, Annales Mycologici, Berlin 10:393-394. 1912. On stems of Aconitum compactum Rchb., Aconitum nappehts L.. Aconitum paniculatum Lam., Ranunculaceae. Germany (Upper Bavaria).

hitschkei H. Relın, Ascomyceten, Fascicle I, No. 15. Anno 1870. Nom. nud. Art. 32.1; Flora, Jena und Regensburg 55:510. 1872. On hard, dry stems of Cacalia sp., Compositae. Austria.

nitscltkei H. Rehm forma adenostylidis H. Rehm, Asconyceten, Fascicle 11, No. 532. Anno 1879. Nom. nud. Art. 32.1. On fallen stems of Adenostyles alhifroms (L. fil.) Reichenb., Compositac. Germany.

uobilis P.A. Saccardo, Nuovo Giomale Botanico Italiano e Bolletino della Società Botanica Italiana. Firenze 7:312. 1875. „Me'osiphuerios nobilis (P.A. Saccardo) P.A. Saccardo. Sylloge Fungorum Omnium Hucusque Cognitorum Digessit P.A. Saccardo 2:169. 1883. On leaves of Lamus nobilis L.. Lauraceae. Italy.

nodormm E. Müller, Phytopathologische Zeitschrilt. Berlin 19:409-410. 1952. EPhucosphaeria nodorum (E. Müller) A. Hedjaroude,

Sydowia. Annales Mycologici, Hom, N.Ö. 22:79. 1968. [Anamorph: Septoria nodorum (M.J. Berkeley) M.J. Berkeley.] On dead culms of Triticum vulgare Vill.. Gramineae. Canada, Kenya, Switzerland.

nodorum E. Müller forma specialis hordei V. Smedegord-Petersen, Friesia. Nordisk Mykologisk Tidsskrift, Kobenhavn 10:262-263. 1974. On leaf lamina and sheaths of Hordeum iulgare L., Gramineae. Denmark.

norfolcia (M.C. Cooke) P.A. Saccardo, Sylloge Fungorum Omnium Hucusque Cognitorum Digessit P.A. Saccardo 2:73. 1883. ESphaeria norfolcia M.C. Cooke, Grevillea, London 5:120-121. 1877. 三Phaeosphaeria norfolcia (M.C. Cooke) A. Leuchtmann. Sydowia. Annales Mycologici. Horn. N.Ö. 37:152. 1984. On stems of Eleocharis sp., Juncus sp., Gramineae, Juncaceae. France, Great Britain.

nonegica E. Rostrup, Norske Ascomyceter i Christiania Universitetets Botaniske Museum. Christiania (Oslo) (I. Kommission Hos Jacob Dybwad), p. 24. 1904. [lssued in Skrifter Udg. af Videnskabsselskabet i Christiania. Mathematisk-naturvidenskabelig klasse. Christiania (Oslo) No. 274.J Non vidi. On stems of Braya sp.. Cruciferae. Poland.

(Clypeosphaeria) notarisii M.C. Cooke, Grevillea. London 17:91. 1889. ESphacria clypeiformis G. de Notaris, Memorie della Accademia delle Scienze di Torino. Series 2. No. 7. p. 113. 1853: Micromycetes Italici Novi vel minus Cogniti Decas 7. p. 113. 1845. Non Sphaeria clypeiformis L.V. de Lacroix in G.L.

Rabenhorst. $\equiv$ Clypeosphacria notarisii L. Fuckel, Symbolae Mycologicae, p. 117. 1870. On barky runners of Rubus fruticosus L.. Rosaceae. Italy.

obesa (M.C. Durieu de Maisonneuve \& J.P.

Montagne) P.A. Saccardo, Michelia Commentarium Mycologicum Fungos in Primis Italicos Illustrans 1:38. 1877: Fungi Italici autographice delineati (additis nonnullis extraitalicis asterisco notatis). Paravii. Table 284. 1878. ESphaeria obesa M.C. Durieu de Maisonneuve \& J.P. Montagne, Exploration Scientilique de L'Algérie Publice Par Ordre du Gouvemenuent. Series Naturelles. Botanique 1. pp. 526-527. 1868. $\equiv \mathrm{Hep}$ tameria obesa (M.C. Duricu de Maisonneuve \& J.P. Montagne) P.A. Saccirdo. Sylloge Fungorum Onnium Hucusque Cognitorum Digessit P.A. Saccardo 2:88. 1883. On stents of Scabiosa urceolua Desf.. Cucurbitaceae. Algeria.

obesula P.A. Saccardo in V. Mouton, Bulletin. Société $R$. de Botanique de Belgique. Bruxelles 26:176. 1857. On stems of Acer pserdeplatamus L. Angelica sp.. Spiraea 
ulmaria L. (=Filipendula dmaria Mixim.). Aceraceie, Umbelliferae, Rosaceat. Belgum. obesula P.A. Saccurdo, Michelia Commentarium

Mycologicum Fungos in Primis Italicos

lllustrans 2:318. 1881. On stems ol most

herbaceous? plants. France.

obiones (P.L. Crouan \& H.M1. Crouan) P.A.

Saccardo, Sylloge Fungorum Ommium

IJucusque Cognitorum Digessit P.A. Saccardo

2:24. 1883. EPleospora obiones P.L. Crouan

\& H.M. Crouan, Florule Du Finistère

Contenant Les Descriptions De 360 Espèces

Nouvelles De Sporogames, De Nombreuses

Observations ef une synonymie des plantes

Collulaires et Vasculaires Qui Croissent

Spontanément Dans ce Département, p. 22.

1867. EPasseriniella obiones (P.L. Crouan \&

H.M. Crouan) K.D. Hyde \& R. Mouzouras,

Transactions of the British Mycological

Society. London 91:183. 1988. On deald stems of Obione sp. (=Alriplex), Chenopodiaccae. France.

ohiones P.A. Saccardo var. evolutior W.B. Grove, Journal of Botany, British and Foreign, Londion 71:281-282.1933. On dead stems of Obione pormlacoides (L.) Moq., Chenopodiaceae. Great Britain.

obusispora C.L. Spegazzini, Anales de la Sociedid Cientifica Argentina, Buenos Aires 12:179.

1881. EParaphaeosphaeria oblusispora (C.L. Spegazzini) O. Eriksson. Arkiv för Botanik. Uppsala, Stockholm, Series 2, 6:406. 1967. On decaying leaves of Yuc'a gloriosa L., Agavaceae. Argentina.

ohtusispora C.L. Spegazzini forma agaves J.J.

Barthelet, Annales des Epiphyties, Paris. New

Series 8:118. 1942. On leaves of Agure ferox C. Koch, Agave striara Zucc. Agavaceae. France.

occidentalis J.B. Ellis \& B.M. Everhart, Erythea. A Journal of Botany, Berkeley 2:20. 1894. $\equiv$ Phacosphaeria occidentalis (J.B. Ellis \& B.M. Everhart) R.A. Shoemaker, Canadian Joumal of Botany. Ottawa 67:1572. 1989. On dead culms of Panicum crus-galli L., Gramineae. U.S.A.

acculta J. Lind, Danish Fungi as Represented in the Herbarium of E. Rosirup, Nordisk Forlag, Copenhagen, p. 218. 1913. EPhaeosphaeria occulsu (J. lind) A. Leuchimann, Sydowia. Annales Mycologici, Hom, N.Ö. 37:128. 1984. On leaves of Carex hira L., Cyperaceae. Denmark.

ocellata G. Nicssl v. Mayendorf in J. Kunzc, Fungi Selecti Exsiccati, Century 4, No. 334. Anno 1880. Nom. nud. Art. 32.1. 三Molasphueria ocellana (G. Niessl v. Mayendorf) ex P.A. Saccardo, Sylloge Fungorum Onnium llucusque Cognitorum Digessit P'.A. Saccardo) 2:157. 1883. ETrichometasphaseria ocellowa (G. Niessl v. Mayendorf ex P.A. Saccardo) L. Holm. Symbolac Botanicae Upsaliconses
14(3): 143. 1957. On dry stems of Hypericum perforutum I... Guttiferae. Sw it/erland.

wimicole S.R. Naphade, Sydowia. Annales

Mycologici, Horn, N.Ö. 24(1-6):242. (1970)

1971. On dead stems of Ocimum sanc $13 m \mathrm{~L}$., Labialate. India.

жopluragmia G.B. Traverso \& R. González Fragoso in G.B. Traverso, Bollettino Della Societá

Botanica Italiana. Firen 2 1915:23. 1915. On dry stems of Alosisia cirriodoro Ort. ex Pers., Verhenacese. Spain.

ocroplwagmia G.B. Traverso \& R. González Fragoso var. major W.B. Grove, Journal of Botany. British and Foreign, London 71:282. 1933. On very old thick dead stem of Lippia (Aloysia) citriodora Kunth. Verbenaceac. Great Britain.

ocfosepuru L.E. Wehmeyer, Lloydia: a quarterly journal of biological science, Manasla 9:239-240. 1946. 三Nodulosphaeria octoseptala (L.E. Wehmeyer) L. Holnt, Symbolac Botanicae Upsalienses, Uppsala 14(3):91. 1957. On stems of Senecio crassulus A. Gray, Compositae. U.S.A. (Wyoming). odora (M.C. Cooke \& H.W. Harkness) A.N. Berlese \& P. Voglino, Sylloge Fungorum Omnium Hucusque Cognitorum Digessit P.A. Saccardo Additamenta Ad Volumina I-IV, p. 137. 1886. ESphueria (Leptosphueria) odora M.C Cooke \& W.H. Harkness, Grevillea, London 13:19. 1885. On branches of Umbellularia sp., Lauraceae. U.S.A. (California).

ugilvicusis (M.J. Berkeley \& C.E. Broome) V. Cesati \& G. de Notaris, Commentario della Societả Crittogamologica ltaliana, Milan 1:235. 1863. ESplacieria (Caulicolae) ogilivensis M.J.

Berkeley \& C.E. Broome, Annals and Magazine of Natural History. London. Series 2. 9:379. 1852. On dead stents of Senecio jacobaea L., Compositae. Canada, Germany. Great Britain, ltaly, Switzerland, U.S.A.

ogilviensis M.J. Berkeley \& C.E. Broome forma achilleae H. Relm, Hedwigia, Dresden 39:(193). 1900. Nom. nud. Art. 32.1. Ascomyceten, Fascicle 27, No. 1336. Anno 1900. Nom, nud. Art. 32.1. On stems of Achillea millefolium auct., Compositae. Germany.

ogilviensis (M.J. Berkeley \& C.E. Broome) V. Cesatti \& G. de Notaris forma lepidii F. Fautrey in C. Roumeguc̀re, Fungi Selecti Gallici Exsiccati. Century 62, No.6138. Anno 1892; Revue Mycologique, Toulouse 14:172. 1892. On capsules of Lepidium compestre (L.) R. Br., Cruciferae. France.

ogilviensis (M.J. Berkeley \& C.E. Broome) V. Cesati \& G. de Notaris forma megalospora $\mathrm{F}$. Fautrey in C. Rouneguère. Fungi Selecti Gallici Exsiccati. Century 61, No. 6035. Anno 1892; Revue Mycologique, Toulouse 14:107. 
1892. On stems of Solidago virgaurea L.. Compositae. France

ogilviensis (M.J. Berkeley \& C.E. Broome) V. Cesati \& G. de Notaris forma myrhis-odorata F. Fautrey in C. Roumeguère, Fungi Selecti Gallici Exsiccati, Century 56, No. 5540. Anno 1891: Revue Mycologique. Toulouse 13:8. 1891. On stems of Myrhis odorala (L.) Scop., Umbelliferae. France.

ogilviensis (M.J. Berkeley \& C.E. Broome) V. Cesati \& G. de Notaris var. plewrospermi O.A. Ade. Allgemeine botanische Zeitschrift f. Systematik, Floristik, Pllanzengeographie, etc.. Karlsruhe 30/31:22(134). 1926. On dead stems of Pleurospermum austriacum (L.) Hoffm., Umbelliferae. Germany.

ogilviensis (M.J. Berkeley \& C.E. Broome) V. Cesati \& G. de Notaris var. senecionis-cordati C.B. Massalongo, Malpighia. Rassegna Mensuale di Botanica. Messina, Genova 8:194. 1894: Nuova Conıribuzione alla Micologia Veronese Genova (Tipografia di Angelo Ciminago), p. 37. 1894. On stems of Senecio cordatus Koch. Compositae. Italy.

dericola (M.J. Berkeley \& M.A. Curtis) P.A. Saccardo, Sylloge Fungorum Omnium Hucusque Cognitorum Digessit P.A. Saccardo 2:21. 1883. ESphaeria olericola M.J.

Berkeley \& M.A. Curtis in M.J. Berkeley, Grevillea, London 4:150. 1876. On stems of Brassica sp. Cruciferae. U.S.A.

oligospora (E.A. Vainio) P.A. Saccardo \& D. Saccardo, Sylloge Fungorum Omnium Hucusque Cognitorum Digessit P.A. Saccardo 17:730. 1905. EXenosphaeria oligospora E.A. Vainio, Meddelanden af Societas pro Fauna et Flora Fennica, Helsingfors 10:203. 1883. On thalli of Solorina crocea (L.) Ach., Lichenes (Peltigeraceae). Finland.

oligotheca F. Petrak \& H. Sydow, Annales Mycologici, Berlin 22:359. 1924. [Described from the type material of Laestadia potentillae $\mathrm{E}$. Rostrup, Botanisk Tidsskrift, Kjobenhavn 25:300. 1903.] On leaves of Potemilla maculata Pour. (=Potentilla anrea L.). Rosaceae. Denmark, Iceland.

olivacea J.B. Ellis, Bulletin of the Torrey Botanical Club (and Torreya), New York 10:53. 1883. $\equiv$ Ophiobolus olivaceus (J.B. Ellis) A.N. Berlese \& P. Voglino, Sylloge Fungorum Omnium Hucusque Cognitorum Digessit P.A. Saccardo Additamenta Ad Volumina 1-1V,p. 186. 1886. 三Nodulosphaeria olivacea (J.B. Ellis) L. Holm, Svensk Botanisk Tidskrift. Stockholm 55:75. 1961. On deid herhaceous stems of unknown host, unknown family. U.S.A. (Utah).

olivaspora (M.J. Berkeley \& M.A. Curtis) P.A. Saccardo, Sylloge Fungorum Omnium Hucusque Cognitorum Digessit P.A. Saccardo 2:85-86. 1883. ESphaeria divaespora M.J.
Berkeley \& .M.A. Curtis in M.J. Berkeley. Grevillea, London 4:148. 1876. On branches of Cormus forida L., Comaceae. U.S.A. onagrae H. Rehm. Annales Mycologici. Berlin 1 1:398. 1913. On dead sems of Onagra sirigosa Rydb.. Onagraceae. U.S.A.

onobrychidicola L. Hollós. Magyar Botanikai Lapok, Budapest (Ungarische Botanische Blatter) 28:50. (1929) 1930. On dried stems of Onobrychis sativa Link. Leguminosae. Hungary.

onobrychidis L. Hollós, Magyar Botanikai Lapok. Budapest (Ungarische Botanische Blatter) 28:50. (1929) 1930. On dry stems of Onobrychis sativa Link. Leguminosae. Hungary.

ophioboloides P.A. Saccardo, Atti del Istituto Veneto di Scienze. Lettere ed Ani. Venezia. Series 6, 2:457. 1884. 三Leptosphaeriopsis ophioboloides (P.A. Saccardo) A.N. Berlese. Icones Fungorum Omnium Hucusque Cognitorum ad usum Sylloges Saccardianae Adcommodatae 1:89. 1892. EOphiobolus ophioboloides (P.A. Saccardo) L. Holm. Svensk Botanisk Tidskrift. Stockholm 42:345. 1948. On stems of Tragopogon sp.. Compositae. Switzerland.

ophiopogonis (P.A. Saccardo) P.A. Saccardo. Nuovo Giomale Botanico Ltaliano e Bolletino della Società Botanica Italiana. Firenze 7:321. 1875. ESphaerella ophiopogonis P.A. Saccardo, Atti dell ${ }^{\circ}$ Accademia Scientifica Veneto-Trentino-1striana, Padova 2(2): 142-143. 1873. On fallen decaying leaves of Opliopogon juponicus Ker-Gawl. Liliaceae. Italy.

ophiopogomis (P.A. Saccardo) P.A. Saccardo var. graminum P.A. Saccardo, Sylloge Fungorum Omnium Hucusque Cognitorum Digessit P.A. Saccardo 2:68. 1883. On rotten leaves of unknown host. Gramineae. France.

opizii T. Nitschke in J. Schröter. Kryptogamen-Flora von Schlesien. Im Namen Der Schlesischen Gesellschalt für vaterländische Culuur herausgegeben von Prof. Dr. Ferdinand Cohn. Secretair der Botanischen Section, Breslau 3(2):358-359. 1894. On dead stems of Solanum dulcumara L., Solanaceae. Poland. opumtice B.O. Dodge. Mycologia. Lancaster. Pennsyluania 29:708. 1937. On stems of Opunta lindheimeri Engelm.. Cactaceae. U.S.A. (Texas).

orac-maris D.H. Linder. Farlowia. A Joumal of Cryptogamic Botany, Camhridge, Massachusetts 1:413. 1944. On driliwood of unknown host. U.S.A. (Califormia).

orindearmm P.A. Karsten. lledwigia. Dresden

26:125. 1887. On dead stems of Gymnadenia comopsea (L.) R. Br., Orchidaceac. Finland oreophila P.A. Saccardo. Michelia Conmentarium Mycologicum Fungos in Primis ltalicos 
Illustrans 1:120.1878. On dead stems of Tofieldia calyculata (L.) Wahlenb., Liliaceae. Austriat.

oreophiloides P.A. Saccardo \& O. Pencig in P.A. Saccardo. Michelia Commentarium Mycologicum Fungos in Primis ltalicos lllustrans 2:599-600. 1882. On dead stems of Laserpitium angustifolium Georgi. Umbellilerae. France.

oreophiloides P.A. Saccardo subsp. scroph/mulariac P.A. Karsten, Hedwigia, Dresden 22:178. 1883: Acta Societatis pro Fauna et Flora Fennica, Helsingforsiae 2(6):51 ( 1884 ) 1885. On dead stems of Scrophularia nodosa L.. Scrophulariaceae. Finland.

ornithogali S.A. Gucevicz, Novosti Sistematiki Nizshikh Rastenij. Novitates Systematicae Plantarum Non Vascularium 4:209-2 10. 1967. On dead stems of Ornichogahm pyenaicum L., Liliaceae. U.S.S.R. (Crimea). orthogramma (M.J. Berkeley \& M.A. Curtis) P.A. Saccardo, Sylloge Fungorum Omnium Hucusque Cognitorum Digessit P.A. Saccardo 2:60. 1883. ESphaeria orthogramma M.J. Berkeley \& M.A. Curtis, Grevillea, London 4:144-145. 1876 [as orthogrammi]. On stems of Erianthus alopecuroides (L.) Ell., Gramineae. U.S.A. (Pennsylvania, South Carolina).

orthrosanthi E. Müller in E. Müller and R.W.G.

Dennis, Kew Bulletin. Royal Botanic Gardens, Kew 19:373-375, 384. 1965 [as orthosamihi]. On leaves of Orhtroscanthus chimboracensis Baker (=Orhrosanhus). Iridaceae. Venezuela.

oryzae A. Cattaneo, Atti dell’ Istituto Botanico della Universiı̀ e Laboratorio Crittogamica di Pavia, Milano 2 \& 3:127. 1879. 三Metasphaeria oryzac (A. Cattaneo) P.A. Saccardo, Sylloge Fungorum Omnium Hucusque Cognitorum Digessit P.A. Saccardo 2:180. 1883. On culms and leaves of Orya sariva L.. Gramineae. China. Italy.

oryzicolo K. Hara. A Monograph of Rice Diseases. p. 113. 1959 [as oryzaecola]. On living plant of Oryza sativa L., Gramineae. Japan.

(Leprosphaerella) oryzina P.A. Saccardo, Atti dell' Accademia Scientificá Veneto-TrentinoIstriana, Padova 10:67-68. 1919. On dead glumes of Oryza sariva L., Gramineae. Philippines.

(Clypeosphaeria) osculanda (C.G.T. Prcuss) M.C. Cooke, Grevillea, London 17:91. 1889. ESphaeria osculanda C.G.T. Preuss. Linnaca. Institut Botanique de l'Université de Genève 26:713. 1853. EClypeosphacria asculanda (C.G.T. Preuss) P.A. Saccardo. Sylloge Fungorum Omnium Hucusque Cognitorum Digessit P.A. Saccardo 2:91. 1883. On branches of Rubus sp., Rosaceac. Germany. oubanguiensis A.M. Saccas, Étude de la Flore Cryptogamique des Cafćicrs en Afrique
Centrale. Bulletin de Institut Français du Café du Cacio et D'Autres Plantes Stimulantes (Bulletin IFCC No. 16), pp. 257-259. 1981. Nom. inval. Art. 37.1. On dead stems of Coffea robusia L. Linden (= Coffea canephora Pierre ex Frochn.), Rubiaceac. Central African Republic.

owanica K. Kalchbrenner \& M.C. Cooke, Sylloge Fungorum Omnium Hucusque Cognitorum Digessit P.A. Saccardo 2:33. 1883 [as Sphaeria omaniae K. Kalchbrenner \& M.C. Cooke, Grevillea, London 9:29. 1880); falsely so cited in P.A. Saccardo]. On stems of Artemisia sp., Compositae. South Africa.

onviae E. Rostrup, Meddelelser om Gronland. Kjobenhavn 3:559. 1888. On dry stems of Oxyria digyna (1.) Hill, Polygonaceae. Greenland.

oxyspora J. Feltgen. Vorstudien zu einer Pilz-Flora des Grossherzogthums, Luxemburg 1(2):165-166. 1901. On dry stems of Rubus idaeus L., Rosaceae. Luxembourg.

pachyoasca G. Niessl v. Mayendorf, Hedwigia, Dresden 21:111. 1882. Non. illegit. Art. 64.1 [as pachyascus]. EMetasphaeria pachyasca (G. Niess] v. Mayendorf) P.A. Saccardo, Sylloge Fungorum Omnium Hucusque Cognitorum Digessit P.A. Saccardo 2:171. 1883. On dead leaves of Campanmla zoysii Wulfen, Campanulaceae. Yugoslavia. pachyasca G. Niessl v. Mayendorf, Öterreichische Botanische Zeitschrift, Wien 31:345-346.

1881 [as pachyascus]. 三Mctasphaeria pachyasca (G. Niess) v. Mayendorf) P.A. Saccardo, Sylloge Fungorum Omnium Hucusque Cognitorum Digessit P.A. Saccardo 2:171-172. 1883. ESphacruhina pachyasca (G. Niess] v. Mayendorf) A.N. Berlese, lcones Fungorum Omnium Hucusque Cognitorum ad usum Sylloges Saccardianae Adcommodatae 1:126. 1894. EPseudosphaeria pachyasca (G. Niessl v. Mayendorf) F. v. Höhnel,

Sitzungsberichte der Akademie der Wissenschalten in Wien, Mathematisch-naturwissenschaftliche Klasse, Abt. I, 1 16:635. 1907. $\equiv$ Saccolhecium pochyascum (G. Niessl v. Mayendorf) W. Kirschstein, Annales Mycologici, Berlin 37:105. 1939. \$Wettstoinina pachlyasca (G. Niessl v. Mayendorf) F. Petrak, Sydowia. Annales Mycologici, Iorn. N.Ö. 1:56. 1947. [Combination not actually made!] On dead or overwintered leaves, on leaves of Androsace lactea L., Campanuka assii Wullen, Primulaceac, Campanulaceate. Yugoslavia.

pachycapua P.A. Saccardo \& E. Marchal, Revuc Mycologique, Toulouse 7:145. 1885. On culm of grass of unknown host, Gramineac. Unknown country.

pachyoheca P.A. Saccardo. Nuovo Giomale Botanico Italiano e Bolletino della Società Botanica Italiana, Firenze, New Series 22:39, 1915. 
Nom. illegit. Ant 64.1. ETrenatosphaeria paclycarpa (P.A. Saccardo \& E. Marchal) R.A. Shoemaker, Canadian Journal of Botany, Ottawa 67:1595. 1989. On dead branches of Amygdalus communis L. [=Prunus dulcis (Mill.) D.A. Webb], Rosaceae. Yugoslavia. pachytheca P.A. Hariot \& P.A. Briard, Revue Mycologique, Toulouse 12:178. 1890. On dry culms and leaves of Nardis stricta L., Gramineae. France.

pacifica $\mathbf{H}$. Rehm, Annales Mycologici, Berlin 9:364. 1911. On leaves of Yucca sp. Ias Yucca colusplei], Agavaceae. U.S.A.

paludosa J. Feltgen, Vorstudien zu einer Pilz-Flora des Grossherzogthums, Luxemburg 1(2):157-158. 1901. On fallen leaves of Carex vesicaria L., Cyperaceae. Luxembourg.

palustris E. Müller, Sydowia. Annales Mycologici, Horn, N.Ö. 4(1-6):207. 1950. 三Massariosphaeria palustris (E. Müller) A. Leuchtmann, Sydowia. Annales Mycologici, Hom, N.Ö. 37:171. 1984. On dead stems of Typha latifolia L., Typhaceae. Switzerland.

palustris J. Schröter, Sylloge Fungorum Omnium Hucusque Cognitorum Digessit P.A. Saccardo 15:191. 1901. [Falsely so cited in P.A.

Saccardo; for Leptospora palustris J. Schröter in F.J. Cohn, Jahresbericht der Schlesischen Gesellschaft für vaterländische Kultur, Breslau 6I:179. 1884.] 三Ophiobolus palustris (J. Schröter) A.N. Berlese \& P. Voglino, Sylloge Fungorum Omnium Hucusque Cognitorum Digessit P.A. Saccardo Additamenta Ad Volumina I-IV, p. 190. 1886. pampaniniana P.A. Saccardo, Annales Mycologici, Berlin 1 1:566. 1913. On decayed leaves of Stipa tenacissina L., Gramineae. Libya. pampini (P.A. Saccardo) P.A. Saccardo, Sylloge Fungorum Omnium Hucusque Cognitorum Digessit P.A. Saccardo 15:191. 1901. ESphaerella (Leptosphacria) pampini P.A. Saccardo, Revue Mycologique, Toulouse 4:1. 1882. 三Metasphacria pampini (P.A.

Saccardo) P.A. Saccardo, Sylloge Fungorum Onnium Hucusque Cognitorum

Digessit P.A. Saccardo 2:164. 1883 [as pampincal. On vines of Vitis sp., Vitaceae. France.

pampini (F. v. Thümen) P.A. Saccardo, Sylloge Fungorum Omnium Hucusque Cognitorum Digessit P.A. Saccardo 2:3 I. 1883. झSphaerella pampini F. v. Thümen, Die Pilze des Weinstockes, p. 27. 1878. On wilted, living runners ol Vitis vinifera L., Vitaceac. Italy.

pandani F. Tassi, Bollettino del R. Orto Botanico, Siena 6:125. 1904. On dry leaves and sheaths of Pandanus utilis Bory. Pandanaceac. Italy. pandanicola K. Sawada, Descriptive Catalogue of Taiwan (Formosan) Fungi, Part XI (Special Publication of the National Tawan University
Taipaei No. 8). pp. 71-72. 1959. Nom. inval. Art. 36.1. On leaves of Pandanus odoratissimus Nor. (=Pandanus lacvis Lowr.). Pandanaceae. Taiwan.

panici H. Sydow, Leaflet of Philippine Botany, Manila 9:3121-3122. 1925. EPhacosphaeria panici (H. Sydow) R.A. Shoemaker, Canadian Joumal of Botany, Ottawa 67:1535. 1989. On dead leaves of Panicum palmaefolium Koen.. Gramineae. Philippines.

paollıccii M. Curzi, Atti dell' Istituto Botanico della Università e Laboratorio Crittogamica di Pavia, Milano, Series 3, 3:204. 1927. In spots on upper surface and margins of leaves of Laurus nobilis L., Lauraceae. Italy.

papaveris E. Rostrup, Botanisk Tidsskrift, Kjøbenhavn 25:305-306. 1930. On stems of Papaver radicalum Rottb., Papaveraceae. Jceland.

papillata (H.F. Bonorden) P.A. Saccardo \& G.B. Traverso, Sylloge Fungorum Omnium Hucusque Cognitorum Digessit P.A. Saccardo 19:1109. 1910. 三Myriocarpium papillantm H.F. Bonorden, Abhandlungen auf dem Gebiete der Mykologie. Halle 1:154-155. 1864. On branchlets of unknown host. unknown family. Germany.

papillosa M. de Sousa da Camara. Agronomia Lusitana, Sacavém 13:127. 1951. On culms of Oryzopsis miliacea (L.) Bentham \& Hooker ex Ascherson \& Graebner, Gramineac. Portugal.

populosa (M.C. Durieu de Maisonneuve \& J.P. Montagne) P.A. Saccardo. Nuovo Giomale Botanico Jtaliano e Bolletino della Società Botanica ltaliana. Firenze 7:313. 1875. ESphacria papulosa M.C. Durieu de Maisonneuve \& J.P. Montagne in M.C. Durieu de Maisonneuve, Exploration Scientifique de L'Algérie Publiée Par Ordre du Gouvernement. Series Naturelles. Botanique I. pp. 536-537. 1868. EMctasphaeria papulosa (M.C. Durieu de Maisonneuve \& J.P. Montagne) P.A. Saccardo, Sylloge Fungorum Omnium Hucusque Cognitorum Digessit P.A. Saccardo 2:168-169. IS\$3. On somewhat rotten leaves of Cirrus arrantium L., Hedera helix L., Smilax maurinanica Poiret, Rutaceae. Araliaceae, Liliaceae. Algeria.

paprri P.A. Saccardo \&. P. Sydow in P.A. Saccardo. Sylloge Fungorum Onnium Hucusque Cognitorum Digessit P.A. Saccardo 16:514. 1902. ELeprosplacria papvicola F. Tassi. Nec J.B. Ellis \& B.M. Everhart. On dry peduncles of Cyperus papyrus L.. Cyperaceae. China.

papricola F. Tassi. Bolletino del R. Orto Botanico. Siena 2:171. 1899. Nom. illegit. Art. 64.1. $\equiv$ Leprosphacria papyri P.A. Saccardo of P. Sydow.

papyricola J.B. Ellis \& B.M. Everhar, Proceedings of the Academy of Natural Sciences of 
Philadelphia 1894:337. 1894. On old pasteboard lying by roadside. U.S.A. papyricola L. Vouaux in M. Bouly de Lesdain, Recherches sur les Lichens des Environs de Dunkerque. p. 73. 1912. Nom, illegit. Art. 64. I. On cardboard. France. paraguariensis A. Maublanc, Bulletin de la Société Mycologique de France, Paris 36:35. 1920. On leaves of llex paraguariensis A. St. Hil., Aquifoliaceae. Brazil.

parietariue P.A. Saccardo, Alti dell Accademia Scientifica Veneto-Trentino-Istriana, Padova 2(2):154. 1873. On rotten stems of Parielaria officinalis L., Urticaceae. Italy.

parietariae P.A. Saccardo forma lamii P.A. Saccardo, Fungi Selecti Gallici Exsiccati. Century 72, No. 7138. Anno 1897: Revue Mycologique. Toulouse 19:149-150. 1897. On dry stems of Lamium album L., Labiatae. France?

parmeliarım (W. Phillips \& C.B. Plowright) P.A. Saccardo. Sylloge Fungorum Omnium Hucusque Cognitorum Digessit P.A. Saccardo 2:83. 1883. ESphaeria parmeliarum W. Phillips \& C.B. Plowright. Grevillea. London 4:124. 1876. On unknown substrate of Parmelia saratilis (L.) Arch., Lichenes (Parmeliaceae). Great Britain.

parıla G. Niessl v. Mayendorf, Verhandlungen des Naturforschenden Vereins in Brünn 10:173-174. (1871) 1872; Hedwigia, Dresden 12:119.1873. IPhaeosphaeria parvula (G. Niessl v. Mayendorf) A. Leuchtmann. Sydowia. Annales Mycologici, Horn, N.Ö. 37:109. 1984. On wilting leaves of / $\mathrm{ris}$ psendacorus L., Iridaceae. Austria, Czechoslovakia, Switzerland.

parula G. Niessl v. Mayendorf var. iridis-germanicae C.B. Massalongo ex C.B. Massalongo, Madona Verona. (Verona. Museo Civico) 2(1):38. 1908. On leaves of /ris germanica L., Iridaceae. Italy.

parula G. NiessI v. Mayendorf var. iridis-germamirae C.B. Massalongo. Novitates Florae Mycologicae Veronensis (Fungi Potissimum in Valle Tremniacensi Detecti) Cum 10 Tabulis Coloratis, Verona, p. 65. 1902. Nom. inval. Art. 33.1. On leaves of Iris germanica L., Iridaceae. Italy.

passeriniana P.A. Saccardo, Sylloge Fungorum Omnium Hucusque Cognitorum Digessit P.A. Saccardo 9:785. 1891. ELeplosphaeria asparagi G. Passerini. Non C.H. Peck. On dry stems of Asparagus officinalis L.. Liliaceae. Italy.

passerinii P.A. Saccardo, Mıchelia Commentarium Mycologicum Fungos in Primis Italicos Illustrans 1:37-38. 1877. On stems of Scabiosa columbaria L., Dipsacaceae. Italy. passerinii P'A. Saccardo, Sylloge Fungorum Omnium Hucusque Cognitorum Digessit P.A.
Saccardo 2:53. 1883. Nom. illegit. An. 64.1. $\equiv$ Leptosphaeria marginalis G. Passerini. On wilted leaves of Achillea prarmica L..

Compositae. Italy.

patellaeformis G. Passerini. Atti dell' Accademia Nazionale dei Lincei, Rendiconti, Roma, Series 4, 4(2):59. 1888. On wilted culms of Zea mays L., Gramineae. Italy.

pancispora A.B. Cribh \& J.W. Cribb, Papers from the Department of Botany, University of Queensland, Brisbane 4(3):41-44. 1960. On wood of unknown host, unknown family. Australia (Queensland).

pelagica E.B. Gareth Jones, Transactions of the British Mycological Society, London 45:105. 1962. On dying culms of Spartina sp., Gramineae. Great Britain.

pelargonii H. Rehm, Hedwigia, Dresden 44:5. 1904 las pelageriniil. On leaves of cultivated Pelargonium sp., Geraniaceae. Brazil.

pellita (F. Currey) P.A. Saccardo. Michelia Commentarium Mycologicum Fungos in Primis Italicos Illustrans 2:63. 1880 [as Leptosphaeria pellita G.L. Rabenhorst \& K. Kalchbrenner]. ESphaeria pellita F. Currey. Transactions of the Linnean Society of London 22:331. 1859. Nec Sphaeria pellita E.M. Fries:E.M. Fries. Nec Pleospora pellita (E.M. Fries:E.M. Fries) E.L.R. Tulasne. On various stems of unknown host, unknown family. Unknown country.

peltigerarum $\mathrm{F}$. Amold, Verhandlungen der Zoologisch-Botanischen Gesellschaft in Wien 28:271. 1878. EOphiobolus pelligerarum ( $\mathrm{F}$. Amold) A.N. Berlese \& P. Voglino, Sylloge Fungorum Omnium Hucusque Cognitorum Digessit P.A. Saccardo Additamenta Ad Volumina I-IV, p. 189. 1886. On thallus of Pelrigera aphthosa (L.) Willd. forma complicata Th. Fries, Lichenes (Peltigeraceae). Italy.

peligerea (G.K. Merrill) ex L. Vouaux, Bulletin de la Société Mycologique de France, Paris 29:119-120. 1913. ETryperhelium (Melanotheca) peligereum G.K. Merrill, Lichenes Exsiccati, Series I, Fascicle 4, No. 85. Anno 1910. Nom. inval. Art. 32.1. On thallus of Peltigera sp.. Lichenes (Peltigeraceae).

Jamaica.

penicillus (E.M. Fries:E.M. Fries) P.A. Saccardo. Atti dell' Accademia Scientifica VenetoTrentino-Istriana, Padova 2(2):258. 1873. $=$ Sphacria penicillus Schmidt, unpublished. =Sphoeria penicillus E.M. Fries:E.M. Fries, Systema Mycologicum Sistens Fungorum 2:508. 1823. ERhaphidospora penicillus (E.M. Fries:E.M. Fries) V. Cesati \& G. de Notaris, Commentario della Società Crittogamologica Italiana, Milan 1:233. 1863. EPyenophora penicillus (E.M. Fries:E.M 
Fries) P.A. Saccardo, Sylloge Fungorum Omnium Hucusque Cognitorum Digessit P.A. Saccardo 2:282. 1883 [as (Schmidt)]. $\equiv$ Pleospora penicillus (E.M. Fries:E.M. Fries) L. Fuckel, Symbolae Mycologicae Dritter Nachtrag, pp. 23-24. 1873. On stem of Peucedanum cervaria (L.) Lapeyr., Umbelliferae. Austria, Germany.

penicillus P.A. Saccardo. Nuovo Giornale Botanico Italiano e Bolletino della Società Botanica Italiana, Firenze 7:316. 1875. Non (E.M. Fries:E.M. Fries) P.A. Saccardo. 1873. Nom. illegit. Art. 64.1. On stems of Pencedanum cervaria (L.) Lapeyr., Umbelliferae. Italy.

penniseti C.G. Hansford, Proceedings of the Linnean Society of London 153:24. 1941. On dead culms of Pennisetum purpureum Schum., Gramineae. Uganda.

pemniseticola F.C. Deighton, Sydowia. Annales Mycologici, Horn. N.Ö. 6(1-4):311. 1952. On living leaves of Pennisetum purpureum Schum., Gramineae. Sierra Leone.

perforans (M.R. Roberge \& J. Desmazières) B. Auerswald in M. Marcucci, Unio Itineraria Cryptogama Exsiccata No. XXXIII. Anno 1866. ESphaeria perforans M.R. Roberge \& J. Desmazières in J. Desmazières, Plantes Cryptogames Du Nord De La France, Edition 1, Fascicle 26, No. 1288. Anno 1843; Annales des Sciences Naturelles. Paris, Botanique, Series 2, No. 19, p. 357. 1843. ESphaerella perforats (M.R. Roberge \& J. Desmazières) V. Cesati \& G. de Notaris, Commentario della Società Crittogamologica Italiana, Milan 1:238. 1863. On leaves of Ammophila arenaria (L.) Link, Gramineae. France, ltaly.

periclymeni C.A. Oudemans, Nederlandsch kruidkundig Archief. Verslagen en Mededeelingen der Nederlandsche Botanische Vereeniging, Leiden, Series 2, 5:168. 1888. On branches of Lonicera periclymenum L., Caprifoliaceae. Netherlands.

periclymeni C.A. Oudemans var. tatarica A.A. Potebnia, Annales Mycologici, Berlin 5:14. 1907. On living twigs of Lonicera tatarica L., Caprifoliaceae. U.S.S.R.

perplexa P.A. Saccardo \& C.E. Fairman in P.A.

Saccardo, Journal of Mycology, Columbus,

Ohio 12:49. 1906. On dead stems ol Solidago sp.. Compositae. U.S.A.

perpusilla (J. Desmazières) P.A. Karsten, Mycologia Fennica Pars 2, Pyrenomycetes, p. 99. 1873. ESphaeria (folliicola) perpusilla J. Desmazières, Anniles des Science Naturelles, Paris, Botanique, Series 3, 6:80. 1846. ELaestadia perpusilla (J. Desmazières) P.A. Saccardo, Sylloge Fungorum Omnium Hucusque Cognitorum Digessit P.A. Saccardo 1:423. 1882. On stems of Phragmites communis Trin., Grannineac. Belgium, France, Great Britain, Italy. perpusilla (J. Desmazières) P.A. Karsten var. typhae P.A. Karsten, Mycologia Fennica Pars 2. Pyrenomycetes, p. 99.1873. 三Sphaeria perpusilla J. Desmazières var. typhae $\mathrm{B}$. Auerswald in G.L. Rabenhorst, Fungi Europaei Exsiccati, Klotzschii Herbarii vivi Mycologici Continuatio Edita Nova, Series Secunda. Century 9. No. 831. Anno 1865. Nom. nud. Art. 32.I. झLeptosphaeria trphae (P.A. Karsten) P.A. Saccardo, Nuovo Giomale Botanico Italiano e Bolletino della Società Botanica Italiana, Firenze 7:321. 1875. [P.A. Saccardo, Fungi Italici autographice delineati (additis nonnullis extra-italicis asterisco notatis), Patavii. Table 491. 1879; P.A. Saccardo, Sylloge Fungorum Omnium Hucusque Cognitorum Diggesit P.A. Saccardo 2:64. 1883.] On rotting culms of Typha latifolia L., Typhaceae. Finland.

personata G. Niessl v. Mayendorf in G.L. Rabenhorst, Fungi Europaei Exsiccati, Klotzschii Herbarii vivi Mycologici Continuatio Edita Nova, Series 2, Century 20, No. 1933. Anno 1875: Botanische Jahrbücher für Systematik. Pflanzengeschichte und Pflanzengeographie. Leipzig 3:262. (1875) 1877. EScleropleella personata (G. Niessl v. Mayendorf) F. v. Höhnel, Annales Mycologici. Berlin 16:158. 1918. $\equiv$ Mycotodea personata (G. NiessI v. Mayendorf) W. Kirschstein, in O.C. Schmidt. Kryptogamentlora der Mark Brandenburg und Angrenzender Gebiete herausgegeben von dem Botanishen Verein der Provinz Brandenburg 7(3):433. 1938. 三Leptosphaerulina personata (G. Niessl v. Mayendorf) M.E. Barr, Contributions from the Univerity of Michigan Herbarium, Ann Arbor 9:542. 1972. On dry stems of Aira cespitosa L.. Gramineae. Austria.

perniana C.L. Spegazzini. Anales de la Sociedad Cientifica Argentina. Buenos Aires 12:179. 188I; Fungi Argentini Pugillus t. No. 168. 1881. =Leptosphacria promontorii P.A. Saccardo. Nec M.C. Cooke.

petiolaris J. Feltgen, Vorstudien zu einer Pilz-Flora des Grossherzogthums, Luxemburg 1(3):216-217. 1903. On petioles of Juglans regia L.. Juglandaceae. Luxembourg.

petiolicola P.A. Saccardo. Atti dell Accademia Scientifica Veneto-Trentino-lstriana. Padova 2(2):153. 1873. On petioles of Robinic pseudacacia L., Leguninosae. Italy.

petkovicensis F. Bubák \& N. Ranojerié in N.

Ranojević, Annales Mycologici. Berlin 8:361. 1910. $\equiv$ Vetasphaeria petkovicensis $(\mathrm{F}$. Bubák \& N. Ranojević) W. Kirschstein. Annales Mycologici, Berlin 37:134. 1939. $\equiv$ Phacosphacria petkoricensis (F. Bubák of N. Ranojević) R.A. Shomaker, Canadian Journal of Botany, Ottawa 67:1508. 1989. On 
dry culms of Jincis effusus L., Juncaceae. Czechoslovakia, Germany, Great Britain, Switzerland. Yugoslavia.

petkovicensis F. Bubák \& N. Ranojević var. elymi L.E. Wehmeyer, Mycologia, Lancaster.

Pennsylvania 55:322. 1963. EPhaeosphacria elymi (L.E. Wehmeyer) R.A. Shoemaker,

Canadian Journal of Botany, Ottawa 67:1503.

1989. On stems of Blysmus compressus (L.)

Panzer ex Link (=Scirpus), Cyperaceae. India.

petrakii P.A. Saccardo in F. Petrak, Annales Mycologici, Berlin 12:287. 1914. On diseased culms of Luzula nemorosa (Pollich) E.H.F. Meyer, Juncaceae. Czechoslovakia.

perri J. Chevaugeon. Encyclopedie Mycologique, Paris 28:24. 1956. On leaves of Maniliot utilissima Pohl, Euphorbiaceae. Ivory Coast.

phacae E. Müller, Sydowia. Annales Mycologici, Horn, N.Ö. 5(3-6):49-50. 1951. On dead culms of Phaca alpina L. (=Astragalus), Leguminosae. Switzerland.

phaeospora E. Müller, Sydowia. Annales Mycologici, Horn, N.Ö. 4(1-6):208. 1950. =Massariosphaeria phaeospora (E. Müller) P.G. Crivelli, Ueber die Heterogene Ascomycetengattung Pleospora Rabh.; Vorschlag für Eine Aufteilung. Abhandlung zur Erlangung des Titels eines Doktors de Naturwissenchaften der Eidgenössischen Technischen Hochschule, Zürich, No. 7318:141. 1983. ETremarosphaeria phaeospora (E. Müller) L. Holm, Symbolae Botanicae Upsalienses, Uppsala 14(3): 165. 1957. EChaetomastia phaeospora (E. Müller) M.E. Barr, Mycotaxon. An International Joumal Designed to Expedite Publication of Research on Taxonomy \& Nomenclature of Fungi \& Lichens, Ithaca, New York 34:512. 1989. On dead stems of Artemisia campestris L. Compositie. Switzerland.

phaeosticta B. Auerswald in W. Gonnermann and G.L. Rabenhorst, Mycologia Europaea, Abbildungen Sämmtlicher Pilze Europa's, Hefte 5 und 6, Synopsis Pyrenomycetum Europaeorum, Table 11, Figure 154. 1869. Nom. nud. Art. 32.1. [Sphaeria phaensticta M.J. Berkeley is a facultative synonym, fide P.A. Saccardo, Michelia Commentarium Mycologicum Fungos in Primis Italicos Illustrans 1:374. 1878.] EAnthostomella phaensticta (M.J. Berkeley) P.A. Saccardo. Michelia Commentarium Mycologicum Fungos in Primis Italicos Illustrans 1:374. 1878. Europe.

phaseoli F. Fautrey \& C. Roumeguère in C. Roumeguère, Revue Mycologique, Toulouse 14:6. 1892; Fungi Selecti Gallici Exsiccati, Century 60. No. 5951. Anno 1892. On dry stems of Phaseolus vulgaris L.. Leguminosae. France. phaseolorum J.B. Ellis \& B.M. Everhart, American Naturalist, Lancaster, Pennsylvania 31:34 I-342. 1897. On old bean vines (stems) of Phaseolus vulgaris L., Leguminosae. U.S.A.

phiala (M.C. Durieu de Maisonneuve \& J.P. Montagne) P.A. Saccardo, Sylloge Fungorum Omnium Hucusque Cognitorum Digessit P.A. Saccardo 2:19. 1883. ESphaeria phiala M.C. Durieu de Maisonneuve \& J.P. Montagne in M.C. Durieu de Maisonneuve, Exploration Scientifique de L'Algérie Publiée Par Ordre du Gouvernement. Series Naturelles. Botanique 1:519. 1868. On cortex of branches of Genista ferox Poir., Leguminosae. Algeria.

phlogis C.A. Oudemans, Verslagen van de Gewone Vergadering der Wis-en Natuurkundige Afdeeling. K. Academie van Wetenschappen Te Amsterdam 9(2):141. 1900. On leaves of Phlox decussata Lyon. Polemoniaceae. Netherlands.

phoenicis S.T. Tilak, Mycopathologia et Mycologia Applicata, Den Haag 28:83-84. 1966. On dried petioles of Phoenix dactylifera L.,

Palmae. India (Aurangabad).

phoradendri L. Bonar, Proceedings of the California Academy of Sciences, San Francisco, Series 4, 22:198-199. 1939. On living leaves of Phoradendron rownsendii Trel., Viscaceae. Chile (Socorro Island).

phormicola M.C. Cooke \& H.W. Harkness, Grevillea, London 14:10. 1885. On unknown substrate of Phormium sp., Agavaceae.

U.S.A.

phormii W.B. Grove, Kew Bulletin. Royal Botanic Gardens, Kew 1921:150. 1921. On dead leaves of Phormium sp., Agavaceae. Great Britain (Scotland).

phragmiticola (P.L. Crouan \& H.M. Crouan) P.A. Saccardo, Sylloge Fungorum Omnium Hucusque Cognitorum Digessit P.A. Saccardo 2:87. 1883. $\equiv$ Pleospora phragmitecola P.L. Crouan \& H.M. Crouan, Florule Du Finistère Contenant Les Descriptions De 360 Espèces Nouvelles De Sporogames, De Nombreuses Observations et une synonymie de plantes Cellulaires et Vasculaires Qui Croissent Spontanément Dans ce Département, p. 23. 1867. On culms of Phragmites communis Trin., Gramineae. France.

phyllachoricola F. Petrak, Sydowia. Annales Mycologici, Horn, N.Ö. 2(1-6):337-338. 1948. On stroma of Phyllachora disseminata Syd.. Fungi (Phyllachoraceac). Ecuador. phyllachorivora F. Petrak. Annales Mycologici. Berlin 20:302-303. 1922. Parasitic on stroma of Phyllachora graminis (Pers.:Fr.) Fuckel. Fungi (Phyllachoraceae). Czechoslovakia.

(Metasphueria) phyllostachydis K. Hara, Botanical Magazine, Tokyo 27(317):250. 1913 las 
phllostachydis]. On unknown substrate of Phyllostachys hambusoides Sieb. \& Zucc. var. bambusoides [as Phyllostachys reticulata], Gramineae. Japan.

physalidis J.B. Ellis \& B.M. Everhart, Bulletin of the Torrey Botanical Club (and Torreya), New York 11:42. 1884. On dirty white round spots on leaves of Physalis puhescens L., Solanaceae. U.S.A.

physostegiae C.E. Fairman, Proceedings of the Rochester Academy of Science, Rochester, New York 4:216. 1906. On dead stems of Physostegia virginiana (L.) Benth., Labiatae. U.S.A.

phyteumatis (L. Fuckel) P.A. Saccardo, Sylloge Fungorum Omnium Hucusque Cognitorum Digessit P.A. Saccardo 2:37. 1883. $\equiv P / e-$ ospora (Nodulosphaeria) phyreumatis L. Fuckel, Fungi Rhenani Exsiccati A Leopoldo Fuckel Collecti, Fascicle XXV(X), No. 2439. Anno 1872; Symbolae Mycologicae Zweiter Nachtrag, p. 25. 1873. On dry stems of Phytema spicatum L., Campanulaceae. Switzerland.

plyteumatis (L. Fuckel) H.G. Winter forma knautiae J. Feltgen, Vorstudien zu einer Pilz-Flora des Grossherzogthums, Luxemburg 1(2):164. 1901. On unknown substrate of Kuatia arvensis (L.) Coulter, Dipsacaceae. Luxembourg.

phytolaccae F. Cavara, Revue Mycologique. Toulouse 11:181. 1889. On old stems of Phytolacca decandra L., Phytolaccaceae. Italy.

picastra (E.M. Fries:E.M. Fries) F. v. Höhnel. Mitteilungen des Botanischen Instituts der Technischen Hochschule, Wien 4:44. 1927. $\equiv$ Trematosphacria morthieri L. Fuckel, Symbolae Mycologicae Nachtrag 1:306. 1871. $\equiv$ Trematosphaeria picastra (E.M. Fries:E.M. Fries) L. Fuckel, Symbolae Mycologicae, p. 162. 1870. ESphaeria picastra E.M. Fries:E.M. Fries, Kungliga Svenska Vetenskapsakademiens Handlingar, Uppsala and Stockholm 38:260-261. 1817; E.M. Fries, Systema Mycologicum Sistens Fungorum $2: 463.1823$. On resin-hardened wood of Pinus syliestris L., Pinaceae. Sweden.

picridis F. Fautrey \& J.B. Lambotte, Revue Mycologique, Toulouse 16:75-76. 1894. On unknown substrate of Picris hierucioides L., Compositae. France.

pilulariac A. Ade. Kryptogramische Forschungen, München 2:26. 1929. On dead leaves of Pilularia sp., Marsileaceae. Gemany (Bavaria).

pimpinellac R. Lowen \& A. Sivanesan, Mycotavon. An International Journal Designed to Expedite Puhlication of Research on Taxomomy \& Nomenclature of Fungi \& Lichens, Ithaca, New York 35:205-209. 1989. On standing dead stems of Pimpinella anisum L.. Umbelliferae. Israel

pini (D. Cruchet) E. Müller, Sydowia. Annales Mycologici, Horn, N.Ö. 4( 1-6):277. 1950. EMetasphaeria pini D. Cruchet, Bulletin de la Société Vaudoise des Sciences Naturelles, Lausanne 55:167. 1923. On cones of Pinus sy/vestris L., Pinaceae. France. Switzerland.

pinnarum G. Passerini. Revue Mycologique.

Toulouse 2:34. 1880. झMelasphaeria pinnarum (G. Passerini) P.A. Saccardo, Sylloge Fungorum Omnium Hucusque Cognitorum Digessit P.A. Saccardo 2:179. 1883. On soft pinnae of Phoenix dactylifera L., Cyperaceae. Italy.

pinnarum G. Passerini var. rachidis G. Passerini. Revue Mycologique, Toulouse 2:34. 1880 [as pinnosum]. $\equiv$ Metasphaeria rachidis $(\mathrm{G}$. Passerini) P.A. Saccardo, Sylloge Fungorum Omnium Hucusque Cognitorum Digessit P.A. Saccardo 2:180. 1883. On pinnae of Phoenix dactrlifera L., Palmae. Italy.

piperis P.C. Hennings in H.G.A. Engler, Die Pflanzenwelt Ost-Afrikas und der Nachbargëbiete, Theil C. p. 33. 1895. On leaves of Piper capense L., Piperaceae. East Africa.

plagia (M.C. Cooke \& G.E. Massee) L. Holm. Svensk Botanisk Tidskrift. Stockholm 62:234. 1968. ECucurbitaria (Melanomma) plagia M.C. Cooke \& G.E. Massee. Grevillea. London 17:8. 1888. EGibberidea plagia (M.C. Cooke \& G.E. Massee) P.A. Saccardo. Sylloge Fungorum Omnium Hucusque Cognitorum Digessit P.A. Saccardo 9:820. 1891. On living twigs of Cassinia aculeara $\mathrm{R}$. Br., Compositae. Australia.

planiuscula (H. Riess) V. Cesati \& G. de Notaris. Commentario della Società Crittogamologica Italiana, Milan 1:236. 1863. ESphaeria planiuscula H. Riess, Hedwigia, Dresden 1. Table IV, Figure 7. 1854. Nom. nud. Art. 32.1; in G.L. Rabenhorst, Klotzschii Herbarium Vivum Mycologicum Sistens Fungorum Per Totam Germaniam Cresentium Collectionem Perfectam. Dresden. Edition I. Century 19. No. 1\$29. 1854. On dead stems of Solidago virganres L.. Compositae. Finland, Germany, Switzerland.

planiuscula (H. Riess) V. Cesati \& G. de Notaris formu cruciferarum H. Rehm. Hedwigia. Dresden 22:55-56. 1883. On stems of Cruciferae. Unknown country:

planiuscula V. Cesati \& G. de Notaris Iomua succisae J. Feltgen, Vorstudien zu einer PilzFlora des Crossherzogthums. Luvemburg 1(2):161. 1901. On unhnown subsirate of Succisa matensis Moench.. Dipsacaceae. Luxemhourg.

plantaginicola (N.T. Patouillard) F. Petrak, Sydowia. Annales Mycologici, Horn, N.Ö. $4(1-6): 496$. 
1950. ESphacrella? plantaginicola N.T. Patouillard in N.T. Patouillard and N.G. de Lagerheim. Bulletin de la Société Mycolo-

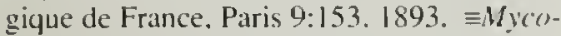
sphaerella plantaginicola (N.T. Patouillard) F.L. Stevens, Illinois Biological Monographs, University of Illinois at Urbana-Champaign 11:199-200. 1927. On leaves of Planiago sp.. Plantaginaceae. Ecuador.

platanicola (E.C. Howe) P.A. Saccardo, Sylloge Fungorum Omnium Hucusque Cognitorum Digessit P.A. Saccardo 2 (Addenda Ad Volumen Secundum), p. LVII. 1883. Esplacria platanicola E.C. Howe, Bullerin of the Torrey Botanical Club (and Torreya). New York 5:43. 1874. On branchlets of Platamus sp., Platanaceate. U.S.A. (New York).

platanicola F. Petrak, Sydowia. Annales Mycologici. Horn, N.Ö. 7(1-4):122. 1953. Nom. illegit. Art. 64.1. On dry stems of Platanus occidenfalis L., Plaranaceae. U.S.A.

plarycarpa P.A. Saccardo. Michelia Commentarium Mycologicum Fungos in Primis Italicos Illustrans 1:342-343. 1878. On branches of Cornus sanguinea L., Comaceae. Italy.

platychorae E. Müller, Sydowia. Annales Mycologici, Horn. N.Ö. 7(1-4):275-276. 1953. On stroma of Plarnchora ulmi (Schleich.:Fr.) Petrak, Fungi (Venturiaceae). Switzerland. platypus (L.D. v. Schweinitz) F. Petrak \& H. Sydow. Annales Mycologici, Berlin 21:349. 1923. Esphaeria platypus L.D. v. Schweinitz, Synopsis Fungorum in America Boreali Media Degentium. Secundum Observationes Ludovici Davidis de Schweinitz, p. 222. 1832. =Macrobasis plarypus (L.D. v. Schweinitz) K. Starback, Bihang till K. Svenska Vetenskapsakidemiens Handlingar. Stockholm 19(Ald. III, No. 2):97-98. 1894. On stems of Anemone virginiana L., Ranunculaceae. U.S.A. (Pennsylvanja).

plectrospora J. Feligen, Vorstudien zu einer PilzFlora des Grossherzogthums, Luxemburg 1(2):162-163. 1901. On dry stems of Galium mollugo L., Rubiaceae. Luxembourg.

plemeliana G. Niessl v. Mayendorf, Österreichische Botanische Zcitschrift, Wien 31:346. 1881; I ledwigia, Dresden 21:1 11-112. 1882. On leaves of Campamula zoysii Wulfen, Campanulaceae. Yugoslavia.

pleosporoides B. Auerswald in G.L. Rahenhorst, Fungi Europaei Exsiccati, Klotsschii Herharii vivi Mycologici Continuatio Edita Nova, Series 2, Century 13, No. 1253. Anno 1869. On runners of Clemaris vifalbo L.. Ranunculaceale. France.

pleurospora G. Niessl v. Mayendorf in H. Rehm. Ascomyccien, Fascicle 19. No. 937. Anno 1888: Hedwigia. Dresden 27:172. 1888. $\equiv$ Phaeosphacria plemenpora (G. Nicsslv.
Mayendor() A. Leuchimann, Sydowia. Annales Mycologici, Horn, N.Ö. 37:162. 1984. ESulcispura pleurnspora (G. Nicssl v. Mayendorf) R.A. Shoemaker, Canadian Journal of Botany, Ottawa 67:1594. 1989. On dry culms of Aira cespitosa L., Gramineac. Austria, Switzerland, U.S.S.R. (Latvia). plocamac F. Petrak, Beiblatt zu den Botanischen Jahrbiichern 62(No. 142), Heft 3:1 10-111. 1928. On decorticated wood of Plocama pendula W. Aiton, Rubiaceate. Canary lslands (Tenerife lsland).

plumbaginis N.T. Patouillard, Revue Mycologique, Toulouse 8:181. 1886. On dead stems of Plambago sp., Plumbaginaceae. China.

plurisepra (L.R. Tehon \& E.Y. Daniels) J.A. v. Arx \& E. Müller, Studies in Mycology. Baarn. Nerherlands 9:76. 1975. EExilispora plurisepıa L.R. Tehon \& E.Y. Daniels, Mycologia, Lancaster. Pennsylvania 19:113. 1927. On stems of Erigeron sp., Compositae. U.S.A.

poae G. Niessl v. Mayendorf in H. Rehm ex G. Niessl v. Mayendorf. Ascomyceten, Century 13. No. 643. Anno 1881. Nons. nud. Art. 32.1; Hedwigia, Dresden 21:83. 1882. $\equiv$ Mcrasphaeria poae (G. Niessl v. Mayendorf ex G. Niessl v. Mayendorf) P.A. Saccardo. Sylloge Fungorum Omnium Hucusque Cognitorum Digessit P.A. Saccardo 2:175 1883. On dry culms of Poa nemoralis L.. Gramineae. Czechoslovakia.

poar G. Niessl v. Mayendorf var. agrostidis F.A. Hazslinszky. Matematikai és Természettudomanyi Közlemenyek Vonatkozólag a Hazaj Viszonyokra, Budapest 25(2):131. 1892. On unknown substrate of Agrostis vulgaris With.. Gramineae. Hungary.

polaris P.A. Saccardo, Sylloge Fungorum Omnium Hucusque Cognitorum Digessit P.A. Saccardo 2:83. 1883. [Published as Sphacria No. 8 by T. Fries, Hedwigia, Dresden 20:59. 1881.] On thalli of Rhizocarpon geographicum (L.) DC.. Lichenes (Rhizocarpaceat). Greenland.

polini S.A. Gucevicz, Trudȳ Gosudarstvennogo Nikitskogo Botanicheskogo Sada, Yalta 29:189-190. 1959. On dried branches of Buddleja davidii Franch., Loganiaceae.

U.S.S.R. (Crimea).

poliris F. Petrak. Sydowia. Annales Mycologici, Horn, N.Ö. I0( 1-6):308-309. (1956) 1957. On dry stems of Asperula sp.. Rubiaceac. Greece.

polygonari E. Müller \& M. Tomaševič, Phytopathologische Zcitschritt. Berlin 29:289. 1957. On dry overwintered stems of Polygomarum sp.. Liliaceate. Switzerland.

polymichina A. Racovilza. Memoires du Múscum Nationale d'Histoire Naturelle, Paris, Series B. Botanique 10:150. 1959. On dead plant of 
Polytrichum juniperinum Willd. ex Hedw. var. alpinum Schimp., Musci (Polytrichaceae). France.

pomiformis (C.H. Persoon:E.M. Fries) V. Cesati \& G. de Notaris, Commentario della Società Crittogamologica Italiana, Milan 1:235. 1863. 三Sphaeria pomiformis C.H. Persoon:E.M. Fries, Synopsis Methodica Fungorum, p. 65. 1801: E.M. Fries, Systema Mycologicum Sistens Fungorum 2:455. 1823. On dry stems of unknown host, unknown family. Europe.

(Leptosphaerella) pomona P.A. Saccardo, Nuovo Giomale Botanico ltaliano e Bolletino della Società Botanica Italiana, Firenze 8:176. 1876. On upper surface of leaves of Pyrus malus L. (=Malus domestica Borkh.), Rosaceae. Italy.

pomona P.A. Saccardo forma transilvanica A. Negru et J. Ditzu, Notulae Systematicae e Sectione Cryptogamica Instituti Botanici Nomine V.L. Komarov Academicae Scientiarum U.R.S.S., Petropolis 16:152-153.1963. On fruits of Crataegus monogyna Jacq., Rosaceae. Romania.

pontiformis (L. Fuckel) P.A. Saccardo. Sylloge Fungorum Omnium Hucusque Cognitorum Digessit P.A. Saccardo 2:78. 1883. $\equiv P l e-$ ospora pontiformis L. Fuckel, Symbolae Mycologicae, p. 139. 1870. EPhaeosphaeria pontiformis (L. Fuckel) A. Leuchtmann, Sydowia. Annales Mycologici, Horn, N.Ö. 37:134. 1984. On culms and rhizomes of Cynodon sp., Secale sp., Triticum sp.. Gramineae. Czechoslovakia, Gennany, Great Britain, Hungary.

porellae M.J. Thirumalachar, Transactions of the British Mycological Society, London 31:11. 1948. On thallus of Porella sp.. Porellaceae. India (Mysore State).

portoricensis L.A. Alvarez Garcia, Joumal of Agriculture of the University of Puerto Rico. Rio Pedras 47(1):56. 1963. On leaves of Asparagus officinalis L.. Liliaceae. Puerto Rico.

poterii G. Passerini, Atti dell Accademia Nazionale dei Lincei. Memoire, Rome. Series 4, 6:459. (1889) 1890. On wilted stems of Poterium sanguisorba L., Rosaceae. Italy.

praeclara P.A. Karsten, Hedwigia. Dresden 23:2. 1884: Acta Societatis pro Fauna el Flora Fennica, Helsingforsiae 2(6):5ł. 1885. On dead stems of Asparagus officinalis $\mathrm{L}$.. Liliaceae. Finland.

proclura P.A. Karsten formal typhiseda (P.A. Saccardo \& A.N. Berlese) A.N. Berlese. Icones Fungorum Omnium Hucusque Cognitorum ad usum Sylloges Saccardianale Adcommodatac 1:75. 1892. ELepeosphicuerio typhiseda P.A. Saccardo \& A.N. Berlese.

maetermissa (P.A. Karsten) P.A. Saccardo, Sylloge Fungorum Onnium Hucusque Cogniorum
Digessit P.A. Saccardo 2:26. 1883. 三Sphaeria praetermissa P.A. Karsten, Fungi Fenniae Exsiccati, Century 9. No. 852. Anno 1869. On dried canes of Rubus idaeus L.. Rubus odoratus L.. Rosaceae. Finland. pratensis P.A. Saccardo \& P.A. Briard. Revue Mycologique, Toulouse 7:209. 1885. On dried stems of Medicago sativa L., Leguminosae. France.

preandina (C.L. Spegazzini) L. Holm. Svensk Botanisk Tidskrift, Stockholm 62:235. 1968. =Gibberidea praeandina C.L. Spegazzini. Anales del Museo Nacional de Historia Natural de Buenos Aires 23:59-60. 1912. On dead branches of Eupatorium saucechicoense Hieron., Compositae. Argentina (Mendoza). primulaecola (H.G. Winter) P.A. Saccardo. Sylloge Fungorum Omnium Hucusque Cognitorum Digessit P.A. Saccardo 2:49. 1883. ESphaerella primulaecola H.G. Winter. Hedw igia. Dresden 19:166. 1880. ESphaerulina primulicola (H.G. Winter) A.N. Berlese, Icones Fungorum Omnium Hucusque Cognitorum ad usum Sylloges Saccardianae Adcommodatae 1:123. 1894. EScleropleella primulicola (H.G. Winter) F. v. Höhnel. Annales Mycologici. Berlin 18:76. 1920. $\equiv$ Mycodotea primulicola (H.G. Winter) W. Kirschstein in O.C. Schmidt, Kryptogamenflora der Mark Brandenburg und Angrenzender Gebiete herausgegeben von dem Botanishen Verein der Provinz Brandenburg. Leipzig 7(3):432. 1938. On leaves and petioles of Primula latifolia Lapeyr. Primulaceae. Switzerland.

primulana A. Allescher. Bericht der Bayerischen Botanischen Gesellschaft zur Erforschung der Heimischen Flora. München 5:14. 1897. On dry stems of Primula elatior (L.) Hill. Primulaceae. Germany.

priuscheggiana F. Petrak. Annales Micologici. Berlin 16:225. 1919. On rotting leaves of Tilia sp.. Tiliaceae. France.

proliferue? J. Feltgen. Vorstudien zu einer Pilz-Flora des Grossherzogthums, Luxemburg 1(4):50. 1905. On dry stems of Diamthus prolifer L. Caryophyllaceae. Luxembourg.

promontorii P.A. Saccardo. Sylloge Fungorum Omnium Hucusque Cognitorum Digessit P.A. Saccardo 2:22-23. 1883. ELeprospluaria peruianu C.L. Spegazzini. Anales de la Sociedad Cientifica Argentina. Buenos Aires 12:179. 1881: Fungi Argentina Pugillus $f$, No. 168. 1881. On fallen decas ing stems of Salicornia peruviana H.B.K., Chenopodiaceae. Argentina.

protedrum 11. Sydow \&. P. Sydow, Annales Mycologici, Berlin 10:4+1-442. 1912. On leaves of Protea mclulesuca R. Br.. Proteaceac. South difrica. 
proteispora C.L. Spegazzini, Anales des Museo Nacional de Buenos Aires 6:282-283. 1898 ; Fungi Argentini Novi vel Critici, No. 629. Anno 1899. On decaying culms of Paspahum gigantem Baldw. ex Vasey. Gramineac. Argentina.

pruni N.N. Woronichin. Vestnik Tiflisskogo Botanicheskogo Sada. Tiflis (Moniteur du Jardin Botanique de Tiflis) 28:17-18. 1913. On living leaves of Prumus demestica L.. Rosaceae. U.S.S.R. (Caucasus).

prumi N.N. Woronichin forma plurivora N.N. Woronichin. Trudȳ Akademiia Nauk SSSR Botanicheskii Muzei 21:125-126. 1927. On leaves of Chamaerops sp.. Palmae. U.S.S.R. (Caucasus).

pseudo-diaporthe C.A. Oudemans. Nederlandsch kruidkundig Archief. Verslagen en Mededeelingen der Nederlandsche Botanische Vereeniging, Leiden, Series 2, 6:34. 1892. On dry stems of Jumcus effusus L., Juncaceae. Netherlands.

pseudohleria C.E. Fairman, Proceedings of the Rochester Academy of Science, Rochester. New York 6:127. 1922. On stems of Typha latifolia L., Typhaceae. U.S.A.

psilospora B. Auerswald, Hedwigia, Dresden 7:184.

1868. On dry stems of Phyteuma scheuchzeri All., Campanulaceae. Germany.

prarmicae P.A. Karsten, Fungi Fenniae Exsiccati, Century 9, No. 888. Anno 1869. On stems of Achillea ptarmica L., Compositae. Finland. pterocelastri E.M. Doidge, Bothalia, National Herbarium, Pretoria 4:859-860. 1948. On leaves of Pterocelastrus trituspidatus Walp., Celastraceae. South Africa.

puccinioides C.L. Spegazzini in P.A. Saccardo, Michelia Commentarium Mycologicum Fungos in Primis Italicos lllustrans 1:459. 1879. 三Metasphaevia puccinioides (C.L. Spegazzini) P.A. Saccardo, Sylloge Fungorum Omnium Hucusque Cognitorum Digessit P.A. Saccardo 2: 182. 1883. On fallen leaves of Carex paludosa Good., Cyperaceae. Italy. puiggarii C.L. Spegazzini, Fungi Puiggariani 1:143-144. No. 281 . 1889. Boletin de la Academia Nacional de Ciencias en Córdoba 1]:521-522.1889. On dead leaves of Andropogon sarcharoides Swartz, Gramineac. Brazil.

pukhra (H.G. Winter) P.A. Saccardo, Sylloge Fungorum Omnium Hucusque Cognitorum Digessit P.A. Saccardo 2:53-54. 1883. Sphacrella pulchra H.G. Winter, Hedwigia, Dresden 11:145-146. 1872. झMycodotea pulchra (II.G. Winter) W. Kirschstein. Annales Mycologici, Berlin 34:201. 1936; Kryptogamenllora der Mark Brandenburg und Angrenzender Gcbiete herausgegchen von dem Botanischen Verein der Provinz. Brandenhurg, Leipzig 7(3):433. 1938.
Eleptosphaerulina pulchra (H.G. Winter)

M.E. Barr, Contributions de l'Institut

Botinic|ue de L'Université de Montréal 73:7.

1959. On dry stems and leaves of Potentilla caulescens L., Rosaceae. Austria.

puncriformis G. Passerini, Atti dell' Reale Accademia Nazionale dei Lincei. Rendiconti, Rome. Series 4, 7(2):45. 1891. On wilted culms of Zea mays L., Gramineae. Italy.

punctiformis V. Mouton, Bulletin. Société R. de Botanique de Belgique, Bruxelles 39:44 45 . 1900. Nom. illegit. Art. 64.1. 三Leptosphacria montoniana P.A. Saccardo \& P. Sydow. On culm of grass of unknown host. Gramincac. Belgium.

punctillum H. Rehm, Annales Mycologici, Berlin 10:356. 1912. EPhaeosphaeria punctillum (H. Rehm) L. Holm, Symbolae Botanicae Upsalienses, Uppsala 14(3): 125.

1957. On leaves of Typha latifolia L., Typhaceae. Canada.

punctoidea P.A. Karsten, Hedwigia, Dresden 23:2. 1884; Acta Societatis pro Fauna et Flora Fennica, Helsingforsiae 2(6):53. 1884. On dead stems of Asparagus officinalis L., Liliaceae. Finland.

puniciae A.K. Kar \& M.K. Maity. Canadian Joumal of Botany, Ottawa 48:1302. 1970. On branches of Punica granatum L.. Punicaceac. India (West Benga]).

punjabensis L.E. Wehmeyer, Mycologia, Lancaster, Pennsylvania 55:322. 1963. On stems of Berberis petiolicola Wall., Berberidaceae. India, Pakistan.

purpurea H. Rehm, Ascomycetes Lojkani Lecti in Hungaria, Transylvania el Galicia, Berlin, p. 54. 1882. EMelanomma purpurea $(\mathrm{H}$. Rehm) L. Holm, Symbolae Botanicae Upsalienses. Uppsala 14(3):64. 1957. On dry stems of Artemisia iw/garis L., Compositae. Hungary. purpurearum B. Auerswald in W. Gonnermann and G.L. Rabenhorst, Mycologia Europaea. Abbildungen Sämmtlicher Pilze Europa's, Hefte 5 und 6, Synopsis Pyrenomycetum Europacorum, Table 12. Figure 168. 1869. Nom, nud. Art. 32.1. EDidymella purpurearum (B. Auerswald) ex P.A. Siccardo, Sylloge Fungorum Omnium Hucusque Cognitorum Digessit P.A. Saccardo 1:549.

1882. On leaves of Salix purpurea L., Salicaceae. Germany.

pusilla (C.L. Spegazzini) P.A. Saccardo, Sylloge Fungorum Omnium Hucusque Cognitorum Digessit P.A. Saccardo 22:999-1000. 1928. 三Leptosphacrella pusilla C.L. Spegazzini. Boletin de la Academia Nacional de Ciencias en Córdoba 23:558-559. 1918. On living leaves of Coffea sp., Rubiaceac. Costa Rica. puteana J.B. Ellis \& W.A. Kellerman in J.B. Ellis and B.M. Everhart, The North American Pyrenomycetes. A Contribution to Mycologic 
Botany, p. 368. 1892. On inside and outside of an oaken well bucket, Quercus sp., Fagaceae. U.S.A.

puttemansii A. Maublanc, Bulletin de la Société Mycologique de France, Paris 21:88-89. 1905. On leaves of Erinhorya japonica (Thunb.) Lindley, Rosaceae. Brazil.

pycnostigma (W. Nylander) P.A. Saccardo \& D. Saccardo, Sylloge Fungorum Omnium Hucusque Cognitorum Digessit P.A. Saccardo 17:731. 1905. EVerrucaria pycnostigma $W$. Nylander, Flora, Jena und Regensburg 52:297. 1869: in A.F. Malbranche. Bulletin de la Société des Amis des Sciences Naturelles de Rouen 6:307. 1870. EPhaeospora pycnostigma (W. Nylander) F. Arnold, Flora, Jena und Regensburg 57:151. 1874. 三Sphaeria pycnostigma (W. Nylander) H. Olivier. Bulletin de Géographie Botanique, LeMans 17:169. 1907. On thallus of Baeomyces rufus (Huds.) Rebent., Lichenes (Baeomycetaceae). France.

Pycnostigma (W. Nylander) P.A. Saccardo \& D. Saccardo var, morhosa (F. Arnold) K. Keissler in Dr. L. Rabenhorst's KryptogamanFlora von Deutschland, Oesterreich und der Schweiz, Second edition, 9(I Abt.. Teil 2):37 I. 1938. ESagedia morbosa F. Arnold. Verhandlungen der Zoologisch-Botanischen Gesellschaft in Wien 21:1133.1871. 三Porina morbosa (F. Arnold) G. Lettau. Hedwigia. Dresden 52:105. 1912. On thalli of unknown host, unknown fanily [originally described as a lichen]. Italy.

pyrenopezizoides P.A. Saccardo \& C.L. Spegazzini in P.A. Saccardo, Michelia Commentarium Mycologicum Fungos in Primis Italicos Illustrans 1:394. 1878. EHeptameria (Leptosphaeria) pyrenopezizoides (P.A. Saccardo \& C.L. Spegazzini) M.C. Cooke, Grevillea, London 18:30. 1889. On decaying twigs of Clematis vitalha L., Ranunculaceac. Italy.

quadriseplala J.W.H. Trail, Transactions and Proceedings of the Botanical Society of Edinburgh 17:491. 1889. On old stems of Lactuca muralis (L.) Gatertner, Rumer acerosa L., Thalictrum minuss L.. Valeriana officinalis L., Compositae. Polygonaceac, Ranunculaceate, Valerianaceac. Norway.

quamoclidii C.E. Fairman, Mycologia. Lancaster. Pennsylvania 10:246. 1918. On old stems of Qummolidion multiflorm Torr. ex A. Gray, Nyctaginaceae. U.S.A. (New Mexico).

quima L.N. Vasil eva, Pirenomitsety i Lokuloaskomitsety Severa Dal'nego Vostoka (Leningrad:"Nauka"), p. 120.1987. 三Pleospora iagans G. Niessi v. Mayendort, Verhandlungen des Naturforschenden Vereins in Brïn 14:174-175. (1875) 1876. On Aira cespitosa L., Calamagrostis syliarica Host. Elymus arenarius L., Gramineae. Germany. rajasthanensis K.S. Panwar \& S. Kaur, Kavaka. Madras 5:41-43. 1977. On dead wood of Lamiana camara L., Verbenaceae. India (Mt. Abu, Rajasthan).

ramalinae (M.R. Roberge) P.A. Saccardo. Sylloge Fungorum Omnium Hucusque Cognitorum Digessit P.A. Saccardo 2:84. 1883 (as (Desm.) Sacc.]. ESphaeria (Subrecta) ramalinae M.R. Roberge in J. Desmazières, Annales des Sciences Naturelles, Paris, Botanique. Series 3. 11:354. 1849. On thallus of Rumalina sp.. Lichenes (Ramalinaceae). France.

ram.sangiensis H. Schmid-Heckel. Pilze in den

Berchtesgadener Alpen. Nationalpark

Berchtesgaden Forschungsberichte No. 15

(Regensberg: Nationalpark Bercht-

esgaden):48. 1988. Non vidi. On stem of Sarifraga paniculaia Miller. Saxifragaceae. Germany.

ramulicola (C.H. Peck) P.A. Saccardo. Sylloge Fungorum Omnium Hucusque Cognitorum Digessit P.A. Saccardo 2:26-27. I883. ESphaeria ramulicola C.H. Peck. Repon. New York State Museum of Natural History. Albany 25:104. 1873. 三Neodeightonia ramulicola (C.H. Peck) M.E. Bar in M.E. Barr. C.T. Rogerson. S.J. Smith. and J.H. Haines, Bulletin of the New York State Museum. Albany 459:39. 1986. $\equiv D o-$ thidothia ramulicola (C.H. Peck) M.E. Barr. Mycotaxon. An Intemational Joumal Designed to Expedite Publication of Research on Taxonomy \& Nomenclature of Fungi \& Lichens, Jthaca. New York 34:524. 1989. On dead twigs of Ulmus sp.. Ulmacene. U.S.A.

ranunculi E. Rostrup, Meddelelser on Gronland.

Kjohenhavn 3:558. 1888. On stenss of

Ranunculus affinis R. Br.. Ranunculaceae.

Greenland.

rammcali-polyanthemi C. Sandu-Ville \& M. Mititiuc in C. Sandu-Ville. V. Jacob. M. Hamanu, and M. Mititiuc. Lucrările Stiintifice. Institutul Agronomic 'Professor Ion lonescu de la Brad', Iasi. Bucharest 1967:173-174. 1967. On dead stems of Ramunculus polsumbemos L., Ranumculaceae. Romani:.

ranunculoides A. Noelli. Nuovo Giomale Botanico Italiano e Bolletino della Societả Botanica Italiana. Firemze, New Series 19:703. 1912. On stems of Bupleurum rammouloides L.. Unbelliferale. Italy:

raphani D. Hawksuorth \& A. Sisanesan. Transactions of the British Migcological Society. London 64:104. 1975. On dead culums of Raphamus maritimus Sm.. Crucilerac. Great Britain.

raphidophora F.A. Hazslinszk!. Matemarihai én Termeszettudományi Közlemenyeh Von- 
atkozólag a Hazai Viszonyokra, Budapest 25(2):147. 1892. On scapes of Anemone narcissiflora L., Ranunculaceae. Hungary.

recessa G. Passerini, Atti dell Accademia Nazionate dei Lincei. Memoire, Rome, Series 4, 6:459. (1889) 1890. EPhaeosphaeria recessa (G. Passerini) R.A. Shoemaker, Canadian Journal of Botany, Ottawa 67:1555. 1989. On wilted stems of Arundo donax L., Gramineae. Haly. recurita (L. Fuckel) J. Lind, Danish Fungi as

Represented in the Herbarium of E. Rostrup, Nordisk Forlag, Copenhagen, p. 219. 1913. Esphaeria recurim L. Fuckel, Fungi Rhenani Exsiccati A Leopoldo Fuckel Collecti, Fascicle 25(10). No. 2434. Anno 1872 [as (Fr.) Fckel.]. 三Sphaeria recutita L. Fuckel. Symbolac Mycologicae 2:2]-22. 1873 [as Sphaeria recurita (Fr.) Fuckel]. Type based on Fungi Rhenani Exsiccati A Leopoldo Fuckel Collecti, Fuscicle 25, No. 2434. Anno 1872. Nom. nud. Art. 32.1. Non Sphaeria recutita E.M. Fries:E.M. Fries, Systema Mycologicum Sistens Fungorum 2:524. 1823. $\equiv$ Metasphaeria recurita (L. Fuckel) P.A. Saccardo, Sylloge Fungorum Omnium Hucusque Cognitorum Digessit P.A. Saccardo 2:176. 1883. On wilted leaves of Dactylis glomerata L.. Glyceria aquatica (L.) Wahlenb., Gramineae. Denmark, Germany. recutita (E.M. Fries:E.M. Fries) E. Müller las (L. Fuckel)], Sydowia. Annales Mycologici. Horn, N.Ö. 4(1-6):269. 1950. ESphaeria recutita E.M. Fries, Systema Mycologicum Sistens Fungorum 2:524. 1823. EMetasphaeria recutita (E.M. Fries:E.M. Fries) P.A. Saccardo, Sylloge Fungorum Omnium Hucusque Cognitorum Digessit P.A. Saccardo 2:176. 1883. On preceding year's stubble of Hordeum distichon L., Triticum vilgare L., Gramineae. Austria, Germany, Switzerland.

rehmiana W. Voss, Mitteilungen des Musealvereines für Krain, Carniola, Laibach 4(2): 19. 1891. On dead leaves of Drypis spinosa L., Caryophyllaceae. Yugoslavia.

rehmii V. Mouton, Bulletin. Société R. de Botanique de Belgique, Bruxelles 39:44. 1900. On leaves of grass of unknown host of Gramineac, Gramineae. Italy.

reidiana H. Sydow. Annales Mycologici, Berlin 22:297. 1924. On dead culms of Radicula sp. (=Rorippa sp.), Cruciferae. New Zealand. resedae G. Passerini, Atti dell' Reale Accademia Nazionale dei Lincei. Rendiconti, Rome, Series 4. 4(2):58. 1888. On wilted stems of Resedu littea L... Resedaceae. Italy.

restionis C.G. Hansford, Proceedings of the Linnean Society of New South Wales, Sydney 79:121. 1954. On stems of Restio (nustralis R. Br., Restionaceae. Austratia.

revocans P.A. Saccardo. Sylloge Fungorum Omnium Hucusque Cognitorum Digessit P.A. Siccardo
11:322. 1895. ELeptosphueria molanommoides A.N. Berlese. Nec F. Fautrey. 1891. On dead branches of Buxis semperirens L., Buxaceae. Belgium.

rhizomatum G. Passerini, Atti dell' Accademia Nazionale dei Lincei, Rendiconti, Roma, Series 4, 4(2):59. 1888. On rhizomes and runners of Agrostis vu/garis With., Cynodon dactylon (L.) Pers., Gramineae. Italy.

rhodiolicola F. Petrak, Annales Mycologici, Berlin 34:441 445. 1936. On stems of Rhodiola rosea L., Crassulaceae. Finland.

(Melanomma) rhododendri (H. Rehm) M.C. Cooke, Grevillea, London 17:91. 1889. 三Melanomma rhododendri H. Rehm, Ascomyceten, Fascicle 4, No. 186. Anno 1873. [三Cucurbitaria rhododendri G. Niessl v. Mayendorf, Verhandlungen des Naturforschenden Vereins in Brünn 10:200. (1871) 1872, is listed as a synonym in H. Rehm.] On dry branches of Rhododendron ferrugineum L., Ericaceae. Italy.

rhododendri P.C. Hennings. Verhandlungen des Botanischen Vereins Der Provinz Brandenburg, Berlin 40:157. 1898. On leaves of Rhododendron sp. Ericaceae. Himalayas.

rhodophaea G. Bizzozero, Atti del Istituto Veneto di Scienze, Lellere ed Arti, Venezia, Series 6. 3:304-305. 1885. IMontagnula rhodophaea (G. Bizzozero) A. Leuchtmann. Sydowia. Annales Mycologici, Horn, N.Ö. 37:176. 1984. On dead culms of Arundo donar L.. Gramineae. Italy.

rhoina J.B. Ellis \& B.M. Everhart, Bulletin of the Torrey Botanical Club (and Torreya), New York 24:131. 1897. On decorticated wood of Rhus glabra L.. Anacardiaceae. U.S.A.

rhopalispora A.N. Berlese, Icones Fungorum Omnium Hucusque Cognitorum ad usum Sylloges Saccardianae Adcommodatae 1:81. 1892. On stems of Crithmum maritimum L., Inula crithmoides L., Umbeltiferae, Compositae. France.

rhopographoides H. Rehm, Hedwigia, Dresden 41:(204). 1902. ERhopographus caulincola C.A. Oudemans, Nederlandsch kruidkundig Archief. Verslagen en Mededeelingen der Nedertandsche Botanische Vereeniging, Leiden, Series 3, 2(3):712. 1902. [Contributions is la Flore Mycologique des Pays-Bas XVIII.] Nec L.D. v. Schweinitz. On stem of unknown host of Umhelliferae. Umbelliferac. Netherlands.

ribis P.A. Karsten, Revue Mycologique. Toulouse 7:106. 1885. On dry branches of Rihes alpimm L.. Saxifragaceae. Finland.

richomi P.A. Saccardo \& P. Sydow, Sylloge Fungorum Omnium Hucusque Cognitorum Digessit P.A. Saccardo 14:571. 1899. $\equiv$ Leptosphasriastellariae C.E. Richon. Nec Leptosphaeria stellaria E. Rostrup. On old 
stems of Siellaria media (L.) Vill., Caryophyllaceae. France.

rimalis G. Niessl v. Mayendorf in J. Kunze, Fungi Selecti Exsiccati, Century 3, No. 337. 1880. Nom. nud. Art. 36.1. 三Leptosphaeria agminalis P.A. Saccardo \& P. Morthier. Nec V. Cesati \& G. de Notaris. On dry runners of Clematis vitallha L., Ranunculaceae. Switzerland.

rimicola (G.H. Otth) P.A. Saccardo, Hedwigia, Dresden 35:XXIX. 1896: Sylloge Fungorum Omnium Hucusque Cognitorum Digessit P.A. Saccardo 11:XXIX. 1896. 三Cladosphaeria rimicola G.H. Otth, Mitteilungen der Naturforschenden Gesellschaft in Bern 1870:106. 1871. Nom. inval. Art. 43.1. [The genus Cladosphaeria was validated in 1894.] On fallen branches of Prumes avium L., Rosaceae. Switzerland.

riofrioi R. González Fragoso, Memorias de la R. Sociedad Española de Historia Natural. Madrid 11:92. 1919. On dry stems and branchlets of Coronilla emerus L.. Leguminosae. Spain.

riparia P.A. Saccardo, Michelia Commentarium Mycologicum Fungos in Primis ltalicos Illustrans 1:39. 1877. On culms of Juncus effusus L.. Juncaceae. Spain.

rivalis J. Feltgen, Vorstudien zu einer Pilz-Flora des Grossherzogthums, Luxemburg 1(2):157. 1901. [Description effectively published: Vorstudien zu einer Pilz-Flora des Grossherzogthums, Luxemburg I:256. 1899.] On dry stems, leaves, and leaf sheaths of Carex paludosa Good., Cyperaceae. Luxembourg.

rivana (G. de Notaris) P.A. Saccardo, Sylloge Fungorum Omnium Hucusque Cognitorum Digessit P.A. Saccardo 2:83. 1883. $\equiv$ Sphaeria rivana G. de Notaris, Commentario della Società Crittogamologica Italiana, Milan 2:485. 1867. On thallus of Peligera canina (L.) Willd., Lichenes (Peltigeraceae). ltaly.

rivana (G. de Notaris) P.A. Saccardo forma solorinae H. Rehm, Österreichische Botanische Zeitschrift, Wien 54:84-85. 1904. On thallus of Solorina crocea (L.) Ach., Lichenes (Peltigeraceae). Italy.

rivularis E.C. Bommer, M.H. Rousscau \& P.A.

Saccardo in P.A. Saccardo, Sylloge Fungorum Omnium Hucusque Cognitoruın Digessit P.A. Saccardo 9:787. 1891. On stems of Alisma plantago L.. Alismataceae. Belgium.

rolnusta (P. Strasser) E. Müller, Sydowia. Annales Mycologici. Horn, N.Ö. 4(I-6):298. 1950. $\equiv$ Leptosphacria derasa (M.J. Berkeley \& C.E. Broome) 13. Auerswald forma rohusta $P$. Strasser, Verhandlungen der ZoologischBotanischen Gesellschaft in Wien 57:315. 1907. $\equiv$ Nodulosphacria rolmusta (P. Strasser) L. Holm, Symbolae Botanicae Upsalienses. Uppsala 14(3):90. 1957. EOphiobolus.s robustus (P. Strasser) R.A. Shoemaker. Canadian Journal of Botany. Ottawa 54:2389. 1976. On dry stems of Senecia nemorensis L., Senecio rupestris Waldst. \& Kit., Compositae. Switzerland.

roseotinctum J.B. Ellis \& B.M. Everhart, The North American Pyrenomyceles. A Contribution to Mycologic Botany, p. 237. 1892. Nom. nud. Art. 32.]. [Apparently an error for Lophiostoma roseotinctum J.B. Ellis \& B.M. Everhart.]

rostruta M.L. Farr \& H.T. Horner, Nova Hedwigia. Zeitschrift für Kryptogramenkunde, Weinheim 15:250-251. 1968. On leaves of Selaginella arenicola subsp. acanthonota (Underwood) R. Tryon. Selaginella rupestris (L.) Spring. Selaginellaceae. U.S.A. (Virginia).

rostrupii P.A. Saccardo \& D. Saccardo in P.A. Saccardo, Sylloge Fungorum Omnium Hucusque Cognitorum Digessit P.A. Saccardo 17:721. 1905. ELeplosphaeria dryadis $\mathrm{E}$. Rostrup. Nec E. Rostrup. 1903. On upper surface of dried leaves of Dryas octopetala L.. Rosaceae. Norway.

rostrupii J. Lind, Annales Mycologici, Berlin 13:17. 1915. Nom. illegit. Art. 64.1. On stems of Daucus carota L.. Umbelliferae. Denmark. Germany.

rothomagensis P.A. Saccardo, Michelia Commentarium Mycologicum Fungos in Primis Italicos Illustrans 2:63. 1880. On stems of Senecio erucifolius L., Compositae. France.

rothomagensis P.A. Saccardo var. artemisiae N. Patouillard. Exploration Scientifique de la Tunisie, p. 13. 1892. On dead stems of Artemisia herha-alha Asso, Compositae. Tunisia.

roumegueri P.A. Saccardo, Michelia Commentarium Mycologicum Fungos in Primis Italicos Illustrans 2:62.1880. EMetasphaeria roumegueri P.A. Saccardo, Sy'lloge Fungorum Omnium Hucusque Cognitorum Digessit P.A. Saccardo 2:181. 1883. झMassariosphacria rotmegueri (P.A. Saccardo) A Leuchemann. Sydowia. Annales Mycologici. Hom. N.Ö. 37:173. 1984. On decaying contex of Phormium sp.. Agavaceac. France.

rousscliana (J. Desmazières) V. Cesati \& G. de Notaris. Commentario della Società Crittogamologica ltaliana. Milan 1:236. 1863. ESphacria (Foliicola) rousseliana J. Desmazières, Annales des Sciences Naturelles. Paris, Botanique. Series 3. 11:355. 1849. $\equiv$ Phacosphacria rousseliana (J. Desmazières) L. Holm, Symholae Botanicac Upsalienses. Uppsala 14(3):114. 1957. On sheath of Phlewm bochmeri Withel. Gramineac. Canada, Czechoslovakia, Germany, Netherlands, Switzerland. 
rubelloides (C.B. Plowright) P.A. Saccardo, Sylloge Fungorum Omnium Hucusque Cognitorum Digessit P.A. Saccardo 2:77. 1883.

Esphaeria rubelloides C.B. Plowright in M.C. Cooke, Grevillea, London 5:120. 1877. On decaying culms of Triticum repens $\mathrm{L}$.. Gramineae. Great Britain.

rubellula (J. Desmazières) F. v. Höhnel, Österreichische Botanische Zeitschrift, Wien 63:377. 1913. ESphueria modesta J. Desmazières var. rubellula J. Desmazières, Annales des Sciences Naturelles. Paris, Botanique. Series 3. 16:312. 1851. On stems of Crepis vesicaria L. subsp. haenseleri (Boiss. ex DC.) P.D. Sell [as Barkhomsia taraxacifolia (Thuill.) DC.]. Compositae. France. rubicunda H. Rehm ex H. Rehm in H.G. Winter. Asconyceten, Fascicle 2. No. 92. Anno 1871. Nom. nud. Art. 32.1. Flora, Jena und Regensburg 55:540. 1872. 三Melanomma rubicundum (H. Rehm ex H. Rehm) L. Holm, Symbolae Botanicae Upsalienses, Uppsala 14(3):65. 1957. On fallen stems of unknown host of Umbelliferae. Umbelliferae. Hungary. rubicunda H. Rehm, Ascomycetes Lojkani Lecti in Hungaria. Transylvania et Galicia, Berlin, pp. 53-54. 1882. Nom. illegit. Art. 64.1. On decayed stems of Conium maculatum L. Umbelliferae. Hungary.

Inbrotincta J.B. Ellis \& B.M. Everhart, Journal of Mycology. Columbus, Ohio 1:150-151. 1885. On dead iwigs of Staphylea trifolia L., Staphyleaceae. U.S.A.

rudbeckiae (L.A. Kirchner) P.A. Saccardo. Sylloge Fungorum Omnium Hucusque Cognitorum Digessit P.A. Saccardo 2:17. 1883. $\equiv$ Pleospora rulheckia L.A. Kirchnes in F. v. Thümen. Fungi Austriaci Exsiccati. Century 9. No. 858. 1874. On dried stems of Rudheckia amplevicaulis Vahl., Compositae. Czechoslovakia.

rugosa J. Dearness \& G.R. Bisby in G.R. Bisby, A. Buller, and J. Dearness. The Fungi of Manitoba, p. 74. 1929. On dead stems of Cornus stolonifera Michx., Cornaceae. Canada.

rulingice F. Tassi, Bolletino del R. Orto Botanico. Siena 3:95-96. 1900). On branchlets of Rulingia prostrata Maiden \& Betche. Sterculiaceac. Australia.

rumicicola S. Ahunad. Monographs, Biological Society of Pakistan. Lahore 5:13. 1969. =Leptosphaeria rumicis A.C. Batista. W.A. Cavalcanti \& S.H. lqbal. Nec Leptosphaeria rumicis F. Fatutrey. Nec Leprosphacria mumicis H. Wegelin. On stems of Rumex nepalensis Sprengel, Polygonaceae. Pakistan. rumicis A.C. Batista. W.A. Cavalcanti \& S.HI. lqbal in A.C. Batista, G.E.P. Peres, and S.I1. Iqbal. Atas do Instituto de Micologia. Recife
4:135-136. 1967. Nom. illegit. Art, 64.1. On stems of Rumex nepalensis Sprengel. Polygonaceae. Pakistan.

rumicis F. Fautrey in C. Roumeguère, Revue Mycologique, Toulouse 13:168. 1891: Fungi Selecti Gallici Exsiccati, Century 59, No. 5848. Anno 1891. On old stems of Rumex patientia L., Polygonaceae. France.

rumicis $\mathrm{H}$. Wegelin, Mitteilungen der Thurgauischen Naturforschenden Gesellschaft, Frauenfeld 12:177. 1896. Nom. illegit. Art. 64.1. =Leprosphaeria negeliniana P.A. Saccardo \& P. Sydow. On dried stems of Rumex sp.. Polygonaceae. Switzerland.

rusci (E.M. Fries) G. Niess! v. Mayendorf. Instituto. Revista Scientifica e Litteraria, Coimbra 31:90. 1883. ESphaeria rusci K.F.W. Wallroth. Flora Cryptogamica Germaniae 2:776. 1833. ELeplosphaeria rusci (E.M. Fries) P.A. Saccardo, Sylloge Fungorum Omnium Hucusque Cognitorum Digessit P.A. Saccardo 2:74. 1883 [as (K.F.W. Wallroth)].

rusci (E.M. Fries) P.A. Saccardo, Sylloge Fungorum Omnium Hucusque Cognitorum Digessit P.A. Saccardo 2:74. 1883. ESphaeria atro-virens E.M. Fries:E.M. Fries var. rusci E.M.

Fries:E.M. Fries, Systema Mycologicum Sistens Fungorum 2:501. 1823. 三Sphaeria rusci (E.M. Fries) K.F.W. Wallroth, Flora Cryptogamica Germaniae 2:776. 1833. ESphaerella musci (E.M. Fries) V. Cesati \& G. de Notaris, Commentario della Società Crittogamologica Jıaliana, Milan 1:237. 1863. $\equiv$ Paruphaeosphaeria rusci (E.M. Fries) O. Eriksson, Arkiv för Botanik, Uppsala, Stockholn, Series 2, 6:406. 1967 [as (Wallroth) O. Erikss.]. On cladodium and siems of Ruscus aculeatus L., Ruscus androgynus L., Ruscus hypoglossum L. Ruscaceae. Austria, Belgium, France, Great Britain. Italy. Portugal.

rusci (E.M. Fries:E.M. Fries) P.A. Saccardo forma caulina D. Saccardo, Mycotheca Italica. Sistens Fungos Venetos Exsiccatos. Century 15, No. 1484. 1905. [Description in P.A. Saccardo, Sylloge Fungorum Omnium Hucusque Cognitorum Digessit P.A. Salccardo 2:74. 1883 suh. Leplosphaeria mustici.] On culms of Rusrus aculecums L., Ruscaceae. lta!y.

Insci (E.M. Fries) P.A. Saccardo lorma fourcroyde P.A. Saccardo. Florula Mycologica Lusitanica Sistens Contributionem Decimam ad Eamden Floram nec non Conspectum Fungorum Omnium in Lusitania Hucusque Observatorum. Coimbra (Imprensa da Universidade). p. 11. 1893. On dead leaves of Furcraea bedinghausii C. Koch. Agavaceae. Portugal. rusci (E.M. Fries) P.A. Saccardo var. hypophylli R.C. Maire, Bulletin de la Socieite Botanique 
de France, Paris 56:CCLXXII-CCLXXIII. (1909) 1911. On dead cladodes of Ruscus hypophyllum L., Ruscaceae. France.

rusci (E.M. Fries) G. Niessl v. Mayendorf var. ruscihypoglossi C. Massalongo, Malpighia. Rassegna Mensuale di Botanica. Messina, Genova 8:194. 1894; Nuovo Contribuzione alla Micologia Veronese Genova (Tipografia di Angelo Ciminago), p. 38. 1894. Nom. nud. Art. 32.1. On leaves of Ruscus hypoglossum L., Ruscaceae. Italy.

ruscicola P. Hariot \& P.A. Karsten, Revue Mycologique, Toulouse 12:128-129. 1890. On rotting stems of Ruscus sp., Ruscaceae. France.

ruscicola P.A. Hariot \& P.A. Karsten forma cladodiicola R. González Fragoso, Trabajos del Museo Nacional de Ciencias Naturales, Madrid. Series Botanica 10:90. 1916. On dead cladodes of Ruscus aculeatus L., Ruscaceae. Spain.

russellii R.A. Shoemaker, Canadian Journal of Botany, Ottawa 62:2711. 1984. On stems of Asclepias sp.?. Asclepiadaceae. Canada.

rustica P.A. Karsten, Fungi Fenniae Exsiccati. Century 10, No. 964. Anno 1870. $\equiv$ Metasphaeria rustica (P.A. Karsten) P.A. Saccardo, Sylloge Fungorum Omnium Hucusque Cognitorum Digessit P.A. Saccardo 2:157. 1883. On stems of Spiraea ulmaria L. (=Filipendula ulmaria Maxim.), Rosaceae. Finland.

ruthenica F. Petrak, Annales Mycologici, Berlin 19:78-79. 1921. On dry stems of Sambucus chulus L., Caprifoliaceae. France.

sabalicola (J.B. Ellis \& G.W. Martin) P.A. Saccardo, Sylloge Fungorum Omnium Hucusque Cognitorum Digessit P.A. Saccardo Additamenta Ad Volumina l-IV, 2:LVII-LVIII. 1882. ESphaeria sahalicola J.B. Ellis \& G.W. Martin, American Midland Naturalist, Notre Dame, Indiana 16:810. I882. झHeptameria sabalicola (J.B. Ellis \& G.W. Marrin) M.C. Cooke, Grevillea, London I8:32. 1889. On rachis of Sabal sermalata Roem \& Schult., Palmae. U.S.A.

(Clypeosphaeria) sabaligera (M.J. Berkeley \& M.A. Curtis) M.C. Cooke, Grevillea. London 17:91. 1889. $\equiv$ Sphacria sabaligera M.J. Berkeley \& M.A. Curtis in M.J. Berkeley. Grevillea. London 4:147. 1876. EClypeosphaeria sahaligera (M.J. Berkeley \& M.A. Curtis) P.A. Saccardo. Sylloge Fungorum Omnium Hucusque Cognitorum Diggesit P'A. Saccardo 2:91. 188.3. On unknown substrate of Sabal sp., Pialmae. U.S.A.

sabmuda C.L. Spegazzini, Revue Myeologique. Toulouse 4:79. 1882. On dead, decaying leaves of Silcue acaulis (L..) Jacy., Caryophyllaceas. France. sabauda C.L. Spagazzini forma arraticae R. González Fragoso, Trabajos del Museo Nacional de Ciencias Naturales. Madrid. Series Botanica 4:17. 1914. On dead or rotting leaves of Silene anatica Lag.. Caryophyllaceae. Spain.

sabulctorum (M.J. Berkeley \& C.E. Broome) J.

Lind. Danish Fungi as Represented in the Herbarium of E. Rostrup. Nordisk Forlag. Copenhagen, p. 219. 1913. 三Metasphaeria sabuletorum (M.J. Berkeley \& C.E. Broome) P.A. Saccardo, Sylloge Fungorum Omnium Hucusque Cognitorum Digessit P.A. Saccardo 2:180. 1883. ESphaeria (Foliicolae) sabuletorum M.J. Berkeley \& C.E. Broome. Annals and Magazine of Natural History, London, Series 2, 9:382. 1852. On dead leaves of Fesinca arundinacea Schreber. Gramineae. Denmark, Great Britain.

sabuletorum (M.J. Berkeley \& C.E. Broome) F. v. Höhnel. Hedwigia. Dresden 60:141. 1918. EShaeria (Foliicolae) sabuleiorum M.J. Berkeley \& C.E. Broome. Annals and Magazine of Natural History, London, Series 2. 9:382. 1852. On dead leaves of Ammophila arundinacea Host. Gramineae. Great Britain.

saccardiana J.H. Fabre, Annales des Sciences

Naturelles, Paris. Botanique. Series 6. 9:89. 1878. 三Requienella saccardiana (J.H. Fabre) J.H. Fabre, Annales des Seiences Naturelles. Paris, Botanique. Series 6, 15:56. 1883. On dried stems of Crataegus oxyacansha L.. Rosaceae. France.

sacchari J. Van Breda De Haan. Mededelingen Van Het Proefstation Voor Suikerriet in WestJava, 'Kagok'. Soerabaia I\$92:25-28. 1892. ELeptosphaerella sacchari (J. Van Breda De Haan) S.C. Teng. Sinensia. Special Bulletin of the Metropolitan Nuseum of Natural History: Nanking 9:224. 1938. झPhacosphacria sacchari (J. Van Breda De Haan) R.A. Shoemaker. Canadian Joumal of Botany. Ottawa 67:1535. 1989. On sheaths and leaves of Saccharum officinarum L.. Gramineae. Java.

sacchari C.L. Spegazzini. Revista. Unisersidad Nacional de La Plata 2(I8):232. 1896: Hongos De La Caña De Azúcar No. 9. Aлno 1896. Nom. illegit. Art. 64.1. =Leptosphueria spegaz-inii P.A. Saccardo of P. Sydow. On wilted sheaths and leaves of Saccharum officinurum L.. Gramineae. Argentinis.

saccharicola P.C. Hennings. Hedw igia. Dresden 39:79. 1900. On leaves of Saccharum officinarum L.. Gramineae. Brazil. sacculus G. Passerini \& V. Beltrani. Atti dell" Accademia Nazionale dei Lincei. Transunti. Rome, Series 3, 7:36. 1\$\$3. EMcpasphucriu saccultus (G. Passerini \& V. Beltrani) P.A. 
Saccardo. Sylloge Fungorum Onniun Hucusque Cognitorum Digessit P.A. Saccardo 2:162. 1882. On dry stem of Euphortica dendroides L.. Euphorbiaceae. Italy (Sicily).

saginatu H. Sydow. Annales Mycologici, Berlin 37:377-378. 1939. 三Mixtmra saginata (H. Sydow) O. Eriksson \& J. Yue, Mycotaxon. An International Journal Designed to Expedite Publication of Research on Taxonony \& Nomenclature of Fungi \& Lichens, lthaca, New York 38:203. 1990. On living or wilted leaves of Chusquea serrulata Pilger, Gramineae. Ecuador.

sulebricola E.C. Bommer, M.H. Rousseau \& P.A. Saccardo in P.A. Saccardo, Sylloge Fungorum Omnium Hucusque Cognitorum Digessit P.A. Saccardo 9:783. 1891. EPhaeosphaeria salchricola (E.C. Bommer. M.H. Rousseau \& P.A. Saccardo) A. Leuchtmann, Sydowia. Annales Mycologici, Horn, N.Ö. 37:156. 1984. On drying leaves of Stellaria gramines L. Caryophyllaceae. Belgium, Germany. salebrosa P.A. Saccardo, Michelia Commentarium Mycologicum Fungos in Primis Italicos Illustrans 2:599. 1882. Nec C.G.T. Preuss. $\equiv$ Leptosphaeria sapeyensis P.A. Saccardo. On stems of Gentiana lutea L., Gentianaceae. France.

sulebrosa (C.G.T. Preuss) P.A. Saccardo, Sylloge Fungorum Omnium Hucusque Cognitorum Digessit P.A. Saccardo 2:20. 1883. Nom. illegit. Art. 64.1. EPhueoderris salebrosa (C.G.T. Preuss) F. v. Höhnel, Sitzungsberichte der Akademie der Wissenschaften in Wien, Mathematisch-naturwissenschaftliche Klasse, Abt. I, 120:462. 1911. ESphaeria salebrosa C.G.T. Preuss, Linnaea. Institut botanique de l'Université de Genève 26:714. 1853. On trunks of Brassica crispa Rafin.. Cruciferae. Germany.

salicaria G. Passerini, Revue Mycologique, Toulouse 2:35. 1880. On dry stems of Lythrum salicaria L., Lythraceae. Italy. salicincarum (G. Passerini) P.A. Saccardo, Sylloge Fungorum Omnium Hucusque Cognitorum Digessit P.A. Saccardo 2:50. 1883. ESphaerella sulicinearum G. Passerini. Nom. nud. Art. 32.1 (herbarium nane). =Sphacrella salicinearum G. Passerini forma populinigrae G.L. Rabenhorst, Fungi Europaei Exsiccati, Klotzschii Herbarii vivi Mycologici Continuatio III, Century 25, No. 2444. Anno 1876; Hedwigia, Dresden 17:172. 1878. ESphacrella salicineturum G. Passerini forma salicis-alhac G.L. Rabenhorst, Fungi Europaci III, Century 25, No. 2445. Anno 1876. On leaves of Populus nigra L., Salix alha L., Salicaceac. Italy.

salsolue L. Hollós. Annales Historico-Naturales Musei Nationalis Hungarici, Budapest 4:335.
1906. On dry stems of Salsola kali L..

Chenopodiaceae. Hungary.

salvicue G. Passerini, Atti dell Reale Accademiat

Nazionale dei Lincei. Rendiconti, Rome.

Series 4, 4(2):59. 1888. On dry branches of

Saliva officinalis L., Labiatae. Italy.

salviae G. Passerini forma minor C. Sandu-Ville.

Academia Republicii Populare Romîne.

Filiala lassi. Studii si Cercetări Stiintifice.

Biologie si Stinte Agricole 13:257. 1962. On unknown substrate of Lavomdula vera DC.

Labiatac. Romania.

salvinii A. Cattaneo, Archivo del Laboratorio di Botanica Crittogamica Universita di Pavia 2/ 3:126. 1879. झMagnaporthe salvinii (A. Cattaneo) R.A. Krause \& R.K. Wehster, Mycologia, Lancaster, Pennsylvania 64:110. 1972. On leaf sheaths of Orya sativa L., Gramineae. Italy.

sambuci F. Fatutrey in C. Roumeguère, Revue Mycologique. Toulouse 14:7. 1892; Fungi Selecti Gallici Exsiccati, Century 60, No. 5960. Anno 1892. Nom. illegit. Art. 64.1. On dry branches of Sambucus nigra L., Caprifoliaceae. France.

sambuci P.A. Saccardo. Atti dell ${ }^{\circ}$ Accademia Scientifica Veneto-Trentino-Istriana. Padova 2(2): 152. I873. On branches below the epidermis of Sambucus migra L.. Caprifoliaceae. ltaly.

sambucina J.B. Ellis \& B.M. Everhart. Proceedings of the Academy of Natural Sciences of Philadelphia 1894:336-337. 1894. 三Chatomastia sambucina (J.B. Ellis \& B.M. Everhart) M.E. Barr. Mycotaxon. An International Journal Designed to Expedite Publication of Research on Taxonomy \& Nomenclature of Fungi \& Lichens. Ithaca. New York 34:513. 1989. On dead plant of Sambucus melanocarpa A. Gray, Capriloliaceare. U.S.A.

sangnisorbae P.A. Karsten in A.N. Berlese, Icones Fungorum Omnium Hucusque Cognitorum ad usum Sylloges Saccardianae Adcommodatac 1:78. 1892. On branches of Sungmisorba officinalis L. Rosaceae. Germany.

samguisorbae P.A. Karsten, Enumeratio Fungorum et Myxonycetum in Lapponia Orientali Acstate I86I Lectorum, p. 2] 4. 1886: Notiser Sallskapets Pro Fauna et Flora Fordhandlingar 8:214. 1866 [as Sphaeria (Leprosphueria) sanguisorhael. On dead stems of Samgnisorha polygama F. Nyl., Rosaceate. U.S.S.R.

sapeyensis P.A. Saccardo, Sylloge Fungorum Omnium Hucusque Cognitorum Digessit P.A. Saccardo 2:38. 1883. Nom. illegit. Art. 63.1. ELeprosphaeria salemrosa l'.A. Saccardo: Michelia Commentarium Mycologicum Fungos in Primis Italicos lllustrans 2:599. 1882. Nec C.G.T. Preuss. On stems of Gentiana lutea L.. Gentianaceae. France. 
suprophilu H. Rehm. Hedwigia. Dresden 21:121. 1882. ĐMerasphaeria saprophila (H. Rehm) P.A. Saccardo, Sylloge Fungorum Omnium Hucusque Cognitorum Digessit P.A. Saccardo 2:172. 1883. On decaying berries of Juniperus nana Willd., Cupressaceae. Austria.

surmenticia P.A. Saccardo. Michelia Commentarium Mycologicum Fungos in Primis ttalicos Illustrans 2:63-64. 1880. On runners of Solanum dulcumara L., Solanaceae. France. surothamni J.B. Lambotte \& F. Fautrey in C. Roumeguère, Revue Mycologique, Toulouse 15:115. 1893: in C. Roumeguère, Fungi Selecti Gallici Exsiccati, Century 64, No. 6356. Anno 1893. On branches of Sarothammus scoparius (L.) Wimmer ex Koch,

Leguminosae. France.

sarraziniana P.A. Saccardo \& C. Roumeguère, Revue Mycologique, Toulouse 7:159. 1885. On nodes of unknown host. Caryophyllaceae. France.

susacola K. Hara, Byôchu-gai Zasschi (Journal of Plant Protection), Tokyo 5:885. 1918. On unknown substrate of $S a s a$ sp.. Gramineae. Japan.

sasue K. Hara, Byôchu-gai Zasschi (Joumal of Plant Protection). Tokyo 5:884-885. 1918. On dead leaves of Sasa sp., Gramineae. Japan. saxonica F. v. Höhnel, Annales Mycologici. Berlin 16:84-86. 1918. झPhaeosphaeria saronica (F. v. Höhnel) R.A. Shoemaker, Canadian Journal of Botany, Ottawa 67:1564. 1989. On dead culms of Scirpus maritimus L., Cyperaceae. Germany.

scabiens (V. Cesati) P.A. Saccardo, Sylloge Fungorum Omnium Hucusque Cognitorum Digessit P.A. Saccardo 2:69. 1883. ESphaeria scahiens V. Cesati, Atti dell' Accademia delle Scienze Fisiche e Matematiche, Napoli 8(2):20. 1879. On culms of unknown host, unknown family. Malaysia.

scabrispora S.C. Teng, Sinensia. Special Bulletin of the Metropolitan Museum of Natural History. Nanking 4:378. 1934. EMassariosphaeria scabrispora (S.C. Teng) R.A. Shoemaker. Canadian Joumal of Botany, Ottawa 67:1589. 1989. On culms of Bamhiras sp.. Gramineae. China (Kiangsu).

scapophila (C.H. Peck) P.A. Saccardo, Sylloge Fungorum Omnium Hucusque Cognitorum Digessit P.A. Saccardo 2:43-44. 1883. ESphaeria (Cumlicolue) scapophila C.H. Peck, Report. New York State Museum of Natural History, Albany 30:66-67. 1878. On dead scapes of Surracenia purpurea L.. Sirtaceniaceae. U.S.A.

scheiderianu (J. Rick) J. Rich in P.A. Saccardo. Sylloge Fungorum Omnium Hucusque Cognitorum Digessit P.A. Saccardo 22:231. 1913. ETrematosphaeria schneideriana J.
Rick. Broteria. Lisboa 5:47. 1906. On unknown substrate of Bambusa sp.. Gramineae. Brazil.

schocnocauli H. Sydow. Annales Mycologici. Berlin 28:84-85. 1930. On dead decaying stems of Schoenocaulon officinale A. Gray. Liliaceae. Portugal.

scirpina H.G. Winter, Hedwigia. Dresden 11:146. 1872. =Metasphaeria scirpina (H.G. Winter) P.A. Saccardo. Sylloge Fungorum Omnium Hucusque Cognitorum Digessit P.A. Saccardo 2:182. 1882. ETrichometasphaeria scirpina (H.G. Winter) L. Holm. Symbolae Botanicae Upsalienses, Uppsala 14(3):142. 1957.

=Massariosphacria scirpina (H.G. Winter) A. Leuchtmann. Sydowia. Annales Mycologici. Hom, N.Ö. 37:174. 1984. On dry culms of Scirpus lacustris L.. Cyperaceae. Austria (Tyrol?), Italy.

scimla H. Sydow. Annales Mycologici. Berlin 36:321-332. 1938. झUclanomma scirulum (H. Sydow) L. Holm. Symbolae Botanicae Upsalienses. Uppsala 14(3):66. 1957. On rotten stems of Galium aparine L. Rubiaceae. Germany.

sclaronica (S. Schulzer v. Müggenburg \& P.A. Saccardo) F.A. Hazslinszky. Matematikai és Természettudományi Közlemenyek Vonatkozólag a Hazai Viszonỵokra, Budapest 25(2):150. 1892. झMetasphaeria sclaronca S. Schulzer v. Müggenburg \& P.A. Saccardo. Revue Mycologique, Toulouse 6:69-70.1884. On decorticated branches of linis vinifera $\mathrm{L}$. subsp. sylvestris (C.C. Gmelin) Hegi.

Vitaceae. Yugoslavia.

scolecosporarum M1. de Sousa da Camara. Revista Agronómica. Lisbon 20(1):23. 1932. On leaves of Querrus suher L.. Fagaceae. Portugal.

scorophila P.A. Saccardo. Nuovo Giomale Botanico Italiano e Bolletino della Società Botanica Italiana. Firenze 7:317. 1875. On stems of Aconirum sp.. Cynanchum sp., Iinceroxicum sp.. Ranunculaceae. Asclepiadaceae. Italy. scotophila P.A. Saccardo Ionna aconti P.A.

Saccardo [falsely so cited in P.A. Saccardo, Sylloge Fungorum Omnium Hucusque Cognitorum Digessit P.A. Saccardo 2:157. 1883. As published in Nuorn Giomale Botanico ltaliano e Bolletino della Società Botanica Italiana, Firenze 7:317. 1875: Fungi Veneti Novi vel Critici. Series 2. p. 317. 1875.] ELepressphucrice soorophila P.A. Saccardo.

scroplunlariuc (J. Desmazières) P.A. Saccardo. Sylloge Fungorum Ommium Hucusque Cognitorum Digessit P.A. Silccardo 2:57. 1883. ESphaeria scrophulariue J. Desmazières. l'lantes Cryptogames Du Nord De Lat France, Edition 1. Fascicle 15. No. 718. 
Anno 1834. Non vidi; Annales des Sciences Naturelles, Paris, Botanique, Series 2. 6:24.5-246. 1836. On capsules of S(rop/mIaria aquarica L., Scrophulariaceae. France.

scurati S.A. Gucevicz, Novosti Sistematiki Nizshikh Rastenij. Novitates Systematicae Plantarum Non Vascularium 6:129-130. 1969 [as sculata]. On dried stems of Rumex scutatus L., Polygonaceate. U.S.S.R. (Ukraine). secalina A. Jaczew'ski. Opredielitel' Gribov '2. Fungi Imperfecti (Petrograd), pp. 606-607. 1917. On unknown substrate of Secale cereale L., Gramineae. U.S.S.R.

secalina Lachevskii in N.N. Lavrov, Trudy Tomskogo Gosudarstvennogo Universiteti. Tomsk. Series Biologicheskii 104:88. 1948. Non vidi. Nom. illegit. Art. 64.1. On dead culms of Secale cereale L., Gramineae. U.S.S.R.

seculis F. Haberlandt in J. Schröter, Just's Botanische Jahresberichte, Berlin 6(2):319. 1878. On unknown substrate of Secale cereale $\mathrm{L}$., Gramineac. Unknown country.

semelina A. Caballero, Anales del Jardin Botánico de Madrid 1:200. 1941. On living cladode of Semele androgyna (L.) Kunth. Liliaceae. Spain.

seminuda (C.I1. Persoon:E.M. Fries) V. Cesati \& G. de Notaris, Commentario della Società Crittogamologica Italiana. Milan 1:235. 1863. 三Sphaeria seminuda C.H. Persoon:E.M. Fries, Synopsis Methodica Fungorum. pp. 70-71. 1801: E.M. Fries, Systema Mycologicum Sistens Fungorum 2:464. 1823. ETrematosphaeria semimula (C.H. Persoon:E.M. Fries) L. Fuckel, Symbolae Mycologicae Beiträge zur Kenntnis Der Rheinischen Pilze. p. 162. 1870. On dry wood of Fagus sp., Fagaceae. Austria. Germany.

senecionis (L. Fuckel) H.G. Winter, Dr. L.

Rabenhorst's Kryptogamen-Flora von Deutschland. Oesterreich und der Schweiz 1(2):469. 1867. EPleospora senecionis L. Fuckel. Fungi Rhenani Exsiccati A Leopoldo Fuckel Collecti Supplement, Fascicle 6, No.

2032. Anno 1867. DMetasphaeria senecionis (L. Fuckel) P.A. Saccardo. Sylloge Fungorum Omnium Hucusque Cognitorum Digessit P.A. Saccardo 2:158. 1883. On dry stems of Senecio nemonensis L., Compositae. Austria. senegalensis G. Segretin. J. Baylet, H. Darasse \& R. Camain. Comptes Rendus hebdomadaire des Sciences de l'Académic des Sciences, Paris, 248:3732. 1959. On /lomo supiens, Honinidae. Senegal.

sepalorum (J.S. Vleugel) J. Lind, Svensk Botanisk Tidskrift. Stockholm 22:66. 1928. 三Metasphareriu sepalorwm J.S. Vleugel, Svensk Botanisk Tidskrift. Stockholm 2:369-370). 1908. EBricoskea sepalormm (J.S. Vleugel)
M.E. Barr. Mycotaxon. An Intemational Journal Designed to Expedite Publication of Rescarch on Taxonomy \& Nomenclature of Fungi \& Lichens, lthaca. New York 15:346347. 1982. On dead sepals of Juncus filiformis L.. Juncus mifidus L.. Juncaceae. Norway (Spitsbergen).

sepincola (E.M. Fries:E.M. Fries) H.G. Winter, Dr.

L. Rabenhorst's Kryptogaman-Flora von Deutschland. Oesterreich und der Schweiz, Second edition, 1(2):473. 1885. ESphueria sepincola E.M. Fries, Observationes Mycologicae. Kjobenhavn 1:181. 1815: Systema Mycologicum Sistens Fungorum 2:-988. 1823 [as saepincola]. 三Saccothecium sepincola (E.M. Fries:E.M. Fries) E.M. Fries, Sumna Vegetabilium Scandinaviae, Seu Enumeratio. Systematica el Critica, Plantarum tum Cotyledonearum, Quam Nemearum Inter Mare Occidentale Et Album, Inter Eidoram et Nordkop, Hactenus Lectorum, una Cum Singulae Distributione Geographica, p. 398. 1849. EPringsheimia sepincola (E.M. Fries:E.M. Fries) F. v. Höhnel. Annales Mycologici. Berlin 18:97. 1920. 三Metusphaeria sepincola (E.M. Fries:E.M. Fries) P.A. Saccardo, Sylloge Fungorum Omnium Hucusque Cognitorum Digessit P.A. Saccardo 2:164. 1883 [as Fr? Fuckel]. ESphaerwlina sepincola (E.M. Fries:E.M. Fries) K. Starbäck, Botaniska Notiser. Lund 1890: 117. 1890: Botanisches Zentralblatt, Jena \& Dresden 46:261. 1891. EPleosphuerulina sepincola (E.M. Fries:E.M. Fries) H. Rehm in F. v. Höhnel. Annales Mycologici. Berlin 18:96. 1920. ESclerodothis sepincola (E.M. Fries:E.M. Fries) F. Petrak, Annales Mycologici, Berlin 19:41. 1921. On dry stems of Rosu sp., Ruhus sp., Rosaceae. Sweden. sepincola (M.J. Berkeley \& C.E. Broome) F.A. Hazslinszky, Matematikai és Természettudományi Közlemenyek Vonatkozólag a Hazaa Viszonyokra, Budapest 25(2):151. 1892. Nom. illegit. Ant 64.1. ESphueria sepincola M.J. Berkeley \& C.E. Broome, Annals and Magazine of Natural History, London, Series 2, 9:326. 1852. EMetasphaeria sepincola (M.J. Berkeley \& C.E. Broome) P.A. Saccardo, Sylloge Fungorum Omnium Hucusyue Cognitorum Digessit P.A. Saccardo 2:164. 1883. [Pro parte fide F.A.

Hazslinszky.] On herbaceous stems of Rume. sp., Polygonaceae. Great Britain.

s'preme'llukan E. Müller, Sydowia. Annales

Mycologici. Horn. N.Ö. 4(1-6):300. 1950. $\equiv$ Noduiospharia sepremcellulata (E. Müller) L. Holm. Symbolac Botanicae Upsalienses, Uppsala 14(3):86. 1957. On dry. dead stems of Buphthatmum salicifolimm L. (=Amhe'mis), Compositae. Switzerland. 
seplorariata A.M. Saccas, Agronomie Tropicale, Nogent-sur-Marne 9:271. 1954. On aerial roots of Sorghum vulgare Pers., Gramineae. French Equatorial Africa.

sequana A.L. Guyot, Revue de Mycologie, Paris 1 1:68-69. 1946. EPhaeosphaeria sequana (A.L. Guyot) R.A. Shoemaker, Canadian Joumal of Botany, Ottawa 67:1573. 1989. On dry seeds of Phlewm boehmeri Wibel., Gramineae. France.

serbica J. Schröter, Hedwigia, Dresden 29:59-60. 1890. On stems of Camphorosma monspeliaca L.. Chenopodiaceae. Yugoslavia.

seriata G. Passerini, Rendiconti della Sedute della $R$. Accademia dei Lincei, Classe di Scienze Fisiche. Matematiche e Natural (=Atti dell" Accademia Nazionale dei Lincei, Rendiconti, Roma), Series 4, 3:91. 1887. On wilted leaves of Zea mays L., Gramineae. Italy.

setosa G. Niessl v. Mayendorf, Verhandlungen des Naturforschenden Vereins in Briinn 10:178. (1871) 1872. On dried stems of Angelica sp., Pastinaca sp., Umbelliferae. Czechoslovakia (near Gratz and Brünn).

setosa (A. Leuchtmann) R.A. Shoemaker, Canadian Journal of Botany, Ottawa 67:1580. 1989. Nom. illegit. Art. 64.1. EPlicseosphaeria serosa A. Leuchtmann, Sydowia. Annales Mycologici, Horn, N.Ö. 37:159-160. 1984. On Yucca elephantipes Hort. ex Regel, Agavaceae. Mexico.

(Hendersonia?) selulosa P.A. Saccardo \& C. Roumeguère in C. Roumeguère and P.A. Saccardo, Revue Mycologique, Toulouse 3:44. 1881; Reliquiae Mycologicae Libertianae, Series Altera Reviserunt C.

Roumeguère \& P.A. Saccardo, Toulouse, No. 123. 1881. =Pocosphaeria setulosa (P.A. Saccardo \& C. Roumeguère) A.N. Berlese, Icones Fungorum Omnium Hucusque Cognitorum ad usum Sylloges Saccardianae Adcommodatae 1:89. 1892. On decayed culms of Secale sp., Gramineae. Germany.

shahvarica F. Petrak, Sydowia. Annales Mycologici, Hom, N.Ö. 3(1-6):279. 1949. On dry stems of Cousinia mulriloba DC., Compositae. Iran. shastensis R.A. Shoemaker. Canadian Journal of Botany, Ottawa 62:2712. 1984. On stems of Lupimus alhicaulis Dougl. ex Hook. var shastensis C.P. Sm. Leguminosae. U.S.A.

sibirica F. v. Thümen, 1. Moskovskoe Obshchestvo lspytatelei Prirody. Bulletin 56(2): 124. 1882. On dead stems of Crepis sibrica L.. Compositae. U.S.S.R.

sibrorpii J. Politis, Akademia Athēnōn, Pragmateiai. Athens 1935:16. 1935. On dry stems of Euphorbia siblhorpii Boiss., Euphorbiaceae. Grecese.

sicula P.A. Saccardo \& V. Beltrani, Michelia Commentarium Mycologicum Fungos in
Primis ltalicos 1llustrans 1:397. 1878. On decayed trunks of Opuntia ficus-indica (L.) Miller, Cactaceae. Italy.

sieversiae (F.E. Clements) F. Petrak, Sydowia. Annales Mycologici, Horn. N.Ö. 6(1-4):6. 1952. 三Massaria sieversiae F.E. Clements, Cryptogamae Formationum Coloradensium. Century 11I. No. 234. Anno 1906. Nom. nud. Art. 36.1. [Combination is not valid because the basionym is an invalidly published species.] On standing. dead stems of Sieversia turbinata (Rydb.) Greene. Rosaceae. U.S.A.

silenes-acaulis G. de Notaris, Commentario della Società Crittogamologica Italiana. Milan. 2:485-486. 1867. EScleropleella silenesacaulis (G. de Notaris) F. v. Höhnel. Annales Mycologici, Berlin 18:76. 1920.

$\equiv$ Phaeosphaeria silenes-acaulis (G. de Notaris) L. Holm, Symbolae Botanicae Upsalienses, Uppsala 14(3):128. 1957. On withering leaves of Silene acaulis (L.) Jacq.. Caryophyllaceae. Italy.

sileris G. Bresadola, Studi Trentini Di Scienze Naturall, Trento, Series 2. 7:66-67. 1926. On stems of Laserpitium siler L.. Umbelliferae. Italy.

silvestris J. Feltgen, Vorstudien zu einer Pilz-Flora des Grossherzogthums, Luxemburg 1(2):162. 1901. On dry stems of Silere inflata Sm.. Caryophyllaceae. Luxembourg.

simillima H. Rehm. Leaflet of Philippine Botany. Manila 6:2203-2204. 1914. On dead branches of Capparis horrida L.. Capparaceae. Philippines.

simmonsii P.A. Saccardo, Nuovo Giomale Botanico 1taliano e Bolletino della Società Botanica Italiana, Firenze. New Series 27:77. 1920. On dead stems of Heracleum lanarum Michx.. Compositae (Umbelliferae?). U.S.A.

sinapis L. Hollós, Matematikai és Természettudományi Közlemenyek Vonatkozólag a Hazai Viszonyokra, Budapest 35:34. 1926. On dry stems of Sinapis arvensis L.. Cruciferae. Hungary.

slovacica R. Picbatuer, Sbornik Výsoké Skoly Zemědĕlské a Lesnické Faculı̣ 1. Beme (Bulletin de L'Ecolo Superieure D’Agronomic Bmo. RCS, Faculte de Silviculture) 1927(D7):7-8. 1927. On sems of Ballora nigra L.. Labiatae. Czcchoslovakia.

smarodsii G. Moesz. Magyar Botanikai Lapok. Budipest 31:37. 1932. On wilted leaves of Convallaria majalis L.. Liliaceae. U.S.S.R. (Latvia).

smilacis T. Petch, Ceylon Joumal of Science. Colombo. Section A (Botany) 6:225. 1917. Nom. illegit. Art. 64.1. On branches of Smilox aspera L. Smilacaceae. Sri Lanka. 
smilucis C.E. Chardon, Farlowia. A Journal of Cryptogamic Botany, Cambridge. Massachusetus 2:466-467. 1946. Nom. illegit. An. 64.1. On dry branches ol (on leaves of?) Smilax sp.. Smilacaceac. Dominican Republic.

smilacis (J.L. Castagne) P.A. Saccardo. Sylloge Fungorum Omnium Hucusque Cognitorum Digessit P.A. Saccardo 2:69. 1883. Ephacria smilacis J.L. Castagne. Caltalogue Des Plantes qui Croissent Naturellement aux Environs de Marseille, p. 169. 1845. On dry branches of Smilax sp., Smilacaceae. France.

soria P.A. Saccardo. Michelia Commentarium Mycologicum Fungos in Primis Italicos Illustrans 2:533. 1882. EMetasplueria socia (P.A. Saccardo) P.A. Saccardo, Sylloge Fungorum Omnium Hucusque Cognitorum Digessit P.A. Saccardo 2:165. 1883. On living vines of l'itis rinifera L., Vitaceae. Italy.

socialis A.N. Berlese, Icones Fungorum Omnium Hucusque Cognitorum ad usum Sylloges Saccardianae Adcommodatae 1:63. 1890. On stems of Asparagus officinalis L., Liliaceae. Finland.

sodumaca (G. de Notaris) P.A. Saccardo, Sylloge Fungorum Omnium Hucusque Cognitorum Digessit P.A. Saccardo 2:20. 1883.

ESphaeria sodomara G. de Notaris, Commentario della Società Critıgamologica Italiana. Milan 2:485. 1867. On dried stems of Solanum sodomeum L.. Solanaceac. Italy.

solani L.G. Romell in A.N. Berlese, lcones Fungorum Omniun Hiucusque Cognitorum ad usum Sylloges Saccardianae Adcommodatae 1:63. 1890. ELeprosplaceria solanicola P.A.

Saccardo. Non Leptosphaeria solani J.B. Ellis \& B.M. Everhart. 1893. 三Melanomma solmii (L. Romel] ex A.N. Berlese) L. Holm. Symbolate Botanicae Upsalienses, Uppsala 14(3):56. 1957. On stems of Solanum dulcamara L. Solanaceae. Sweden.

whan J.B. Ellis \& B.M. Everhart. Proceedings of the Academy of Natural Sciences of Philadelphia 1893:136. 1893. Nom. illegit. Art. 64.1. On living leaves of Solammm duk amara L., Solanaceac. Canada.

solanicrola P.A. Saccardo. Sylloge Fungorum Omnium Hucusque Cognitorum Digessit P.A. Saccardo 24:991. 1928. ELeposphoreria solumi J.B. Ellis \& B.M. Everhart. Non Leprosphaseria solani L.G. Romell in A.N. Berlese. On stems of Solammm dulcamara L.. Solanaceac. Sweden.

solleimii F. Petrak, Sydowia. Annales Mycologici, Hom, N.Ö. 18(1-6):369. 1964. On dry culms of Inucus drummondii E. Mcy.. Juncaceae. U.S.A.

soliduginis (E.M. Fries:E.M. Fries) V. Cesiti \& G. de Notaris, Commentario della Società
Critloganologica Jtaliana, Milan 1:236. 1863. ESphaeria solidaginis E.M. Fries:E.M. Fries. Elenchus Fungorum Sistens Commentarium in Systema Mycologicun 2:106. 1828. On stems of Solidago sp., Compositac. France.

sopliorue E.N. Koschkeloval \& 1.I'. Frolov,

Mikotlora Podgornoi Ravinīy Kopetdaga i

Tsentral nȳkh Karakumov [Mycollora of the Mountainous Plains of Kopet Dagh and Central Kara Kum]. p. 76. 1973. Nom. inval. Art. 34.1, 36.1. On unknown substrate of Sophora jabonica L.. Leguminosae. U.S.S.R. sorbi A. Jaczewski, Annales Mycologici, Berlin

1:30. 1903. On leaves of Subus ancuparia

L., Rosiaceae. U.S.S.R

songho-armudinacei M. Luc, Revue de Mycologie

22. Paris (Supplement Colonial No. 1)

18:15-18. 1953. EPhacosphaeria sorghoarundinacei (M. Luc) R.A. Shoemaker.

Canadian Journal of Botany, Ottawa 67:1535. 1989. On leaves of Sorghum arumdinaceum Roem \& Schult., Gramineae. Canada, lvory Coast, U.S.A.

sorghophila (C.H. Peck) P.A. Saccardo, Sylloge Fungorum Omnium Hucusque Cognitorum Digessit P.A. Saccardo 2:60. 1883. ESphacria (Caulicolae) sorghophila C.H. Peck, Report. New York State Museum of Natural History, Albany 31:51. 1879; Hedwigia, Dresden 20:157. 1881. On brush of an old broom. U.S.A.

sowerbyi (L. Fuckel) P.A. Saccardo, Sylloge Fungorum Omnium Hucusque Cognitorum Digessit P.A. Saccardo 2:78. 1883. इLeptosphaeria maculans (M.J. Sowerby) P.A. Karsten, Mycologia Fennica 2:99. 1873. Non Leprosphaeria maculams. (J. Desnazières) V. Cesati \& G. de Notaris, Commentario dellat Società Critlogamologica Italiana, Milan 1:235. 1863. ESphaeria mar ulans M.J. Sowerby, Coloured Figures of English Fungi or Mushrooms 3:Table 394, Figure 9. 1803: in M.J. Berkeley and C.E. Broome. Annals and Magazine of Natural History. London. Series 2, 9:378. 1852. ESphar") "lla maculams (M.J. Sowerby) B. Auerswald in W. Gonnermamn and G.L. Rabenhorst. Mycologia Europaea. Abbildungen Sämmtlicher Pilze Europa's, Hefte 5 und 6. Synopsis Pyrenomycetum Europacorum. p. 18. 1869. EPleospora somerbi L. Fuckel. Symbolae Mycologicae. Erster Nachtralg. p. 301. 1871. Non Sphaseria maculanas J. Desmazières, Annales des Sciences Naturelles, Paris, Botanicue, Series 3,6:77-78. 1846. EP/arosphaeria somerbyi (L. Fuckel) L. Holm, Symbolae Botanicac Upsalienses, Uppsala 14(3):130. 1957. On culms of Ileleocharis palussris (L.) Roen. \& Schult. (=l:leorharis). Sehoenoplectus lacusris (L.) Palla, Scirpus lacustris L... 
Cyperaceae. Belgium, Canada, Poland, Switzerland.

sparganii (F. Fautrey) A. Munk, Dansk Botanisk Arkiv, Kjøbenhavn 17(1):376. 1957. Nom. illegit. Art. 33.2. $\equiv$ Melasphaeria sparganii $\mathrm{F}$. Fautrey, Revue Mycologique, Toulouse 13:76-77. 1891. [Basionym not completely cited as to place of publication and date.] On dead stems of Sparganium crectum L., Sparganium ramosum Hudson, Sparganiaceae. Ghana.

sparsa (L. Fuckel) P.A. Saccardo, Sylloge Fungorum Omnium Hucusque Cognitorum Digessit P.A. Saccardo 2:77. 1883. EPleospora sparsa L. Fuckel, Symbolae Mycologicae, p. 138. 1870. EPhaeosphaeria sparsa (L. Fuckel) R.A. Shoemaker, Canadian Journal of Botany, Ottawa 67:1556. 1989. On culms and leaves of Agrostis sp., Calamagrostis sp., Poa sp., Gramineae. Switzerland.

sparsa (L. Fuckel) P.A. Saccardo var. elynae A. Ade, Allgemeine Botanische Zeitschrift f. Systematik, Floristik, Pflanzengeographie, etc., Karlsruhe 30/31:22(134). 1926. On unknown substrate of Elyna spicata Schrader, Cyperaceae. Austria.

sparsa (L. Fuckel) P.A. Saccardo var. meizospora J. Feltgen, Vorstudien zu einer Pilz-Flora des Grossherzogthums, Luxemburg I(3):207. 1903. On dry leaves of Carex acutiformis Ehrh., Sparganium ramosum Hudson. Cyperaceae, Sparganiaceae. Luxembourg.

spartii F. Tassi, Bolletino del R. Orto Botanico, Siena 7:60-61. 1905. On culms and sheaths of Lygeum spartum L., Gramineae. Italy.

spartinac J.B. Ellis \& B.M. Everhart, Journal of Mycology, Columbus, Ohio 1:43. 1885. झPhaeosphaeria spartinae (J.B. Ellis \& B.M. Everhart) R.A. Shoemaker, Canadian Journal of Botany, Ottawa 67:1573. 1989. On dead culms of Spartina sp., Gramineae. U.S.A.

spatharum V. Cesati in G.L. Rabenhorst, Fungi Europaei Exsiccati, Klotzschii Herbarii vivi Mycologici Continuatio Edita Nova, Series 2, Century 26, No. 2530. Anno 1881. Nom. nud. Art. 32.1. $\equiv$ Metasphacria spartharum (V. Cesati) ex P.A. Saccardo, Syltoge Fungorum Omnium Hucusque Cognitorum Digessit P.A. Saccardo 2:179. I883. On spath of Chamaerops crcelsior Boj. Palmae. Italy.

spectabilis G. Niessl v. Mayendorf, Verhandlungen des Naturforschenden Vereins in Brünn 10:179-180. (1871) 1872. On dried stems of Laserpitium sp., Umbelliferac. Austria (near Vienna).

spegazzini P.A. Saccardo \& P. Sydow, Sylloge Fungorum Omnium Hucusque Cognitorum Digessit P.A. Saccardo 14:570. 1899. $\equiv$ Leptosphaeria sacchari C.L. Spegalızini. Nec Leprosphacria sacchari H.J. Van Breda.
On wilting or drying leaves and sheaths of Saccharum officinarum L.. Gramineae. Argentina.

spegazzini P.A. Saccardo \& P. Sydow var. minor C.L. Spegazzini, Anales del Museo Nacional de Historia Natural de Buenos Aires 19(Series 3, 12):383. 1909; Mycetes Argentinenses, Series 4, p. 383, No. 629. 1909. On culms of Saccharum officinarum L., Gramineae. Argentina.

sphyridiana (J.G. Lahm) H.G. Winter in Dr. L.

Rabenhorst's Kryptogaman-Flora von Deutschland, Oesterteich und der Schweiz 1(2):442-443. 1887. (As Leptosphaeria?). $\equiv$ Xenosphaeria sphyridiana J.G. Lahm in $\mathrm{F}$. Amold, Flora, Jena und Regensburg 57:150. 1874. On thallus of Sphyridium fungiforme Flotow [=Baeomyces rufus (Huds.) Rebent.]. Lichenes (Baeomycetaceae). Austria, Germany

spiraeae P.A. Karsten, Hedwigia, Dresden 27:260. 1888. On dry fallen branches of Spiraea sorbifolia L. (=Sorbaria sorbifolia A. Br.). Rosaceae. Finland.

sporoboli J.B. Ellis \& B.T. Galloway, Joumal of Mycology, Columbus, Ohio 5:66. 1889. On dead culms of Sporobohis depanperauns Torr. ex Hems]. [=Muehlenbergia richardsonis (Trin.) Rydb.], Gramineae. U.S.A. squamata (M.C. Cooke \& J.B. Ellis) J.B. Ellis. Catalogue of Plants Found in New Jersey. Geological Survey of New Jersey. Final Report of the State Geologist. Trenton 2(1):525. 1889. SSphaeria (Obtectae) squamata M.C. Cooke \& J.B. Ellis. Grevillea. London 7:10. 1878. On bark of dead pine limbs of Pinus sp., Pinaceae. U.S.A. (New Jersey).

staritzii P.C. Hennings in R. Staritz. Verhandlungen des Botanischen Vereins Der Provinz Brandenburg. Berlin 55:78. 1913. On dead stems of Armeria vulgaris Willd.. Plumbaginaceae. Germany.

staritzii R.A. Shoemaker, Canadian Joumal of Botany, Ottawa 62:2713. 1984. Nom. illegit. Art. 64.1. On stems of Achillea millcfolium auct. Compositac. Germany.

staticicola L.M. Unamuno Y'rigoyen. Asociación Espiñola Para el Progresso de las Ciencias. Congreso de Coimbra 1923:40. 1923. On dry leaves of Statice occidentalis Lloyd $(=A \mathrm{rm}$ cria). Plumbaginaceae. Spain.

stcinii (G.W. Koerber) ex H.G. Winter. Dr. L. Rahenhorst's Kryptogaman-Flora von Deutschland, Oesterreich und der Schweiz. Second edition. 1(2):443. 1585. (A) Leptosphacria?). ELcptorhuphis steinii G.W. Koerher. Jalhresberiche der Schlesischen Gesellschaft für vaterländiscle Kultur. Breslau 46:91. I\$69. Nom. nud. Art. 32.1. 三Ophoholus stcinii (G.W. Koerher) A.N. 
Berlese \& P. Voglino, Sylloge Fungorum Omnium llucusque Cognitorum Digessit P.A. Saccardo Additamenta Ad Volumina I-IV, p. 189. 1886. On thallus of Leconora frustulosa (Dicks.) Ach., Lichenes (Lecanoraceae). Czechoslovakia, Germany.

steironemanis J.B. Ellis \& B.M. Everhart, Proceedings of the Academy of Natural Sciences of Philadelphia 1890:237. (1890) 1891. On dead stems of Steironema cilianm (L.) Ratin. (=Lysimachia ciliata L.), Primulaceae. Canada.

stellariac C.E. Richon, Catalogue Raisonné Des Champignons Qui Croissent Dans le Département de la Marne. p. 299. 1889. Nom. illegit. Art. 64.1. ELeptosphaeria richoni P.A. Saccardo \& P. Sydow. Sylloge Fungorum Omnium Hucusque Cognitorum Digessit P.A. Saccardo 14:571, 1899. On old stems of Stellaria media (L.) Vill., Caryophyllaceae. France.

stellariae E. Rostrup, Meddelelser om Gronland. Kjobenhavn 3:557. 1888. EPhaeosphueria stellariae (E. Rostrup) A. Leuchtmann. Sydowia. Annales Mycologici, Horn, N.Ö. 37:157. 1984. On leaves of Sicllaria humifusa Roub.. Caryophyllaceae. Greenland.

stellata N.T. Patouillard in N.T. Patouillard and N.G. de Lagerheim. Bulletin de la Société Mycologique de France. Paris 11:225. 1895. On dead stems of Chusquea sp. Gramineae. Colombia.

stereicola J.B. Ellis. American Naturalist, Lancaster, Pennsylvania 17:317. 1883. On hymenium of Sterenm hicolor (Pers.) Pers. [=Laxire.rum bicolor (Pers.). Lentz]. Fungi (Stereaceae). U.S.A.

stereocaulorum F. Amold. Flora, Jena und Regensburg 57:175. 1874. EMerasphaeria stereocuulorum (F. Anold) P.A. Saccardo, Sylloge Fungorum Omnium Hucusque Cognitorum Digessit P.A. Saccardo 2:183. 1883. Parasitic on thallus of Stereocanton alpinum Laur., Lichenes (Stercocaulaceae). ltaly.

stic fa J.B. Ellis \& B.M. Everhart, Journa! of Mycology, Columbus, Ohio 1:43. 1885. On dead culms of Spartina sp.. Gramineae. U.S.A.

stictoides (M.J. Berkeley \& M.A. Curtis) P.A. Saccardo, Sylloge Fungorum Omnium Hucusque Cognitorum Digessit P'.A. Saccardo 2:86. 1883. ESphacria stictoides M.J. Berkelcy \& M.A. Curtis in M.J. Bcrkeley, Grevillea, London 4:149. 1876. On branches of Liriodendron sp. Magnoliace:te. U.S.A. sticrostoma (M.J. Berkeley \& M.A. Curtis) P.A. Saccardo. Sylloge Fungorum Onınium Hucusque Cognitorum Digessit P.A. Saccardo
2:84. 1883. ESphaeria sticiessoma M.J. Berkeley \& M.A. Curtis in M.J. Berkeley, Grevillea, London 4:151. 1876. On stems of herbaccous plant of unhnown host, unknown family. U.S.A.

stipar-minor L. Lacoste, Revue de Mycologie 22. Paris (Supplement Colonial No. 1) 22:12-13. 1957. On leaves of Stipa tenacissima L., Granincac. Tunisia.

stipae L. Trahur, Etude sur L'Halfa (Stipa e'enacissima) Alger (Adolphe Jourdan...). p. 48. 1889. [Lepesphehacria pampaniniana Sacc. may be a synonym.] On decayed leaves of Stipa senacissima L.. Gramineae. Algeria.

stipa A.l. Lobik, Materialȳ po Floristicheskim i Faunisticheskim Obsledovaniyam Terskogo Okruga [Data from Investigations on the Flora and Fauna of the Ter Region], p. 24. 1928. Nom. inval. Art. 36.1. On leaves of Stipa cupillata L., Gramineae. U.S.S.R.

straminis M.C. Cooke \& H.W. Harkness, Grevillea. London 14:10. 1885. =Pleospora harknessii A.N. Berlese \& P. Voglino. Sylloge Fungorum Omnium Hucusque Cognitorum Digessit P.A. Saccardo Additamenta Ad Volumina I-IV. p. 174. 1886. Nec Pleospora straminis P.A. Saccardo \& C.L. Spegazzini. On culms of straw, Gramineae. U.S.A.

strutiotis C.A. Oudemans. Beihelte zum Botanischen Zentralblatt, Cassel 11:528. 1902. On leaves of Stratiotes aloides L., Hydrocharitaceae. Netherlands.

striata H.G. Winter, Hedwigia, Dresden 11:147. 1872. On dry stems of Veronica latifolia aucl., Scrophulariaceae. Germany.

striolata G. Passerini. Atti della R. Accademia dei Lincei Memoriae. Rome, Series 4, No. 6, p. 459. 1890; Diagnosi Di Funghi Nuovi. Nota IV. p. 5. 1890. EMycotodea striolata (G. Passerini) W. Kirschstein, Kryptogamenllora der Mark Brandenburg und Angrenzender Gebiete herausgegeban von dem Botanischen Verein der Provinz Brandenburg). Leipzig 7(3):434. 1938. On dry stems of Scirpus holoselioemis L.. Cyperaceae. Italy.

striolata G. Passerini var. caricis-glancac L.M. Unamuno Yrigoyen, Anales del Jardin Botánico de Madrid 2:43-44. 1942. On dry leaves of Cares glanca Scop.. Cyperaceae. Spain.

stromatoidea F.A. Hazslinszky. Matematikai én Természettudományi Közlemenyek Vouatkozỏlag a Hazai Viszonyokra, Budapesı 25(2):135. 1892. On culms of Festuca sp.. Gramineate. Hungary.

smaedae C.G. Hlansford, Proceedings of the Linnean Society of New South Wales, Sydncy 82:215. 1957. On dead branches of Sucrecta australis Moq., Chenopodiaceae. Australia.

suhalpina F. Bubák. Botanikai Közlemények, Budapest 14:(59). 1915. EPhaeosphoreria 
subalpina (F. Bubák) R.A. Shoemaker, Canadian Journal of Botany, Ottawa 67:1573. 1989. On crack (cleft) of sheaths of Phragmites communis Trin., Gramineae. Yugoslavia.

subarticulata (F. Arnold) K. Keissler, Dr. L. Rabenhorst's Kryptogamen-Flora von Deutschland, Oesterreich und der Schweiz, Second edition, 9(1 Abt., Teil 2):373. 1937. $\equiv$ Sagedia subarriculara F. Amold, Verhandlungen der Zoologisch-Botanischen Gesellschaft in Wien 21:1133-1134. 1871. $\equiv$ Segestria subarticulata (F. Amold) Dalla Torre \& L. Sarntheim, Flora der Gefürsteten Grafschaft Tirol. des Landes Vorarlberg und des Fürstenthumes Liechtenstein 4:568. 1902. $\equiv$ Porina subarticulara $(\mathrm{F}$. Amold) G. Lettau. Hedwigia, Dresden 52:105. 1912. On thallus of unknown host. unknown family [originally described as a lichen]. Italy.

subcuespitosa M.C. Cooke \& H.W. Harkness,

Grevillea, London 14:10. 1885. On stems of Geranium sp., Geraniaceae. U.S.A.

subcompressa J.H. Miller \& M.G. Burton, Mycologia, Lancaster, Pennsylvania 34:4. 1942. On dead culms of Andropogon glomerutus (Walt.) B.S.P., Gramineae. U.S.A.

subconica (G.W. Clinton \& C.H. Peck) P.A.

Saccardo, Sylloge Fungorum Omnium

Hucusque Cognitorum Digessit P.A. Saccardo 2:15. 1883. ESphaeria (Cauticolae) subconia G.W. Clinton \& C.H. Peck, Report. New York State Museum of Natural History, Albany 29:65. 1878. On dead stems of herbs. unknown host. unknown family. U.S.A.

subcutanea (M.C. Cooke \& J.B. Ellis) J.B. Ellis in

N.L. Britton, Catalogue of Plants Found in New Jersey. Geological Survey of New Jersey, Final Report of the State Geologist. Trenton 2(1):525. 1889. ESphaeria (suhtectac) subcutunca M.C. Cooke \& J.B. Ellis, Grevillea, London 7:41. 1878. 三Metasphaeria subcutanea (M.C. Cooke \& J.B. Ellis) P.A. Saccardo, Sylloge Fungorum Omnium Hucusque Cognitorum Digessit P.A. Saccardo 2:167. 1883. EComisphaeria subcutanea (M.C. Cooke \& J.B. Ellis) M.C. Cooke. Grevillea, London 16:87. 1888. EEndophluea subculanea (M.C. Cooke \& J.B. Ellis) M.C. Coohe. Grevillea, London. 17:89. 1889. On decorticated limbs of Pyrus communis L., Rosaceac. U.S.A. (New Jersey).

subiculffera? C.L. Spegazzini. Anales del Nuseo Nacional de Historia Natural de Buenos Aires 6:283. 1898: Fungi Argentini Novi vel Critici. No. 630. Anno 1899. On decaying leaves of Gymerium argenteum Nees, Gramincae. Argentina.

sublumosa (M.C. Cooke) J.13. Ellis in N.L. Britton, Catalogue of Plants Found in New Jersey. Geological Survey ol New Jersey, Final
Report of the State Geologist. Trenton 2(1):525. 1889. ESphaeria (Oblectae) sublanosa M.C. Cooke in M.C. Cooke and J.B. Ellis, Grevillea, London 7:41. 1878. On decorticated parts of Andromeda sp.. Ericaceae. U.S.A. (New Jersey).

submaculans L. Holm, Symbolae Botanicae Upsalienses, Uppsala 14(3):38. 1957. On dry stems of Berteroa incana (L.) DC., Envimum cheiranthoides L.. Sisymhrium loeselii L.. Cruciferae. Germany.

submodesfa E. Muller, Sydowia. Annales Mycologici. Horn. N.Ö. 5(3-6):53-54. 1951. On dead stems of Tofieldia calyculuta (L.) Wahlenb. Liliaceae. Switzerland.

subriparia V. Mouton. Bulletin. Société R. de

Botanique de Belgique. Bruxelles 39:41. 1900. On stalks of Juncus glaucus Sibth.. Juncaceae. Belgium.

subsimilis (S. Schulzer v. Müggenburg \& P.A.

Saccardo) F.A. Hazslinszky. Matematihai és

Természettudományi Közlemenyek Vonatkozólag a Hazai Viszonyokra. Budapest 25(2):151. 1892. EMetusphacria suhsimilis S. Schulzer v. Müggenburg \& P.A. Saccardo. Revue Mycologique. Toulouse 6:70. 1884. On branches of Carpinus sp., Betulaceae. Yugoslavia.

substerilis C.H. Peck. Bulletin of the Neu York State Museum. Albany 105:24-25. 1906. On living leaves of Mentha piperita L... Labiatae. U.S.A.

subsuperficialis P.A. Saccardo \& P. Sydow in P.A.

Saccardo. Sylloge Fungorum Omnium

Hucusque Cognitorum Digessit P.A. Saccardo 16:516. 1902. \#Monagnula subsuperficialis

(P.A. Saccardo \& P. Sydow) R.A. Shoemaher. Canadian Journal of Botany. Ottawa 67:1591. 1989. $\equiv$ Leprosphaeria melanommoides C.L. Spegazzini. On fallen rotten culms of Panicum gltumosum Nees, Granineae. Argentina.

suhteck H.G. Winter, Hedwigia, Dresden 11:146.

1872. On dry leaf of Erica curnea L..

Ericaceate. Italy.

suffula (C.G. Nees I. Esenteck:E.MI. Fries) G

Niessl $\because$. Mayendorl in G.L. Rabenhorn.

Fungi Europaei Exsiccari. Klotzschii Herbarii vivi Mycologici Continuatio Edita Nova.

Series 2. Century 16. No. 1549. Anno 1872 ESpharia suffuhla C.G. Neew I. Esenbeck:

E.M. Fries. Das System der Pilze und

Schwämme. p. 316. 1816: E.M. Frics.

Systema Mycologicum Sistens Fungorum

2:508-509. 1823. On dry stems ol Melumpynum protense' L., Scrophulariacene. Austria. Italy.

superficiulis F.A. Hazslinszh! Matematıhai és Természettudományi Kiozlemenyeh V'onathozóliag a Itazai Viszonyokra, Budapest 25(2):146. 1892. On unhnown substrate of Porenilla argentea L.., Rosaceac. Hungar. 
surculorm W. Kirschstein. Amnales Mycologici, Berlin 33:212-213. 1935. On hard. living. annual shoots of Lomicera talarica L., Caprifoliaceae. Germany.

swerrice M.J. Thirumalachar, Bulletin of the Torrey Botanical Club (and Torreya), New York 75:176. 1948. Nom. nud. Arts. 32.1 and 34.1. On leaves of Sirertia sp.. Gentianaceae. India.

sydowiona H. Rehm in P. Sydow, Mycotheca Marchica, Century 15, No. 1456. Anno 1887. Nonı. nud. Art. 32.J. On unknown substrate of Artemisia anmua L., Compositae. Germany.

sydowiama H. Rehm in P. Sydow, Mycotheca Marclica, Century 15, No. 1484. Anno 1887. Non. nud. Art. 32.1. On unknown substrate of Aremisia campesfris L., Compositae. Germany.

sydowiana H. Rehm in P. Sydow, Mycotheca Marchica, Century 9, No. 849. Anno 1885. Nom, nud. Art. 32.1. On unknown substrate of Artemisia campestris L., Compositate. Germany.

syliatica G. Passerini in G.L. Rabenhorst. Hedwigia, Dresden 16:118. 1877: Fungi Europaei

Exsiccati. Klotzschii Herbarii vivi Mycologici Continuatio Edita Nova, Series 2, Century 23. No. 2235. Anno 1877. EPhaeosphaeria silvatica (G. Passerini) A. Hedjaroude, Sydowia. Annales Mycologici. Horn, N.Ö. 22(1-4):91. (1968) 1969. On leaves of Brachypodium sylvaticum (Hudson) Beauv.. Gramineae. Jtaly, Switzerland, Turkey. taichungensis J. Yen, Bulletin de la Société Mycologique de France, Paris 89:67-69. 1973. On leaves of Musa sp., Musaceate. Taiwan (Puli).

tainanensis W.Y. Yen \& C.C. Chi, Journal of Sugar Cane Research, Taiwan 6:213. 1952

EDidynclla tainamensis (W.Y. Yen \& C.C. Chi) R.A. Shoemaker, Canadian Journal of Botany, Ottawa 67:1576. 1989. On decaying leaves of Saccharum officinarum L.. Gramineac. Taiwan

tamuricis L. Hollós, Botanikai Közlemények, Budapest 25:130. 1928. Nom. illegit. Art. 64. I. ELeprosphaeria hollosii G. Moesz. On dry branches of Tamarix gallica L.. Tamaricaccae. Hungary.

lamaricis (R.K. Greville) P.A. Saccardo, Sylloge Fungorum Omnium Hucusque Cognitorum Digessit P.A. Saccardo 2:26. 1883. 三Cryplosphareria tamaricis R.K. Greville, Scontish Crypooganic Flora I. Fascicle 9. Tabic 45. 1823. [Text unpaginated; as tamariscimis.] ESphaeria fumaricis (R.K. Greville) F. Currey. Transactions of the Linnean Socicty of London 22:324. 1859 [as tamariscinis). On dead brancles of Tamarix germanica L.. Tamaricaceac. Great Britain.
(Metaspharvia) raminensis H. Wegelin, Miltcilungen der Thurgauischen Naturlorschenden Gesellschaft, Frauenfeld 12:173. 1896. $\equiv$ Trichumetasphaeria aminensis (]).

Wegelin) L. IJolm. Symbolae Botanicae Upsalienses, Uppsala 14(3):142. 1957. On dried culms of Festucasp. Gramineae. Switzerland.

tanaceli (L. Fuckel) L. Holm, Symbolac Botanicae Upsalienses, Uppsala 14(3):49. 1957. Nom. illegit. Art. 64.1. IA later homonym of Leprosphateria tanaceri A. Jaczewski.] $\equiv$ Sphaerwlina tamaceri L. Fuchel, Jalurhuch des Nassauischen Vereins für Naturhunde, Wieshaden 15:77-78. 1860 [as Sphacrolinal. $\equiv$ Ohiobolus tanaceri (L. Fuckel) P.A.

Saccardo. Sylloge Fungorum Omnium Hucusque Cognitorum Digessit P.A. Saccardo 2:348. 1883. On wilted leaves of Achillea sp., Anthemis rinctoria L., Chrysanthemum lewcanhemum L.. Chrysanhemum vulgare (L.) Bernh., Compositae. Austria, Gernany. tanaceri A. Jaczewski, Bulletin de la Société Mycologique de France. Paris 9:217. 1893. On stems of Tanacetum vilgare L., Compositae. U.S.S.R.

tarlarina? (W. Nylander) W. Zopf, Hedwigia. Dresden 35:341. 1896. 三Verracaria lamarina W. Nylander, Flora, Jena und Regensburg 57:318. 1874. On thallus of Oclrolectrio artarea (L.) Massal, Lichenes (Pertusariaceae). Unknown country.

taurica N.A. Naumov \& T.L. Dobrozrakova, Materialy po Mikologii i Fitopatologii Rossi. Petrograd 8(2):134. 1929. On dry stems of Ceplialaria uralensis (Murray) Roemer \& Scultes, Dipsacaceae. U.S.S.R.

aricola (C.H. Peck) P.A. Saccardo, Sylloge

Fungorum Omnium Hucusque Cognitorum

Digessit P.A. Saccardo 2:85. 1883.

ESphaeria lavicola C.H. Peck, Report. New York State Museum of Natural History. Albany 24:99. 1872. EMetasphaeria iavicola (C.H. Peck) C.H. Pech, Report. New York State Museum of Natural History, Albany 39:58. 1886. [Combination not formerly proposed.] ESphaerulina taricola (C.H. Peck) A.N. Berlese, Icones Fungorum Omnium Jucusque Cognitorum ad usum Sylloges Saccardianae Adcommodatic 1:125. 1894. ESacrollecium laxicolum (C.11. Peck) W. Kirschstein, Kryptogamenflora der Mark Brandenhurg und Angrenzender Gebicte herausgegehen von dem Botanischen Verein der Provinz Brandenhurg. Leipzig 7(3):427. 1938. EDothiora fuvicola (C.H. Pech) M.E. Barr, Contributions from the University of Michigan Herbarium, Ann Arbor 9:575. 1972 On dead leaves of Taxus camadensis Marsh. Taxiceac. U.S.A. 
Ienera J.B. Ellis, Bulletin of the Torrey Botanical Club (and Torreya), New York 8:124-125. 1881 [as Leptosphaeria tenera (J.B. Ellis) P.A. Saccardo in Sylloge Fungorum Omnium Hucusque Cognitorum Digessit P.A. Saccardo 2:39. 1883]. On dead herbaceous stems of unknown host, unknown family. U.S.A.

renuis E. Müller, Sydowia. Annales Mycologici, Horn, N.Ö. 4(1-6):221. 1950. On dead stems of Thalictrum aquilegifolium L., Ranunculaceae. Switzerland.

tephrosiac (M.C. Cooke \& J.B. Ellis) P.A. Saccardo, Sylloge Fungorum Omnium Hucusque Cognitorum Digessit P.A. Saccardo 2:27. 1883. ESphaeria (Obtectae) tephrosiae M.C. Cooke \& J.B. Ellis, Grevillea, London 7:10. 1878. On stems of Tephrosia virginiana (L.) Pers., Leguminosae. U.S.A.

temata F. Hazslinszky, Matematikai és Természettudományi Közlemenyek Vonatkozólag a Hazai Viszonyokra, Budapest 25(2): 150. 1892. On branches of Pyrus malus L. (=Malus domestica Borkh.), Rosaceae. Hungary.

tetonensis L.E. Wehmeyer, Lloydia: a quarterly joumal of biological science, Manasha 9:234. 1946. Nom. illegit. Art. 64.1. 三L. jacksonensis R.A. Shoemaker.

tetonensis (J.B. Ellis \& B.M. Everhart) H. Rehm, Annales Mycologici, Berlin 9:289. 1911. $\equiv$ Mclanomma tetonensis J.B. Ellis \& B.M. Everhart, Proceedings of the Academy of Natural Sciences of Philadelphia 1890:240. 1890. $\equiv$ Melanomma occidentale (J.B. Ellis) P.A. Saccardo var. tetonensis J.B. Ellis \& B.M. Everhart, The North American Pyrenomycetes. A Contribution to Mycologic Botany, p. 183. 1892. ESyncarpella ietonensis (J.B. Ellis \& B.M. Everhart) M.E. Bar \& J.R. Boise, Memoirs of the New York Botanical Garden, Bronx 49:303. 1989. On stems of Artemisia frigida Willd., Compositae. U.S.A. (Califomia, Colorado, Montana, Utah).

tctraspora A.M. Saccas, Étude de la Flore Cryptogamique des Caféiers en Afrique Centrale.

Bulletin de Institut Français du Café du Cacao et d'Autres Plantes Stimulantes (Bulletin IFCC No. 16), pp. 260-262. 1981. Nom. inval. Art. 37.1. On dead branches of Coffea robussa L. Linden (=Coffea cancpliora Pierre ex Froehn.), Rubiaceae. Central African Republic.

esucrii (P.L. Crouan \& H.M. Crouan) P.A. Saccardo. Sylloge Fungorum Omnium Hucusque Cognitorum Digessit P.A. Saccardo 2:85. 1883. 三Sphacria I'writi P.L. Crouan \& H.M. Crouan, Florule Du Finistère Contenant Les Descriptions De 360 Espèces Nouvelles De Sporogames, De Nombreuses Observations et une synonymie des plantes Cellulaires et Vasculaires Qui Croissent Spontanément Dans ce Département. p. 28. 1867. On dead stems of Teucrium scorodonia L.. Labiatae. France.

Ihalictri H.G. Winter, Hedwigia, Dresden 11:147. 1872. 三Mctasphacria thaliciri (H.G. Winter) P.A. Saccardo, Sylloge Fungorum Omnium Hucusque Cognitorum Digessit P.A. Saccardo 2:156. 1883. EScleropleella thalictri (H.G. Winter) F. v. Höhnel, Annales Mycologici, Berlin 18:76. 1920. EBuergenerula thalictri (H.G. Winter) E. Müller, Sydowia. Annales Mycologici. Hom, N.Ö 4(1-6):307. 1950. On dry stems of Thalictrum aquile gifolium L.. Ranunculaceae. Italy.

thalictricola L. Hollós, Annales Historico-Naturales Musei Nationalis Hungarici, Budapest 7:51-52. 1909. On dry stems of Thalictrum collinum Wallr., Ranunculaceae. Hungary.

thalictrina L. Hollós, Matematikai és Természettudományi Közlemenyek Vonatkozólag a Hazai Viszonyokra, Budapest 35:34. 1926. On dry stems of Thalictrum aquilegifolium $\mathrm{L}$..

Ranunculaccae. Hungary.

theohromicola R. Ciferri \& R. González Fragoso.

Boletin de la R. Sociedad Española de

Historia Natural, Madrid 26:474-475. 1926:

Publicaciones Estación Agronómica de Moca. Santa Domingo. Series B. Botanica 8:23.

1927. Nom. nud. An. 34.1b. On living leaves of Theobroma cacao L.. Sterculiaceae.

Dominican Republic.

theroplita B. Auerswald in W. Gonnermann and

G.L. Rabenhorst. Mycologia Europaea.

Abbildungen Sämmtlicher Pilze Europa ‘.

Hefte 5 und 6. Synopsis Pyrenomycetum

Europaeorum. Table 12. Figure 158. 1869.

Nom. nud. Art. 32.1. =Sphaeria (Canlicola) therophila J. Desmazières, Annales des

Sciences Naturelles, Paris. Botanique. Series

3, 16:310. 1851. =Phomatospora therophila

(J. Desmazières) P.A. Saccardo. Sylloge

Fungorum Onnium Hucusque Cognitorum

Digessit P.A. Saccardo 1:433. 18S2. On dry culms of .luncus arriculatus L.. Juncaceae. France.

hiclensii (G.D. Westendorp) P.A. Saccardo, Sylloge Fungorum Omnium Hucusque Cognitorum

Digessit P.A. Saccardo 2:46. 1883. $\equiv P / e$ ospora sliclensii (G.D. Westendorp) J.B. Lambote, Flore Mycologique de la Belgique. Comprenant la description des espèces trouvées jusqu a ce Jour. 2:274-275. 1880. $\equiv$ Sphacria thichensii G.D. Westendorp. Bulletin. Société R. de Botanique de Belgique, Bruxelles 5:37. 1866. On dead stems of Tunacetum vilgare L.. Compositae. Belgium. 
thomasiana P.A. Saccardo \& C. Rouneguère. Revue Mycologique, Toulouse 5:236. 1883. On dead runners of Ruhus sp., Rosaceac. France.

thorac O. Jaap, Annales Mycologici, Berlin 6:210. 1908. On living leaves of Ramunculus thora L.. Ranunculaceae. Austria.

Ihuemeniana G. Niessl v. Mayendorf in F. v. Thümen, Instituto. Revista Scientifica e Litteraria. Coimbra 28:29. 1881; Contributiones ad Floram My'cologicam Lusitanicam. Series 3. p. 29. 1881. EHeptameria thuemeniana (G. Niessl v. Mayendorf) P.A. Saccardo. Sylloge Fungorum Omnium Hucusque Cognitorum Digessit P.A. Saccardo 2:89. 1883. On somewhat rotten stems of Amirrhimum mujus L., Scrophulariaceae. Portugal. thujuecola K. Hard. Reports of the Shizuokaken Prefecture Agricultural Society 34:(49)-(50). 1930. [In Japanese.] On leaves of Thuja oriemalis L., Cupressaceae. Japan.

thurgoviensis E. Müller, Sydowia. Annales Mycologici, Horn, N.Ö. $f(1-6): 280.1950$. =Massariosphaeria thurgoviensis (E. Müller) R.A. Shoemaker, Canadian Joumal of Botany, Ottawa 67:1589. 1989. On dead grass culms of unknown host of Gramineae. Gramineae. Switzerland.

thyphicela G. Passerini \& V. Beltrani, Atti dell' Accademia Nazionale dei Lincei. Transunti, Rome, Series 3. 7:36. (1882) 1883. ELeptosphaeria licasensis P.A. Saccardo. Non P.A. Karsten. On dry leaves of Typha latifolia L.. Typhaceae. Italy (Sicily).

figrisoides K. Hara, The Diseases of Trees (ZikkenZyumoku-Byogaihen), p. 331. 1927. On unknown substrate of Bambusa sp., Gramineae. Japan.

timi J.B. Ellis \& B.M. Everhan, Journal of Mycology, Columbus, Ohio 4:64. 1888. On leaves of I ibumum timus L.. Caprifoliaceae. U.S.A. tiroliensis W. Kirschstein. Annales Mycologici, Berlin 33:213. 1935. On dry branches of Sambucus racemosu L.. Caprifoliaceae. Austria.

wfieldiae E. Müller. Sydowia. Annales Mycologici. Hom. N.Ö. 5(3-6):53. 1951. EPhaeosphacria iofieldiae (E. Müller) A. Leuchtmann, Sydowia. Annales Mycologici. Hom. N.Ö. 37: 1 10. 1984. On leaves of Toficldia calvilula (L.) Wahlenb., Liliaceae. Switzerland.

(u)/gorensis F. Petrak. Sydowia. Annales Mycologici, Horn. N.Ö. 3(1-6):281. 1949. On dry stems of Euphorbia sp. Euphorbiaceae. Iran.

Iomphinsii A.S. El-Ani. Mycologia. Lancaster. Pennsylvania 58:409. 1966. On $/ 1 \mathrm{omo}$ sapiens, Ilominidae. Mauritania.

esnduzi C.L. Spegazzini. Boletin de la Academia Nacional de Ciencias en Córdoba 23:562.
1919: Reliquiat Mycologicae Tropicae et Fungi Costaricenses Nonnulli, pp. 200-201. 1919. On living leaves of Coffea sp.. Rubiaceae. Costa Rica.

torbolensis W. Kirschstein, Annales Mycologici. Berlin 37:108. 1939. On dry stems of Scrophularia camina L.. Scrophulariaceae. Italy.

Iormutospora T. Petch, Ceylon Journal of Science. Colombo, Section A (Botany) 9:319.1925. On dead twigs of $C$ amelliu theifera Grilf., Theaceae. Sri Lanka.

romendii G.B. Traverso \& C. Spessa. Boletim da Sociedade Broteriana, Coimbra 25:171. 1910 [as correndi]. On dry hranches of Ricimus communis L., Euphorbiaceac. Portugal.

iorulispora (M.C. Cooke) P.A. Saccardo. Sylloge

Fungorum Omnium Hucusque Cognitorum Digessil P.A. Saccardo 2:42. 1883. ESphaeria (Caulicolae) torulaespora M.C. Cooke. Joumal of the Linnean Society (Botany) London 17:144. 1878. On herbaceous stems of unknown host, unknown fumily. U.S.A.

Irans/ucenss H.G. Winter. Boletim da Sociedade Broteriana. Coimbra 2:42. (1883) 1884. On dry leaves of Furcraea sp.. Labiatae. Portugal.

treatiana P.A. Saccardo. Sylloge Fungorum Omnium Hucusque Cognitorum Digessit P.A. Saccardo 10:923. 1892 [in a footnote]. Nom. illegit. Art. 63.1.

fremarostuma J. Feltgen, Vorstudien zu einer PilzFlora des Grossherzogthums. Luxemburg 1(3):214. 1903. On dry barked branches of Syinga in/garis L.. Oleaceae. Luxembourg.

rrewae C.L. Spegazzini, Fungi Chilenses, p. 79. 1910: Revista de La Facultad de Agronomia Y Veterinaria, Universidad Nacional de Lil Plata. Series 2, 6:79. 1910. On dead branches ol Treva trineria Gill. \& Hook., Rhamnaceale. Chile.

wrichoperygis C.G. Hansford, Proceedings of the Linnean Society of London 153:25. 1941. On dead culms of Trichoplery $<$. [as $T$. afiroflammida], Gramineae. Uganda.

michostoma G. Passerini in F. v. Thümen. Mycotheca Universalis, Century 15. No. 1455. Anno 1879: Flora, Jena und Regensburg 63:324. 1880. EMetasphastia richostoma (G. Passcrini) P.A. Saccardo, Sylloge Fungorum Omnium Hucusque Cognitorum Digessit P.A. Saccardo 2:158. 1883. On stems and hranches of Chomdrilla juncea $\mathrm{L}$. Compositite. Italy.

mifolii J. Feltgen. Vorstudien zu einer Pilz-Flora des Grossherzogthums, Luxemburg 1(.3):210. 1903. On dry stems of Trifolium medium L.. leguminosae. Luxembourg. 
wifolii-alpestris T. Dominick, Acta Societatis Botanicorum Poloniae, Warszawa 1 1:239-240. 1934. On living leaves of Trifolium alpestre L., Leguminosae. Poland.

triglochinicola (F. Currey) P.A. Saccardo, Sylloge Fungorum Omnium Hucusque Cognitorum Digessit P.A. Saccardo 2:69. 1883. EShaeria triglochinicola F. Currey, Transactions of the Linnean Society of London 24:158-159. (1863) 1864. EPhacosphaeria trighochinicola (F. Currey) A. Leuchtmann, Sydowia. Annales Mycologici, Horn, N.Ö. 37:1 11. 1984. On carpels and stems of Triglochin palustris L., Juncaginaceae. Great Britain, Switzerland.

triglochinis J. Schröter, Kryptogamen-Flora Von Schlesien. Im Namen Der Schlesischen Gesellschaft für vaterländische Cultur herausgegeben von Prof. Dr. Ferdinand Cohn. Secretair der Botanischen Section, Breslau 3(2):366. 1894. On dead stems of Triglochin palustris L.. Juncaginaceae. Poland.

rimera P.A. Saccardo. Nuovo Giomale Botanico Italiano e Bolletino della Societá Botanica Italiana. Firenze 7:319. 1875. On dead stems of Andropogon ischaemum L., Gramineae. ltaly.

trimerioides H. Rehm, Annales Mycologici, Berlin 13:2. 1915. On stems of Liatris scariosa (L.) Willd., Compositae. U.S.A.

tritici (S. Garovaglio) G. Passerini in G.L. Rabenhorst, Fungi Europaei Exsiccati, Klotzschii Herbarii vivi Mycologici Continuatio Edita Nova, Series 2, Century 24, No. 2333. Anno 1876. 三Pleospora tritici S. Garovaglio, Rendiconti dell' Istituto Lombardo di Scienze e Lettere, Milano, Series 2, 6:61 1-612. 1873. $\equiv$ Leptosphacria eustoma (E.M. Fries:E.M. Fries) P.A. Saccardo forma tritici (S. Garovaglio) A.N. Berlese, Icones Fungorum Omnium Hucusque Cognitorum ad usum Sylloges Saccardianae Adcommodatae 1:56. 1892 [as (Garov.) Pass.]. 三Phacosphaeria tritici (S. Garovaglio) A. Hedjaroude, Sydowia. Annales Mycologici, Horn. N.Ö. 22:74. 1968. On leaves of Triticum v'u/gare Vill., Gramineae, Italy, Kenya.

mitici (S. Garovaglio) G. Passerini var. pupyricola J.B. Ellis \& B.M. Everhart, Report of the Michigan Academy of Science, Lansing 7:66. 1905 [as pupicola]. Nom. nud. Art. 32.1. On old paper. U.S.A. (Michigan).

tritorulosa (M.J. Berkeley \& C.E. Broome) V. Cesati \& G. de Notaris, Commentario della Societá Crittogamologica Italiana, Milan 1:236. 186.3. ESpluaria (Canlicolae) tritomesa M.J. Berkeley \& C.E. Broome, Annals and Magazine of Natural I Iistory, Lomdon. Series

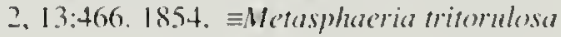

M.J. Berkeley \& C.E. Broome, Sylloge Fungorum Omnium Hucusque Cognitorum Digessit P.A. Saccardo 15:190. 190] [as Metasphaeria bilorulosa]. On dead stems of Epilobium hirsutum L. . Onagraceae. Great Britain.

(Astrosphaeria) trochus (O.A.J. Penzig \& P.A. Saccardo) F. v. Höhnel, Sitzungsberichte der Akademie der Wissenschaften in Wien, Mathematisch-naturwissenschaftliche Klasse 118(Abt. 1):328. 1909. 三Melanomma trochus O.A.J. Penzig \& P.A. Saccardo, Malpighia. Rassegna Mensuale di Botanica. Messina. Genova 11:401. 1897: Icones Fungorum Bambusicolorum Japonicorum, p. I6. 1904. ETrematosphaeria irochus (O.A.J. Penzig \& P.A. Saccardo) S.C. Teng. Sinensia. Special Bulletin of the Metropolitan Museum of Natural History. Nanking 9:257. 1938. 三Asterotheca trochus (O.A.J. Penzig \& P.A. Saccardo) I. Hino, Bulletin Miyazaki College of Agriculture and Forestry 10:57. 1938. 三Asterella trochus (O.A.J. Penzig \& P.A. Saccardo) K. Hara, Nippon Baikin-Gaku, p. 187. 1936. ĐAstrosphaeriella trochus (O.A.J. Penzig \& P.A. Saccardo) D.L. Hawksworth. Journal of the Linnean Society (Botany) London 82:46. 1981. On rachis of palm. Palmae. China, Java.

Irollii (P.A. Karsten) E. Miiller, Sydowia. Annales Mycologici, Horn, N.Ö. 4(1-6):249. 1950. $\equiv$ Metasphaeria trolii P.A. Karsten. Hedwigia. Dresden 23:86. 1884. On dry stems of Cardamine sylvatica Link, Trollius europaeus L. Cruciferae. Ranunculaceae. Switzerland.

Itucumanensis C.L. Spegazzini. Revista. Universidad Nacional de La Plata 2(18):232-233. 1896:

Hongos De La Caña De Azúcar No. 10. Anno 1896. On culms of Saccharum officinarum L.. Gramineae. Argentina.

mmefaciens (J.B. Ellis \& H.W. Harkness) F. Perak, Annales Nycologici. Berlin 32:360-361. 1934. ESphaeria (Momagnella) tumefaciens J.B. Ellis \& H.W. Harkness, Journal of Mycology. Columbus. Ohio 2:41. 1886. DMomtagnella tumefaciens (J.B. Ellis \& H.W. Harkness) A.N. Berlese \& B. Voglino in P.A. Saccardo, Sylloge Fungorum Omnium Hucusque Cognitorum Digessit P.A. Saccardo Additamenta Ad Volunima 1-IV, p. If4. 18S6. $\equiv$ Phacoderris numefaciens (J.B. Ellis \& H.W. Harkness) F. v. Höhnel. Sitzungsberichte der Ahademic der Wissenschaften in Wien. Mathematisch-naturw issenschaftliche Klasse. Abt. 1. 120:462. 1911. ESvicorpella numefaciens (J.B. Ellis \& H.W. I Harkness) F. Theissen \& H. Sydow. Amales Mycologici. Berlin 13:631. 1915. On dead limb. of Artomisia californica 1_ess.. Artemisia sp.. 
Compositae. U.S.A. (Califomia), U.S.S.R. (Turkistin).

fungurahuensis F. Petrak, Sydowia. Annales Mycologici. Hom, N.Ö. 2(1-6):338. 1948. On stroma of Phyllachora tungurahuensis Petrak. Fungi (Phyllachoraceae). Philippines. nupue C.L. Spegazzini. Fungi Chilenses, pp. 79-80. 1910; Revista de La Facultad de Agronomia Y Veterinaria. Universidad Nacional de La Plata, Series 2. 6:79-80. 1910. On year-old shoots of Lohelia rupa L.. Campanulaceae. Chile.

typhae (P.A. Karsten) P.A. Saccardo, Nuovo Giornale Botanico Italiano e Bolletino della Società Botanica Italiana. Firenze 7:321. 1875. =Leprosphaeria perpusilla (J. Desmazières) P.A. Karsten var. typhae P.A. Karsten, Mycologia Fennica Pars 2, Pyrenomycetes. p. 99. 1873: Bidrag till Kännedom om Finlands Natur och Folk. Utgifna af Finska Vetenskaps-Societeten. Helsingfors 23:99. 1873. ESphaeria perpusilla J.

Desmazières var. typhae B. Auerswald in G.L. Rabenhorst, Fungi Europaei Exsiccati, Klotzschii Herbarii vivi Mycologici Continuatio Edita Nova. Series Secunda, Century 9. No. 831. Anno 1865. Nom. nud. Art. 32.1. 三Phaeosphueria typhae (P.A. Karsten) R.A. Shoemaker. Canadian Joumal of Botany, Ottawa 67:1536. 1989. On decaying culms of Typha larifolia L.. Typhaceae. Finland, Germany.

nyphurum (J. Desmazières) P.A. Karsten. Mycologia Fennica Pars 2, Pyrenomycetes, p. 100. 1873; Bidrag till Kännedom om Finlands Natur och Folk. Utgifna af Finska Vetenskaps-Societeten. Helsingfors 23:100. 1873. 三Sphaeria scirpicola A.P. de Candolle:E.M. Fries var. typharum J. Desmazières, Plantes Cryptogames Du Nord De La France, Edition 2. Fascicle 36, No. 1778. Anno 1849. Esphaeria rypharum (J. Desmazières) G.L. Rahenhorst, Klotzschii Herbariun Vivum Mycologicum Sistens Fungorum Per Totam Germaniam Cresentium Collectionem Perfectam. Dresden. Editio Novo, Century 8 , No. 731. Anno 1858. EPleospora typharum (J. Desmazières) L. Fuckel, Fungi Rhenani Exsiccati A Leopoldo Fuckel Collecti, Fascicle 9, No. 858. Anno 1864: Symbolac Mycologicae. p. 137. 1870. EPhaensphaeria typharum (J. Desmazières) L. Holm, Symbolae Botanicae Upsalienses. Uppsala 14(3):126. 1957. On decaying leaves of Typha larifolia L., Typhaceae. Belgium, Canada, Finland. France, Germany, Italy. New Zealind. U.S.A.. U.S.S.R.

typharum (L. Fuckel) P.A. Saccardo, Atti dell' Accademia Scientifica Vencto-TrentinoIstriana, Padova 2(2):154. 1873. EPleospura sypharum (J. Desmazières) L. Fuckel, Fungi Rhenani Exsiccati A Leopoldo Fuckel Collecti. Fuscicle 9, No. 858. Anno 1864. [P.A. Saccardo distinctly attributed the basionym to L. Fuckel.]

rypharum (G.L. Rahenhorst) B. Auerswald in J. Kunze, Fungi Selecti Exsiccati, Series 11, No. 256. Anno 1877 [as Tauschrerein for possible earlier place of publication|. ESphaeria typharum G.L. Rabenhorst, Fungi Europaci Exsiccati, Klotzschii Herbarii vivi Mycologici Continuatio, Edition 2. Century 8. No. 731. Anno 1858. 三Pleospora rypharum (G.L. Rabenhorst) L. Fuckel. Fungi Rhenani Exsiccati A Leopoldo Fuckel Collecti. Fascicle 9. No. 858. Anno 1864: Symbolae Mycologicae, p. 137. 1870. ELeptosphueria kumzeana A.N. Berlese. On dry leaves and standing stems of Typha angustifolia L., Typha latifolia L.. Typhaceae. Germany.

typharum (J. Desmazières) P.A. Karsten forma acori W.B. Grove, Journal of Botany, British and Foreign. London 68:97. 1930. On unknown substrate of Acorus calamus L.. Araceae. Great Britain.

typharum (J. Desmazières) P.A. Karsten subsp. papyrogena P.A. Saccardo. Annales Mycologici, Berlin 6:558. 1908. On putrid paper. France.

Iypharum (J. Desmazières) P.A. Karsten subsp. phragmatina P.A. Karsten, Hedwigia, Dresden 23:5. 1884; Acta Societatis pro Fauna et Flora Fennica, Helsingforsiae 2(6):54. 1885. On decaying leaves of Phragmites communis Trin., Gramineae. Finland.

typhicolı G. Passerini \& V. Beltrani, Atti dell Accadernia Nazionale dei Lincei. Transunti. Rome, Series 3, 7:36. 1883. ELeptosphaeria licatensis P.A. Saccardo, Sylloge Fungorum Omnium Hucusque Cognitorum Digessit P.A. Saccardo 2:70. 1883. Nec Leptosphaeria uphicola P.A. Karsten, fide P.A. Saccardo 1883. ELeptosphacria syphicola P.A.

Karsten. On dry leaves of Typha latifolia L., Typhaceae. Italy.

ryphicola P.A. Karsten, vide P.A. Saccardo, Sylloge Fungorum Onnium Hucusque Cognitorum Digessit P.A. Saccardo 2:70. 1883 sub. Lepresphueria lic carensis P.A. Saccardo. ELeposphaseria moculans (M.J. Sowerhy) P.A. Karsten var. ryphicola P.A. Karsten, Mycologia Fennica Pars 2. Pyrenomycetes, p. 100. 1873. झMassariosphacria ryphirola (P.A. Karsten) A. Leuchtmann, Sydowia. Annales Mycologici. Horn. N.Ö. 37:168. 1984. EPhaeosphueria ryphicola (P.A. Karsten) A. Hedjaroude, Sydowia. Annales Mycologici. Horn. N.Ö. 22:86. 1968. EChaetomastia typhicola (P.A. Karsten) M.E. 
Barr, Mycotaxon. An International Journal Designed to Expedite Publication of Research on Taxonomy \& Nomenclature of Fungi \& Lichens, Ithaca, New York 34:514. 1989. Canada, Czechoslovakia, Switzerland.

lyphiseda F. Petrak, Hedwigia, Dresden 65:220-221. 1925. Nom. illegit. Art. 64.1. On dry flower stalks of Typha angustifolia L., Typhaceae. Poland.

typhiseda P.A. Saccardo \& A.N. Berlese, Revue Mycologique, Toulouse 8:33. 1886. 三Leptosphaeria pracclara P.A. Karsten forma typlriseda (P.A. Saccardo \& A.N. Berlese) A.N. Berlese. On dead leaves of Typha angnisifolia L.. Typhaceae. Algeria.

typhiseda P.A. Saccardo \& A.N. Berlese forma sodoloci $\mathrm{F}$. Fautrey in C. Roumeguère. Fungi Selecti Gallici Exsiccati. Century 54. No. 5357. Anno 1890: Revue Mycologique. Toulouse 12:122. 1890. On leaves of Typha angustifolia L.. Typhaceae. France.

uligimosa (W. Phillips \& C.B. Plowright) P.A. Saccardo, Sylloge Fungorum Omnium Hucusque Cognitorum Digessit P.A. Saccardo 2:47. 1883. ESphaerella wiginosa W. Phillips \& C.B. Plowright, Grevillea, London 10:74. 1881. On leaves of Stellaria uligimosa Murray, Caryophyllaceae. Great Britain. ulmicola C. Massa. Annales Mycologici. Berlin 10:287. 1912. On leaves of Ulmus campestris auct., Ulmaceae. Italy.

umbilicariac (L. Lindsay) P.A. Saccardo \& D. Saccardo in P.A. Saccardo, Sylloge Fungorum Omnium Hucusque Cognitorum Digessit P.A. Saccardo 17:731. 1905. EMicrothelia umbilicariae W.L. Lindsay, Proceedings of the Royal Society of Edinburgh 6:535. 1869. Norr. nud Art. 32.Ic; Transactions of the Royal Society of Edinburgh 25(2):538-539. 1869. Parasitic on sterile thallus of Umbilicaria pustulata (L.) Hoffm., Lichenes (Umbilicariaceae). Great Britain.

umbrosa G. Niessl v. Mayendorl in G.L. Rabenhorst. Fungi Europaei Exsiccati, Klotzschii Herbarii vivi Mycologici Continuatio, Edition 3 (Edita Nova), Series 2, Century 20, No. 1934. Anno 1875; Just 's Botanisch Jahresberichte, Berlin 3:262. 1887. EMassaria Imbrosa (G. Niessl v. Mayendorf) H. Rehm in P.A. Saccardo, Sylloge Fungorum Omnium Hucusquc Cognitorum Digessit P.A. Saccardo 9:761 1891. On unknown substrate of Actaca spicata L., Astramia major L.. Gentiana asclepiadea L.. Solanum dislcamara L.. Spiraca aluncus L. (=Armusus dinicus Ferr.). Ranunculaceac. Umbelliferate. Gentianaceac. Solanaceae, Rosaceac. Austria.

manama (G. Niessl v. Mayendorf) ex P.A. Saccardo. Sylloge Fungorum Omnium Hucusquc Cognitorum Digessit P'A. Saccardo 9:798. 18S1. Elleptameria uncinam G. Niessl v.
Mayendorf in H. Rehm. Ascomyceren.

Fascicle 15. No. 735. Anno 1883. Nom. nud. Art. 32.]b [as (Niess]) Rehm]. On stems of Artemisia vulgaris L.. Compositae. Czechoslovakia.

usneae N.W. Woronichin. Trudy Akademiia Nauk SSSR Botanicheskii Muzei 21:126. 1927. On thallus of Usnea florida (L.) Web.. Lichenes (Usneaceae). U.S.S.R.

utahensis J.B. Ellis \& B.M. Everhart. The Norh American Pyrenomycetes. A Contribution to Mycologic Botany. p. 361. 1892. On dead stems of unknown host of Umbelliferae. Unbelliferae. U.S.A.

vagahunda P.A. Saccardo. Nuovo Giornale Botanico Italiano e Bolletino della Società Botanica Italiana. Firenze 7:318. 1875. ESphaeria fuscella P.A. Saccardo. Atti dell’ Accademia Scientilica Veneto-Trentino-]striana. Padova 2(2):145. 1873 [as Sphaeria fuscella M.J. Berkeley \& C.E. Broome. Non Leprosphueria fuscella (N.J. Berkeley \& C.E. Broome) V. Cesati \& G. de Notaris]. On branches of Abies excelsa (Lam.) Poiret. Acer campesire L.. Alnus glutinosa (L.) Gaertner. Ampelopsis heterophylla (Thunb.) Sieb. \& Zucc.. Clematis vitalba L.. Comus sanguinea L.. Corylus avellana L.. Dulcamara sp.. Hypericum calycinum L.. Kerria japonica (L.) DC.. Quercus pedunculata Ehrh.. Salix purpurea L.. Pinaceac. Aceraceae. Betulaceac. Vitaceae. Ranunculaceac. Comaceae. Solanaceae. Guttiferae, Rosaceae. Fagaceae. Salicaceae. Italy.

vagabunda P.A. Saccardo forma abietis F. Fautrey $m$ C. Roumeguère. Fungi Selecti Gallici

Exsiccati, Century 59. No. 5846. Anno 1891: Revue Mycologique. Toulouse 13:167. 1891. On stems of Abies excelsa (Lam.) Poiret. Pinaceae. France.

vagabunda P.A. Saccardo forma citri-limonii P.A. Saccardo. Fungi Italici autographice delineati (additis nonnullis extra-italicis asterisco notatis). Patavii. No. 520. 1\$79. On stems of Cirms limomum Risso. Rutaceae. Italy.

ragahmina P.A. Saccardo forma daphmes? II. de Sousa da Camara. Agronomia Lusitana. Sacavém 11:172. 1949. On branches of D(t)/me gnidium L.. Thymelaeaceac. Portugal.

lagahumda P.A. Saccardo forma lonicerae F. Fautrey in C. Roumeguère. Fungi Selecti Gallici Exsiccati. Century 61. No. 6040. Anno 1892: Revue Mycologique. Toulouse 14:108. 1892. On young shoots of Periclumenum sp. (=Lomicera sp.). Caprifoliaceate. France.

lagabumda P.A. Saccardo forma salicis-capreac F. Fautrey in C. Roumeguère. Fungi Selecti Gallici Exsiccati, Century 6.5. No. 6440. Anno 1894: Revue Mycologique. Toulouse 16:8. 
1894. On stems of Salix caprea L.. Salicaceae. France.

vagabunda P.A. Saccardo subsp. alvarensis K. Starbäck, Bihang till K. Svenska Vetenskapsakademiens Handlingar. Stockholm, Series 3, 15(2): 13. 1889. On rhizomes on uncovered soil of Plantago maritima L., Plantaginaceae. Sweden.

vagabunda P.A. Saccardo var. caulium P.A. Saccardo, Revue Mycologique, Toulouse 3:44. 1881; Reliquiae Mycologicae Libertianae, Series Altera Reviserunt C.

Roumeguère \& P.A. Saccardo, Toulouse, p. 6 , No. 119. 1881. On stems of Perasites sp., Compositae. France.

vagabunda P.A. Saccardo var. divergens P.A. Saccardo. Fungi Italici autographice delineati (additis nonnullis extra-italicis asterisco notatis), Patavii, No. 518. 1879; Sylloge Fungorum Omnium Hucusque Cognitorum Digessit P.A. Saccardo 2:31. 1883. On branches of Kerria sp., Rosaceae. Italy. vagabunda P.A. Saccardo var. dukcamarae P.A. Karsten, Meddelanden af Societas pro Fauna et Flora Fennica, Helsingfors 16:34. 1888: Symbolae ad Mycologiam Fennicam XXVII:34. 1888. On dead stems of Solanum dulcamara L., Solanaceae. Finland.

vagabunda P.A. Saccardo var. sarmenti P.A. Saccardo, Fungi ltalici autographice delineati (additis nonnullis extra-italicis asterisco notatis), Patavii, No. 518. 1879; Sylloge Fungorum Omnium Hucusque Congitorum Digessit P.A. Saccardo 2:31. 1883. On runners of Rubus sp., Rosaceae. Italy.

ragans P.A. Karsten, Svenska Vetenskapsakademien Stockholm Öfversigt af Förhandlingar,

Stockholm 29(2): 101. 1872. On rotting leaves of Dupontia fisheri R. Br., Gramineae. Norway.

ragans P.A. Karsten forma scirpi P.A. Saccardo, Sylloge Fungorum Omnium Hucusque Cognitorum Digessit P.A. Saccardo 2:59. 1883. On leaves of Dupontia fisheri R. Br., Gramineae. France, Norway.

laginae G. Passerini, Atti dell' Reale Accademia Nazionale dei Lincei. Rendiconti, Rome, Series 4, 7(2):45. 1891. On wet sheaths of Phragmites communis Trin., Gramineae. Italy.

vahlii E. Rostrup, Meddelelser om Gronland, Kjohenhavn 3:557-558. 1888. On dry stems of Melandrium triflorum J. Vahl. ex Liebm., Caryophyllaceae. Greenland.

valdiviensis C.L. Spegazzini, Fungi Chilenses, pp. 80-81. 1910; Revista de Lal Facultad de Agronomia $Y$ Veterinaria, Universidad Nacional de La Plata, Series 2. 6:80-81. 1910.
On decaying shoots of Digiralis purpurea L., Scrophulariaceae. Chile.

raldobbiae T. Ferraris, Malpighia. Rassegna Mensuale di Botanica. Messina, Genova 18:488-489. 1904. On leaves of Fagus syliatica L., Fagaceae. Italy.

valesiaca $\mathrm{H}$. Wegelin, Mitteilungen der Thurgauischen Naturforschenden Gesellschaft. Frauenfeld 12:175. 1896. On preceding year's stems of Artemisia campestris L., Compositae. Switzerland.

ranhoeffeniana A. Allescher in A. Allescher and P.C. Hennings, Biblotheca Botanica, Stuttgart 42(1):47. 1897. [lssued also in Botanische Ergebnisse der von der Gesellschaft für Erdkunde zu Berlin unter Leitung Dr. v. Drygalski’s ausgesandten Grönlandexpedition nach Dr. Vanhöffen's Sammlungen Bearbe itet, A. Kryptogamen...Stuttgart, in 1897.] Non vidi. On dead leaves of Melandrium aperahum (L.) Fenzl, Caryophyllaceae. Greenland.

rariabilis L.M. Unamuno Yrigoyen, Anales del Jardin Botánico de Madrid 1:29-31. 1941. On culms of Juncus sp., Juncaceae. Spain. variegata C.H. Peck, Bulletin of the New York State Museum, Albany 67:31-32. 1903. On dead stems of Phyrolacca americana L., Phytolaccaceae. U.S.A.

variiseptata G.L. Stout, Mycologia, Lancaster, Pennsylvania 22:276-277. 1930.

$\equiv$ Phaeosphaeria variiseptata (G.L. Stout) R.A. Shoemaker, Canadian Journal of Botany, Ottawa 67:1536. 1989. On leaves of Zea mays L., Gramineae. U.S.A.

vectis (M.J. Berkeley \& C.E. Broome) V. Cesati \& G. de Notaris, Commentario della Società Crittogamologica ltaliana. Milan 1:236. 1863. ESphaeria (Caulicolae) vectis M.J. Berkeley \& C.E. Broome, Annals and Magazine of Natural History, London, Series 2, 13:467. 1854. 三Paraphaeosphaeria vectis (M.J.

Berkeley \& C.E. Broome) A. Hedjaroude, Sydowia. Annales Mycologici, Horn, N.Ö. 22(1-4):98. (1968) 1969. On dead leaves of Iris foetidissima L., lridaceae. Great Britain.

veratri F.S. Earle in E.L. Greene. Plantae Bakerianae 2:20-21. 1901. On dead weathered stems of Veratrwm sp., Liliaceae. U.S.A.

vernoerdiana S.J. DuPlessis, South A frican Journal of Science, Cape Town 30:207-208. 1933. On leaves of Aloe affinis A. Berger, Aloe striatula Haw., Liliaceae. South Africa.

viciae E. Müller, Sydowia. Annales Mycologici, Hom, N.Ö. 4(1-6):268. 1950. On dead stems of Vicia cracca L., Leguminosae. Switzerland.

vincae (E.M. Fries) P.A. Saccardo, Michelia Commentarium Mycologicum Fungos in 
Primis Italicos lllustrans 1:37. 1877.

ESphaeria vincae E.M. Fries in J.E. Duby,

Aug. Pyrami de Candolle Botanicon Gallicum seu synopsis plantarum in flora gallica descriptum. Pars Secunda, p. 709. 1830. $\equiv$ phaerella vincae (E.M. Fries) B. Auerswald in W. Gonnermann and G.L. Rabenhorst, Mycologia Europaea, Abbildungen Sämmtlicher Pilze Europa's, Hefte 5 und 6, Synopsis Pyrenomycetum Europacorum, p. 10. 1869. =Mctasphaeria vincac (E.M. Fries) P.A. Saccardo, Sylloge Fungorum Omnium Hucusque Cognitorum Digessit P.A. Saccardo 2:171. 1883. On dry leaves of Vinca minor L., Apocynaceae. France, Germany, ltaly. vindobonensis F. Petrak, Annales Mycologici, Berlin 42:75. 1944. On dead stems of Cynoglossiom afficinale L., Boraginaceae. Austria. vinealis G. Passerini in F. v. Thümen, Die Pilze des Weinstockes, pp. 133-134. 1878. On dry runners of Vitis vimifera L., Vitaceae. Italy.

vinosa C.L. Spegazzini, Anales de la Sociedad Cientifica Argentina, Buenos Aires 10:142. 1880. On decaying leaves and stems of Eryngium agavifolilum Griseb., Umbelliferae. Argentina.

virginica (M.C. Cooke \& J.B. Ellis) P.A. Saccardo, Sylloge Fungorum Omnium Hucusque Cognitorum Digessit P.A. Saccardo 2:35. 1883. ESphaeria (Caulicolae) virginica M.C. Cooke \& J.B. Ellis, Grevillea, London 8:16. 1879. On stems of Lepidiun wirginicum L., Cruciferae. U.S.A.

viridella (C.H. Peck) P.A. Saccardo, Sylloge Fungorum Omnium Hucusque Cognitorum Digessit P.A. Saccardo 2:18. 1883. ESphaeria (Caulicolae) viridella C.H. Peck, Report. New York State Museum of Natural History, Albany 30:66. 1878. EPhaeosphaeria viridella (C.H. Peck) A. Leuchtmann, Sydowia. Annales Mycologici, Horn, N.Ö. 37:108. 1984. On dead stems of Juncus effusus L., Melilotus sp., Cyperaceae, Leguminosae. U.S.A.

vitalhae G. Niessl v. Mayendorf in H. Rehm. Ascomyceten, Fascicle 19, No. 938. Anno 1888; Hedwigia, Dresden 27:172. 1888. On decorticated twigs of Clematis vitalha L., Ranunculaceae. Austria.

vitalbae (G. de Notaris ex V. Cesati \& G. de Notaris) 11.G. Winter in J. Kunze, Fungi Selecti Exsiccati, Century 3, No. 331. Anno 1880. (=Fungi Helvetica, No. 31.) 三Spharria vilalbae G. de Notaris, Memorie della Accademia delle Scienze di Torino, Series 2. 13:124. 1853. Nom. nud. Art. 32.1; Micromycetes Italici Novi vel minus Cogniti Decas 8, p. 124. 1854; Commentario della Società
Crittogamologia Italiana, Milan 1:221-222. 1863. On dry runners of Clematis vitalba L.. Ranunculaceae. Switzerland.

vitalhae G. Niessl v. Mayendorf var. sarmenticola J. Feltgen, Vorstudien zu einer Pilz-Flora des Grossherzogthums, Luxemburg 1(3):221-222. 1903. On dry shoots of Clematis vitalba L.. Ranunculaceae. Luxembourg.

vitensis L.M. Unamuno Yrigoyen, Boletin de la R. Sociedad Española de Historia Natural. Madrid 29:394-395. 1929. On above-ground parts of reeds, bracts, and peduncles of inflorescence of Juncus glaucus Sibth., Juncaceae. Spain.

viticola F. Fautrey \& C. Roumeguère in C. Roumeguère, Revue Mycologique, Toulouse 14:6. 1892; Fungi Selecti Gallici Exsiccati, Century 60. No. 5950. Anno 1892. On vines of Vitis vinifera L., Vitaceae. France.

vitigena P.A. Saccardo, Sylloge Fungorum Omnium Hucusque Cognitorum Digessit P.A. Saccardo 2:29. 1883. ESphaerella vitis S. Schulzer v. Müggenburg, Verhandlungen der ZoologischBotanischen Gesellschaft in Wien 20:643. 1870. Non Leprosphaeria viris (J.L. Castagne) R. Pirotta 1882, non Leptosphaeria vitis S. Schulzer v. Müggenburg, 1870. =Sphaerella sarmentorum R. Pirotta, fide P.A. Saccardo, 1883. On living branches of Vitis vinifera L., Vitaceae. Austria.

vitis (J.L. Castagne) R. Pirotta, Archivo del Laboratorio di Botanica Crittogamica Universita di Pavia 2 \& 3:161. (1877) 1879. Nom. illegit. Art. 63.1. 三Sphaeria vitis J.L. Castagne. Catalogue Des Plantes qui Croissent Naturellement aux Environs de Marseille. p. 166. 1845. Nom. illegit. Art. 64.1. On dried vines of Vitis vinifera L., Vitaceae. France.

vitis S. Schulzer v. Müggenburg. Verhandlungen der Zoologisch-Botanischen Gesellschaft in Wien 20:642-643. 1870. On vines of Vitis vinifera L., Vitaceae. Austria.

volkartiana E. Müller, Sydowia. Annales Mycologici, Horn. N.Ö. 4( 1-6):210. 1950. $\equiv$ Phaeosphaeria volkartiana (E. Müller) A. Hedjaroude, Sydowia. Annales Mycologici. Hom, N.Ö. 22(1-4):84. (1968) 1969. On dead culms of Trisetum distichophy/hm (Vill.) Beauv., Tristeum spicanm (L.) K. Richter, Gramineae. Switzerland.

rrieseae W. Siemaszko, Acta Societatis Botanicorum Poloniae, Warszawa 1:21-22. 1923. On leaves of Vriesea sp., Bromeliaceae. U.S.S.R.

waghorniana H. Rehm, Hedwigia. Dresden 39:324. 1900. On cortex? of Betula sp., Betulaceae. Canida.

weberi C.A. Oudemans, K. Akademie van Wetenschappen Amsterdam, Afdeeling Natuurkund 
Verslagen en Mededeelingen 3 Reeks, Deel

2:156. 1885. On leaves of Rammculus nivalis

L. var. sulp/ureus Wahlenberg, Ranunculaceae. U.S.S.R.

weddellii (J.P. Montagne) P.A. Saceardo. Sylloge Fungorum Omnium Hucusque Cognitorum Digessit P.A. Saccardo 2:86. 1883. ESphatria weddellii J.P. Montagne. Annales des Sciences Naturelles, Paris. Botanique. Series 4. 5:372-373. 1856. On culms of Bambusa sp.. Gramineae. Bolivia.

negeliniana P.A. Saccardo \& P. Sydow, Sylloge Fungorum Omnium Hucusque Cognitorum Digessit P.A. Saccardo 14:567. 1899. $\equiv$ Leptosphaeria mumicis H. IVegelin. Nec F. Fautrey. On dry stems of Rumex sp.. Polygonaceae. Switzerland.

wegeliniana P.A. Saccardo \& P. Sydow forma teucrii J. Feltgen, Vorstudien zu einer PilzFlora des Grossherzogthums, Luxemburg 1(3):222. 1903. On dry stems of Tencrium scorodonia L., Labiatae. Luxembourg.

wehmeveri R.A. Shoemaker. Canadian Joumal of Botany. Ottawa 62:2725. 1984. On stems of Lupimus rubricanlis Greene, L. lutifolius J.G. Agardh. Thermopsis montana Nutt., Leguminosac. Canada, U.S.A.

williamsii C.G. Hansford. Proceedings of the Linnean Society of New South Wales. Sydney 82:216. 1957. On dead leaves of Dianella nolma R. Br., Liliaceae. Australia.

winteri G. Niessl v. Mayendorf in H.G. Winter. Hedwigia. Dresden 22:1. 1883. 三Scleropleella mimteri (G. NiessI v. Mayendorf) F. v. Höhnel. Annales Mycologici. Berlin 18:76. 1920. Nom. inval. Art. 33.1. [Combination not formally proposed.] Wethieinind winteri (G. Niessl v. Mayendorf) E. Müller, Sydowia. Annales Mycologici. Horn. N.Ö. 4(1-6):203. 1950. On dry leaves of Plantago alpina L., Plantaginaceae. Germany.

woodron'-wilsonii [as Woodrowi Wilsoni] L.

Garbowski, Bulletin Trimestriel de la Société Mycologique de France, Paris 39:238. 1924. On soft parts of dried leaves of Eryngium campestre L., Umbellilerae. U.S.S.R. (Crimea).

soroninii E. Docea \& A. Negru in A. Negru, E. Docea, and E. Szasz. Novitates Systematicate Plantarum Non Vascularium (=Novosti Sistematiki Nizshikh Rastenij. Novitates Systematicate Plantarum Non Vascularium) 9:168. 1972. On seeds of Camnahis sativa L.. Cannabaceae. Romania.

rerophylli J.B. Ellis, American Naturalist, Lancaster. Pennsylvania 17(1):316-317. 1883. \$Melosphaeria derephylli (J.B. Ellis) P.A. Saccardo, Sylloge Fungorum Omnium Hucusque Cognitorum Digessit P.A. Saccardo $2(\mathrm{Ad}-$ denda Ad Volumen Secundem):I.XI. 1883;
Sylloge Fungorum Omnium Ilucusque Cognitorum Digessit P.A. Saccardo 9:829. 1891. On dead leaves of X'rophy/lum a.sphodeloides (L.) Nutı., Liliaceae. U.S.A.

riphii G. Passerini, Rendiconti della Sedute della R. Accademia dei Lincei, Classe di Scienze Fisiche, Matematiche e Nutural (=Atti dell" Accademia Nazionale dei Lincei, Rendiconti, Romia), Series 4, 3:90-91. 1887. On wilted leaves of /ris foetidissima L., Iridaceac. Italy.

.y/ogena M. Curzi \& M. Barbani, Atti dell Istituto

Botanico della Universilà e Laboratorio Crittoganica di Pavia, Milano, Series 3. 3:160. 1927. On decorticated branches of Sulix sp.. Salicaceae. Italy.

yerbae C.L. Spegazzini, Anales del Museo Nacional de Historia Natural de Buenos Aires 17(Series 3. 1(1): 126-127. 1908: Hongos de La Yerba Mate. No. 32. 1908. On wilting and dead branches of /lex paraguariensis A. St. Hil., Aquifoliaceae. Argentina.

Inlan P.A. Saccardo, Nuovo Giomale Butanico Italiano e Bolletino della Societa Butanica Italiana. Firenze 7:312. 1875. On wilted leaves of Magnolia yulan Desf.. Magnoliaceae. Italy.

zahlbruckneri P. Sirasser, Verhandlungen der Zoologisch-Botanischer Gesellschalt in Wien 57:315-316. 1907 [as Leplospharia [Pocosphacria Sacc.)]. झAcanthostignella zahlbruckneri (P. Strasser) F. v. Höhnel. Sitzungsberichte der Akademie der Wissenschaften in Wien, Mathematisch-

naturwissenschaftliche Klasse, Abt. I. I 18:1503. 1909. On dry stems of Mentha swhestris L. [=Mentha longifolia (L.) Huds.]. Labiatac. Austria.

zeac G.L. Stout. Mycologia, Lancaster, Pennsylvania 22:277. 1930. On leaves of Zea mays I.. Gramineae. U.S.A. (1llinois).

zeae-maydis A.M. Saccas, Revue de Pathologie Végcitale et D’Entomologie Agricole de France. Paris 30:179-180. 1951. On dead leaves of Lea mays L., Gramineae. French Equatorial Africa.

zeicola A.M. Saccas, Revue de Pathologic Végétale et D'Entomologie Agricole de France, Paris 30:176-177. 1951. On living leaves of Ked mays L... Gramineas. French Ecjuatorial Alrica.

zingiberis (K. Sawada) V. Dhar. I.S. Srivastava \& II.S. Silhambi, Current Science. Bangalore 50:540. 1981. EPhacosplueria zingiberis K. Sawada. Descriptive Catalogue of Taiwan (Formosan) Fungi. Part XI. (Special Publication of the National Taiwan University Taipaei No. 8), p. 67. 1959. On leaves of Zingiber officinak Rosc., Zingiberaceac. Indial. 
zizuniaecola (M.J. Berkeley \& M.A. Curis) P.A. Saccardo, Sylloge Fungorum Omnium

Hucusque Cognitorum Digessit P.A. Saccardo 2:86. 1883. ESphaeria zizaniaecola M.J.

Berkeley \& M.A. Curtis, Grevillea,

London 4:145. 1876. On stems of Zizania sp., Gramineae. U.S.A. zizamnivora K. Hara. A List of Japanese Fungi Hitherto Known, p. 402(2). 1954 [as zizaninaerora]. [There are two pages numbered 402; the description is on the second page.] On living plant of Zizania latifolia Turcz., Gramineae. Japan. 


\section{Host Index}

\section{Abies balsamea Miller}

$$
\text { L. faulii }
$$

Abies excelsa (Lam.) Poiret

L. vagabunda

L. vagabunda forma abietis

Aburilon avicenmue Gaertner

$$
\text { L. abutilonis }
$$

Abutilon indicum (L.) Sweet

$$
\text { L. abutilonis }
$$

Acacia kempeana F. Muell.

$$
\text { L. clelandii }
$$

Acantholimon acerosum Boiss.

$$
\text { L. kotschyana }
$$

Acantholimon melananthum

Boiss.

$$
\text { L. kotschyana }
$$

Acer campestre $\mathbf{L}$.
L. vagabunda

Acer laetum C.A. Mey.

$$
\text { L. aceris }
$$

Acer negundo $\mathrm{L}$.

$$
\text { L. diana }
$$

Acer pseudoplatanus $\mathrm{L}$.
L. dioica
L. obesula

Acer saccharum auct.

Acer sp.
L. inquinans

\section{L. controversa \\ L. leucoplaca \\ L. muelleri}

Achillea millefolium auct.
L. achilleae
L. compressa
L. dolioloides
L. millefolii
L. ogilviensis forma achilleae
L. staritzii

Achillea nana $\mathrm{L}$.

$$
\text { L. nanae }
$$

Achillea prarmica $\mathrm{L}$.
L. marginalis
L. passerinit
L. prarmicue

Achillea sp.
L. doliolum
L. tanaceti

Aconitum compactum Rchb.

L. nigromaculara
Aconinum lycoctonum L.

L. lasiosphaerioides

Aconitum nappelus $\mathrm{L}$.

L. aconili

L. anthostomoides

L. napelli

L. nigromaculata

Aconitum panicularum Lam.

L. nigromaculata

Aconitum sp.

L. scorophila

Acorus calamus L.

L. acorella

L. acori

L. densa

L. microscopica subsp. calami

L. Typharum forma acori

Actaea spicata L.

L. umbrosa

Adenostyles albifrons (L. fil.)

Reichenb.

L. nitschkei forma adenostylidis

Adesmia sp.

L. adesmicola

Adhatoda sp.

L. acanthi

Adonis pyrenaica DC.

L. doliohum var. pachyspora

Acluropus littoralis (Gouan.) Parl.

L. aeluropodis

Agastache urticifolia (Benth.)

Kize.

$$
\begin{aligned}
& \text { L. brightonensis } \\
& \text { L. darkeri }
\end{aligned}
$$

Agave ferox C. Koch

L. obmusispora forma agaies

Agave rigida Mill. var. sisalana

(Perrine) Engelm.

L. agaves

Agave striata Zucc.

L. obtusispora

Agropyron repens (L.) Beauv.

L. anisomere's

L. avenaria

L. lincaris
Agrostis vulgaris With.

L. poae var. agrostidis

L. rhizomatum

Agrostis sp.

L. sparsa

Ailanthus glandulosa Desf.

L. ailanthi

L. endophaena

L. glandulosae

Aira alpina L.
L. insignis

Aira cespitosa $\mathrm{L}$.

L. anarithma

L. insignis forma airaecespitosase

L. lineolaris

L. personala

L. pleurospora

L. quinta

Albizia julibrissin Durz.

L. marginalis

Algae

L. flurianilis

L. lemaneae

L. marina

L. mirabilis

L. mirandac

Alhagi sp.

L. alhagii

Alisma plantago L.

L. rivularis

Alliaria officinalis Andrz ex Bieb.

L. alliariae

Allium validum $\mathrm{S}$. Wats.

L. lassenensis

Almus glutinosa (L.) Gaertner

L. coniothyrium

L. vagabuida

Almus sp.
L. lewcoplaca
L. Ionicerina

Alne affinis $\mathrm{A}$. Berger

L. verwoerdiana

Aloe urborescens Miller

L. aloes

Aloe striatula Haw.

L. verwoerdianes

Alopecurus borealis Trin.

L. alopecuri 
Alopecurus ovatus Knapp
L. insignis
L. microseopica

Aloysia citriodora Ort. ex Pers.
L. octophragmia

Alpinia speciosa K. Sch.

L. alpiniae

Amaranthus sp.

L. eriophora

Ambrosia trifida L.

L. drechisleri

L. fulgida

Ambrosia sp.

L. doliolum

Ammophila arenaria (L.) Link

L. ammophilae

L. ammophilae forma calamagrastidisarenariae

L. lithoralis

L. perforans

Ammophila arundinacea Host

L. sabuletorum

Ammoplila sp.

L. marram

Ammothammus lehmannii Bunge

L. ammothamni

Amorpha fruticosa $\mathrm{L}$.

L. amorphae

Ampelopsis heteroplylla (Thunb.)

Sieb. \& Zucc.

L. vagabunda

Anacylus radialus Loisel.

L. anacycli

Anaphalis sp.

L. doliolum

Anarlimum bellidifolium (L.)

Willd.

L. anarhini

Andromeda tetragona $\mathrm{L}$.

L. andromedae

L. hyperborea

Andromeda sp.

L. sublanosa

Andropogen glomeralus (Walt.)

B.S.P.

L. subcompressa

Andropogon ischaemum L.
L. enstomoides
L. ischaemi
L. trimera

Andropogon muricalss. Retz.

L. muricata

Andropogon saccharoides Swartz

L. Miggarii

Andropogons sp.

L. herponichoides

L. latebrosa

L. michonii

Androsace lactea L.

1. pachyosea
Anemone narcissiflora $\mathrm{L}$.

L. raplidophora

Anemone sylvestris L.

L. anemones

Anemone virginiana L.

L. platypus

Angelica sulvestris L.

L. connidea

L. conoidea forma angelicae

Angelicasp.

L. obesula

L. selosa

Authemis tinctoria L.

L. dnlioloides

L. lanaceri

Anthericum ramosum $\mathrm{L}$.

L. antherici

Anthriscus sylvestris (L.) Hoffm.

L. dolioloides var. inops

Antirrhinum majus $\mathbf{L}$.

L. Imemeniana

Antirhinum siculum Miller

L. galiarum subsp. antirlhini

L. insulana

Apios fortunei Maxim.

L. apios

L. apios-fortunei

Apocynum sp.

L. doliolum

Aquilegia rulgaris L.

L. aquilegiae

Arabis alpina $\mathbf{L}$.

L. arabidis

L. johansonii

Aralia sp.

L. doliolum

Araucaria imbricala Pav.

L. californica

Arbutus unedo $\mathrm{L}$.

L. arbuti

Arciilum sp.

L. doliolum

Areca sapida Soland. ex Hook. f.

L. arecae

Arenaria ciliana L.

L. anerswaldii

Armeria iulgaris Willd.

L. slarilzii

Arhenaherum arenacem

Beauv.

L. arrhenalheri

Arbenatherum clatius (L.)

Beauv. ex J. Presl. \& C. Presl.

L. arrhemalheri

Aremisia absinthimm L.

L. glocespora

Arfemisia amma l.

L. siddomiand
Artemisia austriaca Jacq.

L. compressa

Arlemisia califarnica Less.

L. Iumefaciens

Artemisia campestris L.

L. artemisiae

L. cuespitosa

L. compressa

L. phacospora

L. sydoniana

L. valesiaca

Artemisia camphorata Vill.

L. camplonrala

Artenisia frigida Willd.

L. tetonensis

Aremisia herha-alha Asso

L. rothomagensis var. aremisiae

Artemisia tridentala Nun.

$$
\text { L. lasioderma }
$$

Artemisia vulgaris L.

L. doliolum var. suldisticha

L. grammodes

L. hispanica

L. kalmusii

L. purpurea

L. Incinata

Arremisia sp.

L. cenispora

L. crustacea

L. owaniae

L. Iumefaciens

Aruncus dioicus Fem.

L. Immbrosa

Aruncus silvestris Kostel.

$$
\text { L. arunci }
$$

Arundinaria sp.

$$
\text { L. cemorpha }
$$

Arunda donax L.

L. donacina

L. recessa

L. Hondophaea

Asclepias syriaca L.

L. dearnessii

Asclepias sp.

\section{L. doliolum \\ L. russellii}

Asparagus offucinalis $\mathrm{L}$.

L. asparagi

L. asparagina

L. Passeriniama

L. porforicensis

L. praeclara

L. punchoiden

L. socialis

Asparagus sp.

L. comaliella

L. indica

Asperella japonica Hack.

L. aspertillac 
Asperula sp.

L. politis

Aspicilia calcarea (L.) Korb L. crozalsii

Aspidistra clatior Blume L. aspidistrue

Asplenium septentrionale (L.)

Hoffm.

$$
\text { L. asplenii }
$$

Aster multiflorus Ait.

$$
\text { L. astericola }
$$

Aster saginifolius Wedem. ex

Willd.

$$
\text { L. asteris }
$$

Aster salignus Willd.

$$
\text { L. conoidea forma asteris }
$$

Aster sp.

$$
\begin{aligned}
& \text { L. doliolum } \\
& \text { L. heliopsidis }
\end{aligned}
$$

Astrantia major $\mathrm{L}$.

$$
\text { L. umbrosa }
$$

Atraphaxis muschketowii Krasnov

$$
\text { L. atraphaxidis }
$$

Atriplex hortensis L.

$$
\text { L. calvescens }
$$

Atriplex verrucifera Bieb.

$$
\text { L. arriplicis }
$$

Atriplex sp.

$$
\text { L. echinella }
$$

Avena sativa $\mathrm{L}$.

Avena sp.

$$
\text { L. korrae }
$$

$$
\text { L. avenae }
$$

Avicennia marina (Forsk.) Vierh. var. resinifcrae (Forst.) Bakh.

$$
\text { L. australiensis }
$$

Avicennia nirida Jacq.

$$
\text { L. avicennicie }
$$

Aronopsus compressus (Swartz)

Beauv.

$$
\text { L. korrac }
$$

Azaleu sp. (=Rhododendran sp.)

$$
\text { L. azaleae }
$$

Baccharis sp.

$$
\text { L. bicuspidata }
$$

Bacomyces rufies (Huds.) Rebent.

$$
\begin{aligned}
& \text { L. bacomycearia } \\
& \text { L. neomizans }
\end{aligned}
$$

Baldingera arundinacea (L.)

Dumort.

$$
\begin{aligned}
& \text { L. baldingeras } \\
& \text { L. larseniana }
\end{aligned}
$$

Ballota acetahulosa (L.) Bentham

$$
\text { L. hallotae }
$$

Ballota nigra L.

Bambusa sp.

$$
\text { L. slovacica }
$$
L. amphiloga
L. bambusae
L. bambusicola
L. scabrispora
L. schncideriana
L. ligrisoides
L. weddellii

Baprisia sp.

L. comatella

Bardana sp.

$$
\text { L. hardanae }
$$

Barkhousia taraxacifolia (Thuill.) DC.

L. modesta var. rubellula

Berheris ilicifolia Forst.

L. berberidicola

Berberis petiolicola Wall.

L. punjabensis

Berberis vulgaris L.

L. herberidis forma berberidis

L. coniothyium

Berberis sp.

L. inconspicua

Berleroa incana (L.) DC.

L. submaculans

Betula vermcosa Ehrh.

$$
\text { L. betulina }
$$

Betula sp.

$$
\begin{aligned}
& \text { L. befulina } \\
& \text { L. waghorniana }
\end{aligned}
$$

Biebersteinia emodii Jaub. \& Spach.

L. hollosiana
Blysmus compressus (L.) Panzer
ex Link

L. petkowicensis var. elymi

Bocconia sp.

$$
\text { L. bocconiae }
$$

Bomarea caldasii (HBK) Asch. \& Graebn.

\section{L. homareue}

Barreria lewcomela (L.) Mudd

\section{L. levcomelaria}

Brachypodium sylfaricum

(Hudson) Beauv.

$$
\begin{aligned}
& \text { L. brachypodii } \\
& \text { L. microscopica forma } \\
& \text { brachypodii } \\
& \text { L. syluatica }
\end{aligned}
$$

Brachypodium sp.

$$
\text { L. culmifraga var. linearis }
$$

Brassica campestris $\mathbf{L}$.

$$
\begin{aligned}
& \text { L. alliariae } \\
& \text { L. maculans }
\end{aligned}
$$

Brassica crispe Rafin.

$$
\text { L. salebrosa }
$$

Brassica napus $\mathrm{L}$.

$$
\text { L. napi }
$$

Brassica oleifera Moench

L. maculans forma denudata

Brassica oleracea $\mathrm{L}$.
Brassica rapa L.

$$
\text { L. nigrella }
$$

Brassica sp.

L. vlericola

Braya sp.

$$
\text { L. norregica }
$$

Briza media L.

$$
\begin{aligned}
& \text { L. brizae } \\
& \text { L. media }
\end{aligned}
$$

Bromus asper Murray

$$
\text { L. culmifraga var. }
$$
bramicola

Bromus inermis Leysser

L. avenaria

Broussonetiasp.

$$
\text { L. conionhyrium }
$$

Buddleja daridii Franch.
L. buddlejae
L. davidii
L. polini

Buphthalmum salicifolium L.

L. septemcellulata

Bupleurum falcatum L.

L. agnita var. bupleuri

Bupleurum petraeum L.

L. bupleuri

Bupleurum ranunculoides $\mathrm{L}$.

L. ranunculoides

Buxus sempervirens $\mathrm{L}$.
L. buxina
L. melanommaides
L. revocans

Cacalia hastata L.

L. doliolum var. cacaliae

Cacalia sp.
L. nitschikei

Calamogrostis epigejos (L.) Roth

L. culmorum forma epigeii

Calamagrostis montana Host
L. fuckelii

Calamagrostis sylvatica Host

L. quima

Calamagrostis sp.
L. coccodes
L. graminum
L. intermedia
L. sparsa

Calligonum sp.
L. calligoni

Calluna vulgaris (L.) Hull

L. ericae

Calopogonium mucunoides Desv.

$$
\text { L. calopogonii }
$$

Camellia japonica L.

$$
\text { L. camelliae-japonicae }
$$

Camellia theifera Griff.
L. depressa
L. cornatosporat

Camelliasp.

L. camellias

L. cesatiana 
Campanula zoysii Wulfen
L. pachyasca
L. plemeliana

Camphorosma monspeliaca L.

$$
\text { L. serbica }
$$

Camphorosma perennis Pall.

$$
\text { L. camphorosmae }
$$

Canna indica L.

$$
\text { L. cannae }
$$

Cammabis sativa $L$.

$$
\begin{aligned}
& \text { L. cannabina } \\
& \text { L. woroninii }
\end{aligned}
$$

Capparis aplyylla Roth

$$
\begin{aligned}
& \text { L. ahmadii } \\
& \text { L. capparidicola }
\end{aligned}
$$

Capparis horrida L.
L. simillima

Capparis spinosa L. L. capparidis

Cardamine sylvatica Link L. trollii

Carduus carlinoides Gouan L. carlinoides

Carduns defloratus $\mathrm{L}$
L. centaureae

Carduss muans L.

$$
\text { L. carduina }
$$

Carduus scopulorum Greene L. chrysanthemi

Carduus sp.

$$
\text { L. carduorum }
$$

Carex acutiformis Ehrh.

$$
\text { L. sparsa }
$$

Carex arenaria L.

$$
\text { L. cariciplila }
$$

Carex haldensis L.

$$
\text { L. Kochiana }
$$

Carex firma Host

$$
\text { L. caricis-fimae }
$$

Carer folliculata L.

$$
\text { L. folliculata }
$$

Carex glauca Scop.
L. striolata var. caricisglaucae

Carea gracillima Schwein.

$$
\text { L. folliculata var. oxyspora }
$$

Cares hirta $\mathrm{L}$.
L. caricis
L. occulta

Carci hyperborea Drejer

L. macrotheca

Carer leporina L.

$$
\text { L. culmorum var. palcicola }
$$

Carex paludosa Good.
L. giguspsora
L. puecinioides
L. rivalis

Cares panices $\mathrm{L}$.

L. caricina

Cares pendula Hudson

$$
\text { L. caricicols }
$$

L. caricis
Care. pulla Good.
L. caricinella
L. consobrina

Carex rigida Good.

L. macrotheca

Care. riparia Curtis

L. caricicola

Carex silvatica auct.

L. caricina

Carer vaginata Tausch

$$
\text { L. caricis }
$$

Carer vesicaria L.

L. paludosa

Carex vulpina $\mathrm{L}$.

L. caricis-vulpinae

L. microscopica

L. microscopica var. caricis-vulpinae

Carex sp.
L. clara
L. cumana
L. epicarecta
L. hemicrypia

Carlina acaulis L.

L. anthophila

L. cynaracearmm

Carlina vulgaris $\mathrm{L}$.

L. doliolum

L. doliolum forma carlinae-vulgaris

Carpinus sp.
L. depressa
L. subsimilis

Carya sp.
L. cacumimispora
L. lejostega
L. leucoplaca

Cassia sp.

L. cassiaccolo

Cassinia aculeuta $\mathrm{R}$. Br.

$$
\text { L. Plagia }
$$

Castanea sp.
L. involucralis

Castilleja miniara Doug. ex

Hook.

$$
\begin{aligned}
& \text { L. castillejae } \\
& \text { L. concinna }
\end{aligned}
$$

Castilleja pallida (L.) Sprengel

$$
\text { L. castilleiae }
$$

Catabrosa algiala Fr.

$$
\text { L. algida }
$$

Ceanothus sp.

$$
\text { L. ccanothi }
$$

Cecropia peltata L.

$$
\text { L. cecropiue }
$$

Centamea jucea L.

$$
\text { L. jucene }
$$

Contamea scabiosa L.

$$
\text { L. contarreae }
$$

Centrunthus sp.

$$
\text { L. galiicola var. brachy- }
$$
sporic
Cephalaria uralensis (Murray)

Roemer \& Schultes

L. cephalariai-uralensis

L. taurica

Cerastium biebersteinii DC.

L. biebersteinii

Cercocarpus ledifolius Nutt. ex Torr. \& Gray

$$
\text { L. cercocarpi }
$$

Cereus peruvianus (L.) Miller

$$
\text { L. cerei-peruviani }
$$

Chaerophyllum aureum L.

$$
\text { L. agnita var. major }
$$

Chamaerops excelsior Boj

$$
\text { L. spatharum }
$$

Chamaerops humilis $\mathrm{L}$.
L. algarbiensis
L. chamaeropis
L. debeauxii
L. magnusiana

Chamaerops sp.

L. pruni forma plurivora

Cheiranthus anmuns L.

$$
\text { L. impressa }
$$

Chelidonium majus L.

$$
\text { L. chelidonii }
$$

Chenopodium album $\mathrm{L}$.

$$
\begin{aligned}
& \text { L. calvescens } \\
& \text { L. chenopodii-albi } \\
& \text { L. entypoides } \\
& \text { L. inculta } \\
& \text { L. nigricans }
\end{aligned}
$$

Chenopodium anthelminticum

Bert. ex Steud.

$$
\text { L. anthelmintica }
$$

Chenopodium sp.

$$
\text { L. criophora }
$$

Chimonamhiss sp.

$$
\text { L. coniothrium }
$$

Chondrilla juncea L.
L. hella
L. churnea
L. mirabilis
L. Irichostoma

Chondrus crispus J. Stackhouse

$$
\begin{aligned}
& \text { L. chondri } \\
& \text { L. danica } \\
& \text { L. mavina }
\end{aligned}
$$

Chrsanthemum corvmbosum $\mathrm{L}$.

$$
\text { L. agnitu var. ehrysanthemi }
$$

Chrsanthemum leucanthemum $\mathrm{L}$.

$$
\text { L. Ianaceri }
$$

Chrysunthemum vulgare (L.)

Bernh.

$$
\begin{aligned}
& \text { L. dolioloides } \\
& \text { L. Ianaceri }
\end{aligned}
$$

Chrysothumms groveolens

(Nutt.) Greane

L. arthrophyma 
Chusquea serrulata Pilger

$$
\begin{aligned}
& \text { L. chusqueac } \\
& \text { L. saginata }
\end{aligned}
$$

Chusquea sp.
L. consociata
L. stellata

Cicclidoms fontinaloides (Hedw.)

P. Beauv.

$$
\text { L. cinclidori }
$$

Cinnamomum camphora T. Nees ex Eberm.

$$
\text { L. cinnumomi }
$$

Cinnumonum zey/anicum Blume

$$
\text { L. almeidae }
$$

Cirsium altissimum (L.) Spreng.

$$
\text { L. compressa }
$$

Cirsium arvense (L.) Scop.

$$
\text { L. cirsii-arvensis }
$$

Cirsium lanceolatum (L.) Scop.

$$
\text { L. corynispora }
$$

Cirsium muticum Michx.

$$
\text { L. mesoedema }
$$

Cirsium palustre (L.) Scop.

$$
\begin{aligned}
& \text { L. galionum forma } \\
& \text { cirsiorum }
\end{aligned}
$$

Cirsium sp.

L. dolioloides var. cirsii

L. doliohum

Cistus albidus $\mathbf{L}$.

$$
\text { L. cisti }
$$

Cistus ladanifer $\mathrm{L}$.

$$
\text { L. cistina }
$$

Cistus monspeliensis L.

$$
\text { L. cisticola }
$$

Citrus awrantium L.

$$
\begin{aligned}
& \text { L. bondari } \\
& \text { L. papulosa }
\end{aligned}
$$

Cirrus grandis Osb.

$$
\text { L. hondari }
$$

Cirrus limonia Osb.

$$
\text { L. bondari }
$$

Citrus limonum Risso

$$
\begin{aligned}
& \text { L. vagabunda forma cirri- } \\
& \text { limonii }
\end{aligned}
$$

Citrus medica L.

$$
\text { L. bondari }
$$

Cirrus sinensis Osb.

Cirrus sp.

$$
\text { L. bondari }
$$

L. hondari
L. cirricola

Cladium articulatum R. Br.

$$
\text { L. aliena }
$$

Cladium mariscus (L.) Pohl

$$
\begin{aligned}
& \text { L. cladii } \\
& \text { L. crucheri }
\end{aligned}
$$

Clematis recta $\mathrm{L}$.

$$
\text { L. alucta }
$$

Clemutis vitalba $\mathrm{L}$.

$$
\text { L. agminalis }
$$

L. agminalis forma minor

L. grignomenesis

L. haematites

L. incruenta

L. nectrioides

L. pleosporroides

L. pyrenopezizondes

L. rimalis

L. vagabunda

L. vitulbae

L. vitalbae var. sarmemicola

Clerodendrum foetidi Bunge

L. cleradendri

Coccothrinax argentata (Jacq.)

Bailey

L. coccothrinacis

Cocos romanzoffiama Cham.
L. cocoes
L. molleriana

Coffea arabica $\mathrm{L}$.
L. coffeigena

Coffea canephora Pierre ex

Froehn.

$$
\begin{aligned}
& \text { L. canephorue } \\
& \text { L. coffeigena var. longiros- } \\
& \text { Iata }
\end{aligned}
$$

Coffea excelsa Cheval.
L. centrafricand
L. excelsa
L. lobayensis
L. longispora

Coffea robusta L. Linden
L. africana
L. cylindrospora
L. gigaspora
L. macrorostra
L. oubanguiensis
L. terraspora

Coffea sp.
L. coffacicida
L. pusilla
L. tonduzi

Coix lacryma-jobi L. var. susutama Honda

L. coicis

Coleosanthus renifomis (Gray)

Rydb.

$$
\text { L. coleosanthi }
$$

Collinsonia canadensis $\mathrm{L}$.

$$
\text { L. collinsoniae }
$$

Collomier squarrosa Nutt.

$$
\text { L. collumice }
$$

Colocasia antiquorum Schout

$$
\text { L. colocasiae' }
$$

Comium maculanum $\mathrm{L}$.

$$
\begin{aligned}
& \text { L. conii } \\
& \text { L. conitgena } \\
& \text { L. rubicunda }
\end{aligned}
$$

Comvallaria majalis L.

$$
\text { L. smarodsii }
$$

Comvallaria multiflora $\mathrm{L}$.

L. convallariae

Comvallaria polygonatum $\mathrm{L}$.

L. bellynckii

Cora paronia (Swartz) Fries

$$
\text { L. corae }
$$

Corallorrhiza multiflora Nutt.

$$
\text { L. corallorlizac }
$$

Cordyline dracaenoides Kunth

$$
\text { L. corlylines }
$$

Cormus alha L.

L. limitata

Cormus florida $\mathrm{L}$.

L. olvaespora

Cormus sanguinea $\mathrm{L}$.
L. corricola
L. firdlaeri
L. limitata
L. platycarpa
L. vagabunda

Cornus stolonifera Michx.

$$
\text { L. rugosa }
$$

Cormus sp.

$$
\text { L. mamillana }
$$

Coronilla coronata $\mathrm{L}$.

$$
\begin{aligned}
& \text { L. dumetorum var. } \\
& \text { cormillae }
\end{aligned}
$$

Coronilla emerus $\mathrm{L}$.

$$
\text { L. riofriot }
$$

Coronilla sp. [as $C$. comata L.]

L. cormillae

Corvus comix [Animalia]

$$
\text { L. corvina }
$$

Conylus avellana $\mathrm{L}$.

L. avellanae

L. vagabunda

Corylus sp.
L. leucoplaca

Cosmos sulphureus Cav.

L. cosmicola

Coumarouna punctata S.F. Blake

$$
\text { L. coumarrunas }
$$

Cousinia mutriloba DC.

$$
\text { L. shahnarica }
$$

Cratacgues monogyna Jacq.

$$
\text { L. pomona forma tran- }
$$
silvenicas

Crataegus oryacantha $\mathrm{L}$.

$$
\text { L. saccardiana }
$$

Crepis sibirica $\mathbf{L}$.

$$
\text { L. sibirica }
$$

Crepis vesicaria L. subsp.

hacenseleri (Boiss. ex DC.) P.D.

Sell

L. inbellula

Crithmum maritimum $\mathrm{L}$.
L. Ielminthospora forma crithimi-maritimi
L. hopalispora

Curumis sativus L.

$$
\text { L. alexomdrinis }
$$


Cucurbia pepo L.
L. cucurbitae

Cycas revolula Thunb.

L. cycadis
L. irrepia

Cynanchum sp.

L. modesta var. cibostii

L. scotophila

Cynodon dactylon (L.) Pers.

L. cynodontis-dactyli

L. korrae

L. narmari

L. rhizomatum

Cynodon transvaalensis BurttDavy

L. narmari

Cynodon sp.

L. poniformis

Cynoglossum officinale $\mathbf{L}$.

L. cynoglassi

L. vindobonensis

Cynosurus cristatus $\mathrm{L}$.

L. cymosuri

Cyperus flavescens $\mathrm{L}$.
L. cyperi
L. cypericola

Cyperus monti $\mathrm{L}$.

L. cyperina

Cyperus papyrus L.

L. papyri

Cyperus schoenoides Griseb.

L. ceballosi

Cytisus alpinus Miller

L. corrugans

Cytisus labumum L.

L. lacina

Dactylis glomerata L.

L. caricis

L. culmicola var. nigrans

L. dactylina

L. nigrans

L. recurita

Danthonia frigida Vickery

L. gaubae

Daplme gmidium L.

L. daphnes

L. ragabunda forma daphmes

Daphniphyllum macropodium

Mig.

L. dap/miphylli

Dasyliriom junceum Zucc.

$$
\text { L. dasylirii }
$$

Dasystoma sp.

L. doliolum

Datura stramonitum L.

L. caballeroi

Danarus carola L.

L. lomgipedicellara

l. rastripii
Datucus sp.
L. comatella
L. modesta forma dauci

Daviesia latifolia $\mathrm{R} . \mathrm{Br}$.

L. daviesiae

Desmodium sp.
L. dissiliens
L. distributa

Desmoncus sp.

L. desmonci

Deutzia scabra Thunb.

$$
\text { L. dichroa }
$$

Dianella revolura $\mathrm{R}$. $\mathrm{Br}$.

$$
\text { L. nilliansii }
$$

Dianthus atrorubens All.

$$
\text { L. leptospora }
$$

Dianthus caryophyllus L.

$$
\text { L. dianthi }
$$

Dianthus prolifer L.

L. proliferae?

Dianthus superbus L.

$$
\text { L. dianthi }
$$

Dianthus sp.

$$
\text { L. dianthi }
$$

Dichosciadium ranunculaceum

(F. v. Mueller) Domin

L. dichosciadii

Didymella vincetoxici (de Not.)

Sacc.

L. didymellae-vincetoxici

Digitalis lutea L.

L. modesta forma digitalisluteae

Digitalis purpurea $\mathbf{L}$.

L. valdiviensis

Digiralis sp.

$$
\text { L. digitalis }
$$

Dipsacus sylvestris Hudson

L. bractearum var. bractearum

L. carpophila

L. modesta forma syliestris

Dodonaca viscosa (L.) Jacq.

$$
\text { L. dodonacae }
$$

Dorycnium suffruticosum Vill.

L. cucurbiturioides

Draba alpina L.

L. drabae

Dracuena araco (L.) L.

L. comvallariac

L. comallariae forma dracaenae

L. dracuenae

L. dracomis

Drvas actopetala L.

L. dryadis

L. rostripuii

Drypis spimosa L.

L. relimiana

Dulcamarasp.

L. lagabunda
Duponia fisheri $\mathrm{R}$. Br.

L. insignis

L. vagans

L. vagans forma scirpi

Dupontia psilosantha (Rupr.)

Griseb.

L. insignis

Echium vulgare L.

L. cesatiana

L. echiella

L. echii

Eichhornia crassipes (C.F.P.

Mart.) Solms-Laub.

L. eichhorniae

Elaeis guineensis Jacq.

L. elacidicola

L. elacidis

Eleocharis sp.

L. narfolcia

Elymus arenarius L.

L. arenaria

L. elymi

L. Iarseniana

L. quinta

Elymus? barbatus F. Kurtz.

L. lagenoides

Elymus glaucus Buckley

L. elongata

Elymus sp.

L. elymi

Elyna spicata Schrader

L. sparsa var. elynae

Elyrrigia juncea (L.) Nevski

L. ammophilae

Empetrum nigrum L.

L. emperri

Endiusa hirsura Alef.

L. endiusae

Ephedra distacliva L.

L. ephedrae

Epichloe Iyphina (Pers.:Fr.) Tul.

L. associata

Epilobium angustifolium L.

L. culindrospora

Epilobium dodonaei Vill.

L. cadubriae

Epilabium fleischerj Hochst.

L. epilobii

L. multiseptasta forma alpina

Epilabium hirsutum L.

L. "rinemlosa

Epilobium montanum $\mathrm{L}$.

L. epilobii

Epilobium trigomum Schrank L. cpilohii

Equiserum arrense L.

L. arvensis

Equiseasm hyemale L.

L. borlesei

L. hiemalis 
Equisenum limosum L.

\section{L. limosa}

Equiserum variegatum Schleicher ex Weber \& Mohr
L. equiseli
L. equisericola

Equisetum sp. [as E. pallidum] L. larialis

Eranthemum sp. L. eranthemi

Eremochloa ophiwroides (Munro) Hack.

\section{L. korrae}

Erianthus alopecuroides (L.) El]. L. orthogramma

Erica carnea L.

$$
\text { L. subtecta }
$$

Erigeron canadensis L.
L. agnita var. erigerontis
L. canudensis
L. congesta

Erigeron viscidus Rydb.

L. erigerontis

Erigeron sp.
L. doliolum
L. plurisepta

Eriobotrya juponica (Thunb.)

Lindley
L. eriobotryae
L. miyakeana
L. putremansii

Eryngium agavifolilum Griseb.

$$
\text { L. vinosa }
$$

Eryngium campestre L.
L. eryngii
L. nigrella
L. woodrow'n'ilsonii

Ensimum cheiranthoides $\mathrm{L}$.

$$
\text { L. submaculans }
$$

Erysimum diffusmm Ehrh.

L. acutispora

Erysimum erysimoides (L.)

Fritsch ex Janchen

L. acutispora

Erysimum hieracifolium L.

L. fusispora forma erysimi

Erythrina crista-galli $\mathrm{L}$.

$$
\text { L. aerea }
$$

Erythina sp.

$$
\text { L. erythrinae }
$$

Espeletia neriifolia Sch. Bip. ex Wedd.

$$
\text { L. jahnii }
$$

Espeletia sp. (?E. schultzii

Wedd.)

$$
\text { L. espeleriae }
$$

Eucalypus globulus Labill.

$$
\text { L. molleriana }
$$

Euonymus europaeus L.
L. castagnei
L. constricta

Eurmymuss sp.

$$
\text { L. californiea }
$$

Eupatorium cumnabinum L.
L. agnita var. ambigua

L. cerasiii

Eupatorium capillifolium (Lam.)

Small

L. clavispora

Eupatorium coronopifolium

Willd.

\section{L. mesoedema}

Eupatorium macularum L.

$$
\text { L. jacksonii }
$$

Eupatorium pupureum L.

$$
\text { L. jacksonii }
$$

Eupatorium sancechicoense

Hieron.

$$
\text { L. preandina }
$$

Eupatorium sp.

$$
\text { L. doliolum }
$$

Euphorbia cyparissias L.

$$
\text { L. euphorbiae }
$$

Euphorbia dendroides $\mathrm{L}$.

$$
\text { L. succulus }
$$

Euphorbiu esula L.

L. eluphorbiae forma esulae

Euphorbia osyridea Boiss.

L. euphorbicolla

Euphorbia oxyroidea [authority unknown]

$$
\text { L. depressa }
$$

Euphorbia pilosa L.

$$
\text { L. euphorbiaecola }
$$

Euphorbia sibthorpii Boiss.

$$
\text { L. siburpii }
$$

Euphorbia w'ulfenii Hoppe ex

Koch

\section{L. bresadolaeana}

Euphorbia sp.

L. tolgorensis

Euphrasia lutea L.

L. coniformis

Eustephia sp.

$$
\text { L. australis }
$$

Fagus sylvatica L.

$$
\text { L. vakdobbiae }
$$

Fagus sp.
L. faginea
L. seminuda

Farsetia incana (L.) R. Br.

$$
\text { L. conferta }
$$

Feijod sp. [as F. feijoicola]

$$
\text { L. feijoare }
$$

Ferula badra-kema K. Pol.

$$
\text { L. fermlicola }
$$

Festuca arenaria Osbeck

$$
\text { L. aremaria }
$$

Fesurca arundinacea Schreber L. sabulctorum

Fesuca kerguelcnsis Hook. f. L. kerguelensis
Festuca sp.
L. crastophila
L. enstomella
L. stromatoider
L. taminensis

Ficus elastica Roxb.

$$
\text { L. fici-elasticae }
$$

Filipendula ulmaria Maxim.
L. obesula
L. rustica

Florovia excelsa DC.

$$
\text { L. flotoviae }
$$

Foeniculum vulgare Miller

$$
\begin{aligned}
& \text { L. foeniculacea } \\
& \text { L. foeniculi }
\end{aligned}
$$

Foeniculum sp.

L. doliolum

L. doliolum var. pinquicula

Francoa sonchifoliae Cav.

$$
\text { L. francoare }
$$

Frasera speciosa Dougl. ex Griseb.

$$
\text { L. fraserae }
$$

Frasera? sp.

$$
\text { L. harknessiamma }
$$

Froximus americana L.

$$
\text { L. fravini }
$$

Fraximus sp.
L. controversa
L. leucoplaca

Fungi
L. associata
L. caucana
L. cryptica
L. didynellae-vincetoxici
L. fungicola
L. geasteris
L. phyllachoricola
L. phyllachorivora
L. platvchorae
L. siereicola
L. Iungurahuensis

Furraea bedinghausii C. Koch

L. rusci forma fourcrovae

Furcraea longatia Karw. \& Zucc.

$$
\text { L. infernalis }
$$

Furracasp.

$$
\text { L. translueens }
$$

Galeoblolon lateum Hudson

L. galeobdolonis

Galeopsis speciosa Miller

L. galeopsidicola

Galim aparine L.

$$
\begin{aligned}
& \text { L. aparines } \\
& \text { L. scirnla }
\end{aligned}
$$

Galium boreale $\mathbf{L}$.
L. dumetorum var. galii- borealis

Galium mollugo L.

L. galiicola 


\section{L. molluginis \\ L. plectrospora}

Galium sylvaticum L.

L. galii-silvatici

Galium sp.
L. galii
L. galiorum

Gaultheria s/allon Pursh

L. gaultheriue

Geaster psendolimbanus Hollós

L. geasteris

Genista anglica $\mathrm{L}$.

L. genistue

Gonista ferox Poir.

L. phiala

Genista tinctoria L.

\section{L. fusispora}

L. genistae var. miciospora

Gentiana asclepiadea $\mathrm{L}$.

$$
\text { L. umbrosa }
$$

Gentiana lutea L.
L. galiorum forma gentianae
L. salebrosa
L. sapeyensis

Geranium richardsonii Fisch. \& Trautv.

\section{L. modestula}

Geranium sp.
L. subcacspitosa

Gerardia sp.
L. doliolim!

Ginkgo hiloha L.

$$
\text { L. ginkgo }
$$

Glvceria aquarica (L.) Wahlenb.
L. microscopica forma glveriae
L. recutita

Glyceria fluitans (L.) R. Br.

L. glyeeriac

Glyceria plicata (Fries) Fries

L. glyceriae-plicatae

Glyceria spectabilis Mert. \&

Koch

L. culmorum var. flavolvinnea

Gnaphalium sp.

L. galiorum var, gnaphaliana

L. gnaphalii

Gossypiam sp.

L. gossypii

Grindelia? sp.

L. nigricans var. grindeliae

Guazuma sp.

L. guazumae

Gymmadenia comopsea (L.) R. Br.

L. orchidearum

Gyerium argenteum Nees

L. gynerii

L. subiculifera?
Gypsophila paniculara L. L. gypsophilae

Haloxylon aphyllum (Minkw.) 11 jin

$$
\text { L. haloryli }
$$

Hamemelis virginiana L.

$$
\text { L. hamamelidis }
$$

Hardenbergia violacea

(Schneev.) Stearn

$$
\text { L. hardenhergiae }
$$

Hederu helix L.
L. hederae
L. hedericola
L. helicicola
L. papulosa

Hedvarum obscurum L.

L. chochrjakiovii

Heleocharis palustris (L.) Roem. \& Schult.

L. sowerbyi

Helianthemum sp.

L. helianllemi

Helianthus annuws L.
$L$. drechsleri
L. helianthi
L. lindquistii

Helianthus gigantews L. L. helianthi

Helianthus grosserratus Martens L. drechsleri

Helianthus sp.
L. consessa
L. doliolum
L. heliopsidis

Heliopsis sp.

L. drechsleri

L. heliopsidis

Helleborus foetidus $\mathrm{L}$.

L. Lamonia

Helleborus viridis L.

L. lathonia var. helleborifoetidi

Helonias hullata L.

L. heloniacfolia

Hemerocallis sp.

L. hemerocallidis

Heracleum lanarum Michx.

L. simmonsii

Heracleam palmatum Baumg.

L. doliolum var, dissimilis

Heracleum sphondylium L.

$$
\text { L. massarioides }
$$

Heracleum sp.

L. doliolum

Hemodactrilus mberosus (L.)

Miller

L. hemodactili

Hesperis dinarica $\mathrm{G}$. Bech

L. hesperidicola
Hesperis sp.

L. galiicola var hrachyspora

Hevea brasiliensis Müll. Arg.

$$
\text { L. heveae }
$$

Hicoria sp.

$$
\text { L. exocarpogena }
$$

Hieracium murorum L.

$$
\text { L. brauni }
$$

Hierochloë alpina (Willd.)

Roemer \& Schultes

$$
\text { L. hierochloae }
$$

Hierochlö ëantaretica $\mathrm{R} . \mathrm{Br}$.

$$
\text { L. fuegiana }
$$

Hippophaë rhammoides L.
L. fuscella var. hippophaes
L. fuscella var. sidowiana
L. hippophaes

Homo sapiens

$$
\begin{aligned}
& \text { L. senegalensis } \\
& \text { L. tompkinsii }
\end{aligned}
$$

Hordeum arenarium Ascherson

$$
\text { L. arenaria }
$$

Hordeum distichon L.

$$
\text { L. recuitu }
$$

Hordeum turkestanicum Nevski

$$
\text { L. hordei }
$$

Hordeum vulgare $\mathrm{L}$.

$$
\text { L. narmari }
$$

L. Ilodorum form sp. hordei

Humulus lupulus L.

$$
\text { L. dunetorum }
$$

Hura crepilars L.

$$
\text { L. lintare }
$$

Hidrangea arborescens L.

$$
\text { L. hivdrangeae }
$$

Hydrocoñle asiatica L.

$$
\text { L. nesodes }
$$

Hyparrhenias sp.

$$
\text { L. hyparheniae }
$$

Hypericum alpestris Stev. ex Ledeb.

\section{L. hypericola}

Hyperioum calvinum L.

$$
\text { L. vasabunda }
$$

Hypericum perforatum L.
L. cesatiana
L. dematium
L. Inperici
L. lonkeana
L. acellata

Nex paragwariensis A. Si. Hil.
L. paraguariensis
L. verlate

minla crithmovides $\mathrm{L}$.

L. helminhospora

L. rhopalispora

Imula salicima L.

L. devasa var. francomica
L. framoonica 
Ipomoea batalas (L.) Lam.
L. bataticola
L. ferruginea

Iris arenaria Waldst. \& Kit.

$$
\text { L. iridis }
$$

Iris foctidissima $\mathrm{L}$.
L. iridicola
L. vectis
L. xipliii

Iris germanica L.
L. heterospora
L. parvula var. iridis- germanicale

Iris pscudacortis $\mathrm{L}$.
L. iridigena
L. larseniana
L. longispored
L. microllywioides
L. parvinla

Isatis tinctoria $\mathrm{L}$.

L. allorgei

Jambosa molaccensis DC.

L. eustomoides var. punctata

Jasmimum fruticans $\mathrm{L}$.

L. emiliana

Jubaca spectabilis Humb.,

Bonpl.. \& Kunth
L. briosiana
L. jubacae

Juglans regia $\mathrm{L}$.
L. petiolaris

Juglans sp.

L. leucoplaca

Juncus acuriflorus Ehrh. ex Hoffm.

\section{L. juncorum}

Juncus acumus L.

$$
\text { L. junci-acuri }
$$

Juncus articulatus L.

L. heropliila

Juncus bighmis L.

$$
\text { L. junciseda }
$$

Junc'us drummondii E. Mey.

$$
\text { L. sollicimii }
$$

Juncus effusus L.
L. defodiens
L. lydiophila
L. junci
L. juncina
L. perkovicensis
L. pseado-diaporthe
L. riparia
L. viridella

Juncus filiformis L.

L. sepalorum

Juncus glaucus Sihth.
L. junci
L. junci-glanci
L. subriporio
L. vitensis

Juncus hostï Tausch

$$
\text { L. juncicola }
$$

Juncus lampocarpus Ehrh. ex Hofilm.

$$
\begin{aligned}
& \text { L. lamprocarpi } \\
& \text { L. monilispora }
\end{aligned}
$$

Juncus maritimus Lam.

$$
\begin{aligned}
& \text { L. maritima } \\
& \text { L. neomaritima }
\end{aligned}
$$

Juncus roemeriamus Scheele

$$
\text { L. neomaritima }
$$

Juncus trifidus L.
L. juncicola
L. sepalorum

Juncus sp.
L. michorii
L. norfolcia
L. variabilis

Juniperus communis $\mathrm{L}$.

$$
\text { L. juniperi }
$$

Juniperus nana Willd.
L. juniperina
L. saprophila

Kalmia angustifolia $\mathrm{L}$.

$$
\text { L. kalmiae }
$$

Kerria japonica (L.) DC.

Kerria sp.

$$
\text { L. vagabunda }
$$

L. conioshyrium

L. iagabunda var. divergens

Kigelia pinnata DC.

L. dryadea subsp. lussonicnsis

Knautia arvensis (L.) Coulter L. pliyteumaris

Kocleria albescens DC.

$$
\text { L. clavata }
$$

Kocleria cristata (L.) Pers.

$$
\text { L. herpotrichoides }
$$

Kocleria gracilis Pers.

$$
\text { L. rlavata }
$$

Koerberiella wimmeriana

(Koerber) B. Stein

$$
\text { L. koerheri }
$$

Lacura muralis (L.) Gaertner

Lacuea sp.

$$
\text { L. quadriseptata }
$$

\section{L. doliolum}

Lagoris minor (Willd.) Standley

$$
\text { L. holmii }
$$

Lamium album L.

L. parietariae forma lamii

Lamana camara L.
L. isocellula
L. rajusthanensis

Lappa sp.
L. eriophora
L. galicola var. brachy- spora
L. modesta forma lappac

Lapsana communis L.

L. galiorum var. lapsanae

Luscrpitium angustifolium Georgi

L. oreophiloides

Laserpitum halleri Crantz

L. ladina

Laserpitium larifolium $\mathrm{L}$.

$$
\text { L. etralensis }
$$

Laserpitium siler $\mathrm{L}$.

$$
\begin{aligned}
& \text { L. comura } \\
& \text { L. sileris }
\end{aligned}
$$

Laserpirium sp.

L. spectabilis

Lathyrus latifolius $\mathbf{L}$.
L. latlyrina
L. niessleana

Lathrus niger (L.) Bernh.

L. dolioloides var. lathyri

Lathyrus sylvestris L.
L. lathyri
L. mulriseptata

Laurus nobilis $\mathrm{L}$.
L. lauri
L. nobilis
L. paoluccii

Laurus sp.

$$
\begin{aligned}
& \text { L. Coniothyrium var. } \\
& \text { foliicola }
\end{aligned}
$$

Lavandula stoechas $\mathbf{L}$.

L. lavandulae

Lavandula vera DC.

L. salvige forma minor

Lavandula sp.

L. conomillesii

Lecanora frustulosa (Dicks.) Ach. L. steinii

Lecidea excentrica Roehling L. advenula

Lecideo vernalis (L.) Ach.

L. consocians

Leersia oryzoides (L.) Swartz
L. leersiae
L. lecrsiana

Leleha sp. [as L. simplex] L. lelebae

Lemanca pluvialc C. Aq.
L. fluviatilis

L. lemaneae

Leonurus cardiaca L.

L. doliohm var. leomuri

Lepidium campestre (L.) R. Br.

L. ogilviensis forma lepidii

Lepidium virginicum $\mathrm{L}$.

$$
\text { L. virginica }
$$

Leptorhaphis leprogiophila Minks ex Wint.

\section{L. leprogiophila}

Lepus curopacus

L. fimiscede

Lespedeza bicolor Turcz.

L. lespedezae 
Lespedeza capitata Michx.
L. microspora

Liatris scariosa (L.) Willd.

$$
\text { L. trimerioides }
$$

Libanotis montana Crantz
L. foliicola
L. libanotis

Libanotis sp.

Lichenes
L. longchampsi

L. advenula

L. apocalypta

L. arnoldii

L. baeomycearia

L. caminae

L. clarkii

L. consocians

L. corae

L. crozalsii

L. galligena

L. geographicola

L. inarensis

L. koerberi

L. leptogiophila

L. lencomelaria

L. lichenicola

L. maheui

L. mamillula

L. neottizans

L. oligospora

L. parmeliarum

L. peltigerarum

L. peltigerea

L. polaris

L. pycnostigma

L. ramalinae

L. rivana

L. rivana forma solorinae

L. sphyridiana

L. steinii

L. stereocaulorum

L. tartarina

L. umbilicariue

L. usneae

Lilium martagon $\mathrm{L}$.

L. martagoni

Lilium superbum L.

$$
\text { L. lilii }
$$

Lilium sp.
L. lilicola

Linum tenuifolium L.

L. Irwhyana

Lippia (Aloysia) citriodora Kunth

L. octophragmia var, major

Lippia seriphioides A. Gray

L. hasalduai

Liviodendron mulipifera L.

L. halima

Liriodendron sp.

L. stictoides
Lohelia excelsa Lesch.

$$
\text { L. lobeliae }
$$

Lohelia mpa L.

$$
\text { L. tupae }
$$

Lolium perenne $\mathrm{L}$.

$$
\text { L. eustomoides }
$$

L. lolii

Lomaria obliqua $\mathrm{R}$. Br.

L. chilensis

Lonicera alpigena $\mathrm{L}$.

$$
\text { L. albulac }
$$

Lonicera canadensis Bartr.

$$
\text { L. lonicerae }
$$

Lonicera caprifolium $\mathbf{L}$.

$$
\begin{aligned}
& \text { L. caprifolii } \\
& \text { L. corticola }
\end{aligned}
$$

Lonicera periclymenum L.

$$
\text { L. periclymeni }
$$

Lonicera tatarica L.

$$
\text { L. frondis }
$$

L. periclymeni var. tatarica

L. surculorum

Lonicera nylosteum L.

$$
\text { L. lonicerae }
$$

Lonicera sp.
L. aulica
L. Ionicerae

Lophanthus sp.

L. lophanthi

Lunaria rediviva L.

$$
\text { L. lunariae }
$$

Lupinus sp.

L. barriae

L. byssincola

L. foeniculacea subsp. lupina

L. frigida

L. lupini

L. lupinicola

Lupinis latifolius J.G. Agardh

L. wehmeveri

Lupimus obtusilobus

L. castrensis

Lupinus mbricaulis Greene

L. wehmeveri

Lupumus albicaulis var. shasiensis

L. shastensis

Luzula albilla (Holfm.) DC.

L. culmorum forma hangarica

L. epicalamia var. pleosporoides

L. juncina forma ma(r) spora

Luzula lutea (AII.) DC.

L. cpicalamia

L. microscopica var. alpima

Luzula maxima (Reichard) DC.

L. Iuzulac
Luzula nemorosa (Pollich) E.H.F. Meyer
L. epiculamia
L. petrakii

Luzula sylvatica (Hudson) Gaudin

L. caricis

L. epicalamia

Lycium vulgare Dunal.

L. IVciophila

Lycopodium annorinum $\mathbf{L}$.
L. campisilii
L. crepini
L. lycopodina
L. marcyensis

Lycopodium claratum L.

L. Iycopodiicola

Lycopodium selago $\mathrm{L}$.

L. marcyensis

Lycopodium selago L. var. appressum Desv.

L. arctalaskana

Lvcopus europaeus L.

L. cesatiana

Lygeum spartum L.

$$
\text { L. spartii }
$$

Lythrum alatum Pursh

$$
\text { L. Juthri }
$$

Lythrum salicaria $\mathbf{L}$.

$$
\text { L. salicaria }
$$

Maclura auramiaca Nutt.

$$
\text { L. maclurac }
$$

Magnolia grandiflora L.

$$
\text { L. magnoliae }
$$

Magnolia yulan Desf.

$$
\text { L. yulan }
$$

Malus domestica Borkh.
L. mandshurica
L. pomona
L. ternata

Malus sp.

L. concentrica

Manihot utilissima Poh]

$$
\text { L. perri }
$$

Marama arundinaces L.

L. marantac

Maratia sp.

$$
\text { L. caffra }
$$

Marrubium vulgare L.

L. dumetorum var. marrubii

Matisia paraensis Huh.

$$
\text { L. matisiae }
$$

Medicago falcata $\mathrm{L}$.

$$
\text { L. Homeri }
$$

Medicago sariva L.

$$
\begin{aligned}
& \text { L. circinans } \\
& \text { L. medicaginicola } \\
& \text { L. medicaginis } \\
& \text { L. patensis }
\end{aligned}
$$


Medicago sp.
L. medicaginis
L. medicaginum

Melampyum pratense L.

$$
\text { L. suffulea }
$$

Melandrium apetahum (L.) Fenzl L. vanhoeffeniana

Melandrium Irifforum J. Vahl. ex Licbn.

$$
\text { L. valii }
$$

Melica alrissima L.

L. culmicola forma melicae

L. fuscidula

Melica inaequiglumis Boiss.

$$
\text { L. melicae }
$$

Melica magnolii Gren. \& Godron

$$
\text { L. fuscidula forma }
$$$$
\text { magnolii }
$$

Melica uniflora Retz.

$$
\text { L. melicae }
$$

Melilotus alba Medicus

$$
\begin{aligned}
& \text { L. dumetorum forma } \\
& \text { melilori } \\
& \text { L. melilori }
\end{aligned}
$$

Melilotus sp.

$$
\text { L. viridella }
$$

Menispermum conadense $\mathrm{L}$.

$$
\text { L. coniothyrium }
$$

Mentha piperita L.

$$
\text { L. substerilis }
$$

Mentha sylvestris L.

$$
\begin{aligned}
& \text { L. menthae } \\
& \text { L. zahlbruckneri }
\end{aligned}
$$

Mertensia sp.

$$
\text { L. merrensiae }
$$

Mesogloia leveillei (J. Ag.)

Mengh.

$$
\text { L. mirandae }
$$

Metasequoia glyptostroboides $\mathrm{Hu}$

\& Cheng

$$
\text { L. metasequoiae }
$$

Hikamia sp.

L. agnita var. acheniaram

L. mikaniase

Milium effusum L.

$$
\text { L. linearis }
$$

Minuartia sedoides (L.) Hiern.

$$
\text { L. dennisiana }
$$

Monotes dasyantus Gilg.

$$
\text { L. batmmii }
$$

Moriera sp.

$$
\text { L. moricras }
$$

Morus alba L.

$$
\begin{aligned}
& \text { L. fallax } \\
& \text { L. massariella var. disticha }
\end{aligned}
$$

Mortus sp.

$$
\text { L. japonica }
$$

Muehlemberkia plaryblatos

Meissn.

L. muehlenbeckine
Muehlenbergia filiformis Rydb. L. georgius-fischeri

Muehlenbergia racemosa

(Michx.) Britton, Stern, \& Pogg. L. muchlenbergiae

Musa cavendishii Lamb. ex

Paxion

L. musae

L. musigena

Musa sp.
L. musarum
L. taichungensis

Muscari comosum (L.) Miller

$$
\text { L. muscari }
$$

Musci

L. bryophila

L. cinclidoti

L. heufleri

L. polytrichina

Myrica gale L.

L. myricase

Myrrhis odarata (L.) Scop.

L. ogilviensis forma myrrhis-odoraia

Mytus communis L.

L. myrti

L. myrticola

Nandina domestica Thunb.

L. nandinae

Nardus stricta L.
L. nardi
L. pachyrheca

Obione portulacoides (L.) Moq.

L. obiones var. evolutior

Obione sp.

L. obiones

Ochrolechia fartarea (L.) Massal

L. tartarina?

Ocimum sanctum L.

L. ocimicola

Oenothera biennis L.

L. capsularum

L. ellisiana

Oenothera longiflora L.
L. argentina

Onagra strigosa Rydb.

L. onagrae

Onobrychis sariva Link
L. onobrechidicola

L. onobrvehidis

Ophiopogon japonic'us Ker-Gawl.

L. ophiopogonis

Opuntia ficus-indica (L.) Miller

$$
\text { L. sicula }
$$

Opumia lindho'imeri Engelm.

$$
\text { L. opumiase }
$$

Ornithogalum prenenic'um I.

$$
\text { L. ornithogali }
$$

Orhhrosambus chimboracensis

Baker

L. orthrosanthi
Oryza sariva $\mathrm{L}$.
L. cattanei
L. inecola
L. inamotoi
L. korrae
L. narmari
L. oryzue
L. oryzicola
L. oryzina
L. salvinii

Oryzopsis miliacea (L.) Bentham \& Hooker ex Ascherson \& Graebner

$$
\text { L. papillosa }
$$

Oxalis stricta $\mathrm{L}$.

$$
\text { L. aglaja }
$$

Orvia digyna (L.) Hill

$$
\text { L. onyriae }
$$

Paeonia moutan Sims.

Palm

$$
\text { L. moulan }
$$

L. Hochlus

Pandanus odoratissimus Nor. L. pandanicola

Pandanus utilis Bory

$$
\text { L. pandani }
$$

Panicum crus-galli L.
L. occidentalis

Panicum grumosum Nees
L. anthostomella
L. melanommoides

L. subsuperficialis

Panicum palmaefolium Koen.

$$
\text { L. panici }
$$

Papaver radicatum Rottb.

$$
\text { L. papaveris }
$$

Parietaria officinalis $\mathrm{L}$.
L. muralis
L. parietariae

Parmelia saxatilis (L.) Arch.

$$
\text { L. parmeliarum }
$$

Parmelia sp. [as $P$. arrata

Zahlbr.]

L. galligena

Paspalum giganteum Baldw. ex Vasey

$$
\text { L. proteispora }
$$

Pastinaca sativa L.

$$
\text { L. clivensis }
$$

Pastinaca sp.

$$
\begin{aligned}
& \text { L. doliolum } \\
& \text { L. selosa }
\end{aligned}
$$

Pedicularis brachyodonta

Schlosser \& Vuk. var. fallacis

(Beck) Hayek

$$
\text { L. malyi }
$$

Pedicularis bracteosa Benth. ex llook.

\section{L. jacksonensis}


Pedicularis debilis Franch. ex Maxim.

L. doliolum var. angustispora

Pelargonium capitatum (L.)

L'Her.

\section{L. elaoudi}

Pclargonium sp.

L. pelargonii

Peltigera aphthosa (L.) Willd. forma complicata $T h$. Fries

$$
\text { L. peltigerarum }
$$

Peltigera camina (L.) Willd.
L. caninae
L. mamillula
L. rivana

Peltigera malacea (Ach.) Funck.

$$
\text { L. amoldii }
$$

Pelrigera cf. rufescens (Weis.)

Humb.

Peltigera sp.

$$
\text { L. clarkii }
$$

L. pelingerea

Pennisetum clandestinum Hochst. ex Chiov.

\section{L. narmari}

Pennisetum purpureum Schum.

L. penmiseti

L. penniseticola

Penstemon confertus Dougl. ex

Lindl.

\section{L. lethalis}

Periclymenum sp.

L. vagabunda forma lonicerae

Persea americana P. Mill.

L. grarissima

L. gratissima var. longispora

Persea lingue Nees

$$
\text { L. lingue }
$$

Petasites sp.

L. vagabunda var. caulium Petroselinum hortense auct.

L. melanommoides

Peucedanum cervaria (L.)

Lapeyr.

L. penicillus

Peucedanum longifolium Waldst.

\& Kit.

\section{L. doliolmm forma} syndoliola

Phaca alpina L.

L. phacae

phalaris arundinace $\mathrm{L}$.

L. fuckelii forma filamentifcra

phaseolus vulgaris L.
L. phaseoli
L. phascolorum

Phleum arenarium $\mathrm{L}$.

$$
\text { L. arenaria }
$$

Phleum boctmeri Wibel.

$$
\begin{aligned}
& \text { L. rousseliana } \\
& \text { L. sequana }
\end{aligned}
$$

Phleum pratense L.

$$
\text { L. culmifida }
$$

Phlox decussata Lyon

$$
\text { L. phlogis }
$$

Phoenix canariensis Hort. ex

Chabaud

$$
\text { L. batumensis }
$$

Phoenix dactylifera $\mathrm{L}$.
L. phoenicis
L. pinnarum
L. pinnarum var. rachidis

Phoradendron tonnsendii Trel.

$$
\text { L. phoradendri }
$$

Phormium sp.
L. phormicola
L. phormii
L. roumegueri

Phragmites communis Trin.
L. albo-punctata
L. pimpinellae

L. arundinacea

L. arundinacea var. godini

L. clavicarpa

L. culmicola var. hispalensis

L. culmorum forma phragmitis

L. elaeospora

L. graminis

L. larseniana

L. nigrans forma arundinis

L. perpusilla

L. phragmiticola

L. subalpina

L. 'yphartm subsp. phragmatina

L. vaginae

Phragmites sp.

\section{L. arundinacea}

L. culmicola var. rhizomanum

L. cumulata

L. mosana

Phyllachora disseminata Syd.

$$
\text { L. phyllachericola }
$$

Phyllachora graminis (Pers.:Fr.)

Fuckel

$$
\text { L. phyllachorivora }
$$

Phyllachora mungurahuensis Petrak

L. Imuguahuensis

Phyllachora sp.

$$
\text { L. caucana }
$$

L. cruprica

phollosiachys bamburides

Siebold \& Zuce.

L. bambusiac
Phyllosiachys hambusoides Sieb.

\& Zucc. var. hambusoides

L. minoensis

L. phyllostachydis

Physalis pubescens L.

L. physalidis

Physostegia virginiana (L.)

Benth.

L. physostegiae

Phyreuma scheuchzeri All. L. psilospora

Phyteuma spicalum L.

L. phyteumatis

Phytolacca americana L.
L. clavigera
L. variegata

Phytolacca decandra L. L. phyolaccac

Picris hieracioides L. L. picridis

Pilularia sp.
L. pilulariae

Pimpinella anisum $\mathrm{L}$.

Pinecenectria sp.

L. almeidana

Pimus silvestris $\mathbf{L}$.
L. acicola
L. picastra
L. pini

Pinus sp.
L. squamata

Piper capense L.

$$
\text { L. piperis }
$$

Pircunia dioica Mloq.

$$
\text { L. argentinensis }
$$

Pistacia rerchinthus L.

$$
\text { L. emiliana }
$$

Placodium teicholvimm (Ach.)

DC.

$$
\text { L. crozalsii }
$$

Plantago alpina L.

$$
\text { L. winteri }
$$

Plamago crnops L.

$$
\text { L. cynops }
$$

Plantago macrostachys Decne.

L. austro-americana

Plantago maritima L.

L. vagabunda subsp. alvarensis

Plamfagosp.

L. plamfaginicala

Plaramus accidenalis L.

$$
\text { L. plasmicala }
$$

Platamus sp.

$$
\text { L. plapanicola }
$$

Plarvchoru ulmi (Schleich.:Fr.)

Petrak

\section{Blarichorat}


Pleiohlastus hindsii (Munro)

Nakai

$$
\text { L. hambusae }
$$

Pleioblastus simomi (Carriere)

Nakaj

\section{L. hambusue}

Pleurospermum ausiriacum (L.) Hoffm.

$$
\begin{aligned}
& \text { L. ogilviensis var. } \\
& \text { pleurospermi }
\end{aligned}
$$

Plocama pendula W. Aiton

$$
\text { L. plocamae }
$$

Plumbago sp.

$$
\text { L. plumbaginis }
$$

Poa alpina L.

$$
\text { L. microscopica }
$$

Poa annua L.
L. muirensis

Poa aquatica $\mathrm{L}$.

$$
\begin{aligned}
& \text { L. culmifraga var. } \\
& \text { propinqua }
\end{aligned}
$$

Poa colpodea Th. Fries

L. microscopica

Poa nemoralis $\mathbf{L}$.
L. linearis
L. pode

Poa pratensis $\mathrm{L}$.
L. avenaria

Poa sudetica Haenke Poasp.

L. culmifraga forma poae

$$
\text { L. sparsa }
$$

Poa sp.?

L. anarithmoides

Polygonatum sp.

$$
\text { L. polygonati }
$$

Polygomm bistorta L.

$$
\text { L. altaica }
$$

Polymmis sp.

$$
\text { L. doliolum }
$$

Polytrichum formosum Hedw.

$$
\text { L. heufleri }
$$

Polytrichum juniperinum Willd. ex Hedw. var. alpimm Schimp.

$$
\text { L. polytrichina }
$$

Populus alba L.
L. aegira
L. alcides
L. leucoplaca

Populus balsamifera L.

L. borealis var. populi

Populus nigra $\mathbf{L}$.
L. salicinearum

Popuhus tremula $\mathbf{L}$.
L. immunda

Populus sp.
L. decaisneana

Porellasp.

$$
\text { L. porellat }
$$

Potentilla argentea L.
L. minima
L. superficialis

Potentilla argyrophy/la Wallich ex Lehm.

\section{L. hollosiana}

Potentilla cawlescens L. L. pulchra

Potentilla fragarioides $\mathrm{L}$. L. hollosiana

Porentilla maculata Pour. L. oligotheca

Potentilla sp. L. doliolum

Poterium sanguisorba L. L. poterii

Primula elatior (L.) Hill L. primulana

Primula latifolia Lapeyr. L. primulaecola

Primula sikkimensis Hook.

$$
\text { L. delanayi }
$$

Protea melaleuca $\mathrm{R}$. Br.

$$
\text { L. protearum }
$$

Prunella vulgaris L.
L. brunellae
L. hesperia

Prumus avium L.

L. rimicola

Prumus baccala Borkh.

$$
\text { L. corticola }
$$

Prumus domestica $\mathrm{L}$.
L. corticola
L. prumi

Prumus dulcis (Mill.) D.A. Webb

$$
\text { L. pachylheca }
$$

Prumus mume Sieb. \& Zucc.

$$
\text { L. mume }
$$

Prumus spinosa L.

$$
\begin{aligned}
& \text { L. rorticola } \\
& \text { L. Iycii }
\end{aligned}
$$

Pseadosasa japonica (Siebold \&

Zucc. ex Steudel) Makino

$$
\text { L. bambusae }
$$

Pleridium aquilinum (L.) Kuhn

$$
\text { L. coorgica }
$$

Pleris aquilina $\mathrm{L}$.

$$
\text { L. aquilina }
$$

Plerocelastrus tricuspidatus

Walp.

$$
\text { L. prerocelastri }
$$

Punica granalum $\mathrm{L}$.

$$
\text { L. puniciae }
$$

Pyrola scounda L.

$$
\text { L. marginata }
$$

Pyrus commumis L.

$$
\begin{aligned}
& \text { L. Iucilla } \\
& \text { L. subculanea }
\end{aligned}
$$

Pyrus sinensis Hort.

$$
\text { L. nashi }
$$

Quamoclidion mulifforum Torr.

ex A. Gray

$$
\text { L. quamoclidii }
$$

Quercus pedunculata Ehrh.

L. dryadea

L. vagabunda

Quercus robur L.

L. alcides forma quercina

Quercus suber L.

L. scolecosporarum

Quercus virens Ait.

L. janus

Quercus sp.
L. dryophila
L. lericoplaca
L. puteana

Radicula sp. (=Rorippa sp.)

L. reidiana

Ramalina sp.

$L$. ramalinae

Ramunculus affinis $\mathrm{R} . \mathrm{Br}$.

L. ranunculi

Romunculus nivalis L. var. sulphureus Wahlenberg

L. neheri

Ranunculus polyanthemos $\mathrm{L}$.

L. ramunculi-polyanhemi

Ranunculus thora L.

L. thorae

Ramunculus villarsii $\mathrm{DC}$.

L. andrijevicensis

Raphanus maritimus Sm.

L. raphani

Resedalutea L.

\section{L. resedae}

Resio australis $\mathrm{R}$. Br.

$$
\text { L. restionis }
$$

Rhammus sp.

L. limirata

Rhinanthus sp.

L. affinis

L. dolioloides var. rhinanthi

Rhizocarpon geographicum (L.)

DC.

L. geographicola

L. polaris

Rhodiola rosea $\mathrm{L}$.

L. rhodiolicola

Rhododendron ferrugineum $\mathrm{L}$.

L. rhododendri

Rhododendron sp.

L. californica

L. rhododendri

Rhus glabra L.

1. rhoina

Rlues typhina $\mathrm{L}$.

L. curla

Ribes alpinum $\mathrm{L}$.
L. cladophila
L. ribis

Ribes grossularia L.

L. grossulariae 
Ribes nigrum L.
L. anceps

Ricinus communis L.

L. torrendii

Rinodina collectica (FIk.) Arn.

L. maheui

Robinia pseudecacia $\mathrm{L}$.

L. customa forma leguminosa

L. Iyndonvillae

L. petiolicola

Rosa camina L.

Rosa sp.
L. aculeorum

L. coniothyrium

L. corticola

L. fuscella

L. hazslinszkii

L. lejostega

L. sepincola

Rubia peregrina L.
L. bornmuclleri
L. icositana

Rubus fruticosus L.
L. coniothyrium
L. notarisii

Rubus idaeus L.
L. oryspora
L. praetermissa

Rubus odoralus L.

L. praetermissa

Rubus sp.
L. abbreviala
L. controversa
L. doliolum
L. hendersoniae
L. osculanda
L. scpincola
L. thomasiana
L. vagabunda var. sarmenti

Rudbeckia amplexicanlis Vahl.

L. rudbecticie

Rulingia prostrata Maiden \&

Betche

$$
\text { L. rulingiac }
$$

Rumer acetosa L.

\section{L. quadriseptata}

Rumex nepalensis Sprengel
L. rumicicolo
L. Itumicis

Rumer paticntia L.

$$
\text { L. rmmicis }
$$

Rumer sculatus L.

$$
\text { L. scutati }
$$

Rumcisp.

$$
\begin{aligned}
& \text { L. rumicis } \\
& \text { L. sepincola } \\
& \text { L. wegeliniana }
\end{aligned}
$$

Ruscus aculeatus L.

L. comallariae forma rusci

L. glanco-punctuta
L. rusci

L. rusci forma caulina

L. ruscicola forma cladodiicola

Ruscus androgymus L.

$$
\text { L. rusci }
$$

Ruscus hypoglossum L.
L. rusci
L. rusci var. rusci- hypoglossi

Ruscus hypophy/lum L.

L. rusci var. hypophy/lum Ruscus sp.

$$
\text { L. ruscicola }
$$

Ruta gruveolens L.

$$
\text { L. bu/garica }
$$

Sabal serrulata Roem \& Schult.

Sabal sp

$$
\text { L. sabalicola }
$$

L. sabaligera

Saccharum officinamum $\mathrm{L}$.
L. bicolor
L. kuangfuensis
L. sacchari
L. saccharicola
L. spegazzini
L. spegazini var. minos
L. tainanensis
L. Itucumanensis

Sagitharia sp.

$$
\text { L. duplex }
$$

Salicornia peruviana H.B.K.

$$
\text { L. promontorii }
$$

Salix alba L.

$$
\text { L. salicinearum }
$$

Salix aurita L.

$$
\text { L. cinerea }
$$

Salix caprea L.

$$
\begin{aligned}
& \text { L. vagabunda forma } \\
& \text { salicis-capreae }
\end{aligned}
$$

Salix purpurea L.
L. purpurearum
L. vagabunda

Salix vifellina L.

Salix sp.

$$
\text { L. coniothyrium }
$$

L. baggei

L. borealis

L. consimilis

L. gillotiama

L. hendersoniae'

L. leacoplaca

L. lomicerina

L. malojensis

L. rylogena

Salsolakali L.
L. kali
L. lecanora
L. salsoles

Salvia glatinosa L.

L. momidus
Salvia officinalis L.
L. nicolai
L. saliviae

Salvia pratensis L.

L. caespirosa forma salviac

Sambucus ebulus L.

L. derasa forma macrospora

L. dimetorum forma ehuli

L. megalospora

L. ruthenica

Sambucus melanocarpa A. Gray L. sambucina

Sambucus nigra L.

L. contiothyrium

L. sambuci

Sambucus racomosa $\mathrm{L}$.

L. dumetorum var. dolichospora

L. hiria

L. Inxemburgensis var. dolichespora

L. tiroliensis

Sangmisorba officinalis $L$.

L. sanguisorbae

Samguisorba polygama F. Nyt.

L. sanguisorbae

Sansevicria ehrenbergii

Schweinf. ex Baker

L. haldratiana

Sarohhammus scoparius (L.)

Wimmer ex Koch

L. fuscella forma microsporia

L. sarnihamni

Sarothammus sp.

L. califormica

Sarracenia purpurea L.

L. scapophila

Sasa paniculasa (F. Schmidt)

Makino

L. hambusae

Sasa sp.
L. sasacola
L. sasac

Sasaclla ramosa (Makino)

Makino

L. hambusae

Salureju horensis L.

L. fallaciosa

Saxifraga opposinifolia L. L. hrachiousca

Sarifroga sp.

$$
\text { L. momoris }
$$

Scabiosa collumbaria L.
L. modesia
L. Masserinii

Scabiona ariecolafa Dest.

$$
\text { L. obesas }
$$

Scheuchacria palustris L. L. hacillifera 
Schoenocanlon officinale A. Gray

L. schoenocauli

Schoenoplectus lacustris (L.)

Palla
L. lacustris
L. sowerbyi

Scirpus holosehoenus $\mathbf{L}$.
L. apogon
L. holmii
L. micropogon
L. strioluta

Scirpus lacustris $\mathrm{L}$.
L. culmicola var. aquatica
L. maculans
L. scirpina
L. sowerbyi

Scirpus maritimus L.

L. saxomica

Seirpus sylvaticus L.

L. nardi var. dubiosa

Scirples sp.
L. lithoralis
L. maculans
L. michotii

Serophularia aquatica L.
L. camilla
L. scrop/nulariae

Scrophularia canina L.

L. lorbolensis

Scrophularia nodosa L.

L. oreophiloides subsp. scrophulariae

Scutellaria brevibracteata Stapf
L. davisiana

Secale cereale L.
L. secalina
L. secalis
L. setulosa

Secale sp.

\section{L. pontiformis}

Selaginella arenicola subsp. acanthonota (Underwood) R. Tryon

L. rostrata

Selaginella helvefica (L.) Spring

L. helvetica

L. helvetica forma major

L. lycopodicola var. major

L. major

Selaginella rupestris (L.) Spring

$$
\text { L. rostrata }
$$

Selaginella underwondii llieron.

$$
\text { L. mellispora }
$$

Semele androgyna (L.) Kunth

$$
\text { L. semelina }
$$

Semiarundinaria taneheana Muroi

$$
\text { L. bambusue }
$$

Senceio atrams Greene
L. rlivensis
L. clivensis var. constricta

Senecio cordatus Koch

L. ogilviensis var. senecionis-cordara

Senceio crassulus A. Gray

L. octoseptata

Senceio mucifolius $\mathbf{L}$.

L. rothomagensis

Senerio fuchsii C.C. Gmelin

L. dumeform var. coniformis

Senecio jacolaca L.
L. derasa
L. modesta forma jacobae
L. ogilvionsis

Senecio nemorensis L.
L. macrospora
L. robusta
L. senecionis

Senecio rupestris Waldst. \& Kit.

$$
\text { L. robusta }
$$

Senecio vulgaris L.

$$
\text { L. dolioloides }
$$

Senecio sp.
L. clivensis
L. doliolum

Seseli monramm L.

Seseli sp.
L. montis-bardi

L. niessleand var. starizii

Sieversia turbinata (Rydb.)

Greene

\section{L. sieversiae}

Silene acanlis (L.) Jacq.

L. hausmamiana

L. hausmanniana var. cherlerias

L. sabauda

L. silene's-acaulis

Silene arvatica Lag.

L. sabouda forma arvaticae

Silene inflata Sm.

L. silvestris

Silene rosulata Soyer-Willem. \& Godr.

$$
\text { L. indeprensa }
$$

Sinapis arvensis $\mathrm{L}$.

$$
\text { L. sinapis }
$$

Sisymbrium alliaria (L.) Scop.

$$
\text { L. alliarioe }
$$

Sisymbrium loesclii L.

$$
\text { L. submaculams }
$$

Sisymbrium strictissimum L.

$$
\text { L. acutispora }
$$

Smallamihus medalia (1..)

Micken $/$ ie

$$
\text { L. Iongipedicellara }
$$

Smilax aspera $\mathrm{L}$.

$$
\begin{aligned}
& \text { L. octmensis } \\
& \text { L. canalannica } \\
& \text { L. smilaciss }
\end{aligned}
$$

Similar excelsa L.

$$
\text { L. dobrogica }
$$

Smilax manritanica Poiret

$$
\text { L. papulosa }
$$

Smilar pseudo-china L.

$$
\text { L. nervisequa }
$$

Smilax sp.

$$
\begin{aligned}
& \text { L. doliolum } \\
& \text { L. smilacis }
\end{aligned}
$$

Sobolen'skia lithophila Bicb.

$$
\text { L. lithophilae }
$$

Solanum dulcumara L.

$$
\begin{aligned}
& \text { L. opizii } \\
& \text { L. sarmenticia } \\
& \text { L. solani } \\
& \text { L. solanicola } \\
& \text { L. dumbrosa } \\
& \text { L. vagabunda var. } \\
& \quad \text { duleamarae }
\end{aligned}
$$

Solanum sodomeum L.

$$
\text { L. sodomaea }
$$

Solamum sp.

$$
\text { L. comatella }
$$

Solidago caesia L.

$$
\text { L. longipedicellata }
$$

Solidago virgumea $\mathrm{L}$.
L. ogilviensis forma megalospora
L. planiustula

Solidago sp.
L. aulica
L. doliolum
L. perplexa
L. soliclaginis

Solorina crocea (L.) Ach.
L. lichenicola
L. oligospora
L. rivana forma solorinae

Sonchus sp.

$$
\text { L. doliolum }
$$

Sophora jabonica L.

$$
\text { L. sophorae }
$$

Sophora tetraptera J. Mill.
L. martimianum

Sorbaria sorbifolia A. Br.

$$
\text { L. spiraeae }
$$

Sorbus ancuparia $\mathrm{L}$.

$$
\text { L. sorbi }
$$

Sorbus sp.

$$
\text { L. lencoplaces }
$$

Sarghm arundinacem Room \& Schult.

L. sorgho-arumdimacei

Sorghum vulgare Pers.
L. amplibola
L. culmifraga
L. enstomu
L. grisea
L. seprovariata 
Sparganium erectum L.

L. sparganii

Sparganium ramosum Hudson

L. Iuthiana

L. sparganii

L. sparsa var. meizospora

Sparganium sp.

L. clara

Spartina alterniflora Loisel.

L. neomaritima

Spartina juncea auct.

L. borziana

Spartina tou'nsendii H. Groves \&

J. Groves

L. neomaritima

Spartina sp.

L. discors

L. duplex

L. incarcerata

L. macrosporidium

L. marina

L. neomaritima

L. pelagica

L. spartinae

L. sticta

Spartium junceum L.

L. Iusitanica

Spartium sp.

L. dioica

Splyridium fungiforme Flotow

L. sphyridiana

Spiraca sombifolia $\mathrm{L}$.

L. spiracae

Sporobolus depauperatus Tort. ex

Hemsl.

\section{L. sporoboli}

Staphylea trifolia $\mathbf{L}$.

L. rubrotincta

Statice accidentalis Lloyd

L. staticicola

Steironema cilianum (L.) Rafin.

L. stcironematis

Stcllaria graminca L.

L. isariphora

L. salchricola

Stellaria humifusa Rottb.

L. stcllariae

Stellaria media (L.) Vill.

L. richoni

L. stellariae

Sicllaria uliginosa Murray

L. nliginosa

Stenofaphrum secundatum

(Walter) O. Kuntze

L. Harmari

Stercocoulon alpinum Laur.

L. apocalypta

L. stereocaulorum

Stereum bicolor (Pers.) Pers.

L. stercicola
Stereum subpileatum Berkeley \& Broome

L. fungicala

Stipa capillata L.

$$
\text { L. stipae }
$$

Stipa pennata $\mathbf{L}$.

$$
\text { L. matritensis }
$$

Stipa tenacissima $\mathbf{L}$.
L. macrochloac
L. pampanimiana
L. stipae
L. stipae-minor

Stratiotes aloides $\mathrm{L}$.

$$
\text { L. stratiotis }
$$

Suaeda australis Moq.

$$
\text { L. suacdae }
$$

Succisa pratensis Moench.

Succisa sp.

$$
\text { L. planiuscula }
$$

L. modesta forma succisac

L. morthieriana

Swertio sp.
L. swertiae

Symphytum caucasicum Bieb.

L. dumetorum var. sympliyti

Syringa vulgaris L.
L. Irematostoma

Tamarix gallica L.
L. hollosii
L. meridionalis
L. tamaricis

Tamarix germanica L.
L. ramaricis

Tamarix sp.

$$
\text { L. crozalsiana }
$$

Tanacelum vulgare $\mathbf{L}$.
L. dolioloides
L. tanaceti
L. thielensii

Taxus canalensis Marsh.

$$
\text { L. tavicola }
$$

Tecoma radicans Juss.

$$
\text { L. carpophila }
$$

Tellima grandifloro (Pursh)

Douglas ex Lindley

$$
\text { L. deficiens }
$$

Tepinosia virginiana (L.) Pers.

$$
\text { L. tephrosiae }
$$

Teucrimm scorodonia L.
L. tencrii
L. wegeliniana forma sencrii

Thalictrum aquilegifolium $\mathbf{L}$.

$$
\begin{aligned}
& \text { L. tenwis } \\
& \text { L. thalictri } \\
& \text { L. thalictrina }
\end{aligned}
$$

Thalictrum collimum Wallr.

$$
\text { L. thalicaricola }
$$

Thalictrum dicicum L.

L. houscona
Thalicirum flawum L.

$$
\text { L. cruenta }
$$

Thalicrrum minus L.

$$
\text { L. quadriseprata }
$$

Thea sinensis $\mathrm{L}$.
L. cavarae
L. hotrai

Theobroma cacao L.

L. theobromicola

Thermopsis montana Nutt.

$$
\text { L. wehmeyeri }
$$

Thuja orientalis L.

Tilia sp.

$$
\text { L. thujaecola }
$$

$$
\begin{aligned}
& \text { L. leucoplaca } \\
& \text { L. priuscheggiana }
\end{aligned}
$$

Tillandsia bicolor Brongn.

$$
\text { L. aerea }
$$

Tofieldia calyculara (L.)

Wahlenb.

$$
\begin{aligned}
& \text { L. crastophila forma } \\
& \text { tofieldiae } \\
& \text { L. oreophila } \\
& \text { L. Submodesta } \\
& \text { L. tofieldiae }
\end{aligned}
$$

Tortula tortuosa Ehrh. ex Hedw.

$$
\text { L. hryophila }
$$

Tragopogon sp.

$$
\text { L. ophiaboloides }
$$

Trevoa trineria Gill. \& Hook.

L. trevoae

Trichoptenx sp. [as $T$. afroflammida]

L. trichopteryis

Trifolium alpesire $\mathrm{L}$

L. Irifolii-alpesmis

Trifolium angustifolium L.

L. agnira var. Irifolii

Trifolium kingii S. Wats.

$$
\text { L. manticala }
$$

Trifolium medium L.

$$
\text { L. Irifolii }
$$

Trifolium richocephalum Bieb.

$$
\text { L. balcarica }
$$

Triglochin maritima L.

$$
\begin{aligned}
& \text { L. hollosiama } \\
& \text { L. juncaginearum } \\
& \text { L. maritima }
\end{aligned}
$$

Triglachin palustris L.

L. juncaginearum

L. monilispora forma riglochinis

L. Miglochinicola

L. Iriglochinis

Trisenum distichophyllum (Vill.) Beaus.

$$
\text { L. rolkartiana }
$$

Trisenum longiglume Hachel

L. mendozand

Tiriselum spicalum (L.) K. Richter

L. hollosiana

L. volkartiona 
Triscam subspicaum (L.) Beauv.

$$
\text { L. microscopica }
$$

Triticum aestivum $\mathrm{L}$.

$$
\begin{aligned}
& \text { L. korrae } \\
& \text { L. narmari }
\end{aligned}
$$

Trivicum dumum Desf.

$$
\begin{aligned}
& \text { L. avenaria form } \mathrm{sp} \text {. } \\
& \text { Iriticea }
\end{aligned}
$$

Triticum repens $\mathrm{L}$.

$$
\text { L. rubelloides }
$$

Triticum inlgare Vill.
L. alenaria form $\mathrm{sp}$. triticea
L. nodorum
L. recutita
L. mitici

Triticum sp.
L. pontiformis

Trollius europaens L.

$$
\text { L. Irollii }
$$

Tipha angustala Bory \& Chaub.

$$
\text { L. aquarica }
$$

Typha angustifolia L
L. dematiucela
L. iridigena var. nphae
L. lacustris
L. Iypharum
L. typhiseda
L. iyphiseda forma sodoloci

Typha latifolia L
L. bispora
L. caricis
L. grandispora
L. Kunzeana
L. lacustris
L. licatensis
L. licatensis forma rupefortensis

L. maculans var. typhicola

L. palustris

L. perpusilla var. Isphae

L. psendohleria

L. punctillum

L. iyphue

L. typharim

L. Iyphicola

Typha sp.

$$
\begin{aligned}
& \text { L. duplex } \\
& \text { L. mucosa }
\end{aligned}
$$

Ulmus campesmis auct
L. massariella
L. ulmicole

Ulmus sp.
L. Lencoplaca
L. ramulicola

Umbellulariasp.

L. odora

Umbilicaria pustulata (L.)

Hoffm.

$$
\text { L. umbilicariae }
$$

Urica dioica $\mathrm{L}$
L. acuia
L. acula forma insignis
L. acula forma urticae
L. atropurpurea
L. comiformis
L. convidea forma macrospora

Uricasp.

L. acutiuscula

L. doliohum

Usnea florida (L.) Web.

$$
\text { L. usneare }
$$

l'accinium sp.

$$
\text { L. lejostega }
$$

Valeriana dubia Turcz.

$$
\text { L. dubia }
$$

V'aleriana officinalis L.
L. agnita
L. cameomaculans
L. quadriseprata

l'eratrum sp.

$$
\text { L. veratri }
$$

lerbascum phlomoides L.

$$
\text { L. echinops }
$$

Verbesina virginica L.

$$
\text { L. drechsleri }
$$

Veronica latifolia auct.

$$
\text { L. striata }
$$

Veronica urticifolia Jacq.

$$
\text { L. carpogena }
$$

l'eronica sp.

$$
\text { L. doliolum }
$$

l'iburnum timus L.

$$
\text { L. } r i n i
$$

Vicia cracea $\mathrm{L}$.

$$
\text { L. viciae }
$$

Vicia sativa L.

$$
\text { L. niessleana forma riciae }
$$

l'inca major L.

$$
\text { L. maderensis }
$$

V'inca media Hoffmanns. \& Link

$$
\text { L. demissa }
$$

linca minor $\mathrm{L}$.

$$
\text { L. vincae }
$$

Vincetaxicum officinale Moench

$$
\text { L. molybdina }
$$

Vinceroricum sp.

$$
\text { L. scorophila }
$$

l'iter agmus-castus $\mathrm{L}$.

$$
\text { L. casta }
$$

Vitis rinifera L.

L. ampelina

L. cerletiii

L. chaetosioma

L. cirricola

L. cookei

L. gibelliana

L. pampini

L. socia

L. vinealis

L. vinicola

L. vitigena

L. vitis

Vitis vinifera L. subsp. sylvestris

L. sclavonica

Vitis sp.

I'riesea sp.

L. pampini

L. vieseae

Xamthoxy/um sp.

L. brasiliensis

Xerophylhum asphodeloides (L.)

Nutt.

L. xerophylli

Xerophillum tenax (Pursh) Nutt.

L. hysterioides

Yucca elephamipes

L. seiosa

Yucca filamemosa $\mathrm{L}$.

L. filameniosa

Yacca gloriosa L.

L. obtusispora

Yucca sp. [as Y. colusplei]

L. pacifica

Zea mavs L.
L. bubakii
L. ceratispora
L. hyalospora
L. interspersa
L. Inctuosa
L. maydis
L. patellaeformis
L. punctiformis
L. seriata
L. variiseptata
L. zeac
L. zeae-maydis

L. zeicola

Zingiber officinale Rose.

L. zingiberis

Zizania larifolia Turcz.

Zizania sp.

$$
\text { L. zizamivora }
$$

L. zizaniaces)/a 


\section{Host Family Index}

Acanthaceae
L. acanthi
L. eranthemi

Aceraceae
L. aceris
L. controversa
L. diana
L. dioica
L. inquinans
L. leucoplaca
L. muelleri
L. obesula
L. vagabunda

Agavaceae
L. agaves
L. almeidana
L. baldratiana
L. comvallariae
L. comvallariae forma dracaena

L. cordylines

L. dasylirii

L. dracaenae

L. draconis

L. filamentosa

L. infermalis

L. obtusispora

L. obtusispora forma agaves

L. pacifica

L. phormicola

L. phormii

L. roumegueri

L. insci forma fourcrovac

L. setosa

Alismataceae
L. duplex
L. rivularis

Alstroemeriaceae

L. bomareas

Amaranthaceae

L. criophora

Amaryllidaceae

L. australis

Anacardiaceac
L. brasiliensis
L. cruta
L. cmiliama
L. rhoina

Apocynaceae
L. demissa
L. hardenbergiae
L. maderensis
L. vincae

Aquifoliaceae

$$
\begin{aligned}
& L \text {. paraguariensis } \\
& \text { L. yerbae }
\end{aligned}
$$

Araceae
L. acorella
L. acori
L. colocasiae
L. crucheri
L. densa
L. microscopica subsp. calomi
L. typharum

Araliaceae
L. doliolum
L. hederae
L. hedericolo
L. helicicola
L. papulosa

Araucariaceae

L. californica

Arthropyreniaceae

L. leptogiophila

Asclepiadaceae
L. asclepiadis
L. dearnessii
L. doliolum
L. modesta var. cibostii
L. molydina
L. russellii
L. scotophila

Aspleniaceae

$$
\text { L. asplenii }
$$

Avicenniaceac

$$
\begin{aligned}
& \text { L. anstraliensis } \\
& \text { L. avicenniae }
\end{aligned}
$$

Baeomycetaceate
L. haeomviearia
L. neottizans
L. prenostigma
L. sphlyridiana

Berberidaceae

L. herberidicola

$L$. herberidis

L. comiothrium forma herheridis
L. inconspicua

L. nandinae

L. punjabensis

Betulaceae
L. avellanae
L. hetulina
L. betulina
L. coniothyrium
L. depressa
L. leucoplaca
L. Ionicerina
L. subsimilis
L. vagabunda
L. waghorniana

Bignoniaceae

L. carpophila

L. drvadea subsp. lissoniensis

\section{Boraginaceae}
L. cesatiana
L. cynoglossi
L. dumetorum var. symphyti
L. echiella
L. echii
L. mertensiae
L. vindobonensis

Bromeliaceae
L. acrea
L. irieseac

Buxaceae
L. buxina
L. melanommoides
L. revocans

Cactaceae
L. cerci-permiam
L. opumine
L. sicula

Calycanthaceae

L. conlothyrum

Campanulaceac
L. lobeliae
L. pachivasca
L. phyteumatis
L. plemeliana
L. prilospora
L. "upue

Cannabaceac

L. camnahina

L. woroninii 
Cannaceae

$$
\text { L. cummae }
$$

Capparaceae
L. almadii
L. capparidicola
L. capparidis
L. simillima

Caprifoliaceae
L. albulae
L. aulica
L. cuprifalii
L. coniothyrium

L. corticola

L. derasa forma macrospora

L. dumetorum forma chuli

L. dumetorum var. dolichospora

L. frondis

L. hirfa

L. lonicerae

L. Iuxemburgensis var. dolichospora

L. megalospora

L. periclymeni

L. periclymeni var. iartarica

L. ruthenica

L. sambuci

L. santbucina

L. surculorum

L. rini

L. viroliensis

L. vagahunda forma lonicerae

Caryophyllaceae

L. auerswaldii

L. bieberstemi

L. dianthi

L. gypsophilae

L. hansmannianu

L. hausmanniana var. cherleriae

L. indeprensa

L. isariphora

L. leptospora

L. proliferae?

L. rehmiana

L. richoni

L. sabauda

L. sabanda forma arvaticae

L. sulebricola

L. sarraziniana

L. silenes-acaulis

L. silvestris

L. stellariae

L. stellarias

L. uliginosa

L. vahlii

L. vamhoeffeniana
Celastraceate
L. colifornica
L. castagnei
L. constricta
L. dolioloides var. inops
L. prerciclasmi

Chenopodiaceate
L. anthelmintica
L. atriplicis
L. calvescens
L. camphorosmae
L. chenopodii-albi
L. echinella
L. eriophora
L. entypoides
L. haloxyli
L. inculia
L. kali
L. lecanora
L. nigricans
L. obiones
L. obiones var. evolutior
L. promontorii
L. salsolae
L. serbica
L. suacdae

Chondriellaceae

L. mirubilis

Chordariaceae

L. mirandae

Cistaceae
L. cisti
L. cisticola
L. cistina

Clavicipitaceae

L. associata

Compositae

\section{L. achilleae}

L. agmila var. acheniarum

L. agnira var. ambigua

L. agnita var. chrysanthemi

L. agnita var. erigerontis

L. anacycli

L. amihophila

L. artemisiae

L. arthrophyma

L. astericola

L. asteris

L. anlica

L. hardanae

L. bella

L. bicuspidara

L. hraumi

L. caespitosa

L. camphorata

L. canadensis

L. corduina

L. corducrum

L. carlinoides

L. centanreae

L. cerastii
L. cenvispora

L. chrysanthemi

L. cirsii-arvensis

L. clavispora

L. clivensis

L. clivensis var. comstricen

L. coleasamsti

L. compositarum

L. compressa

L. congesta

L. conoidea forma asteris

L. consessat

L. corynispora

L. cosmicola

L. crustacea

L. cynaracearum

L. derasa

L. derasa forma alpestris

L. derasa forma rohusfa

$L$. derasa var. franconiea

L. dolioloides

L. dolioloides var. cirsii

L. doliolum

L. doliolum forma corlinae-vulgaris

L. doliolum var. cucaliae

L. doliolum var. subdisticha

L. diechsleri

L. dumetorum var. coniformis

L. chumea

L. erigerontis

L. eriophora

L. espeleriae

L. filiformis

L. flotoviae

L. franconica

L. fulgida

L. galiicola var, brachyspora

L. galiorum forma cirsiorum

L. galiorwm var. gnaphaliana

L. galiorum var. lapsanae

L. gloeospora

L. gnaphalii

L. grammodes

L. helianthemi

L. helianthi

L. heliopsidis

L. helminthospora

L. hispanica

L. jaceac

L. jachsonii

L. jahnii

L. Kalmusii

L. Lasioderma

L. lindquistii

L. Iongipedicellata 
L. macrospora

L. marginalis

L. mesnedema

L. mikaniae

L. millefolii

L. modesfa forma jacobaede

L. modesta forma lappae

L. modesta var. rubellula

L. manac

L. Migricans var. grindeliae

L. nitschkei

L. nitschkei forma adenostylidis

L. octoseptata

L. ogilviensis

L. ogilviensis forma achilleae

L. ogilivensis forma megulospora

L. ogilviensis var. senecionis-cordari

L. ophioboloides

L. onamiae

L. passerimii

L. perplexa

L. phacospora

L. picridis

L. plagia

L. planiuscula

L. plurisepta

L. preandina

L. plammicae

L. purpurea

L. quadriscplata

L. rhopalispora

L. robusta

L. rothomagensis

L. rorhomagensis var. artemisiae

L. rubellula

L. rndbeckiae

L. senecionis

L. septemcellulata

L. shalnarica

L. sibirica

L. simmensii

L. solidaginis

L. staritzii

L. sydowiana

L. Pallaceti

L. tetonensis

L. thiclensii

L. Irichostoma

L. trimeriesides

L. Iumefaciens

L. Incinala

L. ragabunda var. coulimm

L. valesiaca
Convolvulaceae

L. butaticola

L. ferruginea

Comaceae

L. fiedlaeri

L. Limitaia

L. mamillana

L. olivaespora

L. platycarpa

L. rugosa

L. vagabunda

Corvidae [Birds]

L. corrina

Crassulaceae

Cruciferae

L. rhodiolicola

L. acutispora

L. alliariue

L. allorgei

L. arabidis

L. cesationa

L. conferta

L. drabae

L. fusispora forma erysimi

L. galiicola vas, brachyspora

L. hesperidicola

L. johansonii

L. lishophilae

L. lumariae

L. maculans

L. muculans forma denudata

L. morierae

L. napi

L. Higrella

L. norvegica

L. ogilivensis forma lepidii

L. olericola

L. planiuscula forma cruciferarum

L. raphani

L. reidiama

L. sulebrosa

L. sinapis

L. submacwlams

L. Irollii

L. virginica

Cucurbitaceate

L. alerandrinis

L. cucurbirue

L. obesa

Cupressaceae

L. jumiperi

L. jumiperina

L. suprephita

L. Imijacola

Cycadaceac
L. cucudis

L. irrepla
Cyperaceae

L. aliena

L. apogon

L. caricicola

L. caricina

L. caricinella

L. cariciphila

L. caricis

L. caricis-firmae

L. caricis-rulpinae

L. ceballosi

L. cladii

L. clara

L. consobrina

L. culmicola vат. aquatica

L. culmorum var paleicola

L. comana

L. cyperi

L. cupericola

L. cuperina

L. epicarecta

L. folliculura

L. folliculata var. orvispora

L. gigaspsora

L. hemicrypta

L. holmii

L. kochiona

L. lacustris

L. litloralis

L. lithoralis forma calamogrostidisarchariae

L. macrotheca

L. maculans

L. michotii

L. micropogan

L. microscopica

L. microscopica var. caricis-ıulpinae

L. occulta

L. paliadosa

L. pupsri

L. perkevicensis var. elymi

L. pinnarum

L. pimnarmm var, rachidis

L. puccinioides

L. rivalis

L. sarimica

L. scirpina

L. sowerhyi

L. spursu var. elymae

L. sporsa var meizospora

L. strinlata

L. strioluta var. caricisglancae

L. viridella

Daphniphyllaccac

L. duplmiphy/li

Dennstaedtiaceae

L. coorgica 
Dipsacaceae
L. bractearum
L. carpophila var. bractearum

L. cephalariai-uralensis

L. modesta

L. modesta forma succisae

L. modesta forma syliestris

L. morthicriana

L. passerinii

L. phyteumatis

L. planiuscula forma succisae

L. laurica

Dipterocarpaceae
L. baumii

Elaeagnaceae

L. fuscella var. hippophaes

L. fuscella var. sydowiana

L. hippophaes

Empetraceae
L. emperri

Ephedraceae

$$
\text { L. ephedrae }
$$

Equisetaceae
L. arvensis
L. berlesei
L. equiseri
L. cquiseticola
L. hicmalis
L. larvalis
L. limosa

Ericaceae
L. andromedae
L. arbuti
L. azaleae
L. californica
L. ericae
L. gaultheriae
L. hyperborea
L. kalmiac
L. lejosiega
L. rhododendri
L. sublanosa
L. subtecta

Euphorbiaceae
L. bresadolacana
L. depressa
L. euphorbiac
L. cuphorbiac forma esulae
L. euphorbiicolla
L. heveae
L. hurae
L. petri
L. sacculus
L. sibtorpii
L. tolgorensis
L. torrendii

Fagaceae

L. alcides forma quercina

L. dryadea
L. dryophila

L. faginea

L. involucralis

L. janus

L. levicoplaca

L. puteuna

L. scolecosporarum

L. seminuda

L. ragabunda

L. valdobbiac

Geastraceae

L. geasteris

Gentianaceae

L. fraserae

L. galiorum

L. galiorum forma gentiance

L. harknessianna

L. salebrosa

L. sapeyensis

L. swertiae

L. umbrosa

Geraniaceae

L. elaoudi

L. hollosiana

L. modestula

L. pelargonii

L. subcacspitosa

Ginkgoaceae

L. ginkgo

Gramineae

L. aeluropodis

L. albo-punctata

L. algida

L. alopecuri

L. ammophilae

L. amphibola

L. amphiloga

L. anarithma

L. anarithmoides

L. anisomeres

L. amhostomella

L. arenaria

L. arenaria

L. arrhenatheri

L. arrhenatheri var. italica

L. arundinacea

L. arundinacea var. godini

L. asperellae

L. arenue

L. avenaria

L. avenaria

L. hambusae

L. bambusicola

L. beaumontii

L. hicolor

L. borziana

L. Irachypodii

L. brizae

L. bubakii
L. caricis

L. cattunei

L. ceratispora

L. chusqueae

L. clavata

L. clavicarpa

L. coccodes

L. coicis

L. conimbricensis

L. consociata

L. crastophila

L. culmicola

L. culmicola forma major

L. culmicola forma melicae

L. culmicola var, hispalensis

L. culmicola var. minor

L. culmicola var, migrans

L. culmicola var. rhizomatum

L. culmifida

L. culmifraga

L. culmifraga forma majuscula

L. culmifraga forma mamuscula

L. culmifraga forma poae

L. culmifraga var alpestris

L. culmifraga var. bromicola

L. culmifraga var. linearis

L. culmifraga var. propinqua

L. culmorim

L. culmorum forma epigeii

L. culmorum forma phragmitis

L. culmorum var. flavobrunnea

L. cumulata

L. cynodontis-dactyli

L. cynosuri

L. dactylina

L. discors

L. disseminata

L. donacina

L. diplex

L. elacospora

L. elongata

L. elymi

L. cumorpha

L. eustoma

L. eustomella

L. eustomoides

L. eustomoides forma lolii

L. fuckelii

L. fuckclii forma filamentifera

L. fuegiana

L. fuscidula 

L. finscidula forma magnolii
L. gaubae
L. georgius-fischeri
L. glyceriae
L. glyceriae-plicatae
L. graminis
L. graminum
L. grisea
L. gynerii
L. hazslinskyana
L. herpotrichoides
L. hierochloae
L. hollosiana
L. hordei
L. hyalospora
L. hyparrheniae
L. incarcerata
L. inecola
L. insignis
L. insignis forma airae- cespitosa

L. intermedia

L. interspersa

L. ischaemi

L. iwamotoi

L. kerguelensis

L. korrae

L. kuangfuensis

L. lagenoides

L. larseniana

L. Iatebrosa

L. leersiae

L. leersiana

L. lelebae

L. linearis

L. lineolaris

L. littoralis

L. Iolii

L. luchosa

L. macrochloae

L. macrosporidium

L. marina

L. marram

L. matritensis

L. maydis

L. media

L. melanommoides

L. melicae

L. mendozuna

L. microscopica

L. microscopica forma brachypodii

L. microscopica forma glyceriac

L. minoensis

L. mosama

L. moutoniana

L. muchlenhergiac

L. muirensis
L. muricata

L. nardi

L. nardi var. dubiosa

L. narmari

L. neglecta

L. neomaritima

L. nigrans

L. nigrans forma arundinis

L. nodortum

L. nodarum form sp. hordci

L. norfolcia

L. occidentalis

L. ophiopogonis var. gramimum

L. orthogramma

L. oryzicola

L. oryzae

L. oryzina

L. pachycarpa

L. pachytheca

L. pampaniniana

L. panici

L. papillosa

L. patellaeformis

L. pelagica

L. penniseticola

L. penniseti

L. perforans

L. perpusilla

L. personatu

L. phragmiticola

L. phyllostachydis

L. plewrospora

L. poae

L. paae var. agrostidis

L. pontiformis

L. proteispora

L. puiggarii

L. puncriformis

L. cluinta

L. recessa

L. recuita

L. rehmii

L. rhizomatum

L. rhodophaed

L. rousseliuna

L. mbelloides

L. sabuletorum

L. sacchari

L. saginutes

L. salvinii

L. susacola

L. sasac

L. scahrispora

L. schmeideriana

L. scculina

L. sccalis

L. septovariand

L. scequanal

L. scriata
L. serulosa

L. sorghi-arundinacei

L. sparsa

L. spartii

L. spartinue

L. spegazzini

L. spegaz=ini var. minor

L. sporoholi

L. stellata

L. stipae

L. stipae-minor

L. straminis

L. stromatoidea

L. subalpina

L. subcompressa

L. subiculifera?

L. suhsuperficialis

L. syliarica

L. tainanensis

L. Iaminensis

L. thurgoviensis

L. tigrisoides

L. trichoptergis

L. trimera

L. tritici

L. Iucumanensis

L. typharwm var. phragmatina

L. vagans

L. vagans forma scirpi

L. vaginae

L. variiseptara

L. volkartiana

L. weddellii

L. zeae

L. zeae-maydis

L. zeicola

L. sizaniaccola

L. zizammilora

Guttiferae

L. cesariana

L. dematium

L. hyperici

L. hypericola

L. lankesnu

L. ocellata

L. vagahunda

Hamamelidaceae

L. hamamelidis

Hominidae

L. senegulensis

L. tompkinsii

Hydrocharitaceas

L. strationis

Iridacese

L. hermodactih

L. heterospora

L. iridicola

L. iridigena

L. iridis 

L. larseniana
L. longispora
L. microthyrioides
L. orthrosamthi
L. parvela
L. parvila var. iridis- germanicae
L. vectis
L. xiphii

Juglandaceae
L. cacuminispors
L. exocarpogena
L. lejostega
L. lencoplaca
L. petiolaris

Juncaceae

L. caricis

L. culmorum forma luangarica

L. defodiens

L. epicalamia

L. epicalamia var. pleosporoides

L. frigida

L. hollosiana

L. hydrophila

L. junci

L. juncicola

L. juncina

L. juncina forma macrospora

L. junciseda

L. junci-acuit

L. junci-glauci

L. juncorum

L. lamprocarpi

L. lizulae

L. marisima

L. michotii

L. microscopica var alpina

L. monilispora

L. neomaritima

L. norfolcias

L. perkovicensis

L. perrakii

L. psendo-diaporthe

L. riparia

L. sepalorim

L. sollheimii

L. subriparia

L. herenphila

L. variabilis:

L. virensis

Juncaginaccac
L. juncogginearum
L. maritima
L. monilispora forma rriglochinis
L. triglos hinicola
L. rriglochinis

Labiatiae
L. ballotae
L. Irighomensis

L. brunellae

L. cuespiesosa forma salviae

L. covamillesii

L. cesationa

L. collinsomiac

L. darkeri

L. davisiana

L. doliolum var. leonuri

L. dumetorum var. marrubii

L. fallaciosa

L. fiumancs

L. galeobdolonis

L. galcopsidicola

L. hesperia

L. lavendulae

L. Iophanthi

L. menthae

L. montana

L. nicolai

L. ocimicols

L. parietariae forma lamii

L. physostegiae

L. salviae

L. salviae forma minor

L. slovacica

L. substerilis

L. tencrii

L. Iranslucens

L. negeliniana forma rencrii

L. zuhlloruckneri

Lauraceale

L. almeidae

L. cimnamomi

L. coniothyrium var. foliicola

L. gratissima

L. gratissima var. longispora

L. lauri

L. lingue

L. nobilis

L. odored

L. paoluceii

Lecanoraceae

L. steinii

Lecideacea

L. adverula

L. comsocions

Leguminosac
L. adesmicola
L. acrea
L. agnita var. Irifolii
L. alhagii
L. ammothamni
L. amorphas
L. apioss

L. apios-fortunci

L. balcarica

L. harriae

L. blumeri

L. byssincole

L. californica

L. calopogenii

L. cassiaecola

L. castrensis

L. chochrjakovii

L. circinans

L. clelandii

L. comatella

L. coronillae

L. corrugans

L. coumarounae

L. cucurbitarioides

L. daviesiae

$L$. dioica

L. dissiliens

L. distributa

L. dolioloides var. lathri

$L$. dumesortum forma meliloti

L. dumesorum var. coronillae

L. endiusae

L.erythinae

L. eustoma forma leguminosa

L. focniculacea subsp. lupina

L. fuscella forma nacrosspora

L. fusisporo

L. genistac

L. genistac var. microspora

L. lathyri

L. lathyrina

L. lespedezae

L. lucina

L. lupini

L. lupinicola

L. lasisanica

L. lyndomillae

L. marginalis

L. martinianum

L. medicaginicola

L. medicaginis

L. medicaginum

L. melilosi

L. microspora

L. monticola

L. multiseptata

L. niessleana

L. niessleanu forma viciae

L. onobrychidicold

L. onobrychidis

L. periolicola

L. phacue

L. phaseoli 
L. phaseolorum

L. phiala

L. pratensis

L. rinfrioi

L. sarothammi

L. shastensis

L. sophorae

L. tephrosiae

L. trifolii

L. trifolii-alpestris

L. viciae

L. viridella

L. welmeyeri

Lemaneaceae

L. fluriatilis

L. lemianeae

\section{Leporidae}

\section{Liliaceae}

$$
\text { L. fimiseda }
$$

\section{L. aloes}

L. antherici

L. asparagi

L. asparagina

L. aspidistrae

L. bellynchii

L. comatella

L. comballariae

L. crastophila forma tofieldiae

L. dobrogica

L. heloniaefolia

L. hemerocallidis

L. hysterioides

L. indica

L. lassenensis

L. lilicola

L. lilii

L. martagoni

L. muscari

L. nervisequa

L. oreophila

L. omithogali

L. papulosa

L. passerimiana

L. polygonati

L. pontoricensis

L. pracclara

L. punctoidea

L. schocnocauli

L. semelina

L. smarodsii

L. socialis

L. submodesta

L. Iofieldiae

L. leratri

L. verwoerdiana

L. williamsii

L. verophylli

Linaceac

L. Irubyana
Loganiaceae
L. huddlejae
L. davidii
L. polini

Lycopodiaceae
L. arcialaskana
L. campisilii
L. crepini
L. lycopodicola
L. lycapodina
L. marcyensis

Lythraceae
L. Iythri
L. salicaria

Magnoliaceae
L. halima
L. magnoliae
L. stictoides
L. yulan

Malvaceae

L. abutilonis

L. gossypii

Marantaceae

L. marantae

Marattiaceae

L. caffra

Marsileaceae

L. pilulariae

Matoniaceae

L. matisiac

Menispermaceae

L. coniothyrium

Moraceae

L. coniothyrium

L. fallax

L. fici-elasticae

L. japonica

L. maclurae

L. massariella var, disticha

Musaceae
L. musae
L. musarum
L. musigena
L. taiclunngensis

Myricaceae
L. myricae

Myrtaceae

L. eustomoides var. punctata

L. ferjoare

L. molleriana

L. myrti

L. myrricola

Nyctaginaceae

L. quamoclidii

Oleaceac

L. controversa

L. emiliuna

L. fraxini

L. leacoplaca

L. Irematostoma
Onagraceae

L. argentina

L. cadubriae

L. capsularum

L. cylindrospora

L. ellisiana

L. epilobii

L. multiseprata forma alpina

L. onagrae

L. Titorulosa

Orchidaceae
L. corallorhizae
L. ophiopaganis
L. orchidcarum

Oxalidaceae

$$
\text { L. aglaja }
$$

Paeoniaceae

Palmae
L. moutan

L. algarbiensis

L. arecae

L. batumensis

L. briosiana

L. chamaeropis

L. coccothrinacis

L. cocoes

L. debeanrii

L. desmionci

L. doliolum var. pachyspora

L. elaeidicola

L. elaeidis

L. jubaeae

L. magnusiana

L. molleriana

L. phoenicis

L. pinnarum

L. pruni

L. sahalicola

L. sabaligera

L. spatharmm

L. trochus

Pandanaceae
L. pandani
L. pandanicola

Papaveraceae

L. bocconiae
L. chelidonii
L. papareris

Pameliaceac

L. gallingena

L. pormeliarmm

Peltigeraceac
L. annoldii
L. caminae
L. clarkii
L. lichenicella
L. mamillula
L. oligospora
L. peltigerea 
L. rivana

L. rivana forma solarinae

Pertusariaceae

L. tartarina

Phaeosphaeriaceae

L. didymella-vinceroxici

Phyllachoraceac

L. caucana

L. cryptica

L. phyllachoricola

L. phyllachorivora

L. tungurahuensis

Physciaceae

L. lewcomelaria

L. mahcui

Phytolaccaceae

L. argentinensis

L. clavigera

L. phytolaccae

L. variegata

Pinaceae

L. acicola

L. faulii

L. picastra

L. pini

L. squamata

L. vagabunda

L. vagabundu forma abietis

Piperaceae

L. piperis

Plantaginaceae

L. austro-americana

L. cynops

L. plantaginicola

L. vagahunda subsp. alvarensis

L. winteri

Platanaceae

L. platanicola

Plumbaginaceae

L. kotschyana

L. plumbaginis

L. staritzii

L. staticicola

Polemoniaceae
L. collumide

L. phlogis

Polygonaceae
L. aliaica
L. atraphaxidis
L. calligoni
L. muehlenbeckiae
L. oxyriae
L. quadriseptata
L. rumicicola
L. rumicis
L. schati
L. sepincola
L. we geliniana

Polypodiaceae

L. impressa

Polytrichaceae

L. henfleri

L. polytrichina

Pontederiaceae

L. cichhorniae

Porellaceae

$$
\text { L. porellac }
$$

Porpidiaceae

$$
\text { L. koerberi }
$$

Primulaceae

L. delawayi

L. pachyasces

L. primulaccola

L. primulana

L. steironematis

Proteaceae
L. chilensis
L. protearum

Pteridaceae

L. aquilina

Punicaceae

L. puniciae

Pyrolaceae

$$
\text { L. marginata }
$$

Ramalinaceae

$$
\text { L. ralmalinae }
$$

Ranunculaceae

L. aconiti

L. agminalis

L. agminalis forma minor

L. andrijevicensis

L. anemones

L. anthostomoides

L. aquilegiae

L. aucta

L. cruenta

L. doliolum var. pachyspora

L. grignonnensis

L. haematites

L. houseana

L. incruenta

L. lasiosphaerioides

L. Iathonia

L. lathonia var. helleborifoetidi

L. napelli

L. nectrioides

L. nigromaculata

L. platypus

L. pleosporoides

L. pyrenopezizoides

L. quadriseptata

L. ranunculi

L. ronunculi-polyanthemi

L. raphidophora

L. rimalis

L. scotophila
L. Tenuis

L. thalictricola

L. thalicirina

L. thalictri

L. thorae

L. trollii

L. umbrosa

L. vagabunda

L. vifalbac

L. vitalbae var. sarmenticola

L. neberi

Resedaceae

L. resedae

Restionaceae

Rhamnaceae

L. restionis

L. ceanothi

L. limitata

L. trevoae

Rhizocarpaceae

L. geographicola

L. polaris

Rosaceae

\section{L. abbreviata \\ L. aculeorum}

L. arunci

L. cercocarpi

L. concentrica

L. coniothyrium

L. controversa

L. corticola

L. doliolum

L. dryadis

L. eriobotryae

L. fuscella

L. hazslinszkii

L. hendersoniae

L. hollosiana

L. lejostega

L. leucoplaca

L. lucilla

L. mandshurica

L. minima

L. miyakeana

L. mume

L. nashi

L. notarisii

L. obesula

L. oligotheca

L. osculanda

L. oxyspora

L. pachytheca

L. pamona

L. pomona forma transilvanica

L. poterii

L. practermissa

L. pruni

L. prumi forma plurivore 
L. pulctira

L. purtemansii

L. rimicola

L. rostrupii

L. rustica

L. saccardiana

L. sanguisorbae

L. sepincola

L. sieversiae

L. sorbi

L. spiraeae

L. subculamea

L. superficialis

L. ternata

L. thomasiana

L. Imimbrosa

L. vagabunda

L. vagabumda var. divergens

L. vagabunda var. sarmenti Rubiaceae

L. africana

L. aparines

L. bormmelleri

L. caneplorae

L. centrafricana

L. coffacicida

L. coffeigena

L. coffeigena var. longirosrata

L. cylindrospard

L. dhmetorum var. galiiborealis

L. excelsa

L. galii

L. galiicola

L. galii-silvatici

L. galiormm

L. gigaspora

L. icositana

L. lobavensis

L. longispara

L. macrarostra

L. molluginis

L. oubanguiensis

L. plectrospora

L. plocamae

L. politis

L. pusilla

L. scimla

L. tetraspora

L. Iomiluzi

Ruscaceae

L. comballariae forma rusci

L. glauco-punctata

L. rusci

L. musci loma canlina

L. rusci var. hylopliylli

L. Musci var, rusciliypoglosii

L. ruscicole
Rutaceae
L. bondari
L. bulgarica
L. citricola
L. papulosa
L. vagabunda forma cirri- limonii

Salicaceae

L. aegire

L. alcides

L. baggei

L. borealis

L. borealis var. populi

L. cinerea

L. coniothyrium

L. consimilis

L. decaisneand

L. gillotiana

L. hendersoniae

L. immunda

L. lencoplaca

L. lonicerina

L. malajensis

L. purpurcarum

L. salicinearum

L. l'agabunda

L. vagahunda forma salicis-capreae

L. rylogena

Sapindaceae

L. dodoncieac

Sarraceniaceae

Saxifragaceae

L. scapopliila

L. anceps

L. brachyasca

L. cladopinila

L. deficiens

L. dichroa

L. francoae

L. grossulariae

L. livdrangeae

L. monotis

L. romsaugiensis

L. ribis

Scheuchzeriaceae

L. hacillifera

Scrophulariaceac

L. affinis

L. anambini

L. aunilana

L. camilla

L. carpogena

L. castilleias

L. castillejae

L. concimus

L. comiformis

L. digilalis

L. doliolovides var. rhinamhli

L. doliolum
L. doliolum var. angustispora

L. echinops

L. galiorum subsp. antirrhini

L. holmii

L. insulana

L. jacksonensis

L. lethalis

L. malyi

L. modesta forma digitalislureae

L. oreophiloides subsp. scropluhlariae

L. scrophulariae

L. striara

L. suffulra

L. thuemeniana

L. corbolensis

L. raldiviensis

Selaginellaceae

L. helvetica

L. helvetica forma major

L. lycopodiucola var. major

L. major

L. mellispora

L. rostrata

Simaroubaceae
L. ailamhi
L. endaphaena
L. glandulosae

Smilacaceae

L. aemensis

L. caralamica

L. doliolum

L. smilacis

Solanaceae

L. caballeroi

L. comatella

L. Iriii

L. leciophila

L. opizii

L. physalidis

L. sammenticia

L. sodomaea

L. solami

L. solanicolo

L. umlrosa

L. vagabanda

L. vasabunda var. dulcamara

Sparganiaceae

L. clara

L. huthiana

L. sparganii

L. sparsa var, meizospora

Stapliyleaceac

L. rubrorincta

Sterculiaceac

I. gucizumac 
L. rulingiae

L. theobromicola

Stereaceae

L. fungicola

L. stereicola

Stereocaulaceae
L. apocalypla
L. stereocaulormm

Tamaricaceae
L. crozalsiana
L. hollosii
L. meridionalis
L. tamaricis

Taxaceae

Taxodiaceae
L. taxicola

L. metasequoiae

Teloschistaceae

$$
\text { L. crozalsii }
$$

Theaceae
L. cannelliac
L. cannelliae-japonicae
L. cavarae
L. depressa
L. hottai
L. Tornatospora

Thelephoraceae

$L$. corae

Thymelaeaceae

L. daphnes

L. vagabunda var. daphmes

Tiliaceae
L. Lencoplaca
L. priuscheggiana

Typhaceae
L. aquatica
L. bispora
L. caricis
L. dematiicola
L. diplex
L. grandispora
L. iridigena var. typhae
L. kunzeana
L. lacustris
L. licatensis
L. licatensis forma rupefortensis

L. maculans var. typhicola

L. mucosa

L. palustris

L. perpusilla var. Iyphae

L. psewdohleria

L. puncrillum

L. typhae

L. typharum

L. typharum forma acori

L. Iyphicola

L. typhiseda

L. typhiseda forma sodoloci
Ulmaceae
L. lencoplac'a
L. massariclla
L. remmilicola
L. ulmicols

Umbelliferae

L. agnita

L. agnita var. bupleuri

L. agnitu var. major

L. bupleuri

L. cibostii

L. clivensis

L. comatella

L. conii

L. coniigena

L. conoidea

L. comoidea forma angelicue

L. cornuta

L. diaporthoides

L. dichosciadii

L. doliolum

L. doliolum forma syndoliola

L. doliolım var. dissimilis

L. doliolum var. pinquicula

L. eryngii

L. entalensis

L. ferulicola

L. foeniculaceo

L. foeniculi

L. foliicola

L. helminthospora forma crithimi-maritimi

\section{L. ladina}

L. libanotis

L. longchamipsi

L. longipedicellara

L. massarioides

L. melanommoides

L. modesta forma danci

L. modesta var. cibostii

L. montis-bardi

L. nesodes

L. niessleanu var. staritzii

L. nigrella

L. obesula

L. ogilviensis forma myrhis-odorata

L. ogilvicnsis var. pleurospermi

L. oreophiloides

L. penicillus

L. pimpinellas

L. ranumculoides

L. ropalispora

L. rhopographoides

L. restrupii

L. rubicunda

L. setoses
L. sileris

L. spectabilis

L. umbrosa

L. Utahensis

L. vinosa

L. woodrow-wilsonii

Umbilicariaceae

L. umbilicariue

Urticaceae

L. acuta

L. acuta forma insignis

L. acuta forma wicae

L. acutiuscula

L. atropurpurea

L. cecropiae

L. coniformis

L. conoidea forma macrospora

L. doliolum

L. dumetorum

L. muralis

L. pariefariae

Usneaceae

L. usneae

Valerianaceae

L. agnita

L. carneomaculans

L. dubia

L. galiicola var. brachyspora

L. quadriseptata

Venturiaceae

L. platychorae

Verbenaceae

L. haldingerae

L. hasalduai

L. casta

L. clerodendri

L. isocellula

L. octoplvagmia

L. octophragmia var. major

L. rajasthanchsis

$\mathrm{V}$ iscaceae

L. phoradendri

Vitaceac

L. anıclina

L. cerlettii

L. chaetostomu

L. cirricola

L. cookei

L. gibelliana

L. pampini

L. sclaronica

L. socia

L. vagabunda

L. vincalis

L. viticola

L. vitigena

L. vitis

Zingiberaceac

L. alpiniae

L. zingiloris 


\section{Substrate Index}

Achenes

Apothecia

L. agnita var. arheniarum

L. inconspicua
L. consocians
L. maheui

Ashes

Bark

$$
\text { L. inconspicua }
$$
L. akagiensis
L. avicenniae
L. buxina
L. clerodendri
L. inquinans
L. inspersa
L. Lencoplaca
L. muelleri
L. squamata

\section{Berries}

Bracts

L. saprophila

\section{L. arcialaskana}

L. bracteamm

L. carduina

L. carpophila var. bractearum

L. crepini

L. lamprocarpi

L. Iycopodina

L. spatharum

L. vitensis

Branches
L. adesmicala
L. acrea
L. acmensis
L. africana
L. ahmadii
L. ailanthi
L. allagii
L. ambiens
L. ammothammi
L. amorphae
L. anceps'
L. arburi
L. avellanae
L. azalear
L. baggei
L. berberidis
L. berulina

L. basalduai

L. biebersteinii

L. borealis var. populi

L. borziana

L. capparidicola

L. capparidis

L. castagnei

L. catatamica

L. cavamillesii

L. chilensis

L. cinerea

L. cimnamomi

L. cisti

L. cisticala

L. clelandii

L. coffeigena var. longirostrato

L. consimilis

L. constricta

L. controversa

L. crosalsiana

L. crucheri

L. cylindrospora

L. daviesiar

L. deraso forma macrospora

L. desmonci

L. dichroa

L. disica

L. dobrogico

L. dodonueae

L. dumetorum var. dolichospora

L. elaoudi

L. emiliana

L. endophaena

L. ephedrue

L. euphorbicalla

L. cricelsa

L. fiedlaeri

L. fuscella forma microspora

L. firscella var. hippophaes

L. firscella var. sydowiand

L. fusispora forma ersimi

L. gibelliana

L. gigasporia

L. gilloriama

L. ginkgo
L. grarissima

L. grossulariae

L. hazslinszkii

L. hendersoniae

L. hippophaes

L. hiria

L. hollosii

L. hydrangeae

L. kuangfuensis

L. lejosiega

L. limirata

L. Jongispora

L. Ionicerae

L. luxemburgensis var. dolichospora

L. macrorostra

L. malojensis

L. mamillana

L. massariella

L. massariella var. brasiliensis

L. melanommoides

L. meridionalis

L. nuchlenbergiae

L. mume

L. miricae

L. mirri

L. mrrricola

L. nigrella

L. adora

L. olivaespore

L. asculanda

L. pachutheca

L. periclimeni

L. phiala

L. plasicarpa

L. polini

L. preandina

L. puniciae

L. revocans

L. rhododendri

L. ribis

L. rimicola

L. riofriori

L. salviale

L. sambuci

L. rarohammi

L. sckaronica

L. simillima 
L. smilacis

L. spiracae

L. stictoides

L. suacdae

L. subcutanca

L. subsimilis

L. Iamaricis

L. temata

L. tetraspora

L. tiroliensis

L. torrendii

L. rematostoma

L. trevoue

L. trichostoma

L. Itmefaciens

L. vagabunda

L. vagabunda forma daplines

L. vagalmunda var. divergens

L. vitigena

L. rylogena

L. jerhae

Branchlets
L. almeidana

L. bella

L. cistina

L. coniothyrium

L. corticola

L. daphnes

L. fusispora

L. juniperi

L. lavandulac

L. papillata

L. platanicola

L. Iulingiae

Broom

L. sorghophila

Canes

L. hendersoniae

L. hippophaes

L. hydrophila

L. nicropogon

L. proctermissa

Capsules

L. capsularmm

L. carpogena

L. carpophila

L. ogilviensis

Carpels

L. serophulariue

Cones

L. triglochinicola

Culms

$$
\text { L. pini }
$$

L. alicna

L. amphiloga

L. anthrostomella

L. apogon

L. arenaria
L. arhenatheri

L. arundinacea

L. baldingerae

L. bambusae

L. bambusicola

L. beatmontii

L. bryzae

L. cattanei

L. cirsii-ariensis

L. clavicarpa

L. coccodes

L. conimbricensis

L. culmicola

L. culmicola var. achuatica

L. calmicola var. minor

L. culmifida

L. culmifraga

L. culmifraga forma majuscula

L. culmorum forma latugarica

L. culmorum var. $f$ avobrumea

L. culmorum var. palcicola

L. cumulata

L. cynosuri

L. dactylina

L. discors

L. donacina

L. epicalamia

L. epicalamia var. pleosporoides

L. eumorpha

L. eustomella

L. eustomoides

L. graminis

L. grisea

L. hazslinskyana

L. helianthi

L. herpotrichoides

L. holmii

L. ischaemi

L. junci

L. juncicola

L. juncina

L. junci-acuti

L. Iarvalis

L. lelebue

L. linearis

L. lineolaris

L. litoralis

L. litrorcalis forma calamagrostidis: arenailae

L. lolii

L. luctuosa

L. marina

L. maritima

L. matritensis

L. melanommoide's
L. michorii

L. microseopica forma brachupodii

L. monilispora

L. ntosana

L. moutoniana

L. nardi

L. neomaritima

L. nigrans forma arundinis

L. nodortm

L. occidentalis

L. oryade

L. pachycarpa

L. pachytheca

L. papillosa

L. patellaeformis

L. pelagica

L. penniseti

L. perpusilla var. typhae

L. petkovicensis

L. petkovicensis var. elymi

L. petrakii

L. phacae

L. phragmiticola

L. plewrospora

L. poac

L. pontiformis

L. proteispora

L. punctiformis

L. raphani

L. reidiana

L. rhodophaca

L. riparia

L. rubelloides

L. rusci

L. rusci forma caulina

L. rusci var. hypophy/li

L. sabauda

L. sanguisorhac

L. saronica

L. scabiens

L. scabrispora

L. scirpina

L. secalina

L. selulosa

L. solleimii

L. souerbyi

L. sparsa

L. spesrtii

L. spartinae

L. spegazzini var. minor

L. sporoboli

L. sticta

L. straminis

L. stromatoidea

L. subcompressa

L. subsuperficialis

L. taminensis

L. therophila

L. thurgoviensis 
Driftwood
L. trichopterygis
L. Iucumanensis
L. typhae
L. variabilis
L. volkartiana
L. weddellii

Exocarp

L. orac-maris

Feathers

L. exocarpogena

Florets

Fronds

$$
\text { L. corvina }
$$

\section{L. lamprocarpi}

L. aquilina

L. asplenii

L. caffra

L. elacidicola

L. motisiae

Fruits
L. pinnarum

L. dryadis

L. genistae

L. pomona forma transilvanica

Glumes

L. oryzina

Hymenium
L. consocians
L. fungicola
L. lichenicola
L. stercicola

Involucre

\section{Leaves}

$$
\text { L. involucralis }
$$

L. abutilonis

L. aceris

L. acicola

L. acorclla

L. acori

L. acgira

L. aeluropodis

L. acrea

L. agaves

L. aglaja

L. alcides

L. alcides forma quercina

L. alexandirinis

L. algarbicusis

L. algida

L. aliena

L. almeidac

L. aloes

L. alopecuri

L. alpiniae

L. ammophilae

L. anarithma

L. amerithmoides

L. andromedac
L. antaretica

L. apios

L. apios-fortunei

L. aquarica

L. arecac

L. arenaria

L. arcnaria form sp. iriticea

L. arrhenatheri var. italica

L. asperellac

L. aspidistrac

L. ancrsuraldii

L. australis

L. anstro-americana

L. avenue

L. avenaria

L. bacillifera

L. haldratiana

L. bambusae

L. bataticola

L. batumensis

L. baumii

L. berberidicola

L. betulina

L. bicolor

L. bispora

L. bomarcae

L. bondari

L. bormmuelleri

L. brachyasca

L. brachypodii

L. brasilicnsis

L. briosiana

L. buddlejae

L. californica

L. calopogonii

L. camelliae

L. camellioe-japonicoe

L. camilla

L. camphorosmac

L. campisilii

L. cancphorae

L. camnabina

L. cammae

L. caricicola

L. caricina

L. caricinclla

L. caricis

L. caricis-firmae

L. caricis-lulpinace

L. cattanci

L. cortarae

L. cetallosi

L. cecropise

L. cercocarpi

L. chamacropis

L. chenopodii-alhi

1. chochrjokesii

L. chusqueac

L. citricola
L. clara

L. clavata

L. coccothrinacis

$L$. cocoes

L. coffaeicida

L. coffeigena

L. coicis

L. collumiae

L. colocasiae

L. concentrica

L. coniothyrium

L. coniothyrium var. folicola

L. consobrina

L. consociata

L. comallariac

L. convallariae forma dracacnae

L. convallariae forma rusci

L. cordylines

L. corrugans

L. coumarounae

L. crastophila

L. crastophila forma tofieldiae

L. cucurbitae

L. culmicola var. hispalensis

L. culmifraga var. alpestris

L. culmifraga var. propinqua

L. culmorum

L. culmorum forma epigeii

L. culmorum forma phragmitis

L. cumana

L. cycadis

L. cynaroccarum

L. cynodontis-dactyli

L. cyperi

L. cypericola

L. daphniphylli

L. dasylirii

L. debcanvii

L. decaisneana

L. dennisiana

L. densa

L. diana

L. dichosciudii

L. dracuenar

L. draconis

L. dryadea

L. dryadea subsp. lussoniensis

L. dryadis

L. drivophila

L. duplex

L. cichlournise

L. clueidis

L. clacospora 
L. elymi

L. empetri

L. epicarecta

L. eriobotryae

L. erythrinae

L. eustomoides var. punctata

L. faulii

L. feijoae

L. ferruginea

L. fici-elasticae

L. filamentosa

L. foliicola

L. folliculata

L. fraxini

L. frigida

L. fuegiana

L. fuscidula

L. fuscidula forma magnolii

L. gaubae

L. georgius-fischeri

L. gigaspsora

L. glandulosae

L. glauco-puncrata

L. glyceriae

L. glyceriae-plicatae

L. gossypii

L. graminum

L. grandispora

L. gratissima

L. gratissima var. longispora

L. guazumae

L. gynerii

L. hardenbergiae

L. hausmamniana

L. hausmarmiana var. cherleriae

L. hederae

L. hedericola

L. helicicola

L. heloniaefolia

L. helverica

L. hemicrypta

L. hermodactyli

L. hesperia

L. heveae

L. honiaraensis

L. hushiana

L. Iyperborea

L. hysterioides

L. immunda

L. indeprensa

L. indica

L. infernalis

L. insignis

L. insignis forma airaecespirosae

L. iridicola
L. iridigena

L. iridigena var. yphac

L. iridis

L. isariphora

L. incumotoi

L. jahmii

L. jamus

L. jubaeae

L. junci

L. juncina forma macrospora

L. juniperina

L. kergaclensis

L. kotschyana

L. lacustris

L. larseniana

L. Lasioderma

L. Iathonia var. hellborifoetidi

L. lauri

L. leersiae

L. leersiana

L. licatensis

L. licatensis forma rupefortensis

L. Iilii

L. lingue

L. livida

L. lobeliae

L. lucilla

L. lucina

L. Iuzulae

L. maclurae

L. macrochloae

L. macrotheca

L. maculans

L. magnoliae

L. magnusiana

L. major

L. malojensis

L. mandshurica

L. marantae

L. marcyensis

L. marginalis

L. marginata

L. maritima

L. matrisensis

L. maydis

L. media

L. melicae

L. mellispora

L. mendozana

L. mertensiae

L. microscopica subsp. calami

L. microscopica var. alpina

L. mikaniae

L. minima

L. miyakeana

L. molleriana
L. monicola

L. morthieriana

L. moulan

L. mucosa

L. muirensis

L. muricula

L. musae

L. musarum

L. musigena

L. nardi var. dubiosa

L. nashi

L. neglecta

L. nervisequa

L. nesodes

L. niessleana

L. nobilis

L. nodorum form sp. hordei

L. obusispora

L. obursispora forma agaves

L. occulta

L. oligotheca

L. ophiopogonis

$L$. ophiopogonis var. graminum

L. orthrosanthi

L. oryzae

L. pachyasca

L. pachytheca

L. pacifica

L. paludosa

L. pampaniniana

L. pandani

L. pandanicola

L. panici

L. paoluccii

L. papulosa

L. paraguariensis

L. parvula

L. parvula var, iridisgermanicae

L. passerinii

L. pelargonii

L. penniseticola

L. perforans

L. petri

L. phlogis

L. phoradendri

L. phormii

L. physalidis

L. pilulariae

L. piperis

L. planfaginicola

L. plemeliana

L. pomona

L. portoricensis

L. primulaecola

L. priascheggiana

L. protearum

L. prumi 
L. pruni forma pluriora

L. pterocelastri

L. purciniorides

L. puiggarii

L. pulchra

L. punctillum

L. purpurearum

L. pusilla

L. putlemansii

L. recurita

L. rehmiana

L. relmii

L. rhododendri

L. rivalis

L. rostrata

L. rostrupii

L. rusci

L. musci forma fourcroycue

L. ruscivar. ruscihypoglossi

L. riescicola

L. sabauda

L. sabauda forma arruricae

L. sabuletorum

L. sacchari

L. saccharicola

L. saginata

L. sulebricola

L. salicinearum

L. sasae

L. scolecosporarum

L. seriata

L. silenes-acaulis

L. smarodsii

L. smilaris

L. solani

L. sorbi

L. sorghi-arundinacei

L. sparsa

L. sparsa var. meizospora

L. spegazzini

L. staticicola

L. stellariae

L. stipae

L. stipae-minor

L. stratiotis

L. striolata var. caricisglancae

L. subiculifera

L. substerilis

L. subrecta

L. swertias

L. sylvatica

L. Irichungensis

L. Iaiwanensis

L. fanareti

L. ervicola

L. theobromicola

L. thorce

L. thujaccola

\section{L. $\operatorname{lini}$}

L. Ioficldiae

L. Iondlazi

L. translucens

L. Irifolii-alpestris

L. Iritici

L. typharum

L. Iypharum subsp. phragmarina

L. typhicola

L. Iyphiseda

L. ryphiseda forma sodoloci

L. uliginosa

L. ulmicola

L. vagans

L. vagans forma scirpi

L. valdobbiac

L. vanhoeffeniana

L. variiseplata

L. rectis

L. verwoerdiana

L. vincae

L. rinosa

L. irieseae

L. weberi

L. williamsii

L. winteri

L. woodrow-nilsonii

L. rerophylli

L. xiphii

L. yulan

L. zeae

L. zeae-maydis

L. zeicola

L. zingiberis

Nuts

L. cacuminispora

Paper

L. fibrincola

L. papyricola

L. Iritici var. puplricola

L. topharmm subsp.

Pedicels papyrogena

L. eramihemi

L. customa

Peduncles

L. bryzare

L. espeletias

L. framsorac

L. Incopodicola

L. papwri

L. rophidophora

L. scopophila

L. typhiseds

L. vitensis

Petioles

L. anemones

L. cinclidoti
L. eryingii

L. monicola

L. periolaris

L. periolicola

L. phocnicis

L. primulaecola

Pods

L. typhiseda

L. endiusae

L. eusrama forma leguminosa

L. impressa

L. Iunariae

L. Jindonillae

Rachis

L. culmifraga forma poae

L. eustamoides forma lolii

L. sahalicola

L. Irochus

Rhizomes

L. culmicola var, rhizomatum

L. heterospora

L. littoralis

L. pontiformis

L. rhizomatum

L. vagabunda subsp. alvarensis

Roots

L. australiensis

L. capparidis

L. circinans

L. cladii

L. Jycii

L. Maculans forma denudata

L. septovariata

Runners

L. cookei

L. grignonnensis

L. hacmatites

L. nectrioides

L. noturisii

L. pampini

L. pleosporvide's

L. rhi=omutum

L. rimalis

L. sommenticia

L. thomessiana

L. Iugabundar var. summeni

L. vine'alis

L. viralhae

Seeds

L. albo-punctura

L. microscopica forma gluceriare

L. sciquamat

L. worrominii

Sepals

L. auerswaldii

L. sepalormm 
Sheaths

\section{L. alsaica}

L. arenaria

L. avenaria form sp. triticest

L. cariciphila

L. contunci

L. cookei

L. culmicola forma melicae

L. culmicola var. hispalensis

L. culmicola var. migrans

L. culmortum

L. fuckelii forma filamentifera

L. interspersa

L. korrae

L. lagenoides

L. latebrosa

L. matritensis

L. media

L. monilispord

L. narmari

L. neomaritima

L. nigrans

L. nodorum form sp. hordei

L. notarisii

L. pandami

L. rivalis

L. rousseliana

L. sacrhari

L. salvinii

L. spartii

L. spegazimi

L. subalpina

L. vaginae

Sporangia

Stems
L. heufleri

\section{L. abbreviata}

L. acumthi

L. achilleac

L. aconiti

L. aculearum

L. acuita

L. acuete forma insignis

L. acuta forma urticae

L. acutispora

L. acutiuscula

L. affinis

L. agminalis

L. agnita

L. agnita subsp. Labens

L. agnita var. ambigna

L. agnita var. bupleuri

L. agnita var. chrysanthemi

L. agnita var. erigeromtis

L. agnita var. major

L. agnita var. trifolii

L. alloulae

L. alliariac
L. allorgei

L. amphilola

L. anacyeli

L. ancarrhini

L. andrijericensis

L. anomala

L. anthelmintica

L. antherici

L. anthostomorides

L. aparines

L. aquilana

L. aquilegiae

L. arubidis

L. argentina

L. artemisiae

L. arthrophyma

L. arumci

L. arvensis:

L. asclepiadis

L. asparagi

L. asparagina

L. astericole

L. asteris

L. atropurpurea

L. aucter

L. aulica

L. balcarica

L. ballotae

L. bardanac

L. bellynckii

$L$. herlesei

L. blumeri

L. bocconiae

$L$. houcera

L. brachyasca

L. hrachysperma

L. branini

L. bresadolaema

L. brightonensis

L. brunellac

L. bryophile

L. butakii

L. buddlejac

L. bulgarica

L. bupleuri

L. caballeroi

L. cadubriae

L. caespitosa

L. caespitosic forma salviae

L. caliesciens

L. camphorala

L. canadensis

L. carduorum

L. carlinoides

L. curneomatulans

L. cassiaceola

L. castilleiae

L. ecastillejac

L. canlincrola

L. centanteae

L. centrafricana
L. cephalariai-tralensis

L. cerastii

L. ceratispora

L. cerei-perwiani

L. cervispora

L. cesstiame

L. chelidonii

L. chrysanthomi

L. cibostii

L. circinams

L. cirricola

L. cladophila

L. clavata

L. clavigera

L. clavispora

L. clivensis

L. clivensis var. constricta

L. colcosanthi

L. collinsoniac

L. comatella

L. complanata

L. compositarmm

L. compressa

L. concinna

L. conferta

L. congesta

L. coniformis

L. conii

L. coniigena

L. coniothyrium

L. conoidica

$L$. conoidea forma angelicae

L. conoidea forma asteris

L. conoidea forma macrospora

L. consessa

L. controversa

L. comallariae

L. coorgicu

L. corallorhizae

L. cormuta

L. coromillae

L. corricola

L. corynispora

L. cosmicold

L. crastophila forma tofieldiae

L. cruenta

L. crustasca

L. cucurbiturioides

L. culmicola forma major.

L. culmifraga var. bromicola

L. culmifraga var. linearis

L. rurta

L. cylindrospora

L. cylindrostoma

L. cynoglossi

L. cynops

L. darkeri 
L. davidii

L. davisiana

L. dearnessii

L. deficiens

L. delawayi

L. dematiicola

L. dematium

L. demissa

L. depressa

L. derusa forma alpestris

L. derasa forma robusta

L. derasa var. franconica

L. desciscens

L. dianthi

L. diaporthoides

L. digiralis

L. dissiliens

L. dolioloides

L. dolioloides var. cirsii

L. dolioloides var, inops

L. dolioloides var. lathyi

L. dolioloides var. rhinanthi

L. doliolum

L. doliolum forma carlinac-vulgaris

L. doliolum forma syndoliola

L. doliolum var. angustispora

L. doliolum var. cacaliae

L. doliolum var, dissimilis

L. doliolum var. leonmri

L. doliolum var. pachyspora

L. doliolum var. pinquicula

L. doliolum var. sublisticha

L. drabae

L. dryadea subsp. lussoniensis

L. dryadis

L. dubia

L. dimberomm

L. Anmetorum forma chuli

L. dumetorum forma meliloti

L. dumetorum var. coniformis

L. dumetormm var. coronillae

L. dumetorum var. galiiborralis

L. dumetorum var. marrubii

L. dumetorim var. swimphyti

L. elorrhes

L. echiclla

L. e'hii
L. echinella

L. echinops

L. ellisiana

L. elomgara

L. endiusae

L. epilobii

L. equiseri

L. equiseticola

L. erigeromis

L. eriophora

L. eryngii

L. ettalensis

L. euphorbiae

L. euphorbiae forma esulae

L. eliphorbiaecola

L. entypoides

L. faginea

L. fallaciosa

L. fallax

L. fermginea

L. ferulicola

L. filiformis

L. fimbriata

L. fiumana

L. foenicularea

L. foeniculacea subsp. liupina

L. foeniculi

L. fiaserae

L. frondis

L. furchelii

L. fuegiand

L. fulgida

L. galeobdolonis

L. galeopsidicola

L. galii

L. galiicola

L. galiicola var. brachyspora

L. galii-silvatici

L. galiorum

L. galiorum forma cirsionmm

L. galionum forma gentianae

L. saliorum subsp. antirr/nin

L. galiorum var. ghaplaliallat

L. guliorum var. Lapsomate

L. ganltheriac

L. genistue var. microspord

L. gigaspora

L. gloesspora

L. gnap/lalii

L. grammodes

L. Bypsoplitae

L. huemurites

L. harknessiamma

L. helianthemi
L. heliopsidis

L. helminhospora forma crithmi-marimi

L. hemerocullidis

L. hesperidicola

L. hiemalis

L. hiria

L. hollosiana

L. holmii

L. houseana

L. Irubsina

L. hurue

L. hyulospora

L. hyparrheniae

L. ivperici

L. icositana

L. incarceraia

L. incruerta

L. inculta

L. indica

L. insulana

L. jaceae

L. jucksonensis

L. jacksonii

L. johlunsomii

L. juncuginearum

L. junci-glauci

L. juncorwm

L. kali

L. Kalmiac

L. kalmusii

L. kochiana

L. kunzeanu

L. lacuseris

L. Iadina

L. lankeana

L. larseniana

L. Iasiosphaerioides

L. lassenensis

L. lathyri

L. lathrina

L. lecanora

L. Ieptospora

L. lespedezae

L. lethalis

L. lihamoris

L. limosa

L. lindquistii

L. lithophilac

L. lobavensis

L. Iongelompsi

L. Iongipedicallata

L. longispora

L. Ionicerins

L. lophammi

L. Inpini

L. lupinicola

L. Insifanica

L. Iyciophila

L. Ivhri 
L. macrospora

L. macrosporidium

L. maculans

L. maculams var. typhicola

L. maderensis

L. malyi

L. marisima

L. marram

L. marfagomi

L. mossariclla var. disticha

L. massariaides

L. media

L. medicaginicola

L. medicaginis

L. medicaginum

L. megalospora

L. melanommaides

L. melicas

L. melilori

L. menthue

L. mesoedema

L. metusequoiae

L. microspora

L. microthrinides

L. mille folii

L. mirabilis

L. modesta

L. modesta forma digitalislileae

L. modesia forma jacobaeae

L. modesia forma lappae

L. modesia forma succisue

L. modesta forma syliestris

L. modesta var. cibostii

L. modesta var. rubellula

L. modestula

L. molluginis

L. molshdina

L. monilispora

L. monilispora forma triglochinis

L. montana

L. montis-hardi

L. morierae

L. muehlenbeckiae

L. muliseprata

L. multiseptata forma alpina

L. muralis

L. muscari

L. nanae

L. napelli

L. napi

L. nicolai

L. niessleana

L. niessleana forma viriae

L. niessleand var. staritii

L. nigrella

L. nigricans
L. nigromaculasa

L. nitschkei

L. nisschkei forma adenostylidis

L. norfolcia

L. norvegica

L. abesa

L. ahesula

L. obione's

L. obiones var. evolutior

L. ocellara

L. ocimicola

L. octophragmia

L. octophragmia var, major

L. octoscptata

L. ogilviensis

L. ogilviensis forma achilleae

L. ogilviensis forma lepidii

L. ogilviensis forma megalospara

L. agilviensis forma mirrhis-odorata

L. ogilviensis var. pleurospermi

L. ogilviensis var. senecionis-cordati

L. olericola

L. olivacea

L. onagrae

L. onobrychidicola

L. onobrychidis

L. ophioboloides

L. opi-ii

L. opuntiae

L. orchidearam

L. oreophila

L. oreophiloides

L. oreophiloides subsp. scrop/unlariae

L. arnillogali

L. arfhagramma

L. aubanguiensis

L. orvaniae

L. oxyrias

L. oxyspora

L. palustris

L. papaveris

L. pariefariae

L. pariefariae forma lamii

L. passeriniana

L. passerinii

L. pellira

L. penicillus

L. perplexa

L. perpusilla

L. personala

L. phacospora

L. phaseali

L. phaseolorwm
L. physostegiae

L. phyteumatis

L. phyolaccae

L. pimpinellae

L. plamiuscula

L. plamiuscula forma cruciferarum

L. plaranicola

L. platypus

L. plecrospora

L. plumbaginis

L. plarisepta

L. polinis

L. palygomati

L. pamiformis

L. poterii

L. praeclara

L. pramontorii

L. pratensis

L. primulana

L. proliferae

L. pseudo-diaporthe

L. psendohleria

L. psilospora

L. ptarmicae

L. pulchra

L. punctoidea

L. punjahensis

L. purpurea

L. quadriseptana

L. quamoclidii

L. ramsangiensis

L. ranunculi

L. ranunculi-polyanthemi

L. ranunculoides

L. recessa

L. resedae

L. restionis

L. rhodiolicola

L. rhopalispora

L. Hopographoides

L. richani

L. riofrioi

L. rivalis

L. rivularis

L. robusta

L. rostrupii

L. rothomagensis

L. rothomagensis var. artemisiae

L. rubellula

L. rubicunda

L. rudbeckiae

L. rugosa

L. numicicola

L. rumicis

L. ruscicola

L. ruscicola forma cladodiicola

L. russellii 

L. rustica
L. mihenica
L. saccardiana
L. sacculus
L. salebrosa
L. salicaria
L. salsolae
L. sanguisorbae
L. sapeyensis
L. sarrazimiana
L. schoenocauli
L. scitula
L. scotophila
L. scutati
L. semelina
L. senecionis
L. sepincola
L. septemcellulata
L. serbica
L. setosa
L. shahvarica
L. shastensis
L. sibirica
L. sibtorpii
L. sieversiae
L. sileris
L. silvestris
L. simmonsii
L. sinapis
L. slovacica
L. socialis
L. sodomaea
L. solani
L. solanicola
L. solidaginis
L. sparganii
L. spectabilis
L. staritzii
L. steironematis
L. stellariae
L. stellata
L. stictostoma
L. striata
L. striolata
L. subcaespitosa
L. subconica
L. subniaculans
L. thalictrina
L. thielensii
L. thuemeniana
L. tolgorensis
L. lorbolensis
L. torulispora
L. trichostoma
L. trifolii
L. triglochinicola
L. triglochinis
L. trimera
L. trimerioides
L. Iritorulasa
L. trollii
L. lupae
L. typharum
L. uncinata
L. utahensis
L. vagabunda
L. vagabunda forma abietis
L. vagabunda forma cirri- limonii
L. vagabunda forma lonicerae
L. vagabunda forma salicis-capreae
L. vagabunda var. caulium
L. vagabunda var. dulcamara
L. vahlii
L. valdiviensis
L. valesiaca
L. variegata
L. veratri
L. viciae
L. vindobonensis
L. vinosa
L. virginica
L. viridella
L. vitalbae var, sarmenti- cola
L. wegeliniana
L. h'egeliniana forma teucrii
L. weluncyeri
L. zahlbruckneri
L. zizaniaecola

L. submodesta

L. subriparia

L. suffulta

L. surculorum

L. tanaceti

L. taurico

L. tenera

L. temuis

L. tephrosiae

L. tetonensis

L. tcucrii

L. thalictri

L. Ihalictricola
Stolons

L. cookei

L. Korrae

L. narmari

L. Inotarisii

Straw

L. culmifruga

L. customa

Stroma

L. caucana

L. cryprica

L. phyllachoricols

L. phyllachorivora
Stubble

L. platychorae

L. tungurahuensis

L. arundinacea

L. arundinacea var. godini

L. cyperina

L. recutita

Thalli

L. adienula

L. apocalypta

L. arnoldii

L. baeomycearia

L. caninae

L. clarkii

L. consacians

L. corae

L. crozalsii

L. galligena

L. geographicola

L. koerberi

L. lemaneae

L. leptogiophila

L. lencomelaria

L. lichenicola

L. mamillula

L. mirandae

L. oligospara

L. peltigerarum

L. peltigerea

$L$. polaris

L. porellae

L. pycnostigma

L. pycnosigma var. morbosa

L. ramalinae

L. rivana

L. rivana forma solorinae

L. sphyridiana

L. steinii

L. stereocaulorum

L. subarticulata

L. Iartarina

L. umbilicariae

L. usneae

Trunks

L. butina

L. coffeigena var. longirostrata

L. ericae

L. hottai

L. Martinianum

L. salcbrosa

L. sicula

Twigs

L. bicuspidata

L. bomlari

L. californica

L. calligomi

L. casia

L. ceanothi 
L. distributa

L. fuscella

L. hispanica

L. hyalina

L. leiostega

L. myricae

L. periclymeni var. tatarica

L. plagia

L. pyrenopezizoides

L. ramulicola

L. rubrotincta

L. tornatospora

L. vitalbae
Vines

L. ampelina

L. caprifolii

L. cerletiii

L. chaetostoma

L. pampini

L. phaseolorum

L. socia

L. viricola

L. vitis

Wood
L. abuensis
L. argentinensis

L. horealis

L. calligoni

L. clerodendri

L. coniecia

L. halima

L. haloryli

L. isocellula

L. paucispora

L. picastra

L. plocamae

L. pureuna

L. rajasthanensis

L. rhoina

L. seminuda 


\section{Geographic Index}

Afghanistan

Algeria

L. morierae

L. crozalsiana

L. debeauxii

L. icositana

L. indeprensa

L. macrochloae

L. obesa

L. papulosa

L. phiala

L. stipae

L. typhiseda

Angola

L. haumii

Argentina

L. untarctica

L. adesmicola

L. aerea

L. anthostomella

L. argentina

$L$. argentinensis

L. anstro-americana

L. basalduai

$L$. berberidicola

L. conii

L. coniigena

L. cordylines

L. cylindrostomo

L. fuegiana

L. gynerii

L. lagenoides

L. lindquistii

L. lycopodicole var. major

L. mendozana

L. obtusispora

L. preandina

L. promontorii

L. proteispora

L. sacchari

L. spegazzini

L. spegazini var. minor

L. suhiculiferd

L. subsuperficialis

L. fucumune'nsis

L. vinosa

L. verbae

Australiat

L. aliena
L. australiensis

L. australis

L. camelliae

L. cannae

L. clelandii

L. daviesiae

L. dichosciadii

L. gauhae

L. hardenbergiae

L. korrae

L. narmari

L. paucispora

L. plagia

L. restionis

L. rulingicse

L. suaedae

L. nilliamsii

Austria

L. acuta

L. aparines

L. artemisiae

L. baggei

L. caricis-firmae

L. cattanei

L. cesatiana

L. cinerea

L. coniformis

L. coniothyrium

L. corticola

L. culmifraga var. alpestris

L. depressa

L. derasa forma robusta

L. customa

L. fusispora

L. gnaphatii

L. inconspicus

L. intermedia

L. juncicola

L. macrospores

L. marginata

L. medicuginis

L. medicaginum

L. monilispora

L. nupi

L. migrans

L. nitschkei forma adenostylidis

L. oreophila

L. parruka
L. penicillus

L. personata

L. pleurospora

L. pulchra

L. recutita

L. rusci

L. saprophila

L. scirpina

L. seminuda

L. senecionis

L. sparsa var. elynae

L. spectabilis

L. sphyridiana

L. suffulta

L. tanaceti

L. thorae

L. tiroliensis

L. umbrosa

L. vindobonensis

L. vitalbae

L. vitigena

L. vitis

L. Eahlbruckneri

Belgium

L. acura

L. alho-punctata

L. arenaria

L. bellynckii

L. carduorum

L. crepini

L. doliolum

L. endophaena

L. gnophalii

L. hacmutrites

L. juncina forma macrospora

L. lihomoris

L. longchampsi

L. maculans

L. mamillana

L. melanommoides

L. michorii

L. mosana

L. moutoniant

L. mucosad

L. nardi

L. nardi var. dubiosa

L. obesula

L. perpusillo 
L. pancriformis

L. revocans

L. rivilaris

L. rusci

L. Salebricola

L. sowerlyi

L. subriparia

L. Ihichensii

L. Iypharum

Bolivia

L. agmita var. achenianum

L. weddellii

Brazil

L. alpiniae

L. bondari

L. brasiliensis

L. desmonci

L. diaporthoides

L. eustrmoides var. punciuta

L. massariella var. brasilicnsis

L. matisiac

L. mikanias

L. paraguaricusis

L. pelargonii

L. puiggarii

L. partemansii

L. saccharicola

L. schneideriana

\section{Bulgaria}

L. bubakii

L. bulgarica

L. dianthi

Canada

L. andromedae

L. anisomeres

L. asclepiadis

L. associana

L. australiensis

L. avenaria form sp. rivicea

L. barriae

L. berlesei

L. bruncllae

L. canadensis

L. coricinells

L. curicis

L. corticola

L. culmifraga forma mimascula

L. culmorum

L. dearnessii

L. doliolum

L. diechsleri

L. clongata

L. equiseti

L. customa

L. faulii

L. folliculara

L. fuckelii
L. gaultheriak

L. hesperia

L. hicmalis

L. insignis

L. jacksomii

L. licatensis

L. lilii

L. Inctuosa

L. Iycopodicicola

L. iveopodina

L. mareyensis

L. marram

L. microscopica

L. microspora

L. neomaritima

L. migrams

L. nodormm

L. ogilviensis

L. punctillum

L. ronsseliana

L. Inssellii

L. Irugosa

L. solani

L. sorgho-amundinacei

L. steironematis

L. Yypharm

L. typlicola

L. waghomiama

L. wehmeveri

Canary Islands

L. plocamae

Cape Verde Islands

L. Larvalis

Central African Republic

L. centrafricana

L. cylindrospora

L. cicelsa

L. gigaspora

L. lobavensis

L. longispora

L. macrorostra

L. Oubangwiensis

L. tetraspora

Central America

L. corae

Chile

L. chilensis

L. flotoviae

L. francoue

L. jubaeae

L. lingue

L. melanommovides

L. phoradendri

L. Mevoue

L. Iipue

L. valdiviensis

China

L. acamthi

L. aspidistrate

L. bambusicola

L. deficiens
L. delawavi

L. dolichlum var angustispora

L. crambemi

L. mandishurica

L. mirakeana

L. oryzac

L. pupyri

L. plambaginis

L. scahrispora

L. trochus

Colombia

L. calucana

L. stellata

Costa Rica
L. coffaricida
L. pusilla
L. Ionduzi

Cuba

L. coffeigena

Czechoslovakia

L. alicta

L. haggei

L. caricis

L. caineomaculans

L. conferta

L. corticola

L. culmorum

L. cylindrospora

L. cynaracearum

L. dumetornm

L. dumetorum forma ebuli

L. euphorbiac

L. fici-clasticae

L. galeopsidicola

L. gigaspsora

L. helerospora

L. hrubvama

L. juncicola

L. juncina

L. Kalmusii

L. Koerberi

L. lycopodina

L. marginata

L. megalospora

L. millefolii

L. miessleana

L. parrula

L. petkovicensis

L. petrakii

L. phyllachorivora

L. pore

L. pomifermis

L. rousseliana

L. rudbeckias

L. se'osa

L. slovacica

L. stcinii

L. Syplicosa

L. Incinulu 
Denmark
L. ammophilat
L. urenaria
L. macillifera
L. borlesei
L. bispora
L. chendri
L. canii
L. carvina
L. culmorum
L. demices
L. dicumthi
L. marina
L. microséopica
L. nodorwni form sp. hordei
L. acculta
L. oligotheca
L. recutita
L. rostrupii
L. sabuletarum

Dominican Republic
L. calopogomii
L. cecropiae
L. coccothrinacis
L. commarounae
L. eichlormiae
L. guazumae
L. smilacis
L. theobromicola

East Africa

Ecuador
L. piperis

\section{L. bomareae \\ L. consociata \\ L. phyllacharicola \\ L. plantaginicola \\ L. saginata}

Ethiopia

Europe

\section{L. baldratiana}

L. auerswaldii

L. phaeosticta

Finland

L. pamiformis
L. acori
L. acura
L. affinis
L. ammophilae
L. asparagina
L. coccodes
L. culmicola var, nigrans
L. culmifida
L. culmorum
L. dolioloides
L. doliolaides var. cirsii
L. dolioloides var. inop.s
L. doliolum
L. elongata
L. fuckelii forma filamon- iffera

L. herpourichuides

L. immunda

L. Iencoplaca

L. lycopodina

L. muculans

L. maculans var. Mphicola

L. microseropica

L. microscopica subsp. calami

L. modesia

L. nigrans

L. nigricans

L. oligospora

L. orchidearum

L. oreophilaides subsp. scrophulariac

L. perpusilla var. typhae

L. planiuscula

L. praeclara

L. practermissa

L. ptarmicac

L. puncroidera

L. rhadialicola

L. ribis

L. ristica

L. socialis

L. spiracae

L. typhae

L. typharum

L. typharum subsp. phragmatina

L. vagabunda var. dulcarnarae

France

L. acuta

L. acuta var. insignis

L. acuta var. urticae

L. agminalis

L. agminalis forma minor

L. cilauthi

L. allorgei

L. arenaria

L. arundinacca

L. arundinacea var. godimi

L. avellanae

L. baldingerae

L. hambusae

L. herberidis

L. bupleuri

L. caliescens

L. caprifolii

L. carduorum

L. caricicola

L. castagnei

L. chelidonii

L. cinclidoti

L. cisti

L. clarues

1. comiothyium

L. conionhyrium forma borberidis
L. conoidea forma angelicae

L. conoidea forma macraspara

L. controversa

L. comallariae

L. crozalsii

L. cruense

L. cucurbitarioides

L. culmicola

L. culmicrsla forma melicae

L. culmicola var. aquarica

L. culmicola var. nigrans

L. culmicola var. rhizamaturm

L. culmifraga

L. culmifraga forma poae

L. culmifiaga var. linearis

L. culmifraga var. propinqua

L. curta

L. cyons

L. decaisneana

$L$. derasa forma macraspora

L. digitalis

L. disica

L. doliolaides

L. doliolum

L. doliolum var. pachyspora

L. doliolum var. pinquicula

L. dumetorum var. marrubii

L. elacospara

L. emiliana

L. empetri

L. ephedrae

L. ericae

L. ervngii

L. euphorbiaccola

L. customoides forma lolii

L. foeniculacea

L. fuscella forma micraspora

L. galiorum

L. galiorum forma cirsiorum

L. galiorum forma gentiance

L. galionum var. lapsancae

L. genistae var. microsporid

L. gilloriana

L. glanco-punctato

L. grignomnersis

L. haematites

L. hansmamniana var. cherleriae 
L. hedericola

L. helicicola

L. helminthospora forma crithmi-maritimi

L. hippophaes

L. inculia

L. insignis forma airaecespirosate

L. iridicola

L. iridigena

L. isariphora

L. juncorum

L. juniperi

L. kali

L. lathyri

L. lauri

L. lecunora

L. lemoinii

L. libanotis

L. licutensis forma rupefortensis

L. limiosa

L. linearis

L. Ionicerae

L. maculans

L. maculans forma denuduta

L. melanommoides

L. menthae

L. michotii

L. microscopica forma brachypodii

L. microscopica forma glyceriue

L. minima

L. modesia

L. modesta forma dauci

L. modesia forma digitalis-luteae

L. modesia forma jacohacae

L. modesta forma lappae

L. modesta forma succisae

L. modesta forma sylvestris

L. modestu var. rubellula

L. molybdina

L. montis-bardi

L. numelleri

L. muralis

L. nardi

L. nigrans

L. nigrans forma arundinis

L. nigrificans

L. norfolica

L. obesula

L. obiones

L. ohtusispora forma agaves

L. ogilviensis forma lepidii
L. ogilviensis forma megalospora

L. agilviensis forma myrhis-odorata

L. ophiopogonis var. gratnitum

L. oreophiloides

L. pachytheca

L. pampini

L. papyricola

L. parietariae

L. parietariae forma Iamii

L. perforats

L. perpusilla

L. phaseoli

L. phragmiticola

L. picridis

L. pimi

L. pleosporoides

L. polytrichina

L. pratensis

L. priuscheggiana

L. pycnostigma

L. ramalinae

L. rhopalispora

L. richoni

L. rothomagensis

L. roumegueri

L. rubellula

L. Inmicis

L. rinsci

L. Iusci var. hypophylli

L. ruscicola

L. Nuthenica

L. sabanda

L. saccardiana

L. salcbiosa

L. sambuci

L. sapeyensis

L. sammenticia

L. sarothammi

L. sarroziniande

L. scrophulariae

L. sequana

L. smilacis

L. solidaginis

L. stellarioe

L. tencrii

L. therophila

L. thomosiana

L. Iypharum

L. typharum subsp. papyrogena

L. typhiseda forma sodoloci

L. vagabunda forma abietis

L. vagabunda forma canlium

L. vagabunda forma lonicerae

\section{L. vagabunda forma} salicis-capreac

L. vagans forma scirpi

L. vincue

L. viticola

L. vitis

French Equatorial Africa

L. heveae

L. septovariata

L. zeae-maydis

L. zeicola

Germany

L. achilleas

L. acicola

L. acula

L. acutiuscula

L. agnita var. anhigua

L. agnita var. clirysumhemi

L. agnita var. mujor

L. alliariac

L. ammophilac

L. anceps

L. aquilegicue

L. arabidis

L. artemisiae

L. asplenii

L. atropurpurea

L. avenae

L. baggei

L. bardanae

$L$. bellynckii

L. berlesei

L. caespitosa

L. carduorum

L. circinans

L. rlara

L. clivensis

L. coniothrium

L. comecta

L. conticola

L. culmornm var. paleicola

L. cummlata

L. dematiicola

L. densa

L. dolioloides

L. doliolum

L. dumetorum forma meliloti

L. duplex

L. endiusas

L. epicalamia

L. ettalensis:

L. fibrincola

L. fimiseda

L. frondis

L. fuckelii

L. fuscella var. sydomiana

L. galii-silvatici

L. geographicola

L. graminis 
L. graminum

L. haemarires

L. harslinskyana

L. helianthemi

L. helminthospora

L. hippophaes

L. hirta

L. huthiana

L. hyperici

L. impressa

L. incruenta

L. isariphora

L. juncaginearum

L. juncina

L. koerberi

L. kunzeana

L. lacustris

L. lemaneae

L. leptogiophila

L. linritata

L. littoralis forma calamagrostidisarenariae

L. lolii

L. Ionicerae

L. lonicerina

L. maculans

L. malojensis

L. medicaginis

L. medicaginum

L. michorii

L. microscopica

L. microscopica var. caricis-vulpinae

L. mulriseptata

L. nardi

L. miessleana var. staritzii

L. nigrans

L. nigrella

L. migromaculara

L. nitschkei

L. ogilviensis

L. ogilviensis forma achilleac

L. ogilviensis var. plewrospermi

L. asculanda

L. papillata

L. penicillus

L. petkovicensis

L. pilulariae

L. plamiuscula

L. ponriformis

L. primularia

L. Dsilospora

L. mupurearum

L. alinina

L. ramsangiensis

L. recurita

L. rostrupii
L. rousseliana

L. salebricola

L. salebrosa

L. sanguisorbae

L. saxomica

L. scilula

L. seminuda

L. setulosa

L. spliyridiana

L. staritzii

L. steinii

L. striata

L. submaculans

L. surculorum

L. sydowiana

L. tanaceti

L. typhae

L. typharum

L. vincae

L. winteri

Ghana

L. sparganii

Great Britain

L. abbreviata

L. acorella

L. acuta

L. anaritlma

L. arundinacea

L. baeomycearia

L. caninac

L. cardworum

L. cesatiana

L. clara

L. clarkii

L. clivensis

L. complanata

L. cookei

L. dennisiana

L. derasa

L. dioica

L. doliolum

L. duplex

L. echinella

L. epicarecta

L. fluviatilis

L. fuscella

L. glauco-punctata

L. glocospora

L. graminis

L. hederae

L. juncina

L. leucomelaria

L. lumariae

L. macrosporidium

L. maculans

L. maritims

L. maram

L. michorii

L. mosama

L. nardi
L. neomaririma

L. neortizans

L. niessleana forma viciae

L. nigrans

L. norfolcia

L. obiones var. evolutior

L. octophragmia var. major

L. ogilivensis

L. parmeliarum

L. pelagica

L. perpusilla

L. petkovicensis

L. phormii

L. pontiformis

L. raphani

L. rubelloides

L. rusci

L. sabulerorum

L. sepincola

L. tamaricis

L. Iriglochinicola

L. tritorulosa

L. Iypharum forma acori

L. uliginosa

L. umbilicariae

L. vectis

Greece

L. ballorae

L. politis

L. sibtorpii

Greenland

L. algida

L. brachyasca

L. hyperborca

L. macroiheca

L. oxvriae

L. polaris

L. ranunculi

L. stellariae

L. vahlii

L. vanhoeffeniana

Guadeloupe

L. hurae

L. Jicii

Himalayas

Hungary

L. rlododendri

L. alliariae

L. ancmones

L. antherici

L. aquilegiae

L. arhhenatheri

L. azaleae

L. berlesei

L. bcrulina

L. compressa

L. consuricia

L. coronillare

L. corricola

L. culmifroga var. hromicola 
L. culmorum forma hungarica

L. cynoglossi

L. cyperi

L. Cypericola

L. demarium

L. dianthi

L. doliolum forma syndoliola

L. dumetorum var. coronillae

L. echinops

L. equisericola

L. fiumana

L. geasteris

L. gypsophilae

L. hollosii

L. iridis

L. irrepla

L. juniperina

L. lineolaris

L. maritima

L. meliloti

L. muscuri

L. onobrychidicola

L. onobrychidis

L. poae var. agrostidis

L. pontiformis

L. purpurea

L. raphidophora

L. mbicunda

L. salsolae

L. sinapis

L. stromatoidea

L. superficialis

L. tamaricis

L. ternata

L. thalictricola

L. Ihalictrina

Iceland

L. dryadis

L. elymi

L. Larseniana

L. oligotheca

India

L. papaveris

L. abuensis

L. agaves

L. aquatica

L. capparidicola

L. coorgica

L. cosmicola

L. eriobotryue

L. helianthi

L. hollosiana

L. hyalina

L. indica

L. isocellula

L. Iobeliae

L. muehlenbeckiae
L. migrans

L. ocimicola

L. petkovicenses var. elymi

L. phoenicis

L. porellae

L. puniciae

L. punjabensis

L. rajasthanensis

L. swertiae

L. zingiberis

Iran

L. cycadis

L. kotschyana

L. shahvarica

lreland

L. tolgorensis

Israel

L. advenula

Italy

$$
\text { L. pimpinellae }
$$

L. aconiri

L. aculeorum

L. acuta

L. adienula

L. aegira

L. aethensis

L. aglaja

L. agminalis

L. agnita

L. alcides

L. alcides forma quercina

L. ampelina

L. anphibola

L. anarithma

L. anarithmoides

L. anthophila

L. anthostomoides

L. apocalypta

L. apogon

L. aquilana

L. arnoldii

L. arrhenatheri var. italica

L. arundinacea

L. arvensis

L. asparagi

L. bella

$L$. bellynckii

L. bornmmelleri

L. borziana

L. brachypodii

L. brachysperma

L. bractearum

L. briosiana

L. bryophila

L. brizae

L. cadubriae

L. comilla

L. camphorata

L. campisilii

L. canadensis
L. cannabina

L. capparidis

L. capsularum

L. carduina

L. carpogena

L. carpophila

L. carpophila var. bractearum

L. callanei

L. calarae

L. cerlettii

L. cesatiana

L. chaetostoma

L. cibostii

L. cirricola

L. coniothyrium

L. coniothyrium forma berberidis

L. conoidea

L. corticola

L. corymispora

L. crastophila

L. crastophila forma tofieldiae

L. crepini

L. cucurbilae

L. culmicola

L. culmicola forma major

L. culmicola var. minor

L. culmifiaga

L. culmorum

L. cumana

L. cyperina

L. dactylina

L. dasylirii

L. derasa var. alpestris

L. diana

L. dichroa

L. disseminata

L. doliolum

L. dryadea

L. epilobii

L. eustoma

L. customella

L. eustomoides

L. faginea

L. fallaciosa

L. fallax

L. fiedlaeri

L. fuscidula

L. galiicola

L. galiicola var. brachyspora

L. geographicola

L. gibelliana

L. glanco-punctata

L. grammodes

L. grandispora

L. grisea 
L. Lausmanniano

L. hederae

L. helverica

L. helvetica forma major

L. hendersoniae

L. hermodactyli

L. herpotrichoides

L. heterospora

L. heufleri

L. hiemalis

L. hippophaes

L. hyalospora

L. Iydrophila

L. involucralis

L. ischaeni

L. juncicola

L. kochiana

L. lamprocarpi

L. lathonia

L. lathonia var. helleborifoetidi

L. lathyri

L. lathyrina

L. leersiae

L. leersiana

L. leptospora

L. licatensis

L. lichenicola

L. lithoralis

L. littoralis forma calamagrostidisarenariae

L. livida

L. licilla

L. lucina

L. lucinosa

L. luzulae

L. maculans

L. magnusiano

L. major

L. mamillula

L. marginalis

L. marginata

L. massariella

L. massariella var. disticha

L. massarioides

L. medicaginis

L. medicaginum

L. melicae

L. meridionalis

L. michotii

L. micropogon

L. microscopica

L. microscopica var. alpina

L. mirandae

L. modesta var. cibostii

L. molluginis

L. monotis

L. momtano

L. mulriseptana forma alpina
L. nectrioides

L. neglecta

L. nigrans

L. nobilis

L. notarisii

L. ogilviensis

L. ogilviensis var. senecionis-cordati

L. ophiopoganis

L. oryzae

L. pampini

L. pandari

L. paoluccii

L. parietariae

L. parvula var. iridisgermanicae

L. passeriniana

L. passerinii

L. patellaeformis

L. peltigerarum

L. penicillus

L. perforans

L. perpusilla

L. petiolicola

L. phytolaccae

L. pinnarum

L. pinharim var. rachidis

L. platycarpa

L. pomona

L. poterii

L. puccinioides

L. punctiformis

L. pycnosigma var. morbosa

L. pyrenopezizoides

L. ranunculoides

L. recessa

L. rehmii

L. resedae

L. rhizomatum

L. rhododendri

L. rhodophoea

L. rivand

L. rivema var solorinae

L. risci

L. musci forma canlina

L. rusci forma rusciliypoglossi

L. sacculus

L. salicaria

L. salicincartm

L. solviae

L. salvinii

L. sambuci

L. scirpina

L. scotopliila

L. seriala

L. sicula

L. silenes-acuulis

L. sileris

L. socia
L. sodomaea

L. spartii

L. spatharum

L. stereacaulorum

L. siriclata

L. subarticulata

L. subrecta

L. suffulta

L. sylvatica

L. Thalictri

L. torbolensis

L. Irichostoma

L. rrimera

L. tritici

L. Sypharum

L. typhicola

L. ulmicola

L. ragabunda

L. Vagabunda fomma critrilimorii

L. vagabunda var. divergens

L. vagabunda var. sarmenti

L. vaginae

L. valdobbiae

L. vincae

L. vinealis

L. xiphii

L. nylogena

L. Iulan

Ivory Coast

L. coffeigena var. longirostrata

L. perri

L. sorgho-arundinacei Jamaica

L. peligerea

Japan

L. akagiensis

L. apios

L. apios-formunei

L. asperellae

L. bambusae

L. buddlejae

L. cimusmoni

L. hothai

L. inecola

L. japonica

L. lelebae

L. Iilicola

L. minoensis

L. numme

L. nandinate

L. nashi

L. orycicola

L. phyllosfachidis

L. sasacola

L. sasue

L. Mujaecola

L. ligrisoides

L. zizammitora 
Java

Kenya
L. sacchari
L. trochus

\section{L. hicolor \\ L. nodorum \\ L. Iritici}

Kerguelen Island

Korea

$$
\text { L. kerguelensis }
$$

Libya

$$
\text { L. inamotoi }
$$

L. pampaniniana

Luxembourg

L. caespitosa forma salviae

L. cerastii

L. culmorum var. flavobrunnea

L. dolioloides var. lathyri

L. dolioloides var. rhinanthi

L. dumetorum var. coniformis

L. dumetorum var. dolichospora

L. dumetorum var. symphyti

L. echiella

L. echii

L. epicalamia var. pleosporoides

L. eluphorbicie forma esulae

L. fuscella var. hippophaes

L. galeobdolonis

L. hemerocallidis

L. iridigena var. typhae

L. junci

L. longispora

L. luxemburgensis var. dolichospora

L. microrhyrioides

L. monilispora forma triglochinis

L. oxyspora

L. paludosa

L. petiolaris

L. phyteumaris forma knautiac

L. planiuscula forma succisae

L. plectrospora

L. proliferac

L. rivalis

L. silvestris

L. sparsa var. meizospora

L. trematostoma

L. trifolii

L. vikalbae var. sarmenticola
L. w'egeliniana forma teucrii

Madeira Archipelago

L. maderensis

Malaysia

L. scabicns

Mauritania

Morocco

L. rompkinsii

L. elaoudi

L. gratissima

L. gratissima var. longispora

L. maheui

Netherlands

L. ammophilae

L. cariciphila

L. desciscens

L. donacina

L. galiicola

L. genistae

L. hemicrypia

L. junci

L. periclymeni

L. phlogis

L. pseudo-diaporthe

L. rhopographoides

L. rousseliana

L. strationis

New Caledonia

L. australis

New Zealand

L. martinianum

L. reidiana

Nigeria

L. typharum

L. elaeidis

Norway

L. andromedae

L. caricinella

L. consobrina

L. dryadis

L. dumetorum var. galiiborealis

L. hyperborea

L. insignis

L. juncisedo

L. quadriseptata

L. rostrupii

L. sepalorum

L. vagans

Pakistan

L. abutilonis

L. ahmadii

L. depressa

L. euphorbiicolla

L. punjabensis

L. rumicicola

L. rumicis

Philippines

L. ambiens

L. amphilogo
L. chusqueae

L. dryadea subsp. liessoniensis

L. erythrinae

L. marantae

L. oryzina

L. panici

L. simillima

L. Imngurahuensis Poland

L. alliariae

L. caricina

L. cerei-permiani

L. coniformis

L. crustacea

L. lycopodina

L. norvegica

L. opizii

L. sowerbyi

L. Irifolii-alpestris

L. Iriglochinis

L. Nophiseda

Portugal

L. algarbiensis

L. almeidae

L. almeidana

L. aloes

L. anacycli

L. anarrhini

L. arbuti

L. arecae

L. huxina

L. cisticola

L. cocoes

L. congesia

L. conimbricensis

L. coniothyrium

L. convallariac forma dracaenae

L. convallariae forma rusci

L. daphmes

L. demissa

L. diaporthoides

L. dolioloides

L. dracacnac

L. foeniculi

L. holmii

L. infernalis

L. junci-acuti

L. juncina

L. lavandulae

L. Iusitanica

L. maculans

L. michorii

L. nolleriana

L. nervisequa

L. papillosa

L. rusci

L. misci var. fourcroyac

L. schocuocauli

L. scolecosporarum 
Puerto Rico

L. thuemeniana

L. correndii

L. Manslescens

L. v'agabunda forma daphnes

Romania

L. portoricensis

L. acurispora

L. alerandrinis

L. derasa var. franconica

L. dobrogica

L. doliolum forma carlinac-vulgaris

L. doliolum var. dissimilis

L. doliolum var. leonuri

L. fuckelii

L. fusispora var, erysimi

L. glyceriac-plicatae

L. hazslinszkii

L. pomona

L. ranunculi-polyanthemi

L. solviae forma minor

L. woroninii

São Tomé

L. fungicola

L. musurtum

Scandinavia

Senegal

L. dolioloides

L. inarensis

L. senegulensis

Sierra Leone

L. penniseticola

Solomon Islands

L. honiaraensis

South Africil

L. africana

L. caffica

L. cervispora

L. collumiae

L. owanias

L. protearmm

L. perocelastri

L. verinocediana

Spain

L. agnita var. mifolii

L. cabulleroi

L. carlinoides

L. catalaninica

L. cavanillesii

L. cebullosi

L. cirsii-arlensis

L. cistina

L. colocasiae

L. culmicola varr hispalensis

L. cyousumi

L. dolioloridess

L. dracomis

L. funscidlula forma mergnolii
L. glyceriae

L. grassulariue

L. hispanica

L. junci-glauci

L. marlagoni

L. matritensis

L. octophragmia

L. riofrioi

L. riparia

L. ruscicola forma cladodiicola

L. sabuida forma anaticae

L. semslina

L. staticicola

L. striolara

L. variabilis

L. vitensis

Sri Lanka
L. depressa
L. lankeana
L. nesades
L. smilacis
L. rornatospora

St. Thomas Island

Sweden

L. musarum

L. associata

L. bellynckii

L. caricis

L. cladophila

L. compressa

L. culmifraga

L. culmifraga forma minuscula

L. culmorim

L. doliolum

L. doliolum var. subdisticha

L. duplex

L. fuckelii

L. hendersoniae

L. hirta

L. jaceae

L. Iasiosphaerioides

L. mucrotheca

L. microscopica

L. nardi

L. migranss

L. piccestre

L. sepincola

L. solani

L. solanicola

L. ragahmola subsp. alvarensis

Switzerland
L. agnitu var, major
1.. cillondac
L. blumeri
L. branni
L. caricicola

L. caricis

L. centawreae

L. cladii

L. composirarum

L. cornuta

L. carticola

L. crucheli

L. culmorum

L. didymellae-vinceroxici

L. epicalamia

L. epilohii

L. elestoma

L. franconica

L. fuckelii

L. galii

L. galiorum var. gentianae

L. hippophaes

L. johansonii

L. juncicala

L. lacustris

L. ladina

L. lihanatis

L. linearis

L. lycopodina

L. maculans

L. morthieriana

L. nanae

L. napelli

L. nardi

L. nigrans

L. nodorum

L. ocellara

L. ogilviensis

L. ophioboloides

L. palustris

L. pariula

L. perkoricensis

L. phacae

L. phaeospora

L. phyteumatis

L. pini

L. planiuscula

L. plarvethorae

L. pleurospara

L. polygonari

L. primulae'cola

L. recurita

L. rimalis

L. rimicola

L. rolussid

L. rousselianu

L. rumicis

L. septemic ellulata

L. sowerbit

L. spersas

L. sulmodessa

L. syluatica

L. Puminensis

L. fenuis

L. thurgoniensis 
Taiwan
L. lofieldiae
L. triglochinicola
L. trollii
L. typhicola
L. valesiaca
L. viciae
L. vitalhae
L. volkartiana
L. wegeliniana

L. coicis

L. kuangfuensis

L. musae

L. musigena

L. pandanicola

L. laichungensis

L. taiwanensis

Tanzania

Tunisia
L. elaeidicola

L. macrochloae

L. rothomagensis var. artemisiae

L. stipae-minor

Turkey

L. darisiana

L. dodonaeae

L. melicae

L. sylvatica

U.S.A.

L. acuta

L. agnita subsp. labens

L. agnita var. erigerontis

L. anomala

L. anthelmintica

L. arctalaskana

L. arthrophyma

L. artunci

L. asclepiadis

L. asparagi

L. associata

L. astericola

L. asteris

L. aulica

L. avenaria

L. avicenniae

L. beaumonii

L. berlesei

L. bicuspidata

L. bocconiae

L. borealis

L. horealis var. populi

L. boucera

L. brightonensis

L. byssincala

L. cacuminispora

L. californica

L. caricis

L. cassiaecola

L. castilleiae

L. castillejae
L. castrensis

L. caulincola

L. ceanathi

L. ceratispora

L. cercocarpi

L. chrysanthemi

L. clavicarpa

L. clavigera

L. clavispora

L. chivensis var. constricta

L. coleosanthi

L. collinsoniae

L. comatella

L. complanata

L. compressa

L. concentrica

L. concinna

L. consessa

L. consimilis

L. corallorhizae

L. culmorum

L. darkeri

L. defodiens

L. discors

L. dissiliens

L. distributa

L. doliolum

L. drechsleri

L. dryophila

L.ellisiana

L. elongata

L. elymi

L. erigerontis

L. eriophora

L. eumorpha

L. eustoma

L. eustoma forma leguminosa

L. eutypoides

L. exocarpogena

L. faulii

L. filamentosa

L. filiformis

L. fimbriata

L. foeniculacea subsp. lupina

L. folliculata var oxyspora

L. fraserae

L. fraxini

L. fuckelii

L. fulgida

L. galiorum var. gnaphaliana

L. galligena

L. georgins-fischeri

L. halima

L. hamamelidis

L. harknessianna

L. helianthi

L. heliopsidis

L. heloniaefolia
L. hendersoniae

L. hesperia

L. hiemalis

L. houseana

L. hysterioides

L. incarcerata

L. inquinans

L. inspersa

L. interspersa

L. janus

L. kalmiae

L. korrae

L. larseniana

L. lasioderma

L. lassenensis

L. latebrosa

L. leiostega

L. Iethalis

L. leucoplaca

L. Iongipedicellara

L. Iophanthi

L. luctuosa

L. lupini

L. Lupinicala

L. lyciophila

L. lycopodiicola

L. lycopodina

L. lyndonillae

L. lythri

L. machurae

L. major

L. marcyensis

L. marina

L. maydis

L. mellispora

L. mertensiae

L. mesoedema

L. modestula

L. monticola

L. muehlentsergiae

L. muricata

L. muirensis

L. muricae

L. neomaritima

L. nigrans

L. nigricans var. grindeliae

L. occidemalis

L. octoseptata

L. odora

L. ogilviensis

L. olericola

L. olivacea

L. olivaespora

L. onagrae

L. opuntiae

L. orae-maris

L. orthogramma

L. pacifica

L. papyricola

L. perplexa

L. phaseolorum 
L. phommicola

L. physalidis

L. pliysostegiae

L. platanicola

L. platypus

L. plurisepla

L. pseudohleria

L. putcana

L. quamoclidii

L. ramulicola

L. rhoina

L. rostrata

L. rubrotincta

L. sabalicola

L. sabaligera

L. sambucina

L. scapophila

L. shastensis

L. sicversiac

L. simmonsii

L. solheimii

L. sorgho-arundinacei

L. sorghophila

L. spartinac

L. sporoboli

L. syuamata

L. stercicola

L. sticta

L. stictoides

L. stictostoma

L. straminis

L. subcacspitosa

L. subcompressa

L. subconica

L. subcutanea

L. sublanosa

L. substerilis

L. taxicola

L. tencra

L. epplosiae

L. tetonensis

L. tini

L. orulispora

L. trimeriosides

L. mitici var. papyricola

L. Iumcfacicns

L. IYphartm

L. Utahensis

L. varicgata

L. variiscptata

L. veratri

L. virginica

L. viridella

L. wehmeveri

L. reropliglli

L. zeae

L. zizamiaccola

U.S.S.R.

L. abutilonis

L. aceris

1. acluropodis
L. agnita var. hupleuri

L. alhagii

L. alopecuri

L. animophile

L. ammothamni

L. amorphae

L. atraphaxidis

L. atriplicis

L. balcarica

$L$. bataticola

L. batumensis

L. betulina

L. biebersteinii

L. buddlejae

L. calligoni

L. camelliac-japonicac

L. camphorosmae

L. caricis-vulpinae

L. casta

L. cephalariai-wralensis

L. cerei-perwiani

L. chanaeropis

L. chenopodii-albi

L. chochrjakovii

L. clerodendri

$L$. coniothyrium var. foliicola

L. consocians

L. culmorum

L. culmorum forma epigeii

L. culmorum forma phragmitis

L. culmorum var. humgarica

L. daphniphylli

L. davidii

L. dianthi

L. doliolum var. cacaliae

L. drabac

L. dubia

L. equiseti

L. feijoae

L. ferruginea

L. ferulicola

L. foliicola

L. frigida

L. ginkgo

L. glandulosac

L. gossypii

L. halo.17li

L. hicrochloae

L. holmii

L. hordei

L. hvelrangeae

L. hypericola

L. lespedesac

L. lithophilac

L. loniccrac

L. magnolias

L. media
L. medicaginicala

L. metasequaiae

L. moutan

L. myrit

L. myrticola

L. ornithogali

L. periclymeni var latarica

L. pleurospora

L. polini

L. prani

L. pruni var. plurivora

L. sanguisorbae

L. scutati

L. secalina

L. sibirica

L. smarodsii

L. sophorac

L. sorbi

L. stipae

L. Ianaceii

L. Raurica

L. Iumefaciens

L. rypharum

L. usneae

L. irieseae

L. neberi

L. woodraw-wi/sonii Uganda

L. hypartheniae

L. penniseti

L. trichopteryis

Venezuela

L. cryptica

L. espeleriae

L. jahnii

L. orthrosanthi

Yugoslavia

L. alraica

L. andrijevicensis

L. bresadolaeana

L. corrugans

L. fuckelii

L. galiorum subsp. anirrhini

L. hesperidicola

L. insulana

L. malyi

L. niculai

L. pachyosca

L. pachytheca

L. petkevicensis

L. plemsliana

L. rehmiana

L. scluronica

L. serbica

L. subalpina

L. subsimilis

Zaire

L. camephorac

L. crnodomis-dactidi 


\section{Appendix 1. Taxonomic Division of Leptosphaeria}

\section{P.A. SACCARDO (1883, 1891)}

Parasites on dicotyledons.

Stem/branch inhabiting.

Leaf inhabiting.

Fruit and flower inhabiting.

Parasites on monocotyledons.

Parasites on acotyledons.

Species of unknown or doubtful spore color.

\section{F. v. HÖHNEL (1918c)}

Scleropleella F. v. Höhnel. Species with typical pseudosphaerialean centra.

Leptosphaeria sensu F. v. Höhnel. Species with typical dothidealean centra.

Nodulosphaeria G.L. Rabenhorst. Species with typical sphaerialean centra.

\section{E. MÜLLER (1950)}

Scleropleella. This section, with few exceptions, corresponds to Höhnel's (1918a) generic concept. Generally it includes smaller forms with relatively few egg-shaped or broadly club-shaped asci that are embedded in a more or less well-developed cellular tissue. The ostiole usually is poorly developed.

Eu-Leptosphaeria. This section contains the largest number of species of the four sections. The inner structure corresponds to a higher (more evolved) stage than Scleropleella; the asci are more numerous and more slender: the paraphysoids are clearly thread-like, although in some forms a cellular arrangement is still noticeable; the ostiole is conspicuous, and early in development is filled with hyaline cells that only litter give way to the pore. Spores are thinner than in Massariosphaeria. and are often fusiform, and they have a mucilaginous coat.

Massariosphaeria. This section is composed of forms whose spores (approaching the Wettsteinina type) are relatively broad. possess a distinct mucilaginous coat when young, and reach maturity relatively late (at least in part), sometimes only outside the asci. Structure of the fruiting hodies corre- sponds to that of section En-Leptosphaeria. These forms may be regarded as transitional to Massaria.

Nodulosphaeria. This section is composed of the most highly evolved forms, designated by $\mathrm{F}$. v. Höhnel as "sphaerial." They are distinguished from all others by the ostiole, which is coated with thread-like, periphyses-like hyphae that usually diverge toward the middle of the mouth-channel and toward the "scheitel." In the region of the "scheitel," these periphyses are often replaced by brown bristles. Peridia of fruiting bodies usually consist of several layers of elongate, sometimes almost rectangular cells. The numerous, usually cylindrical-clavate asci are surrounded by thread-like paraphysoids.

\section{A. MUNK (1957)}

Eu-Leptosphacria. Pseudothecia sclerotioid, thickest at the sides. Asci slender, numerous. Ostiole. generally without a periphysoid structure.

Para-Leptosphaeria. Pseudothecia middle-sized, with a uniform, thin peridium; generally no periphysoid structure in the papilla. Interascicular tissue paraphysoid. This section contains a large and heterogenous group of species.

Scleropleclla. Pseudothecia small; interascicular tissue obsolete.

Nodulosphacria. Pseudothecia with a complicated structure of the papilla. It is covered with brown. spiny hairs that extend into the ostiole. Periphysoid tissue is distinct.

\section{HOLM ( 1957$)$}

Leptosphacria sensu L. Holm. Species similar to the type of the genus Leptosphaeria doliolim: they occur principally on dicotyledons.

Nodulosphacria G.L. Rabenhorst. Species similar to the Nodulosphacria of Müller; they occur on dicotyledons, especially on Compositate.

Phacosphacria 1. Miyakc. Species that correspond essentially to the Scleroplcella section of Müller (1950) and Munk (1957) and that occur on monocotyledons. 
Entodesmium H. Riess. Species with elongated ascospores; they intergrade with Ophiobolus and occur on legumes.

\section{R.A. SHOEMAKER (1984)}

Leptosphaeria. Circumscribed Leptosphaeria in a hroad concept of Holm and Müller: however, many segregate genera were accepted. The genus always lacks erect setac on ascomata. The walls of the ascocarps often have scleroplectenchyma at least near the beak base. The physes are broad, septate with or without guttules and cytoplasmic accumulations and frequently with an external gelatinous coating. Asci are biseriate or, rarely, uniseriate. Ascospores are fusiform, cylindric or clavate, 3 -septate or more, and the first formed septum is constricted and near the middle. These ascospores frequently have globose. terminal appendages that are rarely entirely sheathed. No woody parts of dicotyledonous plants are the usual susbstrates.

M.E. BARR (1987a, 1987b)

Leptosphaeriaceae. Established the family Leptosphaeriaceae based on Leptosphaeria. but also included Curreya. Didymolepia. Heptameria, and Ophioholus. The family was segregated from the Pleosporaceae because of the coelomycetous rather than the hyphomycetous anamorphs and because of the narrow, thin-walled asci. It differs from the Phaeosphaeriaceae in having conoid and applanate or obpyriform or sphaeroid ascomata, whose walls are scleroplecienchymatic. 


\section{Appendix 2. Genera Historically Allied to Leptosphaeria}

Bricookea M.E. Barr, Mycotaxon. An International Journal Designed to Expedite Publication of Research on Taxonomy \& Nomenclature of Fungi \& Lichens, Ithaca, New York 15:346. 1982. Type species: Bricookea sepalorium (J.S. Vleugel) M.E. Barr. Family:

Phaeosphaeriaceae. Ascomata: Clustered, immersed, subepidernal, separate or as locules in crustose stromatic tissues, globose, radiate arrangement of cells of peridium. Asci: Bitunicate, oblong, short-stalked, numerous, basal. Ascospores: Fusiform to narrowly obovoid, 3 -septate, hyaline.

Anamorph: Unknown. Remarks: Barr (1982) erected this genus for a species of Leptosphaeria described from inflorescences of Juncus and considers it to be related to Phaeosphaeria.

Chaetomastia (P.A. Saccardo) A.N. Berlese, Icones Fungorum Omnium Hucusque Cognitorum ad usum Sylloges Saccardianae Adcommodatae 1:38. 1891. Lectotype species: Chaetomastia hirtula (P.A. Karsten) A.N. Berlese. Family: Dacampiaceae. Ascomata: lmmersed-subepidermal, becoming erumpent, scattered or clustered, obpyriform or ovoid to globose; apex wide, blunt, ostiole rounded or slit-like; wall pseudoparenchymatous, cells externally darkened and thickened in upper regions. Asci: Bitunicate, clavate or cylindric, 4-, 6-, or 8-spored. Ascospores: Biseriate or uniseriate; obovoid elongate, 3 to 11 -septate; cell above first septum enlarged; wall wide, dark, smooth or verruculose; yellowish brown becoming dark brown or reddish brown. Anamorph: Coelomycetous where known; conidia hyaline or brown, I- to 2-celled (Aposphaeria-like or Coniothyrium-like). Remarks: This genus is distinguished by the obpyriform or obovoid ascomata with a wide apical papilla, peridium of small dark cells that is widest and 3-layered in the upper region, and by dark asymmetric phragmospores (Barr 1989).

Chactoplea (P.A. Saccardo) F.E. Clements, in F.E. Clements and C.L. Shear, The Genera of Fungi, pp. 74, 275. 1931. ĐPyrenophora subgen. Chaeroplea P.A. Saccardo, Sylloge
Fungorum Omnium Hucusque Cognitorum Digessit P.A. Saccardo 2:279. 1883. Type species: Chaetoplea calvescens (E.M. Fries ex J. Desmazières) F.E. Clements \& C.I. Shear. ESphaeria calvescens E.M. Fries, Scleromyceti Sueciae. Collegit, Digessit et Evulgaviti, No. 401. Unpublished? $\equiv P y-$ renophora calvescens (E.M. Fries ex J. Desmazières) P.A. Saccardo, Sylloge Fungorum Omnium Hucusque Cognitorum Digessit P.A. Saccardo 2:279. 1883. Family: Phaeosphaeriaceae. Ascomata: Seated on a subiculum, globose or depressed globose, dark brown to black, subepidermal becoming superficial, wall composed of polygonal cells, surrounded by stiff dark hyphae. Asci: Bitunicate, numerous, cylindrical, shortstalked with thickened apical walls, 8 -spored. Ascospores: Fusoid-ellipsoid, straight or inequilateral with three transverse septa and with longitudinal septa, yellowish brown or dark brown, smooth or finely verruculose. Remarks: This genus has only recently been accepted by Barr (1981, 1987b), who placed it in the Phaeosphaeriaceae. The type species, Chaetoplea calvescens, has been placed in Pleospora by Webster and Lucas (1959), Wehmeyer (1961), and Shoemaker (1968). Crivelli (1983) transferred Chaetoplea calvescens to Leprosphaeria, and Eriksson and Hawksworth (1986) synonymized Chactoplea with Leposphaeria.

Curreya P.A. Saccardo, Sylloge Fungorum Omnium Hucusque Cognitorum Digessit P.A. Saccardo 2:651. 1883. Type species: Curreya conorum (L. Fuckel) P.A. Saccardo. Family: Leptosphaeriaceae. Ascomata: Scattered, immersed-subepidermal, depressed globose; wall scleroplectenchymatic, melanized black, surface of textura angularis; beak absent. Asci: Many, bitunicate, cylindricil-clavate, thick-walled in apical region, short-stalked, containing eiglut ascospores. Ascospores: Obovarc. constricted at septa, slightly asymmetrical, with onc or more cnlarged cells, 7 -scptilte or more, with longitudinal septa, most cells smooth-walled, surrounded by a gel layer. 
Remarks: The genus is included in the Leptosphaeriaceae by Barr (1987b) and differs from Leptosphaeria in having longitudinally septate ascospores.

Didymolepta A. Munk, Dansk Botanisk Arkiv, Kjobenhavn 15(2):110. 1953. Type species: Didymolepta winteriana (P.A. Saccardo) A. Munk. Family: Leptosphaeriaceae. Ascomata: Scattered, conic, glabrous, black; wall of scleroplectenchymatic cells melanized black; beak ahsent. Asci: Bitunicate, subcylindric, sessile, thick-walled, with eight ascospores. Ascospores: 1-septate, hyaline. Anamorph: Unknown. Remarks: This genus has been placed in the Leptosphaeriaceae by Barr (1987b), and it differs from other genera in the family by having 1-septate ascospores.

Entodesmium H. Riess. Hedwigia, Dresden 1:28. 1854. Type species: Entodesmium rude $\mathrm{H}$. Riess. Family: Phaeosphaeriaceae? Ascomata: Scattered or clustered, more or less lageniform, slightly hairy; beaks very long. Asci: Many, bitunicate, cylindric to narrowly clavate, shor-stalked. Ascospores: Cylindrical, 4-septate or more, with a clearly delimited apical portion and bipolar appendages. Anamorph: Unknown. Remarks: This genus was recognized by Holm (1957) for a small group of Leptosphaeria species found on Leguminosac.

Grophyllum F.E. Clements, Studies in the Vegetation of the State. Nebraska University. Botanical Survey. Report on Recent Collections, Lincoln 5:6. 1901. Type species: Graphyllium chloes F.E. Clements. Family: Phaeosphaeriaceae. Ascomata: Flattened-globose. finally collapsingpezizoid, with more or less dark brown. radiating liyphal tomentum about the base. Asci: Bitunicate, stout-clavate, thick-walled. Ascospores: Strongly llattened in one plane. fusoid-ellipsoid to clavate-ellipsoid, with a single vertical septum running through the central cells but not through the end cells in lace view, yellow-brown to dark red-brown. Anamorph: Unknown. Remarks: Graphyllitum is the earliest name for species formally placed in Platyspora L.E. Wehmeyer and Comoclathris F.E. Clements (Bart 1987b).

Heprameria H. Rehm \& F. v. Thümen, Instituto. Revista Scientilica e Litteriria, Coinhra, Series 2, 27:252. 1879. Type species: Ueptameria elegams H. Rehm \& F. v. Thümen. Family: Leptosphaeriaceac. Ascomata: Clustered, immersed-subepidermal, becoming erumpent, subglobose to conical, rough-surfaced, black. short papillate. Asci: Bitunicate. clavate. shortstalked, with eight ascospores. Ascospores: Fusiform. 7-septate or more, with dark central portion having longitudinal septa. brown. Anamorph: Pycnidial with hyaline, oblong. 1-celled conidia. Remarks: This genus was included in the Leptosphaeriaceae by Barr (1987b), and it differs from Lepiosphaeria by having ascospores with longitudinal septa in the enlarged central cells.

Jaczewski ( 1894 ) considered that Heplameria could be interpreted as a section of Leptosphaeria. but the study of Lucas and Sutton (1971) indicates that the genus is distinct.

Herporrichia L. Fuckel, Fungi Rhenani Exsiccari A Leopoldo Fuckel Collecri, Fascicle 22. No. 2771. Anno 1868. Type species: Herporrichia rubi L. Fuckel. Family: Lophiostomataceae. Ascomata: Globose to conic, immersed, becoming erumpent. or superficial on a subiculum, tomentose. Asci: Bitunicate. cylindrical to clavate. Ascospores: Fusiform to ellipsoidal, 1 - to 3 -septate, hyaline to dull or dark brown, usually with gel coating elongated beyond spore apices. Anamorph: Coclomycetous, Pyrenochacta, or Phomalike. Remarks: Differs from Lepiosphaeria in the tomentose ascocarps on a subiculum and ascospores that can be 1 -septate.

Kalmusia G. Niessl v. Mayendorf. Verhandlungen des Naturforschenden Vereins in Brünn 10:204. 1872. Type species: Kalmusia chuli G. Niessl v. Mayendorf. Family:

Phatesphaeriaceae. Ascomata: Subglobose. immersed in an effuse stroma. Asci:

Bitunicate, clavate. long stipitate. Ascospores: Oblong, curved. 3-septate. brown. Anamorph: Unknown. Remarks: This genus differs from Leptosphacria in having a stroma and long. stipitate asci.

Keisslericlla F. v. Höhnel. Sitzungsberichte der Akademie der Wissenschaften in Wien. Mathematisch-naturwissenschaftliche Klasse. Abt. 1. 128:582. 1919. Type species: Keissleriella aesculi (F. v. Höhnel) F. v. Höhnel. Family: Melannomataceae. Anamorphs: Ascochyra M.A. Libert and Dendrophomu P.A. Saccardo. Remarks: Holm (1957) accepted the genus Trichomerasphaeria A. Munk but stated that there may be earlier generic names for those Ascomycetes with setose pseudothecia placed in the Massarinaceae by Munk (1956). for example. Keissleriella F. V. Höhnel. This later genus differed in having I-septate ascospores is. several septate in Trichometasphaeria. Bose (1961) united the two genera under the earlier name Keissleriella after observing variation in ascospore septation in Reissleriella aesculi.

Lidophid J. Walker \& B.C. Sutton. Transactions of the British Mycological Society. London 62:232. 1974. Type species: Lidophia 
graminis (P.A. Saccardo) J. Walker \& B.C. Sutton. =Dilophia P.A. Saccardo, 1883, non Dilophia T. Thomas, 1953 (Cruciferae). Family: Leptosphaeriaceae. Ascomata: Embedded in a stroma, spherical. Asci: Bitunicate, cylindrical, apically thickened. Ascospores: Narrowly fusiform, widest at the middle and tapering gradually into an elongated fine thread at each end, $\sim 15$ septate, yellow, breaking into equal spore parts at maturity. Anamorph: Unknown, but Dilophospora is found in same stroma.

Remarks: The ascospores ol Lidophia were considered indistinct from those of Leptosphaeria (Müller 1950; v. Arx and Müller 1975); however, Walker (1980) considers Lidophia distinct from Leprosphaeria and close to Ophiobolits in that the ascospores readily break into half-spores at the central septum. Lidophia differs from Ophiobolus in lacking an enlarged central cell in the ascospore.

Lophiostoma V. Cesati \& G. de Notaris, nom. cons., Commentario della Società Crittogamologica Italiana, Milan 1:219. 1863. Type species: Lophiostoma macrostoma (H.J. Tode:E.M. Fries) V. Cesati \& G. de Notaris. Based on Sphaeria macrostoma H.J. Tode:E.M. Fries. Family: Lophiostomataceae. Ascomata: Immersed, subepidermal, globose to subglobose, glabrous, cells of outer wall melanized black, beak laterally compressed. clypeate. Asci: Bitunicate, many, cylindroclavate, wall thin but thick at apex, shortstalked, eight-spored. Ascospores: Obliquely uniseriate, ellipsoidal to narrowly clavate, brown, 3- to 7-phragmoseptate, 1 to 2 longitudinal septa present. Anamorph: Unnamed pycnidial anamorph (Chesters and Bell 1970). Remarks: Lophiostoma differs from Leptosphaeria in having ascomata, with laterally compressed papillae (Chesters and Bell 1970).

Massaria G. de Notaris, Nuovo Giornale Botanico Italiano e Bolletino della Socictà Botanica Italiana, Firenze 1:333. 1844. Type species: Massaria inquinans (H.J. Tode:E.M. Fries) G. de Notaris. Basionym: Sphaeria inquinans H.J. Tode:E.M. Fries. Family: Massariaceae. Ascomata: Immersed in pseudostromatic tissue or a clypeus becoming erumpent or superficial with bases remaining immersed. Asci: Bitunicate. subcylindrical with a short, stout stipe, ocular region low and broad surrounded by a refractive ring. Ascospores: Large, distoseptate, and 3-septate. Anamorph: None reported (Muiller 1979). Remarks: The ascospores of Massaria are generally larger than in Leptosphaeria. Each ascus has an apical cytoplasmic protrusion surrounded by a refractive ring and usually four ascospores at maturity. The ascospores are larger than those in Leptosphaeria and have a thick epispore and a mucilaginous coat (Barr 1979).

Massarina P.A. Saccardo, Sylloge Fungorum Onnium Hucusque Cognitorum Digessit P.A. Saccardo 2:153. 1883. Lectotype species: Massarina ehurnea (L.R. Tulasnc \& C. Tulisne) P.A. Saccardo. Family: Lophiostomataceae. Ascomata: Clustered, immersed, subepidermal, depressed, glabrous, cells of outer ascocarp wall melanized brown, wall tissue of textura prismatica in face view, beak absent, ostiole circular. Asci: Many, bitunicate, cylindric to cylindric-clavate, short-stalked, wall thick at apex. Ascospores: Biseriate, oblong-fusoid, 3-septate, slightly constricted at the septa. symmetrical, hyaline to subhyaline, frequently surrounded by a mucous sheath, which may be evanescent. Anamorphs: Anguillospora C.T. Ingold, Ceratophoma F. v. Höhnel, Coniothyrium A.C. Corda. Diplodia E.M. Fries, Microsphaeropsis F. v. Höhnel, and Stagonospora P.A. Saccardo. Remarks: Massarina is allied with Leptosphaeria species having hyaline ascospores (Metasphaeria sensu P.A. Saccardo, 1883) but differs in lormation of a clypeus and in the ascospores, which form their secondary septa late in development (Munk 1956; Bose 1961; Muiller 1979).

Massariosphueria (E. Müller) P.G. Crivelli,

Dissertation Eigenössischen Technischen Hochschule, Zürich, No. 7318:141. 1983. Type species: Massariosphaeria phaeospora (E. Müller) P.G. Crivelli. Family: Dacampiaceac. Ascomata: Scattered, globose to conic, immersed, subcuticular to suhepidermal, becoming erumpent, beak papillate. conical to longitudinally compressed with a circular or slit-like ostiole. Asci: Bitunicate, numerous, clavate-cylindrical, short-stalked, thick-walled. Ascospores: Phragmoseptate, sometimes with longitudinil septa. hyaline to brown, fusiform to ellipsoid or clavate. Anamorph: Apospharia-like, Phoma-like. Remarks: Müller (1950) segregated species of Leptosphaeria with large, thick-walled ascospores with thick gelatinous sheaths into the subgenus Massariosphaeria. Crivelli (1983) elevated the subgenus to genus and expanded it to include dictyosporous species. It appears to be at natural grouping with casily distinguished feiltures.

Melanomma T. Nitschkc ex L. Fuckel. Symholic Mycologicae, p. 159. 1870. Lectotype species: Melanomma pulvis-prris (C.IH. 
Persoon:E.M. Fries) L. Fuckel. Family: Melanommataceae. Anamorphs: Aposphaeria P.A. Saceardo and Pseudospiropes M.B. Ellis. Remarks: Holm ( I957) maintained Melanomma for certain lignicolous species that ippear to form a unit based on the scleroplectenchymatic walls of the ascocarps. The ascospores are 3-septate. ellipsoidal or frequently cuneiform to nearly clavate and uniformly brown. Melanomma was further subdivided into four groups based on variability and differentiation of the ascocarp wall. A number of species on dead herbaceous stems usually placed in Leptosphaeria were transferred to Melanomma. Holm (1957) considered Melanomma to be elosely related to Leptosphaeria, but Samuels and Müller (1978) believed that Melanomma should be merged with Trematosphaeria (Chesters 1938).

Montugmula A.N. Berlese, lcones Fungorum Omnium Hucusque Cognitorum ad usum Sylloges Saccardianae Adcommodatae 2:68 1896. Type species: Montagmula infernalis (G. NiessI v. Mayendorf) P.G. Crivelli. Family: Phacosphaeriaceae. Ascomata: With or without a clypeus or surrounded by a secondary stromatic development, globose. Asci: Bitunicate, clavate, with filiform basal stalk having a claw-like base, wall thickened. Ascospores: Fusoid, mostly 3-, rarely 4-, 5-, or 7-septate. dark red-brown to opaque, wall often fincly tuberculate, vertical walls in two central cells, symmetric, constricted at the central septum. Anamorph: Unknown. Remarks: Considered a subgenus of Pleospora by Wehmeyer (1961). Montagnula was raised to generic rank by Crivelli (1983). Characteristics distinguishing the group are the tapered or elongate stipitate base of the ascus, the usually very dark pigmentation of the spore wall (which is often tuberculateroughened), the tardy insertion of vulgaris like septa in the central cells, the tendency to form a thick ascostromatic wall, and a surrounding clypeus or stroma.

Nodulosphacria G.L. Rabenhorst, Klotzschii Herbarium Vivum Mycologicum Sistens Fungorum Per Totam Germaniam Cresentium Collectionem Perfectim. Dresden, Edition II. Century 8. No. 725. Anno. 1858. Type species: Nolutosphaeria derasa (M.J. Berkeley \& C.E. Broome) L. Holm. Family: Phaeosphacriaceat. Ascomata: Clustered, immersed-subepidermal, globose. sctose. wall pseudoparenchymatic, melanized brown, of texiura angularis, heak short, with paraphyses. Asci: Many, bitunicate. cylindro-clavate, short-stalked, contaning cight aseospores. Ascospores: Fusilorm. constricted at mid-septum. 8- to 10-septate. with one enlarged cell, with bipolar appendages, pale brownish yellow. Anamorph: Unknown. Remarks: Once considered a subgenus of Leptosphaeria, Nodulosphaeria was reinstated by Holm (1957). This genus can be distinguished from Leptosphaeria by the setose ascomata composed of pseudoparenchyma.

Ophiobolus H. Riess. Hedwigia. Dresden 1:27-28. 1854. Type species: Ophiobohis disseminans H. Riess. Family: Leptosphaeriaceae. Ascomata: Scattered 10 elustered. immersed. becoming erumpent, pyriform, black. glabrous. outer wall of textura globulosa melanized brown, inner wall of textura prismatica, wall pseudoparenchymatic. Asci: Bitunicate. numerous, long-eylindrical to clavate, thick-walled at tips, short-stalked, 4 . to 8-spored. Ascospores: In a single fascicle. yellow or brown, sclolecosporous, multiseptate, often with one or more enlarged cells, sometimes with one or more gelatinous. terminal. globose appendages. Anamorph: Unknown. Remarks: Ophiobolus integrates with Leprosphacria subgenus or section Nodulospliaeria (G.L. Rabenhorst sensu F. v. Höhnel) E. Müller, and authors do not agree on the precise limit between Ophiobolus and subgenus $N$ odulosphaeria. In general. the ascospores of subgenus Nodulosphaeria are only slightly curved or straight and shorter than those in Ophiobolus, and dark periphyses line the ostiole in subgenus $N$ odulosphaeria (Shoemaker 1976. Walker 1980).

Ophiosphaerella C.L. Spegazzini. Anales del Nuseo Nacional de Historia Natural de Buenos Aires 19(Series 3, 12):40I. 1909. Type species: Ophiosphaerella graminicola C.L. Spegazzini. Family: Phaeosphaeriaceae. Ascomata: Scattered to clustered, immersed. subepidermal, globose, glabrous, wall of textura angularis in face view. beak short. papillate, bluntly conical with periphyses. Asci: Bituricate. long. cylindric in a dense basial layer. Ascospores: Filiform (scolecosporous), phragmoseptate, brown, lacking gelatinous sheaths. Anamorph: Unknown. Remarks: A genus close to Ophioholus H. Riess schsu smicto and Phaeosphacrial. Miyake, it is characterized by scolecosporous, brown, septate ascospores and occurs on Gramineate and Cyperaceac.

Paraphacosphucseric O. Eriksson. Arkiv for Botanik. Uppsala. Stockholm. Series 2. 6:405. 1967. Type species: Paraphaensphacria michotii (G.D. Westendorp) O. Eriksson. Famil!: Phaeosphacriaceac. Ascomata: Scattered. intrapidermal. depressed globose 10 globose. without prominent beak. Asci: Bitunicate. 
numerous, cylindrical, short-stalked. Ascospores: Clavate to cylindrical, 2- to 9. septale, lirst-formed septum subtending an enlarged cell, echinulate to rarely smooth. with a thick, gelatinous sheath. Anamorph: Coniothyrim A.C.J. Corda. Remarks: Paraphaeosphaeria is a segregite of Leptosphaeria resembling Phaeosphaeria. but differs in having ascospores with more septa and Coniothyrium anamorphs. In Phoeosphaeria, the anamorphs belong to Hendersonia P.A. Saccardo and Phaeoseptoria C.L. Spegazzini (Eriksson 1967).

Phaeosphaeria I. Miyake, Botanical Magazine, Tokyo 23:93. 1909; Journal of the College of Agriculture, Imperial University of Tokyo 2:246. 1910. Type species: Phacosphaeria oryzae I. Miyake. Family: Phaeosphaeriaceae. Ascomata: Superficial, globose, glabrous, wall pseudoparenchymatic, thin. Asci: Many, bitunicate, cylindro-clavate. containing eight ascospores. Ascospores: Fusiform to cylindrical, 3-septate, yellowish brown, one cell clearly inflated. Anamorph: Coelomycetous, PhyllostictalHendersonia. Remarks: Ascospores of species in this genus are very similar to those of Leptosphaeria species. Phaeosphaeria species are distinguished by small thin-walled, pseudoparenchymatic ascomata, and by their occurrence mostly on monocots (Shoemaker and Babcock 1989).

Platystoma V. Trevisan, Bulletin. Société R. de Botanique de Belgique, Bruxelles 16:16. 1877. Type species: Platystoma compressum (C.H. Persoon:E.M. Fries) V. Trevisan. $\equiv$ Sphaeria compressa C.H. Persoon:E.M. Fries. Family: Platystomaceae. Ascomata: Clustered, immersed-subepidermal, globose, glabrous, outer cells melanized brown, shor papillate, papilla conical with periphyses, ostiole slit-like. Asci: Many, bitunicate, cylindric-clavate, short-stalked. Ascospores: Fusiform to subcylindric, constricted at septa, 5- to 6-septate, with one enlarged cell, brown. Anamorph: Unknown. Remarks: This genus differs from Leptosphaeria in having trabeculate rather than cellular pseudoparaphyses and a slit-like ostiole.

Pleospora G.L. Rabenhorst ex V. Cesati \& G. de Notaris, nom. cons., Commentario della Società Crittogamologica Italiana, Milan 1:217. 1863. Type species: Pleospora herharum (C.H. Persoon:E.M. Fries) G.L. Rabenhorst type cons. Family: Pleosporaceae. Ascomata: Perithecioid, immersederumpent, peridium relatively wide, composed of pseudoparenchyma. Asci: Bitunicate, clavate or oblong, short-stalked, endolunica wide. Ascosporcs: Elliptical, yellow-brown, with transverse and longitudinal septa, with gelattinous sheatl.

Anamorphs: Alernaria C.G. Nees v. Esenbeck, Dendryphion K.F.W. Wallroth. and Stemphylium K.F.W. Wallroth.

Remarks: Pleospora can be separated from Leptosphaeria by ascospore septation and anamorphs. Some species included in Leptosphaeria have ascospores with longitudinal septa, and these species must he examined carefully for ascomatal leatures and cultured lor anamorphic states (Müller 1951, Wehmeyer 1961).

Pyrenophoru E.M. Fries, Summa Vegetabilium Scandinaviae, Seu Enumeratio, Systematica et Critica, Plantarum tum Cotyledonearum. Quam Nemearum Inter Mare Occidentale Et Album, Inter Eidoram et Nordkop, Hactenus Lectorum, una Cum Singulae Distributione Geographica, pp. 397-398. 1849. Type species: Pyrenophora phaeocomes (G.L. Rabenhorst:E.M. Fries) E.M. Fries. Family: Pyrenophoraceae. Ascomata: Immersed to erumpent, medium to large, usually setose or bearing conidiophores. Asci: Bitunicate. few, basal, clavate, oblong or cylindric. Ascospores: Ellipsoidal, broader above the middle, muriform, yellowish-brown, shearhed. Anamorph: Drechslera R.A. Shoemaker. Remarks: Pyrenophora is closely related to Pleospora and differs from Leptosphaeria in having large, muriform ascospores with gelatinous sheaths. The genus is nomenclaturally sound with the conservation of Ceuthospora R.K. Greville, 1826. typified by Ceuthospora lauri (R.K. Greville) R.K. Greville vs. Ceuthospora E.M. Fries, 1825, typified by Ceuthospora phacocomes (J.F. Rebentish:E.M. Fries) E.M. Fries (Shoemaker 1961, Ammon 1963, Barr 1972, Sutton 1972).

Rebentischia P.A. Karsten, Fungi Fenniae Exsiccati, Century 9, No. 881. Anno. 1869; Mycologia Fennica, p. 14. 1873. Type species: Rebentischia pomiformis P.A. Karsten. Family: Tubeufiaceae. Ascomata: Erumpent, globose, roughened with protruding cells and hyphae, pseudoparenchymatic, outer cells melanized black, beak absent. Asci: Many. bitunicate, cylindric-clavate. Ascospores: Obovate, vinaceous brown 4-to 5-septate, with hasal appendage. Anamorph: Unknown. Remarks: Müller (1950) considered the centrum type of this genus to be similar to that of a typical Leptosphaeric. It diliers from Leptosphaeria in the ascospores, which have a hyaline basal appendage (Dennis 1978. Barr 1980).

Sulcispora R.A. Shocmaker, Canadian Journal of Botany, Otlawa 67:1594. 1989. Type 
species: Sulcispora pleurospora (G. Niessl v. Mayendorf) R.A. Shoemaker. Family: Phaeosphaeriaceae. Ascomata: Immersed becoming erumpent, pyriform to globose, smooth. Beak: Composed of brown rectangular cells, the ostiole lacking periphyses. Ascoma wall: Brown pseudoparenchymatic, rectangular cells. Asci: Few, bitunicate, cylindric, 8-spored. Ascospores: Tetraseriate, fusiform, straight, 5- to 6septate, constricted at first septum, reddishbrown, longitudinally sulcate, sheathed.

Trematosphaeria L. Fuckel, Symbolae Mycologicae, pp. 161-162. 1870. Type species: Trematosphaeria pernusa (C.H. Persoon: E.M. Fries) L. Fuckel. Family: Platystomaceae. Ascomata: Superficial to partially immersed, globose to conic, peridium pseudoparenchymatic, melanized black, textura angularis in face view, beak short. papillate. Asci: Bitunicate, cylindricclavate, short-stalked, containing eight ascospores. Ascospores: Fusiform, constricted at mid-septum, 3-septate, brown. Anamorph: Unknown. Remarks: Petrak (1923) considered the structural features of Trematosphaeria to correspond to those of
Leptosphaeria, and he included woodinhabiting Leptosphaeria-like species. Boise (1984) redefined the genus by the presence of trabeculate pseudoparaphyses and included it in the Platystomaceae in the Melanommatales.

Trichometasphaeria A. Munk, Dansk Botanisk Arkiv, Kjøbenhavn 15(2):135. 1953. Type species: Trichometasphaeria diamthi $(\mathrm{E}$. Rostrup) A. Munk. [=Trichometasphaeria gloeospora (M.J. Berkeley \& F. Currey) L. Holm]. Family: Lophiostomataceae. Ascomata: Globose, subepidermal, with ostiole bearing brown setae. Asci: Bitunicate, clavate to cylindrical. Ascospores: Hyaline, fusiform to elliptical, 4- to 6septate. Anamorph: Unknown. Remarks: This genus is similar to Keissleriella. but it differs in having several septate spores versus I-septate spores in Keissleriella. Bose (1961) united the two genera under the earlier name, Keissleriella, after observing variation in ascospore septation in Keissleriella aesculi. Barr (1987b) accepts both genera and places each of them in a different order. 


\section{Appendix 3. Synonyms of Leptosphaeria}

Ampullina L. Quélet (1875). Type: Ampnllina acuta =Leptosphaeria acuta (v. Arx and Müller [975).

Baumiella P.C. Hennings in H. Baum (1903). Type species: Baumiella caespitosa P. Hennings in H. Baum, Kunene-Sambesi Expedition, Berlin, p. 165. 1903; 三Leptosphaeria baumii J.A. v. Arx \& E. Müller (1975) nom. nov., non Leptosphaeria caespitosa G. Niess! v. Mayendorf.

Bilimbiospora B. Auerswald in G.L. Rabenhorst (1860). Nomina generica rejicienda by conservation of Leptosphaeria (Greuter et al. $1988)$.

Chaeroplea (P.A. Saccardo) F.E. Clements in F.E. Clements \& C.L. Shear (1931). =Leptosphaeria (Eriksson \& Hawksworth 1986).

Chironospora E.C. Bommer, M.H. Rousseau, \& P.A. Saccardo in P.A. Saccardo (1891). =Leptosphaeria (Müller 1950).

Dendroleptosphaeria M. de Sousa da Camara (1932). A possible synonym of Leptosphaeria (Eriksson \& Hawksworth 1986).

Dothideopsella F. v. Höhnel (1915). =Leptosphacria (v. Arx \& Müller 1975).

Exilispora L.R. Tehon \& E.Y. Daniels (1927). Type species: Exilispora plurisepta L.R. Tehon \& E.Y. Daniels, Mycologia, Lancaster, Pennsylvania 19:1]2. 1927; =Leptosphaeria plurisepta (L.R. Tehon \& E.Y. Daniels) J.A. v. Arx \& E. Müller (v. Arx \& Müller 1975).

Humboldina C.E. Chardon \& R.A. Toro (1934). =Leptosphaeria (Eriksson \& Hawksworth 1986).

Leptosporopsis F. v. Höhnel (1920). =Leptosphaeria (Eriksson \& Hawksworth 1986).

Macrobasis K. Starbäck (1893). =Leptosphaeria (Petrak and Sydow 1923, Müller 1950).

Metasphaeria P.A. Saccardo (1883). Nomen ambiguum. =Leptosphaeria (Dothideales) $(\mathrm{v}$. Arx and Muiller 1975); a segregate of Lepiosphaeria comprising the hyaline-spored species, otherwise as in Leptosphacria (Petrak 1923. Müller 1950). However, the genus contains both unitunicate and bitunicate Ascomycetes (Barr 1976).
Mycopyrenula E.A. Vainio (1921). =Leptosphaeria (Müller 1950), but considered a good genus by Hawksworth et al. (1983).

Mycotodea W. Kirschstein (1936). According to Petrak (1940). Mycolodea is a synonym of Scleropleella and thus Leptosphacria according to E. Mülles (1950); =Leptosphaeria (Dothideales) (v. Arx and Müller 1975).

Myriocarpium H.F. Bonorden (1864). =Leptosphaeria (Eriksson \& Hawksworth 1986).

Nodulosphaeria G.L. Rabenhorst (1858). Nomina generica rejicienda by the conservation of Leptosphacria (Grueter 1988).

Phaeoderris (P.A. Saccardo) F. v. Höhnel (1907b). =Leprosphaeria (v. Arx and Müller 1975).

Phyllophthalmaria (J. Muller Argov) A. Zahlbruckner in A. Engler \& K. Prantl (1905). A questionable synonym of Leptosphacria (Eriksson and Hawksworth 1986).

Pocosphueria (P.A. Saccardo) A.N. Berlese (1892). =Leptosphaeria (Müller 1950, v. Arx and Müller 1975). Represents Leptosphaeria species with a bristly peridium.

Saccorhecium E.M. Fries (1835). =Pringsheinia $\mathrm{S}$. Schulzer v. Müggenburg in S. Schulzer v Müggenburg. A. Kaniiz, and J.A. Knapp (1866). Placed in the Dothideales (Holm 1975); = Massaria G. de Notaris ( 1844) (Pyrenulales) (Barr 1979).

Sclerodothis F. v. Höhnel (1918a). Based on hyaline spores and therefore identical with Melasphaeria, which is a synonym of Leptosphaeria (v. Arx \& Müller 1975).

Scleropleclla F. v. Höhnel (1918a). =Leprosphaerulina D. McAlpine (1902) (Dothideales) (Barr 1972); considered a good section or subgenus of Leprosphaeria by Müller (1950); species in this group form transitional taxa within Leptosphacria.

Sincarpella F. v. Theissen \& H. Sydow (1915). =Lepiosphaeria (v. Arx and Müller 1975). Accepted by Barr 1987b. 


\section{Appendix 4. Anamorphs of Leptosphaeria}

Ascochytu M.A. Libert (Coelomycete). Connection: Leptosphaeriu pratensis P.A. Saccardo \& P.A. Briard I=Ascochyta meliloti (W. Trelease) J.J. Davis]. Source: Kendrick and DiCosmo 1979. Conidiogenesis: Phialidic with periclinal thickenings of apex of phialide. Conidia: Hyaline, 1- or, rarely, 3septate. Comments: Other anamorphs reported for Leptosphacria pratensis P.A. Saccardo \& P.A. Briard are Phoma meliloni A. Allescher and Stagonospora meliloti (W.G. Lasch) F. Petrak, both Coelomycetes (Lucas and Webster 1967).

Ascochyoula (A.A. Potebnia) H. Diedicke $\mid=$ Psendodiplodia (P.A. Karsten) P.A. Saccardo] (Coelomycete). Connection: Leptosphaeria obiones (H.M. Crouan \& P.L. Crouan) P.A. Saccardo (=Ascoshytula obiones $\mathrm{H}$. Diedicke). Source: Grove 1935 (by association). Conidiogenesis: Phialidic with periclinal thickening of apex of phialide. Conidia: Phaeodidymospores.

Asteromella G. Passerini \& F. v. Thümen (Coelomycete). Connection: Leptosphaeria artemisiue (L. Fuckel) B. Auerswald (=Asteromella artemisiue E. Müller). Source: Müller 1950, Lucis and Webster 1967. Conidiogenesis: Phialidic with periclinal thickening of apex of phialide. Conidia: Hyaloamerospores.

Camarosporium S. Schulzer v. Müggenhurg (Coelomycete). Connection: Leprosphlueria maculans (J. Desmazières) V. Cesati \& G. de Notaris | = Camarosporium affine (P.A. Saccardo) E.C. Bommer \& M.H. Rousseau] (Coelomycete). Source: Müller and Tomiševič 1957: Connection: Leptesphlacria millefolii (L. Fuckel) G. Niessl v. Mayendorf (=Camarosporium sp.). Source: Müller and Tomaševič 1957: Connection: Leptosphaeria ogilviensis (M.J. Berkeley \& C.E. Broome) V. Cesati \& G. de Notaris (=Cumarosperium sp.). Source: Müller and Tomaševič 1957: Connection: Leptosphaeria orthosamthi E. Müller (=Camurosporium sp.). Source: Müller and Dennis 1965; Conidiogenesis: Annellidic. Conidia: Phacodictyospores.
Cladosporium J.H. Link (Hyphomycete). Connection: Leprosphaeria ladina E. Müller (=Cladosporium ladium E. Müller). Source: Müller 1950. Conidiogenesis: Holoblastic. sympodial. Conidia: Brown, o-pluriseptate. Comments: Considered a culture contaminant rather than an anamorph (Kendrick and DiCosmo 1979).

Coniothyrium (A.C.J. Corda) (Coelomycete). Connection: Leprosphaeria hondari A.A. Bitancourt \& A.E. Jenkins (=Coniothyrium sp.). Source: Wehmeyer 1975: Connecrion: Leprosphaeria coniorhyrium (L. Fuckel) P.A. Saccardo (=Coniorhyrium fuckelii P.A. Saccardo). Source: Zeller 1927. Punithalingam 1980: Connection: Leprosphaeria faullii G.D. Darker (=Coniothyrium faullii G.D. Darker). Source: Darker 1964. Wehmeyer 1975: Connection: Leprosphaeria michotii (G.D. Westendorp) P.A. Saccardo $(=$ Paraphaeosphaeria $\mathrm{O}$. Eriksson: $=$ Coniothyrium scirpi J.W. Trail). Source: Wehmeyer 1975: Sivanesan 1984: Connection: Leptosphaeria obiones (H.M. Crouan \& P.L. Crouan) P.A. Saccardo (=Coniothyrium obiones H. Diedicke). Source: Grove 1935: Connection: Leptesphaeria perichymeni C.A. Oudemans (=Coniohlyrimm sp.). Source: Wehmeyer 1975; Connection: Leprosphaeria sparinae J.B. Ellis \& B.M. Everhart (=Coniothyrium sp.). Source: Lucas and Webster 1967: Conidiogenesis: Annellidic. Conidia: Phaeoamerospores. phaeodidymospores.

Diplodina G.D. Westendorp (Coelonycete). Connection: Leptosphaeria marcyensis (C.H. Pech) P.A. Saccardo (=Diplodina sp.). Source: Lucas and Webster 1967. Conidiogenesis: Phialidic, periclinal thichening of apex of phialide, eustomatic. Conidia: Hyaline, 0- to 2-septate.

Hendersomia M.J. Berkeley. Nomina generica rejicienda. $\{=$ Stagonosprora (P.A. Saccardo) P.A. Saccardo) (Coelomycete).

Leptophloma F. v. Höhnel (=Phoma P.A. Saccardo) (Coclomycete).

Microdiplodia A. Alleseher (Coelonicete). Connection: Leprosplateria ohmosispora C.L Spegazini $[=$ licredipledics henriquesii (F. 
v. Thümen) F. Petrak \& H. Sydow]. Source: Lucas 1963. Conidiogenesis: Unknown in Microdiplodia henriquesii (F. v. Thümen) F. Petrak \& H. Sydow. Conidia: Phateodidymospores.

Nakataea K. Hara (Hyphomycete). Connection: Leprosphaeria salvinii A. Cattaneo (=Nakataea sigmoidea $\mathrm{K}$. Hara). Source: Ellis 1971. Conidiogenesis: Holoblastic, sympodial. Conidia: 3-septate, pale brown.

Pestalotia G. de Notaris (Coelomycete). Connection: Leprosphaeria honiarensis T. Matsushima (=Pestalotia sp.). Source: Matsushima 1971. Conidiogenesis: Annellidic. Conidia: Phaeophragmospores with appendages. Comments: Leprosphaeria honiaraensis may belong in Pestalosphaeria M.E. Barr (Amphisphaeriaceae) (Kendrick and DiCosmo 1979).

Pestalotiopsis R.L. Steyaert (Coelomycete). Connection: Leprosphaeria elaeidis C. Booth \& J.S. Robertson. Source: Booth and Robertson 1961. Conidiogenesis: Holoblastic, annellidic. Conidia: Phaeophragmospores with appendages.

Phaeoseproria C.L. Spegazzini (Coelomycete). Connection: Leplosphaeria culmifraga (E.M. Fries:E.M. Fries) V. Cesati \& G. de Notaris (=Phaeoseproria sp.). Source: Wehmeyer 1975; Connection: Leptosphaeria fuckelii $\mathrm{G}$. Niessl v. Mayendorf (=Phaeoseptoria sp.). Source: Webster and Hudson 1957. Sivanesan 1984; Connection: Leptosphaeria lacmosa G. Niessl v. Mayendorf (=Phaeoseptaria sp.). Source: Webster and Hudson 1957. Sivanesan 1984; Connection: Leprosphaeria macrospora (L. Fuckel) F. v. Thümen (=Phaeoseptoria $\mathrm{sp}$.). Source: Lucas and Webster 1967; Connection: Leptosphaeria microscopica P.A. Karsten [=Phaeoseptoria airae (W.B. Grove) R. Sprague]. Source: Sivanesan 1984; Comection: Leptosphaeria nigrans (M.R. Roberge) V. Cesati \& G. de Notaris (=Phacoseptoria sp.). Source: Hughes 1949. Sivanesan 1984; Conidiogenesis: Holoblastic. Conidia: Phaeophragmospores.

Phoma P.A. Saccardo (Coelomycete). Connection: Leptosphaeria acula L. Fuckel (=Phoma acuta L. Fuckel). Source: Grove 1935; Müller and Tomaševič 1957; Connection: Leptosphaeria agnita (J. Desmazières) V. Cesati \& G. de Notaris (=Phoma sp.). Source: Lucas and Webster 1967, Sivanesan 1984; Connection: Leprosphacria albopunctata (G.D. Westendorp) P.A. Saccardo (=Phoma sp.). Source: Sivanesan 1984; Connection: Leptosphaeria conferta G. NiessI v. Mayendorf ex P.A. Saccardo (=Phoma sp.). Source: Lucas 1963, Sivane- san 1984: Connection: Leptosphacria congesta M.T. Lucas (=Phoma sp.). Source: Lucas 1963; Connection: Leprosphaeria cruenta P.A. Saccardo (=Phoma sanguinolenta E. Rostrup). Source: Grove 1935; Connection: Leptosphaeria doliolum (C.H. Persoon:E.M. Fries) subsp. doliolum var. doliolum V. Cesati \& G. de Notaris (=Phoma hoehnelii H.A. Van Kerstern subsp. hoehnelii var, uricae G.H. Boerema \& H.A. Van Kerstern). Source: Lucas and Wehster 1967, Boerema 1976; Connection: Leprosphaeria dumetorum G. Niessl v. Mayendorf (=Phoma sp.). Source: Lucas and Webster 1967: Connection: Leprosphaeria haematies M.R. Roberge ex J. Desmazières) G. Niess] v. Mayendorf (=Phoma sp.). Source: Lucas and Webster 1967, Sivanesan 1984;

Connection: Leptosphaeria libanotis (L. Fuckel) G. Niessl v. Mayendorf (=Phoma sp.). Source: Lucas and Webster 1967. Sivanesan 1984; Connection: Leptosphaeria lindquistii M.J. Frezzi (=Phoma macdonaldii G.H. Boerema). Source: Sivanesan 1984; Connection: Leptosphaeria maculans (J. Desmazières) V. Cesati \& G. de Notaris [=Phoma lingam (H.J. Tode:E.M. Fries)]. Source: Müller and Tomaševič 1957. Sivanesan 1984; Connection: Leprosphaeria millefolii (L. Fuckel) G. Niessl v. Mayendorf (=Phoma sp.). Source: Müller and Tomaševič 1957; Connection: Leptosphaeria ogilviensis (M.J. Berkeley \& C.E. Broome) V. Cesati \& G. de Notaris (=Phoma sp.). Source: Müller 1971: Connection: Leplosphaeria pontiformis (L. Fuckel) P.A. Saccardo (=Phoma sp.). Source: Webster and Hudson 1957. Wehmeyer 1975: Connection: Leptosphaeria purpurea $\mathrm{H}$. Rehm (=Phona sanguinolenta W.B. Grove). Source: Lucas and Webster 1967. Sivanesan 1984; Connection: Leptosphaeria sacchari H.J. Van Breda De Haan (=Phoma sp.; as Phyllosticta sp.). Source: Hudson 1960: Connection: Leprosphaeria solami L.G. Romell (=Phoma sp.). Source: Lucas and Webster 1967: Connection: Leprosphaeria spartinae J.B. Ellis \& B.M. Everhart (=Phoma sp.). Source: Sivanesan 1984; Connection: Leptosphaeria submaculans L. Holm (=Phoma sp.). Source: Lucas and Webster 1967: Connection: Leprosphaeria thomasiana P.A. Karsten (=Phema sp.). Source: Zeller 1927 (by association). Lucas and Webster 1967 (unnamed); Connection: Leptosphaeria lyphicola P.A. Karsten (=Phoma sp.). Source: Lucas and Webster 1967 (unnamed), Sivanesan 1984; Conidiogenesis: Phialidic with periclinal thickening of apex of phialide. Conidia: Phateamerospores. 
Scolecosporiella F. Petrak (Coelomycete). Connection: Leptosphaeria macrospora (L. Fuckel) F. v. Thümen [ =Scolecosporiella bemardiana (P.A. Saccardo) A. Sivanesan]. Source: Sivanesan 1984; Connection: Lcptosphaeria typharum (J. Desmazières) P.A. Karsten [ =Scolecosporiella typhae (C.A. Oudemans) F. Petrak]. Source: Sivanesan 1984: Conidiogenesis: Holoblastic. Conidia: Pale brown, 3- to many-septate, cylindrical.

Septoria P.A. Saccardo (Coelomycete).

Connection: Leptosphaeria avenaria G.F.

Weber (=Septoria avenae B. Frank). Source: Grove 1935, Sivanesan 1984; Connection: Leptosphaeria maydis G.L. Stout (=Septoria zeac G.L. Stout). Source: Stout 1930 (by association); Connection: Leptosphaeria nodorum E. Müller [=Septoria nodorum (M.J. Berkeley) M.J. Berkeley]. Source: Lucas and Webster 1967. Sivanesan 1984; Connection: Leptosphaeria phlogis C.A. Oudemans (=Septoria phlogis P.A. Saccardo \& C.L. Spegazzini). Source: Grove 1935 (by association); Connection: Leptosphaeria sorbi A.L. Jaczewski (=Septoria sorbi W.G. Lasch). Source: Grove 1935 (by association): Conidiogenesis: Three types of conidiogenesis have been found for the pathogenic species of Septoria studied thus far. They are (1) holoblastic, sympodial. (2) simple holoblastic, and (3) phialidic with periclinal thickening of the apex of the phialide. Conidiogenesis has not been determined for any of the Septoria anamorphs of Leptosphaeria. Conidia: Hyaline, multiseptate, filiform.

Stagomospora (P.A. Saccardo) P.A. Saccardo (Coelomycete). Connection: Leptosphacria anemones L. Hollós (=Stagonospora (memones N.T. Patouillard). Source: Sivancsan 1984; Connection: Leptosphaeria arundinaceae P.A. Saccardo (=Siagonospora vexuta P.A. Saccardo). Source: Grove 1935 (by association); Connection: Leptosphacria hicolor D.L. Hawkswont, W. Kaiser \& B.N. Ndimande (=Stagonospora sp.). Source: Kaiser et al. 1979; Connection: Leptosphaeria cisina M.J. de Urríes y Azara (=Stagonospora sp.; as Hendersonia cisti $\mathbf{M}$. de Sousa da Camara). Source: Lucas 1968; Connection: Leptosphaeria dumetorum $\mathbf{G}$. Niessl v. Mayendorf (=Stagonospora sp.: as Hendersonia sp.). Source: Lucas and Webster 1967. Sivanesan 1984: Connection: Leptosphueria eustomoides P.A. Saccardo (=Stagonospora sp.; as Hendersonia sp.). Source: Webster and Hudson 1957;

Connection: Leptosphaeria gigaspora $\mathrm{G}$. Niessl v. Mayendorf (=Siagonospora gigaspora P.A. Saccardo). Source: Grove 1935 (by association); Connection: Leptasphaeria libanotis (L. Fuckel) G. Niessl v. Mayendorf (=Stagonospora sp.: as Hendersonia sp.). Source: Wehmeyer 1975; Connection: Lepiosphaeria polygonati E. Müller \& M. Tomaševič (=Stagonospora sp.: as Hendersonia sp.). Source: Müller and Tomaševič 1957: Connection: Leptosphaeria pontiformis (L. Fuckel) P.A. Saccardo (=Stagonospora sp.: as Hendersonia sp.). Source: Lucas and Webster 1967. Sivanesan 1984: Connection: Leptosphacria pratensis P.A. Saccardo \& P.A. Briard [=Stagonospora melioti (IV.G. Lasch) F. Petrak]. Source: Jones and Weimar 1938, Lucas and Webster 1967; Connection: Leptosphaeria tainanensis W.Y. Yen \& C.C. Chi (=Sragonospora lainanensis W.H. Hsieh. Source: Hsieh 1979, Sivanesan 1984; Connecrion: Leptosphaeria viridella (C.H. Peck) P.A. Saccardo (=Stagonospora sp.; as Hendersonia). Source: Lucas and Webster 1967; Conidiogenesis: Holoblastic. sometines annellidic. Conidia: Hyaline. multiseptate. cylindrical. 


\section{Literature Cited}

Ammon, H.U.v. 1963. Über einige Arten aus den Gattungen Pyrenophora Fries und Cochliobolus Drechsler mit Helminthosporium als Nebenfruchtform. Phytopathologische Zeitschrift, Berlin 47:244-300.

Arx, J.A. v., and E. Müller. 1975. A re-evaluation of the bitunicate Ascomycetes with keys to families and genera. Studies in Mycology, Baarn, Netherlands 9:1-159.

Bar, M.E. 1972. Preliminary studies on the Dothideales in temperate North America. Contributions from the University of Michigan Herbarium. Ann Arbor 9:523-638.

Barr, M.E. 1976. Buergenerula and the Physosporellaceae. Mycologia, Lancaster, Pennsylvania $68: 611-621$.

Barr, M.E. 1979. On the Massariaceae of North America. Mycotaxon. An International Journal Designed to Expedite Publication of Research on Taxonomy \& Nomenclature of Fungi \& Lichens, Ithaca, New York 9:17-37.

Barr, M.E. 1980. On the family Tubeufiaceae (Pleosporales). Mycotaxon. An International Journal Designed to Expedite Publication of Research on Taxonomy \& Nomenclature of Fungi \& Lichens, Ithaca, New York 12:137-167.

Barr, M.E. 1981. The genus Curreya: an example of taxonomic confusion in the Ascomycetes. Mycologia, Lancaster, Pennsylvania 73:599-609.

Barr, M.E. 1982. Leptosphaeria sepalorum. Mycotaxon. An International Journal Designed to Expedite Publication of Research on Taxonomy \& Nomenclature of Fungi \& Lichens, Ithaca, New York 15:345-348.

Barr, M.E. 1987a. New taxa and combinations in the Loculoascomycetes. Mycotaxon. An International Journal Designed to Expedite Publication of Research on Taxonomy \& Nomenclature of Fungi \& Lichens, Ithaca, New York 29:50l-505.

Barr, M.E. 1987b. Prodromus to class Loculoascomycetes. Hamilton I. Newell, Inc., Amherst, Massachusett.s. $168 \mathrm{pp}$.

Barr, M.E. 1989. The genus Chaetomastia (Decampiaceae) in North America. Mycotaxon. An International Journal Designed to Expedite Publication of Research on
Taxonomy \& Nomenclature of Fungi \& Lichens, Jthaca, New York 34:507-5I5.

Baum, H. 1903. Kunene-Sambesi Expedition, Kolonial-Wirtschaftlichen Komitees, herausgegeben von Prof. Dr. O. Warburg, Berlin. $593 \mathrm{pp}$.

Berlese, A.N. 1892. Icones Fungorum Omnium Hucusque Cognitorum ad Usum Sylloges Saccardianae Adcommodatae 1:89.

Boerema, G.H. 1976. The Phoma species studied in culture by R.W.G. Dennis. Transactions of the British Mycological Society, London 67:289-319.

Boise, J.R. 1984. On Trematosphaeria (Loculascomycetes, Fungi) and disposition of heterogenous elements. Ph.D. dissertation, Universiry of Massachusetts, Amherst. $136 \mathrm{pp}$.

Bonorden, H.F. 1864. Abhandlungen aus dem Gebiete der Mykologie. Abhandlungen der Naturforschenden Gesellschaft zu Halle $8: 1-168$.

Booth, C., and J.S. Robertson. 1961. Leptosphaeria elaeidis sp. nov. isolated from anthracnosed tissue of oil palm seedlings. Transactions of the British Mycological Socjety, London 44:24-26.

Bose. S.K. 1961. Studies on Massarina Sacc. and related genera. Phytopathologische Zeitschrift, Berlin 41:151-213.

Brown. P., and G.B. Stratton, eds. 1963. World list of scientific periodicals published in the years 1900-1960. William Clowes and Sons. London. Three volumes.

Camara, M. de Sousa da. 1932. Mycoflora Lusitanica Centuria X. Revista Agronomica, Lisbon 20:24.

Cesati, V., and G. de Notaris. 1863. Schema di Classificazione degli Sferiacei Italici Aschigeri più o meno appertenenti al genere Sphaeria nell 'antico significato attribuitogli da Persoon. Commentario della Societa Crittogamologica Italiana, Milan 1:177-240.

Chardon, C.E., and R.A. Toro. 1934. Sphacriales. Pages 180-194 (Chapter X111) in C.E. Chardon and R.A. Toro, eds. Mycological explorations of Venezuela. Monographs of the University of Pucrto Rico. Series B. Physical and Biological Sciences, San Juan. No. 2. 
Chesters, C.G.C. 1938. Studies on British Pyrenomycetes II. A comparative study of Melanomma pulvis-pyrus (Pers.) Fuckel. Melanomma fuscidulum Sacc. and Thyridaria rubro-notata (B. et Br.) Sacc. Transactions of the British Mycological Society. London 22:116-150.

Chesters, C.G.C., and A. Bell. 1970. Studies in the Lophiostomataceae Sacc. Mycological Papers. Commonwealth Mycological Institute, Kew. Surrey, No. 120. 55 pp.

Christensen, C. 1905-1906. Index Filicum Sive Enumeratio Omnium Generum Specierumque Filicum et Hydropteridum Ab Anno 1753 Ad Finem Anni 1905 Descriptorum Adjectis Synonymis Principalibus, Area Geographica Etc. H. Hagerup. Copenhagen. 744 pp.

Clements, F.E., and C.L. Shear. 1931. The genera of fungi. H.W. Wilson Co.. New York. 496 pp.

Crivelli, P.G. 1983. Ueber die heterogene Ascomycetengattung Pleospora Rabh.: Vorschlag für eine Aufteilung. Abhandlung zur Erlangung des Titels eines Doktors der Naturwissenschaften der Eidgenössischen Technischen Hochschule, Zürich, No. 7318. $231 \mathrm{pp}$.

Cronquist, A. 1981. An integrated system of classification of tlowering plants. Columbia University Press, New York. 1,262 pp.

Dirker, G.D. 1964. A new Leptosphacria species, an agent in the biological control of certain Hypodermataceae. Canadian Journal of Botany, Ottawa 42:1005-1009.

Dennis, R.W.G. 1978. British Ascomycetes. J. Cramer, Vaduz. 585 pp.

De Notaris, G. 1844. Cenno sulla tribù de Pirenomiceti sferiacei e descrizione di alcuni nuovi generi. Giomale Botanico Italiano. Florence 1:322-335.

Ellis, M.B. 1971. Dematiaceous Hyphomycetes. Commonwealth Mycological Institute. Kew. Surrey. 608 pp.

Engler, A., and K. Prantl. 1905. Die natürlichen Planzenfamilien nebst ihren Gattungen und wichtigeren Arten insbesondere den Nutzpflanzen, bearbeitit unter Mitwirkung zahlreicher hervorragender Fachgelehrten. Verlig von Wilhelm Engelmann. Leipzig. I. Abt. I*, Liel. 221:97-144.

Eriksson, O. 1967. On graminicolous pyrenomyceles from Fennoscandia. 2. Phragmosporous and scolecosporous species. Arkiv för Botanik, Uppsala, Stockholm, Series 2. $6: 381-4.40$.

Eriksson, O., and D.I. Hawksworth. 1986. Outline of the Ascomycetes-1986. Systemai Ascomycenm 5:185-324.

Farr, E.R., J.A. Leussink, and F.A. Stafleu. 1979. Index Nominum Genericorum (Plantarum).
Bohn, Scheltema \& Holkema, Utrecht dr. W. Junk b.v. Publishers, The Hague. Three volumes.

Fries, E.M. 1835-1837. Corpus Florarum Provincialium Sueciae 1. Floram Scanicam scripsit Elias Fries. Palmblad. Sebell \& Co.. Uppsala. 394 pp.

Gray Herbarium Card Index. 1894-present. Gray Herbarium. Harvard University. Cambridge. Massachusetts.

Greuter, W., H.M. Burdet, IV.G. Chaloner, V. Demoulin. R. Grolle, D.L. Hawksworth, D.H. Nicolson, P.C. Silva, F.A. Stafleu, E.G. Voss, and J. McNeill. 1988. International code of botanical nomenclature adopted by the Fourteenth International Botanical Congress. Berlin. July-August 1987. Koelrz Scientific Books, Konigstein. Germany (Regnum Vegetabile Volume 118). $328 \mathrm{pp}$.

Grove, W.B. 1935. British stem and leaf-fungi (Coelomycetes). A contribution to our knowledge of the fungi imperfecti belonging to the Sphaeropsidales and the Melanconiales. Cambridge University Press, Cambridge. Volume 1. $488 \mathrm{pp}$.

Halliday, G., and M. Beadle. 1983. Consolidated index to flora Europeae. Cambridge University Press, Cambridge. 210 pp.

Hawksworth, D.L., B.C. Sutton, and G.C. Ainsworth. 1983. Ainsworth and Bisby"s dictionary of the fungi (including the lichens). Seventh edition. Commonwealth Mycological Institute. Kew, Surrey. 445 pp.

Hedjaroude. A. 1969. Etudes [sic] tavonomiques sur les Phaeosphaeria Miyake et leurs formes voisines (Ascomycetes). Sydowia. Annales Mycologici. Hom, N.Ö. 22:57-107.

Höhnel, F. v. 1907a. Fragmente zur Mykologie (IV. Mitteilung, No. 163). Weiteres über Pseudosphaeriaceen. Sitzungsherichte der Akadenie der Wissenschaften in Wien. Mathematisch-naturwissenschaltliche Klasse. Abt. 1. $116: 631-635$.

Hölınel. F. v. 1907b. Mykologisches. IVlll. Uther Leplosphaeria modesta (Desm.) und andere Arten. Österreichische Botanische Zeitschrift. wien 57:321-323.

Hohnel. F. v. 1915. Fragmente zur Mlyhologie ( IVll. Mitteilung, No. 876 bis 943 ). Sitzungsherichte der Akademie der Wissenschalten in Wien. Mathematischnaturu issenschaftliche Klasse. Abt. I, $124: 49-159$.

Höhnel, F. v. 1918a. Mycologische Fragnente. CCX1. Über Sphaeria aggregara Lasch. Amnales Mycologici. Berlin 16:69-70.

Höhnel, F. v. 19/8h. Mycologische Fragnentc. CCLXXY. Über Leprosphacria persomata Niessl. Anmales Mycologici. Berlin $16: 157-1.59$. 
Höhnel, F. v. 1918c. Ueber die Gattung Leptosphaeria Ces. et de Not. Bericht der Deutschen Botanischen Gesellschaft, Berlin 36:135-140.

Höhnel, F. v. 1920. Fragmente zur Mykologie XXIV. Mitteilung Nr. 1211. Über die Gattung Ophiobolus Aut. (non Riess). Sitzungsberichte der Akademic der Wissenschaften in Wien, Mathematischnaturwissenschaftliche Klasse, Abt. I. 129:171-174.

Holm, L. 1957. Études taxonomiques sur les Pléosporacées. Symbolae Botanicae Upsalienses 14(3): 1-188.

Holm, L. 1975. Nomenclatural notes on Pyrenomycetes. Taxon. International Association of Plant Taxonomists. Utrecht 24:475-488.

Hsieh, W.H. 1979. The causal organism of sugarcane leaf blight. Mycologia, Lancaster, Pennsylvania 71:892-898.

Hudson. H.J. 1960. Pyrenomycetes of sugar cane and other grasses in Jamaica 1. Conidia of Apiospora campiospora and Leptosphaeria sacchari. Transactions of the British Mycological Society, London 43:607-616.

Hughes, S.J. 1949. The perithecia and pycnidia of Leptosphaeria nigrans. Transactions of the British Mycological Society, London 32:63-68.

Index Kewensis Plantarum Phanerogamarum Nomina et Synonyma Omnium Genereum et Specierum a Linneao Usque ad Annum MDCCCLXXXV Complectens Nomine Recepto Auctore Partria Unicuique Plantae Subjectis. 1895-present. Clarendon Press, Oxford, England. Two volumes +18 supplements.

Index of Fungi. 1920-present. Commonwealıh Agricultural Bureaux International, Wallingford, Oxon, Great Britain.

Jaczewskj, A. de. 1894. Essai de classification Naturelle des Pyrénomycètes. Bulletin de la Société Mycologique de France, Paris $10: 13-48$.

Jones, F.R., and J.L. Weimer. 1938. Stagonospora leaf spot and root rot of forage legumes. Journal of Agricultural Research, Washington 57:791-812.

Kaiser, W.J., B.N. Ndimande, and D.L. Hawksworth. 1979. Leaf-scorch disease of sugarcane in Kenya caused hy a new species of Leptosphaeria. Mycologia, Lancaster, Pennsylvania $71: 479-492$.

Kartesz, J.T., and R. Kartesz. 1980. A synonymized checklist of the vascular flora of the United States, Canada and Greenland. The University of North Carolina Press. Chapel Hill. $498 \mathrm{pp}$.

Kendrick, B., and F. DiCosmo. 1979. Teleomorphanamorph connections in Ascomycetes. Pages 283-410 in B. Kendrick, ed. The whole fungus. Volume 1. National Museum of Canada. Ottawa.

Kirschstein, W. v. 1936. Beiträge zur Kenntnis der Ascomyceten und ihrer Nebenformen besonders aus der Mark Brandenburg und dem Bayerischen Walde. Annales Mycologici, Berlin 34:180-210.

Koster. C.J.. and J. Gascoigne. 1971. World list of scientific, medical and technical entries from the British Union-Catalogue of Periodicals. New periodical titles. Butterworths, London. 25 pp.

Lamb. I.M. 1963. Index Nominum Lichenum Inter Annos 1932 et 1960 Divulgatorum. The Ronald Press Co., New York. 809 pp.

Lucas, M.T. 1963. Culture studies on Portuguese species of Leptosphaeria I. Transactions of the British Mycological Society, London 46:361-367.

Lucas, M.T. 1968. Culture studies of Portuguese species of Leptosphaeria II. Transactions of the British Mycological Society, London $51: 411-415$.

Lucas, M.T., and B.C. Sutton. 1971. Heptameria Rehm and Thüm. Transactions of the British Mycological Society, London 57:283-288.

Lucas, M.T., and J. Webster. 1967. Conidial states of British species of Leptosphaeria. Transactions of the British Mycological Society, London 50:85-121.

Luttrell, E.S. 1973. Loculoascomycetes. Pages 135-219 in G.C. Ainsworth, F.K. Sparrow, and A.S. Sussman, eds. The fungi. An advanced treatis. Volume IVA. Academic Press, New York.

Matsushima. T. 1971. Microfungi of the Solomon Islands and Papua-New Guinea. Published by the author, Kobe, Japan. $78 \mathrm{pp}$.

McAlpine, D. 1902. Fungus diseases of stone-fruit trees in Australia and their treatment. R.S. Brain, Government Printer. Melbourne. 165 pp.

Müller, E. 1950. Die schweizerischen Arten der Gattung Leptosphaeria und ihrer Verwandten. Sydowia. Annales Mycologici, Hom, N.Ö. 4:185-319.

Müller, E. 1951. Die schweizerischen Arten der Gattungen Clathrospora. Pleospora. Pseidoplea und Pyrenophora. Sydowia. Annales Mycologici, Horn, N.Ö. 5:248-310.

Müller, E. 1971. Imperfect-perfect connections in Ascomycetes. Pages 184-201 in B. Kendrick, ed. Taxonomy of fungi-imperfecti. Proceedings of the First International Specialists' Workshop-Conference on Criteria and Terminology in the Classification of Fungi Imperfecti held at the Environmental Sciences Centre of the University of Calgary. Kananaskis, Alberta, Canada. University of Toronto Press, Toronto. 
Miiller. E. 1979. Report of the bitunicate committee. Pages $396-408$ in B. Kendrick, ed. The whole fungus. The sexual-asexual synthesis. Proceedings of the Second International Mycological Conference held at the Environmential Sciences Centre of the University of Calgary, Kananaskis, Alberta, Canada. Volume 1. 410 pp.

Müller, E.. and R.W.G. Dennis. 1965. Fungi venezuelani VIIl. Plectascales, Sphaeriales. Loculoascomycetes. Kew Bulletin. Royal Botanic Gardens, Kew 19:357-386.

Müller, E., and M. Tomaševič. 1957. Kulturversuche mit einigen Arten der Gattung Leptosphacria Ces. and de Not. Phytopathologische Zeitschrift. Berlin 29:287-294.

Munk, A. 1956. On Metasphaeria coccodes (Karst.) Sacc. and other fungi probably related to Massarina Sacc. (Massarinaceae n. fam.). Friesia. Nordisk Mykologisk Tidsskrift, Kobenhavn 5:303-308.

Munk, A. 1957. Danish Pyrenomycetes. A preliminary flora. Dansk Botanisk Arkiv, Kjobenhavn 17:1-491.

Petrak, F. 1923. Mykologische Norizen v, Nr. 200. Über die Pseudosphaeriaceen v. H. und ihre Bedeutung für die spezielle Systematik der Pyrenomyzeten. Annales Mycologici. Berlin 21:30-69.

Petrak. F. 1940. Mykologische Notizen XIll. Nr. 853. Über die Gattung Mycotodea Kirschst. Annales Mycologici, Berlin 38:185-186.

Petrak, F., and H. Sydow. 1923. Kritischsystematische Originaluntersuchungen über Pyrenomyzeten. Sphaeropsideen und Melanconieen. Annales Mycologici, Berlin 21:349-384.

Porter, K.1.. and C.J. Koster, eds. 1970. World list of scientific periodicals. New periodical titles 1960-1968. Butterworths, London. NPT/603 pp. + indexes.

Punithalingam, E. 1980. Leptosphueria coniothyrium. CMII Descriptions of Pathogenic Fungi and Bacteria. No. 663. Commonwealth Mycological Institute, Kew, Surrey.

Quélet, L. 1875. Les Champignous du Jura et des Vosges. Ménoires de la Société d’Émulation de Montbéliard, Series 2, No. 5. pp. 523-524.

Rabenhorst, G.L. 1858. Klotzschii Herbarium Vivum Mycologicum. Sistens Fungorum Per Totam Germaniam Crescentiun Collectionem Perfectam. Editio Novo, Century 8, No. 725. Dresden.

Rabenhorst, G.L. 1860. Fungi Europaci Exsiccati. Klotzschii Herbarii vivi Mycologici Continuatio. Edition 11I, Century 3, No. 26I. Dresden.

Saccardo. P.A. 1878. Fungi Veneti Novi Vel Critici Vel Mycologiae Venetae Addendi. Series IX. Miclelia Commentarium Mycologicae Italicac $1: 361-4+5$.
Saccardo, P.A. 1882-1931. Sylloge Fungorum

Omnium Hucusque Cognitorum Digessis P.A. Saccardo. Patavii [Padua] (sumptibus auctoris typis seminarii). 25 volumes.

Saccardo. P.A. 1883. Sylloge Fungorum Omnium Hucusque Cognitorum Digessit P.A. Saccardo. Volume 2. Patavii [Padua] (sumptibus auctoris typis seminarii). $882 \mathrm{pp}$. + index.

Saccardo. P.A. 1891. Sylloge Fungorum Omnium Hucusque Cognitorum Digessit P.A. Saccardo. Volume 9. Patavii [Padua] (sumptibus auctoris typis seminarii). 1.141 pp.

Saccardo. P.A. 1895. Sylloge Fungorum Omnium Hucusque Cognitorum Digessit P.A. Saccardo. Volume 11. Patavii [Padua] (sumptibus auctoris typis seminarii). 753 pp. + L pp. Saccardo. P.A. 1899. Sylloge Fungorum Omnium Hucusque Cognitorum Digessit P.A. Saccardo. Volume 14. Patavii [Padua] (sumptibus P.A. Saccardo typis seminarii). 1,316 pp.

Saccardo. P.A. 1913. Sylloge Fungorum Omnium Hucusque Cognitorum Digessit P.A. Saccardo. Volume 22. Sectio 1. Patavii [Padua] (sumptibus P.A. Saccardo typis seminarii). $1.612 \mathrm{pp}$.

Saccardo, P.A. 1928. Sylloge Fungorum Omnium Hucusque Cognitorum Digessit P.A. Saccardo. Volume 24. Secrio JI. Patavii [Padua] (sumptibus Coheredum Saccardo typis Pergola). I.438 pp.

Samuels. G.J.. and E. Müller. 1978. Life-history studies of Brazilian Ascomycetes 3. Melanomma radicans sp. nov. and its Aposphacria anamorph. Trematosphaeria perrumpens sp. nov and Berlesiella fungicola sp. nov. and its Ramichloridium anamorph. Sydowia. Annales Mycologici. Horn. N.Ö. 31:142-156.

Schulzer v. Müggenburg. S., A. Kanitz, and J.A. Knapp. 1866. Die bisher bekannten Pflanzen Slavoniens, Ein Versuch. Verhandlungen der Zoologisch-Botanischen Gessellschaft in Wien 16(Abhandlungen):3-172.

Seltzer, L.E.. ed. 1952. The Columbia Lippincott gazetteer of the world. Columbia University Press. Momingside Heights. New lork. 2.148 pp.

Shoemaker. R.A. 1961. Pyrenophora phaeocomes (Reb. ex Fr.) Fr. Canadian Joumal of Botany. Ottawa 39:901-908.

Shoemaker. R.A. 1968. Type studies of Pleaspora callescens. Pleospora papareracea and some allied species. Canadian Joumal of Borany, Ot:iша 46:1 143-1150.

Shoemaker. R.A. 1976. Canadian and some extralimital Ophobolus species. Canadian Journal of Botany, Ottaw a 54:2365-2404.

Shomaker, R.A. 1984. Canadian and sone extralinital Leposphacria species. Canadian Jotrual of Botany. Ottaw a 62:2685-2729. 
Shoemaker, R.A., and C.E. Babcock. 1989. Phoeosphaeria. Canadian Journal of Botany, Ottawa 67:1500-1599.

Sivanesan, A. 1984. The bitunicate Ascomycetes and their anamorphs. J. Cramer, Vaduz. $701 \mathrm{pp}$.

Smits, R. 1968. Half a century of Soviet serials, 1917-1968. Library of Congress, Washington, DC. Two volumes.

Stalleu, F.A., and R.S. Cowan. 1976-1988. Taxonomic literature. A selective guide to botanical publications and collections with dates, commentaries, and types. Second edition. Bohn, Scheltema \& Holkema, Utrecht/Antwerpen dr. W. Junk b.v. Publishers, The Hague. Seven volumes.

Starbäck, K. 1893. Sphaeriaceae imperfecte cognitae. Botaniska Notiser, Lund 1893:25-31.

Stout, G.L. 1930. New fungi found on the Indian corn plant in Illinois. Mycologia, Lancaster, Pennsylvania 22:271-287.

Sutton, B.C. 1972. Nomenclature of Ceuthospora, Pyrenophora, and Blemnoria (Fungi). Taxon, International Association of Plant Taxonomy, Utrecht 21:319-326.

Tehon, L.R., and E.Y. Daniels, 1927. Notes on the parasitic fungi of Illinois III. Mycologia, Lancaster, Pennsylvania 19:110-129.

Theissen, F. v., and H. Sydow. 1915. Die Dothideales. Kritisch-systematische Originaluntersuchungen. 130. Sincarpella Theiss. et Syd. n. gen. Annales Mycologici, Berlin 13:631-634.

Vainio, E.A. 1921. Lichenographia Fennica I. Pyrenolichenes usque proximi Pyrenomycetes et Lichenes Imperfecti. Acta Societatis pro Fauna et Flora Fennica, Helsingforsiae 49(2): 1-274.

Walker, J. 1980. Gaenmannomyces, Linocarpon, Ophiobolus and several other genera of scolecospored Ascomycetes and Phialophora conidial states, with a note on hyphopodia.
Mycotaxon. An International Joumal

Designed to Expedite Publication of Research on Taxonomy \& Nomenclature of Fungi \& Lichens, lthaca, New York 1 1:1-129.

Webster, J., and H.J. Hudson. 1957. Graminicolous pyrenomycetes V1. Conidia of Ophiobolus herpotrichus, Leptosphaeria lactuosa, $L$. fuckelii, L. pontiformis, and L. eustomoides. Transactions of the British Mycological Society, London 40:509-522.

Webster, J., and M.T. Lucas. 1959. Observations on British species of Pleospora I. Transactions of the British Mycological Society, London 42:332-342.

Wehmeyer, L.E. 1942. Contributions to a study of the fungus llora of Nova Scotia. Canadian Journal of Botany, Ottawa 20:572-594.

Wehmeyer, L.E. 1946. Studies on some fungi of northwestern Wyoming. III. Pleospora and Leprosphaeria. Lloydia. A quarterly Journal of Biological Science, Manasha 9:203-240.

Wehmeyer, L.E. 1961. A world monograph of the genus Pleospora and its segregates. University of Michigan Press, Ann Arbor. 451 pp.

Wehmeyer, L.E. 1975. The pyrenomycetous fungi. Mycologia Memoir No. 6, New York. $250 \mathrm{pp}$.

Wijk, R. Van der, W.D. Margadant, and P.A. Florschutz. 1959-1960. Index Muscorum. Kemink en Zoon N.V., Utrecht. The Netherlands. Five volumes.

Willis, J.C. 1973. A dictionary of the flowering plants and ferns. Eighth edition. Revised by H.K. Airy Shaw, Cambridge University Press, London. 1,245 pp.

Zahlbruckner. A. 1921-1940. Catalogus Lichenum Universalis. Verlag von Gebrüder. Borntraeger, Leipzig. Ten volumes.

Zeller, S.M. 1927. Contributions to our knowledge of Oregon fungi I1. Mycological notes for 1925. Mycologia, Lancaster, Pennsylvania 19:130-143. 
Manuscripts of high quality dealing with any aspect of natural history will be considered for publication in one of the lllinois Natural History Survey series: Bulletin. Biological Notes.

Circular, and Special Publications. Authors who are not employees of the Survey are required to pay printing costs. Manuscripts should follow the recommendations of the Conncil of Biology Editors Style Mamal except that journal names in the literature cited section are to be spelled in full. The Survey expects to publish only one or two manuscripts by non-Surey authors each year. Send three copies of manuscripts to be considered for publication to Office of the Chief. Illinois Natural History Survey, 607 East Peabody Drive, Champaign, 1linois 61 \$20. Before a manuscript is accepted for publication. two or more outside reviewers must recommend it. 

